\title{
SPECTROSCOPIC PROPERTIES OF POLYCYCLIC AROMATIC COMPOUNDS
}

DISSERTATION

\section{Presented to the Graduate Council of the University of North Texas in Partial \\ Fulfillment of the Requirements}

For the Degree of

DOCTOR OF PHILOSOPHY

By

Sheryl A. Tucker, B.S. Denton, TX

MaY, 1994 


\section{SPECTROSCOPIC PROPERTIES OF POLYCYCLIC AROMATIC COMPOUNDS}

DISSERTATION

\section{Presented to the Graduate Council of the University of North Texas in Partial \\ Fulfillment of the Requirements}

For the Degree of

DOCTOR OF PHILOSOPHY

By

Sheryl A. Tucker, B.S. Denton, TX

MaY, 1994 
Tucker, Sheryl A., Spectroscopic Properties of Polycyclic Aromatic Compounds. Doctor of Philosophy (Chemistry), May, 1994, 377 pp., 58 tables, 92

illustrations, bibliography, 333 titles.

The fluorescence spectrum of many polycyclic aromatic compounds (PACs) depends upon solvent polarity. The emission spectrum of PAC monomers consists of several major vibronic bands labeled I, II, etc., in progressive order. Emission intensity enhancement of select bands is observed in polar solvents. The ratio of emission intensities for bands I and III (or II, IV)

$$
\begin{aligned}
& \mathrm{PAC}^{*}+\mathrm{PAC}_{\mathrm{v}_{0}}+\mathrm{hv_{I }} \\
& \mathrm{PAC}^{*}+\mathrm{PAC}_{\mathrm{v}_{2}}+\mathrm{hv} \mathrm{III}_{\text {III }}
\end{aligned}
$$

serves as a quantitative measure of solvent polarity. Experimental data of nearly two hundred PACs are presented. Subclasses include polycyclic aromatic hydrocarbons (PAHs), polycyclic aromatic nitrogen heterocycles (PANHs), polycyclic aromatic sulfur and oxygen heterocycles, acenaphthylene and acephenanthrylene derivatives, cyclopenta-PAHs, fluoranthenoids, fluorenoids and bi-PAHs. The different PACs are classified as probes or nonprobes, depending upon how the emission intensities vary with solvent polarity. Relationships between observed fluorescence properties and molecular structure (point 
group, $\pi$-electrons, Kekule structure counts, planarity, etc.) are carefully examined to ascertain if one can predict which PACs will behave as probe molecules. In light of these studies, six solvent polarity probes are recommended: pyrene, benzo[e]pyrene, benzo[ghi]perylene, 3,4-dihydrobenzo[ghi]perylene, coronene, and ovalene.

Utilization of discriminating solvents can simply observed emission spectra. Experimental measurements on PACs reveal that, in most cases, nitromethane selectively quenches the fluorescence emission of alternant PAHs (pyrene) as opposed to nonalternant PAHs (fluoranthene). Differences in the fluorescence quenching behavior between alternant and nonalterant PAHs and PANHs are rationalized in terms of electron transfer mechanisms and molecular orbital energies. The selective quenching phenomena of nitromethane are examined in four high performance liquid chromatographic solvents and the effect of substituents is also inspected. Differentiating solvents like 2,2,2-trifluoroethanol can be used to protonate PANHs. Protonation of the nitrogen lone pair by a hydrogen ion results in the loss of emission fine structure accompanied by a sizeable redshift in the emission wavelength(s). Degree of protonation should be reflected by both solvent acidity and PANH basicity. As expected protonation is completely reversible. 


\section{ACKNOWLEDGEMENTS}

I would like to express my sincere gratitude to Professor William E. Acree, Jr., my research advisor, for the time, effort, advice, and direction he has given me over the years both as a friend and as a mentor.

I would like to thank my husband, David Beech, for his love, encouragement, and moral support and my parents, Vernon and Grace Tucker, for their interest and encouragement. I would like to thank Dr. John Fetzer, Dr. Maximilian Zander, Dr. Mary Tanga, Dr. Ronald Harvey, Dr. Christopher Upton, Dr. Jan Cornelisse, and Dr. Johan Lugtenburg for supplying most of the noncommercial compounds used in my dissertation research, and I would also like to thank Dr. John Fetzer for making select experimental measurements.

I would like to acknowledge the following sources of financial support: the National science Foundation and the University of North Texas for doctoral fellowships (19901993) and the American Chemical Society Analytical Division for a full-year graduate fellowship which was sponsored by Glaxo, Inc. of Research Triangle Park, North Carolina (19931994). 
TABLE OF CONTENTS

Page

LIST OF TABLES .

LIST OF ILLUSTRATIONS

Chapter

I. INTRODUCTION

Ultraviolet-Visible and Fluorescence

Spectroscopy $\cdot . \quad \cdot \quad \cdot 10$

Fluorescence Probe Techniques . . . 16

Experimental Artifacts Associated with

Solvent Polarity Probe

Determinations . $\quad \cdot \quad \cdot \quad \cdot 27$

Protonation of Polycyclic Aromatic

Nitrogen Heterocycles . . . 39

Selective Quenching Phenomena. . . 40

Chapter References . $\quad . \quad$. $\quad . \quad 53$

II. MATERIALS AND METHODS $\cdot$. $\quad \cdot \quad \cdot \quad \cdot 63$

Chapter References . . . . . . 130

III. RESULTS AND DISCUSSION OF SOLVENT POLARITY

PROBE STUDIES $• \quad \cdot \quad \cdot \quad \cdot \quad \cdot \quad \cdot 136$

Polycyclic Aromatic Hydrocarbons 136

PAH6 Benzenoid Derivatives . . 156

Polycyclic Aromatic Hydrocarbons

Derivatives Containing a

Five-Membered Ring . • . 183

Polycyclic Aromatic Nitrogen

Heterocycles . $\quad . \quad$. 198

Polycyclic Aromatic Oxygen and
Sulfur Heterocycles .

Bi-Polycyclic Aromatic

Hydrocarbons . . . . 229

Chapter References: $: \quad .240$

IV. CORRELATIONS CONCERNING SOLVENT POLARITY

PROBE CHARACTER.$\quad$. . . . 244

Examination of Molecular Structure

Versus Probe Character

Correlations 
Mathematical Functions versus

Probe Character

Correlations.

Recommendations Concerning

. 273

Solvent Polarity Probes . . 290

Chapter References . . . . 297

V. RESULTS AND DISCUSSION OF SELECTIVE

QUENCHING PHENOMENA. . . . . 301

Alternant Polycyclic Aromatic

Hydrocarbons . . . . . 301

Nonalternant Polycycilic Aromatic

Hydrocarbons . . . . . 311

Bi-Polycyclic Aromatic

Hydrocarbons $. \quad . \quad . \quad . \quad .323$

Alternant and Nonalternant

Polycyclic Aromatic

Heterocycles . . . . . 324

Examination of Select High

Performance Liquid

Chromatography Solvents . . . 333

Effect of Substituents on

Nitromethane's Ability to

Selectively Quench Alternant

versus Nonalternant

Polycyclic Aromatic

Hydrocarbons

The Excitation Method

Chapter References 


\section{LIST OF TABLES}

Table

Page

I. Small cuvette results for the variation of PAH fluorescence emission intensity with time in chlorinated solvents

II. Effect of dissolved oxygen on PAH solvent polarity probe ratio values . . . 38

III. Summary of chemical supplier and/or synthetic references for select PAH6 benzenoids

IV. Summary of chemical supplier and/or synthetic references for select acenaphthylene derivatives

v. Summary of chemical supplier and/or synthetic references for select cyclopenta-PAH derivatives

VI. Summary of chemical supplier and/or synthetic references for select PAH6 benzenoids derivatives

VII. Summary of chemical supplier and/or synthetic references for select fluoranthenoids and fluorenoids

VIII. Summary of chemical supplier and/or synthetic references for select fluoranthenoid derivatives

IX. Summary of chemical supplier and/or synthetic references for select PAOHs and PASHs

X. Summary of chemical supplier and/or synthetic references for select PANHS

XI. Summary of chemical supplier and/or
synthetic references for select bi-PAHs 
XII. Summary of chemical supplier and/or synthetic references for select acephenanthrylene derivatives . . . 99

XIII. Addresses of chemical suppliers . . . 100

XIV. Summary of excitation and emission wavelengths of select PAH6 benzenoids

XV. Summary of excitation and emission wavelengths of select PAH6 benzenoid derivatives . . . . . 110

XVI. Summary of excitation and emission wavelengths of select cyclopentaPAH derivatives

XVII. Summary of excitation and emission wavelengths of select acenaphthylene derivatives . . . 113

XVIII. Summary of excitation and emission wavelengths of select PAOHs and PASHS

XIX. Summary of excitation and emission wavelengths of select PANHs

$x X$. Summary of excitation and emission wavelengths of select fluoranthenoids and fluorenoids . 117

XXI. Summary of excitation and emission wavelengths of select fluoranthenoid derivatives . . . 118

XXII. Summary of excitation and emission wavelengths of select bi-PAHs . . . 119

XIII. Summary of excitation and emission wavelengths of select acephenanthrylene derivatives . . . 120

XXIV. Numerical values of primary and secondary inner-filtering correction factors at various absorbances 
XXV. Ratios of fluorescence emission

intensities of pyrene, 3,4-dihydro-

benzo[ghi]perylene, benzo[ghi]-

perylene, coronene, and ovalene

dissolved in select organic

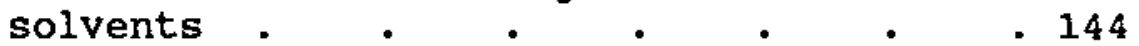

XXVI. Ratios of fluorescence emission

intensities of benzo[e]pyrene

dissolved in select organic

solvents . $\quad . \quad$. $\quad . \quad . \quad 148$

XXVII. Ratios of fluorescence emission

intensities of benzo[a]coronene, naphtho[2,3a]coronene, and

dibenzo[ $a, j]$ coronene dissolved

in select organic solvents . . . . 151

XXVIII. Ratios of fluorescence enission

intensities of dibenzo[fg,ij]-

phenanthro $[2,1,10,9,8,7$ pqrstuv $]$ -

pentaphene dissolved in select

organic solvents $\quad$. $\quad . \quad$. 152

XXIX. Ratios of fluorescence emission

intensities of dibenzo[def, $p]$ -

chrysene and benzo[rst]pentaphene

dissolved in select organic

solvents

XXX. Ratios of fluorescence emission

intensities of naphtho $[8,1,2 a b c]-$

coronene, dinaphtho[8,1,2abc;-

$\left.2^{\prime}, 1^{\prime}, 8^{\prime} \mathrm{k} \cdot \mathrm{m}\right]$ coronene, and

anthra $[2,3 a]$ coronene dissolved

in select organic solvents . . . 154

XXXI. Ratios of fluorescence emission

intensities of benzo[ $v w x]$ hexaphene

and dibenzo[h,rst]pentaphene

dissolved in select organic

solvents

XXXII. Ratios of fluorescence emission

intensities of 1-pyrenebutanoic

acid and $4 \mathrm{H}$-benzo[e]cyclopenta[ 1 ]pyrene dissolved in select organic

solvents

XXXIII. Major fluorescence excitation and emission wavelengths of 1-aminopyrene and 1-pyrenecarboxaldehyde dissolved in select organic solvents 
XXXIV. Ratios of fluorescence emission intensities of 1-methylcoronene and 1,2-dimethylcoronene dissolved

in select organic solvents

XXXV. Ratios of fluorescence emission intensities of benzo[e]aceanthrylene, naphtho $[2,3 b]$ fluoranthene and benzo[k]fluoranthene dissolved in select organic solvents . . . . 185

XXXVI. Ratios of fluorescence emission intensities of $11 \mathrm{H}$-benzo[bc]aceanthrylene, 4H-benzo[b]cyclopenta [mno] chrysene, and $4 \mathrm{H}-\mathrm{cyclo}-$ penta[pqr]picene dissolved in select organic solvents . . . . 186

XXXVII. Ratios of fluorescence emission intensities of $13 \mathrm{H}$-dibenz [bc]aceanthrylene And $4 \mathrm{H}$-cyclopenta[def]chrysene dissolved in select organic solvents

XXXVIII. Ratios of fluorescence emission intensities of 12-azabenzo[a]pyrene, phenanthro $[2,3 h]$ isoquinoline, and phenanthro $[3,2 h]$ isoquinoline dissolved in select organic solvents

XXXIX. Ratios of fluorescence emission intensities of 1-azabenz [a]anthracene, 2-azabenz [a]anthracene, and 9-azabenz [a] anthracene dissolved in select organic solvent

XL. Comparison of the fluorescence excitation and emission wavelengths between the various types of polycyclic aromatic compounds

XLI. Major excitation and emission wavelengths of 9,9'-bianthracene and 9,9'bianthracene-10-carboxaldehyde for various organic solvents.

XLII. Summary of the fluorescence probe character vs. molecular structure for polycyclic aromatic hydrocarbons 
XIIII. Summary of the fluorescence probe character vs. molecular structure for polycyclic aromatic nitrogen heterocycles . . . . . . 250

XIIV. Summary of the fluorescence probe character vs. molecular structure for acenaphthylene derivatives

XLV. Summary of the fluorescence probe character vs. molecular structure for acephenanthrylene derivatives

XLVI. Summary of the fluorescence probe character vs. molecular structure for cyclopenta-PAH derivatives

XLVII. Summary of the fluorescence probe character vs. molecular structure for fluoranthenoids and fluorenoids

XIVIII. Summary of the fluorescence probe character vs. molecular structure for fluoranthenoid derivatives

XLIX. Summary of the fluorescence probe character vs. molecular structure for PAH6 benzenoid derivatives

L. Summary of the fluorescence probe character vs. molecular structure for bi-polycyclic aromatic hydrocarbons

LI. Summary of the fluorescence probe character vs, molecular structure for PAOHs and PASHs. . .

LII. Refractive indices and dielectric constants for various organic solvents

LIII. Summary of emission wavelength and fluorescence quenching data for alternant and nonalternant PAHs . 305

LIV. Effect of nitromethane concentration on the fluorescence emission intensities of select nonalternant fluoranthenoids and fluorenoids 
LV. Summary of emission wavelength and fluorescence quenching data for alternant and nonalternant unprotonated and protonated PANHs . . 326

LVI, Summary of emission wavelength and fluorescence quenching data for alternant polycyclic aromatic hydrocarbon derivatives . . . . 337

LVII. Summary of emission wavelength and fluorescence quenching data for nonalternant fluoranthenoid derivatives

LVIII. Summary of excitation wavelengths and percent reduction in the fluorescence intensity with successive drops of nitromethane for PACs

. 351




\section{LIST OF ILLUSTRATIONS}

Figure

Page

1. Representative structures of the various subclasses of polycyclic aromatic compounds.

2. The tree diagram depicts the relationships among the various subclasses of polycyclic aromatic compounds contained within this dissertation . . 3

3. An example of how polycyclic aromatic hydrocarbons are named . $\quad$. $\quad$ - 8

4. Partial energy-level diagram or Jablonski diagram for a typical photoluminescent molecule . $\quad$ - 11

5. Modified Jablonski diagram showing the

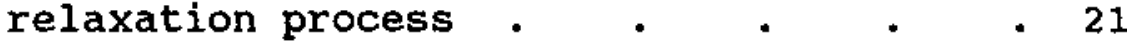

6. Shpol'skii spectra and room temperature fluorescence spectrum of benzo[ghi]-

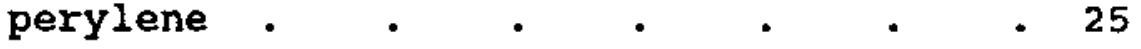

7. Variation of the emission intensity ratios for pyrene and benzo[ghi]perylene with temperature

8. Fluorescence emission spectra of pyrene dissolved in dichloromethane . . . 33

9. Fluorescence enission spectra of benzo[ghi]perylene aissolved in carbon tetrachloride

10. Alternant versus nonalternant . . . . 45

11. Simplified molecular orbital diagram indicating favorable conditions for electron transfer between electron donor alternant PAH and an electron acceptor quenching agent. 
12. Simplified molecular orbital diagram indicating favorable conditions for electron transfer between an electron acceptor nonalternant $\mathrm{PAH}$ and an electron donor quenching agent

13. Molecular structures of PAH6 benzenoids $\mathrm{A}-\mathrm{L} . \quad . \quad . \quad . \quad .64$

14. Molecular structures of PAH6 benzenoids $\mathrm{M}-\mathrm{X}$. $\quad . \quad . \quad . \quad . \quad . \quad 65$

15. Molecular structures of PAH6 benzenoids $\mathrm{Y}-\mathrm{AJ} . \quad . \quad . \quad . \quad . \quad . \quad . \quad 66$

16. Molecular structures of PAH6 benzenoids AK-AV

17. Molecular structures of PAH6 benzenoids AW-BF

18. Molecular structures of PAH6 benzenoids derivatives A-L $\quad \cdot \quad$ • $\quad$ • $\quad$ • $\quad .69$

19. Molecular structures of PAH6 benzenoids derivatives $\mathrm{M}-\mathrm{X}$. $\quad$. . 70

20. Molecular structures of PAH6 benzenoids derivatives $\mathrm{Y}-\mathrm{AC}$. $\quad . \quad$. $\quad$ • 71

21. Molecular structure of cyclopenta-PAH derivatives

22. Molecular structures of acenaphthylene derivatives

23. Molecular structures of acephenanthrylene derivatives

24. Molecular structures of fluoranthenoids and fluorenoids A-J • • $\quad$ • $\quad$ • 75

25. Molecular structures of fluoranthenoids and fluorenoids $\mathrm{K}-\mathrm{Q}$. $\quad . \quad$. $\quad .76$

26. Molecular structures of fluoranthenoid derivatives $\mathrm{A}-\mathrm{H}$

27. Molecular structures of fluoranthenoid derivatives I-P 
29. Molecular structure of PANHs A-L . . . 80

30. Molecular structure of PANHs M-X . . . 81

31. Molecular structures of PANHs Y-AG. . $\quad 82$

32. Molecular structures of bi-PAHs . . 83

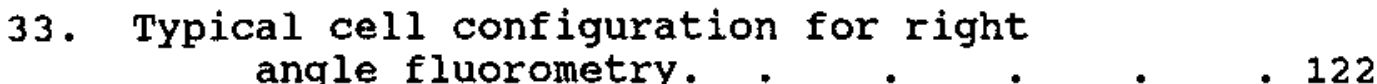

34. Fluorescence emission spectra of pyrene dissolved in $n$-hexadecane, dichloromethane, butyl acetate, and dimethyl sulfoxide . $\quad . \quad$. 137

35. Fluorescence emission spectra of benzo[e]pyrene dissolved in $n$-hexadecane, dichloromethane, butyl acetate, and dimethyl sulfoxide $\quad$. $\quad . \quad$ • $\quad$ • 138

36. Fluorescence emission spectra of benzo[ghi]perylene dissolved in $n$-hexadecane, dichloromethane, butyl acetate, and dimethyl sulfoxide . . 139

37. Fluorescence emission spectra of coronene dissolved in $n$-hexadecane, dichloromethane, butyl acetate, and dimethyl sulfoxide . . . . . 140

38. Fluorescence emission spectra of ovalene dissolved in $n$-hexadecane, dichloromethane, butyl acetate, and dimethyl sulfoxide . . . . . . 141

39. Fluorescence emission spectra of pyranthene dissolved in $n$-hexadecane, dichloromethane, butyl acetate, dimethyl sulfoxide, and binary 1,1,2trichlorotrifluoroethane + perfluoro1,3-dimethylcyclohexane solvent

40. Fluorescence emission spectra of 1-methylpyrene dissolved in $n$-hexadecane, dichloromethane, butyl acetate, and dimethyl sulfoxide . . . . . . 159

41. Fluorescence emission spectra of 3,4dihyrobenzo[ghi] perylene dissolved in $n$-hexadecane, dichloromethane, butyl acetate, and dimethyl sulfoxide 
42. Fluorescence emission spectra of

1-pyrenebutanoic acid dissolved in

n-hexadecane, dichloromethane, butyl

acetate, and dimethyl sulfoxide . . 161

43. Fluorescence emission spectra of

$4 H$-benzo [e]cyclopenta [1] pyrene

acid dissolved in $n$-hexadecane, dichloromethane, butyl acetate, and dimethyl sulfoxide . .

44. Fluorescence emission spectra of 1-aminopyrene dissolved in $n$-hexadecane, dichloromethane, butyl acetate, and dimethyl sulfoxide . . .

45. Fluorescence emission spectra of 1-pyrenecarboxaldehyde dissolved in $n$-hexadecane, dichloromethane, butyl acetate, and dimethyl sulfoxide $. \quad . \quad . \quad . \quad \cdot 168$

46. Fluorescence emission spectra of 1,3-pyrenedicarboxaldehyde dissolved in $n$-hexadecane, dichloromethane, butyl acetate, and dimethyl sulfoxide . . 169

47. Fluorescence emission spectra of 1-methylcoronene dissolved in $n$-hexadecane, dichloromethane, butyl acetate, and dimethyl sulfoxide . . . . . 175

48. Fluorescence emission spectra of 1,2-dimethylcoronene dissolved in $n$-hexadecane, dichloromethane, butyl acetate, and dimethyl sulfoxide . . 176

49. Fluorescence emission spectra of 3methylbenz [ $j]$ aceanthrylene dissolved in $n$-hexadecane, butyl acetate, $N, N-$ dimethyl formamide, and dimethyl

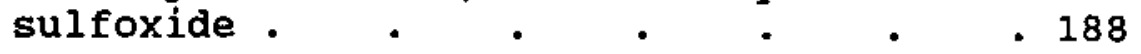

50. Fluorescence emission spectra of naphtho$[2,1 a]$ fluoranthene dissolved in $n$-hexadecane, dichloromethane, butyl acetate, and dimethyl sulfoxide

51. Fluorescence emission spectra of dibenzo$[a, e] f l u o r a n t h e n e$ dissolved in $n$-hexadecane, dichloromethane, butyl acetate, and dimethyl sulfoxide 
52. Fluorescence emission spectra of $11 \mathrm{H}$-benz[bc]aceanthrylene dissolved in $n$-hexadecane, butyl acetate, dichloromethane, and dimethyl sulfoxide . 193

53. Fluorescence emission spectra of $4 \mathrm{H}$-cyclopenta[pqr]picene dissolved in n-hexadecane, butyl acetate, dichloromethane, and dimethyl sulfoxide . 194

54. Fluorescence emission spectra of corannulene dissolved in $n$-hexadecane, butyl acetate, dichloromethane, and dimethyl sulfoxide

55. Fluorescence emission spectra of rubicene dissolved in $n$-hexadecane, butyl acetate, dichloromethane, and dimethyl sulfoxide.

56. Fluorescence emission spectra of 12-azabenzo[a]pyrene dissolved in $n$-hexadecane, butyl acetate, dichloromethane, and dimethyl sulfoxide - 201

57. Fluorescence emission spectra of 1-azapyrene dissolved in $n$-hexadecane, butyl acetate, dichloromethane, and dimethy 1 sulfoxide.

58. Fluorescence emission spectra of 1-azapyrene dissolved dichloromethane determined at approximately 0,10 , and 20 minutes irradiation time

59. Fluorescence emission spectra of the neutral and protonated forms of 12-azabenzo[a]pyrene.

60. Fluorescence emission spectra of 1-azapyrene dissolved in trifluoroethanol, $\mathrm{HClO}_{4}$ trifluoroethanol, n-hexadecane, and dimethyl sulfoxide . . . .

61. Fluorescence emission spectra of benzo[de]isoquino $[1,8 \mathrm{gh}]$ quinoline dissolved in $2,2,2$-trifluoroethanol

62. Fluorescence emission spectra of neutral and protonated forms of diphenanthro$\left[9,10,1\right.$ def $\left.1^{\prime}, 10^{\prime}, 9^{\prime} \mathrm{hij}^{\prime}\right]$ phthalazine 
63. Absorbance spectra of unprotonated and protonated benzo[a]phenazine, and of unprotonated and protonated dibenzo$[a, c]$ phenazine . $\quad$. . . .

64. Absorbance spectra of unprotonated and protonated 11,12 -dimethyldibenzo[a,c]phenazine

65. Fluorescence emission spectra of dinaphtho$\left[1,2 b: 1^{\prime}, 2^{\prime} d\right]$ furan dissolved in $n$-hexadecane, dichloromethane, butyl acetate, and dimethyl sulfoxide . . 220

66. Fluorescence emission spectra of naphtho$\left[1,8 b c: 5,4 b^{\prime} c{ }^{\prime}\right]$ dipyran dissolved in $n$-hexadecane, dichloromethane, butyl acetate, and dimethyl sulfoxide . . 221

67. Fluorescence emission spectra of $1,6-$
dithiapyrene dissolved in methanol

67. Fluorescence emission spectra of 1,6-
dithiapyrene dissolved in methanol and cyclohexane

68. Fluorescence emission spectra of 1,7dithiaperylene dissolved in methanol and cyclohexane

69. Fluorescence emission spectra of 9,9'bianthracene dissolved in $n$-hexadecane, butyl acetate, dichloromethane, and dimethyl sulfoxide.

70. Fluorescence emission spectra of $1,1^{\prime-}$ binaphthacene dissolved in $n$-hexadecane, butyl acetate, dichloromethane, and dimethyl sulfoxide

71. Fluorescence emission spectra of 9,9'bianthracene-10-carboxaldehyde dissolved in $n$-hexadecane, butyl acetate, dichloromethane, and dimethyl sulfoxide

72. Sterically hindered bay-region moieties contained in nonplanar polycyclic aromatic hydrocarbons

73. Coronene derivatives that are classified solvent polarity probes have the length of the center chain of the coronene moiety longer than any other in the compound 
74. Graphical plot of Stoke's shift versus Lippert $\Delta f$ function for 9,9'bianthracene dissolved in 42 nonelectrolyte solvents ..$\quad$. $\quad 277$

75. Graphical plot of stoke's shift versus Biot-Kolwski $F(\epsilon, n)$ parameter for 9,9'-bianthracene dissolved in 42 nonelectrolyte solvents . . . . 278

76. Graphical plot of Stoke's shift versus Lippert $\Delta f$ function for 9,9'bianthracene-10-carboxaldehyde dissolved in 42 nonelectrolyte solvents $\quad . \quad . \quad . \quad . \quad . \quad . \quad 279$

77. Graphical plot of Stoke's shift versus Biot-Kolwski $F(\epsilon, n)$ parameter for 9,9'-bianthracene-10-carboxaldehyde dissolved in 42 nonelectrolyte solvents $. \quad . \quad . \quad . \quad . \quad * \quad 280$

78. Fluorescence emission spectra for $9,9^{\circ}-$ bianthracene dissolved in carbon tetrachloride as a function of irradiation time $\quad . \quad$. $\quad . \quad$. $\quad 288$

79. Correlations between different PAH solvent polarity probes and pyrene . . 291

80. Correlations between different PAH solvent polarity probes and pyrene • 292

81. Major excitation and emission bands of the six recommended solvent polarity probes

82. Fluorescence emission spectra of 4H-benzo$[b] c y c l o p e n t a[j k l]$ triphenylene and $13 H$-dibenzo $[b c, k]$ aceanthrylene dissolved in acetonitrile at various nitromethane concentrations . . 302

83. Effect of nitromethane on the fluorescence emission behavior of benzo[b]fluoranthene dissolved in a binary aqueous-acetonitrile solvent mixture

84. Corrected fluorescence emission spectra of rubicene dissolved in acetonitrile at various nitromethane concentrations 
85. Uncorrected fluorescence emission spectra of aceanthrylene dissolved in acetonitrile at various nitromethane concentrations . . . . .

86. ${ }^{1_{\mathrm{H}}-{ }^{1} \mathrm{H}}$ NMR coupling constants for aceanthrylene, acephenanthrylene, and cyclopenta [cd]pyrene

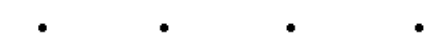

87. Fluorescence emission spectra of dibenzo$[c, f][2,7]$ naphthyridine dissolved in acetonitrile, acetonitrile with one pasteur pipet drop of nitromethane and acetonitrile-perchloric acid protonating solvent

88. Plausible molecular orbital diagram to explain observed quenching of unprotonated and protonated PANH fluorescence emission by nitromethane and 1,2,4-trimethoxybenzene . .

89. Fluorescence excitation spectra of benzo[ $1 \mathrm{mn}][3,8]$ phenanthroline dissolved in

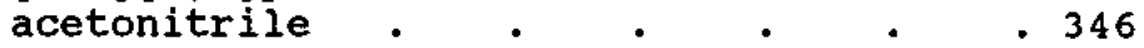

90. Fluorescence excitation spectra of benzo[a]fluoranthene dissolved in acetonitrile at various nitromethane concentrations . $\quad . \quad$. .

91. Fluorescence excitation spectra of naphtho$[2,3 b]$ fluoranthene dissolved in acetonitrile at various nitromethane concentrations . . . .

92. Absorption spectra of nitromethane and 1,2,4-trimethoxybenzene. Superimposed on the absorption spectra are the excitation and emission wavelengths of benzo[ $k]$ fluoranthene, indeno $[1,2,3 i j]-$ isoquinoline, and perylene 
CHAPTER I

INTRODUCTION

The term polycyclic aromatic compound (PAC) is a broad definition for a particular class of compounds that included numerous subclasses, like polycyclic aromatic hydrocarbons (PAHs), PAH6 benzenoids, polycyclic aromatic nitrogen heterocycles (PANHs), polycyclic aromatic sulfur heterocycles (PASHs), polycyclic aromatic oxygen heterocycles (PAOHs), acenaphthylene and acephenanthrylene derivatives, cyclopenta-PAH derivatives, fluoranthenoids, and fluorenoids (see Figure 1 for representative structures). The tree diagram in Figure 2 shows the relationship among the aforementioned subclasses of PACs. The majority of the compounds studied are completely conjugated PAH derivatives. A PAH6 benzenoid is defined as an aromatic molecule containing only six-membered benzo rings. They differ from the cyclopenta-PAHs and acenaphthylene and acephenanthrylene derivatives that contain a five membered cyclopentane or cyclopentene ring, respectively. Initially, the fluoranthenoid and fluorenoid compounds appear to be identical, but PAHs containing an odd number of carbons are called fluorenoids and those containing an even number of carbons are called fluoranthenoids (1). Many of these compounds are ubiquitous environmental pollutants. They can 


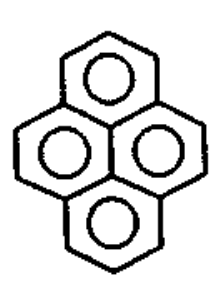

A

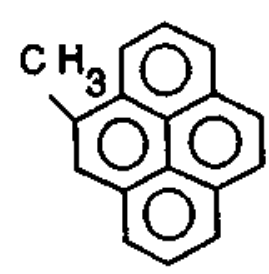

B

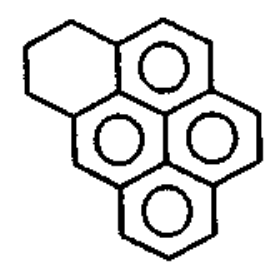

C

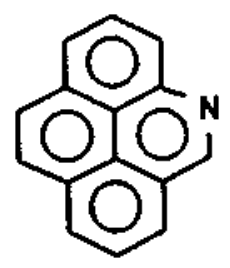

D<smiles>C1=Cc2ccc3c4c(ccc(c24)S1)C=CS3</smiles>

E

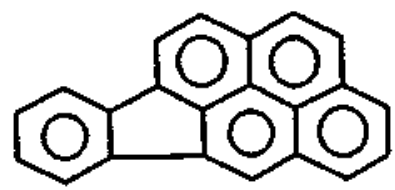

$\mathrm{H}$<smiles>[R]C12C(=C)C=CC=C3C1=CC=CC2=Cc1ccccc13</smiles>

F

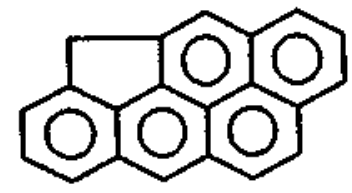

I<smiles>C1=Cc2ccc3c4c(ccc(c24)O1)C=CO3</smiles>

G

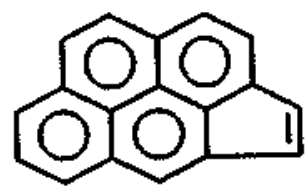

$\mathrm{J}$

FIGURE 1: Representative structures of the various subclasses of polycyclic aromatic compounds: (A) pyrene PAH6 benzenoid, (B) 4-methylpyrene - PAH6 benzenoid derivative, (C) 3,4,5-trihydrobenzo[cd]pyrene - PAH6 benzenoid derivative, (D) 4-azapyrene - PANH, (E) 1,6dithiapyrene - PASH, (F) trans-12b,12c-dihydro-12b, 12cdimethylbenzo[a]pyrene - PAH6 benzenoid derivative ( $R$ groups correspond to methyl groups), (G) naphtho $\left[1,8 b c ; 5,4 b^{\prime} c^{\prime}\right]-$ dipyran - PAOH, (H) indeno $[1,2,3 \mathrm{Cd}]$ pyrene - fluoranthenoid, (I) 4H-benzo[def]cyclopenta [mno] chrysene - cyclopenta-PAH derivative, and (J) cyclopenta[cd]pyrene - acenaphthylene derivative. 


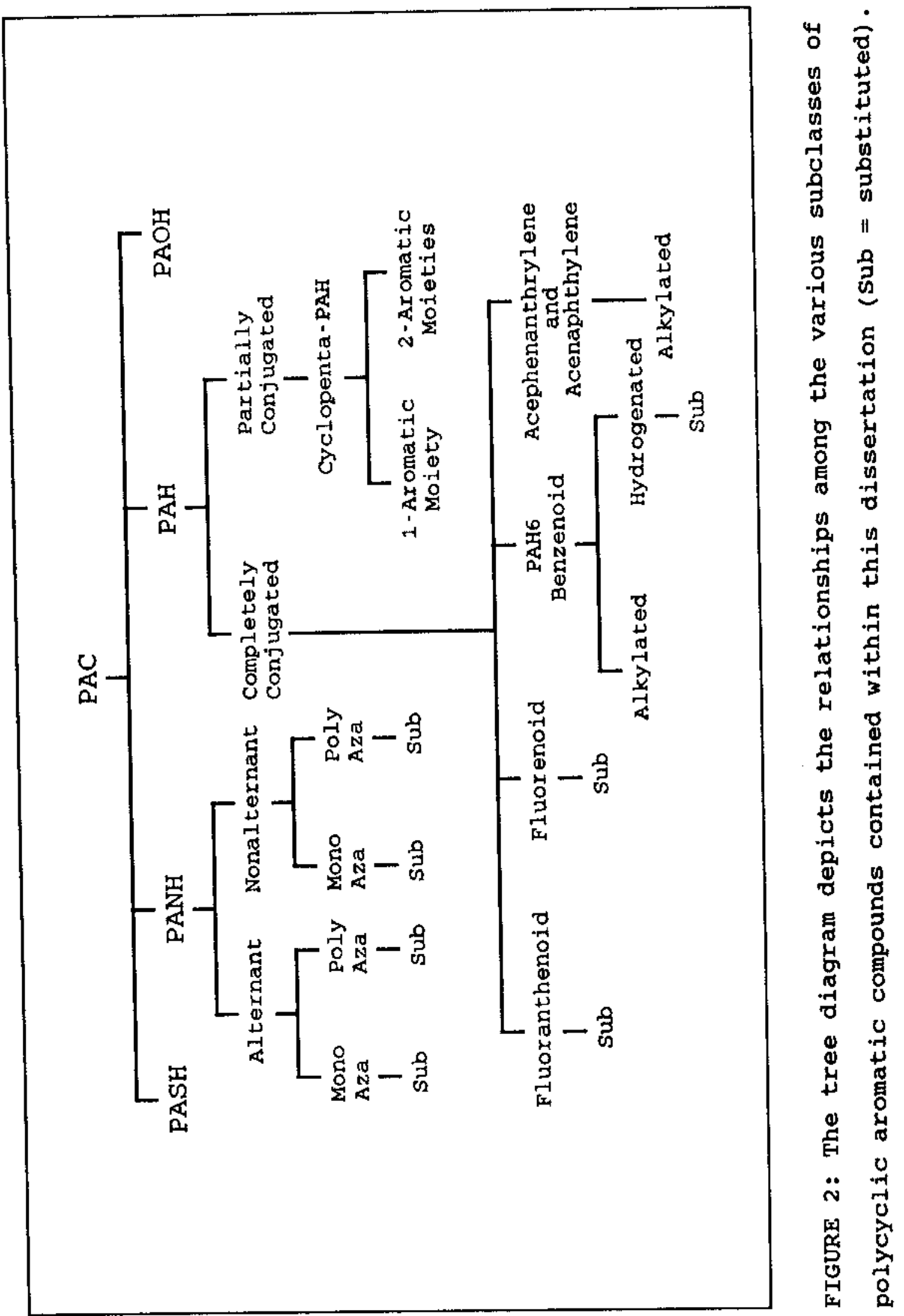


pose significant health concerns due to the fact that many are known or suspected carcinogens and/or mutagens (2-10). Polycyclic aromatic compounds "are formed from the incomplete combustion of fossil fuels, tobacco products, food, and virtually any organic matter" (2). They can be found in coal, coal tars, and coal liquid factions as well as many of the refined coal products $(11-13)$. For example, PAHs are found in automobile exhaust fumes, coal-powered factories and heating facilities' stack emissions, cigarette smoke, diesel fuels, motor oils, lubricating compounds, and even in our streams, lakes, and rivers (2, 11-13). They are priority pollutants due to the significant human exposure (2). These compounds are associated with the causes of such buzz-words as "acid-rain", "fly-ash", and tumors/cancer. For the most part, the toxicity of PACs is well characterized or easily predicted by structure activity analysis. "From the standpoint of structure activity analysis, PAHs represent an intriguing class of compounds for experimental investigation because seemingly small changes in the chemical structure can markedly affect carcinogenic activity" (2). According to Ashwood-Smith, Mitche11, and Kennedy, internal substitution on PAHs can completely alter the carcinogenic activity (4). For example, benzo[a]pyrene is one of the most studied PAH carcinogens, but by internally substituting methyl groups on the $10 b$ and $10 \mathrm{c}$ position the carcinogenic activity was 
completely eliminated (substitution by hydrogen atoms in this case yield an unstable compound) $(4,14)$. One can also add hydrogen atoms to the perimeter $\mathrm{C}=\mathrm{C}$ double bonds. Such partially hydrogenated PAHs are obtained by a catalytic hydrocracking process used in the conversion of crude oil to gasoline (13). The reduction products are not very different in molecular size, although they may differ in shape, from the parent hydrocarbon and from each other. Several reduction products have been prepared in the past and have been shown either to be inactive or much less active than the parent hydrocarbons (7). For example, $7,8,9,10$-tetrahydrobenzo[a]pyrene (7) is inactive; whereas, benzo[a]pyrene is a notorious carcinogen.

one new technique being used to determine the reactivity of carcinogens with target DNA molecules is molecular dosimetry (15). Cancer researchers are using molecular dosimetry to measure the potency of carcinogens and determining if there are threshold levels of exposure. "This technique measures the chemical reactivity of a potential carcinogen, mutagen, or other genotoxin with a host 'target molecule' generally DNA.... Molecular dosimetry may simplify animal studies-it may even shorten them by helping direct their focus on the most likely agents-but ultimately, it's not likely to replace them" (15). Large databases of known experimental mutagenic and carcinogenic properties have been developed for many PACs; 
however, there are still a plethora of environmentally important PACs for which there is little or no information available (2). Utilization of these databases have led to the development of computer programs that will ascertain if a particular PAC is mutagenic and/or carcinogenic. Two particular programs are CASE-SAR (Computer Automated Structure Evaluation-structure Activity Analysis) utilized by Richard and Woo (2) and CASE applied to a Gene-Tox Data Base by Klopman et al. (3). Depending on the "learning set" used to train the database, the predicted accuracy ranges anywhere from 75 to $90+8(2,3)$. The advantage of such programs is that no animal testing must be done and the accuracy is reasonable and being improved daily.

One problem associated with these particular databases as well as the current chemical literature is the nomenclature. Many PACs have common names, International Union of Pure and Applied Chemists' (IUPAC) names as well as names assigned by the Chemical Abstracts Service (published by the American Chemical Society). I, like most others in this particular field, have chosen to use those names assigned by the Chemical Abstract Service to facilitate indexing. Chemical Abstracts has the added advantage in that many new compounds incorporated into several studies still have not been assigned names by IUPAC.

Chemical Abstracts uses a relatively simple method for naming PACs. First, the largest moiety in the compound must 
be determined. There are several bases to choose from, like benzene, naphthalene, anthracene, pyrene, naphthacene, chrysene, coronene, etc. Once the parent base is determined, the substituents must be chosen. Within both moieties the bonds are assigned lower case alphabetic characters, and the atoms around the perimeter of the ring are numbered sequentially with Arabic numbers starting at a assigned point for that moiety (the assigned points to begin numbering and lettering for the moiety can be ascertain by looking up the individual moieties in the Chemical

Abstracts). For example, in Figure 3 naphtho[2,3a]coronene consists of the parent coronene (seven rings) and the substituent naphtho- (two rings). The naphtho- substituent is attached at its number 2 and 3 carbon atoms to the parent coronene's "a" $c-c$ bond or side.

One arawback to using the Chemical Abstracts naming convention is that two or more names can be assigned to a particular $\mathrm{PAH}$ depending upon how the molecules are dissected into smaller, recognizable fragments. For example, the name dibenzo[fg,ij]phenanthro $[2,1,10,9,8,7-$ qrstuvjpentaphene indicated that two benzo-rings are added to pentaphene at positions $f g$ and $i j$, while the phenanthroring is connected via the $2,1,10,9,8$, and 7 phenanthrocarbon atoms and the $q, r, s, t, u$, and $v$ coronene sides. Alternately, perylene could have been selected as the parent compound, in which case the $\mathrm{PAH}$ would have been named 


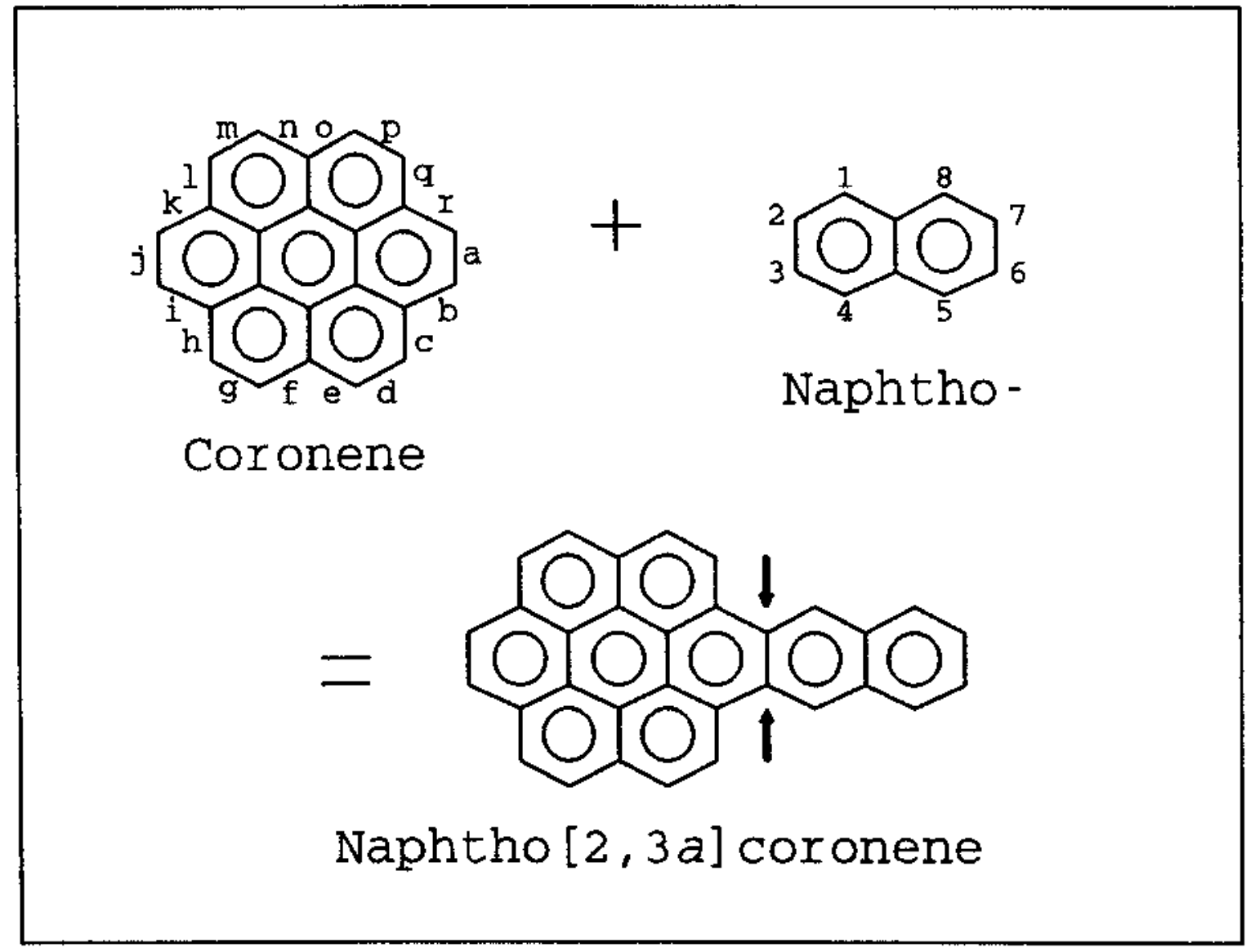

FIGURE 3: An example of how polycyclic aromatic hydrocarbons are named. The $\mathrm{C}-\mathrm{C}$ bonds are designated as lower case letters and the carbon atoms are designated by numbers. The naphtho- substituent is attached at its "2,3" carbons to coronene's "a" $c-c$ bond, hence the name naphtho $[2,3 a]$ coronene. 
dinaphtho $\left[2,3,4 c d ; 2^{\prime}, 3^{\prime}, 4^{\prime} 1 m\right]$ perylene. Both chemical names are correct. While this initially appears to be a disadvantage to the Chemical Abstract naming scheme, it does not present a problem as both names are crossed referenced within the Chemical Abstracts. Another advantage to the Chemical Abstracts naming scheme is that it is simple enough to use for newly-synthesized compounds that previous are unpublished. The easiest way to name most PACs is to use the Chemical Formulas Index of the Chemical Abstracts and simply look them up.

To eliminate the health hazards associated with PACs we must not only identify particular compounds as carcinogens and/or mutagens, but we must be able to remove they from the environment. Currently, they are abundant in the immediate surroundings and are very difficult to identify. Fortunately, it is possible to synthesize many of these compounds or separate them from coal tar mixtures and spectroscopically characterize them. Previously, spectroscopic data available for these compounds have been fragmentary and incomplete. Such characterization of PACs will greatly aid in the identification, quantification and separation of PACs so that their eventual removal from the environment may be complete and less expensive. 


\section{Ultraviolet-Visible and Fluorescence Spectroscopy}

Ultraviolet-visible (UV-Vis) and fluorescence spectroscopy has been successfully employed to characterize most all of the various subclasses of PACs. Figure 4 is a partial energy-level diagram or Jablonski diagram for a typical photoluminescent molecule. It is limited primarily to processes of absorption and fluorescence, the two techniques used in this dissertation research. As shown in Figure 4, excitation of a molecule can result from the absorption of one of two bands of radiation. One at wavelength $\lambda_{1}\left(S_{0} \rightarrow S_{1}\right)$ and one at a shorter wavelength $\lambda_{2}$ $\left(s_{0} \rightarrow s_{2}\right)$. The molecule may end up in any vibrational states within the appropriate excited state (16).

This process of absorption of radiation, particularly UV-Vis radiation, is governed by the Beer-Lambert law. It states that the absorbance is directly proportional to the path length through the solution and the concentration of the absorbing species. The mathematical expression is

$$
A=\epsilon b C
$$

where $A$ is the sample's absorbance of light at a specific frequency $(A=-\log T, T$ is the transmittance), $\epsilon$ is the molar absorptivity, $b$ is the path length the light must travel through the sample in centimeters, and $C$ is the analyte concentration in moles per liter. Equation 1.1 is simplified when the path length is held constant, because A 


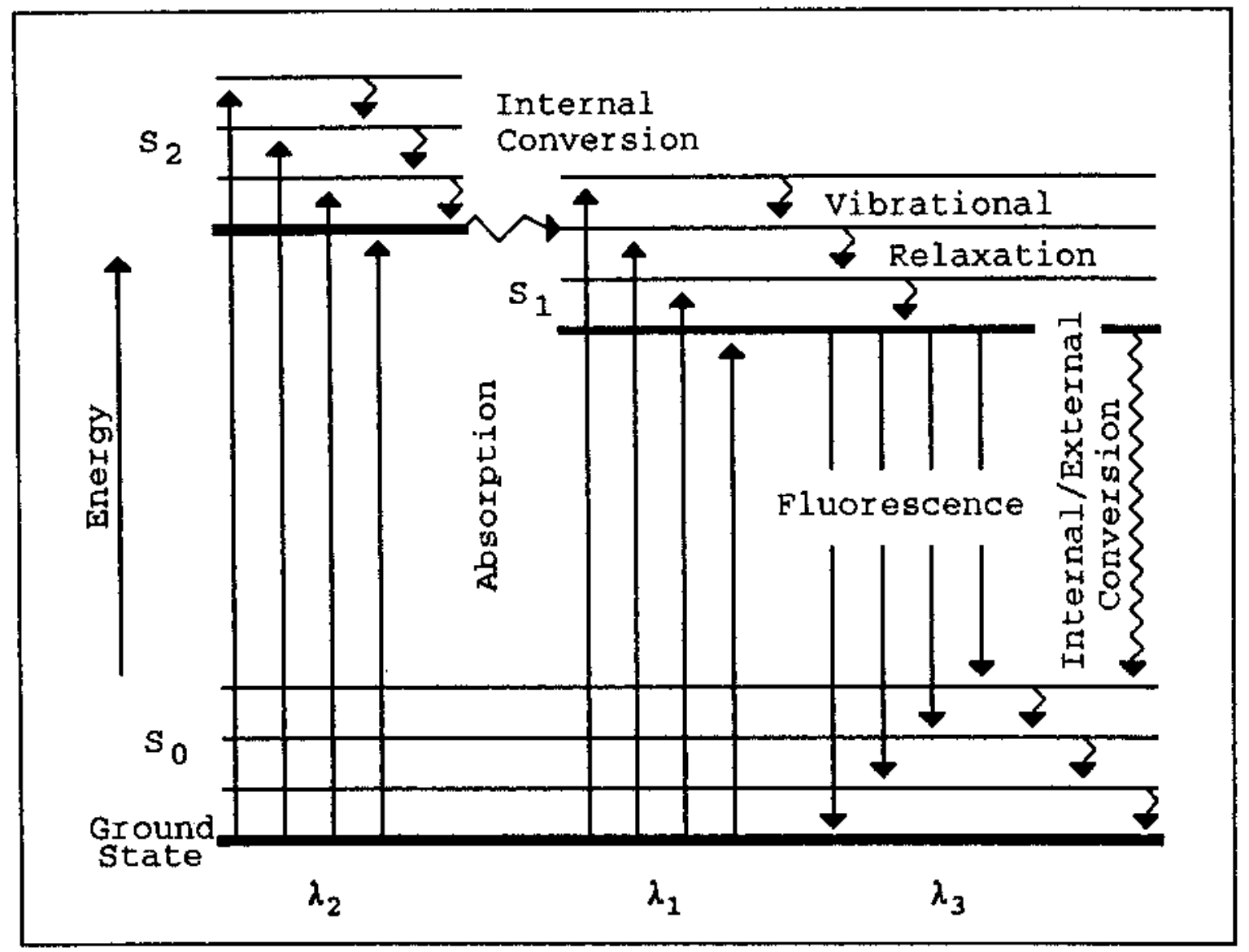

FIGURE 4: Partial energy-level diagram or Jablonski diagram for a typical photoluminescent molecule. The lowest heavy horizontal line represents the ground state energy of the molecule, which is normally a singlet state and is labeled $s_{0}$. The upper heavy lines are energy levels for the ground vibrational states of the two excited electronic states. The two lines represent the first $\left(S_{1}\right)$ and the second $\left(S_{2}\right)$ electronic singlet states. Numerous vibrational energy levels are associated with the electronic states, as suggested by the lighter horizontal lines (reproduced from reference 16 ). 
becomes directly proportional to $c$. The molar absorptivity is defined as the amount of radiation absorbed by one mole of analyte per liter (path length ignored). The molar absorptivity, $\epsilon$, is evaluated through known concentrations of analyte. Molar absorptivity theoretically remains constant with concentration, and in practice one can compensate for minor deviations from linearity by mathematically expressing $\epsilon$ as a function of the measured absorbance.

The Beer-Lambert law is not without limitations. It is only successful in describing the absorption behavior of dilute solutions (concentrations $<0.01$ Molar). There are chemical and instrumental deviations as well. Chemical deviations are caused by association, dissociation or by a reaction of the absorbing species with the solvent. Instrumental deviations are caused when the radiation employed is not truly monochromatic as well as when stray radiation exits the monochromator (16).

Another deviation from the Beer-Lambert law is caused by a change in the concentration of the solution. The molar absorptivity, $\epsilon$, is dependent on the refractive index, $n$, of the solution being analyzed. Concentration changes cause significant changes in the refractive index of the solutions. The correction factor is

$$
(\epsilon n) /\left(n^{2}+2\right)^{2}
$$

Generally the correction factor is minute when the concen- 
trations remain less than 0.01 Molar. Even though there are several sources of possible error, the Beer-Lambert law works very well for the designated concentrations (16). Fluorescence spectroscopy is an extremely versatile, sensitive experimental technique used in identification and quantification of numerous environmentally important PACs. Referring back to Figure 4 , the process of fluorescence can be seen. The absorption process discussed previously is referred to as fluorescence excitation. Prior to fluorescence emission, vibrational relation takes place within the vibrational levels of the various excited states. Internal conversion can also take place leaving the all excited state molecules in the lowest vibrational level of the first excited state $s_{1}$. Now an excited state molecule can return to the ground state, $s_{0}$, by a combination of several mechanistic steps, like intersystem crossing followed by phosphorescence or radiationless relaxation. The favored route to the ground state will be the one that minimizes the lifetime of the excited molecule. If fluorescence is rapid with respect to the radiationless relaxation or intersystem crossing processes, fluorescence emission is observed. From an experimental standpoint, one can monitor the fluorescence excitation or emission radiation.

Fluorescence rarely results from ultraviolet excitation radiation at wavelengths lower than $250 \mathrm{~nm}$. Such radiation 
is sufficiently energetic that it causes deactivation of the excited states by predissociation or dissociation (16). "For example, $200 \mathrm{~nm}$ radiation corresponds to about 140 $\mathrm{kcal} / \mathrm{mole}$; most molecules have at least some bonds that can be ruptured by energies of this magnitude" (16). Therefore, fluorescence emission due to $\sigma^{\star} \rightarrow \sigma$ transition are rarely observed. Most fluorescence emission are confined to the less energetic $\pi^{*} \rightarrow \pi$ and $\pi^{*} \rightarrow \mathrm{n}$ processes. "For the majority of fluorescent compounds, radiation is produced by deactivation of either the $n, \pi^{*}$ or the $\pi, \pi^{*}$ excited state, depending upon which of these is the less energetic" (16).

The quantum yield or quantum efficiency of a fluorescent process is the number of molecules that fluoresce divided by the total number of excited molecules. This ratio approaches unity for highly fluorescent molecules, like fluorescein. Species that do not fluoresce, like cyclohexane, have a quantum efficiency of zero. The quantum efficiency is greater for $\pi^{*} \rightarrow \pi$ transitions than it is for $\pi^{*} \rightarrow \mathrm{n}$ transitions. The greater quantum efficiency can be rationalized in two ways: (1) the molar absorptivity of the $\pi \rightarrow \pi^{*}$ transition is usually 100- to 1000-fold greater, and this quantity represents a measure of the transition probability in either direction; (2) the inherent lifetime associated with the $\pi \rightarrow \pi^{\star}$ transition is shorter $\left(10^{-7}\right.$ to $10^{-9}$ seconds as compared with $10^{-5}$ to $10^{-7}$ seconds for the $\mathrm{n} \rightarrow \pi^{*}$ transition). The shorter lifetime lowers the 
probability that the deactivation processes that compete with fluorescence will occur (16). The fluorescence lifetime is defined as the time required for the population of the excited state to decrease to $1 / \mathrm{e}$ of the original value after the excitation source, such as a xenon arc lamp, is turned off (17).

Published methods assume that the observed emission intensity, F, is directly proportional to the radiant power of the excitation beam that is actually absorbed by the analyte fluorophore situated in the interrogation zone

$$
F=R^{\prime}\left(P_{0}-P\right)
$$

where $P_{0}$ is the power of the beam incident upon the solution and $P$ is its power after transversing the length $b$ of the liquid media. The proportionality constant, $\mathrm{K}^{\prime}$, depends upon the quantum efficiency or quantum yield of the fluorescent process and the response of the photodector at the emission wavelength. For dilute solutions, the absorbance is governed by the Beer-Lambert law, $\mathrm{P} / \mathrm{P}_{0}=10^{-}$ $\epsilon b c$, which enables one to relate the measured fluorescence intensity to the molar concentration of the solute as follows:

$$
F=K^{\prime} P_{0}\left(1-10^{-\epsilon b c}\right)
$$

where $\epsilon$ refers to the solute molar absorptivity at the excitation wavelength. The exponential term in equation 1.4 can be expanded as a Maclaurin series to give 


$$
\begin{aligned}
F=K^{\prime} & P_{0}\left[2.303 \epsilon b c-(2.303 \epsilon b c)^{2} / 2 !\right. \\
+ & \left.(2.303 \epsilon b c)^{3} / 3 !-\ldots \ldots\right]
\end{aligned}
$$

which simples to a linear expression

$$
F=K, P_{0} 2.303 \epsilon b c=\text { Constant *C }
$$

whenever $2.303 \epsilon C<0.05$. Analyte concentrations can be conveniently determined from a working-curve plot of the measured fluorescence intensity versus the known nolar concentration of standard solutions (18). "The fluorescence phenomenon is limited to a relatively small number of systems incorporating structural and environmental features that cause the rate of radiationless relaxation or deactivation processes to be slowed to a point where the emission reaction can compete kinetically" (16). Such compounds are normally planar, rigid, and sterically uncrowded, e.g., PAHs (17).

\section{Fluorescence Probe Techniques}

Fluorescence probe techniques are becoming increasingly popular for examining the surface microenvironment of chromatographic materials (19-21), surfactant micellization/ adsorption (22-28), polymer/surfactant interactions (29-31), cyclodextrin cavities and inclusion complexes (32-38), solgel and inorganic glass formations $(39,40)$, microemulsions $(41,42)$, and other organized solvent media. The method involves the use of a probe molecule (e.g., pyrene) which 
exhibits different characteristics, depending upon the properties of the solubilizing media. The probe molecule selectivity binds to a specific surface site or preferentially partitions into a molecularly organized structure. Appearance of new spectral bands, shifts in the emission wavelengths or changes in the fluorescence emission intensities provide an indication of the environment immediately surrounding the probe.

Pertinent photophysical processes of PACs in fluid solution are summarized below:

$$
\begin{array}{ll}
\mathrm{PAC}+h v_{0} \rightarrow \mathrm{PAC}^{*} & \begin{array}{l}
\text { (absorption-fluorescence } \\
\text { excitation) }
\end{array} \\
\mathrm{PAC}^{*} \rightarrow \mathrm{PAC}+\mathrm{h} v_{1} & \text { (fluorescence emission) } \\
\mathrm{PAC}^{\star}+\mathrm{PAC} \rightarrow(\mathrm{PAC})_{2}^{*} & \text { (excimer formation) } \\
(\mathrm{PAC})_{2}^{*} \rightarrow 2 \mathrm{PAC}+\mathrm{hv_{2 }} & \text { (excimer emission) }
\end{array}
$$

The emission spectrum of the PAC monomer consists of several major vibronic bands labeled I, II etc., in progressive order, starting with the 0-0 vibronic band. Earlier reports have been primarily concerned with measuring the photophysical behavior of dissolved PAH solutes in order to identify probe molecules for future applications (43-52).

Some PACs act as solvent polarity probes. A polar solvent possesses some degree of charge imbalance, like the very polar dimethyl sulfoxide (DMSO). DMSO has a partial positive charge residing on its sulfur atom and a partial negative charge on its oxygen atoms, thus a charge imbalance 
caused by the unequal sharing of bond electrons; whereas, cyclohexane is nonpolar because any charge is balanced throughout the molecule. In the case of a probe molecule, such as pyrene, the ratio of the fluorescence emission bands varies systematically with solvent polarity. The ratio of the I band/III band is 1.95 in the very polar DMSo and only 0.58 in the nonpolar cyclohexane. The absolute dynamic range (ADR) is defined as the difference in the two ratio values e.g.

$$
A D R=\text { Ratio polar }_{\text {- Rationonpolar }}
$$

Pyrene has a dynamic range of 1.37 . Dynamic ranges greater than about 0.60 are considered useful.

The current chemical literature does contain another definition of dynamic range; it is also possible to define a relative dynamic range (RDR):

$$
\text { RDR }=\left(\text { Ratio }_{\text {polar }}-\text { Rationonpolar }_{\text {notio }} /\right. \text { Ranpolar }
$$

As with the ADR, the higher the RDR the better the probe. RDR values as low as 0.25 have been used (53). Nakashima and Tanaka (53) and several other researchers chose to use the RDR, but the true meaning of the relative dynamic range can be misleading. For example, it the ratio of the I/III bands of a compound is 0.20 in the most polar solvent and 0.10 in the least polar solvent, the $A D R=0.10$ indicating the compound would not be a good solvent polarity probe particularly in light of experimental error which can range from $0.02-0.20$ or more. The RDR $=1.0$ erroneously indicates 
the compound would serve as an excellent solvent polarity probe. Because very misleading results can be ascertained from the RDR, only absolute dynamic ranges are employed in this dissertation.

As typical examples of how solvent polarity probe molecules with high absolute dynamic ranges are utilized, street (33) estimated the effective polarity of cyclodextrin cavities on the basis of the measured fluorescence properties of pyrene. Hartner and coworkers (54) detected pyrene absorbed from solution onto an octadecylsilane derivatized fused-silica plate using a total internal reflection method. From the measured ratio intensities for the I and III vibronic bands, the authors concluded that the interfacial environment of $c_{18}$ alkyl layers on optically flat fused silica was more ordered than that of similar layers bound to porous, particulate silica supports. Ramis Ramos et al. (55), Malliaris (56), and more recently Zana and Lang (57) reviewed probe methods used to characterize physicochemical structures of binary and ternary alcohol/ water/surfactant systems, microemulsions, and micelles. Fluorescence measurements in organized solvent media yield information regarding critical micelle concentrations (cmc), aggregation size, and interphase properties.

Solvent-induced fluorescence spectral changes can be rationalized qualitatively in a relatively straightforward manner. To understand these solvent-induced spectral 
changes it is necessary to examine the mechanism of

fluorescence a little closer. The following explanation is

taken from a discussion by Lakowicz (58), Cecil and Rutan

(59), and Cecil (60). Referring to a modified Jablonski

diagram in Figure 5 ,

The dipole moments are represented by the arrows and the electronic configurations of the solvent molecules are represented by the surrounding ovals. Initially, solute and solvent molecules are in the ground state electronic level $\left(S_{0}\right)$ and in the ground state configuration. In the ground state, time averaged configuration, the dipole moments of the solvent molecules in the solvational sphere are aligned with the dipole moments of the solute. Upon absorption of a photon, the molecule is excited to the $s_{1}^{\prime}$ electronic level. When the solute is excited, the dipole moment of the solute changes. This change has been portrayed by a lengthening of the dipole moment vector and a change in the direction of the dipole moment. In the case of PAHs, the ground state dipole moment is usually very small and excitation to the $s_{i}$ level can induce a dipole moment. On the same time scale as absorption of the photon (the transition from $s_{0} \rightarrow s_{1}^{\prime}$ ), there is a redistribution of the electrons in the molecules in the solvation sphere to better align with the solute's new dipole moment. However, the time scale of the absorption of a photon of light is too short to allow the physical reorientation of the molecules in the solvation sphere. The relaxation from the excited $s_{1}$ level to the excited $s_{1}$ level is called solvent relaxation and occurs when the solvent molecules rotationally reorient to a more stable configuration over the lifetime of the excited state. After the emission of a photon resulting in a return to the ground state, $s_{0}^{\prime}$, from the excited state $s_{1}$, the dipole moment of the solute and the electronic configuration of the molecules in the solvation sphere return to the ground state. The solvent molecules, however, have not returned to the ground state spatial orientation. The molecules will subsequently rotate to the ground state orientation, thereby returning the system to the ground energy state. The mechanism of 


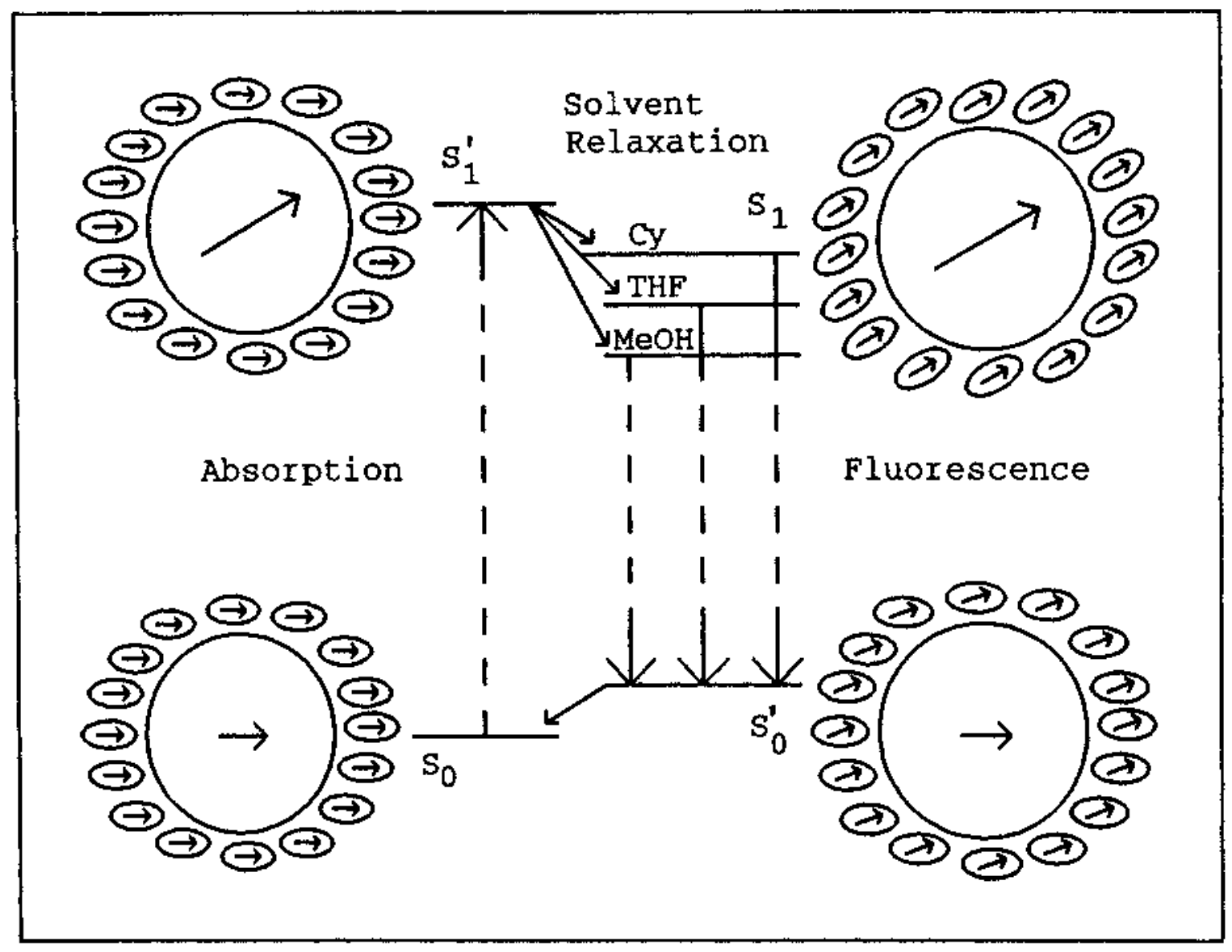

FIGURE 5: Modified Jablonski diagram showing the relaxation process: dashed lines refer to radiative processes and solid lines refer to nonradiative processes. $s_{0}$ is the ground singlet state, $s_{1}$ is the excited singlet state, $C y$ is cyclohexane, THF is tetrahydrofuran, and MeOH is methanol. The large circle and arrow depict the solute and the small ovals and arrows depict the solvent electronic configuration and dipole moment, respectively (reproduced from references $59,60)$. 
fluorescence is the same for every molecule in every solvent, but the separation between the energy levels varies with each solute and solvent (6I). The size of the energy stabilization that occurs during solvent relaxation appears to be the most important factor in determining the size of the fluorescence spectral response shifts. Therefore, when a fluorescence spectrum of a PAH is collected in a polar solvent, the energy stabilization caused by the solvent relaxation is larger that the stabilization found for a nonpolar solvent. This change in the stabilization energy gives rise to fluorescence emission at longer wavelengths, or red shift, of PAHs in polar solvents $(60)$.

Solvent-induced fluorescence changes provide valuable information regarding the microenvironment immediately surrounding the PAH solute probe. To prevent misidentification, the data file should include both polar and nonpolar solvents since electronic interactions between a solvent dipole and an excited PAH solute can lead to spectral distortions, wavelength shifts, and/or intensity ratio variations.

Another class of solvent polarity probe molecules involves redshifts in the fluorescence emission wavelength, rather than intensity changes in select emission bands. A few PACs contain two chromophores of similar energy resulting from transitions from the higher energy $\pi \rightarrow \pi^{*}$ and/or the lower energy $n \rightarrow \pi^{*}$ (62). In light of the previous discussion, the resulting fluorescence emission behavior can be understood. In the nonpolar solvents, like $n$-hexadecane and cyclohexane, the typical fluorescence emission spectrum has two or three resolvable bands at 
higher energy and lower wavelength due to the high energy $\pi$ $+\pi^{*}$ transition. In the more polar solvents, like dimethyl sulfoxide and dimethylformamide, solvational relaxation occurs lowering the energy of the transition and a mixing of $\pi \rightarrow \pi^{*}$ and the lower energy $n \rightarrow \pi^{*}$ transitions occurs resulting in a single unstructured broad emission band that is significantly redshifted to lower energies (62). wavelength shifts of up to $50 \mathrm{~nm}$ going from nonpolar to polar solvents are not uncommon when examining the first and usually most intense emission peak.

Compounds like 1-pyrenecarboxaldehyde and 1-aminopyrene have been shown to exhibit wavelength shifted solvent polarity probe behavior due to the mixing of the $\pi \rightarrow \pi^{*}$ and $n \rightarrow \pi^{*}$ transitions in the more polar solvents. Unfortunately, they have only been examined in alcohol-water binary solvent systems (63) as well as being utilized extensively in the determination of aqueous critical micelle concentrations (27). Part of studies contained in this dissertation will examine the applicability of wavelength shifted solvent polarity probes to more diverse media involving approximately fifty solvents.

9,9-Bianthracene (also called 9,9'-bianthryl) is an atypical fluorescence emission wavelength shifted solute that has been extensively studied to date (64-70), in part because the molecule exhibits dual fluorescence resulting from "twisted intramolecular charge transfer" (TICT) excited 
states, and strong spectral overlap of the TICT band and the anthracene-like "locally excited" (LE) fluorescence band. In nonpolar solvents, such as cyclohexane, 9,9'-bianthracene has strong emission signals near 395 and $414 \mathrm{~nm}$, which are indicative of the LE state. Significant redshifts are observed in the $s_{1} \rightarrow s_{0}$ fluorescence in more polar solvents, like acetonitrile, and are attributed to formation of an excited-state dipole with a full electronic charge separation. Because of the perpendicular ground-state geometry (71-73), the two anthracene moieties in the 9,9'bianthracene are orbitally decoupled (74) and fulfill the "minimum overlap rule" (74-77), a precondition for full electron transfer. A more extensive study of the fluorescence properties of 9,9'-bianthracene and other biPAHs will be examined.

At cryogenic temperatures and in polycrystalline $n$ alkane solvent matrices, emission spectra of dissolved PAHs become concentrated into much narrower wavelength regions, yielding an "atomic-like" quasi-line Shpol'skii spectrum ( $0.1 \mathrm{~nm}$ band widths) superimposed on a weak continuous fluorescence spectrum $(78-80)$. The five band emission spectrum of pyrene observed at room temperature is resolved further into circa 60 and 200 lines at $77 \mathrm{~K}$ and $4 \mathrm{~K}$, respectively, depending upon the $n$-alkane chain length (81). Figure 6 compares the fluorescence emission spectrum of benzo[ghi]perylene at $298 \mathrm{~K}$ (room temperature) and $63 \mathrm{~K}$ in 


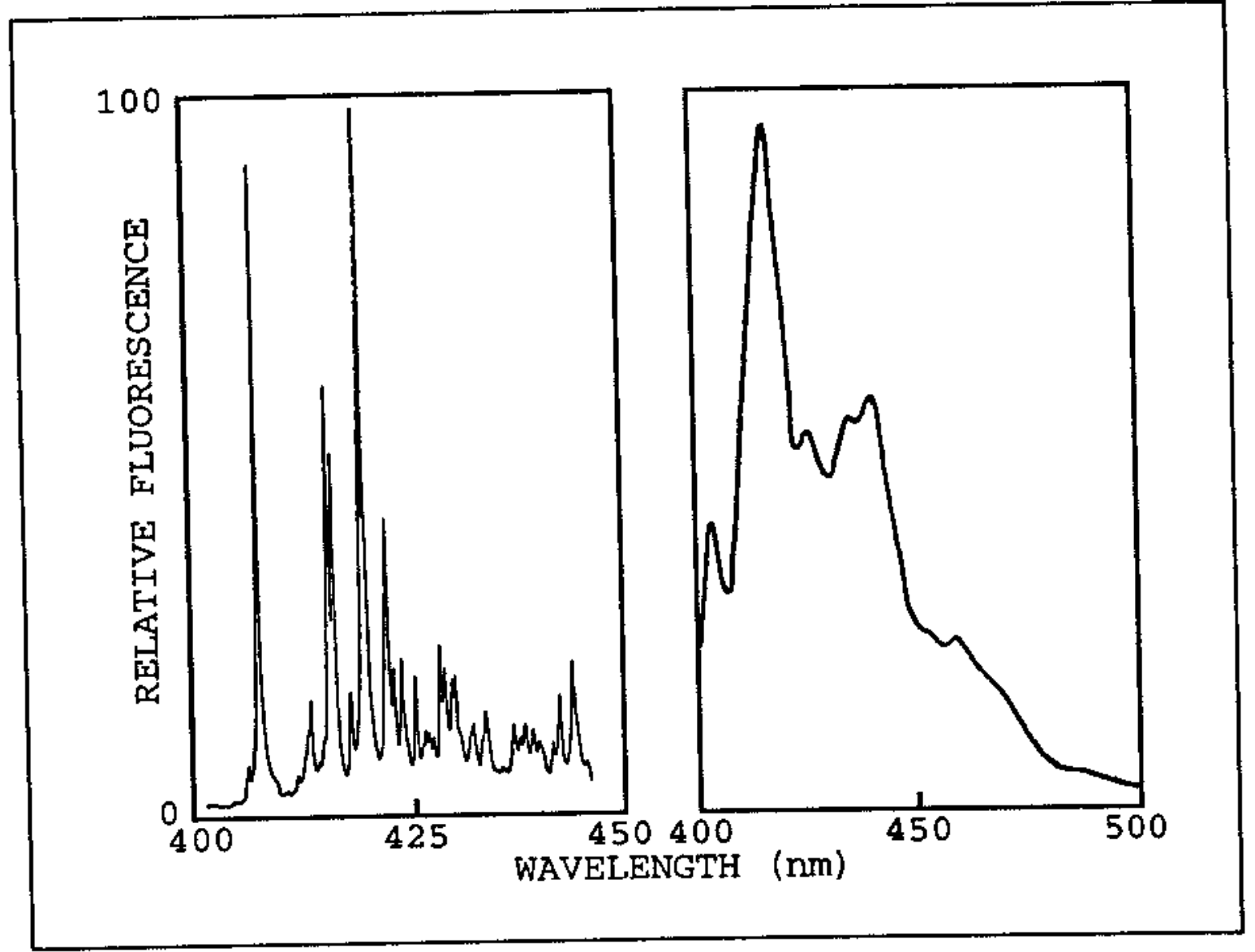

FIGURE 6: Shpol'skii (low temperature) fluorescence spectrum of benzo[ghi]perylene at $63 \mathrm{~K}$ in an $n$-alkane (left hand side: reproduce from reference 82 ). Major peaks occur at $405.5,414.7,415.4,419.2,422.2,428.6,430.5,434.3$, 443.5, and 445.3 nm. Room temperature fluorescence spectrum of benzo[ghi]perylene at $298 \mathrm{k}$ in $n$-heptane (right hand side). Major peaks occur at circa $404,417,428,437,442$, and $461 \mathrm{~nm}$. 
an $n$-alkane. The room temperature spectrum of benzo[ghi]perylene contains only five emission peaks while the low temperature spectrum has approximately eighteen major emission peaks (82). While low temperature fluorescence spectroscopy appears advantageous, the low-temperature instrumentation can be cost prohibitive and problematic.

To understand the Shpol'skii phenomena, it is necessary to examine how a PAH band widths are affected by solvent molecules. First, any spectral transition has an inherent energy uncertainty, related to the excited state lifetime through constraints imposed by the Heisenberg uncertainty principle. This inherent homogenous broadening contribution defines the ultimate limit of spectral resolution obtainable, assuming that all PAH solute molecules interact identically with surrounding solvent molecules. Most solvent media are not absolutely perfect, rigid crystals. Dissolved PAH molecules experience slightly, or perhaps even drastically, different molecular environments. Spectral broadening results from different electronic transitions caused by environmental heterogeneity at the molecular level. Cryogenic temperatures significantly restrict molecular motion, to the point where the $n$-alkane solvent matrix begins to behave more like a perfect crystal. Recent studies by Fetzer and Zander $(83,84)$ indicate that perhydrocoronene matrices also give signal intensification and improved spectral resolution at slightly 
higher temperatures, though not sharp quasi-line emission spectrum characteristic of shpol'skii effects. PAH solvent polarity probes and nonprobes generally exhibit considerable fluorescence emission fine structure at cryogenic temperatures based upon published Shpol'skii spectra (8293). Selective emission intensity enhancement responsible for probe behavior probably results from solvent-induced changes in the transition probabilities associated with one or more of these otherwise "hidden" emission bands. Unfortunately, knowledge of the various vibrational modes is not sufficient to enable a priori prediction of which PAHs are likely to possess probe character.

Experimental Artifacts Associated With Solvent Polarity Probe Determinations

Solvent classifications based on PAH scales require that the calculated fluorescence emission intensity ratio values be free of both chemical and instrumental artifacts. Such artifacts include, but are not limited to, primary (absorption of appreciable excitation radiation) and secondary (absorption of appreciable emission radiation) inner-filtering, excitation and emission spectral slit widths, solvent blank corrections, temperature, dissolved oxygen, halogenated solvents, and data manipulations e.g., smoothing or weighted digital filtering. 
The most basic and easiest to overcome artifact is solvent blank correction. Most solvents have a negligible fluorescence emission spectrum, but in a few instances the corrections are greater than the experimental error (43). For example, the solvent benzene fluoresces when excited at $338 \mathrm{~nm}$, the excitation wavelength for pyrene. The profile of the benzene solvent alone in the emission region of 380 $480 \mathrm{~nm}$ (pyrene's emission region) is a steeply descending peak. If one does not subtract the solvent from the solvent + solute fluorescence emission spectrum, in this case, one will produce erroneously high I band (at $372 \mathrm{~nm}$ )/III band (at $382 \mathrm{~nm}$ ) ratio. The high ratio is due to the fact that the solvent benzene contributes a greater amount to the fluorescence intensity of band I then it does to band III. As a rule of thumb, one must always back subtract the particular solvent of interest from the solvent + solute fluorescence emission spectrum.

Errors associated with primary and secondary innerfiltering are also easy to eliminate. Inner-filtering occurs when solute concentrations are unusually high. While primary inner-filtering does not affect solvent polarity probe ratios because all emission wavelengths are effected by the same amount, this is not the case for secondary inner-filtering. Secondary inner-filtering artifacts can occur through preferential absorption of a particular emission wavelength of light. For example, if secondary 
inner-filtering occurs at the fluorescence emission wavelength of $372 \mathrm{~nm}$ for pyrene, it will effectively reduce the fluorescence intensity of the I band. The I band/III band ratio, in this case, will be too low (43). By working at optically dilute concentrations (absorbance $\mathrm{cm}^{-1}<0.01$ ) at all analytical wavelengths, inner-filtering artifacts become negligible. Normal concentrations for most PACs that give optically dilute solutions are less than $10^{-5}$ or $10^{-6}$ Molar.

For dilute solutions, noisy fluorescence emission spectra are not uncommon. When such phenomena occur, computer manipulation i.e., smoothing, becomes extremely attractive. Unfortunately, erroneous solvent polarity probe ratios can result. For example, street and Acree (43) examined the effect of smoothing on the fluorescence emission spectrum of pyrene dissolved in chloroform. After a single emission scan that was solvent blank corrected, the III band could not be exactly located. It possible to either smooth the spectrum, average a number of emission scans, or generate the emission spectrum with the detector set on high sensitivity utilizing a slow scan rate (one or more options are available on most research grade spectrofluorometers). While it is much faster to smooth the single emission scan, the results can be disastrous. Street and Acree smoothed the emission spectrum of pyrene by a 5, 9, 13, and 19 point function and found that the weaker or less 
intense emission bands are completely removed from the emission spectrum (43). The effect on solvent polarity probe ratios demonstrated that it is by far better to take the time and signal average emission scans or scan at a very slow scan rate with the detector set at high sensitivity. Moreover, spectral averaging does give larger signal-tonoise ratios, $s / \mathrm{N}$, the increase being proportional to the square-root of the number of observations averaged.

The effect of slit widths was also examined by street and Acree (43). When trying to reproduce pyrene solvent polarity ratios published in the literature by Dong and Winnik (94), they found the ratios were consistently low by $10 \%$ on a Perkin Elmer LS-5 spectrofluorometer equipped with a 3600 Data station, but the ratios were consistently high by $10 \%$ on a Perkin Elmer MPF-44B spectrofluorometer. Due other effects such as detector response, it becomes necessary to check the accuracy of solvent polarity probe ratios when comparing to other literature values.

The effect of temperature on the fluorescence emission spectra was examined by Waris, Acree, and street (44). They reported that pyrene emission intensity ratio of the I band/III band does show a solvent-dependent variation with temperature. In nonpolar solvents the temperature effect was small, but in highly polar solvents, such as propylene carbonate, it became much more pronounced. As seen in Figure 7 (44) the pyrene ratio values decrease with 


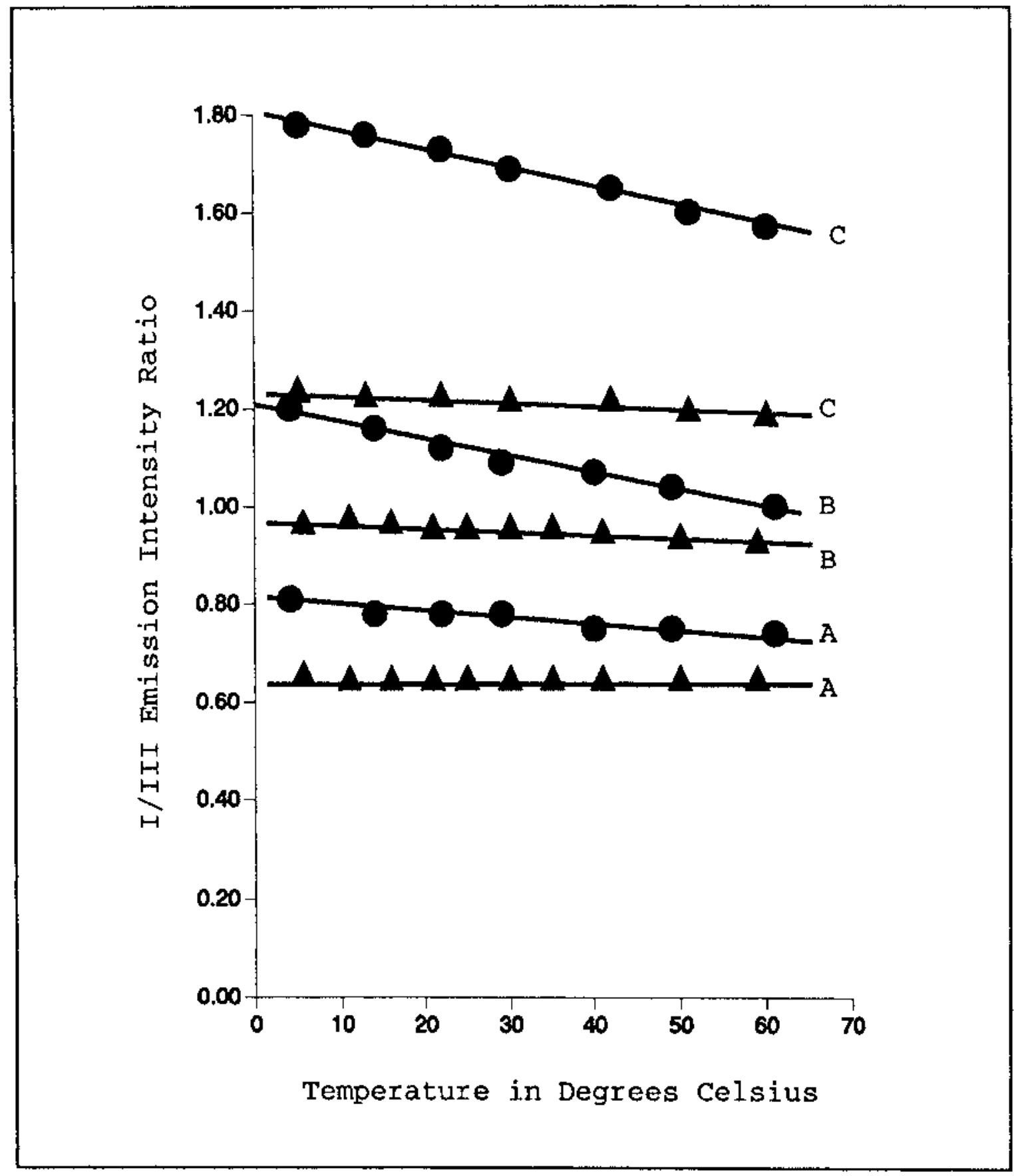

FIGURE 7: Variation of the emission intensity ratios for pyrene (A) and benzo[ghi]perylene (A) dissolved in (A) dibutyl ether, (B) butyl acetate, and (C) aqueous dimethyl sulfoxide $(1+1, V / V)$ with temperature (reproduced from reference 44 ). 
increasing temperature due primarily to a decrease in the emission intensity of band $I$. Interestingly, they found that the emission intensity ratios of band I (at 405 $\mathrm{nm}$ )/band III (at $417 \mathrm{~nm}$ ) for benzo[ghi]perylene were nearly independent of temperature for the five solvents studied. Results of this study indicate it is necessary to obtain all spectra at ambient room temperature when reporting solvent polarity probe ratios or use a thermostated cell holder. Previously, Tucker et al. have examined the effect of chlorinated solvents on the solvent polarity probe values (52). For several PAH probes, the observed fluorescence emission intensities decreased with increasing number of scans averaged. This was particularly true with the solvent carbon tetrachloride. The effect was studied with solvent polarity probes, pyrene, benzo[ghi]perylene, coronene, and ovalene, dissolved in dichloromethane, chloroform, and carbon tetrachloride. The studies revealed that the emission intensity was indeed time dependent. The observed spectra (see representative Figures 8 and 9) fall into one of the following three categories: (1) negligible or very minor decrease in emission intensity with time, (2) significant decrease in emission intensity with time, and (3) significant spectral distortion accompanied in many cases by the appearance of new spectral bands (Figure 9) or considerable enhancement of previously weak emission intensities. Results of the experimental observations are 


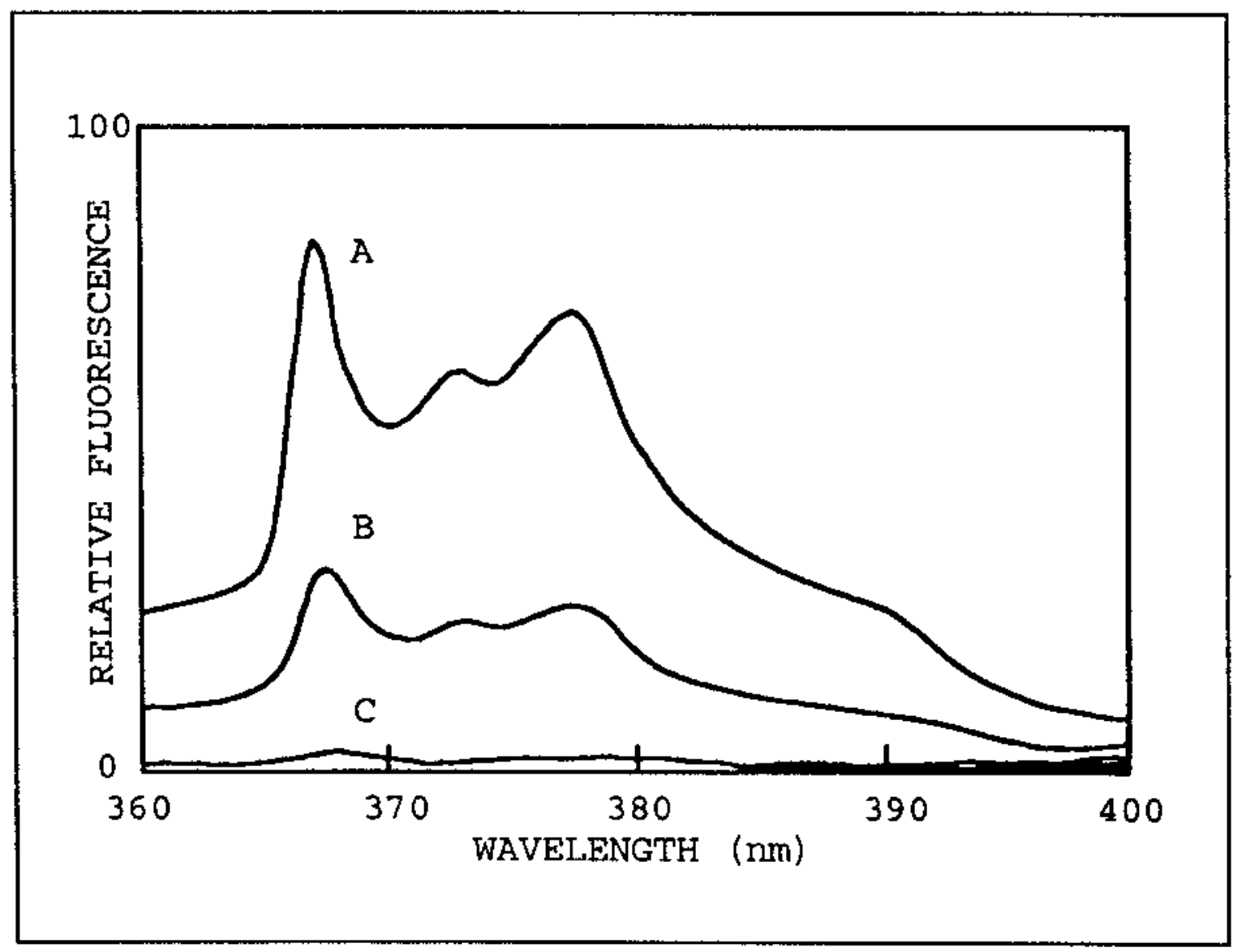

FIGURE 8: Fluorescence emission spectra of pyrene dissolved in dichloromethane determined at 2, 20, and 60 minutes irradiation time in a small rectangular quartz cuvette (52). 


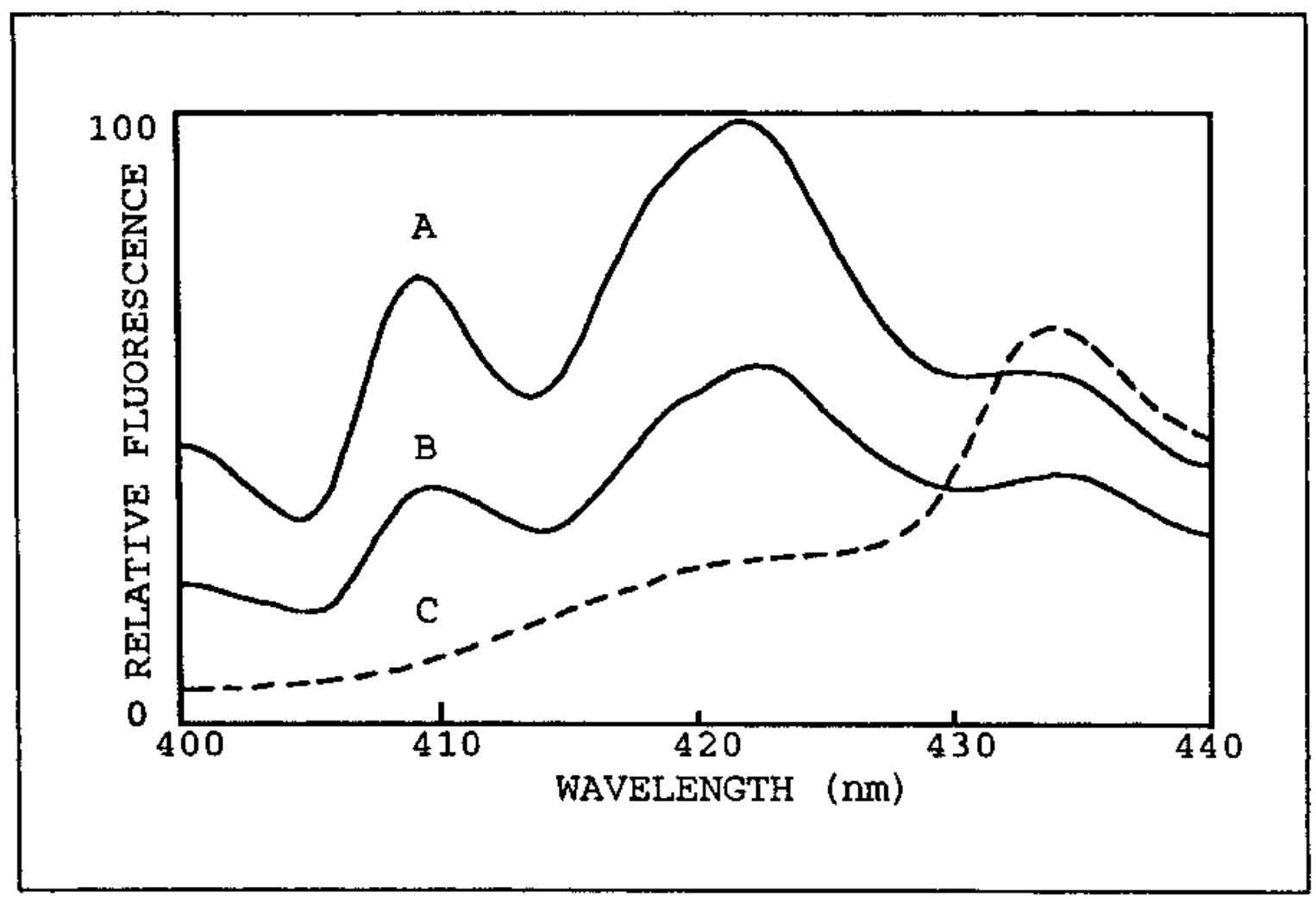

FIGURE 9: Fluorescence emission spectra of benzo[ghi]perylene dissolved in carbon tetrachloride determined at (A) 0 , (B) 10 , and (C) 60 minutes irradiation time in a small rectangular quartz cuvette (52). 
TABLE I. Small cuvette $(2 \times 10 \mathrm{~mm})$ results for the variation of $\mathrm{PAH}$ fluorescence emission intensity with time in chlorinated solvents.

\begin{tabular}{lccc}
\hline PAH Probe & $\mathrm{CCl}_{4}$ & $\mathrm{CHCl}_{3}$ & $\mathrm{CH}_{2} \mathrm{Cl}_{2}$ \\
\hline Pyrene & $\mathrm{SD}^{\mathrm{a}}$ & $\mathrm{DE}$ & $\mathrm{DE}$ \\
Benzo[ghi]perylene & $\mathrm{SD}$ & $\mathrm{NE}$ & $\mathrm{NE}$ \\
Coronene & $\mathrm{SD}$ & $\mathrm{NE}$ & $\mathrm{NE}$ \\
Ovalene & $\mathrm{DE}$ & $\mathrm{DE}$ & $\mathrm{DE}$ \\
\hline
\end{tabular}

a Abbreviations used: SD is severe spectral distortion, DE is decrease of emission intensity with spectral integrity preserved, and NE is negligible effect (52).

summarized in Table I for the four PAH probes examined. Abnormal fluorescence behavior of PAH solute probes in select chloroalkanes can be rationalized in terms of photochemical reactions. Carbon tetrachloride, and to a much lesser extent chloroform and dichloromethane, is known to undergo irreversible photochemical reactions with several PAH solutes (95-99). The photochemical reaction of carbon tetrachloride with anthracene, $A$, is believed to involve the formation of two free radical species, as suggested by the following reaction mechanism (95)

$$
\begin{gathered}
A+h v \rightarrow{ }^{1} A^{*} \\
{ }^{1}{ }^{*}+\operatorname{CCl}_{4}-{ }^{1}\left(\mathrm{ACCl}_{4}\right)
\end{gathered}
$$




$$
\begin{aligned}
& { }^{1}\left(\mathrm{ACCl}_{4}\right)^{\star}+{ }^{3}\left(\mathrm{ACCl}_{4}\right)^{\star} \rightarrow{ }^{3} \mathrm{~A}^{\star}+\mathrm{CCl}_{4} \\
& { }^{1}\left(\mathrm{ACCl}_{4}\right)^{\star} \rightarrow{ }^{\cdot} \mathrm{ACl}+\mathrm{CCl}_{3}+\text { Products }
\end{aligned}
$$

where the superscripts 1 and 3 denote excited singlet and triplet states, respectively. The isolated major product was identified as 9-chloro-10-trichloromethyl-9,10dihydroanthracene. Reaction mechanisms for the larger PAH molecules are excepted to be more complex and to include several major products. In fact, Wiczk and Latowski (100) noted that reaction products resulting from irradiation of pyrene and perylene in carbon tetrachloride had molecular formulas of $\mathrm{C}_{16} \mathrm{H}_{9} \mathrm{Cl}, \mathrm{C}_{17} \mathrm{H}_{9} \mathrm{Cl}_{3}, \mathrm{C}_{33} \mathrm{H}_{19} \mathrm{Cl}_{3}, \mathrm{C}_{17} \mathrm{H}_{10} \mathrm{Cl}_{4}, \mathrm{C}_{18} \mathrm{H}_{8} \mathrm{Cl}_{6}$, $\mathrm{C}_{33} \mathrm{H}_{16} \mathrm{Cl}_{4}$ and $\mathrm{C}_{34} \mathrm{H}_{20} \mathrm{Cl}_{6}$, and of $\mathrm{C}_{20} \mathrm{H}_{11} \mathrm{Cl}, \mathrm{C}_{20} \mathrm{H}_{10} \mathrm{Cl}_{2}, \mathrm{C}_{20} \mathrm{H}_{9} \mathrm{Cl}_{3}$, $\mathrm{C}_{22} \mathrm{H}_{10} \mathrm{Cl}_{4}$ and $\mathrm{C}_{22} \mathrm{H}_{12} \mathrm{Cl}_{4}$, respectively. The authors, however, did not attempt to identify the various products formed. The initial decrease in the fluorescence spectrum of benzo[ghi]perylene dissolved in carbon tetrachloride (Figure 9), later followed by a significant increase in the emission intensity at $433 \mathrm{~nm}$, suggests the formation of a nonfluorescent or weakly fluorescent intermediate which then reacts to form a molecule having an intense $433 \mathrm{~nm}$ fluorescent signal. Further discussion of the reaction mechanisms requires a complete analysis of the photoproducts, which are currently unavailable (52).

Readers are reminded that the fluorescence spectra in Figures 8 and 9 were recorded in a small cuvette (2x10 mm) filled with minimal solution in order to enhance the 
expected spectral changes. For solvent polarity determinations in a $1 \mathrm{~cm}^{2}$ cuvette, the time-dependent changes in emission intensities are not as pronounced. The excitation radiation typically irradiates less than $10 \%$ of the larger cell's total solution volume. Diffusion of unreacted $\mathrm{PAH}$ solute molecules into the fluorescent interrogation zone, along with the outward diffusion of photochemical reaction products, helps maintain a fairly constant probe concentration near the center of the cuvette (52).

Unlike the aforementioned artifacts, the effect of dissolved oxygen of the fluorescence emission spectra seems to be negligible. Table II illustrates the effect of dissolved oxygen on the calculated emission intensity ratios. Numerical $\mathrm{PAH}$ values for oxygen-containing and deoxygenated solutions are identical to within experimental uncertainties. This observation is consistent with the idea that the dissolved oxygen serves only to affect the population of the excited state, and does not alter the relative probabilities associated with transitions from the excited state to various ground state vibronic levels.

The previous discussion on the artifacts associated with solvent polarity probe determination stresses the importance of reporting experimental conditions. Ignoring such artifacts can give erroneous solvent polarity ratio values. Most all of the undesired artifacts can be 
TABLE II. Effect of dissolved oxygen on PAH solvent polarity probe ratio values.

\begin{tabular}{llr}
\hline PAH Probe & Solvent & $\Delta$ PAH $^{\mathrm{a}}$ \\
\hline Pyrene & Dimethyl sulfoxide & 0.00 \\
& Methanol & +0.02 \\
& Water & +0.02 \\
Benzo[ghi]perylene & Dimethyl sulfoxide & -0.02 \\
& Methanol & 0.00 \\
Coronene & Dimethyl sulfoxide & 0.00 \\
& Methanol & -0.01 \\
& Dimethyl sulfoxide & -0.01 \\
& Methanol & +0.02
\end{tabular}

a $\triangle \mathrm{PAH}=\mathrm{PAH}$ value for the deoxygenated solvent minus the PAH value for the aerated solvent. PAH values is calculated as the ratio of the fluorescence intensity of I emission band divided by the intensity of the III band (52).

effectively eliminated, if one pays careful attention to experimental parameters. Literature values are only useful if they contain experimental conditions and can be reasonably reproduced. 
Protonation of Polycyclic Aromatic Nitrogen Heterocycles

Burnell and Hurtubise (101-103) previously reported fluorescence emission intensification whenever neutral solutions of acridine, benzo[f]quinoline, benzo[h]quinoline, and benz $[c]$ acridine were passed through or absorbed onto silica gel chromatographic columns. The fluorescence excitation spectra remained essentially identical, while the emission spectra were redshifted to much longer wavelengths (i.e., $v_{1}>v_{2}$ ) because of protonation of the nitrogen lone electron pair by a hydrogen ion coming from the acidic silanol group. Pertinent photophysical processes of PANHs dissolved in fluid solution differ slightly from those mentioned previously:

Unprotonated

$$
\begin{aligned}
& \text { PANH }+h v_{0}+\text { PANH }^{*} \quad \text { (absorption-fluorescence } \\
& \text { excitation) } \\
& \text { PANH }^{*} \rightarrow \mathrm{PANH}+\mathrm{hv}_{1} \text { (fluorescence emission) } \\
& \mathrm{PANH}^{*}+\mathrm{PANH}+(\mathrm{PANH})_{2}^{*} \text { (excimer formation) } \\
& (\mathrm{PANH})_{2}^{\star} \rightarrow 2 \mathrm{PANH}+\mathrm{h} \mathrm{v}_{2} \quad \text { (excimer emission) } \\
& \text { PANH-H }{ }^{+}+\mathrm{hv}_{0} \rightarrow \mathrm{PANH}-\mathrm{H}^{+*} \quad \text { (absorption-fluorescence } \\
& \text { excitation) } \\
& \mathrm{PANH}-\mathrm{H}^{+*} \rightarrow \mathrm{PANH}-\mathrm{H}^{+}+\mathrm{h} \mathrm{v}_{3} \text { (fluorescence emission) }
\end{aligned}
$$


Note that it is not thought that the protonated PANHs form excimers. Degree of protonation should be reflected by both solvent acidity and PANH basicity.

Selective Quenching Phenomena

Identification and quantification of unknown PAC mixtures require accurate fluorescence emission intensity measurements and availability of a large spectral data file for comparing the unknown's spectrum against PAH standards. Mixtures of environmental/industrial importance rarely contain a single component. The majority of mixtures commonly encountered contain several isomeric pairs or structurally similar PAHs, which emit in approximately the same spectral regions. Kalman filtering and Gaussian or other curve-fitting techniques (104-109), alone or in combination with phase-resolved (110-112) or synchronous scanning fluorescence spectroscopy (113-115), theoretically allow uncoupling of overlapped spectra. Such methods become less reliable, however, as the number of mixture components increases. High performance liquid chromatographic (HPLC) separation prior to fluorometric analysis affords a viable alternative.

Current analytical methods employing high-resolution chromatographic techniques combined with preconcentration via supercritical fluid $(116-120)$, Soxhlet extraction (120- 
122), relatively sophisticated or lengthy isolation, or group separation schemes to obtain fractions from environmental or biological samples suitable for chromatographic analysis. Gas chromatographic separations utilizing liquid crystalline (123-126) and/or polymeric liquid crystalline stationary phases $(127-130)$ or using specially deactivated and stabilized thin-film open-tubular columns (131, 132) enable concentrations of several (though by no means all) of the aromatic constituents to be determined experimentally. Unfortunately, even these high-efficiency columns cannot completely resolve all polycyclic aromatic compound isomers found in environmental samples, and because of volatility constraints, quantification of the higher molecular weight PACs is not generally possible through gas chromatographic measurements.

Mixtures containing larger, nonvolatile polycyclic aromatic hydrocarbons can be conveniently analyzed by HPLC. Conventional chemically-bonded monomeric $C_{18}$ stationary phases (133-137) (i.e., phases prepared with monofunctional silanes) can generally separate the various PAH molecules present according to overall size and number of aromatic rings. Monomeric $c_{18}$ phases possess only limited inherent ability to separate PAH isomers on the basis of molecular shape. Enhanced shape recognition is achieved on either a "charge transfer" (138) or a polymeric $\mathrm{C}_{18}$ stationary phase $(133-137,139,140)$ (i.e., phase prepared with either 
difunctional or trifunctional silanes in the presence of water), the latter of which is believed to be molecularly ordered to some extent. Relative retention values for similar PAH structures of increasing size (i.e., naphthalene, pyrene, benzo[ghi]perylene, benzo[pqr]naphtho $[8,1,2$ bcd $]$ perylene, benzo[ rst $]$ dinaphtho $[2,1,8,7$ defg;$\left.2^{\prime}, 1^{\prime}, 8^{\prime} 7^{\prime} i j k 1\right]$ pentaphene, and tetrabenzo[def, $1 \mathrm{~m}$, grs, yz $]-$ pyranthene) using different polymeric $c_{18}$ stationary and mobile phase combinations, along with the elution strengths of the eleven common HPLC solvents, are reviewed in detail elsewhere (140).

Conventional thin-layer chromatographic, TLC (141-147) and high-performance thin-layer chromatographic, HPTLC (148152) methods have been employed with limited success in the analysis of unknown PAC samples. In many of the published studies, the various polycyclic aromatic compounds were separated on alumina, silica gel, 3-cyanopropyl-silanized silica gel, 3-aminopropylsilanized silica gel, octadecylsilanized silica gel, or partially acetylated cellulose plates. The spots or zones corresponding to expected PACs were then excised, and the concentrations of the individual PAC isomers determined by elution and solution fluorometry. As in the case of HPTLC, resolution in thin-layer chromatography is determined to a large extent by the stationary and mobile phase combination used. Even under optimum experimental conditions, it is very difficult (if 
not impossible) to completely separate all of the different polycyclic aromatic compounds present in the environmental/ biological samples commonly encountered.

Problems associated with coeluting components in HPICC and TLC can be overcome in part by making the detector selective for each given PAC subclass. Of the four most common HPLC detectors on the market, fluorescence affords the most selectivity in that the excitation and emission wavelengths can be varied independently. While several polycyclic aromatic compounds may absorb at the same excitation wavelength, not all will emit at the wavelength(s) monitored by the detector. HPLC separations while very useful are extremely time-consuming whenever large numbers of isomeric compounds are present.

Utilization of selective fluorescence quenching agents further simplify observed emission spectra by eliminating signals from undesired chemical interferences having only slightly different molecular structures. On the basis of limited fluorescence measurements for perylene, dibenzo$[b, k]$ chrysene, dibenzo[h, rst $]$ pentaphene, naphtho[ $1,2 b]-$ fluoranthene, indeno[1,2,3cd]pyrene, and 10,11(perinaphthylene)fluoranthene, Blïmer and Zander (153) recommended that nitromethane and/or nitrobenzene could be added to an aqueous-acetonitrile (20:80 percent by volume) binary mobile phase to selectively suppress fluorescence signals of alternant PAHs. Emission intensities of 
nonalternant PAHs would remain unchanged. Solutes are classified as alternant PAHs because every alternant carbon atom in the aromatic ring system can be "starred." Nonalternant PAHs, on the other hand, would have at least one pair of adjacent starred atoms $(154,155)$ (see Figure 10). For the purposes of this dissertation aromatic carbon atoms and perimeter $-\mathrm{N}=$ atoms are treated identically.

There are three possible types of quenching that can take place: (1) static quenching, (2) dynamic quenching, or (3) a combination of static and dynamic quenching. static quenching occurs in the ground state, [PAH], while dynamic quenching occurs in the excited state, [PAH ${ }^{*}$.

static quenching occurs when a nonfluorescent ground state complex is formed

$$
\mathrm{PAH}+\text { Quencher } \sim \mathrm{PAH}-\mathrm{Q}
$$

The equilibria can be described by an association or binding constant

$$
\mathrm{K}_{\mathrm{PAH}-\mathrm{Q}}=[\mathrm{PAH}-\mathrm{Q}] /[\mathrm{PAH}][\mathrm{Quencher}]
$$

If the quencher and complex do not fluoresce, the fluorescence signal is directly proportional to the free $\mathrm{PAH}$. A mass balance on the total molar concentration of the PAH fluorophore is

$$
[\mathrm{PAH}]=[\mathrm{PAH}]_{\text {free }}+[\mathrm{PAH}-\mathrm{Q}]
$$

The measured fluorescence emission intensity in terms of the association/binding constant is

$$
F_{O}=F\left\{1+K_{\text {PAH }-Q}[Q \text { Quencher }]\right\}
$$



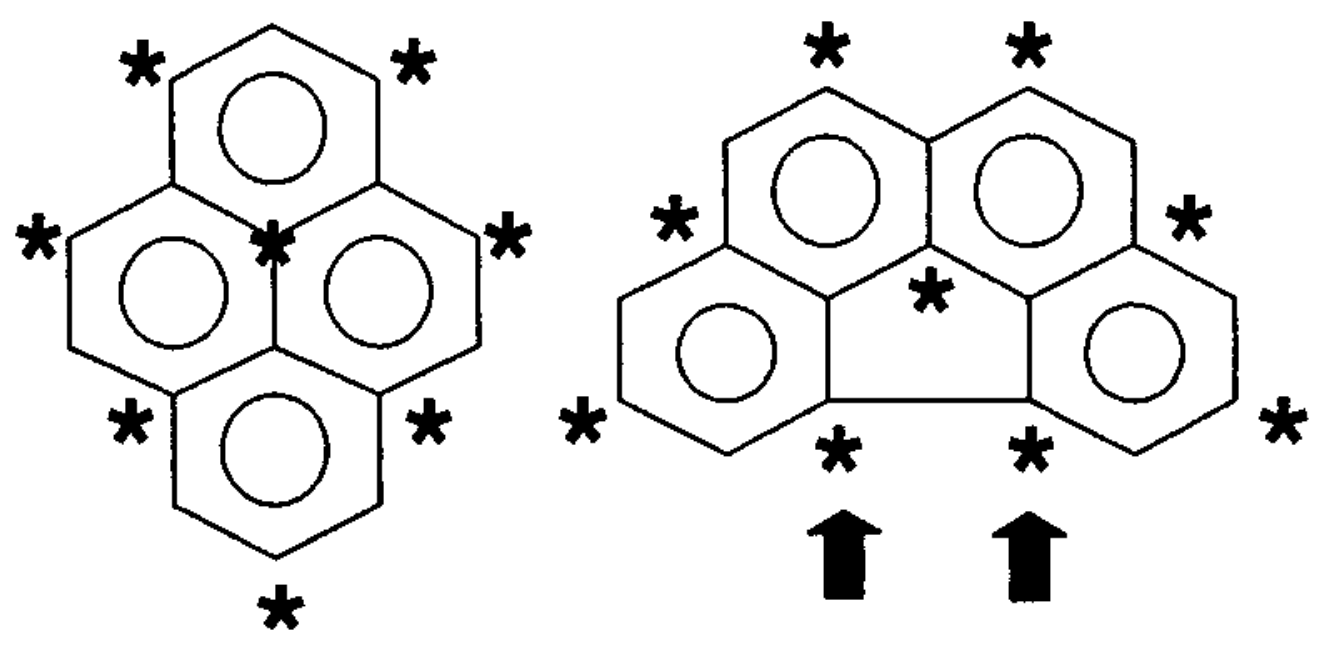

FIGURE 10: Alternant versus nonalternant: pyrene on the left is classified as an alternant PAC because every alternant carbon atom in the aromatic ring system can be "starred". On the other hand, benzo[ghi]fluoranthene, on the right, is classified as a nonalternant PAC because there is at least one pair of adjacent "starred" atoms $(154,155)$. 
where $F_{0}$ is the initial fluorescence intensity equal to [PAH] and $F$ is the observed fluorescence emission intensity at any time (equal to $[\mathrm{PAH}]_{\text {free }}$ (156). Equation (1.11) is one form of the stern-volmer equation for static quenching. Dynamic quenching causes a decrease in fluorescence emission through collisional deactivation involving the excited fluorophore. The nonradiative decay mechanism for returning the fluorophore back to it original ground state is

$$
\mathrm{PAH}^{*}+\text { Quencher } \stackrel{\mathrm{k}_{\mathrm{Q}}}{\rightarrow} \mathrm{PAH}+\text { Quencher }
$$

where $\mathrm{k}_{\mathrm{Q}}$ refers to the second-order rate constant for quenching.

In the absence and presence of the quenching agents, the change in the molar concentration of the excited fluorophore species with time is given by

$$
\begin{aligned}
\mathrm{d}\left[\mathrm{PAH}^{*}\right] / \mathrm{dt}=\mathrm{k}_{\mathrm{abs}}[\mathrm{PAH}]-\mathrm{k}_{\mathrm{fluor}}\left[\mathrm{PAH}^{*}\right] \\
-\mathrm{k}_{\text {int } \mathrm{con}}\left[\mathrm{PAH}^{*}\right]-\mathrm{k}_{\text {int }} \mathrm{cross}\left[\mathrm{PAH}^{*}\right] \\
\mathrm{d}\left[\mathrm{PAH}^{*}\right] / \mathrm{dt}=\mathrm{k}_{\mathrm{abs}}[\mathrm{PAH}]-\mathrm{k}_{\mathrm{f} \text { luor }}\left[\mathrm{PAH}^{*}\right]-\mathrm{k}_{\text {int con }}\left[\mathrm{PAH}^{*}\right] \\
-\mathrm{k}_{\text {int }} \text { cross }\left[\mathrm{PAH}^{*}\right]-\mathrm{k}_{\mathrm{Q}}[\mathrm{Quencher}]\left[\mathrm{PAH}^{*}\right]
\end{aligned}
$$

where $k_{a b s}$, $k_{f l u o r}, k_{\text {int con, }}$ and $k_{\text {int cross }}$ refer to the rate constants for absorbance, fluorescence, internal conversion, and intersystem crossing, respectively. Under constant illumination or steady-state conditions $d\left[\mathrm{PAH}^{*}\right] / d t=0$, and equations 1.13 and 1.14 are solved for the molar concentration of the excited fluorophore $\left[\mathrm{PAH}^{*}\right]=\mathrm{k}_{\mathrm{abs}}[\mathrm{PAH}] /\left(\mathrm{k}_{\text {fluor }}+\mathrm{k}_{\text {int con }}+\mathbf{k}_{\text {int cross }}\right)$ 


$$
\begin{gathered}
{\left[\mathrm{PAH}^{\star}\right]=k_{\mathrm{abs}}[\mathrm{PAH}] /\left(\mathrm{k}_{\text {fluor }}+\mathrm{k}_{\text {int con }}+\mathrm{k}_{\text {int cross }}\right.} \\
\left.\left.+\mathrm{k}_{\mathrm{Q}} \text { [Quencher }\right]\right)
\end{gathered}
$$

which must be directly proportional to the emission signal, F, since the fluorescence process begins with absorption of excitation radiation. Through suitable mathematical manipulation of equations 1.15 and 1.16 a relatively simple expression is derived for relating the measured fluorescence emission to the quencher concentrations

$$
\begin{gathered}
{\left[\mathrm{PAH}^{\star}\right]=\mathrm{k}_{\text {abs }}[\mathrm{PAH}]\left(\mathrm{k}_{\mathrm{fluor}}+\mathrm{k}_{\text {int con }}+\mathrm{k}_{\text {int cross }}\right)^{-1}} \\
*\left\{1+\mathrm{k}_{\mathrm{Q}}[\mathrm{Quencher}] /\left(\mathrm{k}_{\mathrm{f} \text { luor }}+\mathrm{k}_{\text {int con }}+\mathrm{k}_{\text {int cross }}\right)\right\} \\
\mathrm{F}_{\mathrm{O}} / \mathrm{F}-1=\mathrm{k}_{\mathrm{Q}}[\text { Quencher }] \\
*\left(\mathrm{k}_{\mathrm{fluor}}+\mathrm{k}_{\text {int con }}+\mathrm{k}_{\text {int cross }}\right)^{-1} \\
\mathrm{~F}_{\mathrm{O}} / \mathrm{F}-1=\mathrm{k}_{\mathrm{Q}} \text { [Quencher] }
\end{gathered}
$$

where $F_{0}$ refers to the measured fluorescence intensity in the absence of quenching agents. Equation 1.19 is one form of the stern-volmer equation for dynamic quenching.

The numerical value for the $k_{0}$ coefficient is

determined by preparing one standard solution having a known quencher concentration, in much the same fashion that one experimentally determines the molar absorptivity coefficient in the Beer-Lambert law $(A=\epsilon b C)$, except one is monitoring fluorescence emission as opposed to absorbance of the solution. Inherent in the above treatment is the assumption that the stoichiometric concentration of the fluorophore is constant for all of the solutions, and that the quenching 
process results from collisions between the excited fluorophore and quenching reagents (18).

The third possible quenching mechanism is a combination of the two previously mentioned - static and dynamic quenching. There are two reactions taking place: the formation of a ground state complex

$$
\mathrm{PAH}+\text { Quencher } \rightarrow \mathrm{PAH}-\mathrm{Q}
$$

and the collisional deactivation of the vibrational relaxed excited state fluorophore

$$
\mathrm{PAH}^{*}+\text { Quencher } \stackrel{k_{\mathrm{Q}}}{\rightarrow} \mathrm{PAH}+\text { Quencher }
$$

Once again the equilibrium of the complex formed by static quenching can be described by equation 1.9, and the steadystate conditions for dynamic quenching can be described by equation 1.16. Keeping in mind the fluorescence emission intensity is directly proportional to the excited state $\left[\mathrm{PAH}^{*}\right]$, substitution of equation 1.9 into equation 1.16 arrives at the general result (157)

$$
\begin{aligned}
& F=k_{\text {fluor }} k_{\text {abs }}[\mathrm{PAH}]_{\text {total }}\left\{\left(1+[\text { [Quencher }] k_{Q}\right)\right. \\
& \left.\left.*\left(k_{Q} \text { [Quencher }\right]+k_{\text {fluor }}+k_{\text {int con }}+k_{\text {int cross }}\right)\right\}^{-1}
\end{aligned}
$$

which as expected, is a more complex model.

Quenching behavioral differences between alternant and nonalternant PAHs can be rationalized in terms of processes originating from the vibrationally relaxed first electronic excited singlet state, ${ }^{1} \mathrm{PAH}^{*}$, a dynamic quenching mechanism. Deactivation from the ${ }^{1} \mathrm{PAH}^{*}$ state is governed by the competition between radiative and nonradiative processes. 
Rate constants of fluorescence decay, $\mathrm{k}_{\text {fluores, }}$ for PAH fluorophores are generally insensitive to molecular environment. Efficiencies of nonradiative processes, on the other hand, depend to a large extent upon external perturbations resulting from interactions involving PAH solutes with solvent/quenching molecules. Three possible dynamic quenching mechanisms include reduction of fluorescence emission intensities through: (1) intersystem crossing processes involving external "heavy atom" quenchers :

$$
{ }^{1} \mathrm{PAH}^{*}+{ }^{1} \text { Quencher } \rightarrow{ }^{3} \mathrm{PAH}^{\star}+{ }^{1} \text { Quencher }
$$

(2) intermolecular transfer (or partial transfer; $\delta+/ \delta-$ ) of a single electron to form free ions (or charge transfer complex) :

$$
\begin{gathered}
{ }^{1} \mathrm{PAH}^{*}+{ }^{1} \text { Quencher } \rightarrow\left(\mathrm{PAH}^{ \pm}--\right. \text {Quencher } \\
\end{gathered}
$$

and (3) intermolecular electronic energy transfer:

$$
{ }^{1} \mathrm{PAH}^{*}+{ }^{1} \text { Quencher }-{ }^{1} \mathrm{PAH}+{ }^{1} \text { Quencher }{ }^{*}
$$

with the quenching agent promoted from a ground singlet state to an excited singlet state, requiring Energy ( $\left.{ }^{1} \mathrm{PAH}^{*}\right)$ $>$ Energy ( ${ }^{1}$ Quencher $\left.{ }^{\star}\right)$.

of the aforementioned mechanisms, only the second could discriminate between alternant and nonalternant PAHs. Breymann et al. (158) attributed nitromethane's selectivity to an electron/charge transfer reaction. As argued by the authors, reduction potentials of nonalternant PAHs are 
generally $0.4 \mathrm{eV}$ (electron volts) more positive than those of alternant PAHs. Quantum mechanical computations show the highest occupied molecular orbital (HOMO) and lowest unoccupied molecular orbital (LUMO) energies of nonalternant PAHs to be lowered against those of alternant PAHs of equal HOMO-LUMO separation. For the electron transfer reaction

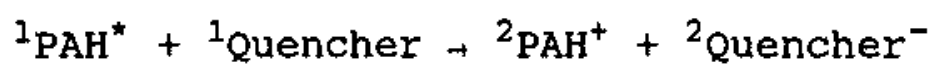

the change in free energy is (158)

$\Delta \mathrm{G} \approx \operatorname{Energy}\left({ }^{1} \mathrm{PAH}^{*} /{ }^{2} \mathrm{PAH}^{+}\right)-$Energy $\left({ }^{2}\right.$ Quencher ${ }^{-} /{ }^{1}$ Quencher $)$

$$
+ \text { other } \operatorname{term}(s)
$$

$\Delta G \approx \operatorname{Energy}\left({ }^{1} \mathrm{PAH} /{ }^{2} \mathrm{PAH}^{+}\right)-\operatorname{Energy}\left({ }^{1} \mathrm{PAH} /{ }^{1} \mathrm{PAH}^{\star}\right)$

- Energy ( ${ }^{2}$ Quencher $-1{ }^{1}$ Quencher $)+$ other term(s) (1.22) expected to be more negative in the case of alternant PAHs (see Figure 11). A more favorable electron transfer process results in larger quenching rate constants which if comparable to (or larger than) $k_{\text {fluores }}$ would cause a significant reduction in $\mathrm{PAH}$ emission intensity. Slow electron transfer reactions (i.e., $k_{\text {quench }}<k_{\text {fluores }}$ ) are not expected to affect fluorescence intensities as the photon is emitted long before electron transfer from the PAH donor to the quencher acceptor can occur. A similar argument could be made for electron donor quenching agents. Here, the quenching agent is more likely to affect fluorescence emission of the nonalternant PAHs (see Figure 12). 


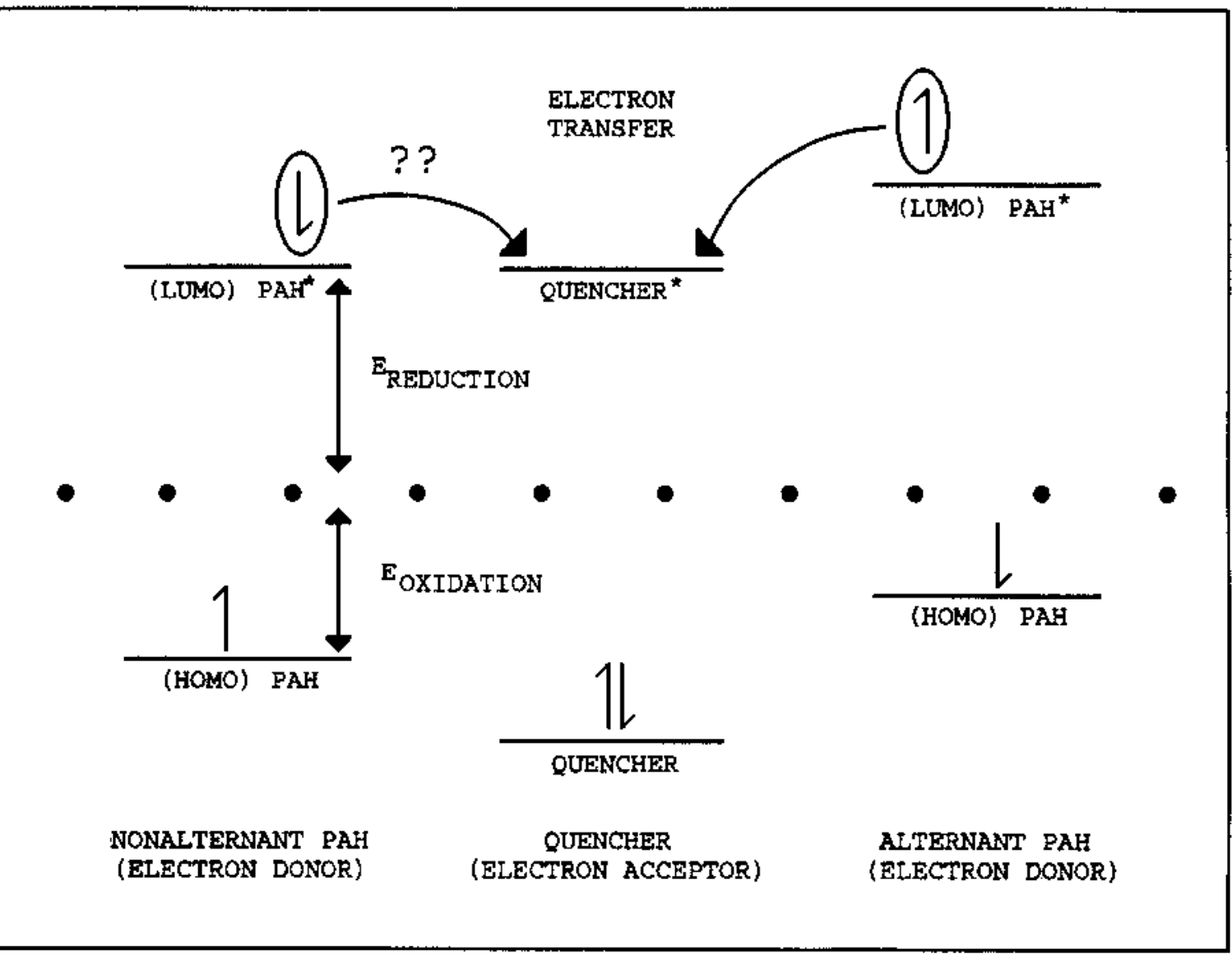

FIGURE 11: Simplified molecular orbital diagram indicating favorable conditions for electron transfer between an electron donor alternant PAH and an electron acceptor quenching agent, such as nitromethane. The quencher's LUMO and nonalternant PAH's LUMO were placed at energies so as to discourage electron transfer. The dotted line represents the potential of a reference electrode. More detailed molecular orbital diagrams are given elsewhere (158). 


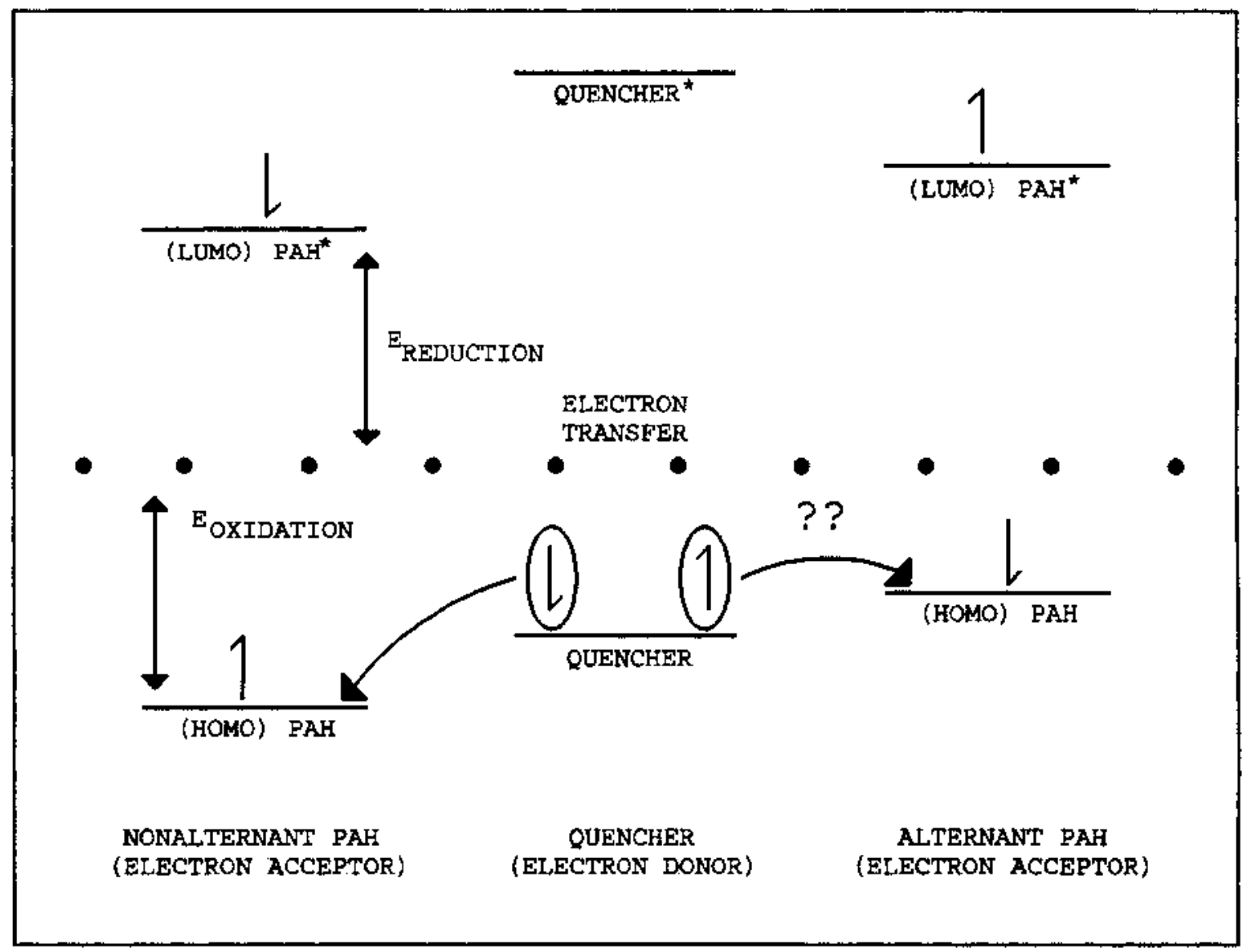

FIGURE 12: Simplified molecular orbital diagram indicating favorable conditions for electron transfer between an electron acceptor nonalternant $\mathrm{PAH}$ and an electron donor quenching agent, such as 1,2,4-trimethoxybenzene. The quencher's HOMO and alternant PAH's HOMO were placed at energies so as to discourage electron transfer. The dotted line represents the potential of a reference electrode. More detailed molecular orbital diagrams are given elsewhere (158). 
CHAPTER REFERENCES

1. Dias, J. R. J. Chem. Inf. Comput. Sci. 1992, 32, 2-11.

2. Richard, A. M.; Woo, Y. Mutat. Res. 1990, 242, 285-303.

3. Klopman, G.; Frierson, M. R.; Rosenkranz, H. S. Mutat. Res. 1990, 228, 1-50.

4. Ashwood-Smith, M. J.; Mitchell, R. H.; Kennedy, A. Mutat. Res. 1978, 57, 123-125.

5. Lijinsky, W.; Garcia, H.; Terracini, B.; Saffiotti, U. $J$. Nat. Cancer Inst. 1965, 34, 1-6.

6. Lijinsky, W.; Garcia, H.; Saffiotti, U. J. Nat. Cancer Inst. 1970, 44, 641-649.

7. Lijinsky, W.; Saffiotti, U. Ann. It. Derm. Clin. Sper. $1965,19,34-44$.

8. Andrews, A. W.; Thibault, L. H.; Linjinsky, W. Mutat. Res. 1978, 51, 311-318.

9. Merch-Sundermann, V.; Mochayedi, S.; Kevekordes, S. Mutat. Res. 1992, 278, 1-9.

10. Nagoa, M.; Sugimura, T.; Yang, S. K.; Gelboin, H. V. Mutat. Res. 1978, 58, 361-365.

1I. Grimmer, G.; Jacobs, J.; Naujack, K.-W. Fresenius $Z$. Anal. Chem. 1981, 306, 347-355.

12. Mason, G.; Gustafsson, J.-A.; Westerholm, R. N.; Li, H. Environ. Sci. Technol. 1992, 26, 1635-1638.

13. Sullivan, R. F.; Boduszynski, M. M.; Fetzer, J. C. Energy \& Fuels 1989, 3, 603-612.

14. Mitchell, R. H. Adv. Theor. Interesting Mol. 1989, 1, 135-199.

15. Nobel, D. Anal. Chem. 1993, 65, 353A-355A.

16. Skoog, D. A; West, D. M. Principles of Instrumental Ananysis, 2nd ed.; Saunders College: Philadelphia, 1980; Chapters 6 and 9 . 
17. Willard, H. H.; Merritt, L. L., Jr.; Dean, J. A.; Settle, F. A., Jr. Instrumental Methods of Analysis, 7th ed.; Wadsworth: Belmont, 1988; Chapter 8 .

18. Tucker, S. A.; Acree, W. E., Jr. J. Chem. Educ, in press.

19. Stahlberg, J.; Almergen, M. Anal. Chem. 1985, 57, 817821 .

20. Carr, J. W.; Harris, J. M. Anal. Chem. 1986, 58, 626631.

21. Carr, J. W.; Harris, J. M. Anal. Chem. 1987, 59, 25462550 .

22. Kalyanasundaram, K.; Thomas, J. K. J. Am. Chem. Soc. 1977, 99, 2039-2044.

23. Kalyanasundaram, K.; Thomas, J. K. J. Phys. Chem. 1977, $81,2176-2180$.

24. Turro, N. J.; Okubo, T. J. Phys. Chem. 1982, 86, 159161 .

25. Turro, N. J.; Okubo, T. J. Am. Chem. Soc. 1981, 103, $7224-7228$.

26. Levitz, P.; van Damme, H.; Keravis, D. J. Phys. Chem. $1984,88,2228-2235$.

27. Ananthapadmanabhan, K. P.; Goddard, E. D. ; Turro, N. J.; Kuo, P.-L. Langmuir 1985, 1, 352-355.

28. Turro, N. J.; Kuo, P.-L. J. Phys. Chem. 1986, 90, 837841.

29. Turro, N. J.; Pierola, I. F. Macromolecules 1983, 16, 906-910.

30. Turro, N. J.; Baretz, B. H.; Kuo.; P.-L. Macromolecules $1984,17,1321-1324$.

31. Ananthapadmanabhan, K. P.; Leung, P.S.; Goddard, E. D. Colloid Surf. 1985, 13, 63-72.

32. Street, K. W., Jr.; Acree, W. E., Jr. Appl. Spectrosc. $1988,42,1313-1318$.

33. Street, K. W., Jr. J. Liq. Chromatogr. 1987, 10, 655662 , 
34. Nelson, G.; Patonay, G.; Warner, I. M. Anal. Chem. $1988,60,274-279$.

35. Nelson, G.; Patonay, G.; Warner, I. M. J. Incl. Phenom. $1988,6,277-289$.

36. Nelson, G.; Warner, I. M. J. Phys. Chem. 1990, 94, 576581 .

37. Kusumoto, Y. Chem. Phys. Lett. 1987, 136, 535-538.

38. Woodberry, R.; Ransom, S.; Chen, F.-M. Anal. Chem. $1988,60,2621-2625$.

39. Matsui, K.; Usuki, N. Bull. Chem. Soc. Jpn. 1990, 63, 3516-3520.

40. Matsui, K.; Nakazawa, T. Bull. Chem. Soc. Jpn. 1990, $63,11-16$.

41. Zana, R.; Yiv, S.; Strazielle, C.; Lianos, P. $J$. Colloid Interface Sci. 1981, 80, 208-223.

42. Thompson, R. B.; Shaw McBee, S. E. Langmuir 1988, 4, 106-110.

43. Street, K. W., Jr.; Acree, W. E., Jr. Analyst 1986, 111, 1197-1201.

44. Waris, R.; Acree, W. E., Jr.; street, K. W., Jr. Analyst 1988, 113, 1465-1467.

45. Street, K. W., Jr.; Acree, W. E., Jr.; Poole, C. F.; Shetty, P. H. Analyst 1988, 113, 1869-1871.

46. Street, K. W., Jr.; Acree, W. E., Jr. J. Liq. Chromatogr. 1986, 9, 2799-2808.

47. Waris, R.; Rembert, M. A.; Sellers, D. M.; Acree, W. E., Jr.; Street, K. W., Jr.; Poole, C. F.; Shetty, P. H.; Fetzer, J. C. Appl. Spectrosc. 1988, 42, 1525-1531.

48. Waris, R.; Rembert, M. A.; Sellers, D. M.; Acree, W. E. Jr.; Street, K. W., Jr.; Fetzer, J. C. Analyst $1989,114,195-199$.

49. Tucker, S. A.; Acree, W. E., Jr.; Street, K. W., Jr .; Fetzer, J. C. Appl. Spectrosc. 1989, 43, 162-164.

50. Waris, R.; Street, K. W., Jr.; Acree, W. E., Jr.; Fetzer, J. C. Appl. Spectrosc. 1989, 43, 845-850. 
51. Street, K. W., Jr.; Acree, W. E., Jr.; Fetzer, J. C.; Shetty, P. H.; Poole, C. F. Appl. Spectrosc. 1989, 43, 1149-1153.

52. Tucker, S. A.; Cretella, L. E.; Waris, R.; Street, K. W., Jr.; Acree, W. E., Jr.; Fetzer, J. C. Appl . Spectrosc. 1990, 44, 269-273.

53. Nakashima, K.; Tanaka, I. Langmuir 1993, 9, 90-95.

54. Hartner, K. C.; Carr, J. W.; Harris, J. M. Appl . Spectrosc. $1989,43,81-87$.

55. Ramis Ramos, G.; Garcia Alvarez-Coque, M. C.; Bertod, A. ; Winefordner, J. D. Anal. Chim. Acta 1988, 208, 119 .

56. Mallaris, A. Int. Reviews in Phys. Chem. 1988, 7, 95121.

57. Zana, R.; Lang, J. Colliod Surf. 1990, 48, 153-171.

58. Lakowicz, J. R. Principles of Fluorescence Spectroscopy; Plenum: New York, 1983.

59. Cecil, T. L.; Rutan, S. C. Anal. Chem. 1990, 62, 19982004 .

60. Cecil, T. L. Ph.D. Dissertation, Virginia Commonwealth University, 1990, p 12-15.

61. West, w. Chemical Application of Spectroscopy; Interscience: New York, 1968; Chapter 4.

62. Pankasem, S.; Thomas, J. K. J. Phys. Chem. 1991, 95, $7385-7393$.

63. Hite, P.; Thomas, J. K. J. Phys. Chem. 1986, 90, 57955799 .

64. Schneider, F.; Lippert, E. Ber. Bunsen-Ges. Phys. Chem. $1968,72,1155$.

65. Visser, R. J.; Weisenborn, P. C. M.; van Kan, P. J. M.; Huizer, B. H.; Varma, C. A. G. O.; Warman, J. M. ; DeHaas, M. P. J. Chem. Soc., Faraday Trans. 1985, 81, 689-704 .

66. Lueck, H.; Windsor, M. W.; Rettig, W. J. Phys. Chem. $1990,94,4550-4559$. 
67. Jarzeba, W.; Kahlow, M; Barbara, P. F. J. Imaging Sci. $1989,33,53-57$.

68. Kabouchi, B.; Benali, B.; Fadouach, M.; Kadiri, A.; Cazeau-Dubroca, C.; Nouchi, G. Spectrochim. Acta 1992, $48 A, 1015-1020$.

69. Bystryak, S. M.; Liktenshten, G. I.; Kotel'niko, A. I. Khim.Fiz. 1992, 11, 374 .

70. Wortmann, R.; Lebus, S.; Elich, K.; Assar, S.; Detzer, N.; Liptay, w. Chem. Phys. Lett. 1992, 198, 220-228.

71. Rettig, W.; Zander, M. Ber. Bunsen-Ges. Phys. Chem. $1983,87,1143-1149$.

72. Yamasaki, K.; Arita, K.; Kajimoto, O.; Hara, K. Chem. Phys. Lett. 1986, 123, 277-281.

73. Khundkar, L. R.; Zewail, A. H. J. Chem. Phys. 1986, 84, 1302-1311.

74. Rettig, w. In Modern Models of Bonding and Delocalization; Liebman, J.; Greenberg, A., Eds.; VCH: New York, 1988; Chapter 5.

75. Grabowski, 2. R.; Rotiewicz, R.; Siemiarczuk, A.; Cowley, D. J.; Baumann, W. Nouv. J. Chim. 1979, 3, 443.

76. Rettig, w. Agnew. Chem., Int. Ed. Engl. 1986, 25, 971988 and references therein.

77. Grabowski, Z. R.; Dobkowski, J. Pure Appl. Chem. 1983, $55,245-252$.

78. Shpol'skii, E. V.; Il'ina, A.A.; Klimova, L.A. Dok1. Akad. Nauk SSSR 1952, 87, 935-938.

79. Shpol'skii, E. V.; Klimova, L.A. Bull. ACad. SCi.SSSR Ser. Fiz, 1954, 18, 357.

80. Shpol'skii, E. V. Soviet Phys. Usp. 1959, 2, 378.

81. Parker, C. A. Photoluminscence of Solutions: With Application to Photocemistry and Analytical Chemistry; Elesevier: Amsterdam, 1968; Chapter 4.

82. Colmsjo, A. L.; Stenberg, U. Anal. Chem. 1979, 51, 145150 . 
83. Fetzer, J. C.; Zander, M. Z. Naturforsch. 1990, 45A, 814-816.

84. Fetzer, J. C.; Zander, M. Z. Naturforsch. 1990, 45A, 727-729.

85. Bowen, E. J.; Brocklehurst, B. J. Chem. Soc. 1954, $3875-3878$.

86. Bowen, E. J.; Brocklehurst, B. J. Chem. Soc. 1955, $4320-4331$.

87. Colmsjo, A. L.; Stenberg, U. Chem. Scr. 1970, 11, 220.

88. Colmsjo, A. L.; Wise, S. A. Anal. Chim. Acta 1986, 187, 129-137.

89. Garrigues, P.; Ewald, M. Int. J. Environ. Anal Chem. $1985,21,185-197$.

90. Colmsjo, A. L. Anal Chim. Acta 1987, 197, 65-70.

91. Colmsjo, A. L. Anal. Chim. Acta 1987, 197, 71-75.

92. Colmsjo, A. L.; Ostman, C. E. Anal. Chim. Acta 1988, $208,183-193$.

93. D'Silva, A. P.; Fassel, V. A. Anal. Chem. 1984, 56, $985 \mathrm{~A}$ and reference therein.

94. Dong, D. C.; Winnik, M. A. Can. J. Chem. 1984, 62, 2560-2565.

95. Selvarajan, N.; Panicker, M. M.; Vaidyanathan, S.; Ramakrishnan, V. Indian $J$. Chem. 1979, 18A, 23-26.

96. Encinas, M. V.; Rubio, M. A.; Lissi, E. A. Photochem. Photobiol. 1983, 37, 125-130.

97. Encinas, M. V.; Rubio, M. A.; Lissi, E. A. J. Photochem. 1982, 18, 137-150.

98. Wiczk, W. M.; Latowski, T. Z. Naturforsch. 1987, 42A, 1290-1295.

99. Bowen, E. J.; Rohatgi, K. K. Disc. Faraday Soc. 1953, $14,146-150$.

100. Wiczk, W. M.; Latowski, T. Polish J. Chem. 1990, 64, 373 . 
101. Burrell, G. J.; Hurtubise, R. J. Anal. Chem. 1988, 60, 2178-2182.

102. Burrel1, G. J.; Hurtubise, R. J. Anal. Chem. 1988, 60, 564-568.

103. Burrell, G. J.; Hurtubise, R. J. Anal Chem. 1987, 59, 965-970.

104. Kalman, R. E. J. Basic Eng. 1964, 82, 34 .

105. Rutan, S. C. J. Chemom. 1987, 1, 7-18.

106. Brown, S. D. Anal. Chim Acta 1986, 181, 1-29.

107. Rutan, S. C.; Gerow, D. D.; Hartmann, G. Intell Lab. Sys. 1988, 3, 61.

108. Gampp, H.; Maeder, M.; Meyer, C. J.; Zuberbuhler, A. D. Talanta 1985, 32, 1133-1139.

109. Maeder, M. Anal. Chem. 1987, 59, 527-530.

110. Millican, D. W.; McGown, L. B. Appl. Spectrosc. 1992, $46,28-33$.

111. Millican, D. W.; McGown, L. B. Anal. Chem. 1989, 61, 580-583.

112. Millican, D. W.; McGown, L. B. Anal. Chem. 1990, 62, 2242-2247.

113. Vo-Dinh, T. In Modern Fluorescence spectroscopy; Wehry, E. L., Ed.; Plenum: New York, 1981; Vol. 4, pp 167-192.

114. Vo-Dinh, T.; Martinex, P. R. Anal. Chim. Acta 1981, $125,13-19$.

115. Vo-Dinh, T.; Gammage, R. B.; Hawthrone, A. R. ; Thronegate, J. H. Environ. Sci. Technol. 1978, 12, 1297-1302.

116. Rein, J.; Cork, C. M.; Furton, K. G. J. Chromatogr. $1991,545,149-160$.

117. Wright, B. W.; Frye, S. R.; McMinn, D. G.; Smith, R. D. Anal. Chem. 1987, 59, 640-644.

118. Hawthorne, S. B.; Miller, D. J. J. Chromatogr. 1987, $403,63-76$. 
119. Hawthorne, S. B.; Langenfeld, J. J.; Miller, D. J.; Burford, M. D. Anal. Chem. 1992, 64, 1614-1622.

120. Wright, B. W.; Wright, C. W.; Gale, R. W.; Smith, R. D. Anal. Chem. 1987, 59, 38-44.

121. Fowlie, P. J. A.; Bulman, T. L. Anal . Chem. 1986, 58, $721-723$.

122. Junk, G. A.; Richard, J. J. Anal. Chem. 1986, 58, 962965.

123. Janini, G. M.; Johnston, K.; Zielinski, W. L., Jr. Anal. Chem. 1975, 47, 670-674.

124. Janini, G. M.; Muschik, G. M.; Zielinski, W. L., Jr. Anal. Chem. 1976, 48, 809-813.

125. Zielinski, W. L., Jr., Scanlan, R. A.; Miller, M. M. J. Chromatogr. 1981, 209, 87-90.

126. Haky, J. E.; Muschik, G. M. J. Chromatogr. 1981, 214, 161-170.

127. Wise, S. A.; Sander, L. C.; Chang, H. K. ; Markides, K. E.; Lee, M. L. Chromatographia 1988, 25, 473-479.

128. Kong, R. C.; Lee, M. L.; Tominaga, Y.; Pratrap, R.; Iwao, M.; Castle, R. N. Anal. Chem. 1982, 54, 18021806 .

129. Apfel, M. A.; Finkelmann, H.; Janini, G. M.; Laub, R. J.; Lühmann, B.-H.; Price, A.; Roberts, W. L. ; Shaw, T. J.; Smith, C. A. Anal. Chem. 1985, 57, 651-658.

130. Markides, K. E. ; Nishioka, M.; Tarbet, B. J.; Bradshaw, J. S.; Lee, M. L. Anal . Chem. 1985, 57, 1296-1299.

131. Lee, M. L.; Yang, F. J.; Bartle, K. D. Open Tubular Column Gas Chromatography: Theory and Practice; John Wiley and Sons: New York, 1984; pp 249, 312.

132. Bertsch, w. In Applications of Glass Capillary Gas Chromatography; Jennings, W. G., Ed.; Marcel Dekker: New York, 1981; p 71.

133. Wise, S. A.; Sander, L. C. ; Lapouyande, R.; Garrigues, P. J. Chromatogr. 1990, 514, 111-122.

134. Sander, L. C.; Wise, S. A. Anal. Chem. 1987, 59, 23092313. 
135. Sander L. C.; Wise, S. A. J. High Resolut. Chromatogr. Chromatogr. Commun. 1988, 11, 383-387.

136. Sander, L. C.; Wise, S. A. Anal. Chem. 1989, 61, 17491754 .

137. Fetzer, J. C. In Chemical Analysis of Polycyclic Aromatic Compounds; Vo-Dinh, T., Ed.; John Wiley and Sons: New York, 1989; Chapter 3.

138. Sander, L. C.; Parris, R. M.; Wise, S. A.; Garrigues, P. Anal. Chem. 1991, 63, 2589-2597.

139. Fetzer, J. C.; Biggs, W. R. J. Chromatogr. 1987, 386, 87-101.

140. Fetzer, J. C.; Biggs, W. R. Chromatographia 1989, 27, 118-122.

141. Poole, S. K.; Dean, T. A.; Poole, C. F. J. Chromatogr. $1987,400,323-341$.

142. Lee, M. L.; Novotny, M. V.; Bartle, K. D. Analytical Chemistry of Polycyclic Aromatic Compounds; Academic: New York, 1981.

143. Hurtubise, R. J.; Phillip, J. D.; Skar, G. T. Anal. Chim. Acta 1978, 101, 333-338.

144. Kraft, J.; Hartung, A.; Lies, K.-H.; Schulze, S. J. J. High Resolut. Chromatogr. Chromatogr. Commun. 1982, 5, 489-494.

145. Katz, M.; Chau, C. Environ. Sci. Technol. 1980, 14, $838-843$.

146. Miguel, A. H. Int. J. Environ. Chem. 1982, 12, 17-25.

147. Matsushita, H.; Esumi, Y.; Suzuki, A.; Handa, T. Bunseki Kagaku1972, 21, 1471-1478.

148. Poole, C. F.; Butler, H. T.; Coddens, M. E.; Khatib, S.; Vandervennet, R. J. Chromatogr. 1984, 302, 149-158.

149. Bruggeman, W. A.; van der steen, J.; Hutzinger, O. J. Chromatogr. 1982, 238, 335-346.

150. Seifert, B. J. Chromatogr. 1977, 131, 417-421.

151. Ho, S. S. J.; Butler, H. T.; Poole, C. F. J. Chromatogr. 1983, 281, 330-339. 
152. Butler, H. T.; Coddens, M. E.; Khatib, S.; Poole, C. F. J. Chromatogr. Sci. 1985, 23, 200-207.

153. Blümer, G.-P.; Zander, M. Fresenuis Z. Anal. Chem. $1979,296,409-410$.

154. Zimmerman, H. E. Quantum Mechanics for Organic Chemists; Academic: New York, 1975; pp 145-146.

155. March, J. Advanced Organic Chemistry: Reactions, Mechanisms and Structure; McGraw-Hill: New York, 1968; pp 46-48.

156. Acree, W. E., Jr. Environ. Sci. Technol. 1993, 27, 757758 .

157. Chin, Y.-P.; Gschwend, P. M. Environ. Sci. Technol. $1993,27,759-760$.

158. Breymann, U.; Dreeskamp, H.; Koch, E.; Zander, M. Chem. Phys. Lett. 1978, 59, 68-70. 
CHAPTER II

MATERIALS AND METHODS

Molecular structures of the various polycyclic aromatic hydrocarbons (PACs) examined are depicted in Figures 13-32. All PAC solutes were obtained from commercial sources or various researchers throughout the world. Those synthesis not contained in the present literature or purification methods performed are as follows: Tetrabenzo[de, $h i, m n, q r]-$ naphthacene was obtained by heating the twin $[09,24]-$ [2.1.2.1] metacyclohapane-9-ene with palladium/charcoal and was purified by high performance liquid chromatography (HPLC) in accordance with the method of Gritzmacher and Husemann (1). Phenanthro $[5,4,3,2$ efghi $]$ perylene, 1-methylcoronene, and 1,2-dimethylcoronene were isolated from a hydrocracker deposit material (2), and 3,4,5-trihydrobenzo[cd]pyrene was prepared from naphthanthrone (3) . 3,4Dihydrobenzo[ghi]perylene and $5,6,7,8,9,10$-hexahydrobenzo[ghi]perylene were prepared by hydrogenation of benzo[ghi]perylene with platinum in glacial acetic acid and purified by chromatography. 11,12-Dimethyldibenzo[a,c]phenazine was prepared from 4,5-dimethyl-o-phenylenediamine, and 9,10phenanthraquinone was prepared according to the method used by Hall et al. (4) for dibenzo[a,c]phenazine. Crystallization from ethanol gave 11,12-dimethyldibenzo[a,c]- 


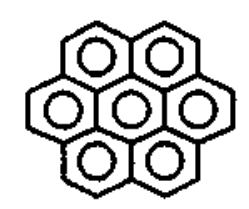

A
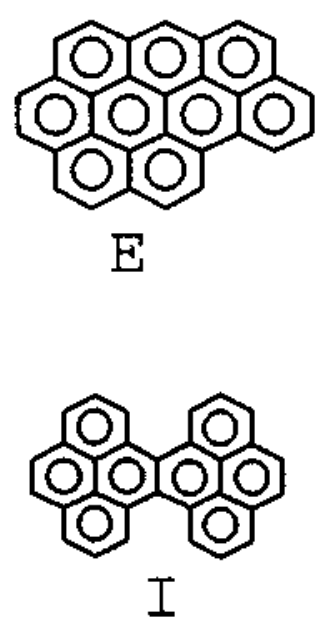

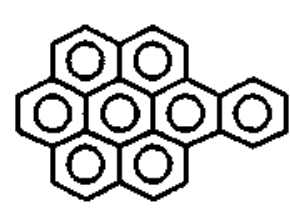

$B$

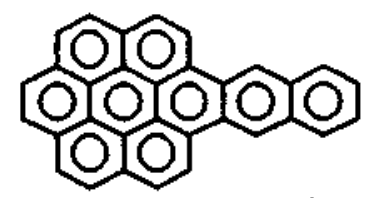

C

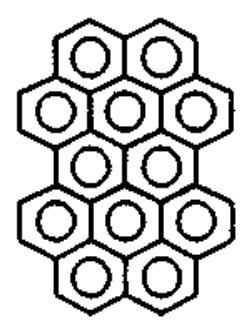

$\mathrm{F}$

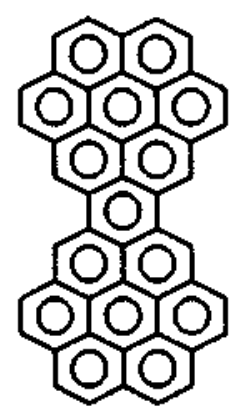

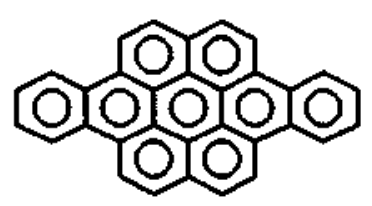

D

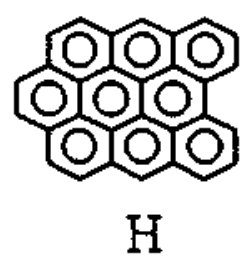

$G$
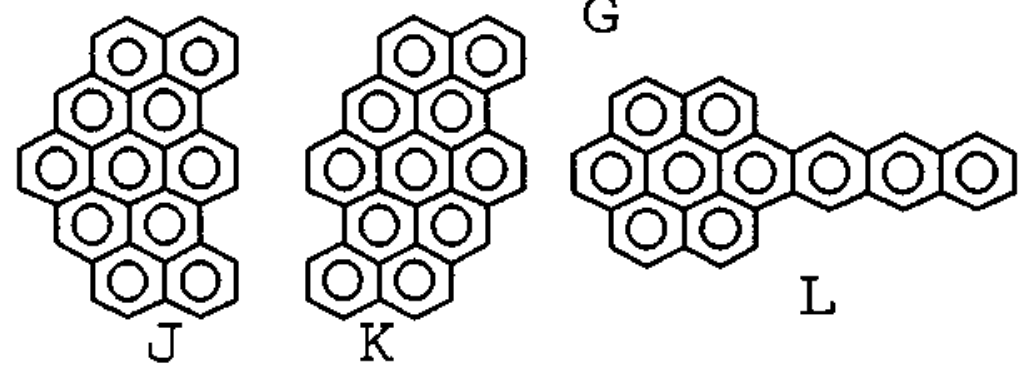

FIGURE 13: Molecular structures of PAH6 benzenoids: (A) coronene, (B) benzo[a]coronene, (C) naphtho[2,3a]coronene, (D) dibenzo[a,j]coronene, (E) naphtho $[8,1,2 a b c]$ coronene, naphth $\left[2^{\prime}, 1^{\prime}, 8^{\prime}, 7^{\prime}: 4,10,5\right]$ anthra $[1,9,8 a b c d]$ coronene, (G) benzo $\left[1,2,3 b c: 4,5,6 b^{\prime} c^{\prime}\right]$ dicoronene, (H) dibenzo[bc,ef]coronene, (I) tetrabenzo[de,hi,mn, gr]naphthacene, (J) dinaphtho $\left[8,1,2 a b c ; 2^{\prime}, 1^{\prime}, 8^{\prime} k 1 m\right]$ coronene, (K) dinaphtho$\left[8,1,2 a b c ; 2^{\prime}, 1^{\prime}, 8^{\prime} j k 1\right]$ coronene, and (L) anthra $[2,3 a]-$ coronene. 
$\frac{10}{0.0}$

M

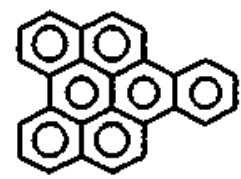

Q

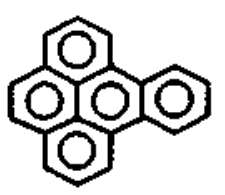

U

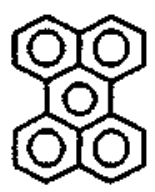

$\mathrm{N}$

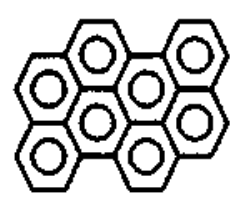

$\mathrm{R}$

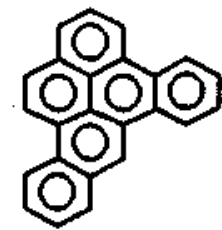

V

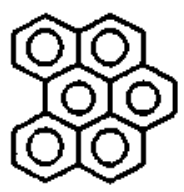

O

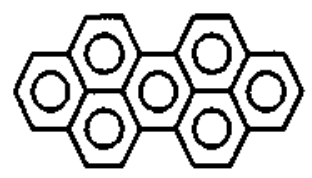

S

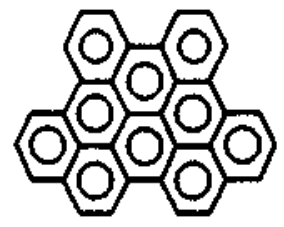

W

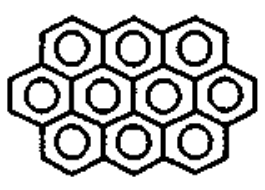

$\mathrm{P}$

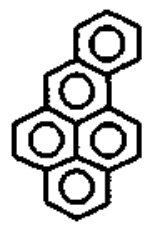

$\mathrm{T}$

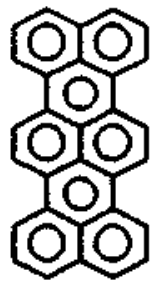

$\mathrm{X}$

FIGURE 14: Molecular structures of PAH6 benzenoids:

pyrene, (N) perylene, (O) benzo[ghi]perylene, (P) ovalene, (Q) naphtho $[1,2,3,4 \mathrm{ghi}]$ perylene, (R) benzo[pgr]naphtho$[8,1,2 b c d]$ perylene, (S) dibenzo $[c d, 1 m]$ perylene, (T) benzo[a]pyrene, (U) benzo[e]pyrene, (V) dibenzo[a,e]pyrene, (W) dibenzo[fg,ij]phenanthro $[2,1,10,9,8,7$ pgrstuv $]$ pentaphene, and (X) tribenzo[de, $k l$, rst $]$ pentaphene. 


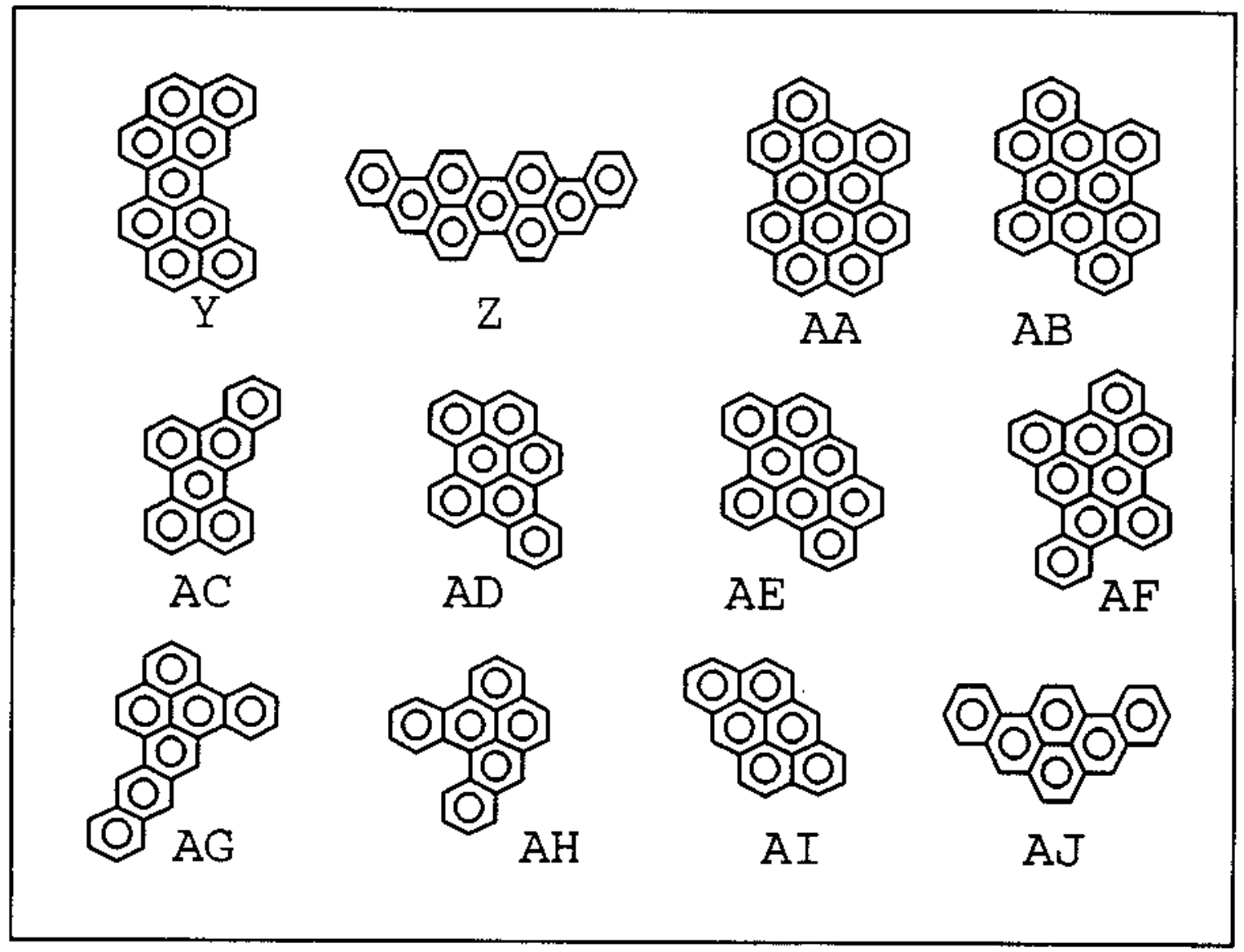

FIGURE 15: Molecular structures of PAH6 benzenoids:

dinaphtho $\left[2,1,8,7 \operatorname{defg} ; 2^{\prime}, 1^{\prime}, 8^{\prime}, 7^{\prime} i j k 1\right]$ pentaphene,

benz [rst] anthra $[9,1,2$ cde]pentaphene, (AA) benz [4, 10] anthra$[1,9,8 a b c d]$ coronene, (AB) benzo[cd]chryseno $[4,5,6,7 f g h i j k]-$ perylene, (AC) benzo[b]perylene, (AD) dibenzo[e,ghi]perylene, (AE) phenanthro $[5,4,3,2$ efghi]perylene, (AF) dibenzo[cd,k]naphtho $[8,1,2 f g h i]$ perylene, (AG) benzo[a]naphtho $[8,1,2 c d e]$ naphthacene, (AH) dibenzo[def, $p]$ chrysene, (AI) anthranthrene, and (AJ) benzo[rst]pentaphene. 


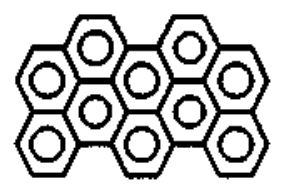

AK

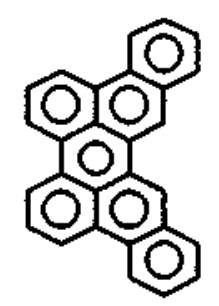

$\mathrm{AO}$

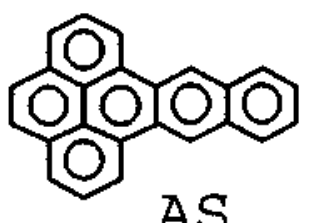

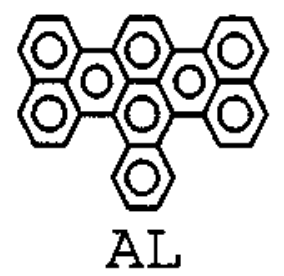
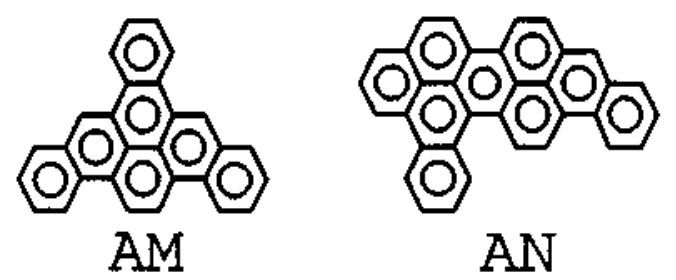

AN
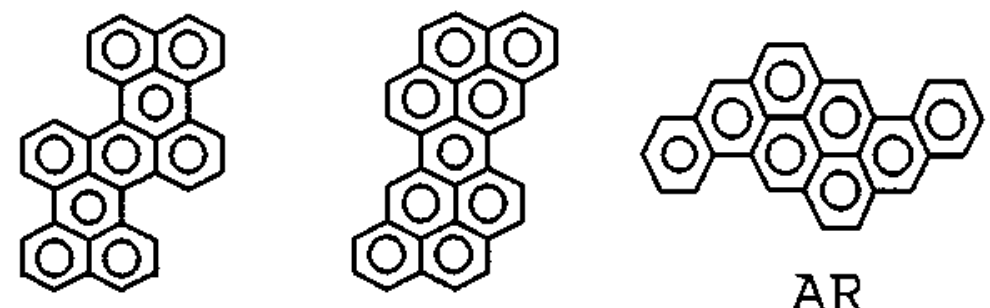

AR
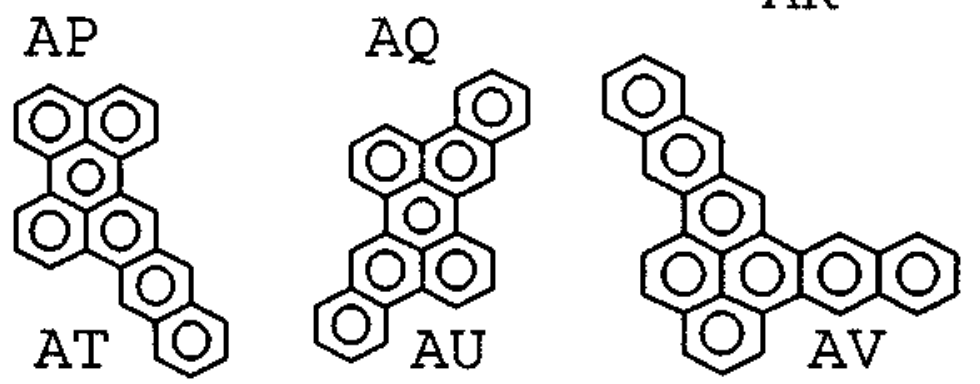

FIGURE 16: Molecular structures of PAH6 benzenoids: (AK) benzo[ rst $]$ dinaphtho $\left[2,1,8,7\right.$ defg $\left.; 2^{\prime}, 1^{\prime}, 8^{\prime}, 7^{\prime} j j k 1\right]$ pentaphene, (AL) dibenzo[de, $k 1]$ naphtho $[1,2,3,4$ rst $]$ pentaphene, (AM) dibenzo[h,rst]pentaphene, (AN) dibenzo[a,rst]naphtho$[8,1,2 c d e]$ pentaphene, (AO) dibenzo $[f g, i j]$ pentaphene, (AP) tetrabenzo[de,hi,op,st]pentacene, (AQ) dinaphtho $[2,1,8,7-$ defg;2',1',8',7'opqr] pentacene, (AR) pyranthene, (AS) dibenzo[de, $q r]$ naphthacene, (AT) dibenzo[de,st]pentacene, (AU) dibenzo $[f g, q r]$ pentacene, and (AV) naphtho $[8,1,2 h i j]-$ hexaphene. 


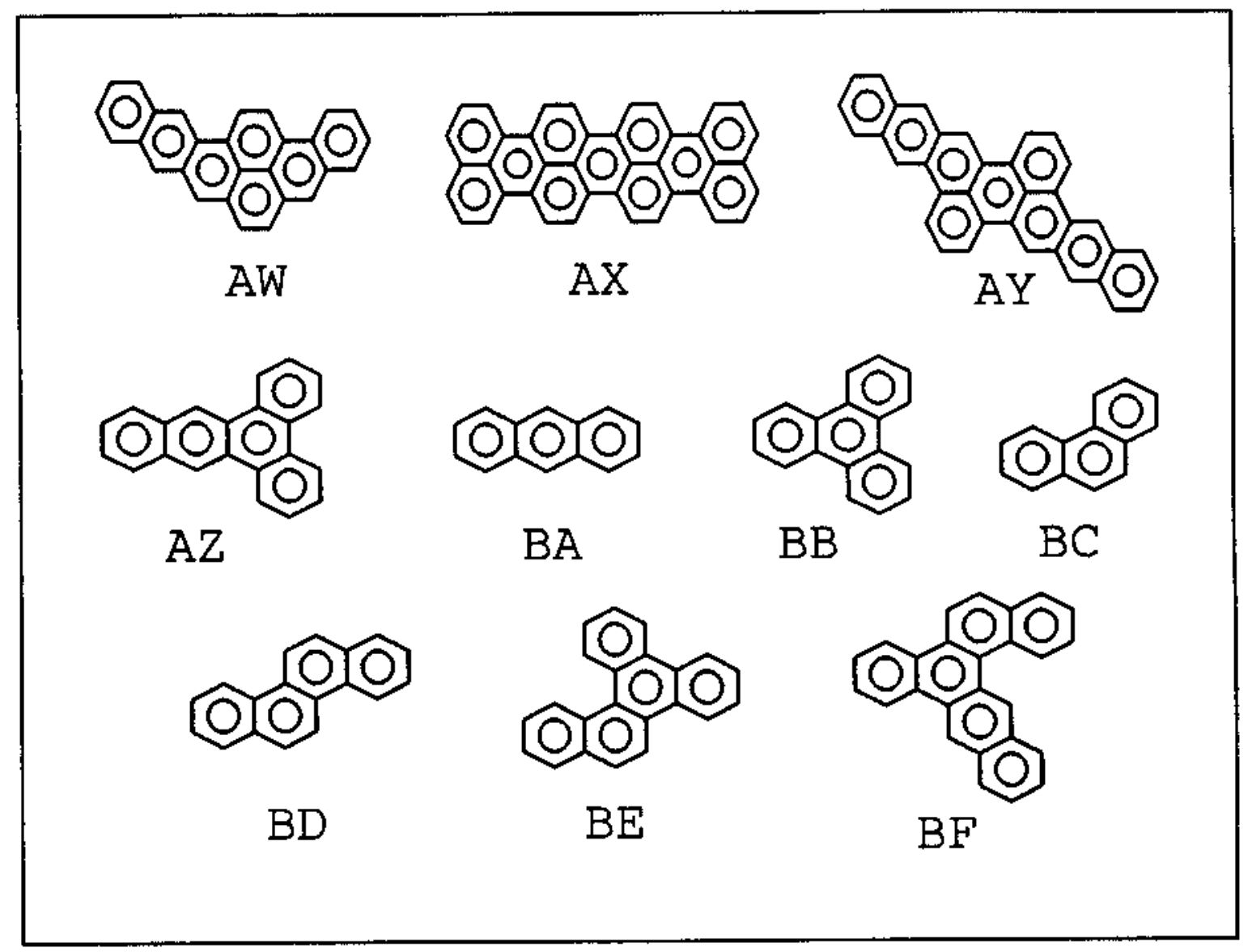

FIGURE 17: Molecular structures of PAH6 benzenoids: (AW) benzo[ $\mathrm{vwx}]$ hexaphene, (AX) benzo[ $\left.1,2,3 c d ; 4,5,6 \mathrm{c}^{\prime} d^{\prime}\right]-$ diperylene, (AY) dibenzo[hi,wX]heptacene, (AZ) benzo[b]triphenylene, (BA) anthracene, (BB) triphenylene, (BC) phenanthrene, (BD) chrysene, (BE) benzo[g]chrysene, and (BF) naphtho $[2,3 g]$ chrysene. 

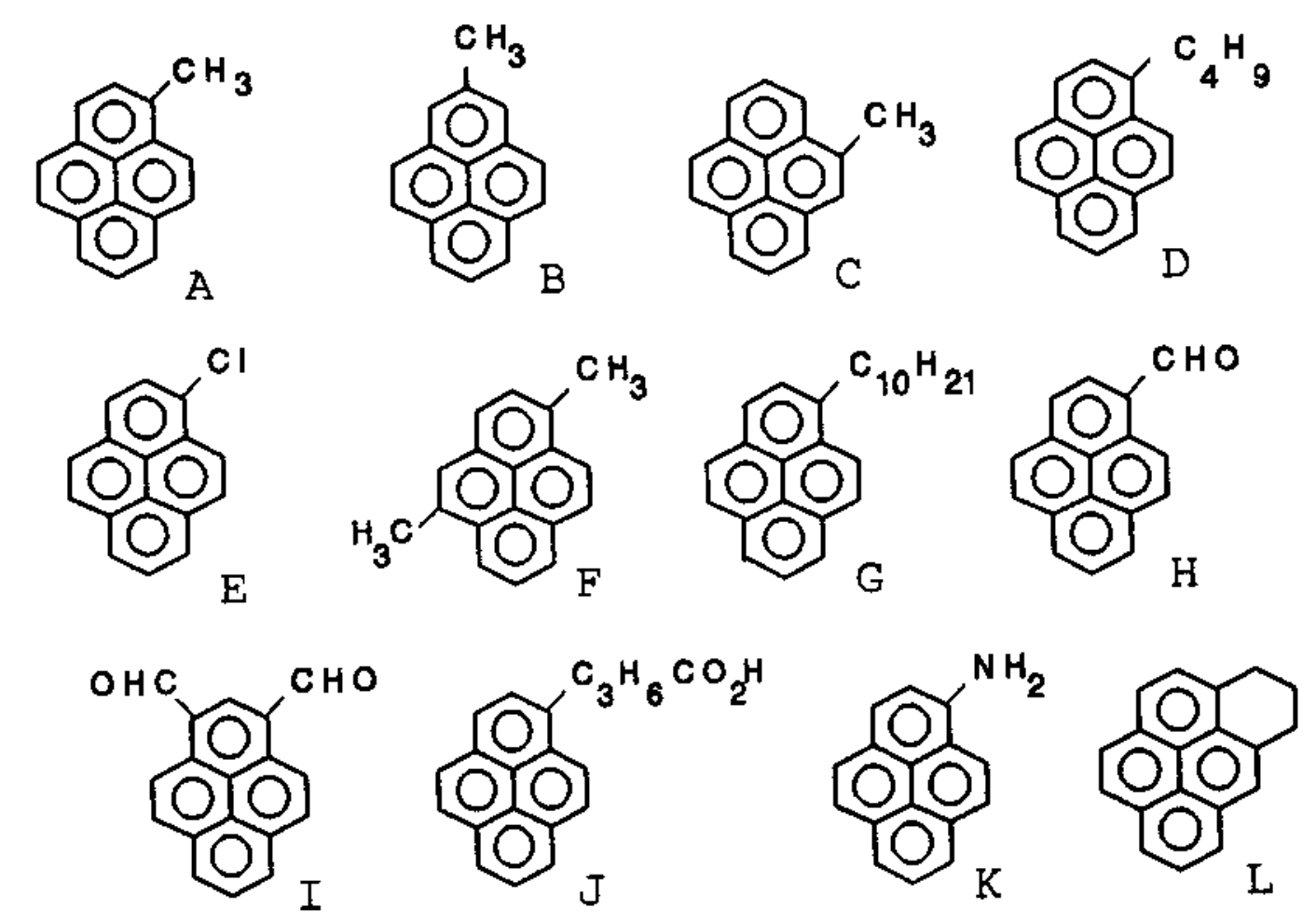

FIGURE 18: Molecular structures of PAH6 benzenoid

derivatives: (A) 1-methylpyrene, (B) 2-methylpyrene, (C) 4-methylpyrene, (D) 1-butylpyrene, (E) 1-chloropyrene, 1,5-dimethylpyrene, (G) 1-decylpyrene, (H) 1-pyrenecarboxaldehyde, (I) 1,3-pyrenedicarboxaldehyde, (J) 1pyrenebutanoic acid, (K) 1-aminopyrene, and (L) 3,4,5-trihydrobenzo[cd]pyrene. 


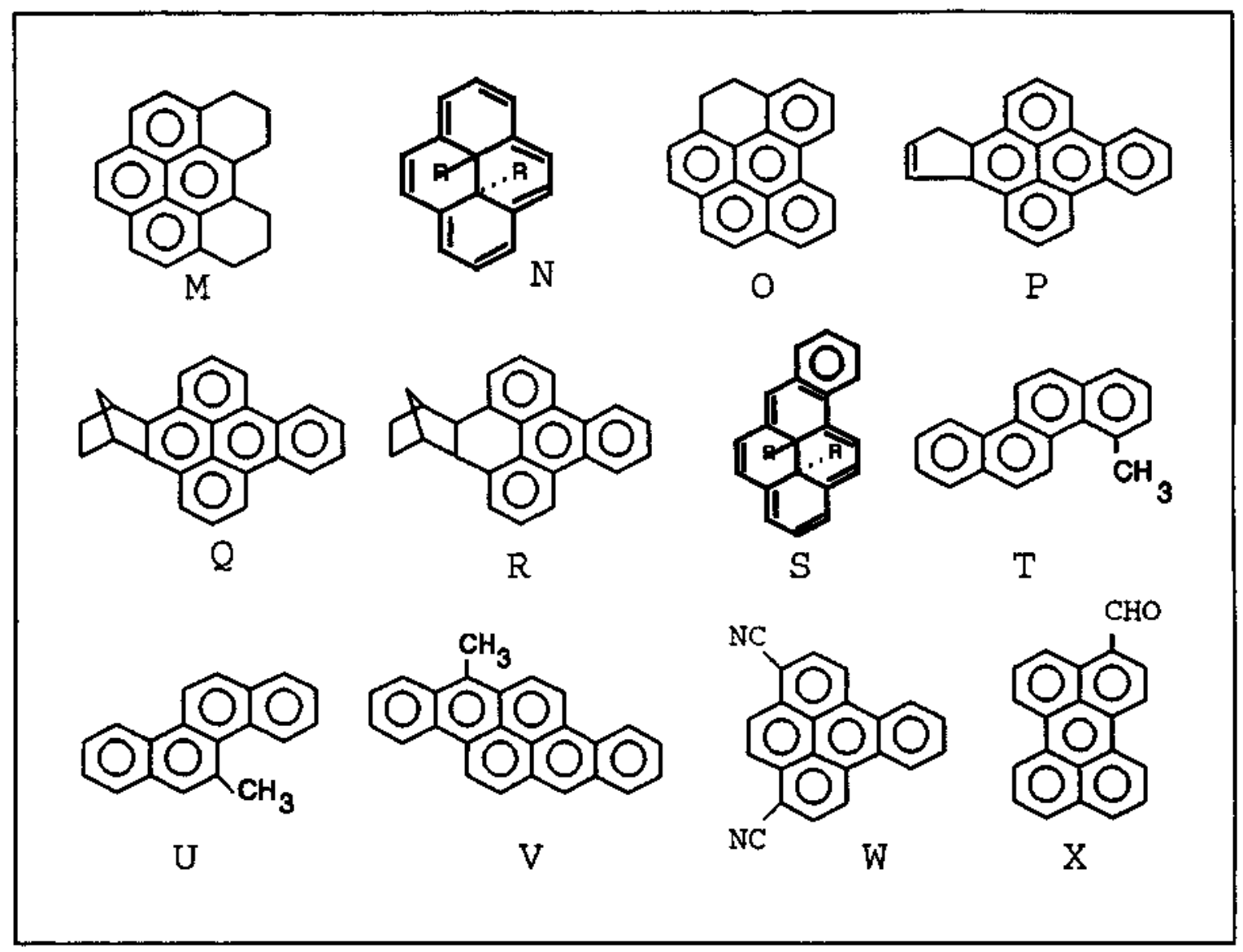

FIGURE 19: Molecular structures of PAH6 benzenoid derivatives: (M) $5,6,7,8,9,10$-hexahydrobenzo[ $g h i$ ]perylene, (N) trans-10b,10c-dihydro-10b, 10c-dimethylpyrene, (0) 3,4-dihydrobenzo[ghi]perylene, (P) 4H-benzo[e]cyclopenta[1]pyrene, (Q) 4,5,6,7-tetrahydro-4,7-methanodibenzo[fg,op]naphthacene, (R) $8 b, 9,10,11,12,12 a$-hexahydro-9, 12-methanobenzo[e]pyrene, (S) trans-12b,12c-dihydro-12b,12c-dimethylbenzo[a]pyrene, (T) 4-methylchrysene, (U) 5-methylchrysene, (V) 5-methyldibenzo[b, def]chrysene, (W) 3,6-dicyanobenzo[e]pyrene, and (X) 3-perylenecarboxaldehyde. The $R$ groups in the solutes correspond to methyl groups. 

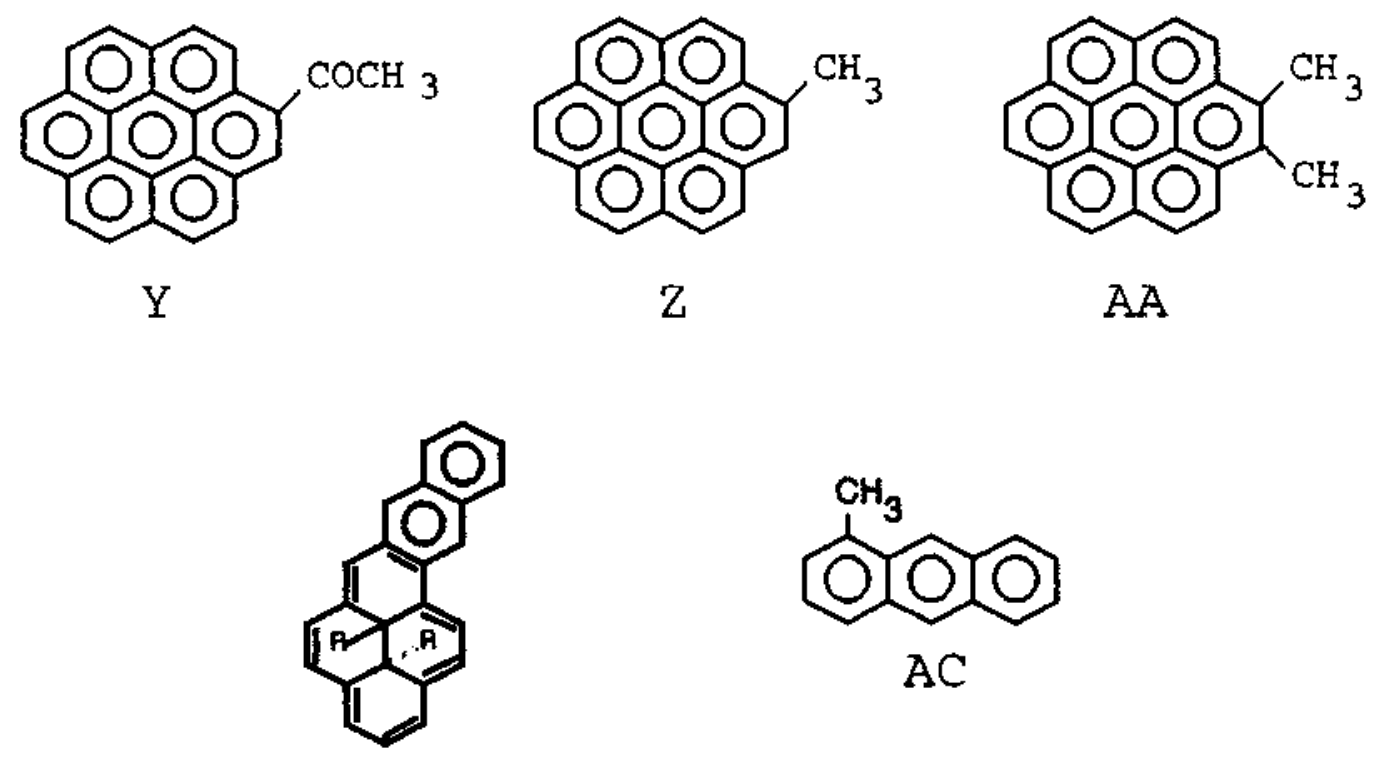

$\mathrm{AB}$

FIGURE 20: Molecular structures of PAH6 benzenoid derivatives: (Y) 1-acetylcoronene, (Z) 1-methylcoronene, (AA) 1,2-dimethylcoronene, (AB) trans-14b,14c-dihydro$14 b, 14 c-d i m e t h y l n a p h t h o[2,1,8 q r a]$ naphthacene, and (AC) 2methylanthracene. The $\mathrm{R}$ groups in the solutes correspond to methyl groups. 


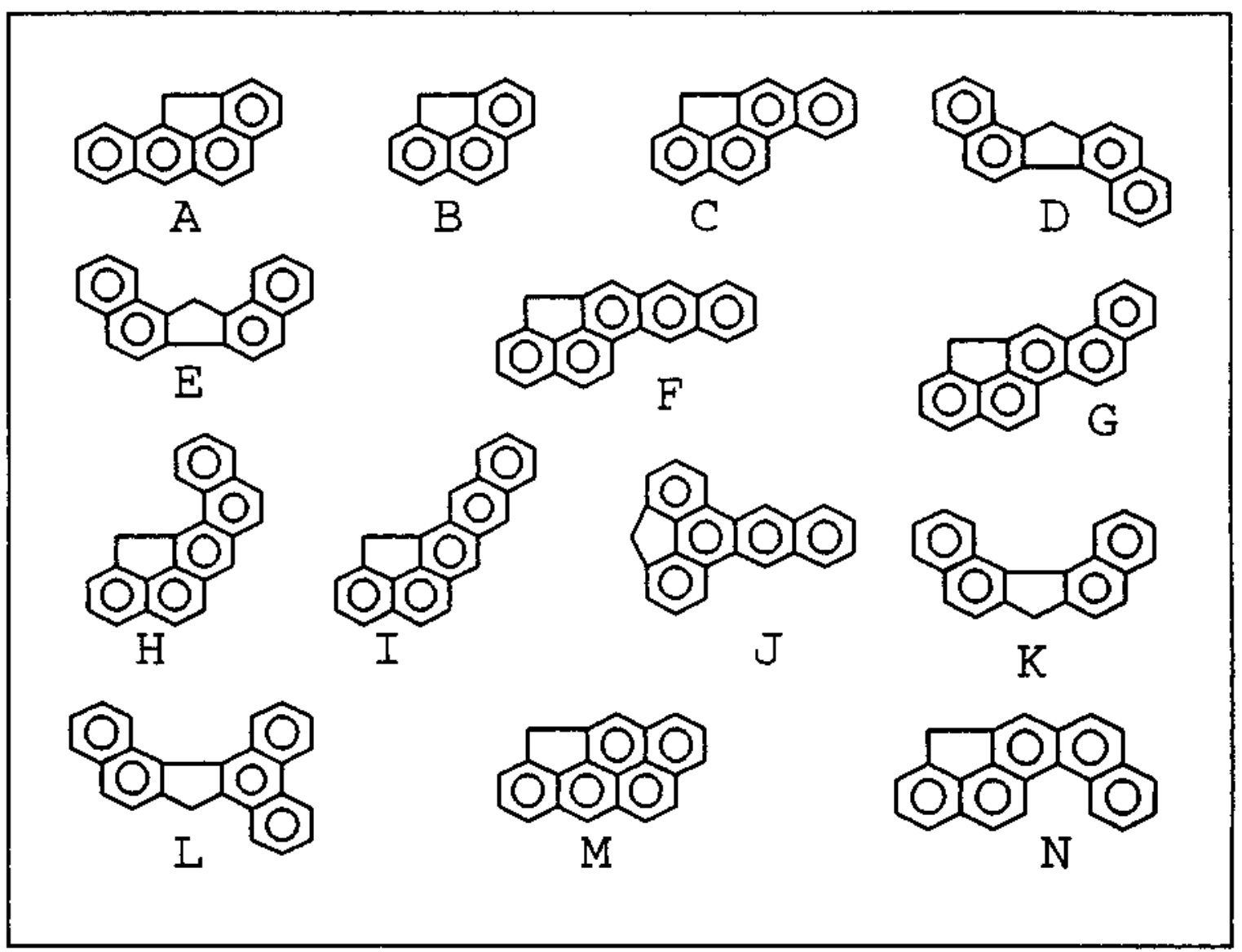

FIGURE 21: Molecular structures of cyclopenta-polycyclic aromatic hydrocarbon derivatives: (A) $11 H$-benzo[bc]aceanthrylene, (B) 4H-cyclopenta[def]phenanthrene, (C) 4H-cyclopenta[def]chrysene, (D) $13 H$-dibenzo $[a, g]$ fluorene, (E) $13 H$-dibenzo[a,i]fluorene, (F) $4 H$-benzo[b]cyclopenta[mno]chrysene, (G) 4H-cyclopenta [pqr]picene, (H) 13H-dibenz [bc, 1] aceanthrylene, (I) $13 H$-dibenz $[b c, k]$ aceanthrylene, (J) $4 H$-benzo[b]cyclopenta $[j k 1]$ triphenylene, (K) $7 H$-dibenzo $[C, g]$ fluorene, (L) $9 H$-benz $[6,7]$ indeno $[1,21]-$ phenanthrene, (M) 4H-benzo[def]cyclopenta[mno]chrysene, and (N) $4 \mathrm{H}$-benzo[ $\mathrm{C}$ ] cyclopenta [mno] chrysene. 


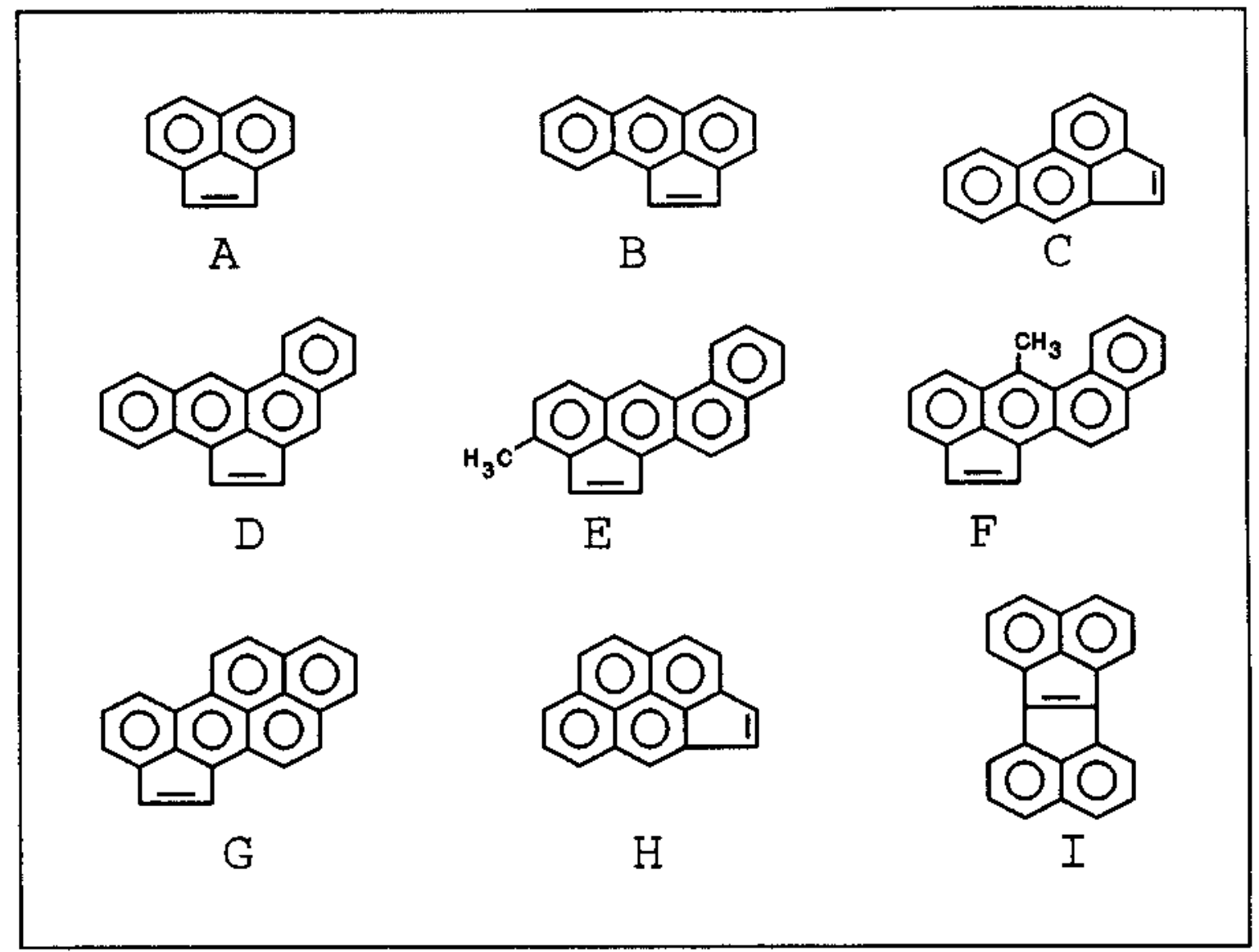

FIGURE 22: Molecular structures of acenaphthylene derivatives: (A) acenaphthylene, (B) aceanthrylene, (C) acephenanthrylene, (D) benz[e]aceanthrylene, (E) 3-methylbenz [j]aceanthrylene, (F) 6-methylbenz [j] aceanthrylene, (G) benzo[def]cyclopenta[hi]chrysene, (H) cyclopenta[cd]pyrene, and (I) acenaphth $[1,2 a]$ acenaphthylene. 
<smiles>Cc1ccc2c3c(cc4ccccc4c13)C=C2</smiles><smiles>CC1=CC=c2c1cc1c2cccc2cc3ccccc3c21</smiles><smiles></smiles><smiles>CC1=Cc2cccc3c2c1cc1ccccc13</smiles><smiles></smiles><smiles>Cc1cccc2c1cc1c3c(cccc32)C=C1</smiles><smiles></smiles>
$G$<smiles></smiles><smiles>Cc1ccc2c(c1)C=c1ccc3c(c1-2)C=CC=3</smiles>

FIGURE 23: Molecular structures of acephenanthrylene derivatives: (A) 1-methylacephenanthrylene, (B) 3methylacephenanthrylene, (C) 4-methylacephenanthrylene, 5-methy lacephenanthrylene, (E) 6-methylacephenanthrylene, (F) 7-methylacephenanthrylene, (G) 8-methylacephenanthryIene, (H) 9-methylacephenanthrylene, and (I) 10-methylacephenanthrylene. 


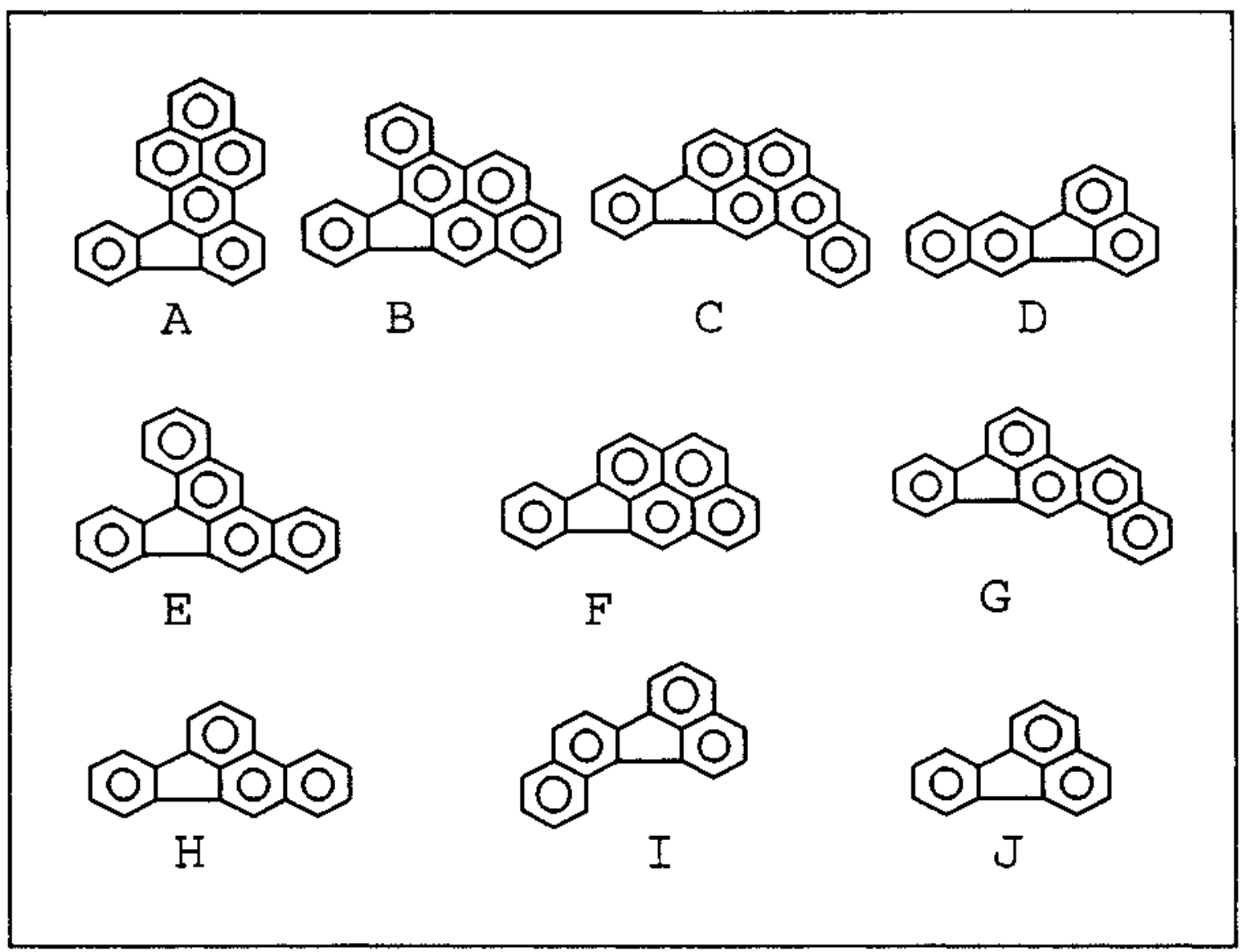

FIGURE 24: Molecular structures of fluoranthenoids and

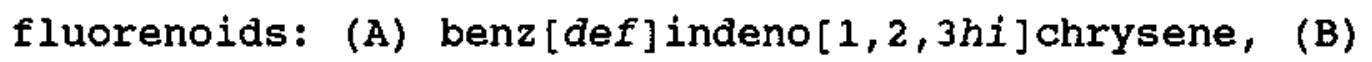
fluoreno $[2,3,4,9 d e f g]$ chrysene, (C) benz $[$ def $]$ indeno $[1,2,3 q r]-$ chrysene, (D) benzo[k]fluoranthene, (E) dibenzo[a,e]fluoranthene, (F) indeno $[1,2,3 c d]$ pyrene, (G) naphtho $[1,2 b]-$ fluoranthene, (H) benzo[b]fluoranthene, (I) benzo[j]fluoranthene, and (J) fluoranthene. 

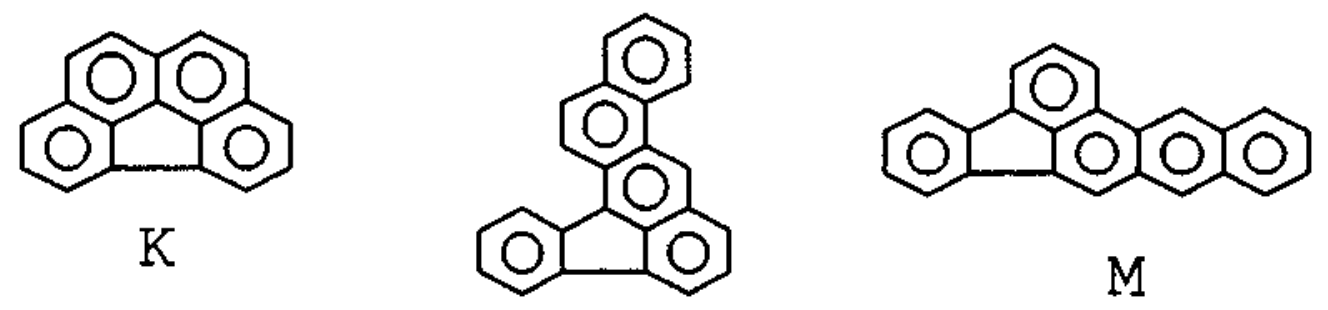<smiles></smiles>

I

$\mathrm{N}$

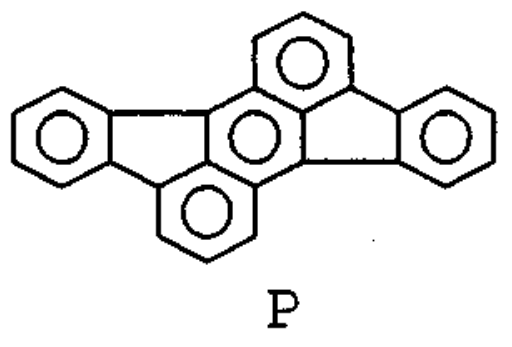

O
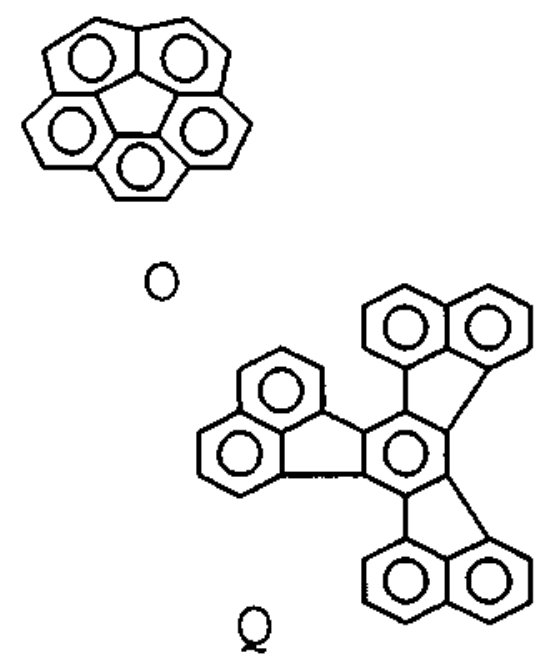

FIGURE 25: Molecular structures of fluoranthenoids and fluorenoids: (K) benzo[ghi]fluoranthene, (L) naphtho[2,1a]fluoranthene, (M) naphtho $[2,3 b] f l u o r a n t h e n e,(N)$ benzo[a]fluoranthene, (O) dibenzo[ghi, mno]fluoranthene, (P) rubicene, and (Q) diacenaphtho $\left[1,2 j: 1^{\prime}, 2^{\prime} 1\right]$ fluoranthene. 
<smiles>Cc1ccc2cccc3c2c1-c1ccccc1-3</smiles>

A<smiles>Cc1cc2c3c(cccc3c1)-c1ccccc1-2</smiles>

B<smiles>Cc1ccc2c3c(cccc13)-c1ccccc1-2</smiles>

C<smiles>Cc1cccc2c1-c1cccc3cccc-2c13</smiles>

D<smiles>Cc1ccc2c(c1)-c1cccc3cccc-2c13</smiles>

E

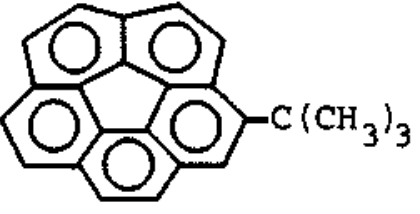

F<smiles>Cc1ccc2c(c1)cc1c3c(cccc32)-c2ccccc2-1</smiles><smiles>Oc1ccc2c(c1)cc1c3c(cccc32)-c2ccccc2-1</smiles>

FIGURE 26: Molecular structures of fluoranthenoid derivatives: (A) 1-methylfluoranthene, (B) 2-methylfluoranthene, (C) 3-methylfluoranthene, (D) 7-methylfluoranthene, (E) 8-methylfluoranthene, (F) t-butyldibenzo[ghi, mno] fluoranthene, (G) 10-methylbenzo[b]fluoranthene, and (H) 10-hydroxybenzo[b]fluoranthene. 
<smiles>COc1ccc2c(c1)cc1c3c(cccc32)-c2ccccc2-1</smiles><smiles>COc1cccc2cc3c4c(cccc4c12)-c1ccccc1-3</smiles>

J

HO<smiles>Cc1ccc2c3c(ccc2c1)-c1cccc2cccc-3c12</smiles>

K

$\mathrm{H}_{3} \mathrm{CO}$<smiles>Cc1ccc2c3c(ccc2c1)-c1cccc2cccc-3c12</smiles>

I

HO<smiles></smiles>

M<smiles>Oc1ccc2cc3c(cc2c1)-c1cccc2cccc-3c12</smiles>

N<smiles>COc1ccc2c3c(cccc13)-c1cc3ccccc3cc1-2</smiles>

0<smiles>COc1ccc2cc3c(cc2c1)-c1cccc2cccc-3c12</smiles>

FIGURE 27: Molecular structures of fluoranthenoid derivatives: (I) 10-methoxybenzo[b]fluoranthene, (J) $12-$ methoxybenzo[b]fluoranthene, (K) 10-hydroxybenzo[j]fluoranthene, (L) 10-methoxybenzo[j]fluoranthene, (M) 3hydroxybenzo $[k]$ fluoranthene, (N) 9-hydroxybenzo $[k]-$ fluoranthene, (O) 3-methoxybenzo[k]fluoranthene, and (P) 9methoxybenzo[ $k$ ] fluoranthene. 


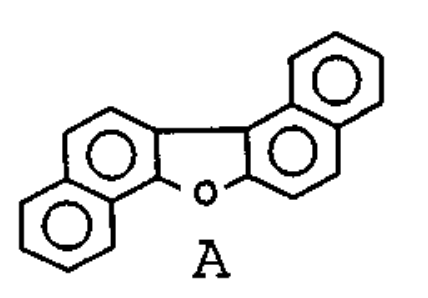

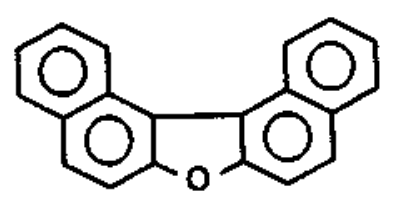

B
Or.

C<smiles>C1=Cc2ccc3c4c(ccc(c24)O1)C=CO3</smiles>

D<smiles>C1=Cc2ccc3c4c(ccc(c24)O1)C=CO3</smiles>

E<smiles>C1=Cc2ccc3c4c(ccc(c24)S1)C=CS3</smiles>

F<smiles>c1cc2c3c(cccc3c1)-c1sccc1-2</smiles>

G<smiles>C1=Cc2c(ccc3ccsc4cccc2c4=3)=c2ccsc2=C1</smiles>

$\mathrm{H}$

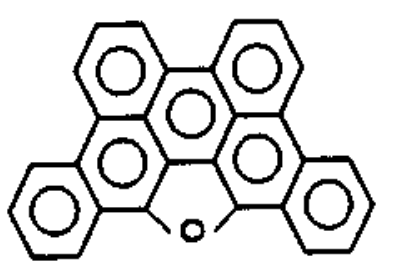

I

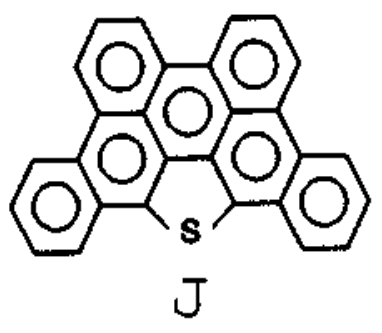

$\mathrm{OT}_{\mathrm{s}}^{\mathrm{s}} \mathrm{O} \mathrm{K}$

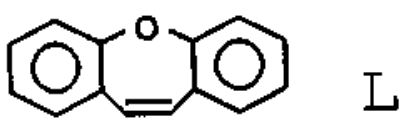

FIGURE 28: Molecular structures of polycyclic aromatic oxygen heterocycles (PAOHs) and polycyclic aromatic sulfur heterocycles PASHs: (A) dinaphtho $\left[1,2 b: 1^{\prime}, 2^{\prime} d\right]$ furan, dinaphtho $\left[2,1 b: 1^{\prime}, 2^{\prime} d\right]$ furan, (C) benzo $[b]$ naphtho $[2,3 d]$ furan, (D) naphtho $\left[1,8 b c ; 5,4 b^{\prime} c^{\prime}\right]$ dipyran, (E) naphtho $[1,8 b c ;-$ 4,5b'C']dipyran, (F) 1,6-dithiapyrene, (G) 1,7-dithiaperylene, (H) 3,10-dithiaperylene, (I) dibenzo[2,3:10,11]perylo $[1,12 b c d]$ furan, (J) dibenzo $[2,3: 10,11]$ pery $10[1,12 b c d]-$ thiophene, (K) thianthrene, and (L) dibenzo $[b, f]$ oxepin. 


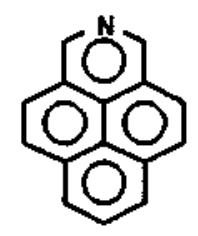

A

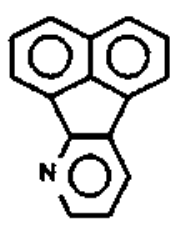

E

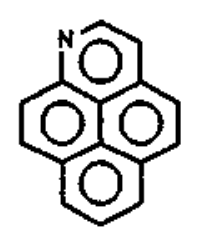

B

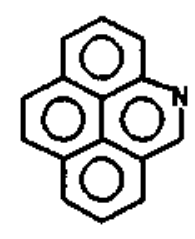

C

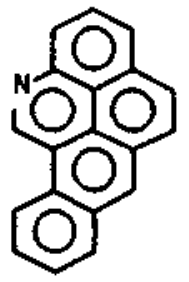

D

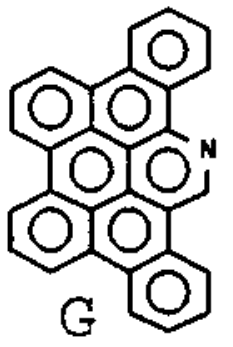<smiles></smiles>
$\mathrm{H}$ F<smiles></smiles><smiles>c1ccc2c(c1)ccc1cc3ccc4ccncc4c3cc12</smiles><smiles></smiles><smiles>[R]Cc1cccc2c1ccc1cccnc12</smiles>

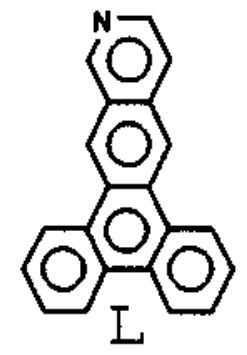

FIGURE 29: Molecular structures of polycyclic aromatic nitrogen heterocycles (PANHs): (A) 2-azapyrene, (B) 1azapyrene, (C) 4-azapyrene, (D) 12-azabenzo[a]pyrene, (E) acenaphtho $[1,2 b]$ pyridine, (F) indeno $[1,2,3 i j]$ isoquinoline, (G) dibenzo[c,i] phenanthro $[1,10,9,8 \mathrm{anm} I k]$ phenanthridine, phenanthro $[2,3 h]$ isoquinoline, (I) phenanthro $[3,2 h]$ isoquinoline, (J) 1-azabenz[a]anthracene, (K) 4-azachrysene, and (L) phenanthro $[9,10 \mathrm{~g}]$ isoquinoline. 


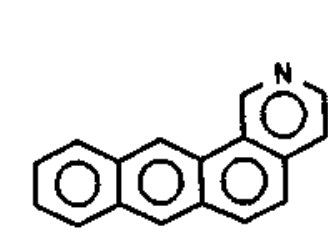

M
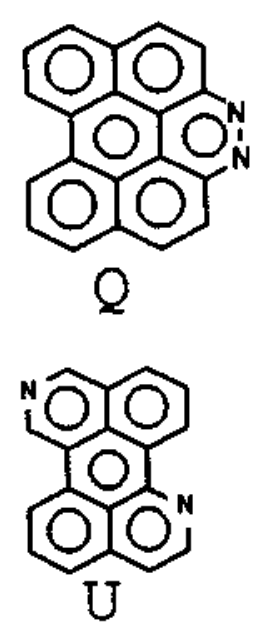

$\mathrm{U}$

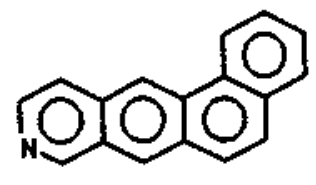

$\mathrm{N}$

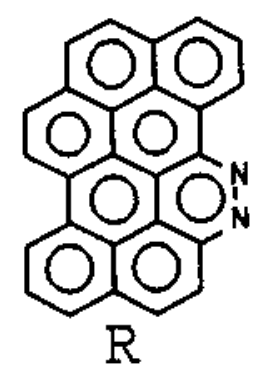

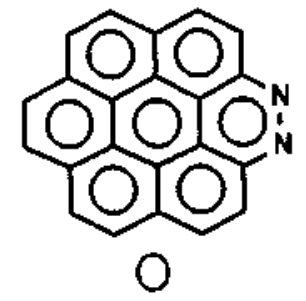

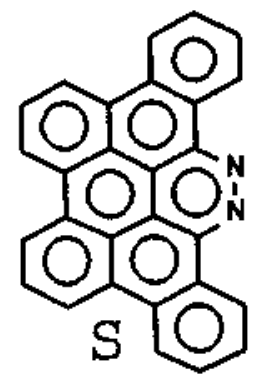

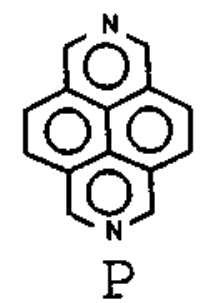

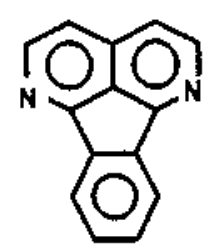

$\mathrm{T}$

FIGURE 30: Molecular structures of PANHs: (M) 2azabenz [a] anthracene, (N) 9-azabenz [a]anthracene, (0) naphth $\left[2^{\prime}, 1^{\prime}, 8^{\prime}, 7^{\prime}: 4,10,5\right]$ anthra $[1,9,8 c d e f]$ cinnoline, benzo [Imn] $[3,8]$ phenanthroline, (Q) benz $[4,10]$ anthra $[1,9,8-$ cdef $]$ cinnoline, (R) naphtho $[8,1,2 h i j]$ pyreno $[9,10,1$ def $]$ phthalazine, (S) diphenanthro $[9,10,1$ def:1',10',9'hij]phthalazine, (T) indeno[ $1,2,3 i j][2,7]$ naphthyridine, benz [ de] isoquino $[1,8 g h$ ]quinoline, (V) benzo[a]phenazine, tricycloquinazoline, and (X) dibenzo[a,c]phenazine. 

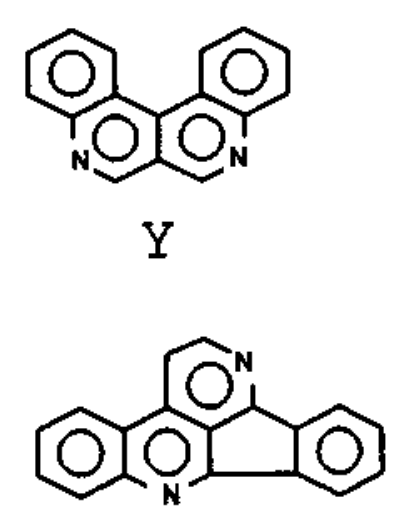

$\mathrm{AB}$

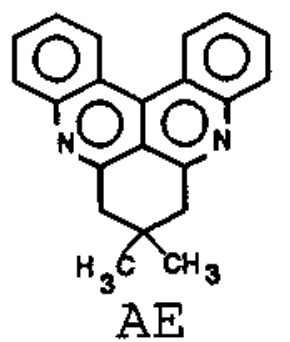

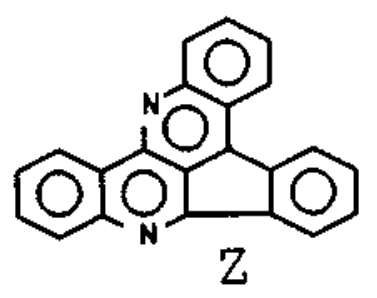

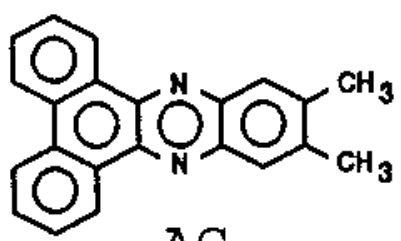

$\mathrm{AC}$

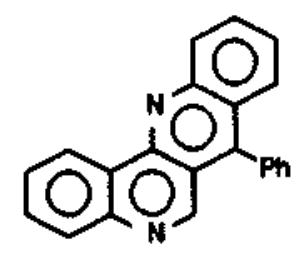

$\mathrm{AF}$
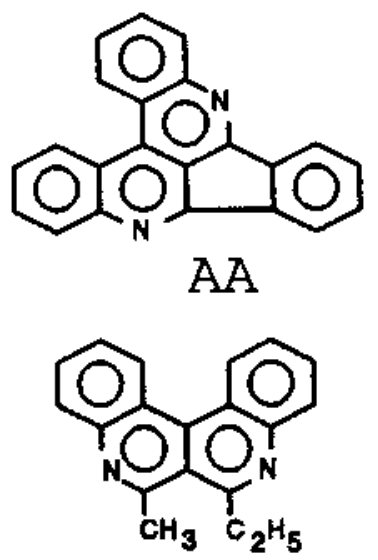

$A D$

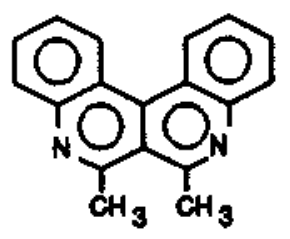

AG

FIGURE 31: Molecular structures of PANHs: (Y) dibenzo$[c, f][2,7]$ naphthyridine, (z) dibenz $[b, h]$ indeno $[1,2,3 d e]-$ $[1,6]$ naphthyridine, (AA) dibenz $[c, f]$ indeno $[1,2,3 i j]-$ $[2,7]$ naphthyridine, $(\mathrm{AB})$ benzo[c] indeno $[1,2,3 i j][2,7]-$ naphthyridine, (AC) 11,12-dimethyldibenzo[a,c]phenazine, (AD) 6-ethyl-7-methyldibenzo $[c, f][2,7]$ naphthyridine, 7,8-dihydro-7,7-dimethyl-6-H-quino $[2,3,4 k 1]$ acridine, (AF) 7phenyldibenzo $[b, h][1,6]$ naphthyridine, and (AG) 6,7-dimethyldibenzo[ $c, f][2,7]$ naphthyridine. 
<smiles>c1ccc2c(-c3cccc4ccccc34)cccc2c1</smiles>

A<smiles>c1ccc2c(Cc3cccc4ccccc34)cccc2c1</smiles>

$\mathrm{E}$<smiles>[GeH3]</smiles>

B<smiles>c1cc2c3c(ccc(-c4ccc5c6c(cccc46)CC5)c3c1)CC2</smiles>

C<smiles></smiles>

D

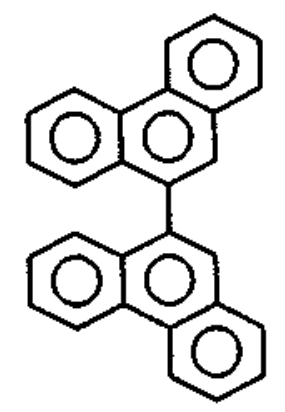

F<smiles>c1ccc2c(-c3c4ccccc4cc4ccccc34)c3ccccc3cc2c1</smiles>

G<smiles>O=Cc1c2ccccc2c(-c2c3ccccc3cc3ccccc23)c2ccccc12</smiles>

$\mathrm{H}$

FIGURE 32: Molecular structures of bi-PAHs (bi-polycyclic aromatic hydrocarbons): (A) 1,1'-binaphthalene, (B) 2,2'binaphthalene, (C) 1,1',2,2'-tetrahydro-5,5'-biacenaphthylene, (D) 5,5'-biacenaphthylene, (E) 9,9'-biphenanthrene, (F) 1,1'-methylenebisnaphthlene, (G) 9,9'-bianthracene, and (H) 10-carboxaldehyde-9, $9^{\prime}$-bianthracene. 
phenazine as pale yellow needles (79\% yield), m.p. 296-298 (uncorrected), $\mathrm{M}^{+} 308(61 \%)$, found: $\mathrm{C}, 86.0 ; \mathrm{H}, 5.3_{5} ; \mathrm{N}$, 9.0 , and calculated: $C, 85.7 ; \mathrm{H}, 5.2 ; \mathrm{N}, 9.1 \%$. The five methylfluoranthenes were synthesized via procedures described in the literature (5) as follows: 1-amino-, 2amino-, 3-amino-, 7-amino-, and 8-aminofluoranthene were synthesized and purified. From each of these aminofluoranthenes the corresponding methylfluoranthene was prepared via diazotization $\left(\mathrm{NaNO}_{2}, \mathrm{H}_{2} \mathrm{SO}_{4}\right)$, replacement of the diazo group by bromine (CuBr), replacement of the bromine by cyanide (CucN, dimethylformamide), conversion of the cyanide into the aldehyde (diisobutylaluminium hydride, tetrahydrofuran), and finally conversion of the aldehyde into the methyl derivative (Wolff-Kishner reduction with $\mathrm{H}_{2} \mathrm{NNH}_{2}$ and $\mathrm{KOH}$ in diethylene glycol). The five methylfluoranthenes were purified by means of column chromatography on silica and each of them was fully characterized by ${ }^{1} \mathrm{H}$ NMR (nuclear magnetic resonance), UV (ultraviolet) and IR (infrared) spectroscopy, and mass spectrometry $(6,7)$.

Pyrene, coronene, chrysene, and benzo[ghi]perylene were purchased commercially from Aldrich Chemical Company and recrystallized several times from methanol or absolute ethanol, and 1-pyrenecarboxaldehyde, purchased from Aldrich, was recrystallized several times from cyclohexane. Acenaphthylene was also purchased from Aldrich and purified by vacuum sublimnation. The remaining PACs were synthesized 
and purified by procedures described in the chemical literature. The chemical supplier and/or synthetic references are given in Tables III-XII. Those solutes obtained from commercial sources not mentioned previously were used as received. The addresses of the commercial and noncommercial chemical suppliers are given in Table XIII.

Stock solutions were prepared by dissolving 1,3-pyrenedicarboxaldehyde, 1-aminopyrene, naphtho $\left[1,8 b c ; 5,4 b^{\prime} c^{\prime}\right]-$ dipyran, and naphtho $\left[1,8 b c ; 4,5 b^{\prime} c^{\prime}\right] d i p y r a n$ in cyclohexane as they are known to be fairly reactive with dichloromethane and by dissolving trans-10b,10c-dihydro-10b,10c-dimethylpyrene, trans-12b,12c-dihydro-12b,12c-dimethylbenzo[a]pyrene and trans-14b,14c-dihydro-14b,14c-dimethylnaphtho[2,1,8qra]naphthacene in deoxygenated cyclohexane because they readily oxidize in chlorinated solvents. All other solutes were dissolved in dichloromethane. Solutions were placed in amber glass bottles and stored in the dark to retard any photochemical reactions between the PAC solute and dichloromethane solvent. Carbon tetrachloride and chloroform (to a much lesser extent) are reported to react with PAHs via a hypothesized concerted tranannular addition with free radical formation (8-12). Small aliquots of the stock solutions were transferred into test tubes, allowed to evaporate, diluted with the solvent of interest, vortexed, sonicated, and vortexed again to redissolve the solute. Limited solubility of the larger PAC solutes did present a 
TABLE III. Summary of chemical supplier and/or synthetic references for select PAH6 benzenoids.

Letter ${ }^{a}$

Chemical supplier
Synthetic

References
A

B

$\mathrm{C}$

D

E

$\mathbf{F}$

G

H

I

$\mathrm{J}$

$\mathrm{K}$

L

$\mathbf{M}$

$\mathrm{N}$

0

$\mathbf{P}$

Q

R

$\mathrm{S}$

$\mathrm{T}$

Aldrich Chemical co.

John C. Fetzer, Ph.D.

$(13,14)$

John C. Fetzer, Ph.D.

$(13,14)$

John C. Fetzer, Ph.D.

$(13,14)$

John C. Fetzer, Ph.D.

$(13,14)$

John C. Fetzer, Ph.D.

$(13,14)$

John C. Fetzer, Ph.D.

$(13,14)$

John C. Fetzer, Ph.D.

$(13,14)$

John C. Fetzer, Ph.D.

$(13,14)$

Hans-Fr. Grützmacher, Ph.D.

John C. Fetzer, Ph.D.

John C. Fetzer, Ph.D.

John C. Fetzer, Ph.D.

$(13,14)$

Aldrich Chemical co.

Aldrich Chemical co.

Alarich Chemical Co.

John C. Fetzer, Ph.D.

$(13,14)$

John C. Fetzer, Ph.D.

$(13,14)$

John C. Fetzer, Ph.D.

$(13,14)$

John C. Fetzer, Ph.D.

$(13,14)$

Aldrich Chemical co. 
TABLE III. continued.

Letter $\quad$ Chemical Supplier

Synthetic

References

$\mathrm{U}$

Aldrich Chemical co.

John C. Fetzer, Ph.D.

$(13,14)$

V

Accustandard

$\mathbf{W}$

John C. Fetzer, Ph.D.

$(13-16)$

John C. Fetzer, Ph.D.

$(13-16)$

John C. Fetzer, Ph.D.

$(13-16)$

John C. Fetzer, Ph.D.

$(13-16)$

John C. Fetzer, Ph.D.

$(13,14)$

AA

John C. Fetzer, Ph.D.

$(13,14)$

AB

John C. Fetzer, Ph.D.

$(13,14)$

AC

John C. Fetzer, Ph.D.

$(13,14)$

$A D$

John C. Fetzer, Ph.D.

AF

John C. Fetzer, Ph.D.

$(13,14)$

AG

John c. Fetzer, Ph.D.

$(13,14)$

AH

John C. Fetzer, Ph.D.

$(13,14)$

AI

John C. Fetzer, Ph.D.

$(13,14)$

AJ

John C. Fetzer, Ph.D.

$(13,14)$

AK

John C. Fetzer, Ph.D.

$(13,14)$

AL

John C. Fetzer, Ph.D.

$(13,14)$

$\mathrm{AM}$

John C. Fetzer, Ph.D.

$(13,14)$

AN

John C. Fetzer, Ph.D.

$(13,14)$

AO

Maximilian Zander, Ph.D.

(17) 
TABLE III. Continued.

\begin{tabular}{|c|c|c|}
\hline Letter ${ }^{a}$ & Chemical supplier & $\begin{array}{l}\text { Synthetic } \\
\text { References }\end{array}$ \\
\hline AP & John C. Fetzer, Ph.D. & $(13,14)$ \\
\hline $\mathrm{AQ}$ & John C. Fetzer, Ph.D. & $(13,14)$ \\
\hline AR & John C. Fetzer, Ph.D. & $(13,14)$ \\
\hline AS & John C. Fetzer, Ph.D. & $(13,14)$ \\
\hline AT & Maximilian Zander, Ph.D. & $(18)$ \\
\hline $\mathrm{AU}$ & Maximilian Zander, Ph.D. & $(19)$ \\
\hline AV & Maximilian Zander, Ph.D. & $(20)$ \\
\hline $\mathrm{AW}$ & Maximilian Zander, Ph.D. & $(21)$ \\
\hline $\mathrm{AX}$ & Maximilian Zander, Ph.D. & $(22)$ \\
\hline$A Y$ & Maximilian Zander, Ph.D. & $(18)$ \\
\hline AZ & Aldrich Chemical Co. & \\
\hline $\mathrm{BA}$ & Aldrich Chemical Co. & \\
\hline $\mathrm{BB}$ & Aldrich Chemical co. & \\
\hline $\mathrm{BC}$ & Aldrich Chemical co. & \\
\hline BD & Aldrich Chemical co. & \\
\hline $\mathrm{BE}$ & Ronald G. Harvey, Ph.D. & $(23)$ \\
\hline $\mathrm{BF}$ & Ronald G. Harvey, Ph.D. & $(24)$ \\
\hline
\end{tabular}

a The letters refer to compounds in Figures 13-17. 
TABLE IV. Summary of chemical supplier and/or synthetic references for select acenaphthylene derivatives.

\begin{tabular}{clr}
\hline Letter & \multicolumn{1}{c}{ Chemical supplier } & $\begin{array}{r}\text { Synthetic } \\
\text { References }\end{array}$ \\
\hline A & Aldrich Chemical Co. \\
B & Ronald G. Harvey, Ph.D. \\
C & Ronald G. Harvey, Ph.D. \\
D & Ronald G. Harvey, Ph.D. \\
E & Ronald G. Harvey, Ph.D. \\
F & Ronald G. Harvey, Ph.D. \\
G & Ronald G. Harvey, Ph.D. \\
H & CTC organics & $(25-27)$ \\
I & Gerald Dyker, Ph.D.
\end{tabular}

a The letters refer to compounds in Figure 22. 
TABLE V. Summary of chemical supplier and/or synthetic references for select cyclopenta-PAH derivatives.

\begin{tabular}{llr}
\hline Letter & Chemical supplier & $\begin{array}{r}\text { Synthetic } \\
\text { References }\end{array}$ \\
\hline A & Ronald G. Harvey, Ph.D. & $(29)$ \\
B & Ronald G. Harvey, Ph.D. \\
C & Ronald G. Harvey, Ph.D. & $(29)$ \\
D & Ronald G. Harvey, Ph.D. \\
E & Ronald G. Harvey, Ph.D. & $(29)$ \\
F & Ronald G. Harvey, Ph.D. \\
G & Ronald G. Harvey, Ph.D. & $(29)$ \\
H & Ronald G. Harvey, Ph.D. & $(29)$ \\
I & Ronald G. Harvey, Ph.D. & $(29)$ \\
J & Ronald G. Harvey, Ph.D. & $(29)$ \\
K & Ronald G. Harvey, Ph.D. & $(29)$ \\
L & Ronald G. Harvey, Ph.D. & $(29)$ \\
M & Ronald G. Harvey, Ph.D. & $(29)$ \\
\hline
\end{tabular}

a The letters refer to compounds in Figure 21 . 
TABLE VI. Summary of chemical supplier and/or synthetic references for select PAH6 benzenoid derivatives.

Letter ${ }^{a}$

Chemical supplier

Synthetic

References

A

B

C

$\mathrm{D}$

$\mathbf{E}$

$\mathbf{F}$

G

$\mathrm{H}$

I

J

$\mathbf{K}$

L

M

N

0

$\mathbf{P}$

Q

$\mathbf{R}$

$S$

$\mathrm{T}$

U
The Thermodynamics Research Center

The Thermodynamics Research Center

The Thermodynamics Research Center

The Thermodynamics Research Center

Frinton Laboratories

The Thermodynamics Research Center

The Thermodynamics Research Center

Aldrich Chemical Co.

Ichiro Murata, Ph.D.

Aldrich chemical co.

Aldrich Chemical Co.

John C. Fetzer, Ph.D.

John C. Fetzer, Ph.D.

Reginald H. Mitchell, Ph.D.

John c. Fetzer, Ph.D.

Armin de Meijere, Ph.D.

Armin de Meijere, Ph.D.

Armin de Meijere, Ph.D.

Reginald H. Mitchell, Ph.D.

Jürgen. Jacob, Ph.D.

$(34,35)$

Jürgen. Jacob, Ph.D. 
TABLE VI. Continued.

\begin{tabular}{clr} 
Letter & \multicolumn{1}{c}{ Chemical Supplier } & $\begin{array}{r}\text { Synthetic } \\
\text { References }\end{array}$ \\
\hline V & The Thermodynamics Research Center \\
W & Maximilian Zander, Ph.D. \\
X & Maximilian Zander, Ph.D. \\
Y & Maximilian Zander, Ph.D. \\
Z & John C. Fetzer, Ph.D. \\
AA & John C. Fetzer, Ph.D. \\
AB & Reginald H. Mitchell, Ph.D. \\
AC & Aldrich Chemical Co. \\
\hline
\end{tabular}

a The letters refer to compounds in Figures 18-20. 
TABLE VII. Sumary of chemical supplier and/or synthetic references for select fluoranthenoids and fluorenoids.

Letter ${ }^{a}$

Chemical Supplier

Synthetic

References
A
Ronald G. Harvey, Ph.D.
B
Ronald G. Harvey, Ph.D.
C
Ronald G. Harvey, Ph.D.
D
Community Bureau of Reference
$\mathbf{E}$
Ronald G. Harvey, Ph.D.
$\mathbf{F}$
Ronald G. Harvey, Ph.D.
Jürgen Jacob, Ph.D.
G
Ronald G. Harvey, Ph.D.
H
Bureau of Community Reference
Ronald G. Harvey, Ph.D.
I
Bureau of Community Reference
$J$
Bureau of Community Reference
K
Bureau of Community Reference
L.
Ronald G. Harvey, Ph.D.
M Ronald G. Harvey, Ph.D.
N Ronald G. Harvey, Ph. D.
O Lawrence T. Scott, Ph.D.
P
John C. Fetzer, Ph.D.
$Q$
John C. Fetzer, Ph.D.

$(42-44)$

a The letters refer to compounds in Figures 24 and 25 . 
TABLE VIII. Summary of chemical supplier and/or synthetic references for select fluoranthenoid derivatives.

\begin{tabular}{|c|c|c|}
\hline Letter ${ }^{a}$ & Chemical supplier & $\begin{array}{l}\text { Synthetic } \\
\text { References }\end{array}$ \\
\hline
\end{tabular}

\begin{tabular}{|c|c|c|}
\hline A & Johan Lugtenburg, Ph.D. & $(5-7)$ \\
\hline B & Johan Lugtenburg, Ph.D. & $(5-7)$ \\
\hline C & Johan Lugtenburg, Ph.D. & $(5-7)$ \\
\hline $\mathrm{D}$ & Johan Lugtenburg, Ph.D. & $(5-7)$ \\
\hline $\mathbf{E}$ & Johan Lugtenburg, Ph.D. & $(5-7)$ \\
\hline F & Lawrence T. Scott, Ph.D. & $(45)$ \\
\hline G & Mary J. Tanga, Ph.D. & $(46)$ \\
\hline H & Mary J. Tanga, Ph.D. & $(47)$ \\
\hline I & Mary J. Tanga, Ph.D. & $(47)$ \\
\hline J & Mary J. Tanga, Ph.D. & $(47)$ \\
\hline K & Mary J. Tanga, Ph.D. & $(48)$ \\
\hline $\mathrm{L}$ & Mary J. Tanga, Ph.D. & $(48)$ \\
\hline M & Mary J. Tanga, Ph.D. & $(49)$ \\
\hline $\mathbf{N}$ & Mary J. Tanga, Ph.D. & $(49)$ \\
\hline 0 & Mary J. Tanga, Ph.D. & $(49)$ \\
\hline $\mathbf{P}$ & Mary J. Tanga, Ph.D. & $(49)$ \\
\hline
\end{tabular}

a The letters refer to compounds in Figures 26 and 27 . 
TABLE IX. Summary of chemical supplier and/or synthetic references for select polycyclic aromatic oxygen heterocycles and polycyclic aromatic sulfur heterocycles.

Letter ${ }^{a} \quad$ Chemical supplier

A

B

C

D

E

F

G

H

I

J

K

L
Maximilian Zander, Ph.D.

Maximilian Zander, Ph.D.

Rütgerwerke AG

Pierre Demerseman, Ph.D.

Pierre Demerseman, Ph.D.

Kazuhiro Nakasuji, Ph.D.

Ichiro Murata, Ph.D.

Kazuhiro Nakasuji, Ph.D.

Ichiro Murata, Ph.D.

Kazuhiro Nakasuji, Ph.D.

Ichiro Murata, Ph.D.

Maximilian Zander, Ph.D.

Maximilian Zander, Ph.D.

Aldrich Chemical Co.

Peter Wan, Ph.D.
(50)

$(51,52)$

$(55,56)$

$(55,56)$

$(55,56)$

a The letters refer to compounds in Figure 28 . 
TABLE X. Summary of chemical supplier and/or synthetic references for select polycyclic aromatic nitrogen heterocyles.

Letter ${ }^{\mathrm{a}}$

Chemical Supplier

Synthetic

References

A

B

C

D

E

F

G

$\mathrm{H}$

\section{I}

丁

K

L

M

$\mathrm{N}$

0

P

Q

R

S

T
Mary J. Tanga, Ph.D.

Mary J. Tanga, Ph.D.

Mary J. Tanga, Ph.D.

Mary J. Tanga, Ph.D.

Maximilian Zander, Ph.D.

Maximilian Zander, Ph.D.

Sumio Tokita, Ph.D.

Mary J. Tanga, Ph.D.

Mary J. Tanga, Ph.D.

Mary J. Tanga, Ph.D.

Mary J. Tanga, Ph.D.

Mary J. Tanga, Ph.D.

Mary J. Tanga, Ph.D.

Mary J. Tanga, Ph.D.

Sumio Tokita, Ph.D.

Jean-Marie Lehn, Ph.D.

Sumio Tokita, Ph.D.

Sumio Tokita, Ph.D.

Sumio Tokita, Ph.D.

Alice M. Clark, Ph.D.
(60)

(60)

$(60)$

(69)

(66)

(74) 
TABLE X. Continued.

\begin{tabular}{clr}
\hline Letter & \multicolumn{1}{c}{ Chenical Supplier } & $\begin{array}{r}\text { Synthetic } \\
\text { References }\end{array}$ \\
\hline U & Heinz Langhals, Ph.D. & $(75)$ \\
V & Erich C. Meister, Ph.D. \\
W & Christopher Upton, Ph.D. \\
X & Christopher Upton, Ph.D. & $(76)$ \\
Y & Christopher Upton, Ph.D. \\
Z & Christopher Upton, Ph.D. \\
AA & Christopher Upton, Ph.D. \\
AB & Henry N. C. Wong, Ph.D. \\
AC & Christopher Upton, Ph.D. & $(78)$ \\
AD & Christopher Upton, Ph.D. & $(79)$ \\
AE & Christopher Upton, Ph.D. & $(81)$ \\
AF & Christopher Upton, Ph.D. & $(78)$ \\
AG & Christopher Upton, Ph.D. & $(78)$ \\
\hline
\end{tabular}

a The letters refer to compounds in Figures 29-31. 
TABLE XI. Sumary of chemical supplier and/or synthetic references for select bi-polycyclic aromatic hydrocarbons. Letter ${ }^{a}$ Chemical Supplier Synthetic References

\begin{tabular}{ll} 
A & Maximilian Zander, Ph.D. \\
B & Maximilian Zander, Ph.D. \\
C & Reginald H. Mitchell, Ph.D. \\
D & Reginald H. Mitchell, Ph.D. \\
E & Maximilian Zander, Ph.D. \\
F & Maximilian Zander, Ph.D. \\
G & Maximilian Zander, Ph.D. \\
H & Maximilian Zander, Ph.D. \\
\hline
\end{tabular}

a The letters refer to compounds in Figure 32 . 
TABLE XII. Summary of chemical supplier and/or synthetic references for select acephenanthrylene derivatives.

Letter ${ }^{a}$

Chemical Supplier

Synthetic

References

\begin{tabular}{ccc}
\hline A & Johan Lugtenburg, Ph.D. & $(84)$ \\
B & Johan Lugtenburg, Ph.D. & $(84)$ \\
C & Johan Lugtenburg, Ph.D. & $(84)$ \\
D & Johan Lugtenburg, Ph.D. \\
E & Johan Lugtenburg, Ph.D. \\
F & Johan Lugtenburg, Ph.D. \\
G & Johan Lugtenburg, Ph.D. \\
H & Johan Lugtenburg, Ph.D. \\
I & Johan Lugtenburg, Ph.D. & $(84)$ \\
\hline
\end{tabular}

a The letters refer to compounds in Figure 23 . 
TABLE XIII. Addresses of chemical suppliers.

\begin{tabular}{|c|c|}
\hline Chemical supplier & Address \\
\hline Accustandard & $\begin{array}{l}25 \text { Science Park, Suite } 687 \\
\text { New Haven, CT 06511, USA }\end{array}$ \\
\hline Aldrich Chemical co. & $\begin{array}{l}1001 \text { West Saint Paul Avenue } \\
\text { Milwaukee, WI 53233, USA }\end{array}$ \\
\hline Alice M. Clark, Ph.D. & $\begin{array}{l}\text { Dept. of Pharmacognosy } \\
\text { University of Mississippi } \\
\text { University, MS } 38677 \text {, USA }\end{array}$ \\
\hline $\begin{array}{l}\text { Community Bureau of } \\
\text { Reference }\end{array}$ & $\begin{array}{l}\text { Directorate General XII } \\
\text { Commission of the European } \\
\text { Communities, } 200 \text { rue de la Loi } \\
1049 \text { Brussels, Belgium }\end{array}$ \\
\hline CTс organics & $\begin{array}{l}792 \text { windsor street } \\
\text { Atlanta, GA } 30315 \text {, USA }\end{array}$ \\
\hline Pierre Demerseman, Ph.D. & $\begin{array}{l}\text { URA CNRS } 1387 \\
\text { Section de Biologie } \\
\text { Institut Curie, F75231 } \\
\text { Paris Cedex 05, France }\end{array}$ \\
\hline Gerald Dyker, Ph.D. & $\begin{array}{l}\text { Institut for Organic Chemistry } \\
\text { TU Braunschweig, Hagenring } 30 \\
\text { W-3300 Braunschweig, Germany }\end{array}$ \\
\hline
\end{tabular}


TABLE XIII. Continued.

Chemical supplier

John C. Fetzer, Ph.D.

Frinton Laboratories

Hans-Fr. Grützmacher, Ph.D.

Ronald G. Harvey, Ph.D.

Jürgen Jacob, Ph.D.

Heinz Langhals, Ph.D.

Jean-Marie Lehn, Ph.D.
Address

Chevron Research and

Technology center

Richmond, CA 94802-0627, USA

W. Grant Ave.

Vineland, NJ 08360, USA

Dept. of Chemistry

University of Bielefeld

D-4800 Bielefeld 1, Germany

Ben May Institute

University of Chicago

Chicago, IL 60637, USA

Biochemisches Institut

für Umweltcarcinogene

D-2070 Großhansdorf, Germany

Institut für organische Chemie

der Universität München

Karlstrasse 23

D-8000 München, Germany

Universite Louis Pasteur de

Strasbourg, Institut de Chimie

1 rue Blaise Pascal

67000 Strasbourg Cedex, France 
TABLE XIII. Continued.

Chemical Supplier

Address

Johan Lugtenburg, Ph.D.

Gorlaeus Laboratories

Leiden University

P.O. Box 9502, 2300 RA Leiden

The Netherlands

Armin de Meijere, Ph.D.

Institut für Organische chemie Georg-August Universität

Tammannstraß 2

D-37077 Göttingen, Germany

Erich Meister, Ph.D.

Physical Chemistry Laboratory

Swiss Federal Institute

of Technology

Universitätstrasse 22

CH-8092 Zurich, Switzerland

Reginald H. Mitchell, Ph.D. Dept. of Chemistry

University of Victoria

Victoria, BC, Canada V8W, 3P6

Ichiro Murata, Ph.D. Dept. of Chemistry

osaka University

Toyonaka, Osaka 560, Japan

Kazuhiro Nakasuji, Ph.D. Institute for Molecular

Science, Myodaiji

Okazaki 444, Japan 
TABLE XIII. Continued.

Chemical supplier Address

NCI Chemical Carcinogen

Reference Standard

Repository

Rütgerswerke AG

Mary J. Tanga, Ph.D.

The Thermodynamics Research Center

Sumio Tokita, Ph.D.

Christopher Upton, Ph.D.
Midwest Research Institute

425 Volher Boulevard

Kansas City, MO 64110, USA

P.O. Box $200241 / 51 / 61$

44558 Castrop-Rauxel, FRG

Chemistry Department

Boston College

Chestnut Hill, MA 02167, USA

SRI International

333 Ravenswood Avenue

Menlo Park, CA 94025, USA

Texas A\& M University

College station, TX 77840 USA

Dept. of Applied Chemistry

Saitama University, Shimo-

Ohkubo, Urawa-shi, 338 Japan

School of Pharmacy and

Pharmacology, University of

Bath, Claverton Down,

Bath, BA2 7AY, England, UK 
TABLE XIII. Continued.

\begin{tabular}{ll}
\hline Chemical Supplier & \multicolumn{1}{c}{ Address } \\
\hline Peter Wan, Ph.D. & $\begin{array}{l}\text { Department of Chemistry } \\
\text { P.O. Box } 3055 \\
\text { University of Victoria } \\
\text { Victoria B.C., Canada V8W 3P6 }\end{array}$ \\
Henry N. C. Wong, Ph.D. & Dept. of Chemistry \\
& The Chinese University of Hong \\
& Kong, Shatin, NT, Hong Kong \\
& Rütgerswerke AG \\
Maximilian Zander, Ph.D. & P.0. Box 2002 41/51/61 \\
& 44558 Castrop-Rauxel, FRG \\
\hline
\end{tabular}


problem in many of the solvents studied. The resulting solutions had to be vigorously vortexed and warmed to about $40-50^{\circ} \mathrm{C}$ in order to redissolve the PAC molecule back into solution from the test tube walls. Final solute concentrations were sufficiently dilute to minimize innerfiltering artifacts $\left(<10^{-6}\right.$ Molar). A dilute $\mathrm{HClO}_{4}-$ trifluoroethanol solution used in the PANH protonation studies was prepared by dissolving one Pasteur pipette drop of concentrated perchloric acid in $150 \mathrm{~mL}$ of $2,2,2-$ trifluoroethanol. Solvents were of HPLC, spectroquality or AR grade, purchased commercially from either Aldrich, Burdick and Jackson, Fisher Scientific, or Lancaster and the resulting solutions were optically dilute (absorbances $\mathrm{cm}^{-1}$ $<0.01$ ) at all wavelengths, except for the nitromethane and 1,2,4-trimethoxybenzene quenching studies.

Absorption spectra were recorded on a Bausch and Lomb Spectronic 2000, Milton Roy Spectronic 1001 Plus, and/or a Hewlett-Packard 8450A photo-diode array spectrophotometer in the usual manner using a $1-\mathrm{cm}^{2}$ quartz cuvette. The excitation and emission wavelengths and solvent polarity probe behavior results for PAH6 benzenoid solutes F, G, and $S$ were taken from waris et al. (85) and for solutes $W, X, Y$, and $\mathrm{Z}$ were taken from waris et al. (86). Fluorescence spectra for PASH solutes F, G, and $H$ were run on a PerkinElmer Model LS-5 spectrofluorometer equipped with a Model 3600 data station (Kent State University, KSU) with 
excitation and emission slit width settings of $15 \mathrm{~nm}$ and 5 nm, respectively. For PASH compounds F, G, and $H$ the larger $5 \mathrm{~nm}$ emission slit width was necessary because of the much weaker fluorescence intensities. All studies involving PAH6 benzenoid solutes $A A, A B, A D, A E, A L, A P$, and $A S$ were run on a Perkin-Elmer MPF-66 spectrofluorometer equipped with a thermostated sample cell (Chevron) with excitation and emission slit width settings of 15 and $2 \mathrm{~nm}$, respectively. All other PAC solutes contained in Tables III-XII were run on a Shimadzu RF-5000U spectrofluorometer with the detector set at high sensitivity (University of North Texas, UNT) with excitation and emission slit width settings of 15 and 3 $\mathrm{nm}$, respectively. In the case of pyrene (solvent polarity probe measurements only) the emission slit width was closed to $1.5 \mathrm{~nm}$ so that calculated emission intensity ratios would be consistent with published values taken from the chemical literature $(87,88)$. Street and Acree had previously shown that the intensity ratios of select vibronic bands in the pyrene fluorescence spectra are a function of emission slit width (89). This was not necessary in the case of the other PAC solutes as all measurements were preformed at UNT. Listed in Tables XIV-XXIII are excitation and emission wavelengths for all PAC solutes examined in their respective solvents. The peak maximum generally tend to be shifted to slightly longer wavelengths in the more polar solvents like dimethyl sulfoxide. Fluorescence data were accumulated in a 
TABLE XIV. Summary of major excitation and emission wavelengths of select PAH6 benzenoids.

\begin{tabular}{|c|c|c|c|c|c|}
\hline Letter ${ }^{a}$ & $\lambda_{\text {ex }}(\mathrm{nm})$ & & $\lambda_{\text {em }}$ & $(\mathrm{nm})$ & Solvent \\
\hline A & 334 & 426 & 433 & $\begin{array}{lll}444 & 453 & 472\end{array}$ & $\mathrm{ACN}$ \\
\hline $\mathrm{B}$ & 316 & 430 & 438 & $448 \quad 457$ & $\mathrm{ACN}$ \\
\hline c & 333 & 442 & 454 & $461 \quad 470$ & $\mathrm{ACN}$ \\
\hline $\mathrm{D}$ & 324 & 432 & 451 & 459 & $\mathrm{ACN}$ \\
\hline $\mathbf{E}$ & 322 & 420 & 460 & $480 \quad 490$ & $\mathrm{ACN}$ \\
\hline $\mathbf{F}$ & 425 & 486 & 510 & 519 & $\mathrm{BA}$ \\
\hline G & 440 & 505 & & & $\mathrm{DCM}$ \\
\hline $\mathrm{H}$ & 336 & 534 & 542 & 572 & $\mathrm{BA}$ \\
\hline$I$ & 318 & 420 & 439 & & $\mathrm{ACN}$ \\
\hline $\mathfrak{J}$ & 425 & 467 & 493 & $512 \quad 527$ & $\mathrm{ACN}$ \\
\hline $\mathrm{K}$ & 370 & 472 & 482 & $500 \quad 514$ & $\mathrm{ACN}$ \\
\hline $\mathrm{L}$ & 357 & 488 & 525 & & $A C N$ \\
\hline $\mathbf{M}$ & 338 & 371 & 382 & $391 s-413$ & $\mathrm{ACN}$ \\
\hline $\mathrm{N}$ & 403 & 437 & 465 & 496 & $\mathrm{ACN}$ \\
\hline 0 & 380 & 405 & 417 & 428 & $\mathrm{ACN}$ \\
\hline $\mathbf{P}$ & 445 & 484 & $s-49$ & 91504 & DMSO \\
\hline$Q$ & 316 & 416 & 442 & 470 & $\mathrm{ACN}$ \\
\hline $\mathbf{R}$ & 325 & 420 & 440 & & $\mathrm{ACN}$ \\
\hline$s$ & 324 & 442 & 473 & 510 & $\mathrm{CY}$ \\
\hline $\mathrm{T}$ & 350 & 402 & 427 & 453 & $\mathrm{ACN}$ \\
\hline U & 335 & 376 & 386 & $396 \quad 407 \quad 418$ & $\mathrm{ACN}$ \\
\hline
\end{tabular}


TABLE XIV, Continued.

\begin{tabular}{|c|c|c|c|c|c|c|}
\hline Letter ${ }^{a}$ & $\lambda_{\text {ex }}(\mathrm{nm})$ & & $\lambda_{\mathrm{em}}$ & $(\mathrm{nm})$ & & Solvent ${ }^{b}$ \\
\hline $\mathrm{V}$ & 360 & 394 & 417 & 442 & & $\mathrm{ACN}$ \\
\hline $\mathrm{W}$ & 393 & 468 & 485 & 498 & & $\mathrm{CY}$ \\
\hline $\mathrm{x}$ & 510 & 568 & 616 & & & $\mathrm{CY}$ \\
\hline $\mathrm{Y}$ & 410 & 486 & 522 & & & $\mathrm{BA}$ \\
\hline $\mathrm{z}$ & 380 & 404 & 425 & 450 & $s-480 \quad 492 \quad 515$ & CY \\
\hline $\mathrm{AA}$ & 370 & 470 & 510 & 525 & 540 & $\mathrm{BA}$ \\
\hline $\mathrm{AB}$ & 330 & 485 & 518 & 555 & & BA \\
\hline$A C$ & 312 & 438 & 466 & 499 & & $\mathrm{ACN}$ \\
\hline$A D$ & 330 & 430 & 440 & 450 & $460 \quad 490$ & $\mathrm{BA}$ \\
\hline $\mathrm{AE}$ & 365 & 455 & 470 & 485 & $500 \quad 510 \quad 520$ & $\mathrm{HXD}$ \\
\hline $\mathrm{AF}$ & 321 & 451 & 482 & 515 & & $\mathrm{ACN}$ \\
\hline AG & 411 & 444 & 474 & $s-50$ & & $\mathrm{ACN}$ \\
\hline AH & 310 & 419 & $\mathrm{~s}-43$ & $30 \quad 44$ & $44 \quad 472$ & $\mathrm{ACN}$ \\
\hline AI & 306 & 431 & 457 & 488 & & $\mathrm{ACN}$ \\
\hline $\mathrm{AJ}$ & 307 & 432 & 446 & 459 & 490 & $\mathrm{ACN}$ \\
\hline$A K$ & 315 & 438 & 467 & 498 & & $\mathrm{ACN}$ \\
\hline AL & 322 & 388 & 451 & 481 & 514 & $\mathrm{HXD}$ \\
\hline$A M$ & 366 & 416 & 441 & 469 & & $A C N$ \\
\hline AN & 300 & 413 & 438 & 483 & $s-514$ & $\mathrm{ACN}$ \\
\hline AO & 300 & 441 & 470 & 501 & & $\mathrm{BA}$ \\
\hline $\mathrm{AP}$ & 318 & 351 & 367 & 384 & & HXD \\
\hline $\mathrm{AQ}$ & 410 & 486 & 522 & & & $\mathrm{ACN}$ \\
\hline
\end{tabular}


TABLE XIV. Continued.

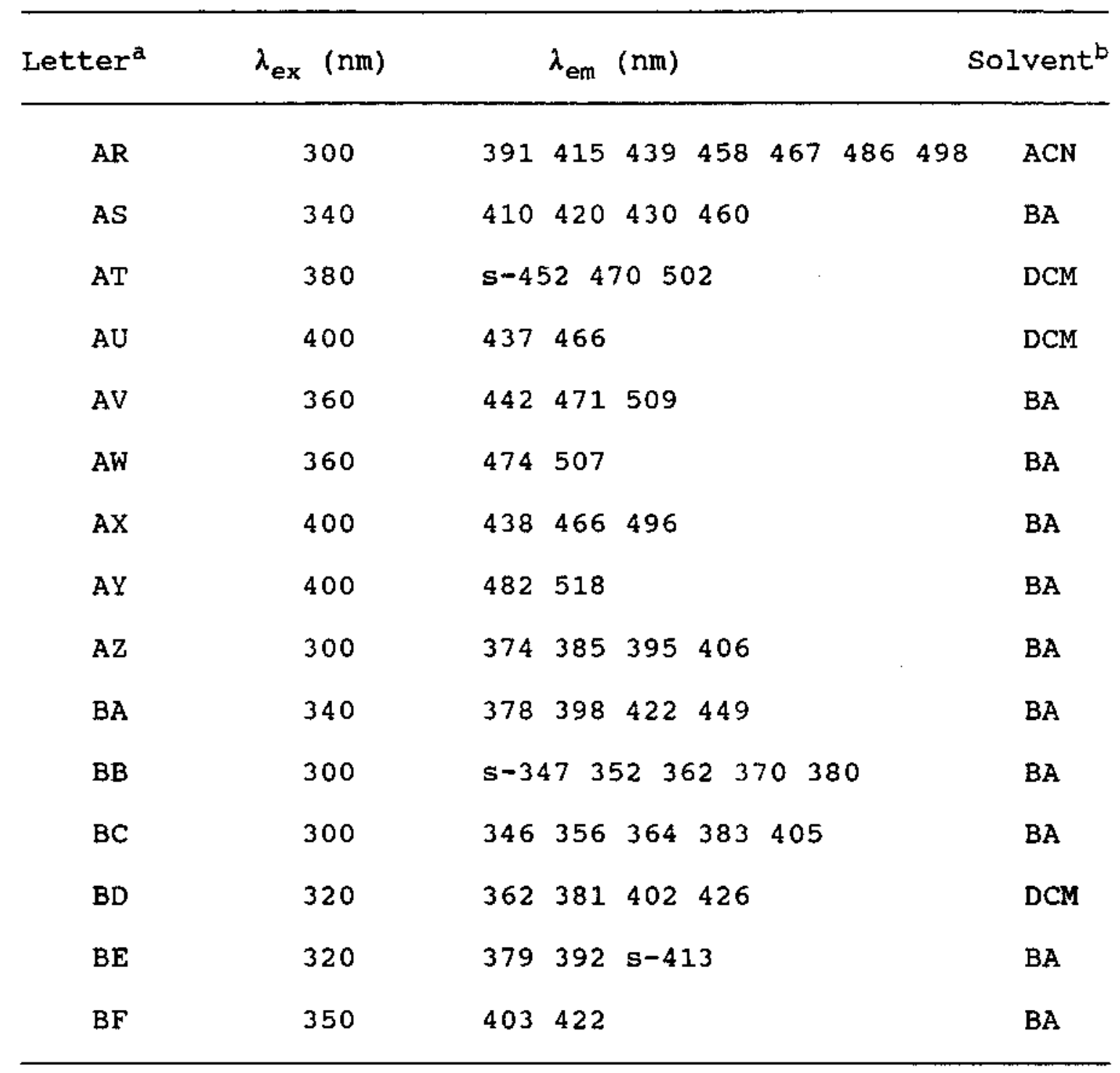

a The letters refer to compounds in Figures 13-17, $\mathrm{b} A C N=$ acetonitrile, $\mathrm{BA}=$ buty 1 acetate, $\mathrm{CY}=$ cyclohexane, $\mathrm{DCM}=$ dichloromethane, DMSO $=$ dimethyl sulfoxide, $\operatorname{HXD}=n-$ hexadecane, and "s-" denotes a shoulder. 
TABLE XV. Summary of major excitation and emission wavelengths of select PAH6 benzenoid derivatives.

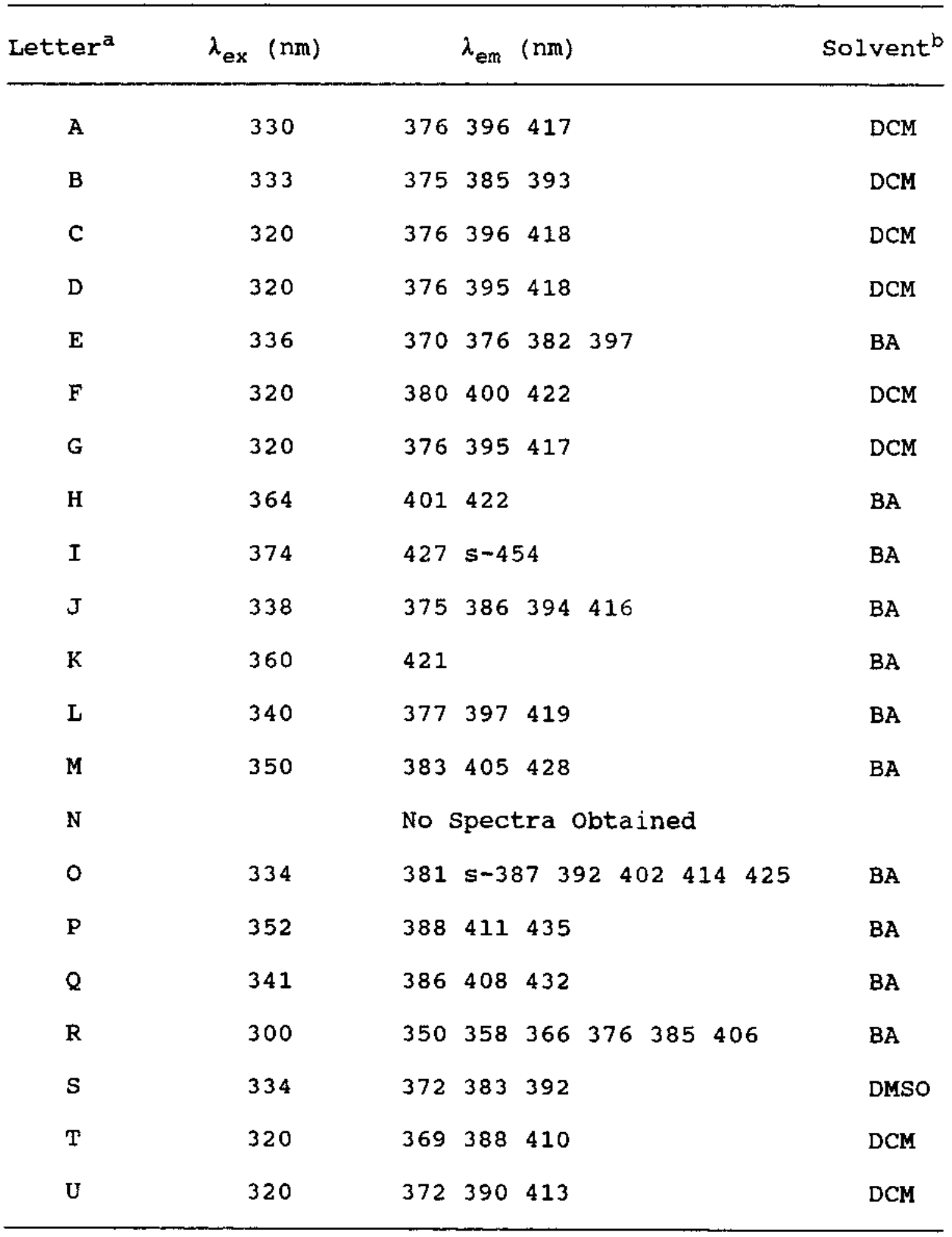


TABLE XV. Continued.

\begin{tabular}{|c|c|c|c|}
\hline Letter ${ }^{a}$ & $\lambda_{\mathrm{ex}}(\mathrm{nm})$ & $\lambda_{\text {em }}(\mathrm{nm})$ & Solvent \\
\hline $\mathrm{V}$ & 300 & 462493 & DCM \\
\hline $\mathrm{W}$ & 358 & $\begin{array}{lllll}393 & 403 & 415 & 438 & 464\end{array}$ & BA \\
\hline $\mathrm{x}$ & 400 & $438 \quad 466 \quad 492$ & $\mathrm{BA}$ \\
\hline $\mathrm{Y}$ & 304 & $s-426 \quad 441 \quad 468 \quad 499$ & $\mathrm{BA}$ \\
\hline $\mathrm{z}$ & 340 & $\begin{array}{llllll}427 & 434 & 446 & 454 & 475 & 484\end{array}$ & BA \\
\hline $\mathrm{AA}$ & 305 & $430 \mathrm{~s}-437 \quad 448 \quad 456 \quad 478 \quad 487$ & BA \\
\hline \multirow[t]{2}{*}{$\mathrm{AB}$} & 320 & $\begin{array}{llll}374 & 392 & 418 & 432\end{array}$ & DMSO \\
\hline & & $451 \quad 459 \quad 480 \quad 490$ & \\
\hline$A C$ & 340 & $382 \quad 404 \quad 428 \quad s-454$ & $\mathrm{BA}$ \\
\hline
\end{tabular}

a The letters refer to compounds in Figures 18-20. b $\mathrm{BA}=$ butyl acetate, DCM = dichloromethane, DMSO = dimethyl sulfoxide, and "s-" denotes a shoulder. 
TABLE XVI. Summary of major excitation and emission wavelengths of select cyclopenta-polycyclic aromatic hydrocarbons derivatives.

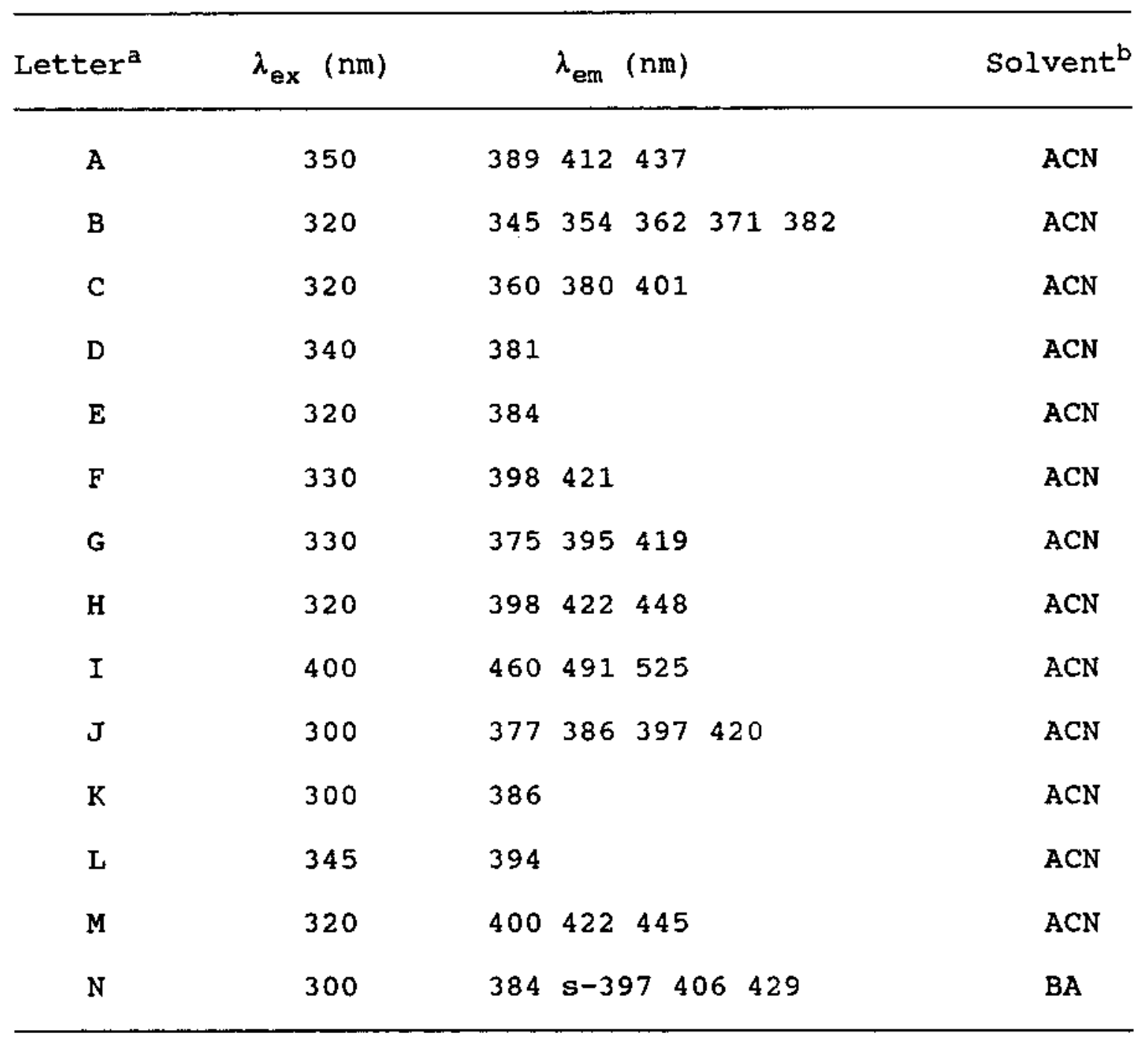

a The letters refer to compounds in Figure $21 . \mathrm{b} A C N=$ acetonitrile, $\mathrm{BA}=$ butyl acetate, and "s-" denotes a shoulder. 
TABLE XVII. Summary of major excitation and emission wavelengths of select acenaphthylene derivatives.

\begin{tabular}{|c|c|c|c|c|c|}
\hline Letter ${ }^{a}$ & $\lambda_{\text {ex }}(\mathrm{nm})$ & & $\lambda_{\text {em }}$ & $(\mathrm{nm})$ & Solvent \\
\hline $\mathrm{A}$ & 288 & 320 & 326 & $336 \quad 353$ & $\mathrm{BA}$ \\
\hline B & 360 & 402 & 426 & 450 & $\mathrm{BA}$ \\
\hline $\mathrm{C}$ & 300 & 354 & 374 & 393 & $\mathrm{BA}$ \\
\hline D & 360 & 398 & 423 & 448 & $\mathrm{BA}$ \\
\hline$E$ & 370 & 395 & 421 & 445 & BA \\
\hline $\mathbf{F}$ & 300 & 405 & 427 & & $\mathrm{BA}$ \\
\hline G & 300 & 410 & 432 & 459 & $\mathrm{BA}$ \\
\hline $\mathrm{H}$ & 336 & 378 & 398 & $422 \quad 479$ & $\mathrm{BA}$ \\
\hline$I$ & 406 & 438 & 466 & 501 & $\mathrm{BA}$ \\
\hline
\end{tabular}

a The letters refer to compounds in Figure $22 . \quad \mathrm{b}$ BA $=$ butyl acetate. 
TABLE XVIII. Summary of major excitation and emission wavelengths of select polycyclic aromatic oxygen heterocycles and polycyclic aromatic sulfur heterocycles.

\begin{tabular}{|c|c|c|c|c|}
\hline Letter ${ }^{a}$ & $\lambda_{\text {ex }}(\mathrm{nm})$ & & $\lambda_{\text {em }}(n m)$ & Solvent \\
\hline A & 300 & 351 & $369 s-388$ & $\mathrm{ACN}$ \\
\hline B & 300 & 357 & 375 & $\mathrm{ACN}$ \\
\hline $\mathrm{C}$ & 315 & 353 & 370 & $\mathrm{BA}$ \\
\hline D & 358 & 454 & $480 \quad 515$ & $\mathrm{ACN}$ \\
\hline$E$ & 406 & 448 & 515 & $A C N$ \\
\hline $\mathbf{F}$ & 400 & 470 & 505605 & CY \\
\hline G & 380 & 575 & 625 & $\mathrm{CY}$ \\
\hline $\mathrm{H}$ & 400 & 480 & 525565 & $\mathrm{CY}$ \\
\hline I & 370 & 430 & $s-452$ & $\mathrm{BA}$ \\
\hline $\mathrm{J}$ & 380 & 414 & 438 & BA \\
\hline $\mathrm{K}$ & 250 & 433 & $444 \quad 529$ & $\mathrm{CY}$ \\
\hline L & 300 & 445 & $475 \quad 505$ & $\mathrm{BA}$ \\
\hline
\end{tabular}

a The letters refer to compounds in Figure 28 . $\mathrm{b} A C N=$ acetonitrile, $\mathrm{BA}=$ butyl acetate, $\mathrm{CY}=$ cyclohexane, and "s-" denotes a shoulder. 
TABLE XIX. Summary of major excitation and emission wavelengths of select polycyclic aromatic nitrogen heterocycles.

\begin{tabular}{|c|c|c|c|c|c|}
\hline Letter $a$ & $\lambda_{\mathrm{ex}}(\mathrm{nm})$ & & $\lambda_{\text {en }}$ & $(\mathrm{nm})$ & Solvent ${ }^{b}$ \\
\hline A & 338 & 376 & 396 & 419 & $\mathrm{ACN}$ \\
\hline B & 332 & 369 & 377 & $388 \quad 409$ & $\mathrm{ACN}$ \\
\hline $\mathrm{c}$ & 331 & 371 & 379 & $390 \quad 411$ & $\mathrm{ACN}$ \\
\hline $\mathrm{D}$ & 364 & 401 & 424 & 449 & $\mathrm{ACN}$ \\
\hline $\mathbf{E}$ & 340 & 461 & & & DCM \\
\hline$F$ & 360 & 470 & & & $\mathrm{DCM}$ \\
\hline$G$ & 350 & 397 & 420 & 446 & $\mathrm{BA}$ \\
\hline $\mathrm{H}$ & 300 & 393 & 416 & 442 & $\mathrm{BA}$ \\
\hline$I$ & 330 & 391 & 414 & 440 & $\mathrm{ACN}$ \\
\hline $\mathrm{J}$ & 340 & 388 & 410 & 435 & $\mathrm{BA}$ \\
\hline$K$ & 300 & 363 & 381 & 402 & $\mathrm{BA}$ \\
\hline $\mathrm{L}$ & 300 & 380 & 400 & 422 & $\mathrm{BA}$ \\
\hline $\mathbf{M}$ & 340 & 383 & 406 & 430 & BA \\
\hline $\mathbf{N}$ & 300 & 394 & 416 & 442 & $\mathrm{BA}$ \\
\hline 0 & 317 & 414 & 434 & $461 \quad 490$ & $\mathrm{BA}$ \\
\hline $\mathbf{P}$ & 328 & 382 & 403 & 426 & $\mathrm{BA}$ \\
\hline$Q$ & 374 & 429 & 454 & & $\mathrm{DCM}$ \\
\hline $\mathbf{R}$ & 395 & 444 & 470 & & $\mathrm{DCM}$ \\
\hline $\mathrm{S}$ & 400 & 442 & 484 & & BA \\
\hline $\mathrm{T}$ & 354 & 475 & $s-49$ & & $\mathrm{DCM}$ \\
\hline
\end{tabular}


TABLE XIX. Continued.

\begin{tabular}{|c|c|c|c|c|}
\hline Letter ${ }^{a}$ & $\lambda_{\text {ex }}(n m)$ & $\lambda_{\text {em }}(r$ & $(\mathrm{nm})$ & Solvent ${ }^{b}$ \\
\hline $\mathrm{U}$ & 410 & $439467 \quad 4$ & 498 & $\mathrm{BA}$ \\
\hline v & 370 & 430 & & DCM \\
\hline W & 390 & $454 \quad 4775$ & $512 \quad 547$ & $\mathrm{BA}$ \\
\hline$x$ & 365 & 421 & & BA \\
\hline $\mathrm{Y}$ & 300 & 3663854 & $406 \quad 432$ & $\mathrm{BA}$ \\
\hline $\mathrm{z}$ & 400 & $474 \quad 499$ & & $\mathrm{BA}$ \\
\hline AA & 300 & $s-427443$ & & BA \\
\hline$A B$ & 350 & 467 & & $\mathrm{BA}$ \\
\hline AC & 360 & $399 \quad 421$ & & BA \\
\hline $\mathrm{AD}$ & 300 & $379397 \mathrm{~s}$ & $s-420$ & $\mathrm{BA}$ \\
\hline $\mathrm{AE}$ & 300 & $370 \quad 390 \quad 4$ & $412 \quad 437$ & $\mathrm{BA}$ \\
\hline $\mathrm{AF}$ & 355 & 397421 & & $\mathrm{BA}$ \\
\hline$A G$ & 300 & $376 \quad 394$ & 414 & BA \\
\hline
\end{tabular}

a The letters refer to compounds in Figures 29-31. b $\mathrm{ACN}=$ acetonitrile, $\mathrm{BA}=$ butyl acetate, $\mathrm{DCM}=$ dichloromethane, and "s-" denotes a shoulder. 
TABLE XX. Summary of major excitation and emission wavelengths of select fluoranthenoids and fluorenoids.

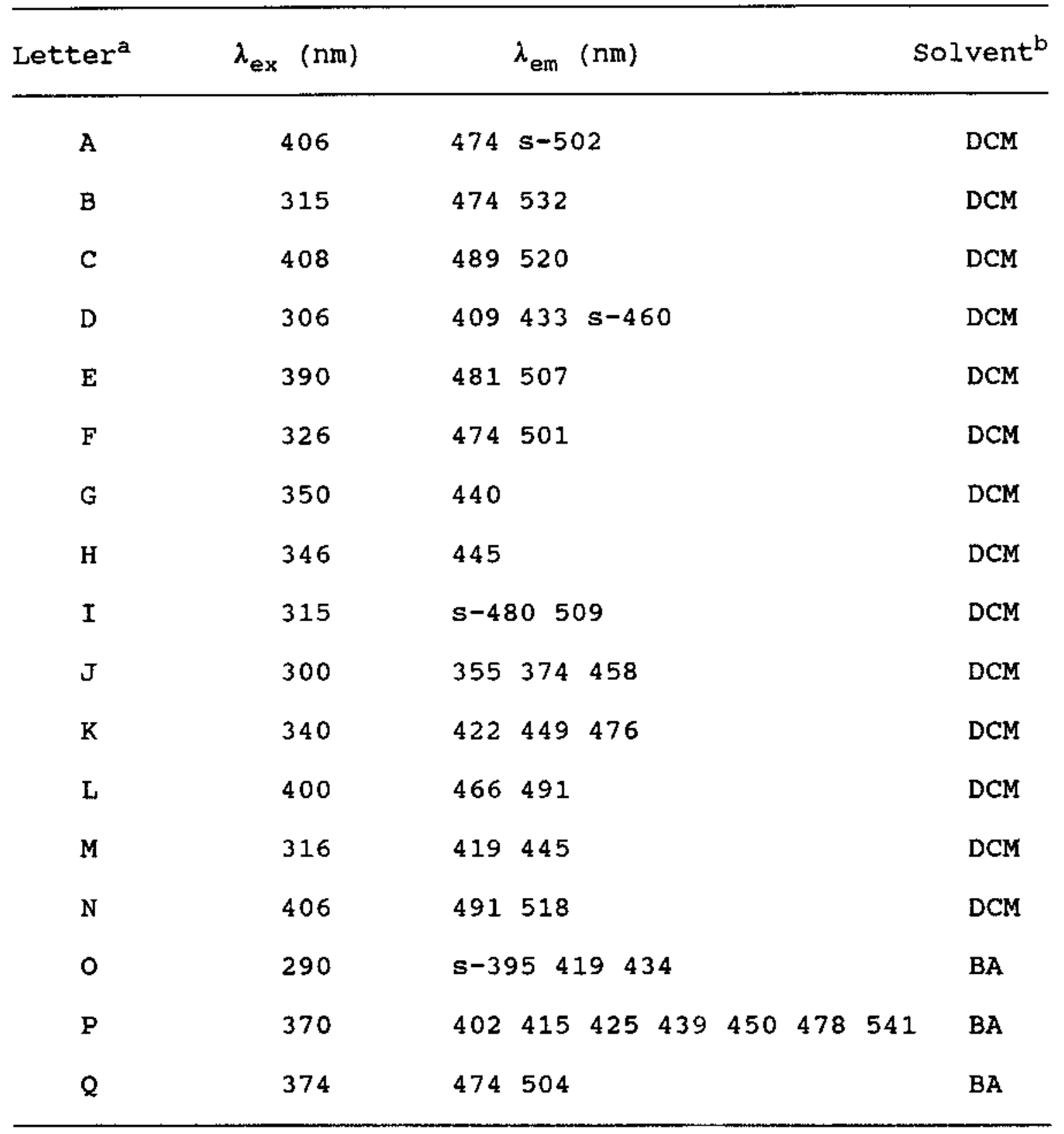

a The letters refer to compounds in Figures 24 and $25 .{ }^{b}$ BA = butyl acetate, $\mathrm{DCM}=$ dichloromethane, and "s-" denotes a shoulder. 
TABLE XXI. Summary of major excitation and emission wavelengths of select fluoranthenoid derivatives.

\begin{tabular}{|c|c|c|c|}
\hline Letter ${ }^{a}$ & $\lambda_{\text {ex }}(\mathrm{nm})$ & $\lambda_{\mathrm{em}}(\mathrm{nm})$ & Solvent \\
\hline $\mathrm{A}$ & 360 & $s-445 \quad 459$ & $\mathrm{BA}$ \\
\hline B & 355 & 462 & $\mathrm{BA}$ \\
\hline$c$ & 355 & $446 \quad 461$ & $\mathrm{BA}$ \\
\hline $\mathrm{D}$ & 357 & $s-446 \quad 464$ & BA \\
\hline $\mathrm{E}$ & 355 & 454 & $\mathrm{BA}$ \\
\hline$F$ & 300 & $422 \quad 437$ & $\mathrm{BA}$ \\
\hline G & 347 & 443 & BA \\
\hline $\mathrm{H}$ & 346 & 398426 & BA \\
\hline I & 347 & $400 \quad 427 \quad 448$ & $\mathrm{BA}$ \\
\hline $\mathbf{J}$ & 347 & $398 \quad 426$ & $\mathrm{BA}$ \\
\hline $\mathrm{K}$ & 325 & $s-499 \quad 525$ & $\mathrm{BA}$ \\
\hline L & 320 & 520 & $\mathrm{BA}$ \\
\hline $\mathbf{M}$ & 310 & 459 & $\mathrm{BA}$ \\
\hline $\mathrm{N}$ & 310 & $434 \quad 453$ & BA \\
\hline o & 312 & $430 \quad 453$ & $\mathrm{BA}$ \\
\hline $\mathbf{P}$ & 380 & $426 \quad 446$ & BA \\
\hline
\end{tabular}

a The letters refer to compounds in Figures 26 and 27 . b BA = butyl acetate and "s-" denotes a shoulder. 
TABLE XXII. Summary of major excitation and emission wavelengths of select bi-polycyclic aromatic hydrocarbons.

\begin{tabular}{|c|c|c|c|}
\hline Letter ${ }^{a}$ & $\lambda_{\text {ex }}(\mathrm{nm})$ & $\lambda_{\text {em }}(\mathrm{nm})$ & Solvent ${ }^{b}$ \\
\hline A & 300 & 362 & $\mathrm{BA}$ \\
\hline $\mathbf{B}$ & 304 & $353 \quad 370$ & $\mathrm{BA}$ \\
\hline $\mathrm{c}$ & 300 & 378 & $\mathrm{BA}$ \\
\hline $\mathrm{D}$ & & No Spectra Obtained & \\
\hline $\mathbf{E}$ & 290 & 324336 & $\mathrm{BA}$ \\
\hline $\mathbf{F}$ & 300 & $356 \quad 374$ & $\mathrm{BA}$ \\
\hline$G$ & 360 & 424 & $\mathrm{BA}$ \\
\hline $\mathrm{H}$ & 370 & 435 & $\mathrm{BA}$ \\
\hline
\end{tabular}

a The letters refer to compounds in Figure 32. b $\mathrm{BA}=$ butyl acetate. 
TABLE XXIII. Summary of major excitation and emission wavelengths of select acephenanthrylene derivatives.

\begin{tabular}{|c|c|c|c|c|c|}
\hline Letter ${ }^{a}$ & $\lambda_{\text {ex }}(n m)$ & & $\lambda_{\mathrm{em}}$ & $(\mathrm{nm})$ & Solvent ${ }^{b}$ \\
\hline A & 304 & 359 & 378 & 397 & $\mathrm{BA}$ \\
\hline B & 300 & 353 & 372 & 391413 & $\mathrm{BA}$ \\
\hline$c$ & 300 & 354 & 372 & 391 & $\mathrm{BA}$ \\
\hline $\mathrm{D}$ & 315 & 354 & 368 & $376 \quad 387410$ & $\mathrm{BA}$ \\
\hline $\mathrm{E}$ & 300 & 359 & 378 & 398 & $\mathrm{BA}$ \\
\hline $\mathbf{F}$ & 304 & 358 & 377 & $397 \quad 418$ & $\mathrm{BA}$ \\
\hline G & 300 & 356 & 374 & $s-391$ & $\mathrm{BA}$ \\
\hline $\mathrm{H}$ & 300 & 358 & 377 & $s-397$ & $\mathrm{BA}$ \\
\hline I & 306 & 358 & 377 & 396 & $\mathrm{BA}$ \\
\hline
\end{tabular}

a The letters refer to compounds in Figure $23 . \quad \mathrm{b} B=$ butyl acetate and "s-" denotes a shoulder. 
quartz $1-\mathrm{cm}^{2}$ cuvette at $19-25^{\circ} \mathrm{C}$, ambient room temperature. The fluorescence spectra of PACs depicted in chapters 3, 4, and 5 represent a single scan when run at UNT which was then solvent blank corrected and verified by repetitive measurements. Those solutes run at $\mathrm{kSU}$ or Chevron represent an average of 9-100 scans which was solvent blank corrected. Utilization of selective quenching reagents, such as nitromethane and trimethoxybenzene, can significantly simplify observed emission spectra. To prevent misidentification, experimentally determined spectra must be free of chemical and instrumental artifacts that might unexpectedly reduce emission intensities. Inner-filtering is a major problem associated with obtaining correct fluorescence data, which assumes that the sample is optically dilute (absorbances $\mathrm{cm}^{-1} \leq 0.01$ ) at all analytical wavelengths. The spectrofluorometers utilized in all studies, like most commercial instruments, employ rightangle fluorometry, which reduces stray radiation by placing the emission detector at $90^{\circ}$ with respect to the incoming excitation beam (see Figure 33). Only fluorescence emission originating from the center interrogation zone of the sample cell is actually collected. Attenuation of the excitation beam before reaching the region viewed by the fluorescence detection optics (pre-filtering region) and through the interrogation volume element is denoted as primary innerfiltering. The correction factor, $f_{\text {prim, for }}$ 


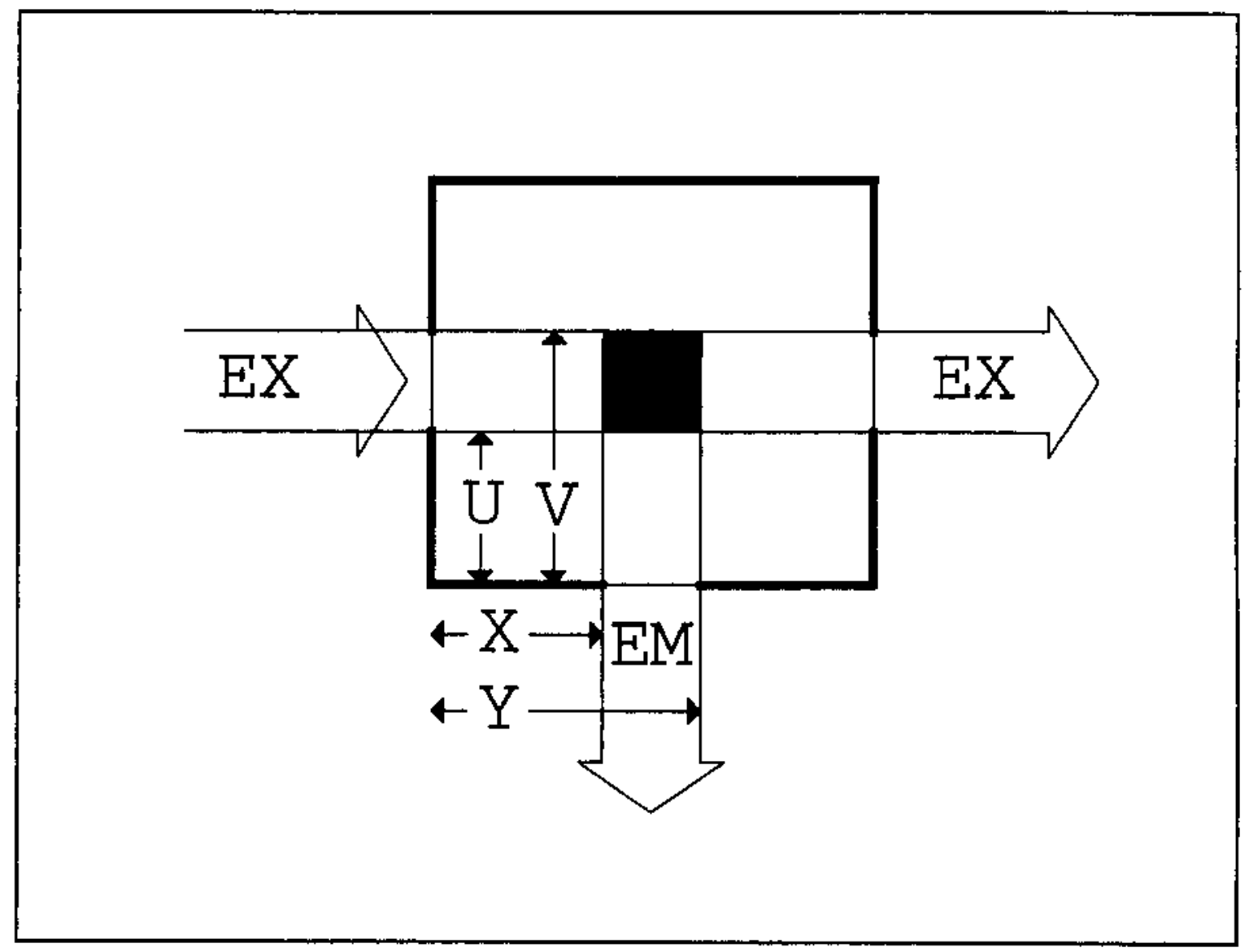

FIGURE 33: Typical cell configuration for right angle fluorometry. Window parameters $(x, y)$ and $(u, v)$ are determined by masking apertures or some other limiting aperture in emission and excitation beam, respectively. 
primary inner-filtering is given by the following expression $(90-95)$

$$
f_{\text {prim }}=\frac{F^{\text {cor } x}}{F^{o b s}}=\frac{2.303 A(y-x)}{10^{-A x}-10^{-A y}}
$$

which differs slightly from the approximate form (93)

$$
f_{\text {prim }} \approx 10^{0.5 \mathrm{~A}}
$$

where $\mathrm{F}^{\text {corr }}$ and $\mathrm{F}^{\mathrm{obs}}$ refer to the corrected and observed fluorescence emission signal, respectively, A is the absorbance per centimeter of pathlength at the excitation wavelength, and $x$ and $y$ denote distances from the boundaries of the interrogation zone to the excitation plane, as shown in Figure 33. Equation 2.1 strictly applies to monochromatic light, which from an experimental standpoint is never achievable, even with the finest spectrofluorometers having small spectral bandpasses. Yappert and Ingle (92) derived a more rigorous mathematical treatment for nonmonochromatic excitation and emission beams.

Primary inner-filtering can often be ignored in solvent polarity experiments requiring determination of intensity ratios as the excitation wavelength remains constant (i.e., A in equation 2.1 remains constant). Emission intensities of both bands are thus affected by the same relative amount. Selective quenching studies are another matter, however, as absorption of the excitation beam by the quenching reagent would reduce emission intensities of every fluorophore having the given excitation wavelength. In the case of 
nitromethane, inner-filtering would reduce emission intensities of both alternant and nonalternant PAHs by the same relative amount. To determine whether selective quenching really occurred, observed emission intensities, $F^{\text {obs }}$, must be multiplied by the inner-filtering correction factor, $f_{\text {prim, }}$ in order to eliminate the undesired effects from this chemical artifact. Failure to correct the observed intensities may lead to erroneous conclusions regarding $P A C$ identification (alternant versus nonalternant), particularly if excitation wavelengths of 300 nm or less are employed. Many of the PACs have excitation wavelengths in the 300-320 $\mathrm{nm}$ spectral region, and a few drops of nitromethane (or trimethoxybenzene) gives solutions with appreciable absorbances.

Secondary inner-filtering results from absorption of large quantities of emitted fluorescence and the correction factor, $f_{\text {sec }}$, contains (95)

$$
\mathrm{f}_{\text {aec }}=\frac{\mathrm{F}^{\mathrm{corr}}}{\mathrm{F}^{\mathrm{obs}}}=\frac{(\mathrm{v}-\mathrm{u})(1 / \mathrm{b}) \ln \mathrm{T}}{\mathrm{T}_{\mathrm{at} \mathrm{v} / \mathrm{b}}-\mathrm{T}_{\mathrm{at} \mathrm{u} / \mathrm{b}}}
$$

the sample transmittance (T) across the entire cell pathlength (b) at the emission wavelength. Transmittances at the two interrogation zone boundaries, $T_{a t ~} \mathrm{v} / \mathrm{b}$ and $\mathrm{T}_{\mathrm{at}} \mathrm{u} / \mathrm{b}$, are calculated from the measured absorbances at the emission wavelength via the Beer-Lambert law. Remember that $v / b$ and $\mathrm{u} / \mathrm{b}$ now serve as the new cell pathlengths. 
For PAC solvent polarity determinations, secondary inner-filtering is a primary concern if the solution preferentially absorbs one of the PAC emission bands, thus leading to different transmittances ( $T$ values in equation 2.3) at the various emission wavelengths and erroneously low intensity ratios. Selective quenching experiments are not generally affected by secondary inner-filtering artifacts. PAC emission bands often appear in the 370-550 nm spectral region where nitromethane's absorbance is greatly diminished. Readers are reminded that only a few Pasteur pipette drops of quenching reagent is used in this type of experiment.

The corrected fluorescence emission intensity is given by

$$
F^{\text {corr }}=f_{\text {prim }} f_{\text {sec }} F^{\text {obs }}
$$

assuming that primary and secondary inner-filtering are independent processes. As a general rule-of-thumb, innerfiltering corrections work well for $f_{p r i m}$ and $f_{\text {sec }}$ values less than three. Calculations of each correction factor requires a prior knowledge of interrogation zone volume and dimensions. Realizing that most instrument manufacturers rarely supply information regarding sample compartment aperture slit widths, particularly for the less expensive spectrofluorometers and fluorescence detectors used in HPLC, $f_{\text {prim }}$ and $f_{\text {sec }}$ computations are based upon assumed values of $\mathbf{x}=\mathbf{u}=0.45 \mathrm{~cm}$ and $\mathrm{y}=\mathrm{v}=0.55 \mathrm{~cm}$. These numerical values 
were previously found to provide accurate inner-filtering corrections for quinine fluorescence inner-filtered by potassium dichromate (96). Use of other numerical values, or use of equation 2.2 , had very little effect on the corrected fluorescence emission intensities. Table XXIV lists numerical values of $f_{p r i m}$ and $f_{\text {gec }}$ as a function of solution absorbance.

"Apparent" acid dissociation constants of protonated PANHs dissolved in a mixed aqueous-acetonitrile solvent (30\% acetonitrile by weight) were estimated from solution absorbances as a function of $\mathrm{pH}$ while holding the stoichiometric moles of PANH constant. Dissolved PANHs were initially protonated by addition of a few drops of concentrated perchloric acid. Absorbances were monitored at 427 and $425 \mathrm{~nm}$, which are the wavelengths corresponding to the dibenzo[a,c]phenazonium and benzo[a]phenazonium ions, respectively. All absorbances were corrected for dilution resulting from addition of a sodium hydroxide solution required to change the $\mathrm{pH}$. Nonaqueous $\mathrm{pH}$-values were measured indirectly as a millivolt reading using a Model 501 Orion Research digital ionalyzer equipped with a standard combination glass electrode. Millivolt readings were converted to pH-values via

$$
\mathrm{pH}_{\mathrm{x}}=\mathrm{pH}_{\mathrm{s}}-\left(\mathrm{E}_{\mathrm{x}}-\mathrm{E}_{\mathrm{g}}\right) / \mathrm{S}
$$

where $\mathrm{pH}_{\mathrm{x}}$ and $\mathrm{pH}_{\mathrm{g}}$ refer to the $\mathrm{pH}$ of the sample $(\mathrm{x})$ and standard (s), respectively, $E_{\mathbf{x}}$ and $E_{s}$ are the millivolt 
TABLE XXIV. Numerical values of primary and secondary inner-filtering correction factors at various absorbances for $x=u=0.45 \mathrm{~cm}$ and $y=v=0.55 \mathrm{~cm}$.

\begin{tabular}{|c|c|c|}
\hline $\mathrm{A} \mathrm{cm}^{-1}$ & $f_{\text {prim }}$ & $f_{\text {sec }}$ \\
\hline 0.000 & 1.000 & 1.000 \\
\hline 0.500 & 1.059 & 1.059 \\
\hline 0.100 & 1.122 & 1.122 \\
\hline 0.150 & 1.189 & 1.188 \\
\hline 0.200 & 1.259 & 1.259 \\
\hline 0.250 & 1.334 & 1.333 \\
\hline 0.300 & 1.413 & 1.412 \\
\hline 0.350 & 1.496 & 1.496 \\
\hline 0.400 & 1.585 & 1.584 \\
\hline 0.450 & 1.678 & 1.678 \\
\hline 0.500 & 1.778 & 1.777 \\
\hline 0.550 & 1.883 & 1.882 \\
\hline 0.600 & 1.994 & 1.994 \\
\hline 0.650 & 2.112 & 2.112 \\
\hline 0.700 & 2.237 & 2.236 \\
\hline 0.750 & 2.369 & 2.368 \\
\hline 0.800 & 2.509 & 2.508 \\
\hline 0.850 & 2.657 & 2.656 \\
\hline 0.900 & 2.814 & 2.813 \\
\hline 0.950 & 2.980 & 2.979 \\
\hline
\end{tabular}


TABLE XXIV. Continued.

\begin{tabular}{ccc}
\hline $\mathrm{A} \mathrm{cm}^{-1}$ & $f_{\text {prim }}$ & $f_{\text {sec }}$ \\
\hline 1.000 & 3.156 & 3.155 \\
1.050 & 3.342 & 3.342 \\
1.110 & 3.539 & 3.359 \\
1.150 & 3.748 & 3.747 \\
1.200 & 3.969 & 3.968 \\
1.250 & 4.203 & 4.202 \\
1.300 & 4.451 & 4.450 \\
1.350 & 4.731 & 4.713 \\
1.400 & 4.991 & 4.990 \\
\hline
\end{tabular}


values of the sample and standard as read by the $\mathrm{pH}$ meter, and $s$ is the Nernstian slope, which is normally $57.18 \mathrm{mv} / \mathrm{pH}$ unit at $15^{\circ} \mathrm{C}(97-99)$. A 0.05 molal solution of potassium hydrogen phthalate in the aqueous-acetonitrile solvent mixture served as the reference standard buffer, $\mathrm{pH}_{\mathrm{s}}=5.030$ at $15^{\circ} \mathrm{C},(100)$ for all of the $\mathrm{pH}$ measurements. 


\section{CHAPTER REFERENCES}

1. Grützmacher; H. F.; Husemann, W. Tetrahedron Lett. $1985,26,2431-2434$.

2. Sullivan, R. F.; Boduszynski, M. M.; Fetzer, J. C. Energy \& Fuels 1989, 3, 603-612.

3. Clar, E.; Fell, G. S.; Ironside, C. T.; Balsillie, A. Tetrahedron, 1960, 10, 26-36.

4. Hall, D. M.; Ladbury, J. E. ; Lesslie, M. S.; Turner, E. E. J. Chem. SoC. 1956, 3475-3482.

5. van Haeringen, C. J.; Aten, N. F.; Cornelisse, J.; Lugtenburg, J. Recl. Trav. Chim. Pays-Bas 1992, 111, 335 .

6. van Haeringen, C. J. Ph.D. Thesis, Leiden University, 1993.

7. van Haeringen, C. J.; Cornelisse, J.; Lugtenburg, J. unpublished results.

8. Selvarajan, N.; Panicker, M. M.; Vaidyanathan, S.; Ramakrishnan, V. Indian J. Chem. 1979, 18A, 23-26.

9. Encinas, M. V.; Rubio, M. A.; Lissi, E. A. Photochem. Photobiol. 1982, 37, 125-130.

10. Encinas, M. V.; Rubio, M. A.; Lissi, E. A. Photochem. Photobiol. 1982, 18, 137-150.

11. Wiczk, W. M. ; Latowski, T. Z. Naturforsch. 1987, 42A, 1290-1295.

12. Bowen, E. J.; Rohatgi, K. K. Disc. Faraday Soc. 1953, $14,146-150$.

13. Fetzer, J. C.; Biggs, W. R. J. Chromatogr. 1984, 295, 161-169.

14. Fetzer, J. C.; Biggs, W. R. J. Chromatogr. 1985, 322, 275-286.

15. Fetzer, J. C.; Biggs, W. R. J. Chromatogr. 1987, 386, 123-135. 
16. Fetzer, J. C.; Biggs, W. R. J. Chromatogr. 1987, 392, 75-82 .

17. Clar, E.; Zander, M. J. Chem Soc. 1958, 1861-1865.

18. Clar, E.; Ironside, C. T.; Zander, M. Tetrahedron 1966, $22,3527-3533$.

19. Clar, E. Ber. Dtsch. Chem. Ges. 1932, 65, 846.

20. Zander, M.; Franke, W. Chem. Ber. 1961, 94, 446-450.

21. Clar, E.; Zander, M. Tetrahedron 1963, 19, 521-526.

22. Clar, E.; Kelly, W.; Laird, R. M. Mh. Chem. 1956, 80, 391.

23. Harvey, R.G.; Yang, C.; Lee, H. manuscript in preparation.

24. Yang, C.; Yang, D. T. C.; Harvey, R. G. Synlett 1992, 799-800.

25. Lee, H.; Harvey, R. G. J. Org. Chem. 1990, 55, 37873791.

26. Konieczny, M.; Harvey, R. G. J. Org. Chem. 1979, 44, 2158-2160.

27. Di Raddo, P.; Hahn, J.-T.; Harvey, R. G. Polycyclic Aromat. Cmpds. 1991, 2, 1-11.

28. Dyker, G. Tetrahedron Lett. 1991, 32 7241-7242.

29. Harvey, R. G.; Pataki, J.; Cortez, C.; Di Raddo, P.; Yang, C. J. Org. Chem. 1991, 56, 1210-1217.

30. Goltz, M.; Murata, I. Bull. Chem. Soc. Jpn, 1988, 61, 3767-3769.

31. Mitchell, R. H.; Boekelheide, V. J. Am. Chem. Soc. $1974,96,1547-1557$.

32. Albrecht, K.; Reiser, O.; Weber, M.; de Meijere, A. Synlett 1992, 521-523.

33. Mitchell, R. H.; Carruthers, R. J.; Mazuch, L.; Dingle, T. W. J. An. Chem. Soc. 1982, 104, 2544-2551.

34. Bachmann, W. E.; Struve, W. S. J. Org. Chem. 1939, 4, 456-463. 
35. Bachmann, W. E.; Edgerton, R. O. I. Am. Chem. SoC. $1940,62,2550-2553$.

36. Lang, K. F.; Zander, M. Chem. Ber. 1964, 97, 218.

37. Lohberger, c. Thesis, Technical University of clausthal, 1983.

38. Clar, E.; McAndrew, B. A.; Zander M., Tetrahedron 1967, $23,985-993$.

39. Mitchel1, R. H.; Zhou, P. Tetrahedron Lett. 1990, 31, 5277-5280.

40. Cho, B. P.; Harvey, R. G. J. Org. Chem. 1987, 52, 56685678 .

41. Scott, L. T.; Hasehemi, M. M.; Meyer, D. T.; Warren, H. B. J. Am. Chem. SoC. 1991, 113, 7082-7084.

42. Dziewoński, K.; Leyko, Z. Ber. Dtsch. Chem. Ges. 1914, $47,1679-1690$.

43. Schönberg, A.; Brosowski, K.-H. Chem. Ber. 1960, 93, 2149-2151.

44. Schönberg, A.; Brosowski, K.-H.; Singer, E. Chem. Ber. $1962,95,1910-1916$.

45. Cheng, P.-C. M.S. Thesis, University of Nevada, Reno, 1992 .

46. Tanga, M. J.; Bupp, J. E. J. Org. Chem. 1993, 58, 41734174 .

47. Amin, S.; Huia, K.; Hussain, N.; Balanikas, G.; Carmella, S. G.; Hecht, S. S. J. Org. Chem. 1986, 51, 1206-1211.

48. Rice, J. E.; Shih, H. C.; Hussain, N.; La Voie, E. J. J. Org. Chem. 1987, 52, 849-855.

49. Weyand, E. H.; Geddie, N.; Rice, J. E.; Czech, A.; Amin, S.; La Voie, E. J. Carcinogenesis 1988, 9, 1277.

50. Peck, D. W. U.S. Patent 3095426, 1963; Chem. Abstr. $1963,59,12761 \mathrm{f}$.

51. Eckstein, 0. Ber. Dtsch. Chem. Ges. 1905, 38, 36603663 . 
52. Pummerer, R.; Prell, E.; Rieche, A. Ber. Dtsch. Chem. Ges. 1926, 59, 2159-2161.

53. Buisson, J.-P.; Demerseman, P. J. Heter. Chem. 1990, $27,2213-2214$.

54. Buisson, J.-P.; Kotzyba, J.; Lievremont, P.-P.; Demerseman, P.; Platzer, N.; Bideau, J.-P.; Cotrait, M. $\mathrm{J}$. Heter. Chem. 1993, 30, 739-747.

55. Nakasuji, K.; Kubota, H.; Kotani, T.; Murata, I. ; Saito, G.; Enoki, T.; Imaeda, K.; Inokuchi, H.; Honda, M.; Katayama, C.; Tanaka, J. J. Amer. Chem. Soc. 1986, $108,3460-3466$.

56. Nakasuji, K.; Oda, A.; Murata, I.; Imaeda, K.; Inokuchi, H.; J. Chem. SoC., Chem. Commun. 1989, 15531554 .

57. Zander, M. Chemikerztg. 1988, 112, 142-142.

58. Zander, M.; Franke, W. Chem. Ber. 1973, 106, 2752-2754.

59. Shukla, D.; Wan, P. J. An. Chem. Soc. 1993, 115, 29902991.

60. Tanga, M. J.; Reist, E. J. J. Heterocyclic Chem. 1986, $23,747-752$.

61. Phillips, R. E.; Drub, G. H.; Hunt, J. A. J. Org. Chem. $1972,37,2030-2033$.

62. Oberkobusch, R. Chem. Ber. 1953, 86, 975-979.

63. Kruber, O. Chem. Ber. 1949, 82, 199-201.

64. Tokita, S.; Hiruta, K.; Yaginuma, Y.; Ishikawa, S.; Nishi, H. Synthesis 1983, 270-271.

65. Tanga, M. J.; Reist, E. J. J. Heterocyclic Chem. 1991, $28,29-32$.

66. Tanga, M. J.; Almquist, R. G.; Smith, T. H.; Wu, H. Y.; Reist, E. J. J. Heterocyclic Chem. 1985, 22, 1597-1598.

67. Gore, P. H. J. Org. Chem. 1957, 22, 135-138.

68. Tanga, M. J.; Davis, R. F.; Reist, E. J. J. Heterocyclic Chem. 1987, 24, 39-41. 
69. Tanga, M. J.; Reist, E. J. J. Org. Chem. 1982, 47, 1365-1366.

70. Tokita, S.; Hiruta, K.; Kitahara, K.; Nishi, H. Bull. Chem. Soc. Jpn. 1982, 3933-3934.

71. Hünig, S.; Gross, J.; Lier, E. F.; Quast, H. Liebigs Ann. Chem. 1973, 339-358.

72. Tokita, S.; Hiruta, K.; Kitahara, K.; Nishi, H. Synthesis 1982, 299-231.

73. Tokita, S.; Hiruta, K.; Ishikawa, S.; Kitahara, K.; Nishi, H. Synthesis 1982, 854-855.

74. Bowden, B. F.; Picker, K.; Ritchie, E.; Taylor, w. E. Aust. J. Chem. 1975, 28, 2681-2701.

75. Naumann, C.; Langhals, H. Chem. Ber. 1990, 123, 18811884 .

76. Kehrmann, F.; Mermond, C. Helv. Chim. Acta 1927, 10, $62-66$.

77. Partridge, M. W.; Slorach, S. A.; Vipond, H. J. J. Chem. Soc. 1964, 3670-3673.

78. Partridge, M. W.; Vipond, H. J. J. Chem. Soc. 1962, 632-635.

79. Upton, C. J. Chem. Soc., Perkin Trans. I 1986, 12251229.

80. Bloomfield, D. G.; Upton, C.; Vipond, H. J. J. Chem. Soc., Perkin Trans. I 1986, 857-860.

81. Tong, T. H.; Wong, H. N. C. Synth. Commun. 1992, 22, 1773-1782.

82. Jaeda, M. I.; Parfitt, R. T.; Upton, C. Chung-Hua Yao Hseuh Tsa Chih, 1990, 42, 403-410.

83. Mitchell, R. H.; Chaudhary, M.; Williams, R. V.; Fyles, R.; Gibson, J; Ashwood-Smith, M. J.; Fry, A. J. Can. J. Chem. 1992, 70, 1015-1022.

84. Mulder, P. P. J.; Boere, B. B.; Baart, A.; Cornelisse, J.; Lugtenburg, J. Rec1. Trav. Chim. Pays-Bas 1993, $112,22-32$. 
85. Waris, R.; Rembert, M. A.; Sellers, D. M.; Acree, W. E., Jr.; Street, K. W., Jr.; Fetzer, J. C. Analyst $1989,114,195-199$.

86. Waris, R.; Street, K. W., Jr.; Acree, W. E., Jr.; Fetzer, J. C. Appl. Spectrosc. 1989, 43, 845-850.

87. Acree, W. E., Jr.; Tucker, S. A.; Fetzer, J. C. Polycyclic Aromat. Compds. 1991, 2, 75-105.

88. Dong, D. C.; Winnik, M. A. Can. J. Chem. 1984, 62, 2560-2565.

89. Street, K. W., Jr.; Acree, W. E., Jr. Analyst 1986, $111,1197-1201$.

90. Parker, C. A.; Barnes, W. J. Analyst 1957, 82, 606-618.

91. Holland, J. F.; Teets, R. E.; Kelly, P. M.; Timnick, A. Anal. Chem. 1977, 49, 706-710.

92. Yappert, M. C.; Ingle, J. D. Appl. Spectrosc. 1989, 43, $759-767$.

93. Lawokicz, J. R. Principles of Fluorescence Spectroscopy; Plenum: New York, 1983.

94. Acree, W. E., Jr.; Tucker, S. A.; Cretella, L.; Zvaigzne, A. I.; Street, K. W., Jr.; Fetzer, J. C. ; Nakasuji, K.; Murata, I. Appl. Spectrosc. 1990, 44, 951-957.

95. Acree, W. E., Jr.; Tucker, S. A.; Zvaigzne, A. I.; Street, K. W., Jr.; Fetzer, J. C.; Grützmacher, H.-F. App1. Spectrosc. 1990, 44, 477-482.

96. Tucker, S. A.; Amszi, V. L.; Acree, W. E., Jr. J. Chem. Educ. 1992, 69, A8, A11, and A12.

97. Westcott, c. c. pH Measurements; Academic Press: New York, 1978; pp 112-123.

98. Skoog, D. A; West, D. M. Principles of Instrumental Analysis; Saunders College: Philadelphia, 1980; sixth ed. ; pp 430-432.

99. Laitinen H. A.; Harris, W. E. Chemical Analysis; McGraw-Hill: New York, 1960; pp 84-88.

100. Longhi, P.; Mussini T.; Rondinini, S. Anal. Chem. 1986, 58, 2290-2292. 
CHAPTER III

RESULTS AND DISCUSSION OF SOLVENT POLARITY PROBE STUDIES

Polycyclic Aromatic Hydrocarbons

Room temperature fluorescence emission spectra of the polycyclic aromatic hydrocarbons (PAHs) or PAH6 benzenoids monomers consists of several major vibronic bands labeled I, II, etc., in progressive order. Representative spectra taken from the large data base are depicted in Figures 34-38 for pyrene, benzo[e]pyrene, benzo[ghi]perylene, coronene, and ovalene, respectively, dissolved in $n$-hexadecane, butyl acetate, dichloromethane, and dimethyl sulfoxide. The four nonelectrolyte solvents were selected so as to encompass the entire range of solvent polarity, from the nonpolar $n$ hexadecane saturated hydrocarbon to the moderately polar butyl acetate and dichloromethane solvents to the very polar dimethyl sulfoxide. Dichloromethane serves as the recommended chlorinated hydrocarbon because PAH6 benzenoids are the less likely to undergo rapid irreversible photochemical reactions as compared with chloroform or carbon tetrachloride (1-10). Reactions between PAH solutes and dichloromethane appear to be much slower and are not expected to significantly affect emission intensity ratios measured in $1 \mathrm{~cm}^{2}$ quartz cuvettes (1). 


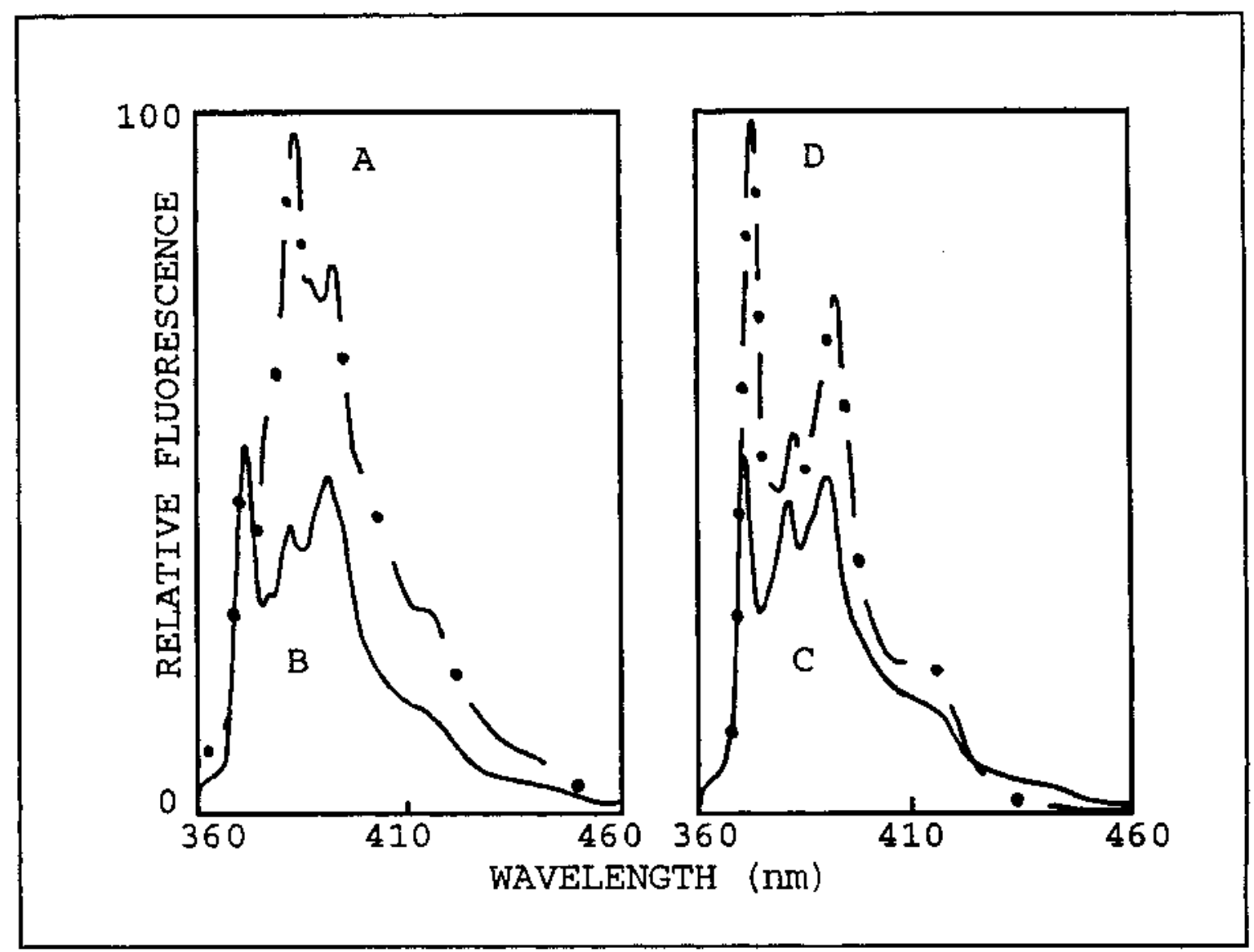

FIGURE 34: Fluorescence emission spectra of pyrene dissolved in $[A(-O-)] n$-hexadecane, [B (-)] butyl acetate, [C (-) ] dichloromethane, and [D (- - ) ] dimethyl sulfoxide. In butyl acetate emission bands occur at about $370,381,390$, and s-413 $\mathrm{nm}$. 


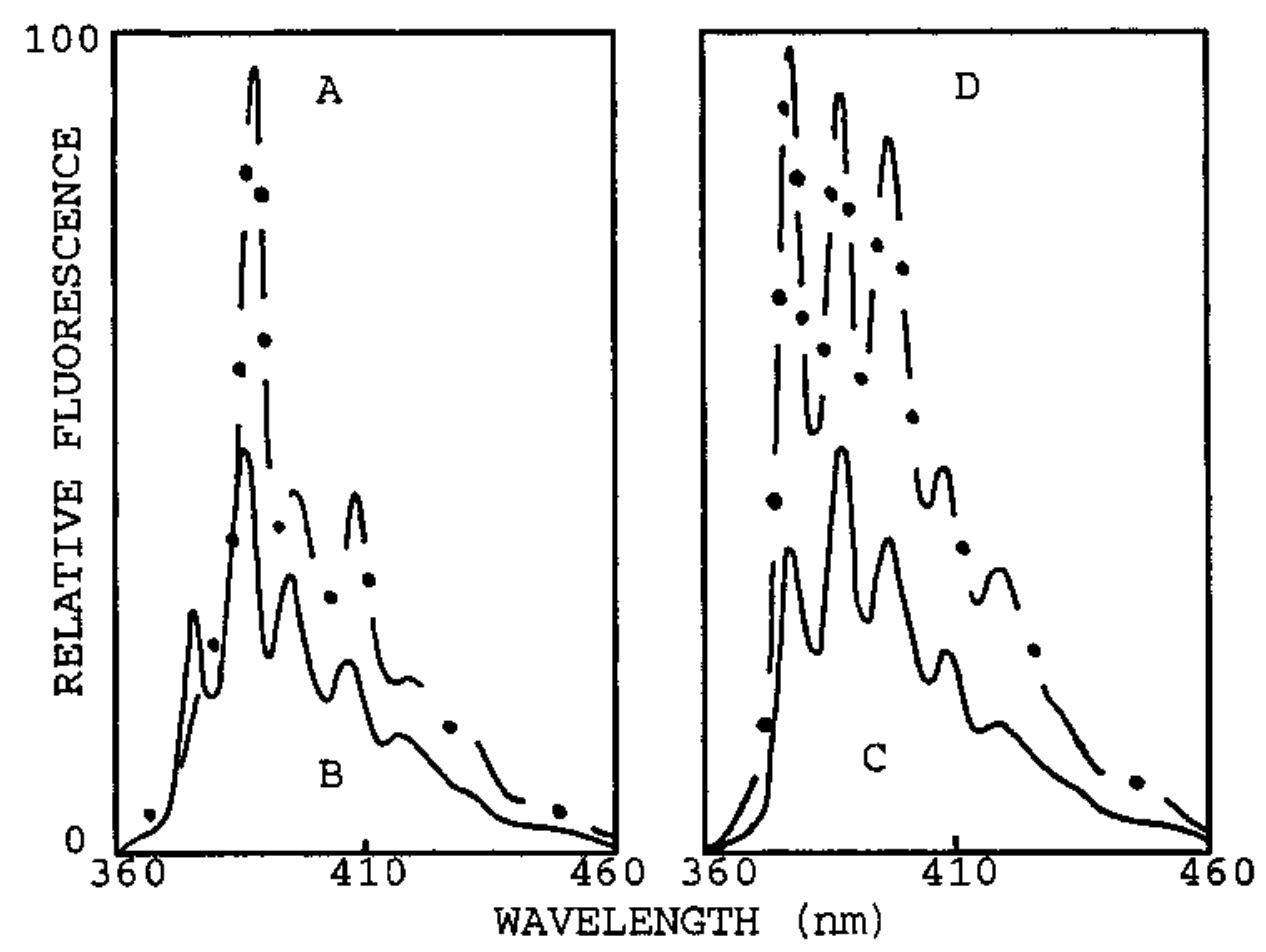

FIGURE 35: Fluorescence emission spectra of benzo[e]pyrene dissolved in [A (- -) ] n-hexadecane, [B (-)] butyl acetate, [C (-)] dichloromethane, and [D (- -)] dimethyl sulfoxide. In butyl acetate emission bands occur at about $376,387,396$, and $408 \mathrm{~nm}$. 


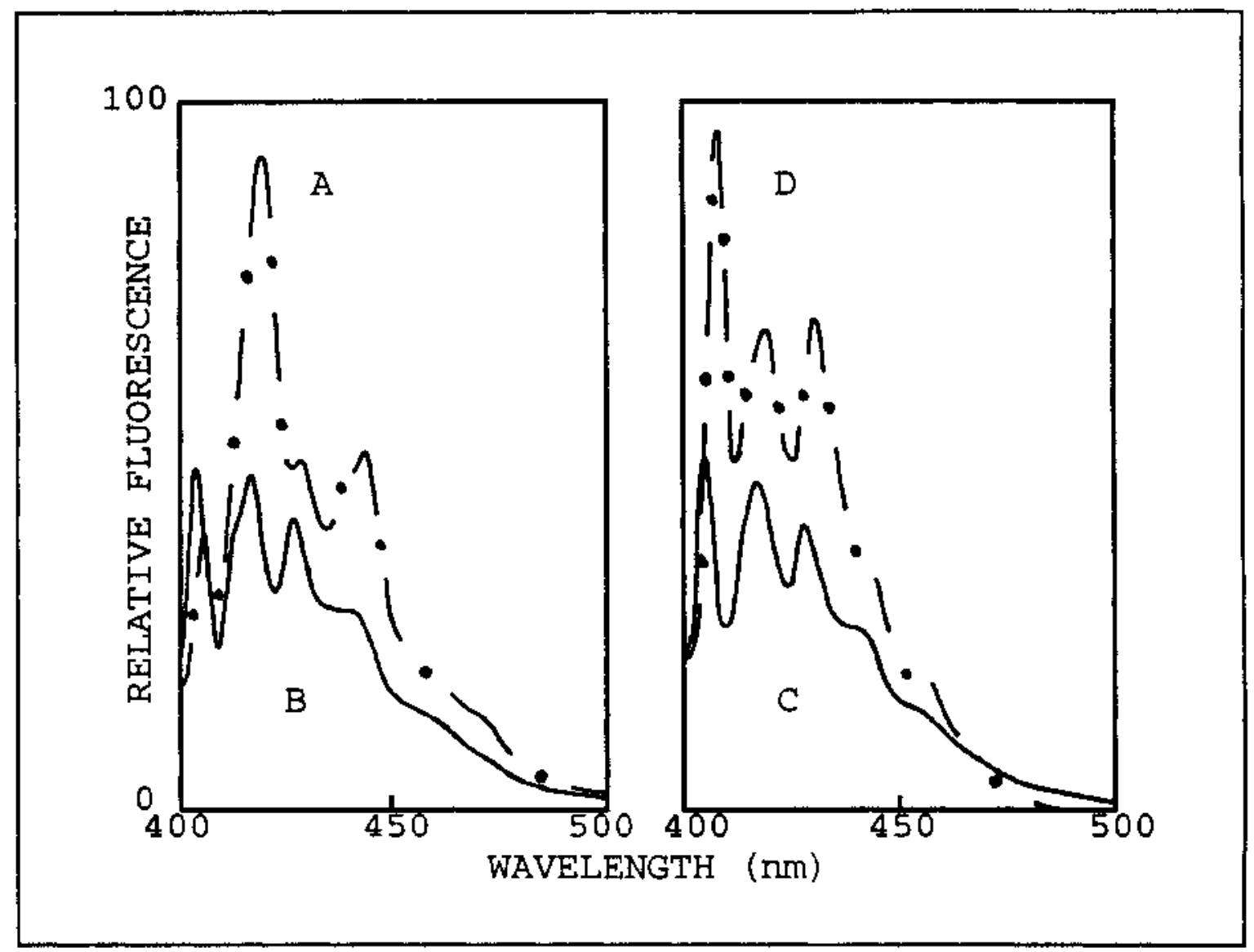

FIGURE 36: Fluorescence emission spectra of benzo[ghi]perylene dissolved in [A (- - -)] $n$-hexadecane, [B ( butyl acetate, [C (- $)$ ] dichloromethane, and [D (- - -)] dimethyl sulfoxide. In butyl acetate emission bands occur at about $405,418,428$, and $\mathrm{s}-443 \mathrm{~nm}$. 


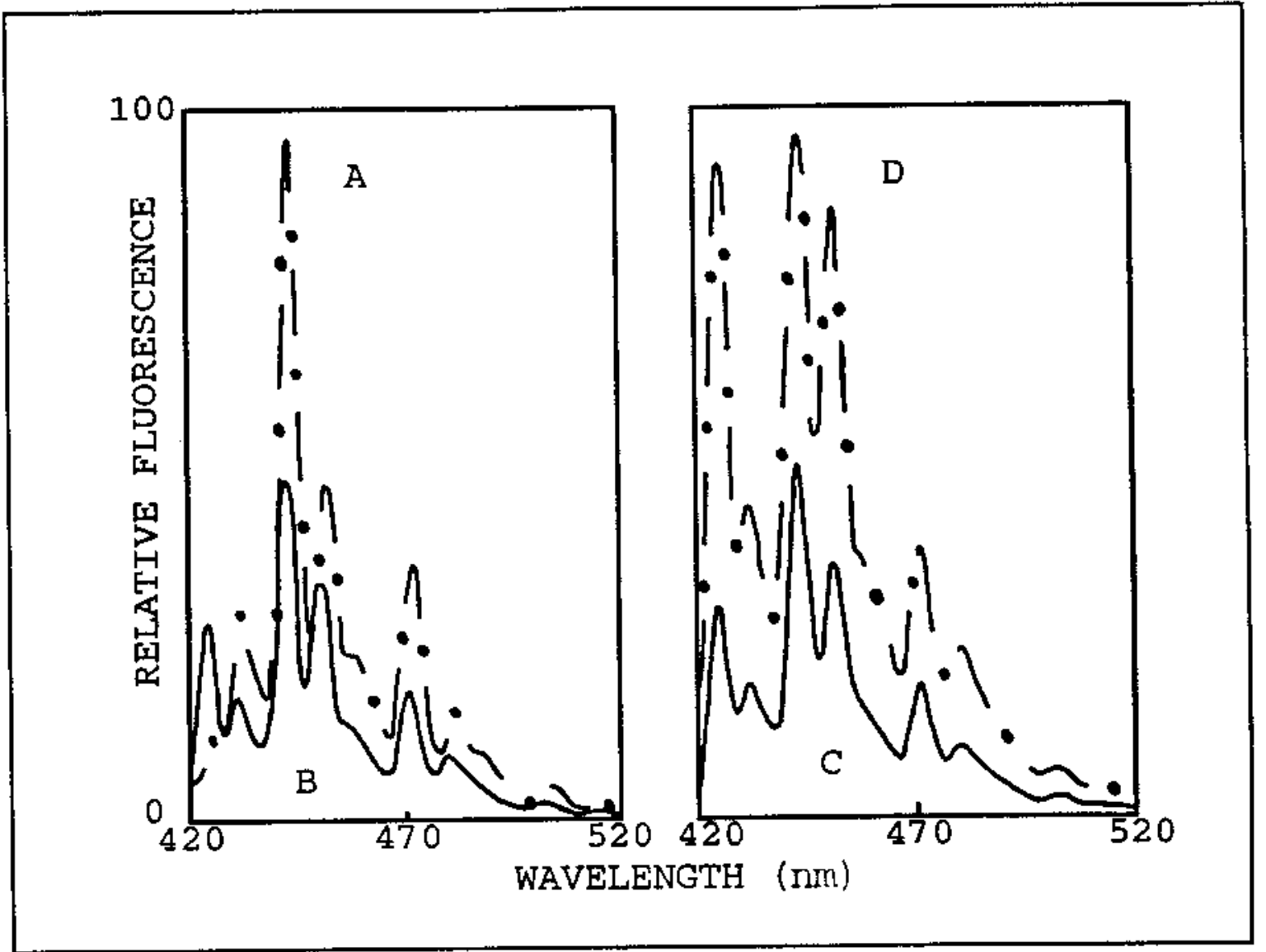

FIGURE 37: Fluorescence emission spectra of coronene dissolved in [A (- -) ] n-hexadecane, [B (- - )] butyl acetate, [C (- $)]$ dichloromethane, and $[D(-0-)]$ dimethyl sulfoxide. In butyl acetate emission bands occur at about $424,432,443,451$, and $472 \mathrm{~nm}$. 


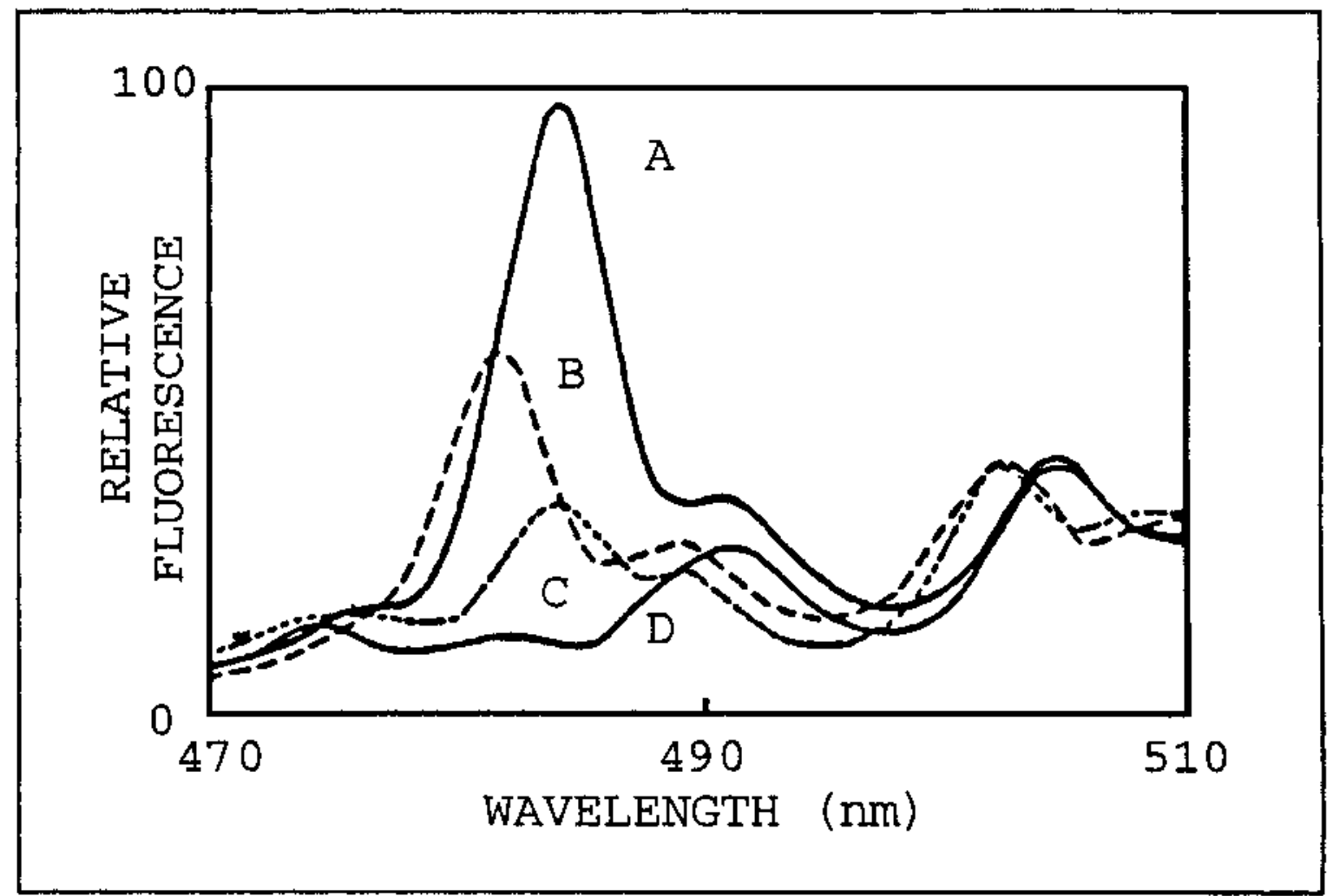

FIGURE 38: Fluorescence emission spectra of ovalene dissolved in $[A(-)]$ dimethyl sulfoxide, [B (- - ) ] methanol, [C (- - ) ] carbon tetrachloride, and [D (- $)$ ] cyclohexane. In dimethyl sulfoxide emission bands occur at about 483,492 , and $504 \mathrm{~nm}$. 
Examination of the entire spectral database of more than 200 polycyclic aromatic compounds (PACs) reveals that PACs can be divided into two categories, solvent polarity probe or nonprobe molecules, depending upon whether the ratio of emission intensities systematically change with solvent polarity. For pyrene (Py), benzo[ghi]perylene (BPe), coronene (Co), ovalene (OV), benzo[e]pyrene (BPy), benzo $[a]$ coronene (BCo), naphtho $[2,3 a]$ coronene (NCO), dibenzo $[a, j]$ coronene (DCo), dibenzo[fg,ij]phenanthro$[2,1,10,9,8,7$ pqrstuv $]$ pentaphene (DBPP), dibenzo[def, $]]^{-}$ chrysene (DBCh), benzo[rst]pentaphene (BPp), naphtho$[8,1,2 a b c]$ coronene (NaCo), dinaphtho $\left[8,1,2 a b c ; 2^{\prime}, 1^{\prime}, 8^{\prime} k 1 m\right]-$ coronene (DNCo), anthra[2,3a]coronene (ACo), benzo[vwX]hexaphene (BHPh), and dibenzo[h,rst]pentaphene (DBPPh) selective enhancement of the I band is observed in the polar solvent. Ratios of bands $I$ and III (or $\mathrm{BPY}=\mathrm{I} / \mathrm{IV}, \mathrm{DCO}=$ $\mathrm{I} / \mathrm{IV}, \mathrm{BPp}=\mathrm{I} / \mathrm{IV}, \mathrm{NaCo}=I / I I, \mathrm{ACO}=\mathrm{I} / \mathrm{II}, \mathrm{BHPh}=I / I I$, $\mathrm{DBPPh}=I / V)$ serve as a quantitative measure of solvent polarity and structure. Benzo[e]pyrene $(B P y=I / I I, I / I I I$ and I/IV), dibenzo[fg,ij]phenanthro $[2,1,10,9,8,7$ pqrstuv $]-$ pentaphene $($ DBPP $=I / I I$ and I/III), dibenzo $[$ def,p]chrysene $(\mathrm{DBCh}=\mathrm{I} / \mathrm{II}$ and I/III), and dibenzo[h,rst]pentaphene (DBPPh $=I / I V$ and $I / V$ ) have two or more intensity ratios which could be used to define a possible polarity scale. In a few instances, the intensity ratio giving the larger dynamic 
range was selected as to enable better discrimination of solvent polarity.

Tables XXV-XXXI summarize the actual numerical values for the aforementioned solvent polarity probe molecules. All PACs are examined in approximately nine initial screening solvents - cyclohexane, n-hexadecane, benzene, butyl acetate, methanol, dichloromethane, acetonitrile, dimethylformamide, and dimethyl sulfoxide. Those that show solvent polarity probe possibilities are run in twenty solvents of varying polarity. It is only those that are recommended for general use, like pyrene, that are examined in approximately fifty solvents (see Table XXV). Estimated uncertainties in the measured intensity ratios are believed to be on the order of \pm 0.05 (or less) on the basis of replicate measurements over at least a five day period for most PAH probes except for ovalene which is on the order of \pm 0.10 due to the extremely high dynamic range and low solubility. Examination of Tables $X X X$ and $X X X I$ also reveals that only anthra[2,3a]coronene and benzo[vwx] hexaphene have observed intensity ratios that decrease systematically with increasing solvent polarity, all other PAH6 benzenoids increase with increasing solvent polarity. Tables XXV-XXXI also show the presence of the so-called chlorinated effect discussed previously in Chapter 1 .

Surprisingly, not all PAH6 benzenoids behave in an identical manner. Only 18 of the 64 PAH6 benzenoid solutes 


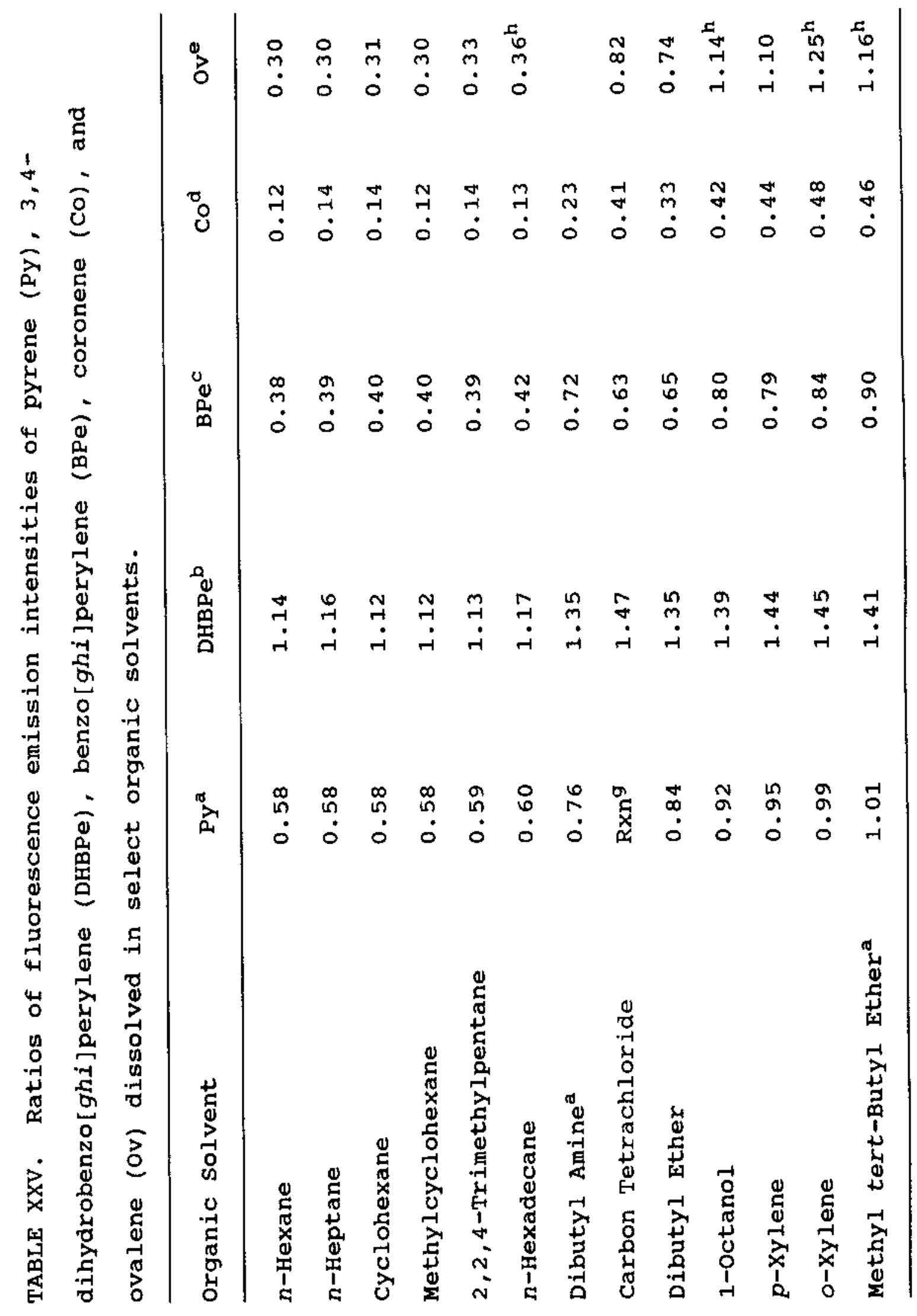




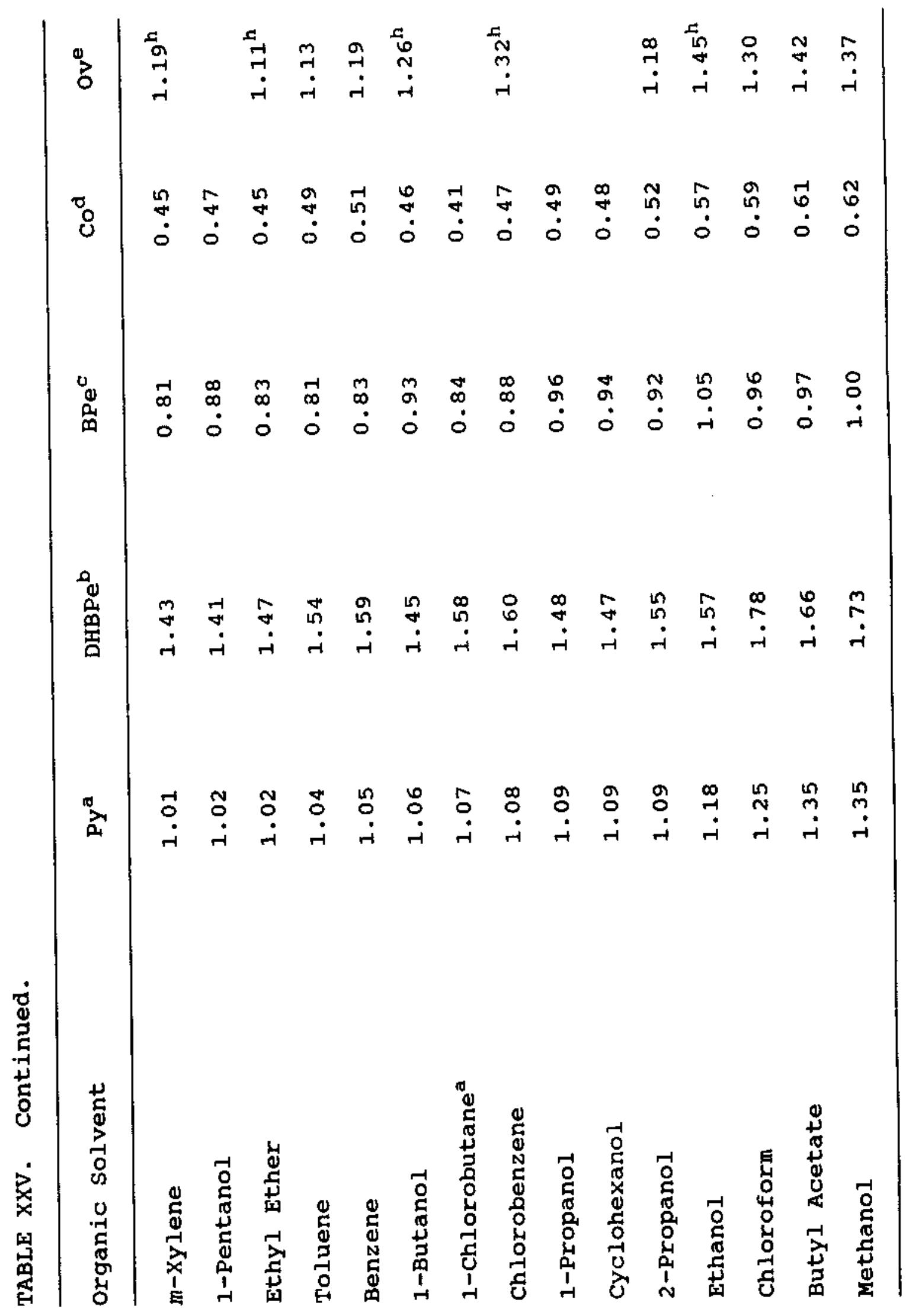




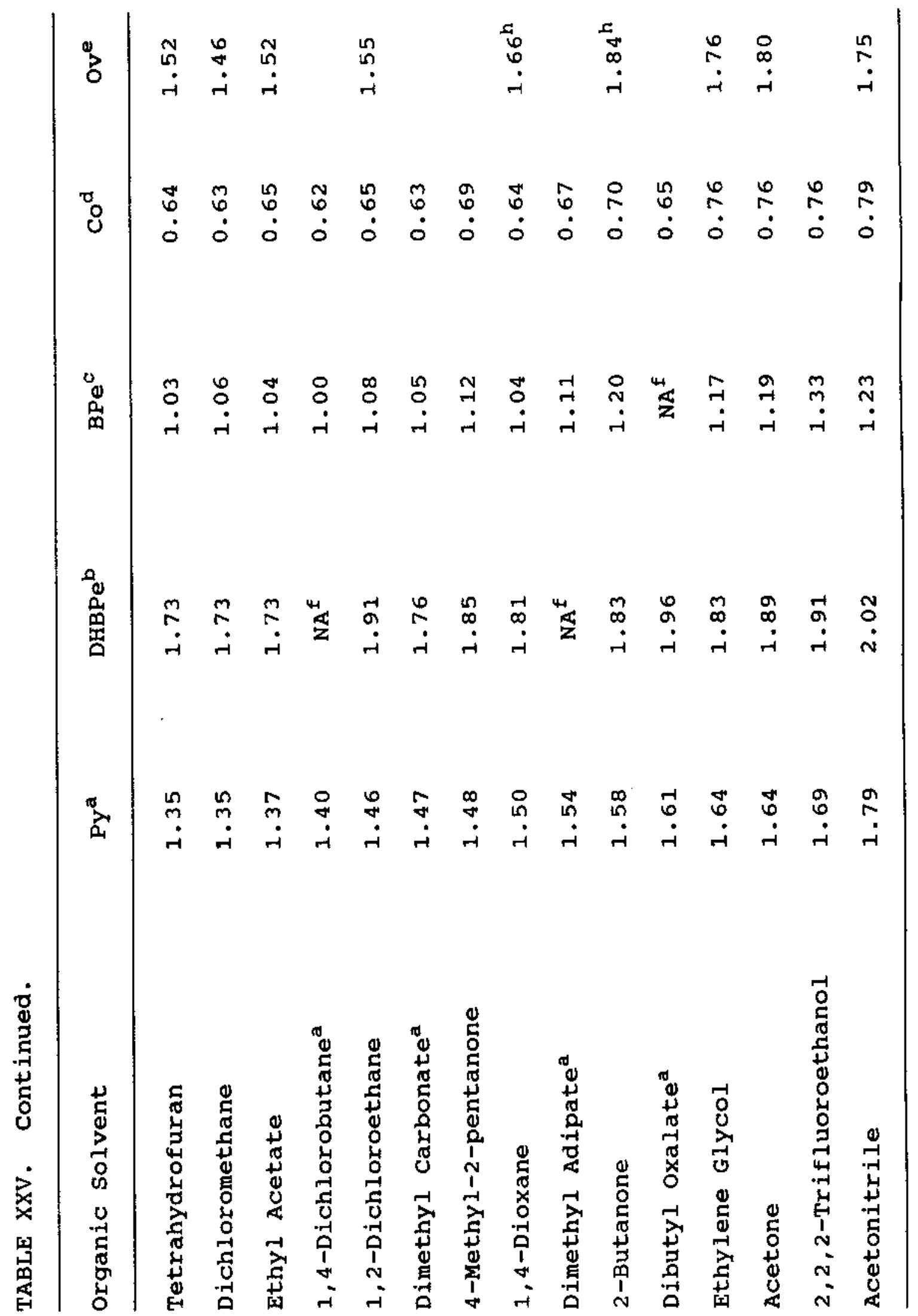




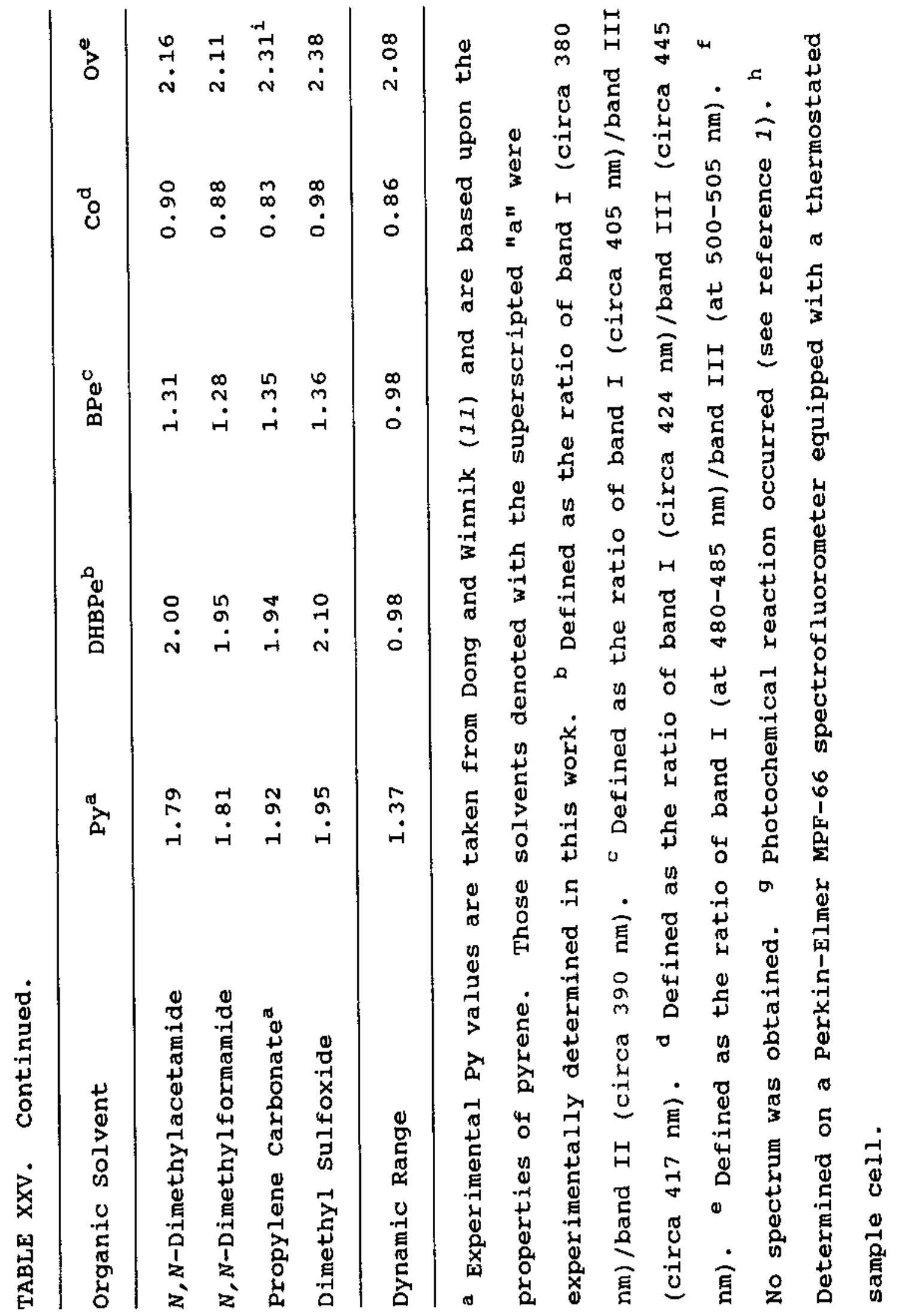


TABLE XXVI. Ratios of fluorescence emission intensities of benzo[e]pyrene (BPY) dissolved in select organic solvents.

\begin{tabular}{|c|c|c|c|}
\hline Organic solvent & $\mathrm{BPy}^{\mathrm{a}}$ & $\mathrm{BPy}^{\mathrm{b}}$ & $\mathrm{BPy}^{\mathrm{C}}$ \\
\hline$n$-Hexane & 0.23 & 0.50 & 0.50 \\
\hline$n$-Heptane & 0.24 & 0.51 & 0.52 \\
\hline Cyclohexane & 0.24 & 0.51 & 0.51 \\
\hline Methylcyclohexane & 0.24 & 0.51 & 0.51 \\
\hline $2,2,4$-Trimethylpentane & 0.24 & 0.50 & 0.50 \\
\hline$n$-Hexadecane & 0.25 & 0.52 & 0.53 \\
\hline Dibutyl Amine & 0.41 & 0.66 & 0.77 \\
\hline Carbon Tetrachloride & 0.50 & 0.89 & 1.11 \\
\hline Dibutyl Ether & 0.37 & 0.67 & 0.77 \\
\hline 1-octanol & 0.43 & 0.73 & 0.90 \\
\hline$p$-xylene & 0.50 & 0.77 & 0.98 \\
\hline o-xylene & 0.51 & 0.81 & 1.04 \\
\hline Methyl tert-Butyl Ether & 0.48 & 0.76 & 0.97 \\
\hline m-Xylene & 0.47 & 0.77 & 0.98 \\
\hline 1-Pentanol & 0.48 & 0.77 & 1.00 \\
\hline Ethyl Ether & 0.47 & 0.77 & 0.95 \\
\hline Toluene & 0.52 & 0.81 & 1.03 \\
\hline Benzene & 0.53 & 0.81 & 1.08 \\
\hline 1-Butanol & 0.50 & 0.79 & 1.04 \\
\hline 1-Chlorobutane & 0.52 & 0.79 & 1.09 \\
\hline Chlorobenzene & 0.59 & 0.85 & 1.17 \\
\hline
\end{tabular}


TABLE XXVI. Continued.

\begin{tabular}{|c|c|c|c|}
\hline organic solvent & $\mathrm{BPy}^{\mathrm{a}}$ & BPy $^{b}$ & BPy $^{\mathrm{c}}$ \\
\hline 1-Propanol & 0.53 & 0.81 & 1.08 \\
\hline Cyclohexanol & 0.51 & 0.80 & 1.08 \\
\hline 2-Propanol & 0.52 & 0.80 & 1.05 \\
\hline Ethanol & 0.59 & 0.85 & 1.19 \\
\hline Chloroform & 0.69 & 0.96 & 1.42 \\
\hline Butyl Acetate & 0.61 & 0.87 & 1.26 \\
\hline Methanol & 0.68 & 0.88 & 1.33 \\
\hline Tetrahydrofuran & 0.63 & 0.90 & 1.28 \\
\hline Dichloromethane & 0.75 & 0.97 & 1.49 \\
\hline Ethyl Acetate & 0.65 & 0.89 & 1.35 \\
\hline 1,2-Dichloroethane & 0.76 & 0.97 & 1.58 \\
\hline Dimethyl Carbonate & 0.70 & 0.89 & 1.35 \\
\hline 4-Methyl-2-pentanone & 0.69 & 0.92 & 1.43 \\
\hline 1,4 -Dioxane & 0.70 & 0.93 & 1.42 \\
\hline 2-Butanone & 0.78 & 0.99 & 1.56 \\
\hline Dibutyl Oxalate & 0.64 & 0.86 & 1.29 \\
\hline Ethylene Glycol & 0.80 & 0.96 & 1.57 \\
\hline Acetone & 0.81 & 0.99 & 1.70 \\
\hline $2,2,2$-Trifluoroethanol & 0.92 & 0.95 & 1.72 \\
\hline Acetonitrile & 0.92 & 1.04 & 1.81 \\
\hline$N, N$-Dimethylacetamide & 0.93 & 1.04 & 1.83 \\
\hline$N, N$-Dimethyl formamide & 0.93 & 1.05 & 1.84 \\
\hline
\end{tabular}


TABLE XXVI. Continued.

\begin{tabular}{lccc}
\hline Organic solvent & $\mathrm{BPy}^{\mathrm{a}}$ & $\mathrm{BPY}^{\mathrm{b}}$ & $\mathrm{BPy}^{\mathrm{c}}$ \\
\hline Propylene Carbonate & 0.95 & 1.05 & 1.86 \\
Dimethyl Sulfoxide & 1.05 & 1.11 & 2.05 \\
\hline Dynamic Range & 0.82 & 0.61 & 1.55 \\
\hline
\end{tabular}

a Defined as the ratio of band I (at 375-378 nm)/band II (at 385-388 nm) . b Defined as the ratio of band I (at 375-378 $\mathrm{nm}$ )/band III (at 394-398 nm). c Defined as the ratio of band I (at 375-378 nm)/band IV (at 406-410 nm). 
TABLE XXVII. Ratios of fluorescence emission intensities of benzo[a]coronene (BCo), naphtho[2,3a]coronene (NCo), and dibenzo[a,j]coronene (DCo) dissolved in select organic solvents.

\begin{tabular}{|c|c|c|c|}
\hline organic Solvent & $\mathrm{BCO}$ & $\mathrm{NCO}$ & $\mathrm{DCo}^{\mathrm{d}}$ \\
\hline$n$-Hexane & 0.38 & 0.57 & 1.23 \\
\hline Cyclohexane & 0.38 & 0.59 & 1.25 \\
\hline $2,2,4-T r i m e t h y l p e n t a n e$ & 0.39 & 0.57 & 1.25 \\
\hline Dibutyl Ether & 0.56 & 0.92 & 1.34 \\
\hline Benzene & 0.79 & 1.23 & 1.64 \\
\hline 2-Propanol & 0.77 & 1.31 & 1.46 \\
\hline Butyl Acetate & 0.85 & 1.35 & 1.59 \\
\hline Methanol & 0.85 & 1.41 & 1.46 \\
\hline Dichloromethane & 0.99 & 1.58 & 1.89 \\
\hline Acetonitrile & 1.12 & 1.77 & 1.81 \\
\hline$N, N$-Dimethylformamide & 1.13 & 1.82 & 1.76 \\
\hline Dimethyl sulfoxide & 1.25 & 1.86 & 2.00 \\
\hline Dynamic Range & 0.87 & 1.29 & 0.77 \\
\hline
\end{tabular}

a Values taken from waris et al. (12). b Defined as the ratio of band I (at: 432-435 nm)/band III (at 450-452 nm). c Defined as the ratio of band I (at 444-447 nm)/band III (at 462-466 nm). d Defined as the ratio of band I (at 432-436 $\mathrm{nm}$ )/band IV (at 452-456 nm). 
TABLE XXVIII. Ratios of fluorescence emission intensities of dibenzo[fg, $i j]$ phenanthro $[2,1,10,9,8,7$ pqrstuv $]$ pentaphene (DBPP) dissolved in select organic solvents. ${ }^{a}$

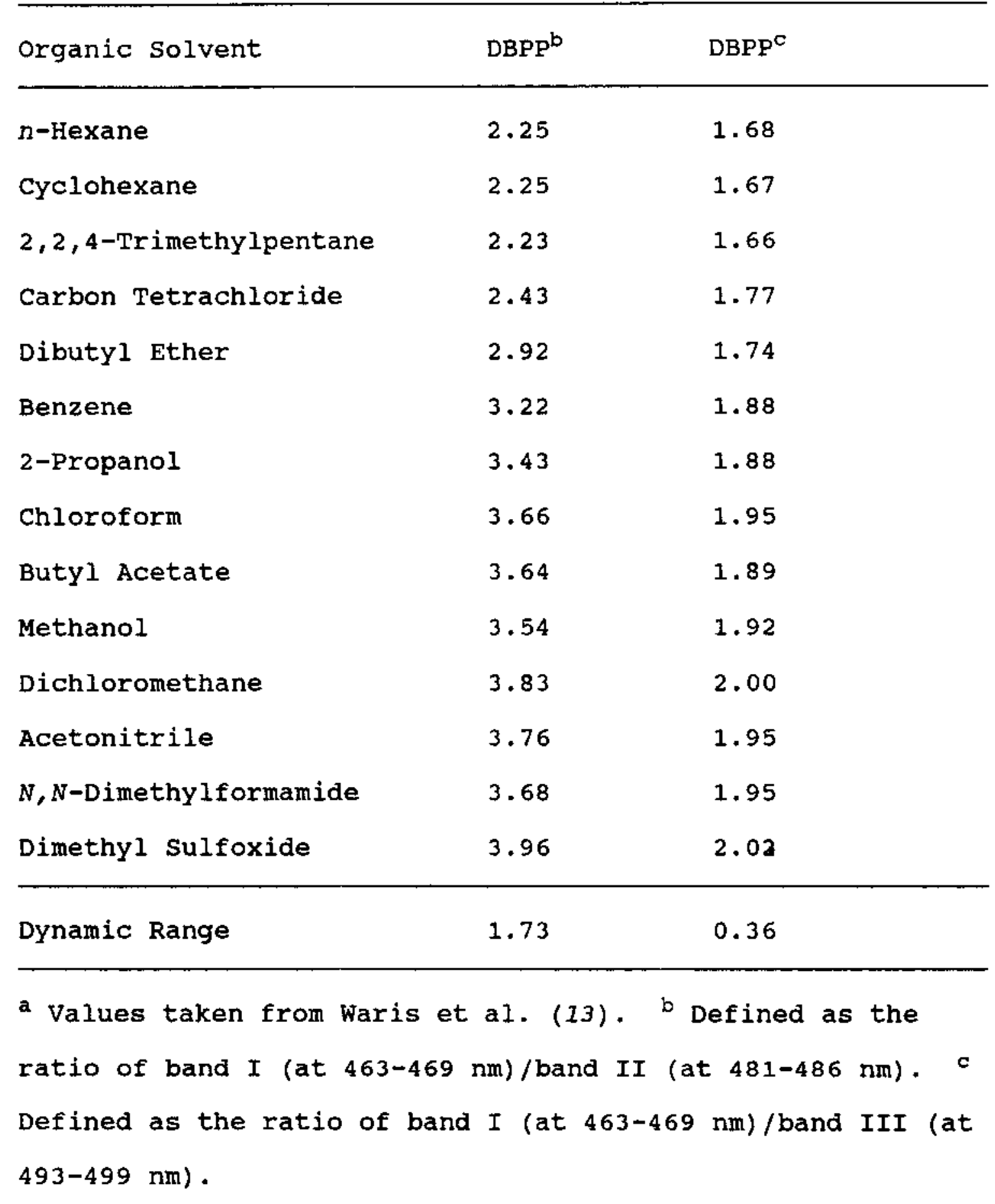


TABLE XXIX. Ratios of fluorescence emission intensities of dibenzo[def,p]chrysene (DBCh) and benzo[rst]pentaphene (BPp) dissolved in select organic solvents. ${ }^{a}$

\begin{tabular}{|c|c|c|c|}
\hline Organic solvent & $\mathrm{DBCh}^{\mathrm{b}}$ & $\mathrm{DBCh}^{\mathrm{C}}$ & $\mathrm{BPp} \mathrm{p}^{\mathrm{d}}$ \\
\hline Cyclohexane & 1.01 & 1.01 & 2.29 \\
\hline $2,2,4-T r$ imethylpentane & 0.99 & 1.00 & 2.32 \\
\hline$n$-Hexadecane & 1.10 & 1.07 & 2.30 \\
\hline Benzene & 1.55 & 1.24 & 2.97 \\
\hline 2-Propanol & 1.62 & 1.27 & 3.14 \\
\hline Butyl Acetate & 1.80 & 1.40 & 3.21 \\
\hline Methanol & 1.81 & 1.29 & 3.18 \\
\hline Dichloromethane & 1.80 & 1.35 & 3.25 \\
\hline Acetonitrile & 1.96 & 1.34 & 3.46 \\
\hline$N, N$-Dimethylformamide & 2.01 & 1.42 & 3.48 \\
\hline Dimethyl sulfoxide & 2.06 & 1.48 & 3.48 \\
\hline Dynamic Range & 1.07 & 0.48 & 1.19 \\
\hline
\end{tabular}

a Values taken from Acree et al. (14). b Defined as the ratio of band I (at 418-422 nm)/band II (at 432-435 nm). c Defined as the ratio of band I (at 418-422 nm)/band III (at 443-447 nm). d Defined as the ratio of band I (at 430-435 $\mathrm{nm}$ )/band IV (at 445-449 nm) . 
TABLE XXX. Ratios of fluorescence emission intensities of naphtho $[8,1,2 a b c]$ coronene (NaCo), dinaphtho $[8,1,2 a b c ;-$ $\left.2^{\prime}, 1^{\prime}, 8^{\prime} \mathrm{k} 1 \mathrm{~m}\right]$ coronene (DNCo), and anthra $[2,3 \mathrm{a}]$ coronene (ACo) dissolved in select organic solvents.

\begin{tabular}{llll}
\hline Organic Solvent & $\mathrm{NaCo}^{\mathrm{a}}$ & $\mathrm{DNCO}^{\mathrm{b}}$ & $\mathrm{ACO}^{\mathrm{C}}$ \\
\hline Cyclohexane & 1.33 & 1.60 & 2.93 \\
$2,2,4$-Trimethylpentane & 1.32 & 1.69 & 2.90 \\
$n$-Hexadecane & 1.35 & 1.63 & 3.00 \\
Benzene & 2.05 & 2.61 & 2.73 \\
2-Propanol & 2.25 & 2.64 & 2.37 \\
Butyl Acetate & 2.65 & 2.64 & 2.44 \\
Methanol & 2.60 & 2.80 & 2.22 \\
Dichloromethane & 2.84 & 2.82 & 2.29 \\
Acetonitrile & 3.19 & 2.88 & 2.03 \\
\hline Dynamic Range & 1.87 & 1.28 & 0.97 \\
\hline
\end{tabular}

a Defined as the ratio of band I (at $458-462 \mathrm{~nm}$ )/band II (at 480-484 nm). b Defined as the ratio of band I (at 491-494 $\mathrm{nm}$ )/band III (at $524-528 \mathrm{~nm}$ ). C Defined as the ratio of band I (at 484-492 nm)/band II (at 521-530 nm). 
TABLE XXXI. Ratios of fluorescence emission intensities of benzo[VwX]hexaphene (BHPh) and dibenzo[h, rst]pentaphene (DBPPh) dissolved in select organic solvents.

\begin{tabular}{|c|c|c|c|}
\hline organic solvent & $\mathrm{BHPh}^{\mathrm{a}}$ & $\mathrm{DBPPh}^{\mathrm{b}}$ & $\mathrm{DBPPh} \mathrm{h}^{\mathrm{C}}$ \\
\hline$n$-Hexane & & 0.73 & 1.90 \\
\hline Cyclohexane & 2.90 & 0.72 & 1.90 \\
\hline Methylcyclohexane & & 0.73 & 1.91 \\
\hline $2,2,4$-Trimethylpentane & 2.96 & 0.73 & 1.90 \\
\hline$n$-Hexadecane & 2.94 & 0.75 & 1.95 \\
\hline Dibutyl Ether & 2.62 & 0.97 & 2.78 \\
\hline Benzene & 2.59 & 1.10 & 3.37 \\
\hline 2-Propanol & 2.36 & 1.13 & 3.45 \\
\hline Butyl Acetate & 2.39 & 1.13 & 3.49 \\
\hline Methanol & 2.27 & 1.18 & 3.73 \\
\hline Dichloromethane & 2.31 & 1.20 & 3.62 \\
\hline Acetonitrile & 2.15 & 1.23 & 3.77 \\
\hline$N, N$-Dimethyl formamide & 2.18 & 1.27 & 4.09 \\
\hline Dimethyl sulfoxide & 2.15 & 1.31 & 4.24 \\
\hline Dynamic Range & 0.81 & 0.59 & 2.34 \\
\hline
\end{tabular}

a Defined as the ratio of band I (at $470-480 \mathrm{~nm}$ )/band II (at 502-512 nm). b Defined as the ratio of band $I$ (at 414-418 $\mathrm{nm}$ )/band IV (at 439-444 $\mathrm{nm}$ ). C Defined as the ratio of band I (at 439-444 nm)/band V (at 469-473 nm). 
studied to date showed an enhancement effect. The various intensity ratios for all other PAHs not previously mentioned remained essential constant, did not vary systematically with solvent polarity, or did not have a common set of bands in all solvent studied. For example, pyranthene has seven resolvable bands at $392,415,439,458,468,486$, and $498 \mathrm{~nm}$ in acetonitrile (not pictured) and in binary 1,1,2trichlorotrifluoroethane + perfluoro-1,3-dimethylcyclohexane solvent, but only 3 bands at 460,467 and $493 \mathrm{~nm}$ in $n-$ hexadecane (see Figure 39).

\section{PAH6 Benzenoid Derivatives}

Most PAHs have very limited water solubility with the exception of pyrene. To increase solubility, particularly in aqueous solvent media, alkyl and/or polar functional groups are attached to known PAH solvent polarity probes. Ring substituents are expected to weaken solute-solute interactions and lower the melting point temperature. As a general rule-of-thumb mole fraction solubility increases with decreasing melting point temperature, provided that all other interactions remain unchanged. An alkyl substituent does increase the hydrophobicity of the parent PAH, but it is hoped that this effect could be favorably offset by the lower melting point contribution. 


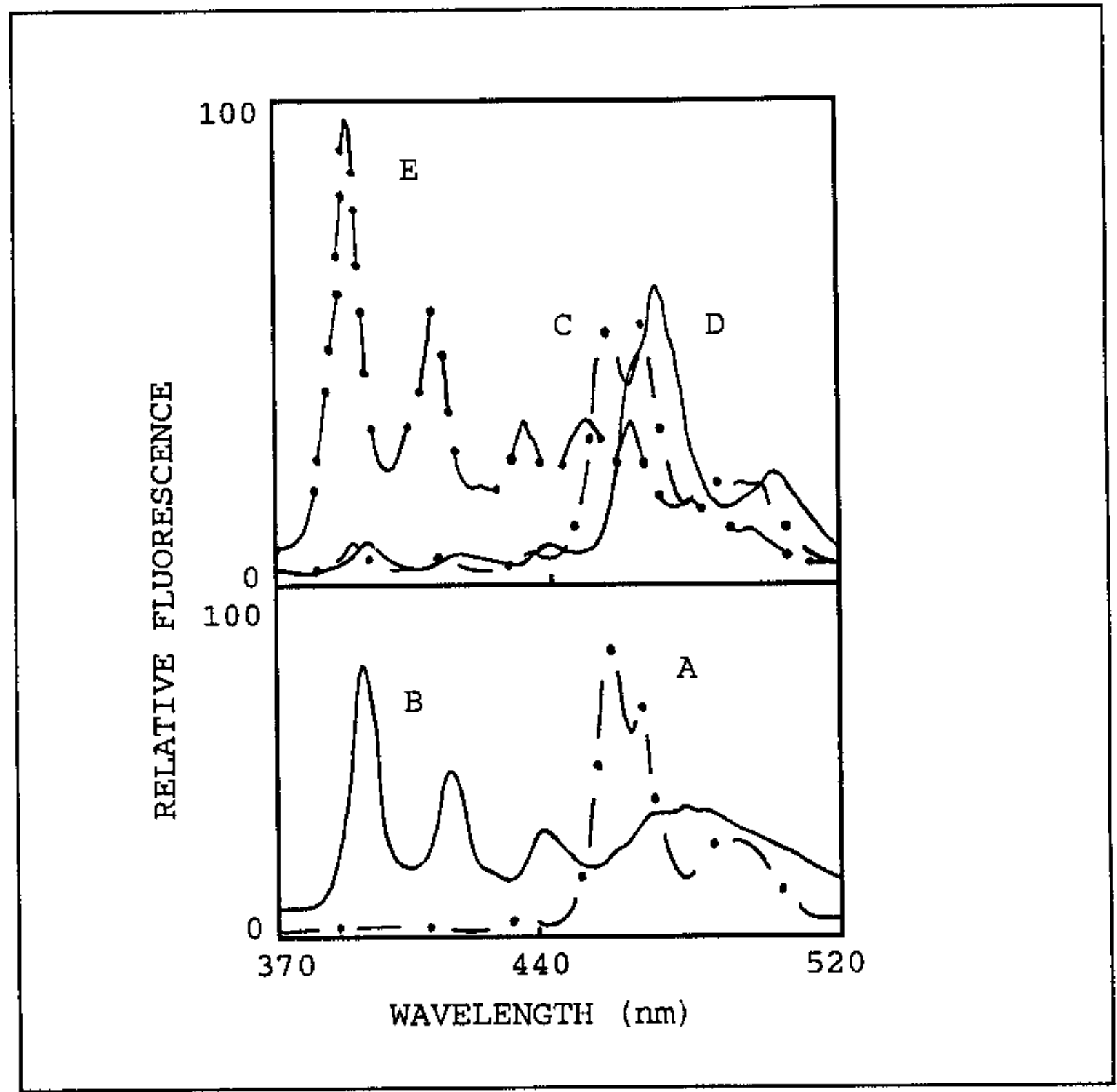

FIGURE 39: Fluorescence emission spectra of pyranthene dissolved in $[A(--)] n$-hexadecane, $[B(-)]$ dichloromethane, [C (- - -)] butyl acetate, [D ( dimethyl sulfoxide, and [E (O)] binary 1,1,2-trichlorotrifluoroethane + perfluoro-1,3-dimethylcyclohexane solvent. In dichloromethane major emission bands occur at about 394 , 416, and $441 \mathrm{~nm}$, with a broad unresolved band located in the 460-520 nm spectral region. 
Representative fluorescence emission spectra of 1 methylpyrene, 1-pyrenebutanoic acid, 4H-benzo[e]cyclopenta[1]pyrene, and 3,4-dihydrobenzo[ghi]perylene dissolved in $n-$ hexadecane, butyl acetate, dichloromethane, and dimethyl sulfoxide are depicted in Figures 40-43. Examination of the spectral data file reveals that only 3,4-dihyrobenzo[ghi]perylene (DHBPe), 4H-benzo[e]cyclopenta[I]pyrene (BCPY), and 1-pyrenebutanoic acid (PyBA) exhibit probe character. Calculated emission intensities are DHBPe $=1.14, \mathrm{BCPY}=$ 4.04, and PyBA $=4.47$ in $n$-hexane and DHBPe $=2.10, \mathrm{BCPY}=$ 5.33 , and PYBA $=6.20$ in dimethyl sulfoxide. Actual numerical values for DHBPe, PyBA, and BCPY in all solvents examined are tabulated in Tables XXV (DHBPe) and XXXII (PYBA and $\mathrm{BCPY}$ ).

Examination of Table XXXII reveals inconsistencies in the numerical values of 1 -pyrenebutanoic acid and $4 H$-benzo[e]cyclopenta[1]pyrene in many solvents. In the chlorinated solvents, like dichloromethane, chloroform, carbon tetrachloride, 1-chlorobutane, and chlorobenzene, the socalled chlorinated effect is evident. All chlorinated values are unusually low with the exception of 1,2-dichloroethane. Another inconsistency in the PyBA data involves all of the alcoholic solvents. In alcoholic solvents, like 1-octanol and methanol, the emission intensity ratio values are extremely high, with the exception of 2,2,2-trifluoroethanol which has a very low ratio. It is thought that there is an 


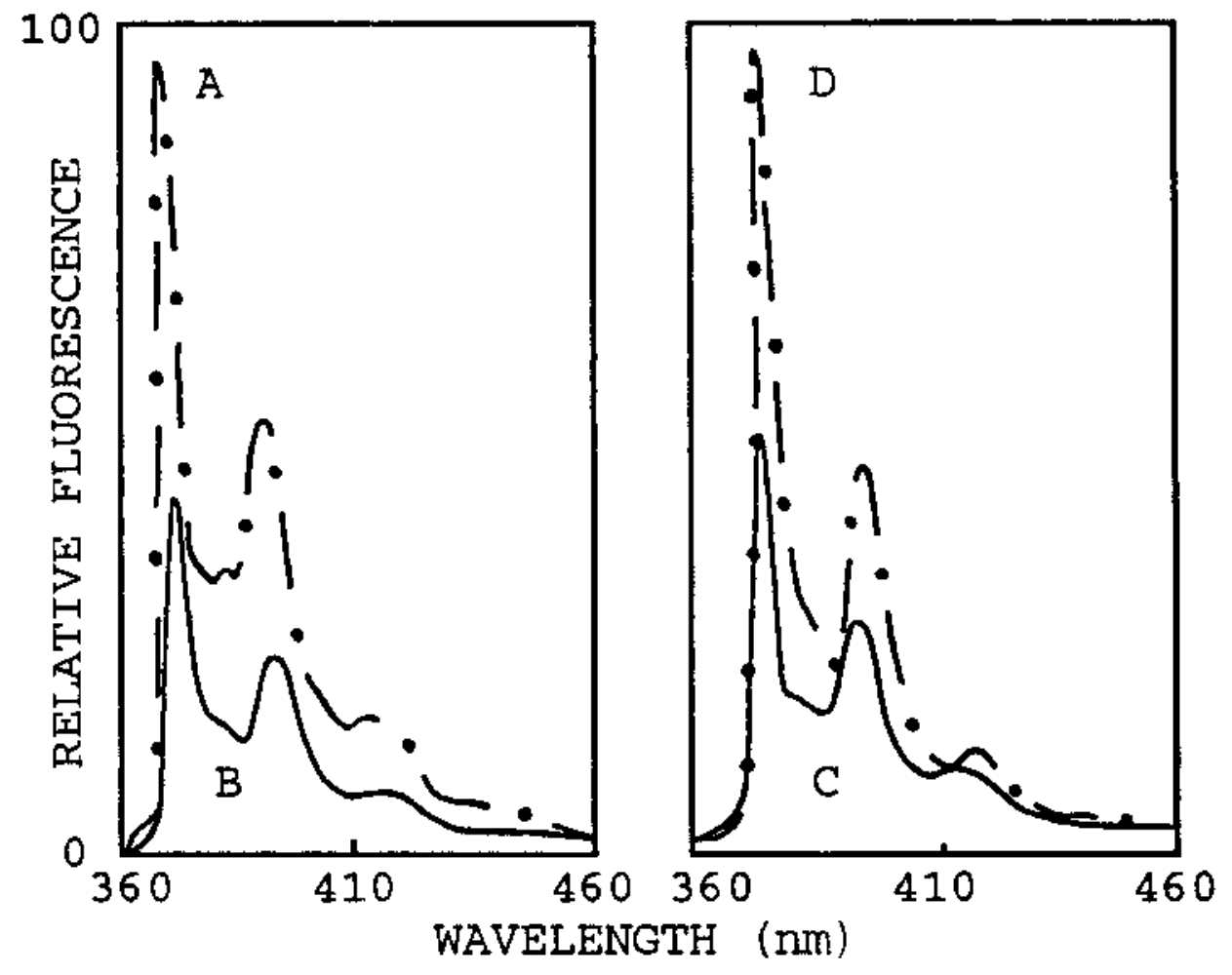

FIGURE 40: Fluorescence emission spectra of 1-methylpyrene dissolved in [A (- -) ] $n$-hexadecane, [B (-)] dichloromethane, [C (-)] butyl acetate, and [D (- O-)] dimethyl sulfoxide. In butyl acetate emission bands occur at about 375,395 , and $415 \mathrm{~nm}$. 


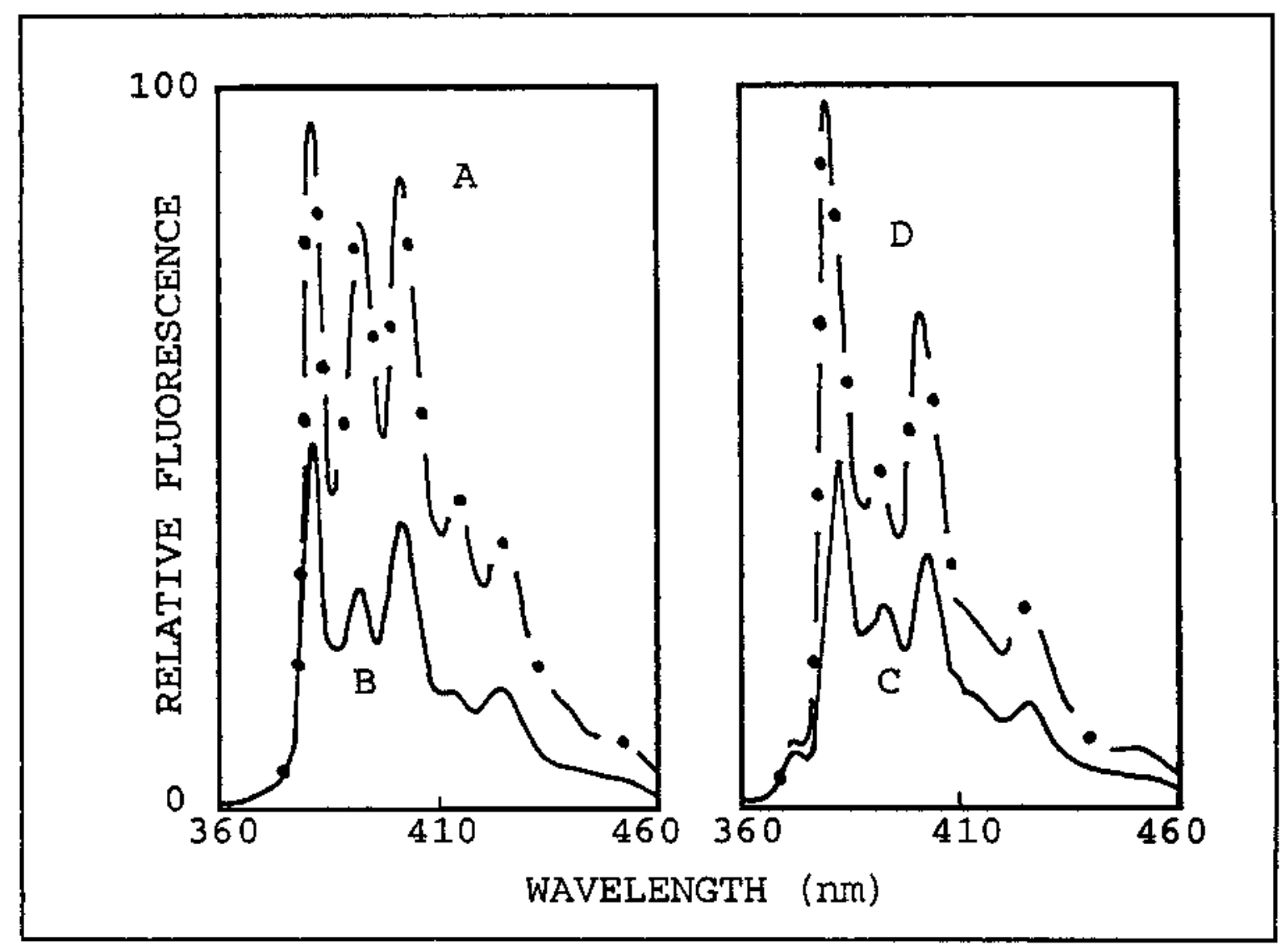

FIGURE 41: FIuorescence emission spectra of 3,4-dihyrobenzo[ghi]perylene dissolved in [A (- -)] n-hexadecane, [B (-) ] dichloromethane, [C (- $)]$ butyl acetate, and [D ( - -)] dimethyl sulfoxide. In butyl acetate emission bands occur at about $381, \mathrm{~s}-387,392,402,414$, and $425 \mathrm{~nm}$. 


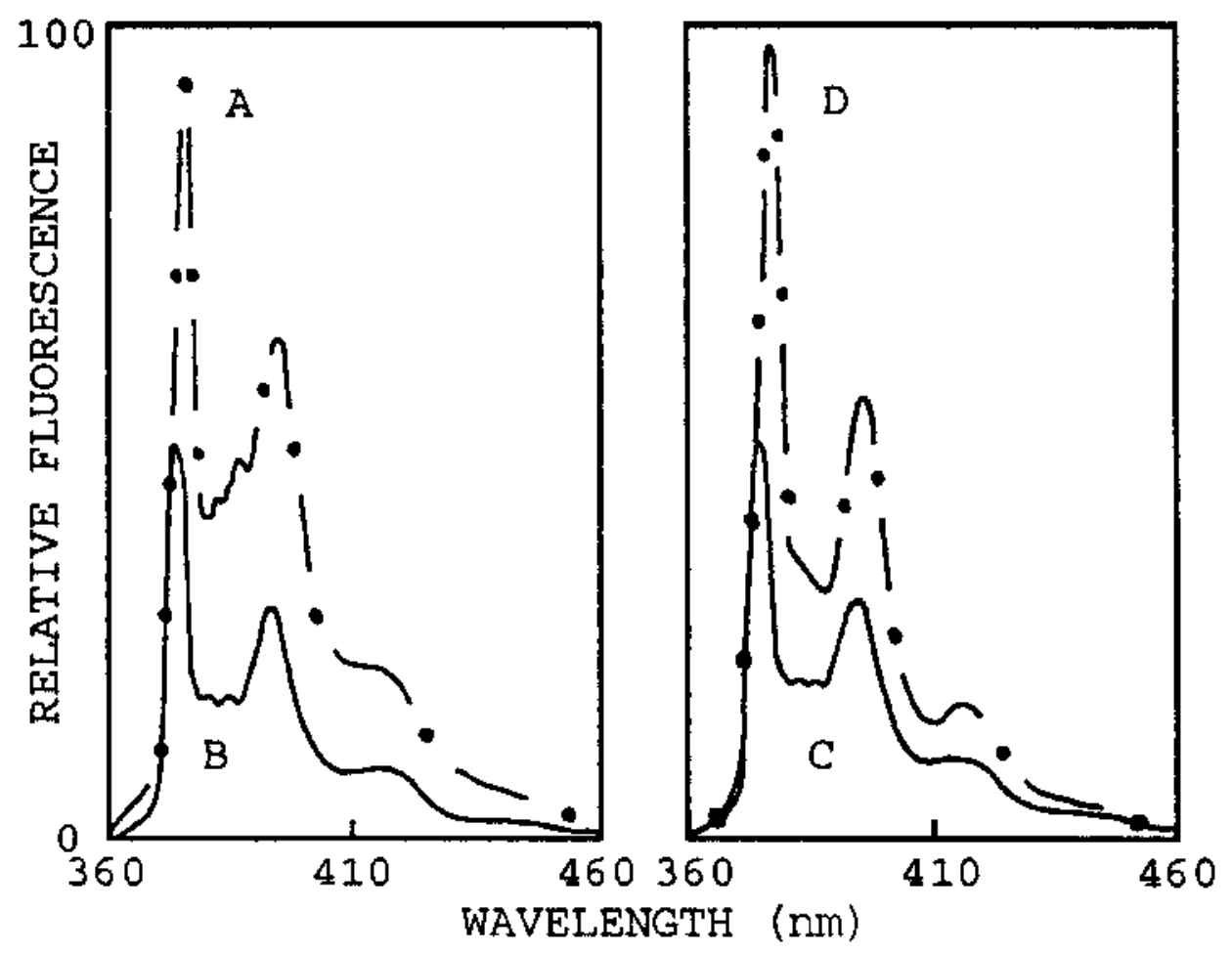

FIGURE 42: Fluorescence emission spectra of 1-pyrenebutanoic acid dissolved in [A (- -)] $n$-hexadecane, [B (-) ] dichloromethane, [C (- - $)$ ] butyl acetate, and [D (- -)] dimethyl sulfoxide. In butyl acetate emission bands occur at about $375,386,394$, and $416 \mathrm{~nm}$. 


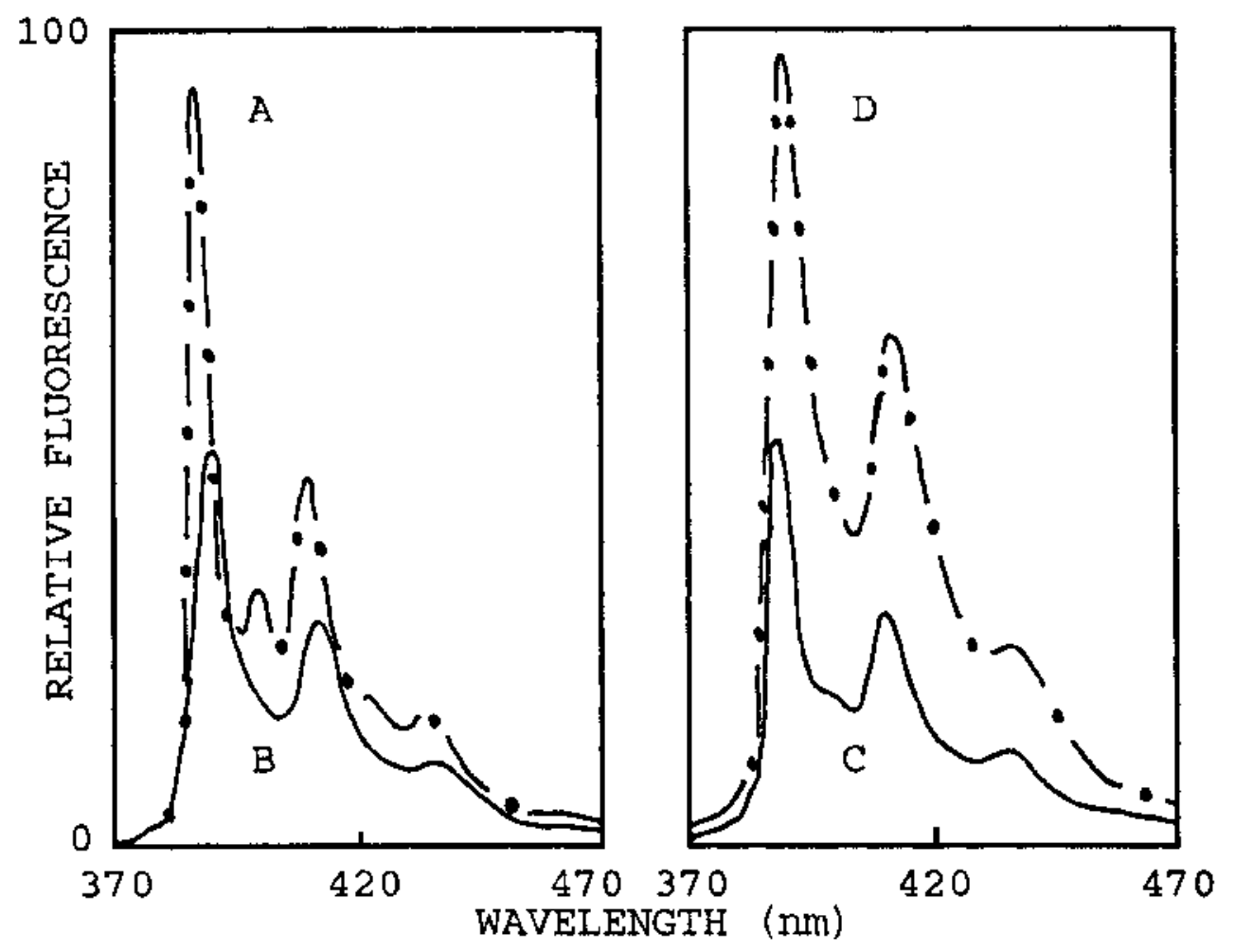

FIGURE 43: Fluorescence emission spectra of 4H-benzo[e]cyclopenta[1]pyrene dissolved in [A (- -)] n-hexadecane, [B (-)] dichloromethane, [C (- - ] butyl acetate, and [D (- -)] dimethyl sulfoxide. In butyl acetate emission bands occur at about 388,411 , and $435 \mathrm{~nm}$. 
TABLE XXXII. Ratios of fluorescence emission intensities of 1-pyrenebutanoic acid (PyBA) and 4H-benzo[e]cyclopenta[1]pyrene (BCPY) dissolved in select organic solvents.

\begin{tabular}{|c|c|c|}
\hline organic Solvent & PyBA $^{a}$ & $\mathrm{BCPY}^{\mathrm{b}}$ \\
\hline$n$-Hexane & 4.47 & 5.33 \\
\hline$n$-Heptane & 4.53 & 5.43 \\
\hline Cyclohexane & 4.55 & 5.34 \\
\hline Methylcyclohexane & 4.60 & 5.30 \\
\hline $2,2,4$-Trimethylpentane & 4.69 & 5.38 \\
\hline$n$-Hexadecane & 4.76 & 5.22 \\
\hline Dibutyl Amine & 5.58 & NA \\
\hline Carbon Tetrachloride & 4.05 & 3.04 \\
\hline Dibutyl Ether & 4.96 & 5.20 \\
\hline 1-octanol & 5.66 & 5.23 \\
\hline$p$-Xylene & 5.01 & 4.68 \\
\hline o-Xylene & 4.92 & 4.69 \\
\hline Methyl tert-Butyl Ether & 5.24 & 4.74 \\
\hline$m-X y l e n e$ & 5.16 & 4.67 . \\
\hline 1-Pentanol & 5.77 & 4.79 \\
\hline Ethyl Ether & 5.15 & 4.90 \\
\hline Toluene & 5.08 & 4.82 \\
\hline Benzene & 5.15 & 4.85 \\
\hline 1-Butanol & 6.07 & 4.72 \\
\hline 1-Chlorobutane & 5.18 & 4.70 \\
\hline
\end{tabular}


TABLE XXXII. Continued.

\begin{tabular}{|c|c|c|}
\hline Organic Solvent & PYBA ${ }^{\mathrm{a}}$ & $\mathrm{BCPy}^{\mathrm{b}}$ \\
\hline Chlorobenzene & 5.11 & 4.72 \\
\hline 1-Propanol & 5.93 & 4.73 \\
\hline Cyclohexanol & 5.24 & 4.86 \\
\hline 2-Propanol & 6.22 & 4.70 \\
\hline Ethanol & 5.96 & 4.47 \\
\hline Chloroform & 5.39 & 4.63 \\
\hline Butyl Acetate & 5.41 & 4.73 \\
\hline Methanol & 5.55 & $4 \cdot 30$ \\
\hline Tetrahydrofuran & 5.45 & 4.46 \\
\hline Dichloromethane & 5.35 & 4.55 \\
\hline Ethyl Acetate & 5.43 & 4.62 \\
\hline 1,2-Dichloroethane & 5.43 & 4.39 \\
\hline Dimethyl Carbonate & 5.33 & 4.80 \\
\hline 4-Methy 1-2-pentanone & 5.28 & 4.37 \\
\hline 1,4-Dioxane & 5.77 & 4.85 \\
\hline 2-Butanone & 5.72 & 4.43 \\
\hline Dibutyl oxalate & 6.32 & 5.52 \\
\hline Ethylene Glycol & 6.12 & 4.29 \\
\hline Acetone & 5.71 & $4 \cdot 34$ \\
\hline $2,2,2$-Trifluoroethanol & 4.84 & 3.88 \\
\hline Acetonitrile & 5.68 & 4.07 \\
\hline$N, N$-Dimethylacetamide & 5.93 & 4.19 \\
\hline
\end{tabular}


TABLE XXXII. Continued.

\begin{tabular}{lcc}
\hline organic Solvent & PyBA $^{\mathrm{a}}$ & $\mathrm{BCPY}^{\mathrm{b}}$ \\
\hline$N, N$-Dimethylformamide & 5.76 & 4.16 \\
Propylene Carbonate & 5.92 & 4.14 \\
Water & 5.71 & $\mathrm{NA}$ \\
Dimethyl Sulfoxide & 6.20 & 4.04 \\
\hline
\end{tabular}

a Defined as the ratio of band I (at 374-376 nm)/band $V$ (at 414-418 nm). b Defined as the ratio of band I (at 387-391 $\mathrm{nm}$ )/band III (at 432-439 $\mathrm{nm}$ ).

interaction between the acid group of PyBA and the hydrogen atom on the alcoholic solvent. These inconsistencies significantly restrict the future use of 1-pyrenebutanoic acid and $4 \mathrm{H}$-benzo[e]cyclopenta[ $I]$ pyrene.

Calculated intensity ratios in the larger emission bands for 1 -methylpyrene (I/II $=1.97 \pm 0.15), 2$-methylpyrene $(I / I I=1.0 \pm 0.04$ and $I / I I I=1.12 \pm 0.04)$, 4methylpyrene $(\mathrm{I} / \mathrm{II}=1.83 \pm 0.08), 1,5$-dimethylpyrene $(\mathrm{I} / \mathrm{II}$ $=1.89 \pm 0.08), 1$-butylpyrene $($ I $/$ II $=1.80 \pm 0.07), 1-$ decylpyrene $($ I/II $=1.80 \pm 0.08), 4$-methylchrysene $(I / I I=$ $0.97 \pm 0.10), 5$-methylchrysene $(I / I I=1.03 \pm 0.08), 5-$ methyldibenzochrysene $($ I $/$ II $=2.16 \pm 0.16), 3,4,5$-trihydrobenzo[cd]pyrene, THBPy (I/II $=2.23 \pm 0.05), 5,6,7,8,9,10-$ hexahydrobenzo(ghi]pyrene, HHBPe $(I / I I=2.28 \pm 0.07), 1-$ 
chloropyrene $(I / I I=1.40 \pm 0.08), 8 b, 9,10,11,12,12 a-$ hexahydro-9, 12-methanobenzo[e]pyrene (II/III $=1.10 \pm 0.10)$, $4,5,6,7$-tetrahydro-4,7-methanodibenzo $[f g, o p]$ naphthacene, THMDBN $(\mathrm{I} / \mathrm{II}=1.77 \pm 0.03), 3,6$-dicyanopyrene $(\mathrm{I} / \mathrm{II}=1.33$ $\pm 0.08)$, and 3-perylenecarboxaldehyde $(I / I I=1.52 \pm 0.05)$ were nearly identical in all solvents examined. Estimated uncertainties in the measured intensity ratios are once again believed to be on the order of \pm 0.05 (or less) on the basis of replicate measurements over at least a five day period for all PAH6 benzenoid derivatives except for 1pyrenebutanoic acid and $4 \mathrm{H}$-benzo[e]cyclopenta[1]pyrene which is on the order of \pm 0.20 due to the extremely high dynamic range and lack of spectral averaging capabilities. Significant loss of the "pyrene-like" five-band emission was observed for many of the alkylpyrenes. Most of the pyrene derivatives possessed only three resolvable bands; whereas, pyrene's emission spectrum normally contains at least five emission bands.

Figures 44-46 document that 1-pyrenecarboxaldehyde (PyCA), 1,3-pyrenedicarboxaldehyde (PyDCA), and 1-aminopyrene (APY) all show solvatochromic behavior as evidenced by rather pronounced changes in the location of their fluorescence emission band. In nonpolar solvents, such as cyclohexane, the first and usually most intense emission band occurs at circa 398,393 , and $418 \mathrm{~nm}$ for APY, PyCA, and PyDCA, respectively. Significant redshifts were noted in 


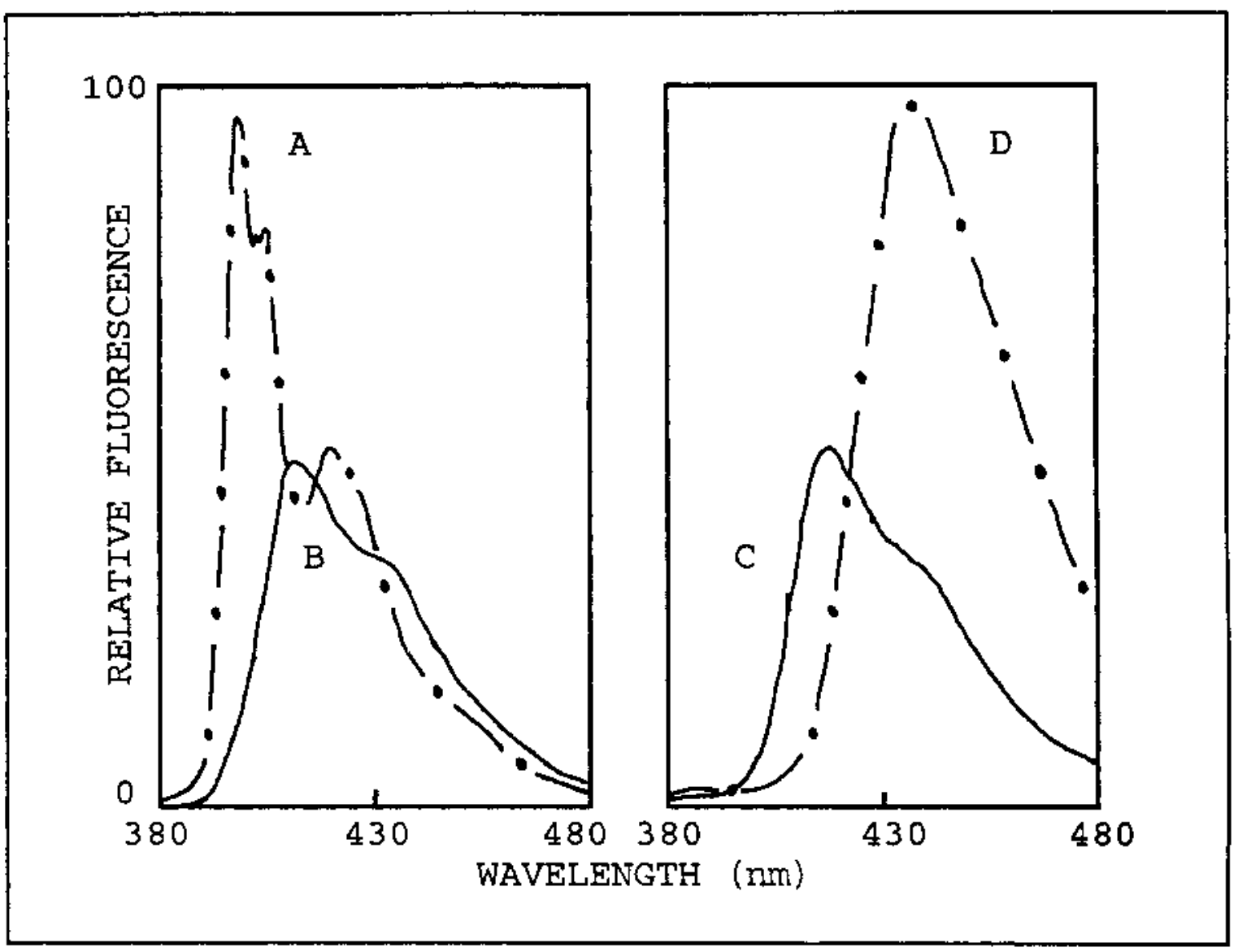

FIGURE 44: Fluorescence emission spectra of 1-aminopyrene dissolved in [A (- - -)] $n$-hexadecane, [B (- - $)$ ] dichloromethane, [C (-)] butyl acetate, and [D (- -)] dimethyl sulfoxide. In butyl acetate the emission band occurs at about $421 \mathrm{~nm}$. 


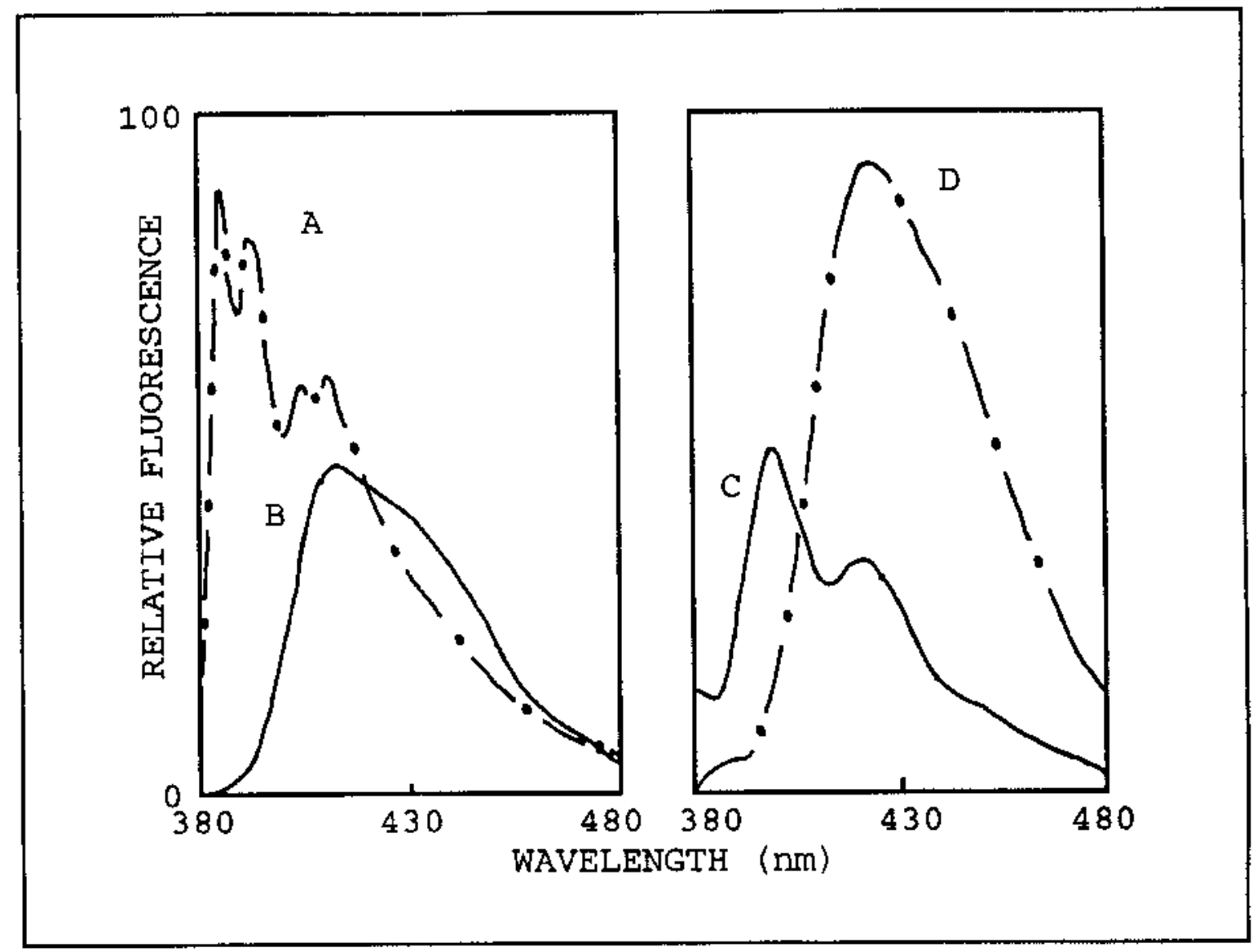

FIGURE 45: Fluorescence emission spectra of 1-pyrenecarboxaldehyde dissolved in [A (- -) ] $n$-hexadecane, [B (dichloromethane, [C (- $)$ ] butyl acetate, and [D (- - -)] dimethyl sulfoxide. In butyl acetate emission bands occur at about 401 and $422 \mathrm{~nm}$. 


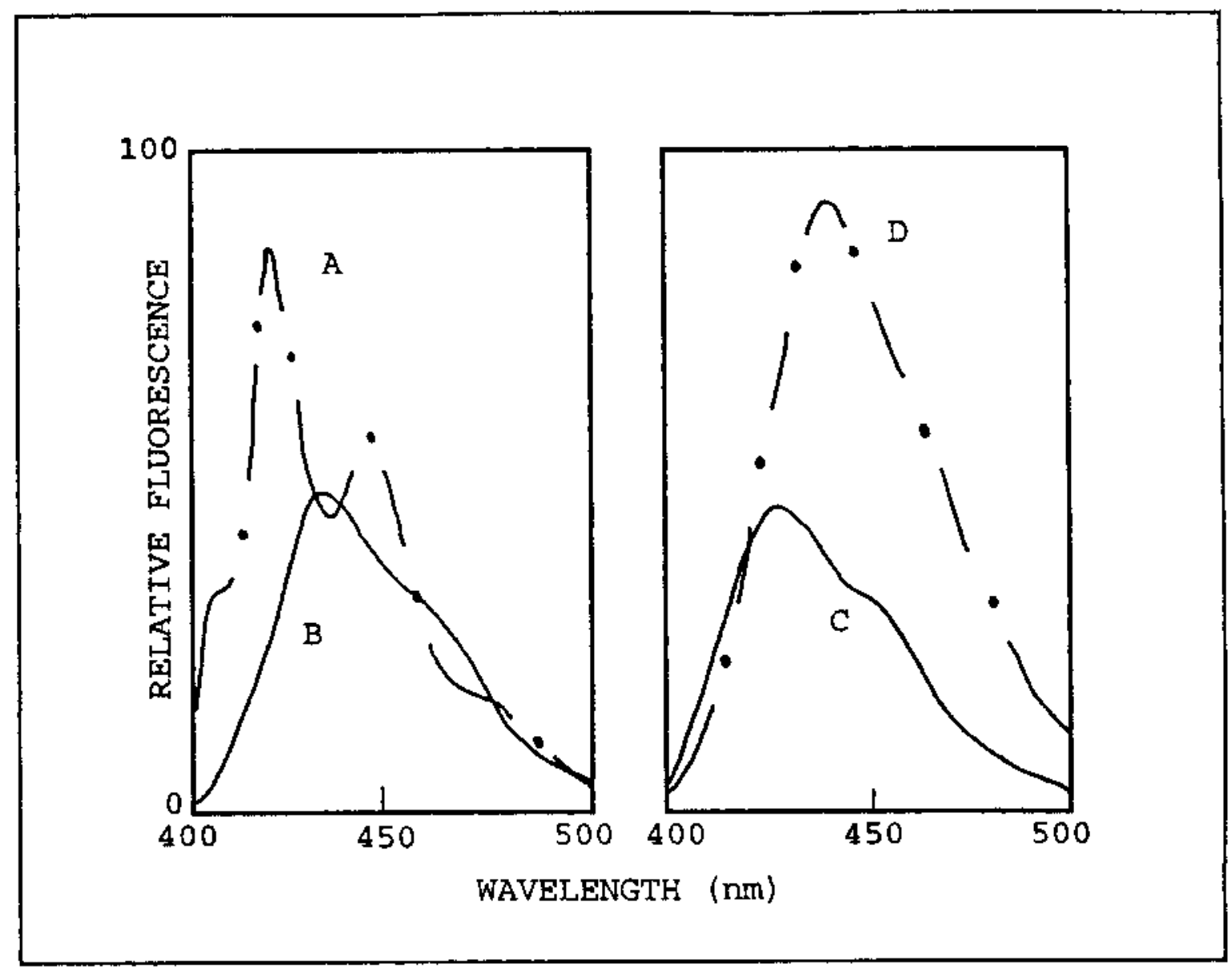

FIGURE 46: Fluorescence emission spectra of 1,3-pyrenedicarboxaldehyde dissolved in [A (- -) ] n-hexadecane, [B (- $)$ ] dichloromethane, [C (- - ) ] butyl acetate, and [D (- -)] dimethyl sulfoxide. In butyl acetate emission bands occur at about 402 and $428 \mathrm{~nm}$. 
the $s_{1} \rightarrow s_{0}$ fluorescence in the more polar dimethyl sulfoxide solvent, e.g. 440, 422, and $438 \mathrm{~nm}$ for APY, PYCA, and PyDCA, respectively. These particular observations have been reported in several previous publications in the case of APY and PYCA (15-18); however, the 42 solvents in Table XXXIII represents the most comprehensive steady-state fluorescence studies completed to date. The pronounced wavelength shifts of APY, PyCA and presumably PyDCA are due to two chromophores of similar energy resulting from the excited $\pi \pi^{*}$ (in polar solvents) and $n \pi^{*}$ (in nonpolar solvents) states (16).

Our fluorescence wavelengths of circa $\lambda_{\mathrm{em}} \approx 450 \mathrm{~nm}, \lambda_{\mathrm{em}}$ $\approx 456 \mathrm{~nm}$, and $\lambda_{\mathrm{em}} \approx 445 \mathrm{~nm}$ for PyCA in methanol, ethylene glycol, and propanol, respectively, are in good agreement with values of $\lambda_{\mathrm{em}} \approx 450 \mathrm{~nm}, \lambda_{\mathrm{em}} \approx 454 \mathrm{~nm}$, and $\lambda_{\mathrm{em}} \approx 442 \mathrm{~nm}$, cited in a paper by Kalyanasundaram and Thomas (15). Also, values of $\lambda_{\mathrm{em}} \approx 403$ and $419 \mathrm{~nm}, \lambda_{\mathrm{em}} \approx 430 \mathrm{~nm}, \lambda_{\mathrm{em}} \approx 440 \mathrm{~nm}$, and $\lambda_{\text {em }} \approx 429 \mathrm{~nm}$ for APy in $n$-hexane, benzene, water, and methanol, respectively, are also in good agreement with cited literature values of $\lambda_{\mathrm{em}} \approx 406$ and $420 \mathrm{~nm}, \lambda_{\mathrm{em}} \approx 430$ $\mathrm{nm}, \lambda_{\mathrm{em}} \approx 438 \mathrm{~nm}$, and $\lambda_{\mathrm{em}} \approx 428 \mathrm{~nm}$ contained in a publication by Hite, Krasanansky and Thomas (16). Inspection of Table XXXIII reveals that aminopyrene's fluorescence emission wavelength is highly redshifted in most all of the hydroxylic solvents. This artifact is probably due to the hydrogen bond formed between the solvent 


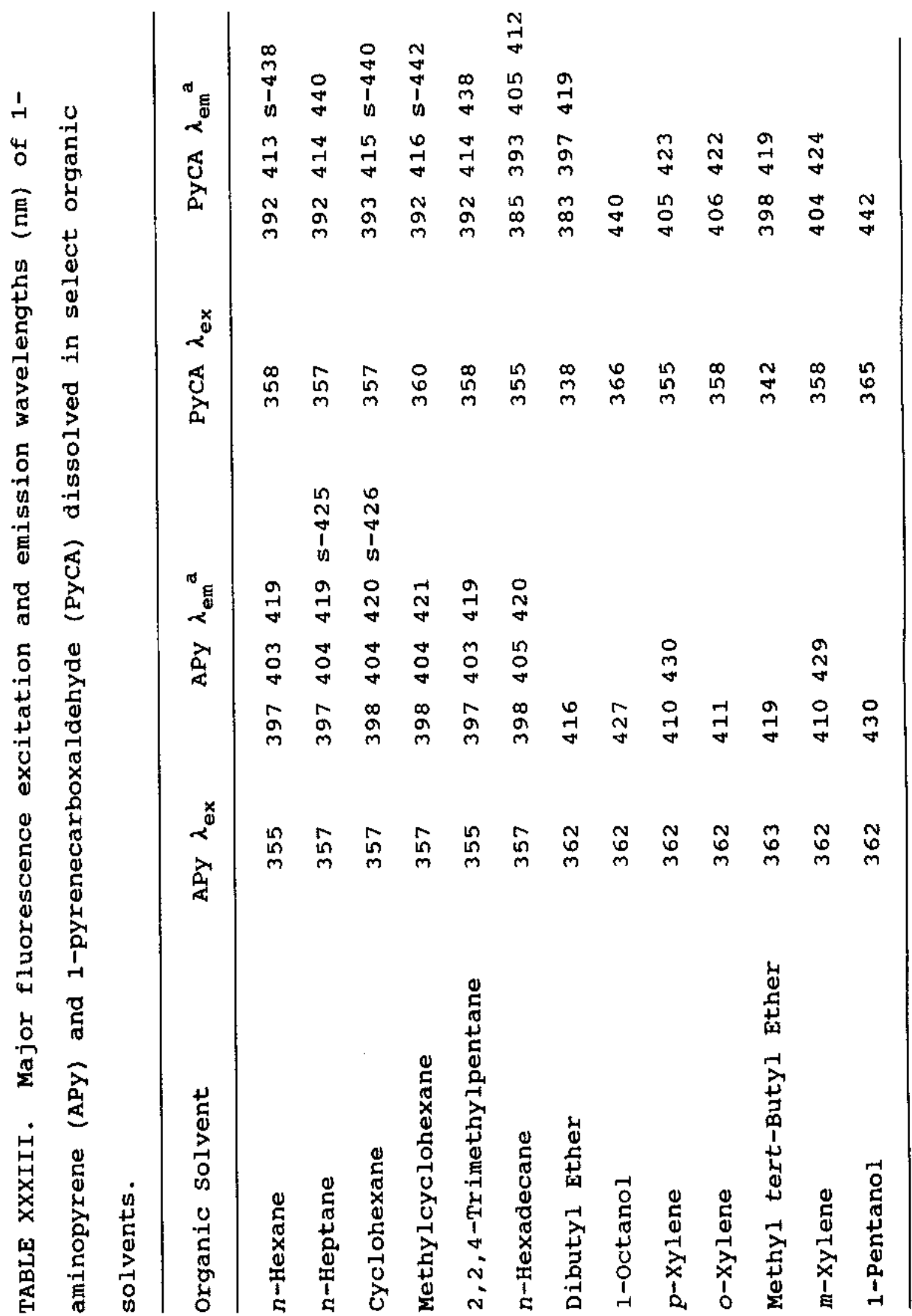




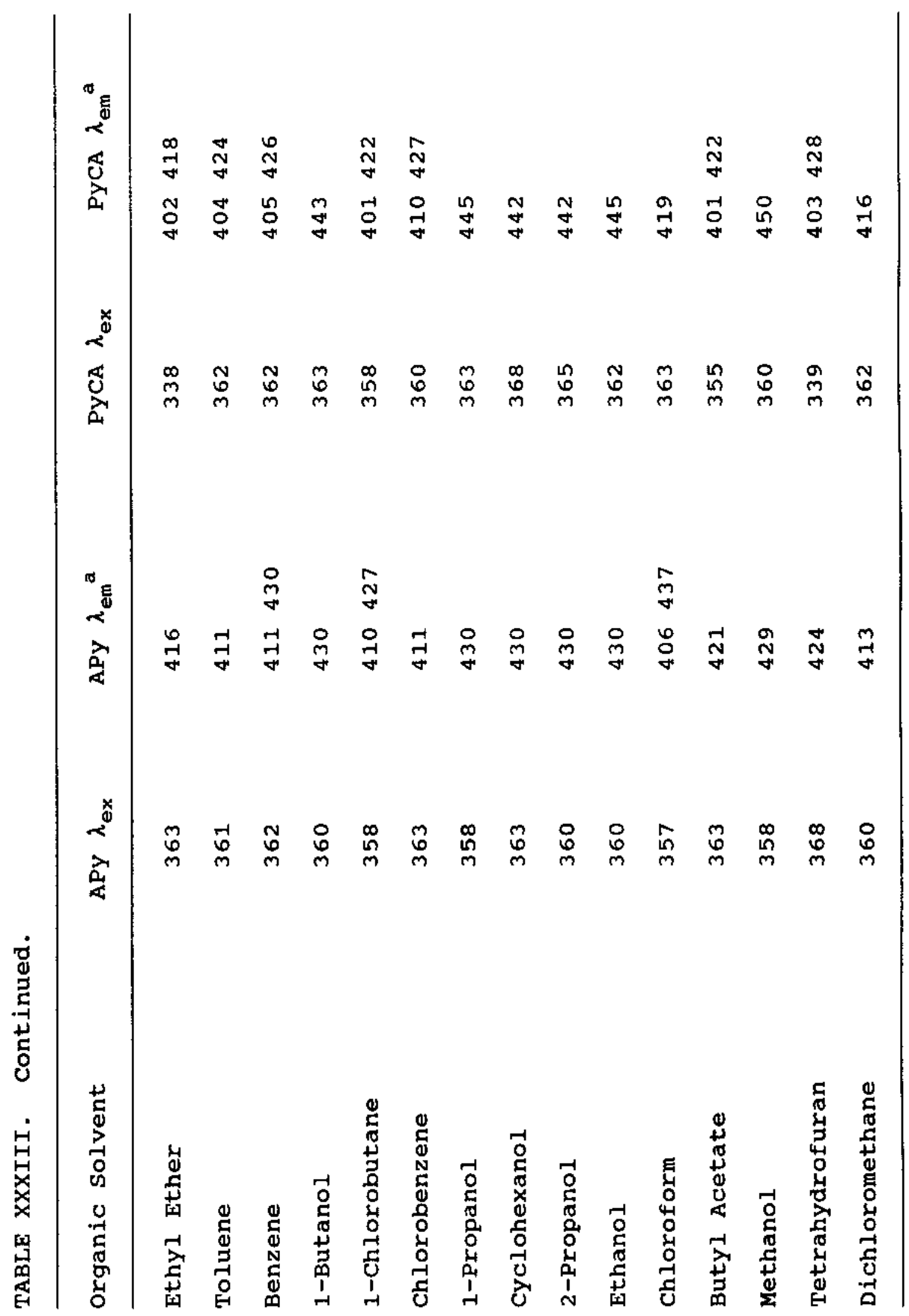




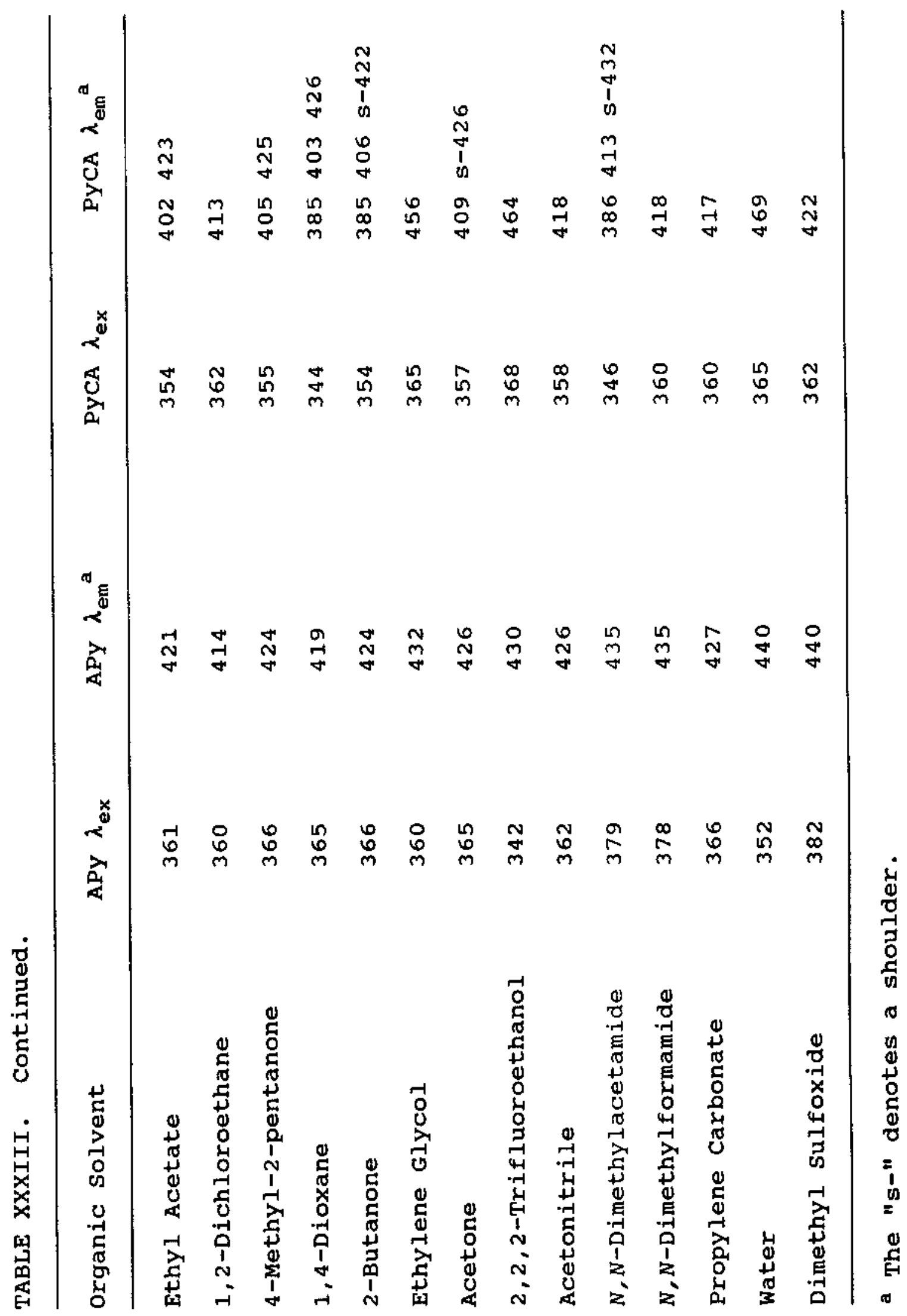


and the lone pair of electrons on the amino group in the ground state that is broken upon excitation with a hydrogen bond being formed between the amino proton and the lone pair of the solvent molecule (19). Table XXXIII also shows that 1-pyrenecarboxaldehyde and 1,3-pyrenedicarboxaldehyde (not shown) also have significantly redshifted emission wavelengths. This phenomena could also be due to hydrogen bonding in the ground state or to the enhanced dipolar character in the excited state from the oxygen atom of the PYCA or PYDCA becoming more negative due to the alignment with the carbonyl-functional group with the hydroxylic solvents (18). Even if the chlorinated and hydroxylic solvents are excluded in the case of ApY, PYCA, and PyDCA, their applicability as general solvent polarity is still Iimited.

The very interesting behavior among the coronene derivatives deserves special attention. Both 1-methylcoronene and 1,2-dimethylcoronene show solvent polarity probe character as seen in Figures 47 and 48 . In fact, inspection of Table XXXIV shows that 1-methylcoronene and 1,2-dimethylcoronene are solvent polarity probe molecules. This behavior is very interesting in light of the fact that coronene and all but two of the coronene derivatives show probe character. Benzo[a]coronene, naphtho[2,3a]coronene, dibenzo $[a, j]$ coronene, naphtho $[8,1,2 a b c]$ coronene, dinaphtho[ $\left.8,1,2 a b c ; 2^{\prime}, 1^{\prime}, 8^{\prime} k 1 m\right]$ coronene, dinaphtho- 


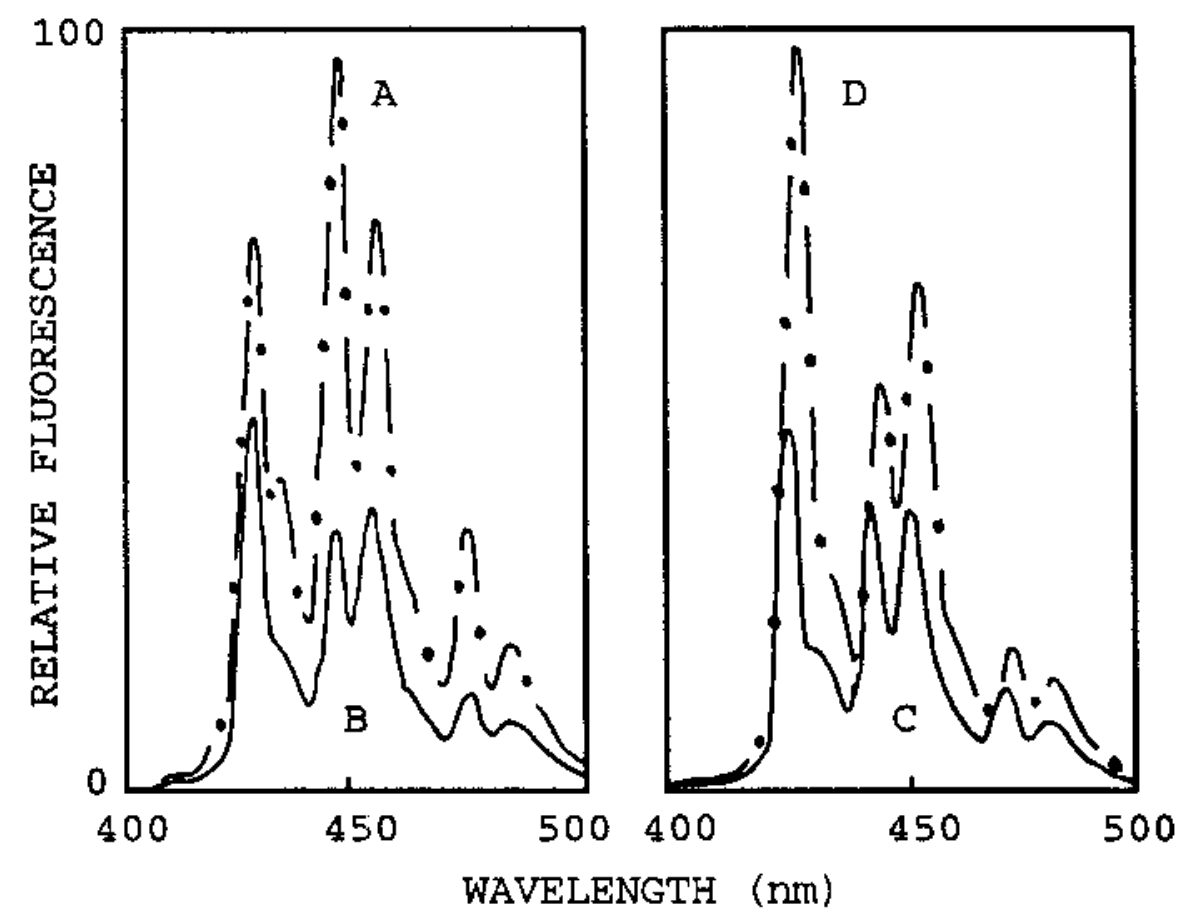

FIGURE 47: Fluorescence emission spectra of 1-methylcoronene dissolved in [A (-O -) ] n-hexadecane, [B (dichloromethane, [C (- $)$ ] butyl acetate, and [D (- -)] dimethyl sulfoxide. In butyl acetate emission bands occur at about $427,434,446,454,475$, and $484 \mathrm{~nm}$. 


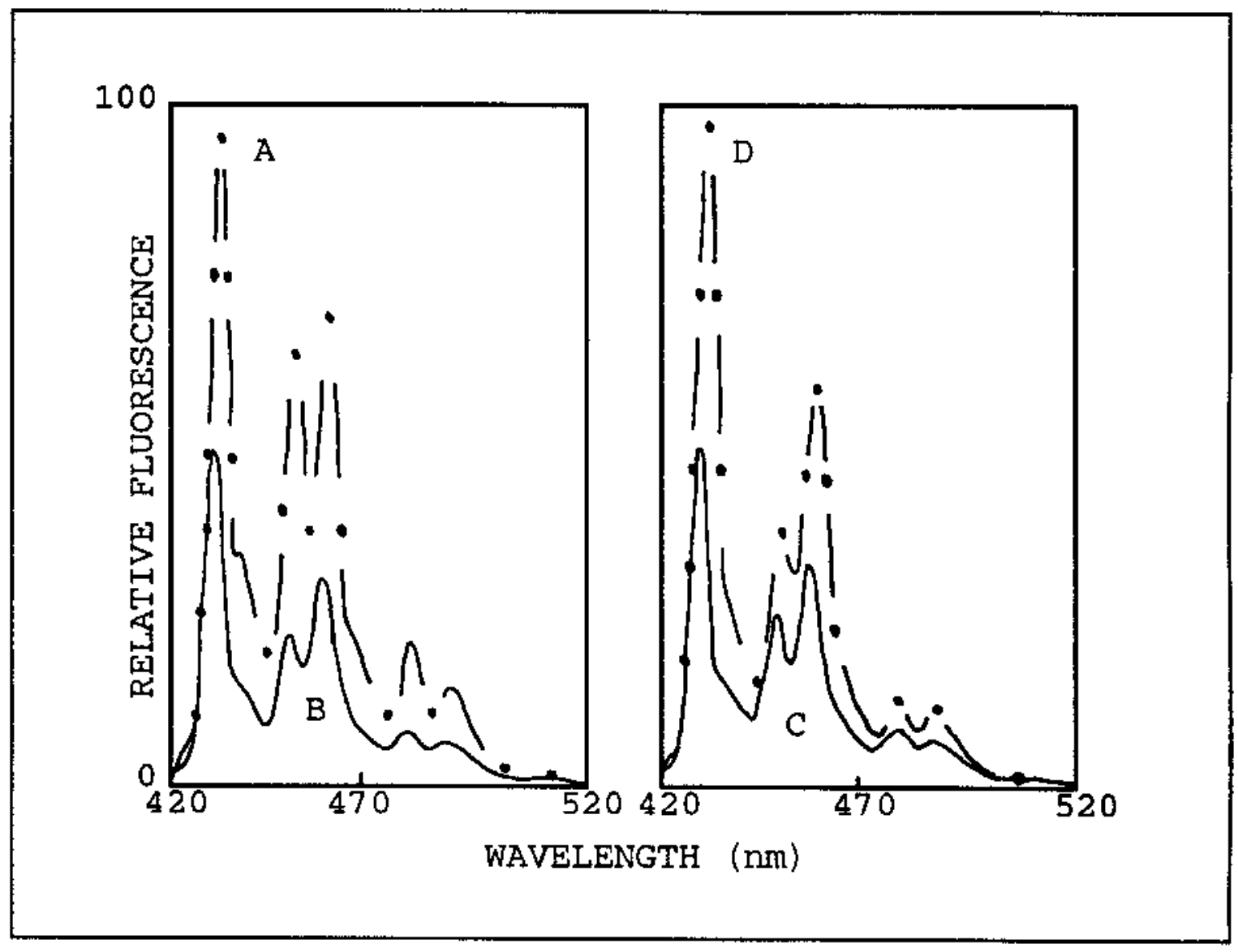

FIGURE 48: Fluorescence emission spectra of 1,2-dimethylcoronene dissolved in [A (- -) ] $n$-hexadecane, [B (- -$)$ ] dichloromethane, [C (- $)]$ butyl acetate, and [D (- - -)] dimethyl sulfoxide. In butyl acetate emission bands occur at about $430, \mathrm{~s}-437,448,456,478$, and $487 \mathrm{~nm}$. 
TABLE XXXIV. Ratios of fluorescence emission intensities of 1-methylcoronene (MeCo) and 1,2-dimethylcoronene (DMeCo) dissolved in select organic solvents.

\begin{tabular}{|c|c|c|}
\hline Organic Solvent & $\mathrm{MeCo}^{\mathrm{a}}$ & $\mathrm{DMeCo}^{\mathrm{b}}$ \\
\hline$n$-Hexane & 0.72 & 1.40 \\
\hline$n$-Heptane & 0.74 & 1.44 \\
\hline Cyclohexane & 0.72 & 1.50 \\
\hline Methylcyclohexane & 0.74 & 1.47 \\
\hline $2,2,4$-Trimethylpentane & 0.74 & 1.42 \\
\hline n-Hexadecane & 0.76 & 1.47 \\
\hline Dibutyl Amine & 0.99 & 1.83 \\
\hline Carbon Tetrachloride & 1.03 & 1.37 \\
\hline Dibutyl Ether & 0.95 & 1.65 \\
\hline 1-octanol & 1.07 & 1.80 \\
\hline$p-X y l e n e$ & 1.13 & 1.79 \\
\hline o-xylene & 1.17 & 1.86 \\
\hline Methyl tert-Butyl Ether & 1.09 & 1.71 \\
\hline m-Xylene & 1.15 & 1.81 \\
\hline 1-Pentanol & 1.16 & 1.87 \\
\hline Ethyl Ether & 1.08 & 1.79 \\
\hline Toluene & 1.15 & 1.86 \\
\hline Benzene & 1.22 & 1.90 \\
\hline 1-Butanol & 1.13 & 1.86 \\
\hline 1-Chlorobutane & 1.10 & 1.87 \\
\hline
\end{tabular}


TABLE XXXIV. Continued.

\begin{tabular}{|c|c|c|}
\hline Organic solvent & $\operatorname{MeCo}^{a}$ & $\mathrm{DMeCo}^{\mathrm{b}}$ \\
\hline Chlorobenzene & 1.16 & 1.92 \\
\hline 1-Propanol & 1.20 & 1.90 \\
\hline Cyclohexanol & 1.14 & 1.83 \\
\hline 2-Propanol & 1.14 & 1.85 \\
\hline Ethanol & 1.23 & 2.02 \\
\hline Chloroform & 1.33 & 2.05 \\
\hline Butyl Acetate & 1.30 & 1.97 \\
\hline Methanol & 1.33 & 2.08 \\
\hline Tetrahydrofuran & 1.37 & 2.07 \\
\hline Dichloromethane & 1.41 & 2.25 \\
\hline Ethyl Acetate & 1.32 & 2.05 \\
\hline 1,4-Dichlorobutane & 1.34 & 2.05 \\
\hline 1,2-Dichloroethane & 1.43 & 2.19 \\
\hline Dimethyl Carbonate & 1.36 & 2.14 \\
\hline 4-Methyl-2-pentanone & 1.36 & $\mathrm{NA}^{\mathrm{C}}$ \\
\hline 1,4-Dioxane & 1.43 & 2.16 \\
\hline 2-Butanone & 1.45 & $N A^{C}$ \\
\hline Dibutyl oxalate & 1.42 & 1.95 \\
\hline Ethylene Glycol & 1.50 & 2.23 \\
\hline Acetone & 1.50 & $\mathrm{NA}^{\mathrm{C}}$ \\
\hline $2,2,2-\operatorname{Tr}$ ifluoroethanol & 1.59 & 2.28 \\
\hline Acetonitrile & 1.61 & 2.34 \\
\hline
\end{tabular}


TABLE XXXIV. Continued.

\begin{tabular}{lcl}
\hline organic Solvent & MeCo $^{\mathrm{a}}$ & DMeCo $^{\mathrm{b}}$ \\
\hline$N, N$-Dimethylacetamide & 1.63 & 2.42 \\
$N, N$-Dimethylformamide & 1.64 & 2.41 \\
Propylene Carbonate & 1.63 & 2.41 \\
Dimethyl Sulfoxide & 1.80 & 2.57 \\
\hline Dynamic Range & 1.08 & 1.17 \\
\hline
\end{tabular}

a Defined as the ratio of band I (at $426-429 \mathrm{~nm}$ )/band III (at 444-448 nm). b Defined as the ratio of band I (at 428$432 \mathrm{~nm}$ )/band III (at 447-450 nm). " No signal could be obtained.

$\left[8,1,2 a b c ; 2^{\prime}, 1^{\prime}, 8^{\prime} j k 1\right]$ coronene, anthra $[2,3 a]$ coronene, 1methylcoronene, and 1,2-dimethylcoronene are all labeled as solvent polarity probe probes. Naphth $\left[2^{\prime}, 1^{\prime}, 8^{\prime}, 7^{\prime}: 4,10,5\right]-$ anthra $[1,9,8 a b c d]$ coronene is a nonprobe molecule because a constant set of fluorescence emission bands could not be defined in all solvents examined. New spectral bands may appear or emission bands used to defined the solvent polarity emission band ratio may not be present in all solvents. 1-Acetylcoronene is also a nonprobe because the dynamic range is not between polar and nonpolar solvents, but the range is mainly between dimethylformamide and dimethyl sulfoxide - two solvents of similar polarity. 
Benzo $\left[1,2,3 b c: 4,5,6 b^{\prime} c^{\prime}\right]$ dicoronene and dibenzo[bc,ef]coronene are really the only two coronene derivatives that do not show signs of solvent polarity probe character. Incidentally, they are the only two coronene derivatives that have no detailed fine structure in their fluorescence emission spectra.

The discovery of solvent polarity probes in the methylated coronene derivatives is quite surprising given the fact that most alkylated pyrene derivatives are nonprobes with significantly reduced fine structure in the fluorescence emission spectra. 1-Methylcoronene (MeCo) and 1,2-dimethylcoronene (DMeCo) both have detailed emission fine structure equivalent to the parent coronene's (CO) as well as having almost identical emission peaks. However, the relative intensity of the I band with respect to the III band is very different in all three compounds. For example, the I/III ratio for Co $=0.12$, MeCo $=0.72$, and $\mathrm{DMeCO}=1.40$ in $n$-hexane and for $\mathrm{Co}=0.98, \mathrm{MeCo}=1.80$, and $\mathrm{DMeCO}=2.57$ in dimethylsulfoxide, but their dynamic ranges are nearly identical - 0.86, 1.08, and 1.17 for Co, MeCo, and DMeCo, respectively. The extremely low intensity of the $I$ band at circa $424 \mathrm{~nm}$ for coronene in nonpolar solvents introduces greater uncertainty in the nonpolar solvent ratios. This is not the case for 1-methylcoronene or 1,2-dimethylcoronene, which would make them more desirable to use as solvent polarity probes over the parent coronene. It should 
be noted that I was still unable to overcome the solubility problems experiences in trying to accurately measure the fluorescence emission spectra of coronene dissolved in water. 1-Methylcoronene and 1,2-dimethylcoronene are only slightly soluble in water. With the Shimadzu RF-5000U spectrofluorometer (xenon excitation source) only a very noisy emission spectrum was obtained for both compounds dissolved in water.

THBPy, HHBPe, DHBPe, and THMDBN are all hydrogenated PAH derivatives (or "di-connected alkyl-PAHs" if the hydrogenated rings are viewed as cyclopentane, $-\mathrm{CH}_{2} \mathrm{CH}_{2}-$ and $-\mathrm{CH}_{2} \mathrm{CH}_{2} \mathrm{CH}_{2}-$ groups) containing an intact, known solvent polarity probe moiety, pyrene versus benzo[e]pyrene, respectively. of all the alkylpyrene or dialkylpyrene derivatives examined, none have been classified as a useful probe molecule except the alkylbenzo[e]pyrene (DHBPe). These results suggests that alkyl substitution, and its effects on molecular shape and size, play an important role in determining how the ground and excited state fluorophores interact with their surrounding solvation cage.

The fluorescence behavior of the internally substituted PAHs is markedly different from the hydrogenated PAHs. In fact, no fluorescence emission spectrum could be obtained for trans-10b, 10c-dihydro-10b,10c-dimethylpyrene (DHDMPY). trans-12b, 12c-Dihydro-12b, 12c-dimethylbenzo [a]pyrene (DHDMBPy), and trans-14b,14c-dihydro-14b,14c-dimethyl- 
naphtho $[2,1,8$ gra $]$ naphthacene (DHDMNN) displayed very weak signals in one or two of the more viscous solvents. DHDMPY has an almost planar (20), rigid skeleton that is a little strained, and as such acts as a "perfect" [14]annulene (21). The lack of the central pyrenoid double bond (22) in comparison to its highly fluorescence parent benzo[a]pyrene may account for the nonfluorescent behavior of this compound. While DHDMBPY and DHDMNN do have more aromatic character than DHDMPY from the additional benzene ring(s) and do fluorescence, their fluorescence quantum yields are much too low to be screened as possible solvent polarity probes. It is unfortunate that the internally substituted PAHs do not appear to have reasonable fluorescence quantum yields as there have been many of these compounds synthesized for determination of structure activity relationships leading to carcinogenicity. For example, DHDMBPy and DHDMNN have the same gross structure and the same electronic periphery as benzo[a]pyrene, but with some alteration in the complete electronic structure (22). All three compounds were found to lack mutagenicity and, therefore, putative carcinogenicity (22) unlike the highly carcinogenic parent compound benzo[a]pyrene.

Earlier structure activity relationships reasoned that a phenanthrene nucleus and, therefore, a K-region, were essential for carcinogenic activity in PAHs (23-25), but later studies indicated that structural characteristics 
other than the phenanthrene structure were important in conferring carcinogenic activity on the PAH (26). AshwoodSmith, Mitchell, and Kennedy (22) concluded that "it is not sufficient for a molecule to have the same electronic periphery as a known carcinogen (mutagen) for it to induce mutagenicity (and probably carcinogenicity). cross conjugation or some degree of benzenoid character may also be necessary" (22). Because of the aforementioned health concerns and known reduced carcinogenicity of the highly fluorescent hydrogenated PAH subclass in general (27), future studies should include additional derivatives.

\section{Polycyclic Aromatic Hydrocarbon Derivatives Containing a Five-Membered Ring}

Like the PAH6 benzenoids derivatives, some of the fluoranthenoid, fluorenoids, cyclopenta-PAHs, acephenanthrylene, and acenaphthalene derivatives show modest probe character. Examination of spectral data file reveals that naphtho[ $2,3 b] f l u o r a n t h e n e(N F A)$, benzo[k]fluoranthene (BFA), 11H-benzo[bc] aceanthrylene (BAA), 4H-benzo[b]cyclopenta[mno]chrysene (BCyCh), 4H-cyclopenta[pqr]picene (CyPi), 13Hdibenz[bc] aceanthrylene (DBAA), and 4H-cyclopenta[def]chrysene (CyCh) show solvent polarity probe behavior as indicated by the systematic variation of the fluorescence emission intensity ratio with solvent polarity (see Tables 
$X X X V-X X X V I I)$. The estimated uncertainties in the measured intensity ratios remains at \pm 0.05 or less. Once again, it is only a few of the solutes that exhibit this effect.

Inspection of Table XXXV reveals that while benzo[e]aceanthrylene $(B[e] A A)$ does show some signs of probe character, the emission intensity ratios do not vary systematically with solvent polarity as the numerical $B[e] A A$ values for benzene, toluene, $p$-xylene and o-xylene (latter three not given) are far too small. Even if the benzene derivatives values are ignored, the dynamic range of 0.62 is significantly smaller than those of pyrene (1.37), benzo[e]pyrene (I/IV, 1.55), benzo[ghi]perylene (0.98), 3,4-dihydrobenzo[ghi]perylene $(0.98)$, coronene $(0.86)$, and ovalene (2.08). To be consistent with the classification scheme, $B[e] A A$ is labelled as a solvent polarity nonprobe molecule because of the unexplained, abnormally low $B[e] A A$ value measured for the benzene derivatives. Tables XXXVXXXVII also show that five of seven solvent polarity probes have intensity ratios that decrease systematically with increasing solvent polarity, the majority of PAHs increase with increasing solvent polarity.

One of the alkylated acenaphthylene derivatives, 3methylbenz[j]aceanthrylene, (see Figure 49) initially appeared to exhibit solvent polarity behavior as evidence by a changing emission intensity ratio, but upon closer examination it was noted that there existed no correlation 
TABLE XXXV. Ratios of fluorescence emission intensities of benzo[e]aceanthrylene (BeAA), naphtho $[2,3 b]$ fluoranthene (NFA), and benzo[k]fluoranthene (BFA) dissolved in select organic solvents.

\begin{tabular}{|c|c|c|c|}
\hline Organic Solvent & $B e A A^{a}$ & $\mathrm{NFA}^{\mathrm{b}}$ & $\mathrm{BFA}^{\mathrm{C}}$ \\
\hline Cyclohexane & 1.62 & 1.48 & 1.80 \\
\hline $2,2,4$-Trimethylpentane & 1.60 & 1.46 & 1.87 \\
\hline n-Hexadecane & 1.58 & 1.43 & 1.84 \\
\hline Carbon Tetrachloride & $\mathrm{Rxn}^{\mathrm{d}}$ & 1.31 & 1.63 \\
\hline Dibutyl Ether & 1.36 & 1.24 & 1.44 \\
\hline Benzene & 1.16 & 1.13 & 1.37 \\
\hline 2-Propanol & 1.27 & 1.10 & 1.24 \\
\hline Butyl Acetate & 1.21 & 1.11 & 1.28 \\
\hline Methanol & 1.20 & 1.08 & 1.14 \\
\hline Dichloromethane & 1.17 & 1.04 & 1.25 \\
\hline Acetonitrile & 1.11 & 1.04 & 1.12 \\
\hline$N, N$-Dimethyl formamide & 1.06 & 1.00 & 1.18 \\
\hline Dimethyl sulfoxide & 1.00 & 1.00 & 1.09 \\
\hline Dynamic Range & & 0.48 & 0.78 \\
\hline
\end{tabular}

a Defined as the ratio of band I (at 394-406 nm)/band II (at 418-406 nm). b Defined as the ratio of band I (at 402-413 nm)/band II (at 427-437 nm). C Defined as the ratio of band I (at 410-427 nm)/band II (at 436-448 nm). d Photochemical reaction. 
TABLE XXXVI. Ratios of fluorescence emission intensities of 11H-benzo[bc] aceanthrylene (BAA), 4H-benzo[b]cyclopenta[mno]chrysene (BCyCh), and 4H-cyclopenta[pqr]picene (CyPi) dissolved in select organic solvents.

\begin{tabular}{|c|c|c|c|}
\hline Organic solvent & $\mathrm{BAA}^{\mathrm{a}}$ & $\mathrm{BCyCh}^{\mathrm{b}}$ & CyPic \\
\hline Cyclohexane & 1.80 & 1.48 & 0.42 \\
\hline $2,2,4$-Trimethylpentane & 1.80 & 1.47 & 0.41 \\
\hline$n$-Hexadecane & 1.73 & 1.41 & 0.43 \\
\hline Carbon Tetrachloride & 1.61 & 1.37 & 0.69 \\
\hline Dibutyl Ether & 1.68 & 1.33 & 0.53 \\
\hline Benzene & 1.46 & 1.26 & 0.69 \\
\hline 2-Propanol & 1.53 & 1.16 & 0.64 \\
\hline Chloroform & 1.37 & 1.17 & 0.79 \\
\hline Butyl Acetate & 1.54 & 1.25 & 0.69 \\
\hline Methanol & 1.51 & 1.19 & 0.72 \\
\hline Dichloromethane & 1.37 & 1.15 & 0.77 \\
\hline Acetonitrile & 1.39 & 1.08. & 0.83 \\
\hline$N, N$-Dimethylformamide & 1.36 & 1.06 & 0.90 \\
\hline Dimethyl sulfoxide & 1.33 & 1.00 & 0.95 \\
\hline Dynamic Range & 0.57 & 0.48 & 0.54 \\
\hline
\end{tabular}

a Defined as the ratio of band I (at 385-392 nm)/band II (at 408-415 nm). b Defined as the ratio of band I (at 390-400 $\mathrm{nm}$ )/band II (at 415-425 nm). C Defined as the ratio of band I (at 373-378 nm)/band II (at 387-395 nm). 
TABLE XXXVII. Ratios of fluorescence emission intensities of $13 H$-dibenz [bc] aceanthrylene (DBAA) and $4 H$-cyclopenta[def]chrysene (Cych) dissolved in select organic solvents.

\begin{tabular}{|c|c|c|}
\hline Organic Solvent & $\mathrm{DBAA}^{\mathrm{a}}$ & $\mathrm{CyCh}^{\mathrm{b}}$ \\
\hline Cyclohexane & 1.83 & 0.72 \\
\hline$n$-Hexadecane & 1.82 & 0.75 \\
\hline Benzene & 1.60 & 0.86 \\
\hline Butyl Acetate & 1.62 & 0.82 \\
\hline Methanol & 1.58 & 0.86 \\
\hline Dichloromethane & 1.49 & 0.91 \\
\hline Acetonitrile & 1.49 & 1.08 \\
\hline$N, N$-Dimethylformamide & 1.43 & 1.08 \\
\hline Dimethyl sulfoxide & 1.44 & 1.09 \\
\hline Dynamic Range & 0.40 & 0.37 \\
\hline
\end{tabular}

a Defined as the ratio of band I (at 395-401 nm)/band II (at 422-427 nm). b Defined as the ratio of band $I$ (at 360-365 nm) /band II (at 378-384 nm). 


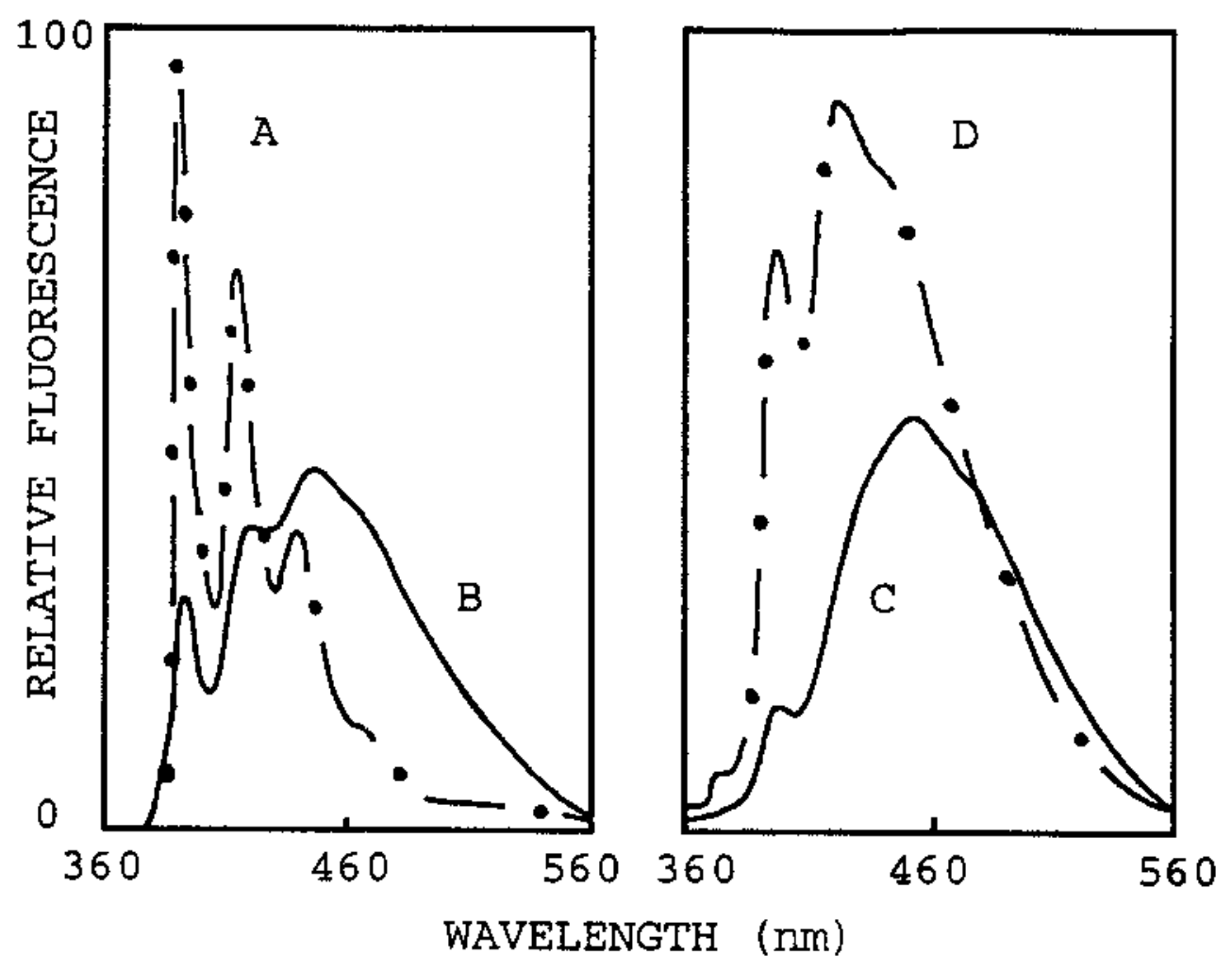

FIGURE 49: Fluorescence emission spectra of 3-methylbenz[j]aceanthrylene dissolved in [A (-O -)] n-hexadecane, [B (一)] butyl acetate, [C (- $)] N, N$-dimethyl formamide, and [D (- -)] dimethyl sulfoxide. In butyl acetate emission bands occur at about 395, 421, and $448 \mathrm{~nm}$. 
between the observed ratios and solvent polarities. For example, I/III band intensity ratios for dimethylformamide and dimethyl sulfoxide were I/III $=0.31$ and I/III $=0.79$, respectively, despite the fact that these two solvents are approximately of equal solvent polarity. It was also found that a common set of emission bands cannot be assigned in all solvents, i.e., in dimethylformamide two resolved bands were observed, the I and the III band, as opposed to two bands and a shoulder in dimethyl sulfoxide and three bands in $n$-hexadecane. Only seven of the 65 five-membered ringcontaining PAH solutes are solvent polarity probes.

Further examination of Tables XXXV-XXXVII reveals another interesting artifact. While the chlorinated effect is once again evident, it is the dynamic ranges that are very different than the aforementioned PAH subclasses. In general the dynamic ranges are much smaller than any seen previously. The only solvent polarity probe molecule to have a dynamic range greater than 0.60 is benzo[k]fluoranthene (BFA). Most of the other dynamic ranges are far too small for the solute to be effectively used as a solvent polarity probe molecule.

Inspection of Figures 50 and 51 also reveals that the fluorescence emission spectra of the five-membered ringcontaining solutes is also different than the PAHs with the exception of rubicene. The emission spectra of naphtho$[2,1 a] f l u o r a n t h e n e(F i g u r e ~ 50)$ and dibenzo $[a, e]$ fluoranthene 


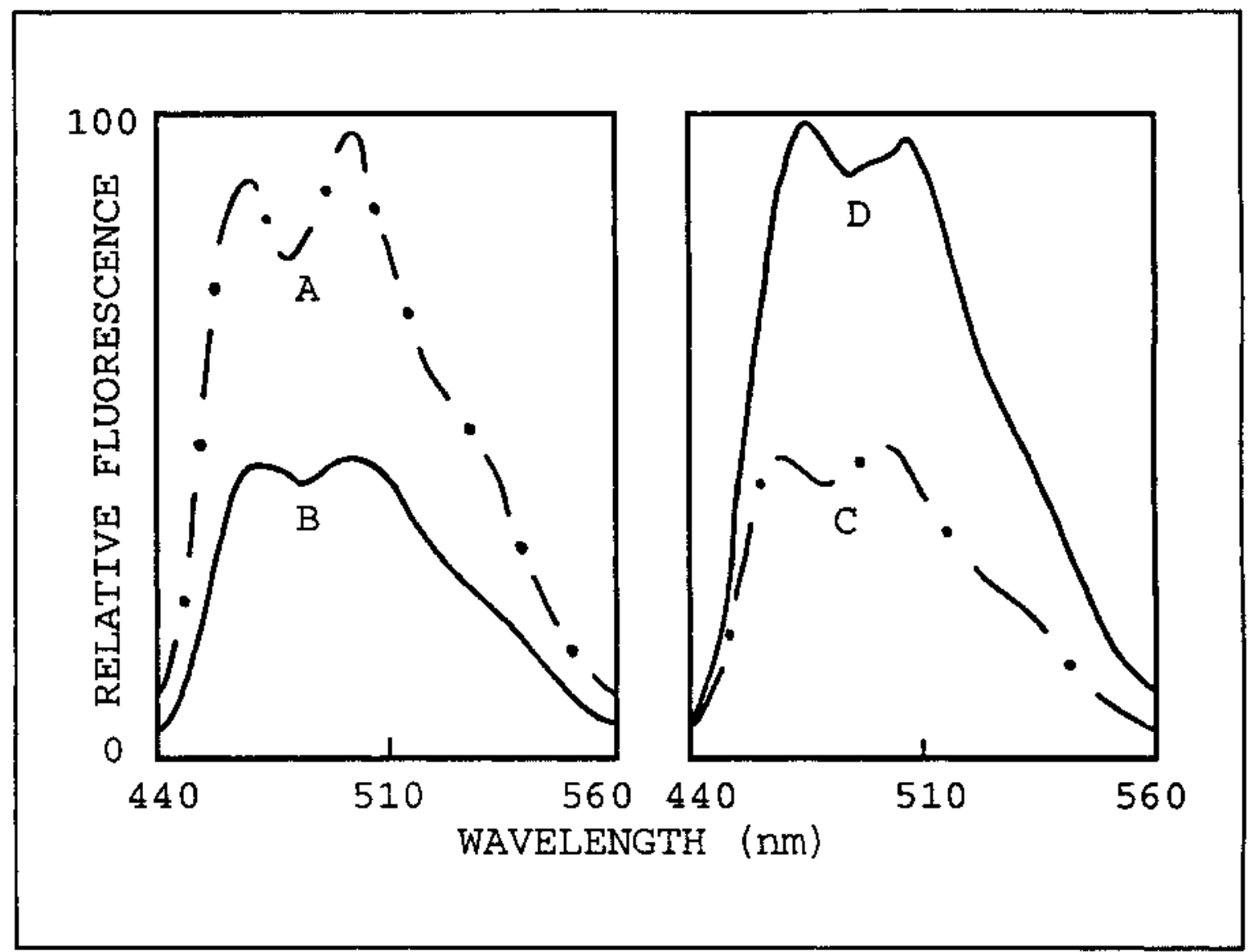

FIGURE 50: Fluorescence emission spectra of naphtho[2,1a]fluoranthene dissolved in $[A(-)-)] n$-hexadecane, [B ((-)] dimethyl sulfoxide. In butyl acetate emission bands occur at about 464 and $491 \mathrm{~nm}$. 


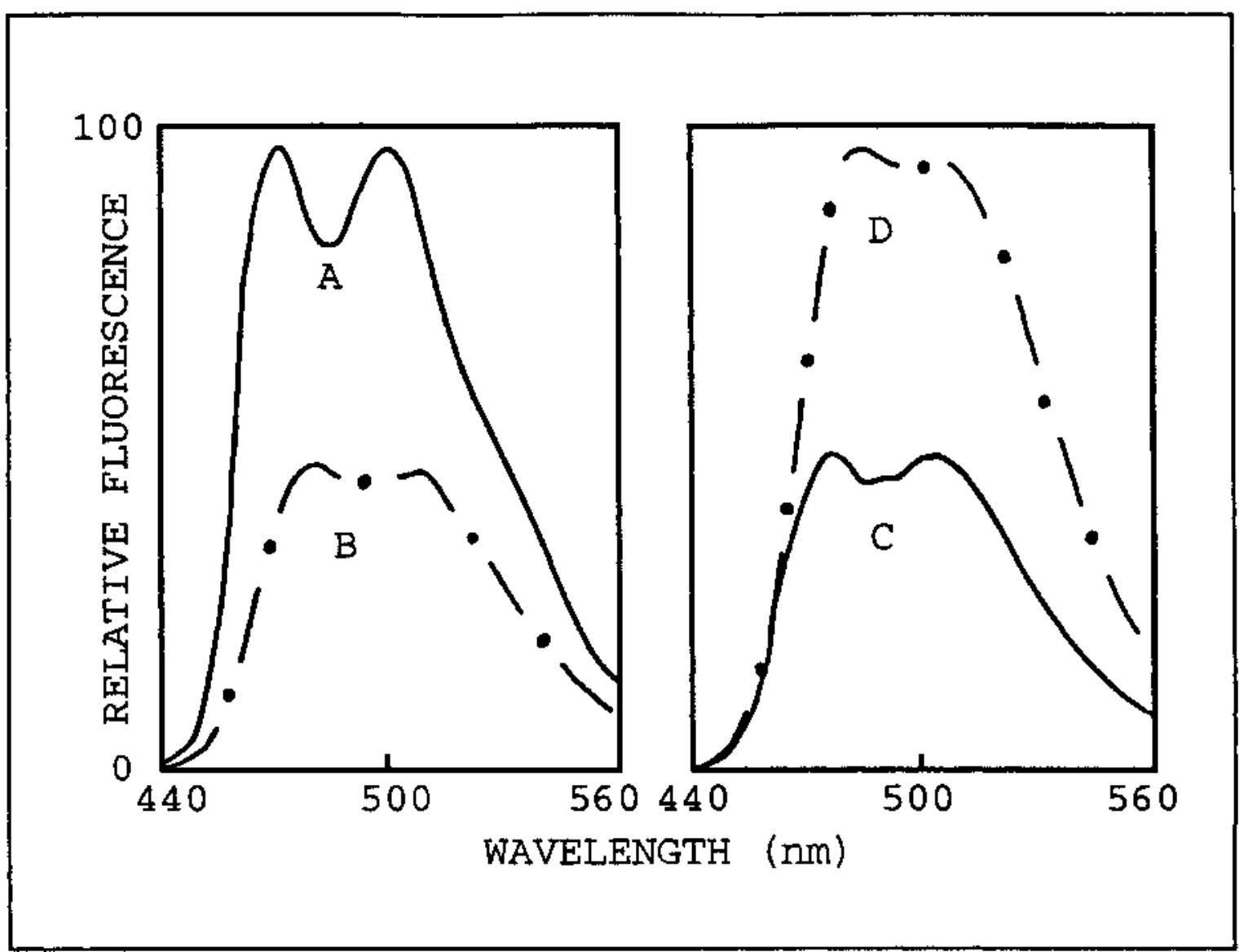

FIGURE 51: Fluorescence emission spectra of dibenzo[a,e]fluoranthene dissolved in [A (-) ] n-hexadecane, [B (-) ] dichloromethane, [C (-)] dimethyl sulfoxide. In butyl acetate emission bands occur at about 477 and $502 \mathrm{~nm}$. 
(Figure 51) show a complete lack of fine structure. While most PAH have more than three emission bands, most of the five-membered ring-containing PAHs have only one or two emission bands. It should be noted that those cyclopentaPAHs that exhibited solvent polarity probe behavior had three or more emission bands, (Figures 52 and 53) while their nonprobe counterparts only had one or two resolvable bands. This lack of fine structure was also seen in some of the PAH6 benzenoids derivatives.

The fluorescence emission spectra of corannulene (dibenzo[ghi, mno]fluoranthene) and rubicene both members of the fluoranthenoid subclass are particularly interesting. The five-membered ring in the center of corannulene introduces curvature, giving it the shape of a bowl. The geometry of corannulene is reminiscent of the carbon cage molecules called fullerenes. Recent studies by Scott and coworkers suggest that its bowl inverts more than 200,000 times per second at room temperature (28). The fluorescence lifetime for most organic molecules is about $10^{-7}$ to $10^{-9}$ seconds; therefore, corannulene should not have time to complete a full inversion during the fluorophore's lifetime. The fluorescence spectra of corannulene in Figure 54 are thought to be an average of the constantly inverting molecule. Although it is completely different in its molecular geometry than any other fluoranthenoid studied to date, its fluorescence emission spectra is typical for a 


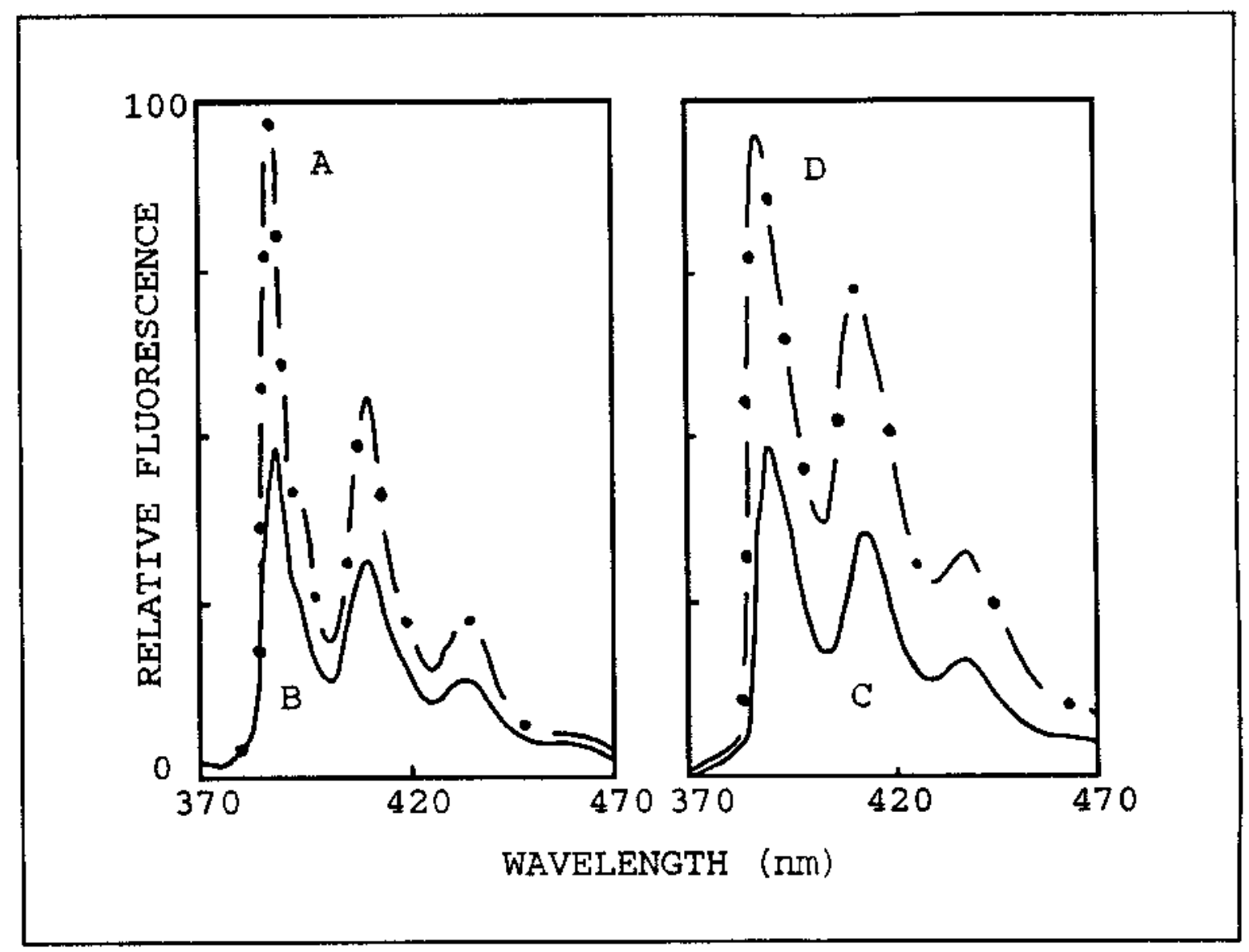

FIGURE 52: Fluorescence emission spectra of 11H-benz[bc]aceanthrylene dissolved in [A $(-O-)] n$-hexadecane, [B (—)] butyl acetate, [C (-)] dichloromethane, and [D (- -)] dimethyl sulfoxide. In dimethyl sulfoxide emission bands occur at about 392,415 , and $439 \mathrm{~nm}$. 


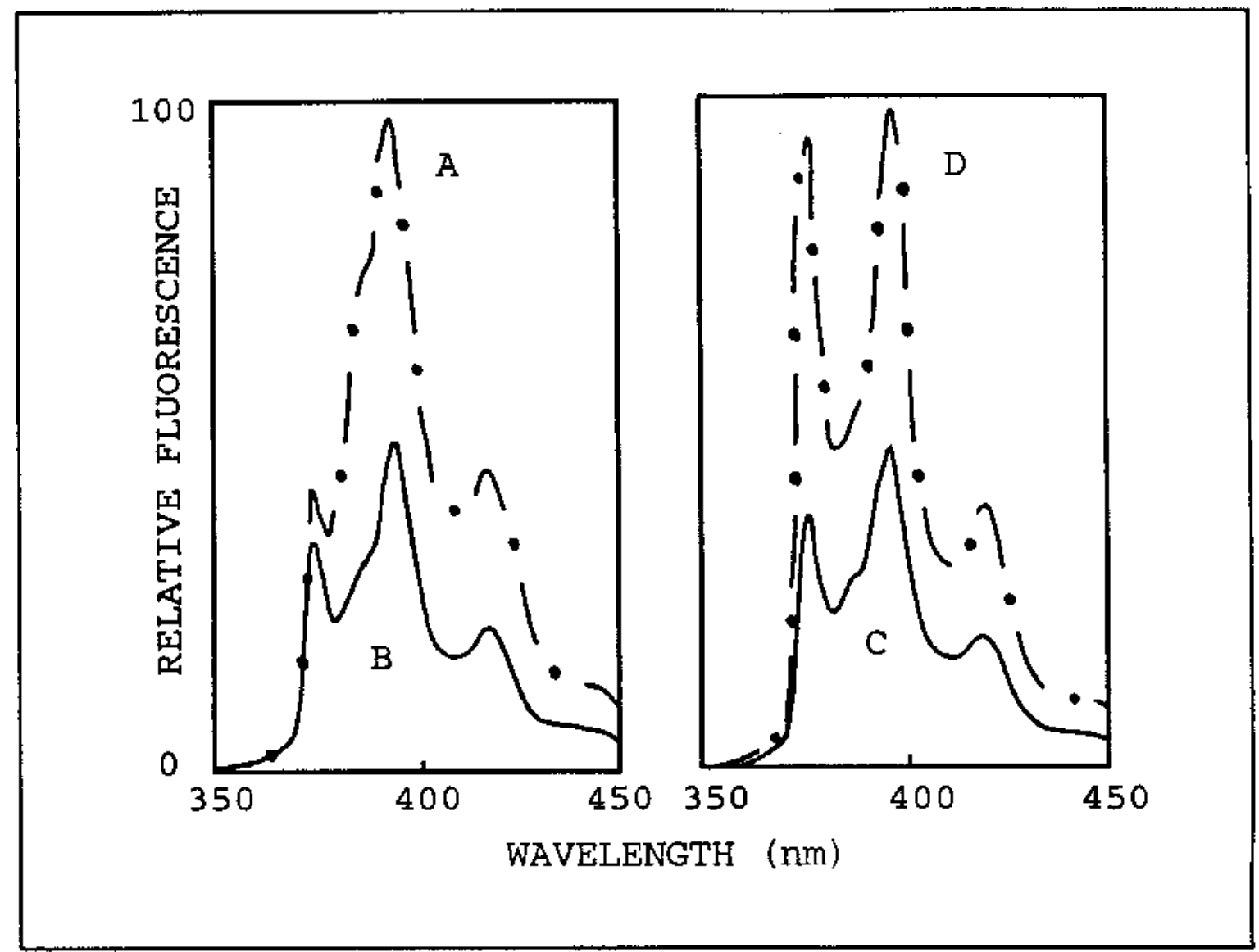

FIGURE 53: Fluorescence emission spectra of $4 \mathrm{H}$-cyclopenta[pqr]picene dissolved in [A (-O -)] n-hexadecane, [B (-)] butyl acetate, [C (- - ) ] dichloromethane, and [D (- -)] dimethyl sulfoxide. In dimethyl sulfoxide emission bands occur at about $377,397,421$, and $448 \mathrm{~nm}$. 


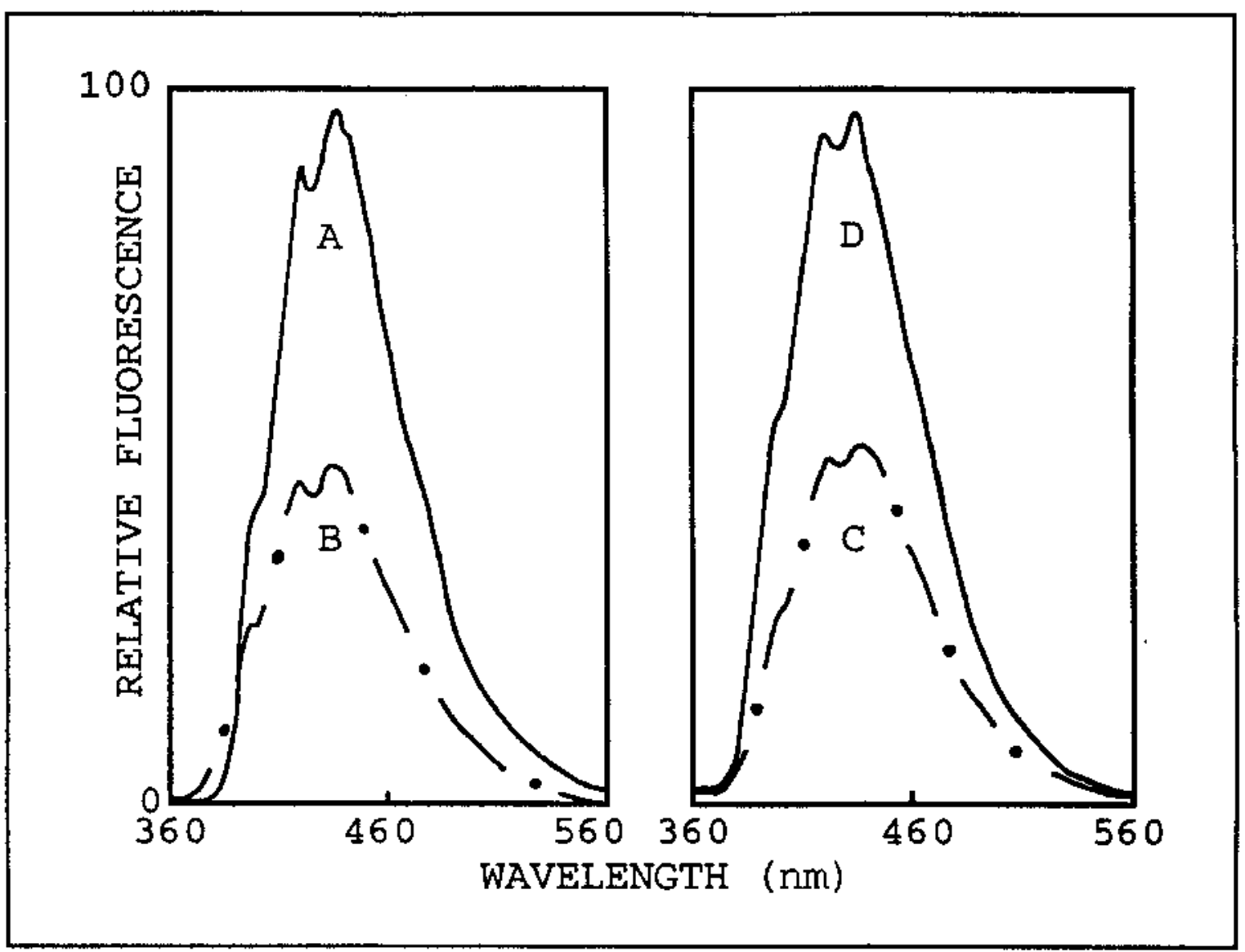

FIGURE 54: Fluorescence emission spectra of corannulene (dibenzo[ghi, mno]fluoranthene) dissolved in [A (-) ] $n$ hexadecane, [B (- -) ] butyl acetate, [C (- -)] dichloromethane, and $[D(-)]$ dimethyl sulfoxide. In dimethyl sulfoxide emission bands occur at about s-395, 419, and $434 \mathrm{~nm}$. 
member of this PAH subclass. The fluorescence emission spectra show little or no fine structure and consisted primarily of 1 to 3 very broad bands for most of the fluoranthenoid and fluorenoid compounds studied.

Figure 55 reveals that rubicene's fluorescence emission spectra is very different from any other solute in the fluoranthenoid and fluorenoid subclass. To date, no compounds in this particular subclass have had more than three fluorescence emission peaks. Rubicene has seven and sometimes eight emission peaks and most are very well resolved. Initially, rubicene showed signs of probe character as evidenced by variation of emission intensity ratios with solvent polarity. Calculated emission intensities for rubicene (RUB; I $403 \mathrm{~nm} / \mathrm{VII}$ \& $544 \mathrm{~nm}$ ) ranged from RUB $=0.80$ for $n$-hexadecane, $\mathrm{RUB}=0.58$ butyl acetate, $R U B=0.96$ for dichloromethane to $R U B=2.16$ for dimethyl sulfoxide. The emission intensity ratios, however, do not vary systematically with solvent polarity regardless of how the emission peak ratios are defined, thus it is classified as a nonprobe. In light of nitromethane quenching behavior (Chapter 5), the fluorescence characteristics of rubicene will be examined in greater detail. 


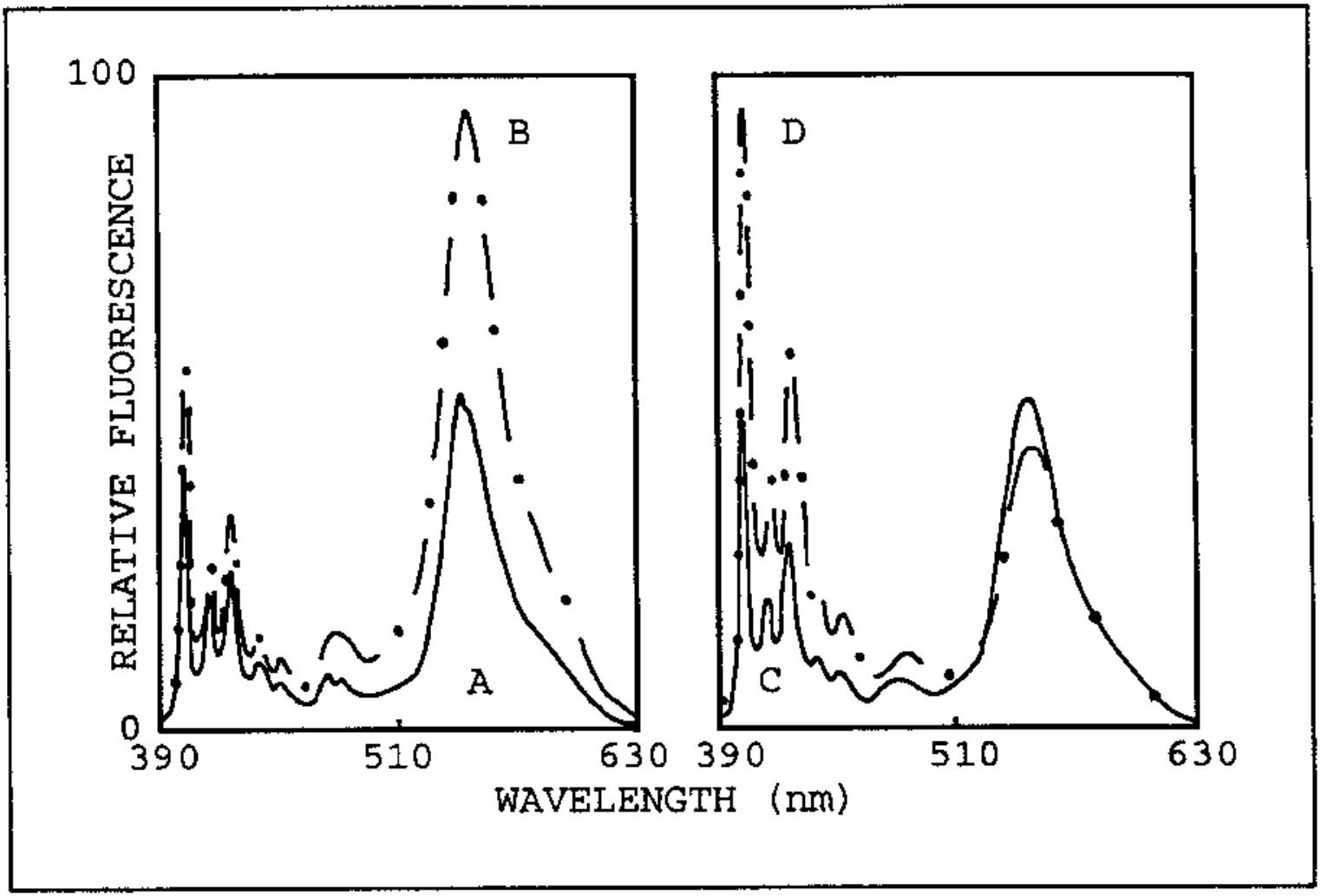

FIGURE 55: Fluorescence emission spectra of rubicene dissolved in [A (- - ] $]$-hexadecane, [B (- - -)] butyl acetate, [C (- $)$ ] dichloromethane, and [D (-O -) ] dimethyl sulfoxide. In dimethyl sulfoxide emission bands occur at about $402,415,425,439,450,478$, and $541 \mathrm{~nm}$. 
Polycyclic Aromatic Nitrogen Heterocycles

Six of the 33 polycyclic aromatic nitrogen heterocycles (PANHs) are classified as solvent polarity probe molecules due to the systematic variation of the intensity ratios with solvent polarity. The ratios of the fluorescence emission intensities for 12-azabenzo[a]pyrene (12-AzBPy), phenanthro$[2,3 h]$ isoquinoline $(2,3-\mathrm{PIQ})$, phenanthro $[3,2 h]$ isoquinoline $(3,2-\mathrm{PI}), 1$-azabenz [a] anthracene (1-AzBA), 2-azabenz [a]anthracene (2-AzBA), and 9-azabenz[a]anthracene (9-AzBA) dissolved in select organic solvents are seen in Tables XXXVIII and XXXIX. Like the five-membered ring-containing PAHs, the PANHs dynamic ranges are much smaller than those of the PAHs. Dynamic ranges are typically on the order of 0.60 or less for these PANH solvent polarity probes and contrary to the general trend of the PAHs, all the intensity ratios decrease with increasing solvent polarity for the PANHs solvent polarity probes and the majority of the PAH subclasses mentioned earlier (e.g., the fluoranthenoids, fluorenoids, acenaphthylene derivatives, etc.).

Representative fluorescence emission spectra for 12azabenzo[a]pyrene and 1-azapyrene are depicted in Figures 56 and 57. Examination of these two figures reveals that the PANH compounds do exhibit several (not all) of the spectral features common to their pyrene and benzo[a]pyrene PAH counterparts, having three and sometimes four resolvable 
TABLE XXXVIII. Ratios of fluorescence emission intensities of 12-azabenzo[a]pyrene (12-AzBPy), phenanthro[2,3h] isoquinoline $(2,3-P I Q)$, and phenanthro $[3,2 h]$ isoquinoline $(3,2-$ PIQ) dissolved in select organic solvents.

\begin{tabular}{|c|c|c|c|}
\hline Organic Solvent & $12-\mathrm{AzBPy}^{\mathrm{a}}$ & $3,2-P I Q^{b}$ & $2,3-\mathrm{PIQ}$ \\
\hline Cyclohexane & 1.90 & 2.36 & 2.02 \\
\hline $2,2,4$-Trimethylpentane & 1.94 & 2.34 & 2.02 \\
\hline n-Hexadecane & 1.92 & $2 \cdot 30$ & 2.00 \\
\hline Dibutyl Ether & 1.85 & 2.24 & 1.93 \\
\hline Benzene & 1.79 & 2.14 & 1.82 \\
\hline 2-Propanol & 1.18 & 1.81 & 1.46 \\
\hline Chloroform & $\operatorname{Rxn}^{\mathrm{d}}$ & $\operatorname{Rxn}^{\mathrm{d}}$ & $\operatorname{Rxn}^{\mathrm{d}}$ \\
\hline Butyl Acetate & 1.65 & 2.06 & 1.73 \\
\hline Methanol & 1.07 & 1.80 & 1.31 \\
\hline Dichloromethane & $\operatorname{Rxn}^{\mathrm{d}}$ & $\operatorname{Rxn} n^{d}$ & $\operatorname{Rxn} n^{\alpha}$ \\
\hline Acetonitrile & 1.47 & 1.91 & 1.55 \\
\hline$N, N$-Dimethylformamide & 1.44 & 1.90 & 1.55 \\
\hline Dimethyl sulfoxide & 1.27 & 1.85 & 1.40 \\
\hline Dynamic Range & 0.67 & 0.51 & 0.62 \\
\hline
\end{tabular}

a Defined as the ratio of band I (at 398-408 nm)/band II (at 420-430 nm). b Defined as the ratio of band I (at 389-393 nm)/band III (at 411-417 nm). c Defined as the ratio of band I (at 391-395 nm)/band III (at 412-419 nm). d Photochemical reaction. 
TABLE XXXIX. Ratios of fluorescence emission intensities of 1-azabenz [a] anthracene (1-AzBA)，2-azabenz [a] anthracene (2$A z B A)$, and 9-azabenz[a]anthracene (9-AzBA) dissolved in select organic solvents.

\begin{tabular}{|c|c|c|c|}
\hline Organic Solvent & $1-\mathrm{AzBA}^{\mathrm{a}}$ & $2-\mathrm{AzBA}^{\mathrm{b}}$ & $9-\mathrm{AzBA}^{\mathrm{C}}$ \\
\hline Cyclohexane & 1.81 & 1.39 & 1.74 \\
\hline $2,2,4-\operatorname{Tr}$ imethylpentane & 1.80 & 1.39 & 1.74 \\
\hline$n$-Hexadecane & 1.75 & 1.33 & 1.68 \\
\hline Dibutyl Ether & 1.64 & 1.22 & 1.52 \\
\hline Benzene & 1.53 & 1.11 & 1.50 \\
\hline 2-Propanol & 1.28 & 0.92 & 1.20 \\
\hline Chloroform & $\operatorname{Rxn} n^{d}$ & $\operatorname{Rxn} \mathrm{n}^{\mathrm{d}}$ & $R \times n^{d}$ \\
\hline Butyl Acetate & 1.54 & 1.06 & 1.38 \\
\hline Methanol & 1.16 & 0.88 & 1.10 \\
\hline Dichloromethane & 1.37 & 1.00 & 1.38 \\
\hline Acetonitrile & 1.36 & 0.97 & 1.33 \\
\hline$N, N$-Dimethylformamide & 1.31 & 0.97 & 1.33 \\
\hline Dimethyl sulfoxide & 1.31 & 0.98 & 1.18 \\
\hline Dynamic Range & 0.50 & 0.42 & 0.56 \\
\hline
\end{tabular}

a Defined as the ratio of band I (at 384-396 nm)/band II (at 406-418 nm). b Defined as the ratio of band I (at 381-391 $\mathrm{nm}$ )/band II (at 404-412 nm). C Defined as the ratio of band I (at 389-402 nm)/band II (at 412-425 nm). d Photochemical reaction. 


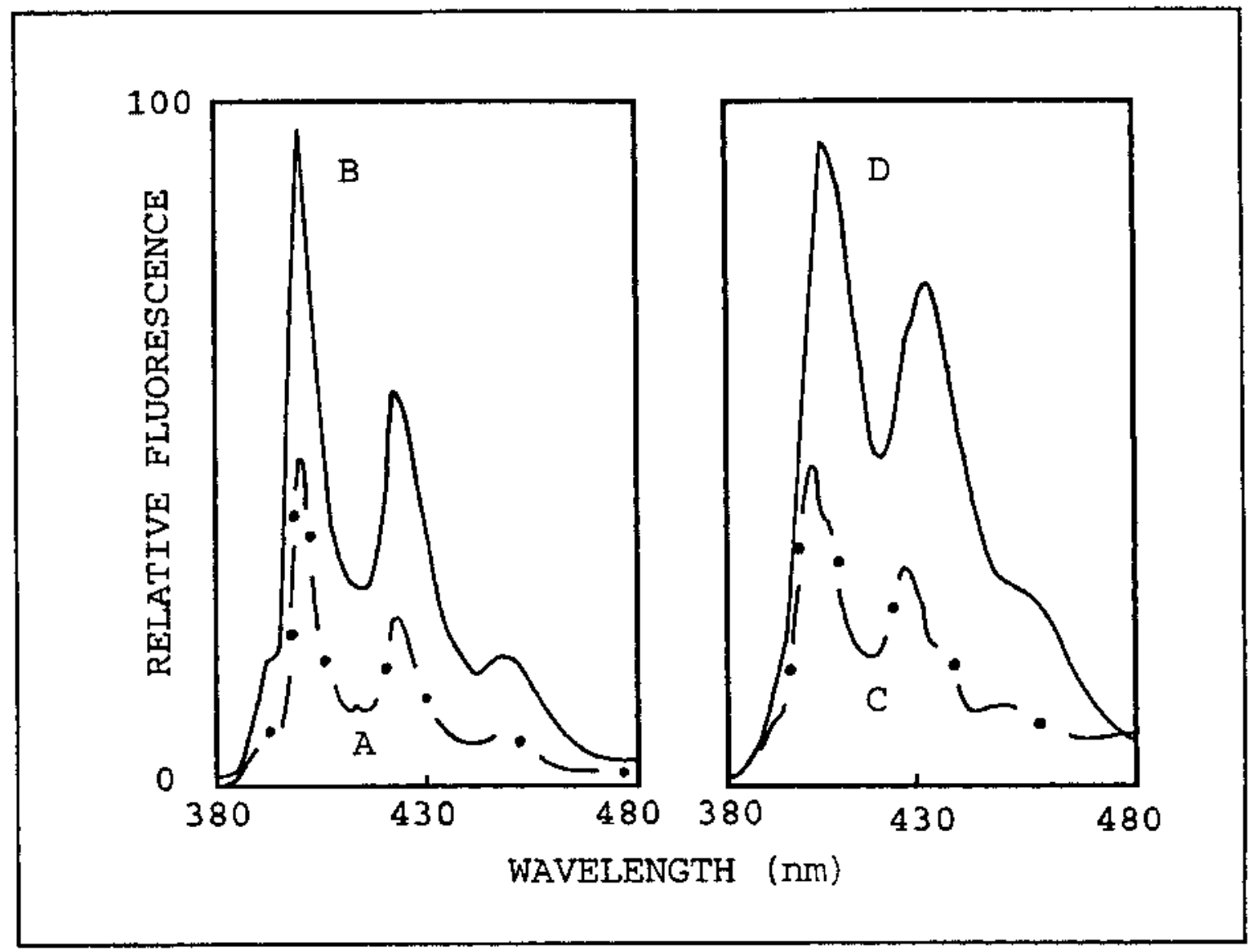

FIGURE 56: Fluorescence emission spectra of 12-azabenzo[a]pyrene dissolved in [A (-O -) ] $n$-hexadecane, [B (-)] butyl acetate, [C (- -) ] dichloromethane, and [D (-)] dimethyl sulfoxide. In n-hexadecane emission bands occur at about 400, 423, and $448 \mathrm{~nm}$. 


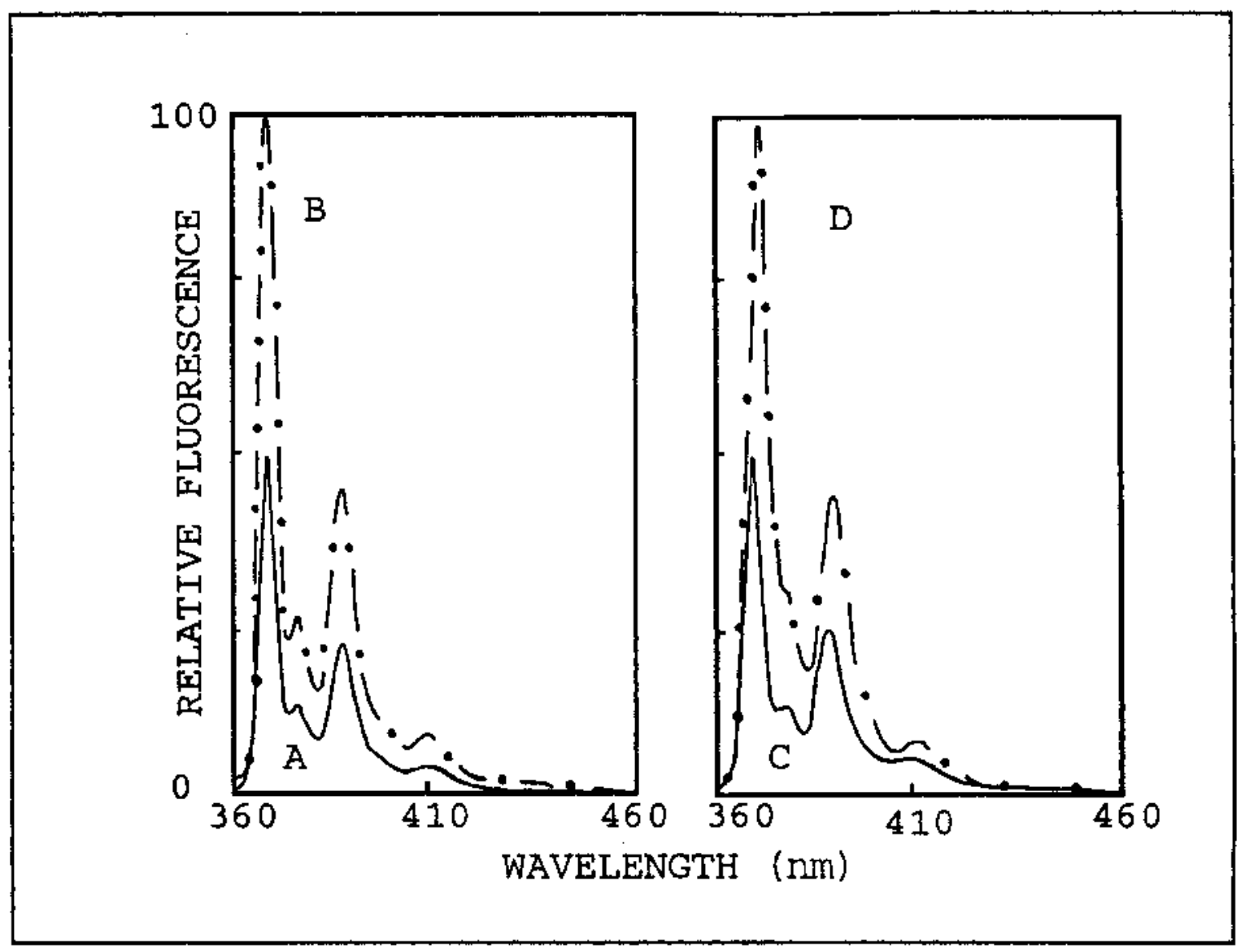

FIGURE 57: Fluorescence emission spectra of 1-azapyrene dissolved in [A (-)] $n$-hexadecane, [B (- -)] buty] acetate, [C (-)] dichloromethane, and [D (- - -)] dimethyl sulfoxide. In butyl acetate emission bands occur at about $369,376,387$, and $408 \mathrm{~nm}$. 
bands in the $360-480 \mathrm{~nm}$ spectral region. Pyrene has five vibronic bands, though not all are resolvable. Placement of the nitrogen in the various ring positions appears to have little effect on the compounds spectral characteristics as 1-azapyrene, 2-azapyrene, and 4-azapyrene have similar excitation and emission wavelengths. Strong fluorescence signals and the emission wavelengths of PANHs suggest that the observed bands correspond to $\pi^{\star} \rightarrow \pi$ transitions, like PAHs, rather than $\pi^{*} \rightarrow \mathrm{n}$ transitions. This will not necessarily be the case with every PANH because a number of compounds have their bonding $\pi$-orbital and nonbonding $n-$ orbital in reverse order. The PANHs are very fluorescent at the $10^{-6}$ Molar solute concentrations. Surprisingly, the presence of the one nitrogen heteroatom destroys the "pyrene-like" solvent polarity probe behavior in the azapyrenes. Similar behavior was seen in the alkylated pyrene derivatives.

Loss of probe behavior could result from alteration of the PANH $\pi$-electron cloud density, specific solute/solvent interactions via the nitrogen lone electron pair, or reduction in the overall molecular symmetry. For example, the pyrene probe molecule ( $D_{2 h}$ point group) has several twofold rotation axes, both vertical and horizontal mirror planes and a center of symmetry. In comparison, the azapyrene nonprobes have fewer symmetry elements and depending on the placement of the heteroatom, the molecule 
would belong to either the $c_{2 v}$ (2-azapyrene) or $C_{s}$ (1azapyrene and 4-azapyrene) point groups. Point group assignments assume a planar molecular structure, which seems to be reasonable on the basis of published $x$-ray crystallographic data for the smaller 2,3-diazanaphthalene (29), 2,7-diazanaphthalene (30), and 1,4-diazaphenanthrene (31) PANHs. It is conceivable that correlations might exist within the small PAH/PANH structural families.

Examination of the spectral data reveals that tricycloquinazoline had four observable emission bands in the 420-650 $\mathrm{nm}$ spectral region, situated at approximately $456,477,512$, and $547 \mathrm{~nm}$. Emission bands near 602 and 650 $\mathrm{nm}$, which appear in the published corrected spectra reported by Cundall and coworkers (32), were not observed. At the present time there is no explanation as to why these latter two bands are missing; however, the reason may be related to differences in chemical purities, concentrations, and/or spectrofluorometers used. The authors' absorption spectrum was correctly reproduced. Calculated emission intensity ratios for both tricycloquinazoline varied only slightly in the fourteen solvents considered.

During the course of these measurements, abnormal fluorescence behavior in chlorinated solvents was noted for the PANH solutes like that previously discussed for PAHs and all PAH derivatives. For example, as shown in Figure 58 the 1-azapyrene fluorescence emission spectrum in dichloro- 


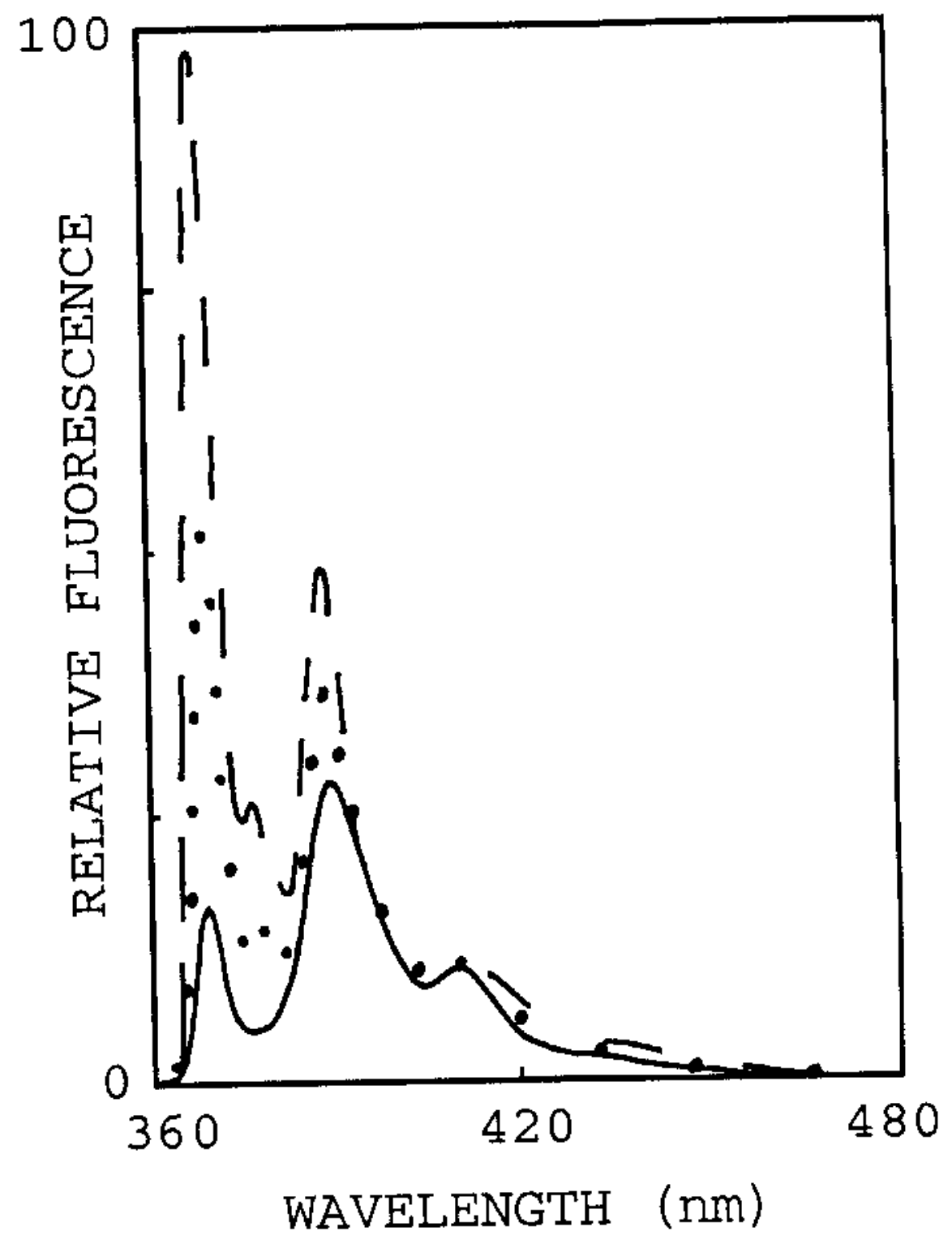

FIGURE 58: Fluorescence emission spectra of 1-azapyrene dissolved dichloromethane determined at approximately 0 $(---), 10(0 \circ 0)$, and $20(\longrightarrow)$ minutes irradiation time. 
methane varied significantly with irradiation time, to the point where there was a complete reversal in the I/III emission intensity ratio. Initially, the I/III ratio was 2.02 , but after approximately 20 minutes of continuous excitation radiation the ratio had decreased to I/III = 0.60 . Emission scans over a much broader 350-650 $\mathrm{nm}$ range failed to show the appearance of any new spectral bands which would indicate the formation of a fluorescence product with the same excitation wavelength.

Examination of PANH Tables XXXVIII and XXXIX reveals abnormally low emission intensity ratios in alcoholic solvents as well as the aforementioned chlorinated effect. For example, the I/II ratio for 12-azabenzo[a]pyrene (12AzBPy) in methanol $(I / I I=1.07)$ and in 2 -propanol $(I / I I=$ 1.18) should be on the order of $1.50-1.60$ and 1.75-1.65, respectively. The abnormally low intensity ratios noted in the alcoholic solvents can be rationalized in terms of acidbase equilibria or strong hydrogen bonding. Protonation of the nitrogen lone electron pair by a hydrogen ion results in the loss of emission fine structure accompanied by a sizeable redshift in emission wavelength(s). Degree of protonation should be reflected by both solvent acidity and PANH basicity.

Bortolus et al. first recommended 2,2,2-trifluoroethanol as a solvent for discriminating the fluorescence of PANHs from that of the corresponding PAH (33). For example, 
trifluoroethanol completely protonates 12-azabenzo[a]pyrene (12-AzBPy) (see Figure 59). The original three-band emission spectrum of 12-AzBPy at circa $395-450 \mathrm{~nm}$ disappeared and is replaced by a fairly broad, single band redshifted by over $60 \mathrm{~nm}$ into the 470-560 $\mathrm{nm}$ spectral region. The spectrum remained unchanged whenever a small drop of perchloric acid was added to the solvent. As expected the protonation is completely reversible. Addition of sodium hydroxide to a $\mathrm{HClO}_{4}$-propanol solution restored the original "unprotonated" PANH spectrum, though a slight loss in the emission intensity was observed. Some PANH solutes, Iike 1-azapyrene and 2-azapyrene, are only partially protonated in trifluoroethanol. For example, Figure 60 shows the presence of both the neutral $(370-380 \mathrm{~nm}$ band) and the protonated (405-415 $\mathrm{nm}$ band) forms in the emission spectrum of 1-azapyrene. Though, in this case, the exhibited redshift was only 20 to $30 \mathrm{~nm}$.

Partial protonation of a PANH solute in an alcoholic solvent would more likely give erroneously low intensity ratios, particularly if the redshift emission spectrum overlapped or selectively tailed into the second band used in defining the intensity ratio (e.g. the II band of a I/II ratio). Strong hydrogen-bonding between the PANH and the alcohol affords a second plausible reason for the erroneously low ratio since the fluorescence spectrum of the resulting PANH-H-OR intermediate would be redshifted to a 


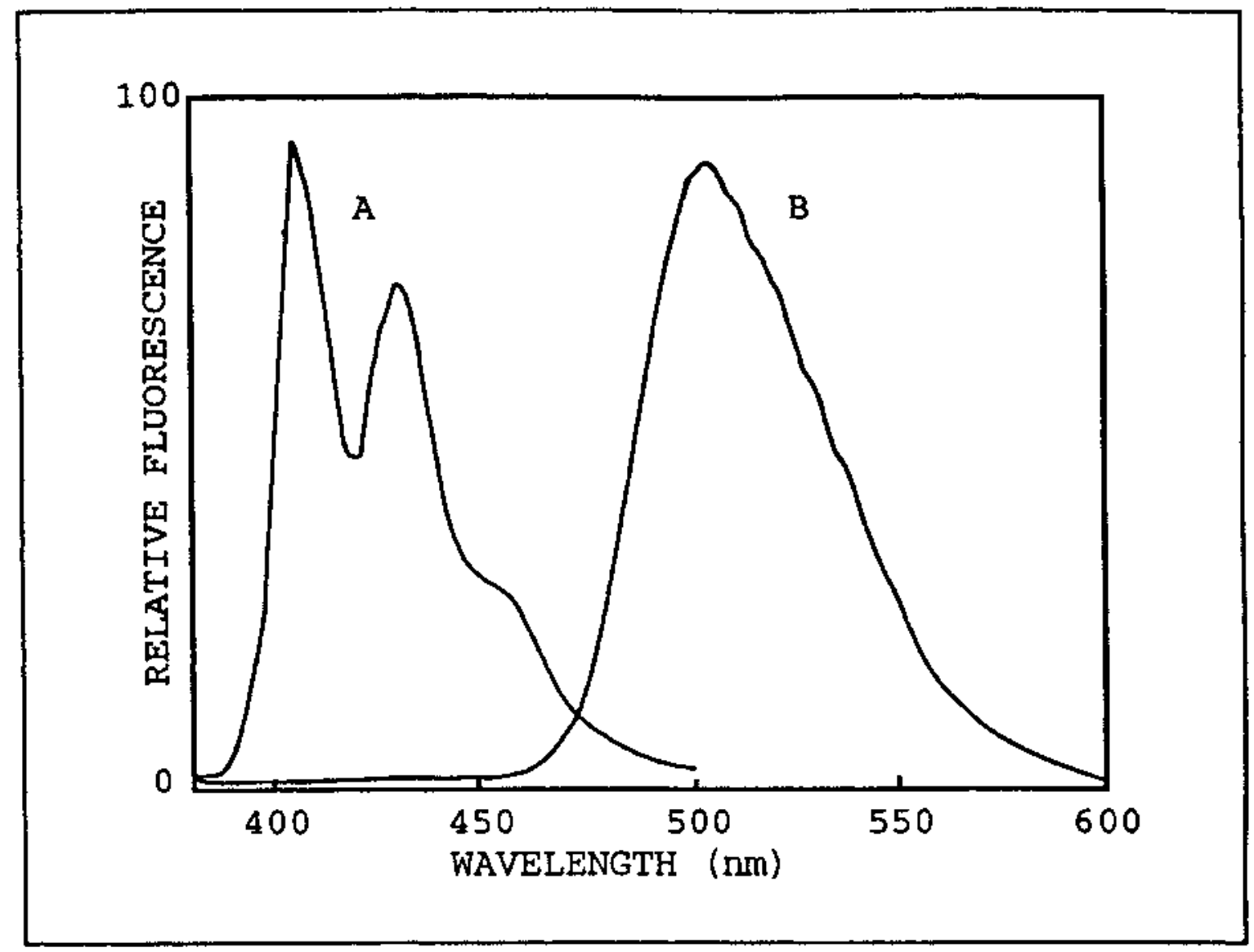

FIGURE 59: Fluorescence emission spectra of the neutral (A, in dimethyl sulfoxide) and protonated (B, in 2,2,2-trifluoroethanol and in $\mathrm{HClO}_{4}$-trifluoroethanol) forms of 12azabenzo[a]pyrene. Protonation of the nitrogen heteroatom results in loss of emission fine structure accompanied by a sizeable redshift in emission wavelengths. 


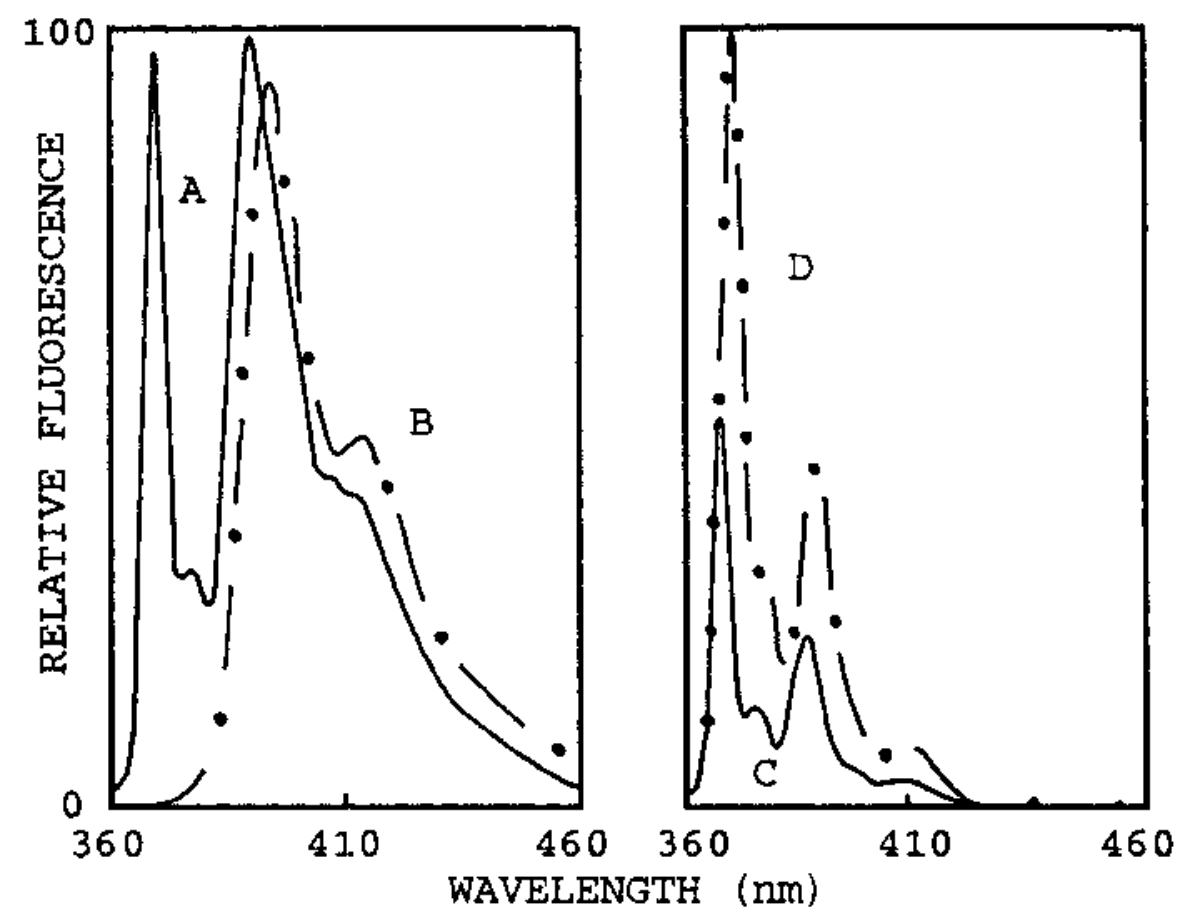

FIGURE 60: Fluorescence emission spectra of 1-azapyrene dissolved in [A (- $)$ ] trifluoroethanol, [B (- - -)] $\mathrm{HClO}_{4}$-trifluoroethanol, [C (- $\left.)\right]$-hexadecane, and [D (-)] dimethyl sulfoxide. Neutral and protonated forms of 1-azapyrene were observed in 2,2,2-trifluoroethanol. The addition of perchloric acid results in loss of the $369 \mathrm{~nm}$ emission peak, indicating "complete" protonation of the nitrogen heteroatom. 
much lesser extent. For methanol and 2-propanol, the entire 350-600 $\mathrm{nm}$ spectral region was scanned, but no new bands were observed to prove conclusively that protonation had indeed occurred.

Previously, no distinction between the monoaza- and diaza-PANHs has been made because their solvent polarity behavior is the thought to be the same. Although no diazaPANH has been classified as a solvent polarity probe, some have shown solvent probe behavior that was not systematic. The behavior of diaza-PANHs in acidic medium is slightly different than monoaza-PANHs due to the extra nitrogen. For example, it is possible to see both the mono- and diprotonated forms of benzo[de] isoquino[1,8gh]quinoline (BIQQ) (Figure 61). Trifluoroethanol only partial protonates BIQQ. Both the neutral (440-450 $\mathrm{nm}$ band) and the protonated (broader 510-550 $\mathrm{nm}$ band) species are present in the BIQQ spectra. Protonation is complete upon the addition of the perchloric acid. The smaller first emission bands disappeared and a new spectral emission band appeared near $483 \mathrm{~nm}$ for the presumed diprotonated $\mathrm{PANH}^{+2}$ cation. The four spectra in Figure 61 were obtained by titrating dropsize amounts of a $\mathrm{HClO}_{4}$-trifluoroethanol solution into the original PANH-trifluoroethanol sample. Curve d corresponds to a constant spectrum recorded after $5,6,7$, and 8 drops of $\mathrm{HClO}_{4}$-trifluoroethanol solution. On the basis of the fact that it was impossible to eliminate the $510-530 \mathrm{~nm}$ peak 


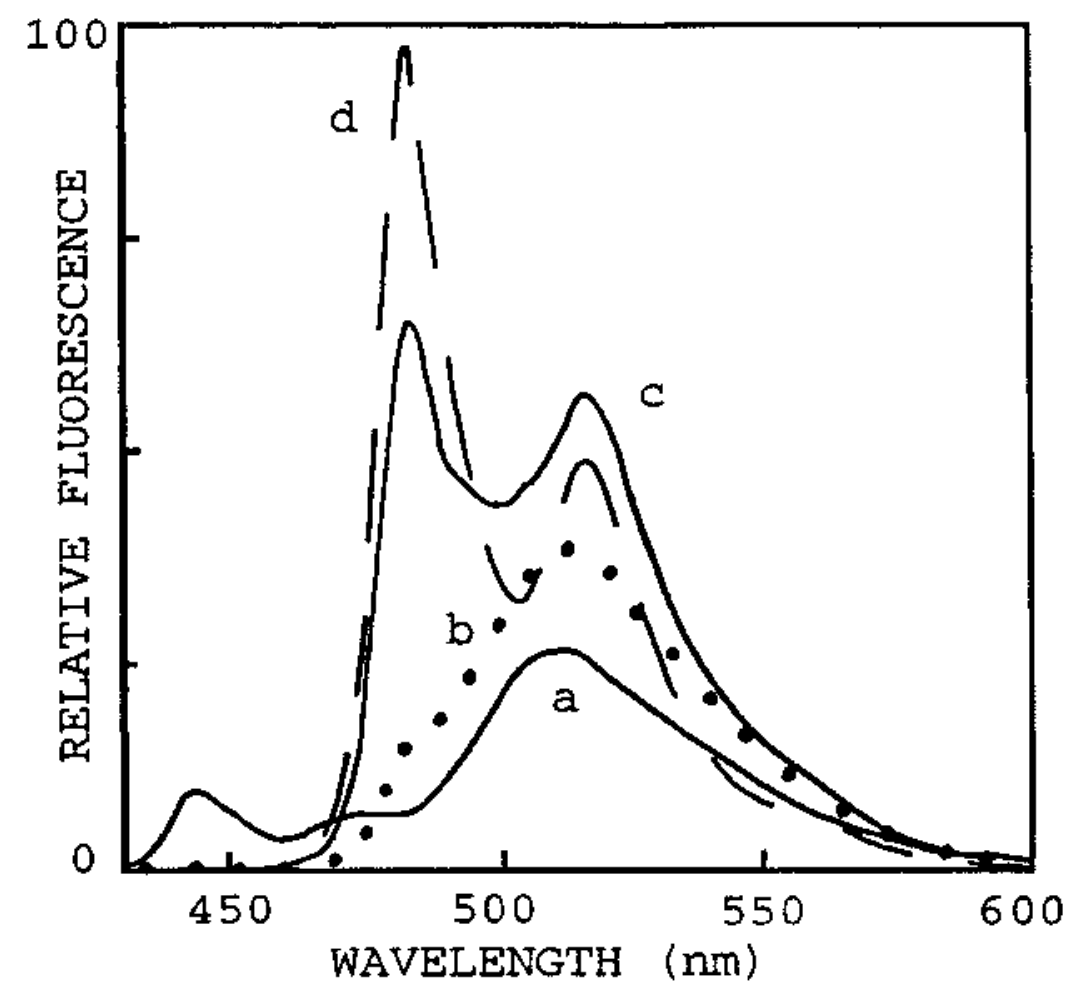

FIGURE 61: Fluorescence emission spectra of benzo[de]-

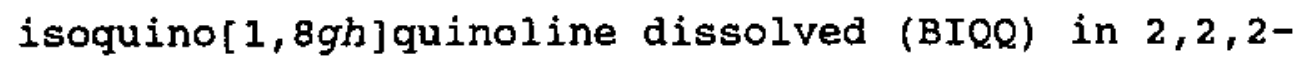
trifluoroethanol at concentrations of $\mathrm{HClO}_{4}$ of (a) neat trifluoroethanol, (b) 1 pasteur pipet drop, (c) 3 pasteur pipet drops, and (d) 5, 6, 7, and 8 pasteur pipet drops of $\mathrm{HClO}_{4}$-trifluoroethanol solution. Curves $\mathrm{b}$ and $\mathrm{d}$ are believed to correspond to the mono- and diprotonated forms of $B I Q Q$, respectively. 
by adding $\mathrm{HClO}_{4}$, it must be concluded that the $\mathrm{BIQQ}$ dication really does have two emission bands in its fluorescence spectra. As mentioned previously, the protonation is completely reversible. Addition of sodium hydroxide to the $\mathrm{HClO}_{4}$-trifluoroethanol-PANH solution restores the original "unprotonated" PANH spectrum with a slight loss in the emission intensity, probably caused in part by dilution.

placement of the two nitrogen heteroatoms in the PANH affects the ability to see the dications. For example, diphenanthro $\left[9,10,1\right.$ def; $\left.1^{\prime}, 10^{\prime}, 9^{\prime} h i j\right]$ phthalazine (DPP) is partially protonated by trifluoroethanol in Figure 62. The neutral (485-495 $\mathrm{nm}$ band) and the protonated (broader 510 $550 \mathrm{~nm}$ band) species are present. Protonation is complete upon the addition of perchloric acid. DPP, like BIQQ, posses two nitrogen heteroatoms along the external perimeter of the aromatic ring system (refer back to Figure 30 ); however, only a single broad band was noted upon addition of $\mathrm{HClO}_{4}$. In all likelihood, the broad 510-550 $\mathrm{nm}$ band belongs to the DPP monocation. The second protonation step should be significantly suppressed because it places "repelling positive charges" on two adjacent nitrogen atoms and the lack of available resonance structures will not allow stabilization of the second positive charge.

of the seven PANHs that contain two nitrogen heteroatoms in the same benzene ring, only five, naphth- 


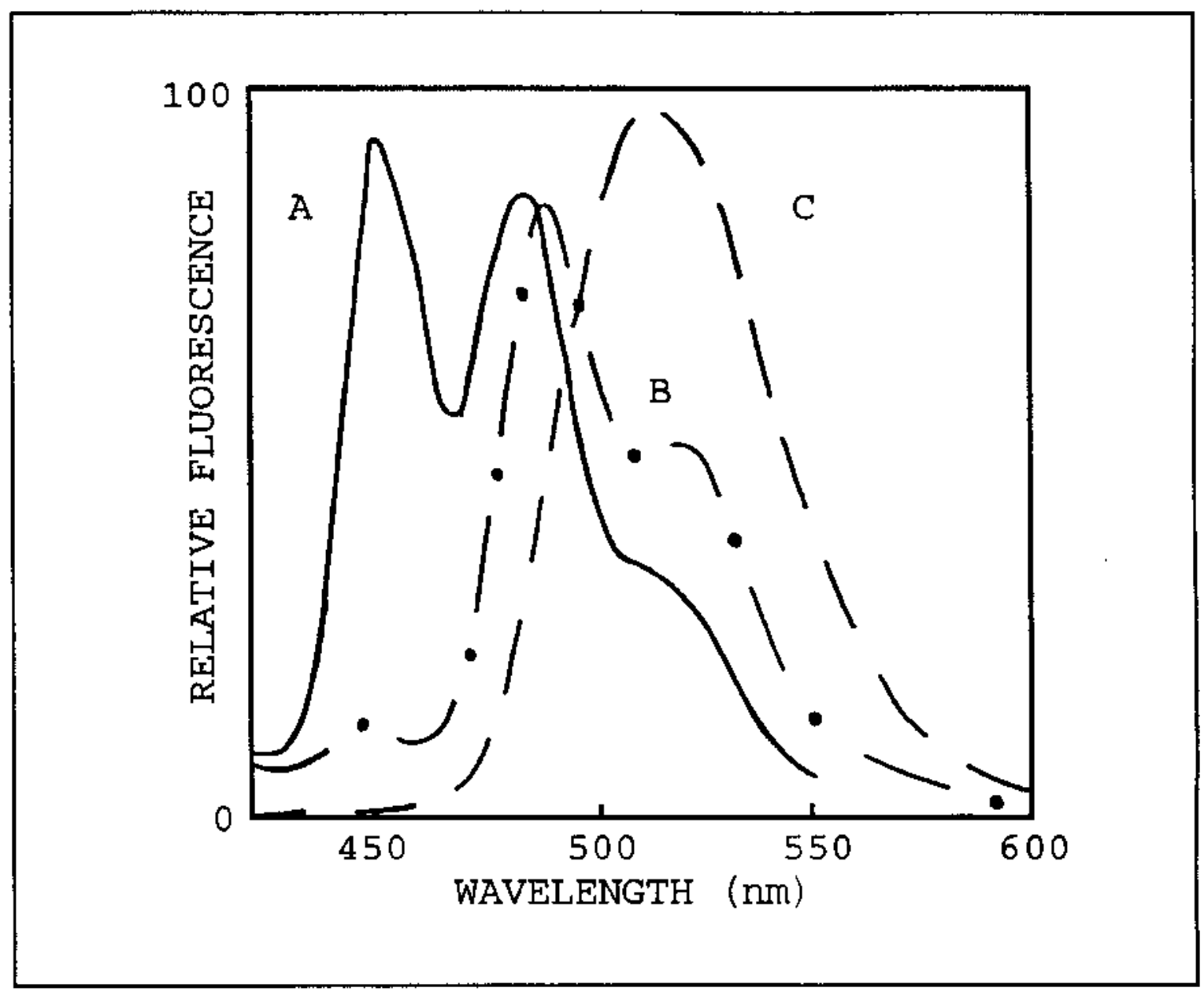

FIGURE 62: Fluorescence emission spectra of neutral (A, in dimethyl sulfoxide) and protonated (C, in $\mathrm{HClO}_{4}$-trifluoroethanol) forms of diphenanthro $\left[9,10,1 \text { def; } 1^{\prime}, 10^{\prime}, 9^{\prime} h_{j j}\right]_{-}$ phthalazine. Curve $B$ was recorded in trifluoroethanol, and only shows partial protonation. Protonation of the nitrogen heteroatom results in loss of emission fine structure accompanied by a redshift in emission wavelengths. 
$\left[2^{\prime}, 1^{\prime}, 8^{\prime}, 7^{\prime}: 4,10,5\right]$ anthra $[1,9,8$ cdef $]$ cinnoline, benz $[4,10]-$ anthra $[1,9,8 c d e f] c i n n o l i n e, ~ n a p h t h o[8,1,2 h i j]$ pyreno$[9,10,1 d e f]$ phthalazine, diphenanthro $[9,10,1$, def:1',10',9'hij]phthalazine, and benzo[a]phenazine, are partially protonated in trifluoroethanol. The other two, dibenzo $\{a, c\}$ phenazine and 11,12-dimethyldibenzo[a,c]phenazine, turned yellow and no emission signal was observed (see Figures 29-31 for molecular structures). The experimental data suggests that the second protonation step is indeed prohibited when the nitrogen heteroatoms are contained in the same benzene rings due to the solutes inability to stabilize the second cation.

Four of the 33 PANHs had no emission signal when dissolved in acidic media. No emission signal was obtained for acenaphtho[1,2b]pyridine, dibenzo[a,c]phenazine, 11,12dimethyldibenzo $[a, c]$ phenazine, and dibenz $[b, h]$ indeno$[1,2,3$ de $][1,6]$ naphthyridine dissolved in an acetonitrileperchloric acid solution. Protonation did occur; however, as these solutions turned visually yellow in color when the acid was added like many of the protonated PANHs. Figures 63 and 64 show the changes in the UV/Visible absorption spectra for benzo[a]phenazine, dibenzo[a,c]phenazine, and 11,12-dimethyldibenzo[a,c]phenazine. Loss of emission signal most likely resulted from a shift in the excitation wavelength and/or a decreased quantum efficiency, combined with inner-filtering artifacts caused by absorption of 


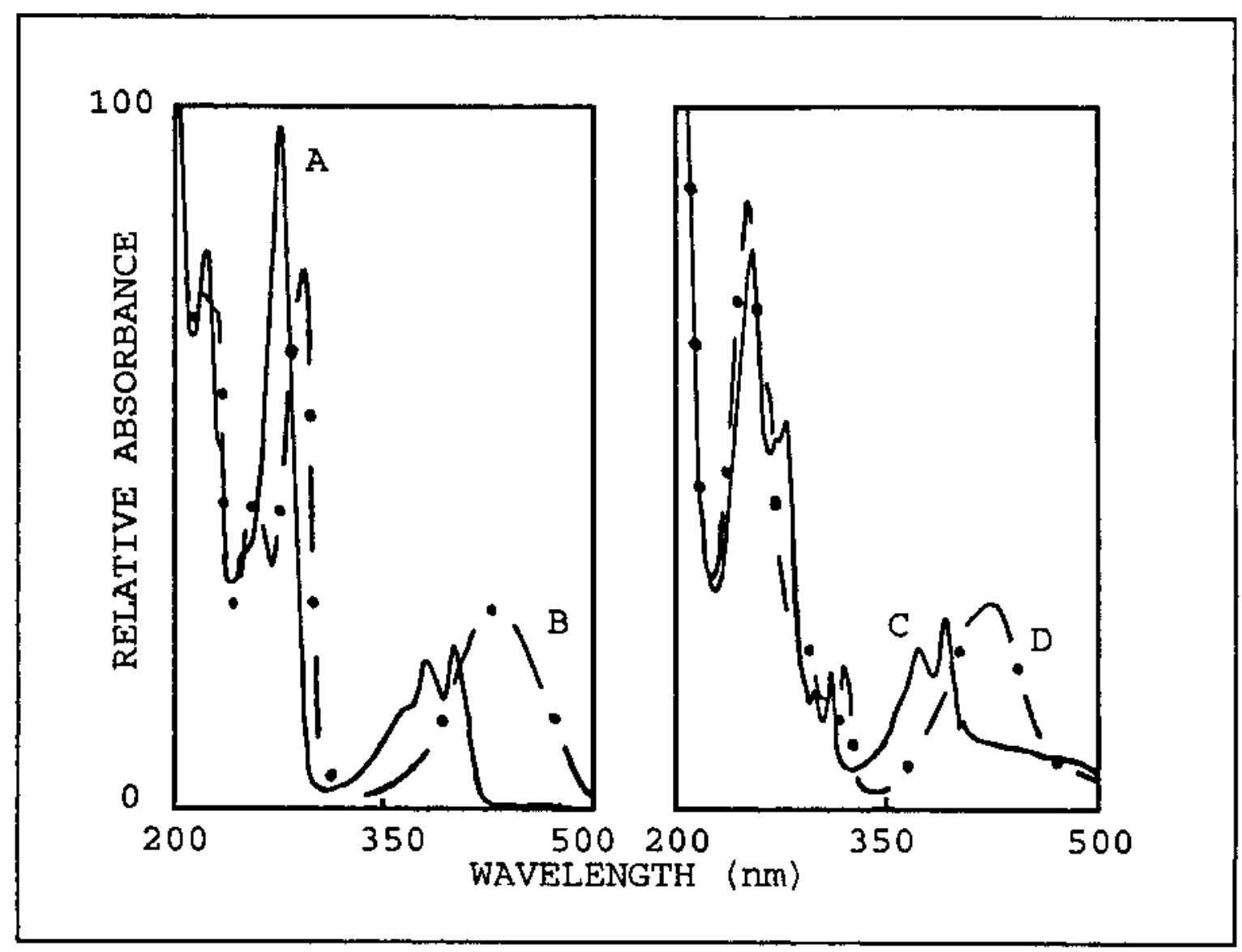

FIGURE 63: Absorbance spectra of unprotonated [A (- $\longrightarrow$ )] and protonated [B (- -)] benzo[a]phenazine, and of unprotonated [C (- $)$ ] and protonated [D (- - -)] dibenzo[a, c]phenazine. 


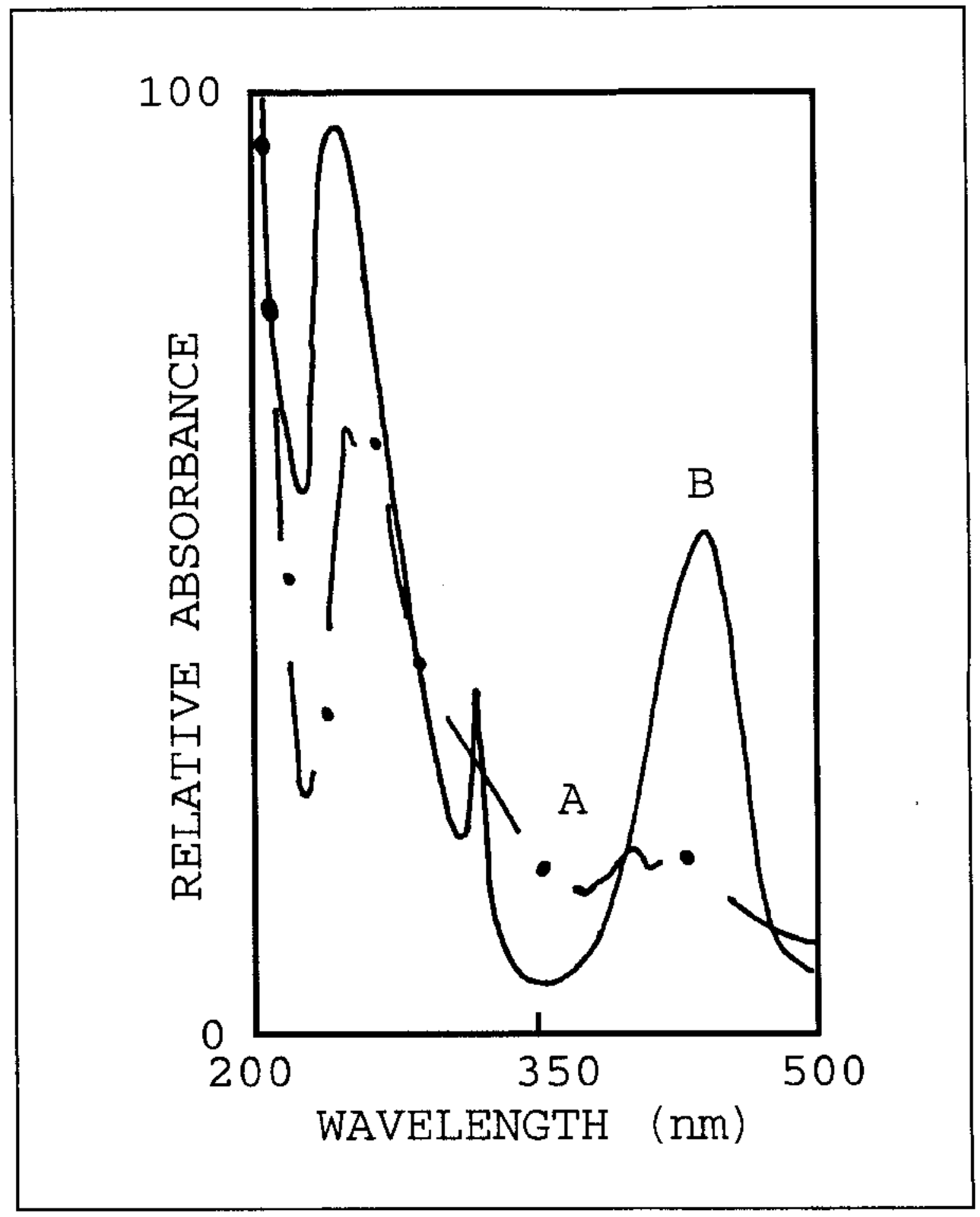

FIGURE 64: Absorbance spectra of unprotonated [A (- - -)] and protonated [B (-) ] 11,12-dimethyldibenzo $[a, c]-$ phenazine. 
significant quantities of excitation/emission radiation by the protonated PANH. The original fluorescence spectrum was restored by addition of sodium hydroxide, indicating that protonation was again reversible.

"Apparent" acid dissociation constants were estimated for two PANHs, dibenzo[a,c]phenazine and benzo[a]phenazine, which gave yellow solutions upon addition of perchloric acid. Note that while solutions benzo[a]phenazine do turn yellow upon protonation, an emission spectrum was still obtained. A suitable wavelength could not be found in the case of dibenz $[b, h]$ indeno $[1,2,3 d e][1,6]$ naphthyridine, $11,12-$ dibenzo[a,c]phenazine, or acenaphtho[ $1,2 b]$ pyridine. Assuming that the yellow color corresponds to the protonated PANH,

$$
\mathrm{PANH}+\mathrm{H}^{+}-\mathrm{PANH}-\mathrm{H}^{+} \quad \mathrm{K}_{\mathrm{a}}=[\mathrm{PANH}]\left[\mathrm{H}^{+}\right] /\left[\mathrm{PANH}-\mathrm{H}^{+}\right]
$$

then the $\mathrm{K}_{\mathrm{a}}$ can be experimentally obtained by measuring the solution absorbance as a function of $\mathrm{pH}$ at a wavelength where only PANH $-\mathrm{H}^{+}$absorbs radiation. The Beer Lambert law states that absorbance is airectly proportional to the light-absorbing species, i.e., $\mathrm{A}=$ constant $\left[\mathrm{PANH}-\mathrm{H}^{+}\right]$. At one-half the maximum absorbance (corrected for dilution) the molar concentration of $\mathrm{PANH}^{-\mathrm{H}^{+}}$and PANH are identical, and the hydrogen ion concentration equals $\mathrm{K}_{\mathrm{a}}$ (alternatively expressed as $\mathrm{pH}=\mathrm{pK}_{\mathrm{a}}$ ). The experimental procedure becomes more involved if both the protonated and unprotonated forms absorb radiation. Experimental acid dissociation constants 
were $\mathrm{pK}_{\mathrm{a}} \approx 0.50 \pm 0.15$ and $\mathrm{pK}_{\mathrm{a}} \approx 1.35 \pm 0.15$ for dibenzo$[a, c]$ phenazine and benzo[a]phenazine, respectively, dissolved in mixed aqueous-acetonitrile solvent $(30 \%$ acetonitrile by weight). These values are in line with tabulated values of Markgraf and Katt (34) for phenazine $\left(\mathrm{pK}_{\mathrm{a}}=1.23\right)$ and dibenzo $[a, c]$ phenazine $\left(\mathrm{pK}_{\mathrm{a}}=0.30\right)$ dissolved in acetic anhydride. The authors attributed the difficulty in protonating dibenzo[a,c]phenazine to steric inhibition of solvation of the PANH $-\mathrm{H}^{+}$ion caused by the two added benzo-rings. Similar effects have been observed in the reactivity of benzo[a]phenazine in that the nitrogen atom nearest to the added benzo-ring is least reactive (35). Inspection of the protonation data in reveals that PANHs can be divided into two categories based upon their observed fluorescence behavior in trifluoroethanol. The first category would include the more basic PANHs, like 4azapyrene and 12-azabenzo[a]pyrene molecules, which are completely protonated by trifluoroethanol. 1-Azapyrene and 2-azapyrene fall into the second category because trifluoroethanol only partially protonates the nitrogen heteroatom. Different basicities for the various PANHs suggest the possibility of utilizing the "neutral" to "protonated" emission intensity ratio to define a new PANH acidity scale for the subsequent characterization of high performance liquid chromatographic silica-silanol surfaces. 
Polycyclic Aromatic oxygen and Sulfur Heterocycles

Representative fluorescence emission spectra of dinaphtho[1,2b:1',2'd]furan and naphtho[1,8bc:5,4b'c']dipyran dissolved in $n$-hexadecane, butyl acetate, dichloromethane, and dimethyl sulfoxide are depicted in Figures 65 and 66 , respectively. The fluorescence emission spectra of 1,6-dithiapyrene, 1,7-dithiaperylene dissolved in methanol and cyclohexane are shown in Figures 67 and 68 , respectively. Examination of the spectral data reveals that only dinaphtho[1,2b:1'2'd]furan shows very limited probe character (see Figure 65). Calculated intensity ratios of $I / I I=1.34$ in cyclohexane and $I / I I=0.96$ in dimethyl sulfoxide gave a dynamic range $\approx 0.36$ which is significantly smaller than those mentioned previously. The extremely narrow dynamic range is too small to classify dinaphtho$\left[1,2 b: 1^{\prime}, 2^{\prime} d\right]$ furan as a solvent polarity probe.

1,6-Dioxapyrene initially appeared to exhibit solvent polarity probe character. Upon closer examination it was found that a common sets of emission bands cannot be assigned in all solvents, $i . e .$, in $n$-hexadecane three resolved bands were observed as opposed to two bands and a shoulder in dimethyl sulfoxide. It was also noted that 1,6dioxapyrene is very heat and light sensitive as evidenced by the sizeable blue shift and complete spectral change in methanol when the solution was allowed to sit overnight 


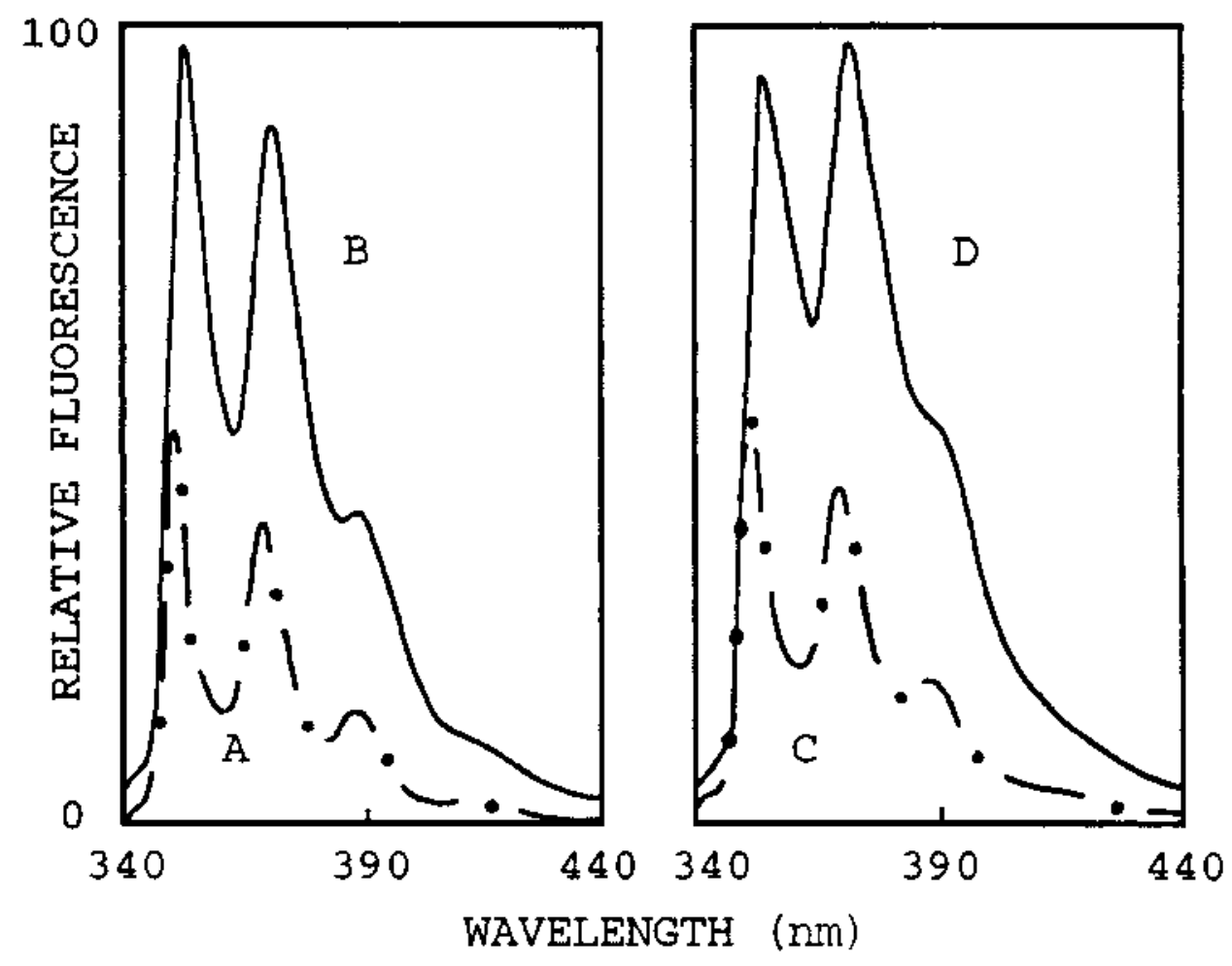

FIGURE 65: Fluorescence emission spectra of dinaphtho$\left[1,2 b: 1^{\prime}, 2^{\prime} d\right]$ furan dissolved in $\left.\left[A(-)^{-}\right)\right] n$-hexadecane, [B (-)] dichloromethane, [C (- -) ] butyl acetate, and [D (—) dimethyl sulfoxide. In butyl acetate emission bands occur at about 351, 369, and $388 \mathrm{~nm}$. 


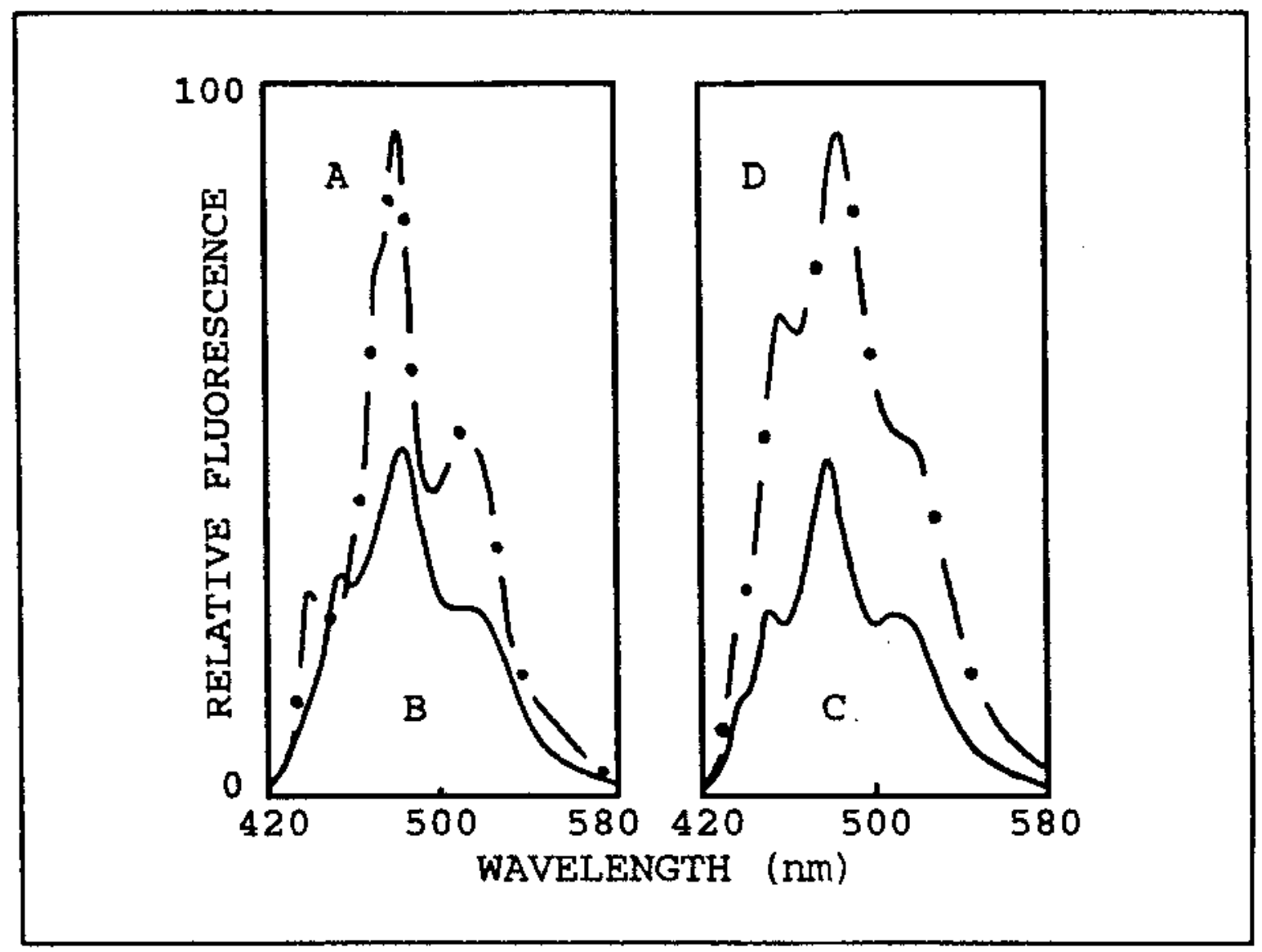

FIGURE 66: Fluorescence emission spectra of naphtho$\left[1,8 b c: 5,4 b^{\prime} c^{\prime}\right]$ dipyran dissolved in [A (-O -)] n-hexadecane, [B (- $)$ ] dichloromethane, [C (acetate, and [D(- -)] dimethyl sulfoxide. In dichloromethane emission bands occur at about 456, 483, and $517 \mathrm{~nm}$. 

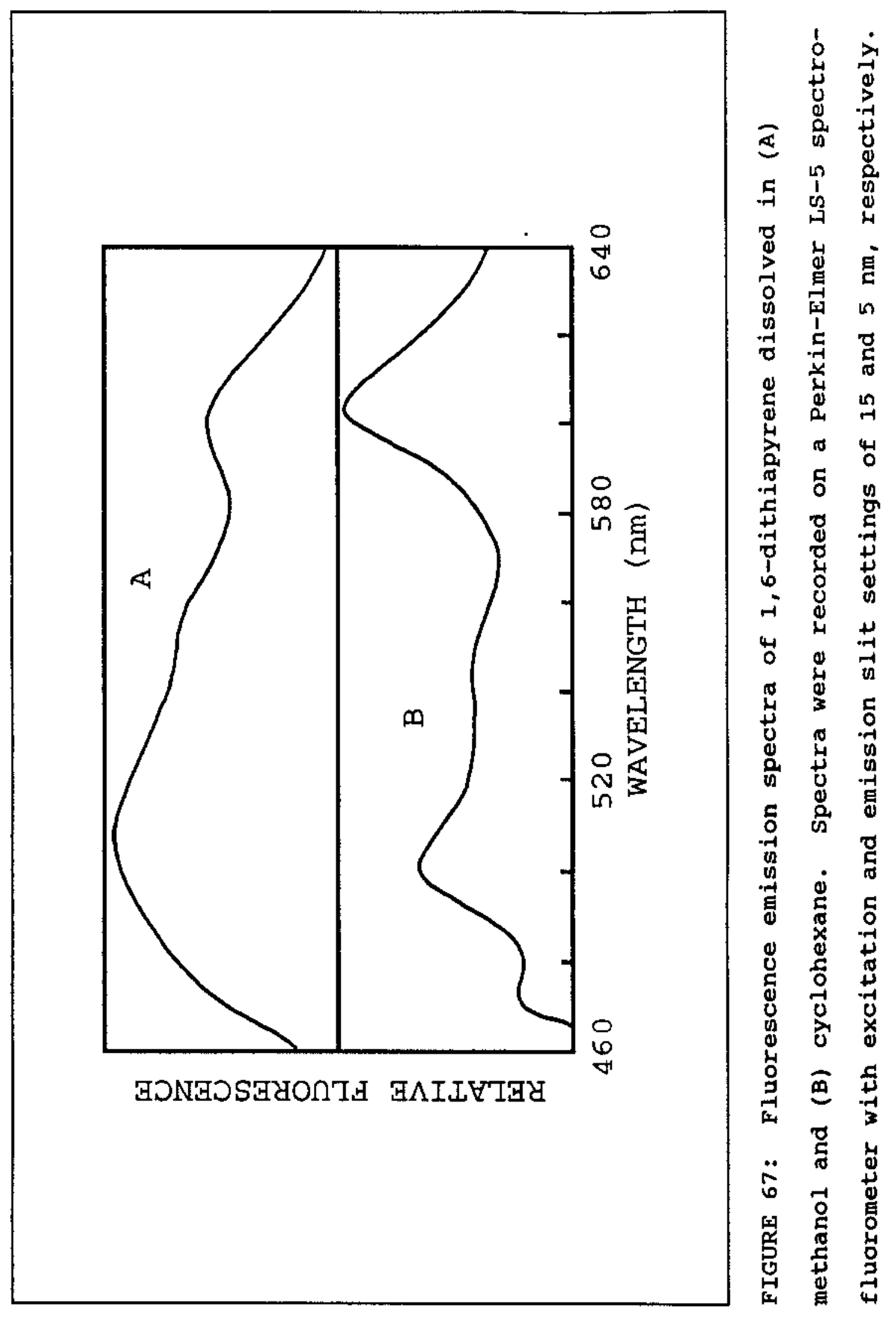

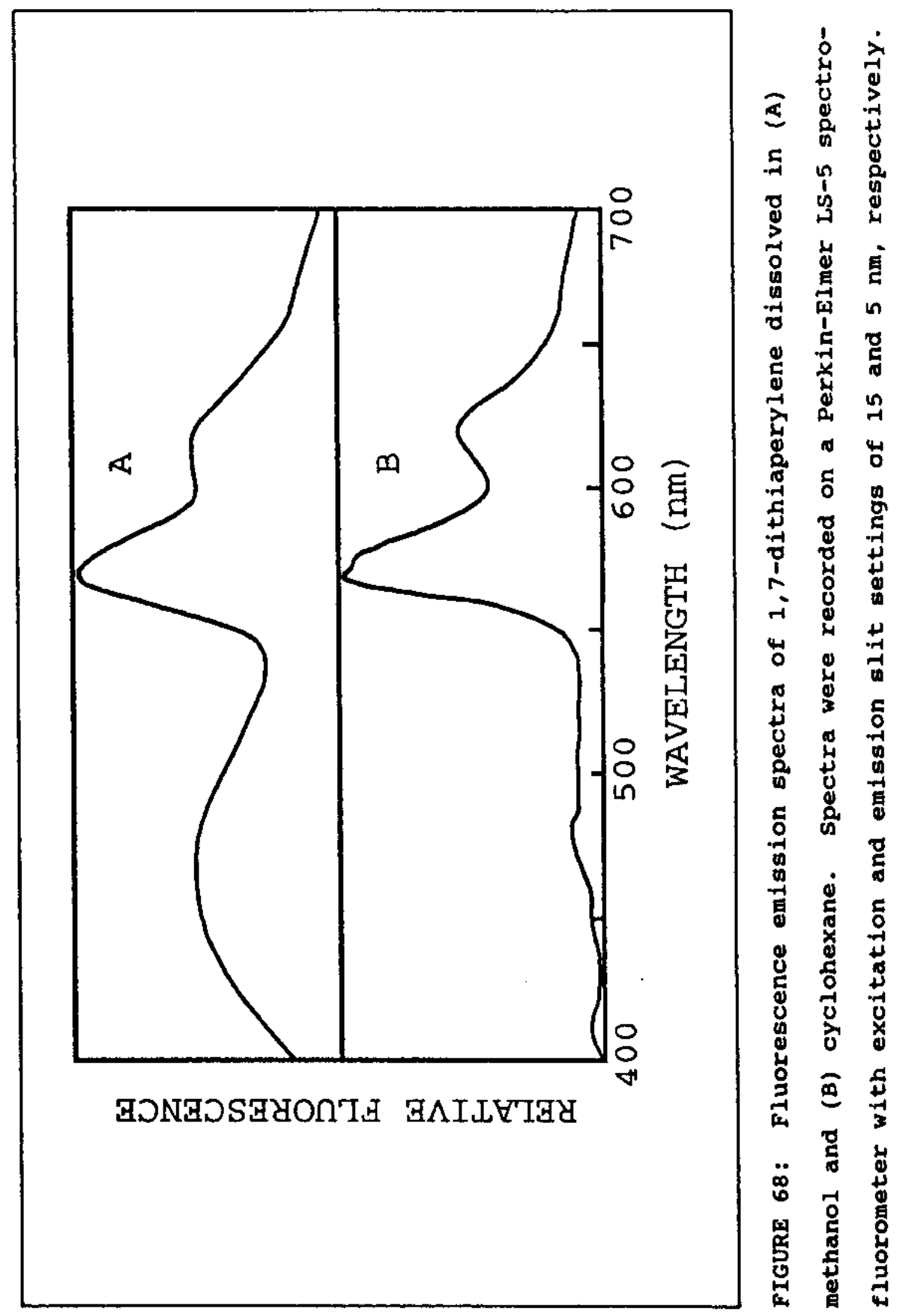
exposed to fluorescence lighting. Interestingly, 1,6dioxapyrene's fluorescence emission spectra essentially remained unchanged under the same set of conditions in all other solvents examined.

Inspection of Figures 67 and 68 reveals that the fluorescence properties of the polycyclic aromatic sulfur heterocycles (PASHs) are considerable different from their PAH counterparts. Surprisingly, very few emission bands are found in the PASH fluorescence spectra. With the exception of thianthrene and dibenzo $[2,3: 10,11]$ perylo $[1,12 b c d]-$ thiophene, significant band broadening and loss of spectral integrity are observed in the PASH spectra with increasing solvent polarity. Only a slight loss in spectral integrity was observed in the nonpolar solvents when the emission slit width was increased to 5 or $10 \mathrm{~nm}$. For PASH solutes, the larger slit width setting is preferred, as the measured emission intensities are very weak, even at solute concentrations approaching $10^{-4}$ Molar.

It is doubtful whether one will be able to use fluorescence measurements in the more polar solvent to identify which PASH compounds are present in an unknown mixtures. This latter comment is based on the complete spectral profile of PASH in various other polar solvents, like dimethyl sulfoxide, acetonitrile, and dimethyl formamide. Significant band broadening and poor band resolution, noted in all of the polar solvents examined, 
make these PASH compounds unsuitable as polarity probe molecules.

One should note in examining the various molecular structures in Figure 28 that the oxygen and sulfur atoms form only two bonds, and thus the polycyclic aromatic oxygen heterocycles (PAOHs) and PASHs will not have the "doublebond conjugation" extending over the entire molecule, as is the case in the PAH6 benzenoid compounds. For example, thianthrene has six double bonds, whereas its PAH6 benzenoid counterpart anthracene has seven double bonds. Published $\mathbf{x}-$ ray crystallographic data $(36-38)$, dipole moments $(39,40)$, and photoelectron spectra $(41,42)$ indicate that thianthrene is not a planar molecule, but rather is folded along the imaginary line connecting the two sulfur atoms. The folded "butterfly" structure prevents any overlap of the two aromatic $\pi$-ring system, regardless of the position sulfur's two nonbonding $3 p$-orbitals. Surprisingly, crystallographic data (43) further suggest that 1,6-dithiapyrene and 3,10dithiaperylene are nearly planar molecules, and it is believed that the planarity results in part from the partial overlap of the sulfur's nonbonding $3 p$-orbitals with the aromatic naphtho-ring (1,6-dithiapyrene) and phenanthro-ring (3,10-dithiaperylene) systems.

Table XI compares the fluorescence excitation and emission wavelengths of structurally similar PACs between the various subclasses. Examination of Table XL reveals 


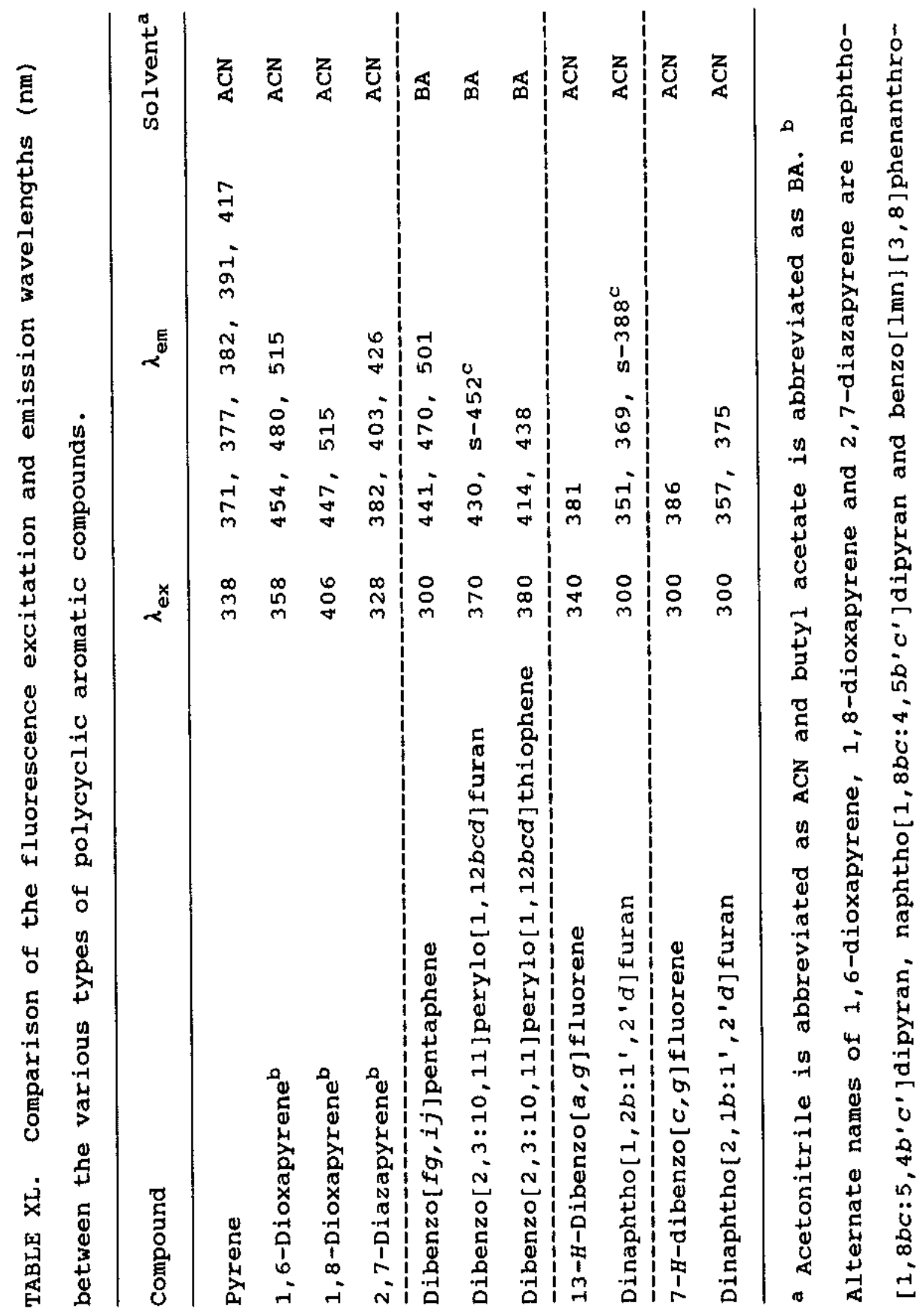


Table XL. Continued.

line, respectively. "The "s-" denotes a shoulder on a peak.

that PAH6 benzenoids have more fine structure than their heteroatom analogs. For example, pyrene has five emission bands whereas the dioxapyrenes and the diazapyrene have only two or three emission bands. Also note that the excitation and emission wavelengths for the dioxapyrenes are significantly redshifted when compared to pyrene and diazapyrene. On the contrary, the furans tend to have one or two more emission bands than their structurally similar cyclopenta-PAH analogs (two bands and a shoulder for dinaphtho[1,2b:1',2'd]furan versus one band for $13-H-$ dibenzo[a,g]fluorene).

Comparing all the subclasses of polycyclic aromatic hydrocarbons, PAH6 benzenoids have the most fluorescence emission fine structure, followed by the PANHs. The cyclopenta-PAHs and the PASHs seem to have the least emission fine structure and the PAOHs fall somewhere in the middle. Fluorescence behavior of the different subclasses can be rationalized in terms of the extent of aromatic character and electronic transition possibilities. Polycyclic aromatic benzenoids are aromatic throughout the molecule, and their electronic transitions are $\pi^{*} \rightarrow \pi$, hence 
the strong fluorescence emission signals and the considerable amount of fine structure. The polycyclic aromatic nitrogen heterocycles can have either a $\pi^{*} \rightarrow \pi$ or a $\pi^{*} \rightarrow \mathrm{n}$ electronic transition depending upon the ordering of the molecular orbitals in the particular compound, but the aromaticity is approximately the same as the analogous PAH6 counterpart; therefore, the fluorescence emission intensity and fine structure may be reduced. The weak, unstructured emission signal of the smaller PASHs results from either less intense $\pi^{*} \rightarrow \mathrm{n}$ transitions, reduced extended aromaticity at the divalent sulfur atom (44) or intraannular internal heavy-atom effect of the sulfur atom (45, 46). Cyclopenta-PAHs also have $\pi^{*} \rightarrow \pi$ transitions, but as in PASHs, the emission signal is weak and lacks fine structure because of reduced aromaticity. The bridging $\mathrm{CH}_{2}$ group divides the molecule into only weakly interacting aromatic moieties. Following this analogy, and because of the orbital energy spacing at $2 p$ versus $3 p$ level, it can be argued that the greater emission intensity and fine structure observed in the dioxapyrenes and furan derivatives suggest a $\pi^{*} \rightarrow \pi$ electronic transition. Dioxapyrenes and diathiapyrenes are reported to possess less aromatic character than their PAH and PANH counterparts (47). 


\section{Bi-Polycyclic Aromatic Hydrocarbons}

Representative fluorescence emission spectra of $9,9^{\prime}-$ bianthracene, 1,1'-binaphthalene, and 9,9'-bianthracene-10carboxaldehyde dissolved in n-hexadecane, butyl acetate, dichloromethane, and dimethyl sulfoxide are depicted in Figures 69-71, respectively. Inspection of the Figures reveals that the fluorescence emission spectra of bi-PAHs, unlike that of their PAH counterparts, show little detailed emission fine structure. For many of the forty-five nonelectrolyte solvents considered, 1,1'-binaphthalene, 2,2'-binaphthalene, 9,9'-bianthracene, 9,9'-bianthracene-10carboxaldehyde, 9,9'-biphenanthrene, 1,1'-methylenebisnaphthalene, and $1,1^{\prime}, 2,2^{\prime}$-tetrahydro-5,5'-biacenaphthalene exhibited only a single, fairly broad emission band. Two simpler PAH molecules, anthracene and phenanthrene, have four and five emission bands in their fluorescence spectrum in the 320-480 nm spectral region, respectively.

Figure 69 documents that 9,9'-bianthracene does show solvatochromic behavior as evidenced by rather pronounced changes in the location of its emission band. In nonpolar solvents, such as cyclohexane, two bands of comparable intensity were observed at circa 395 and $414 \mathrm{~nm}$. Significant redshifts were noted in the $s_{1} \rightarrow s_{0}$ fluorescence in the more polar dimethyl sulfoxide solvent. Excitation wavelength remained essentially constant and unaffected by 


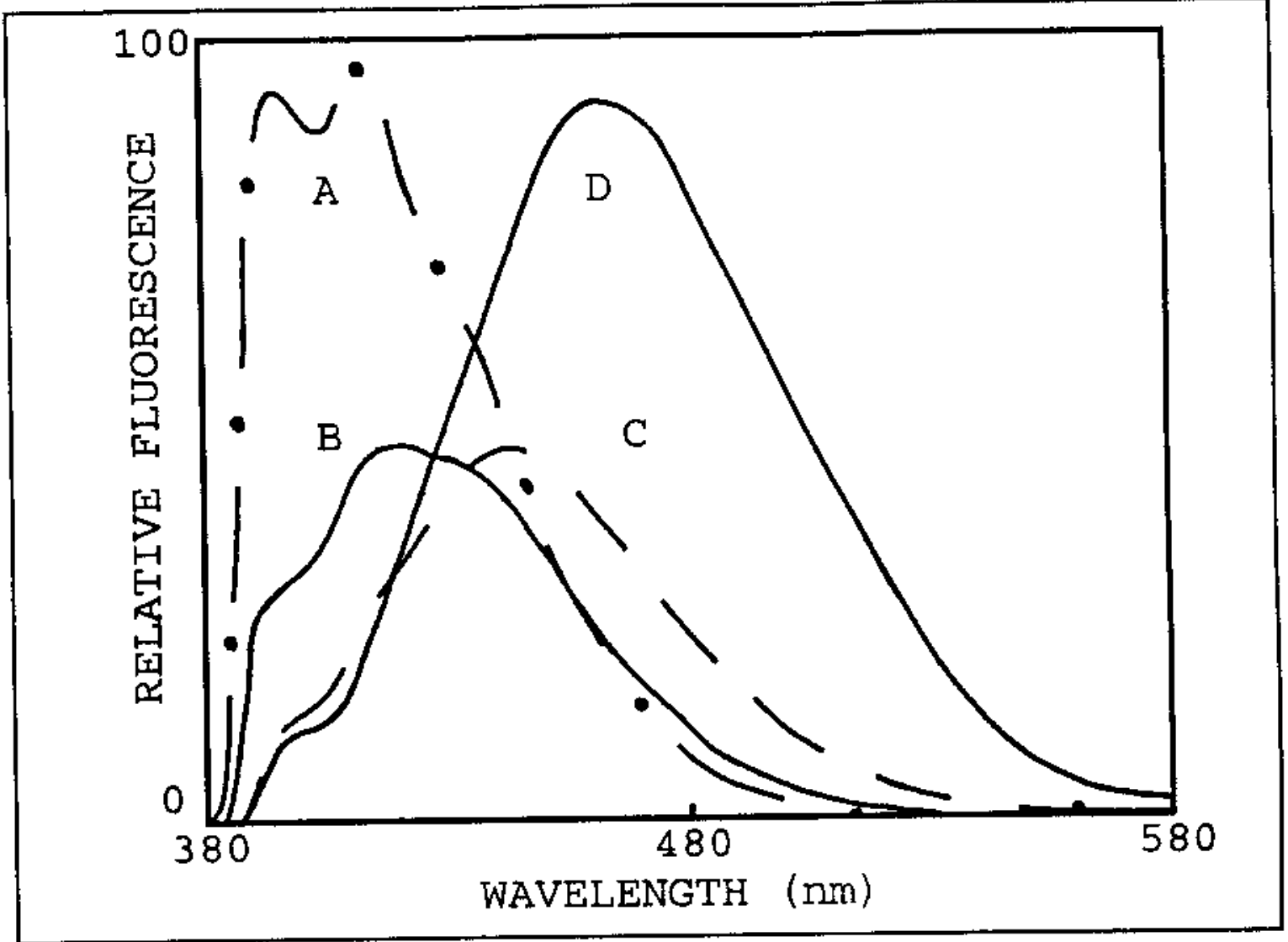

FIGURE 69: Fluorescence emission spectra of 9,9'bianthracene dissolved in [A (- -)] n-hexadecane, [B (- $(-)]$ butyl acetate, [C (- - -)] dichloromethane, and [D (—) dimethyl sulfoxide. 


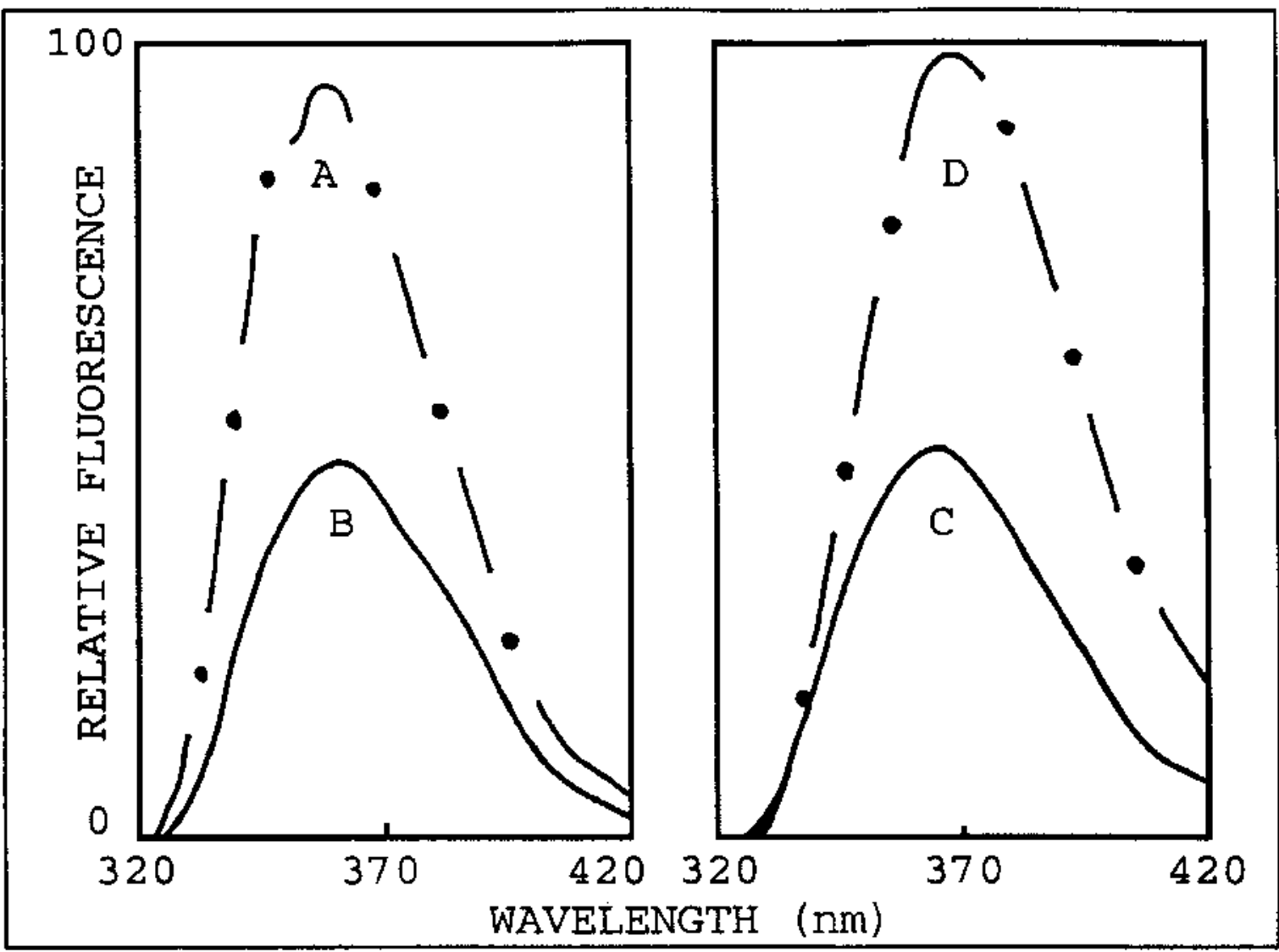

FIGURE 70: Fluorescence emission spectra of 1,1'-binaphthacene dissolved in [A (- -) ] $n$-hexadecane, [B (-)] butyl acetate, [C (- $)$ ] dichloromethane, and [D (- - -) ] dimethyl sulfoxide. 


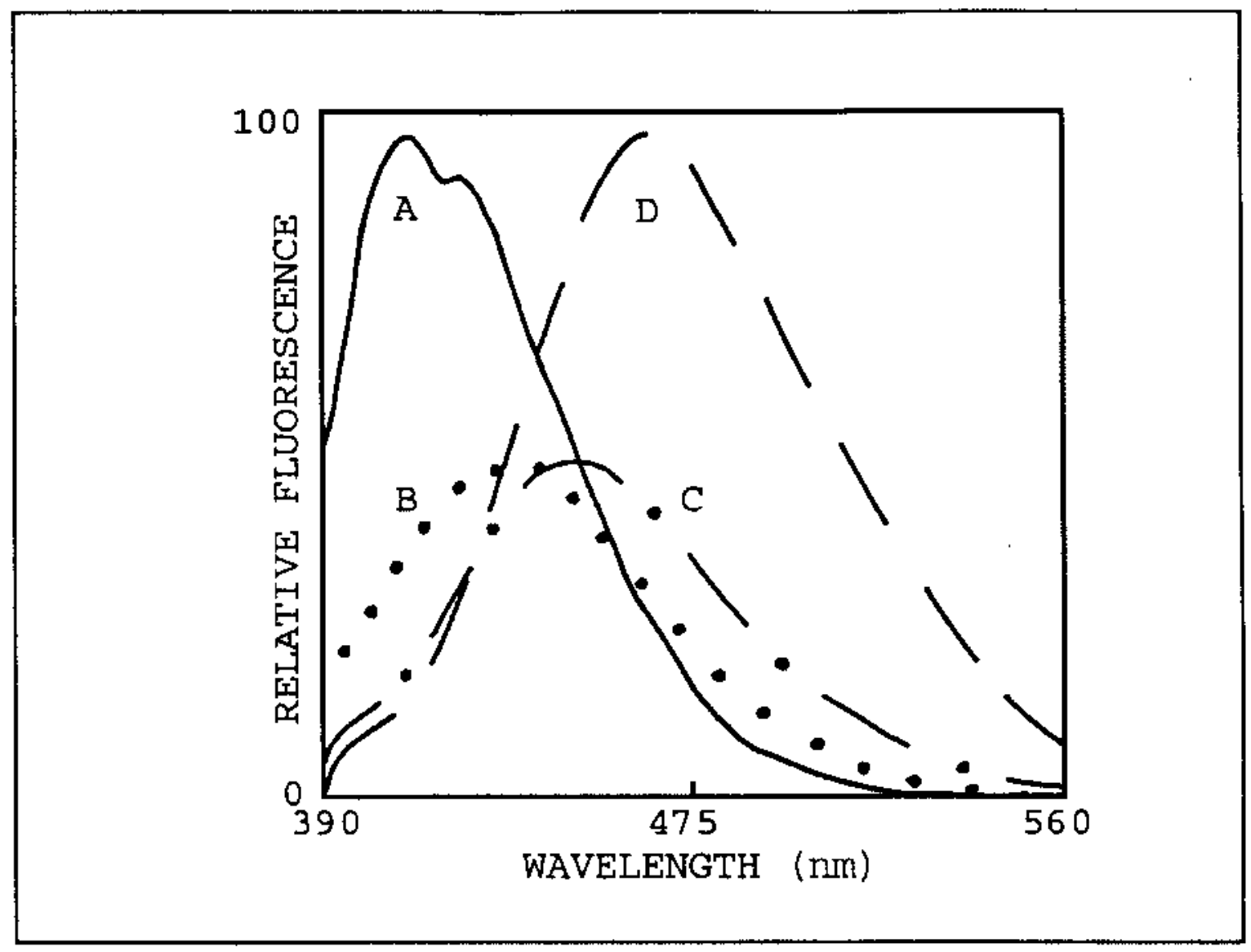

FIGURE 71: Fluorescence emission spectra of 9,9'-bianthracene-10-carboxaldehyde dissolved in [A (- - ) $n$ hexadecane, [B $(O)$ ] butyl acetate, $[\mathrm{C}(-\mathrm{O}-)]$ dichloromethane, and [D (- - -)] dimethyl sulfoxide. 
solvent polarity (see Table XII). As stated in Chapter 1 , these particular observations have been reported in several previous publications $(48-55)$; however, the forty-five solvents in Table XLI represent the most comprehensive steady-state fluorescence studies completed to date. Fluorescence wavelengths of circa $\lambda_{\mathrm{em}} \approx 413 \mathrm{~nm}$ and $\lambda_{\mathrm{em}} \approx 416$ $\mathrm{nm}$ for methylcyclohexane and 1-chlorobutane, and stoke's shifts of $\Delta v=\left(1 / \lambda_{\text {ex }}\right)-\left(1 / \lambda_{\text {em }}\right) \approx 4.9 \times 10^{3} \mathrm{~cm}^{-1}$ and $\Delta v \approx$ $5.4 \times 10^{3} \mathrm{~cm}^{-1}$ for acetone and acetonitrile, respectively, are in good agreement with values of $\lambda_{\mathrm{em}} \approx 422 \mathrm{~nm}, \lambda_{\mathrm{em}} \approx 425$ $\mathrm{nm}, \Delta v \approx 3.50 \times 10^{3} \mathrm{~cm}^{-1}$ and $\Delta v \approx 4.30 \times 10^{3} \mathrm{~cm}^{-1}$ cited in a recent paper by Kabouchi et al. (52). Slight variation in wavelength and frequency shifts are to be expected when comparing fluorescence data recorded on different instruments, due to differences in wavelength calibration, wavelength-dependent photomultiplier detector response, optical component efficiencies, excitation/emission slit widths and/or solvent blank corrections. Also 9,9'bianthracene's broad emission band and shoulders make exact determination of $\lambda_{\text {em }}$ subject to greater experimental uncertainty, and contributes in part to the differences noted between our stoke's shifts and those reported in the chemical literature. For any given spectrofluorometer measured $\Delta v$ values for a series of solvents should be internally consistent. 


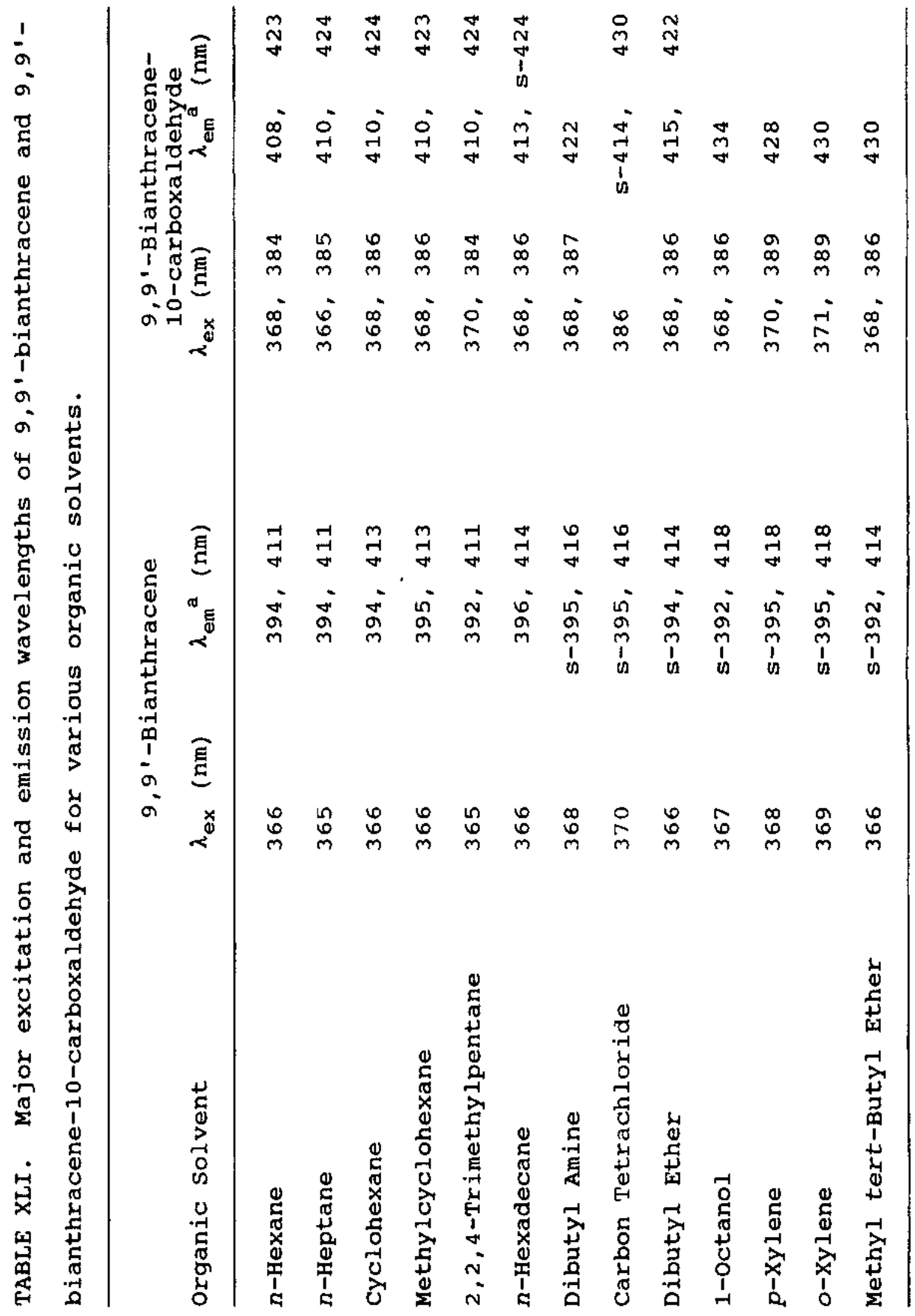




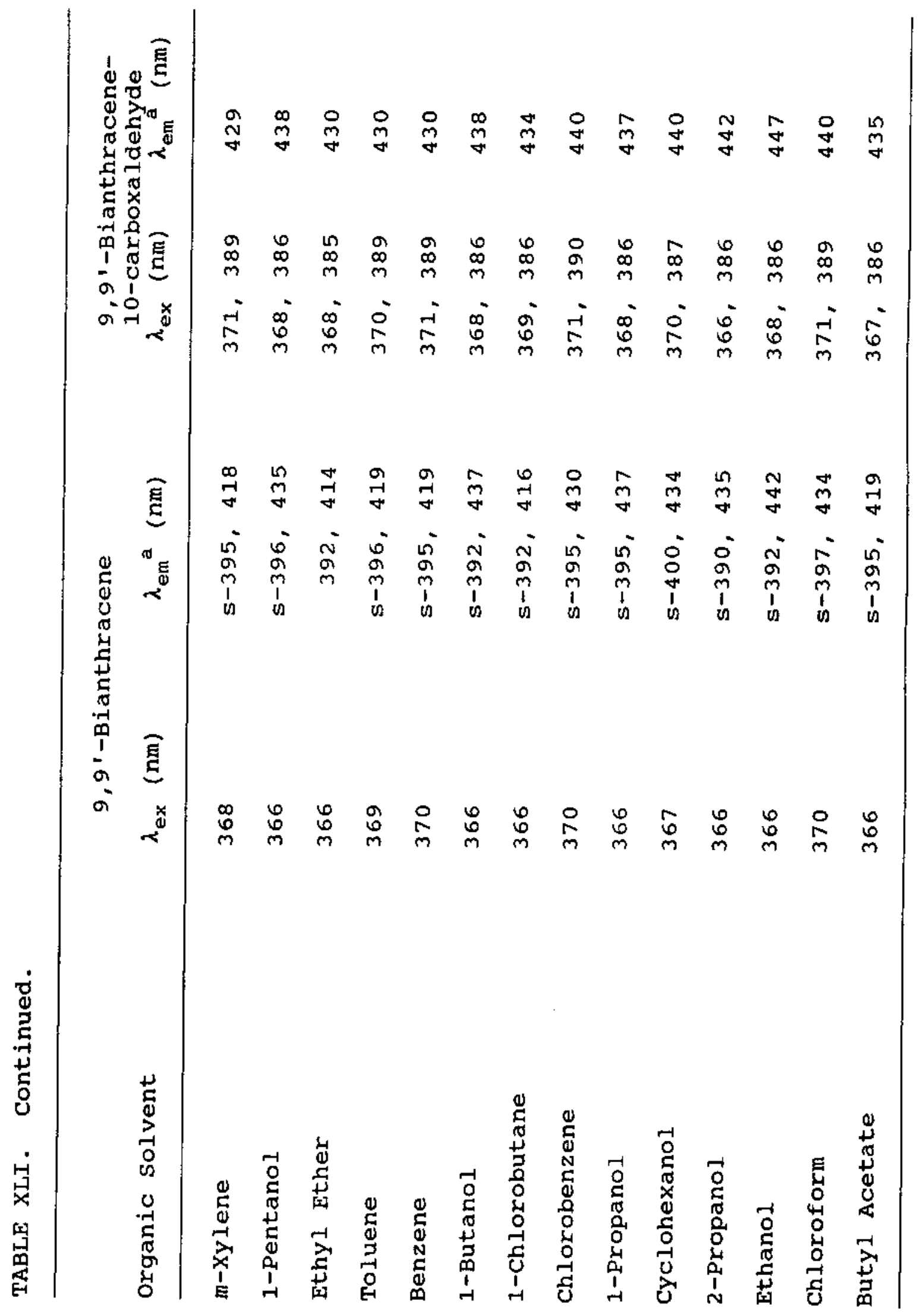




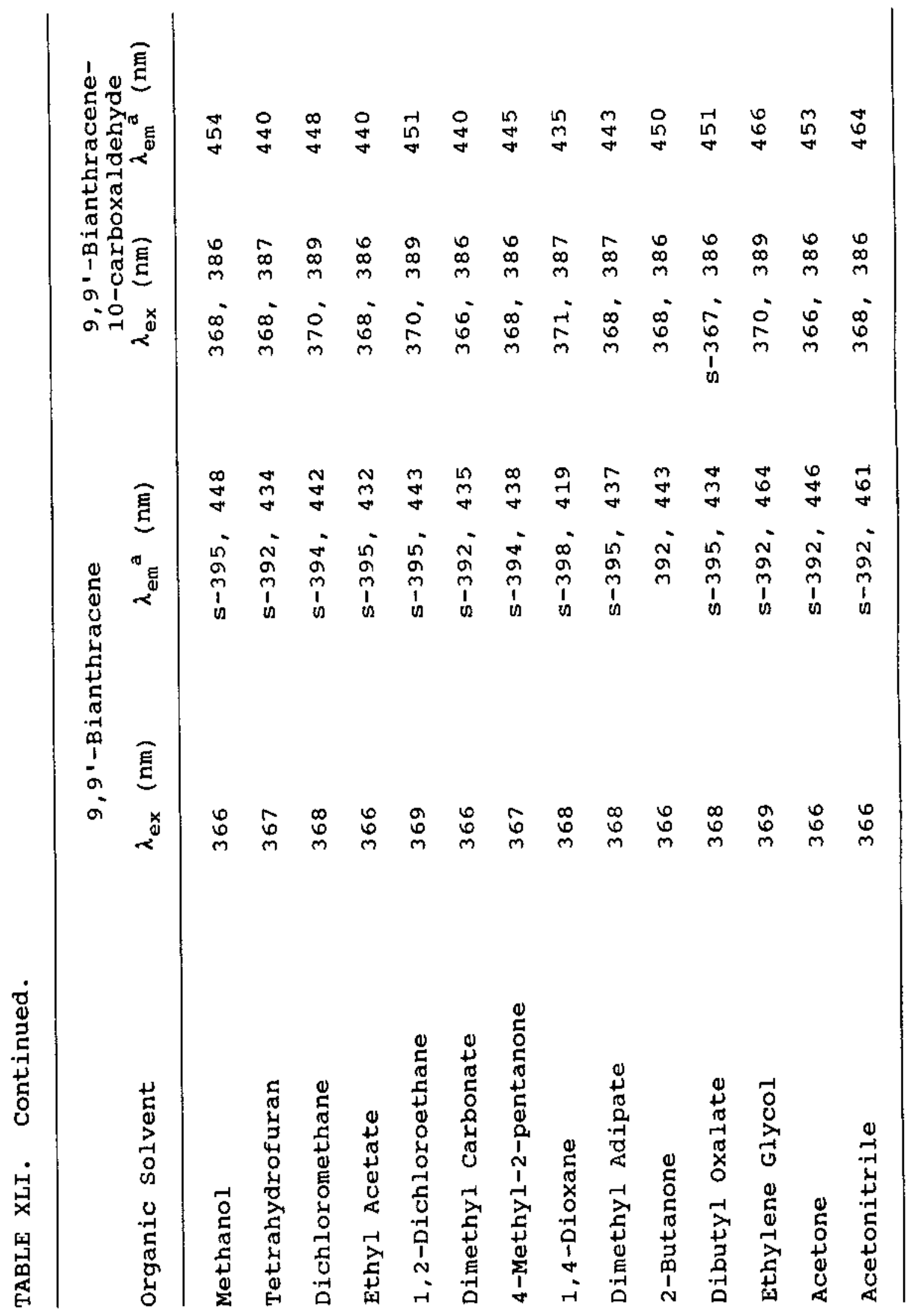




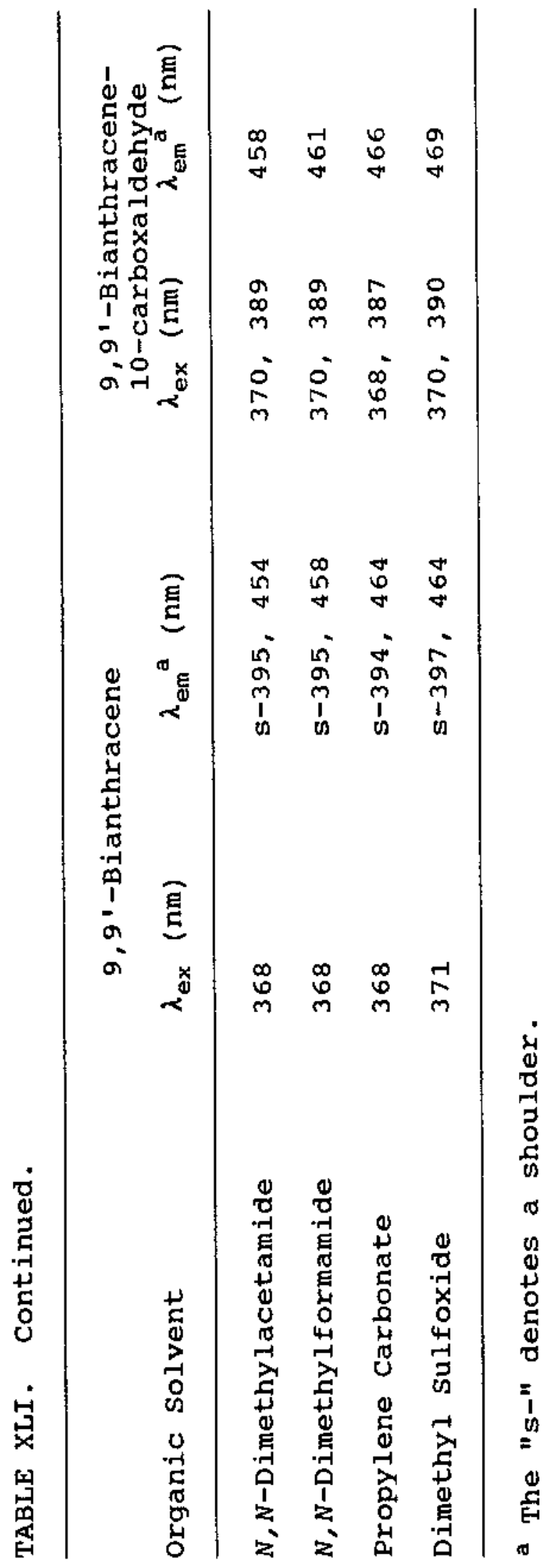


Rettig and Zander (56) reported that 9,9'-bianthracene-10-carboxaldehyde fluoresces in alcohols only. Figure 71 shows that this is not the case. A fluorescence spectrum was observed in all 45 solvents in Table XLI, and in terms of visual appearance, the emission behavior of 9,9'-bianthracene-10-carboxaldehyde is quite similar to that of the parent 9,9'-bianthracene bi-PAH molecule, except that the emission wavelength(s) are generally redshifted by 10-15 nm. Smaller shifts occurred in the case of the more polar solvents and in the alcohols. This latter observation is consistent with the earlier study of Rettig and Zander. Here, the authors argued that specific hydrogen-bonding interactions between the alcohol and 9,9-bianthracene-10carboxaldehyde (at the -cho site) reduce the dipole moment of the fluorophore's excited state, and would lead to a much smaller wavelength shifts.

1,1'-Binaphthalene shows only marginal solvatochromic behavior. The observed $10 \mathrm{~nm}$ shift in emission wavelength; however, was much too small for it to be recommended as a useful probe molecule in fluid solution. Pankasem and Thomas (17) have successfully used 1,1'-binaphthalene as a room temperature luminescent probe for monitoring active sites on $\gamma$-alumina. When absorbed onto a solid surface, free rotation of the two naphthyl moieties about the c-c single bond connecting both the aromatic ring systems is hindered. 1,1'-Binaphthalene thus exists in two distinct 
planar conformations, each of which exhibits different photophysical behavior. In solution rotation is significantly faster. The observed emission spectra in Figure 70 likely corresponds to some "equilibrium average" of the two planar spectrum plus all intermediate rotational conformers, rather than that of a single conformer. The solvatochromic shift will be less pronounced in fluid solution.

A recent study involving bi- and trichromic fluorophores, containing anthracene and/or pyrene aromatic rings connected via short spacer arms, suggests that electron transfer only occurs if the aromatic centers are directly connected to each other by a single $C-C$ bond (57). Fluorescence emission spectra showed no indication of formation of a "twisted intramolecular charge transfer" (TICT) complex in the case of $-\mathrm{CH}_{2} \mathrm{CH}_{2} \mathrm{O}_{2} \mathrm{CCH}_{2} \mathrm{CH}_{2} \mathrm{CO}_{2} \mathrm{CH}_{2}-$, $-\mathrm{CH}_{2} \mathrm{CH}_{2} \mathrm{CH}_{2}-$, and $-\mathrm{CH}_{2} \mathrm{CH}_{2} \mathrm{O}_{2} \mathrm{CCH}_{2} \mathrm{CH}_{2} \mathrm{CO}_{2} \mathrm{CH}_{2} \mathrm{CH}_{2}$ - spacer arms. For the most part, the lower wavelength detailed emission resembled what would be expected for alkyl-substituted anthracene and pyrene derivatives, as discussed earlier in this Chapter. The authors did observe at longer emission wavelengths, however, several interesting homogenous excimers as well as a heterogenous exciplex between two dissimilar aromatic centers and possibly a trimolecular complex involving all three aromatic centers on the trichromic fluorophore (57). 


\section{CHAPTER REFERENCES}

1. Tucker, S. A.; Cretella, L. E.; Waris, R.; Street, K. W., Jr.; Acree, W. E., Jr.; Fetzer, J. C. Appl. spectrose. 1990, 44, 269-273.

2. Selvarajan, N.; Panicker, M. M.; Vaidyanathan, S.; Ramakrishnan, V. Indian J. Chem. 1979, 18A, 23-26.

3. Encinas, M. V.; Rubio, M. A.; Lissi, E. A. Photochem. Photobiol. 1983, 37, 125-130.

4. Encinas, M. V.; Rubio, M. A.; Lissi, E. A. J. Photochem. 1982, 18, 137-150.

5. Wiczk, W. M.; Latowski, T. Z. Naturforsch. 1987, 42A, 1290-1295.

6. Bowen, E. J.; Rohatgi, K. K. Disc. Faraday Soc. 1953, $14,146-150$.

7. Ivanoff, M. I. Bull. Soc. Chim. Belg. 1962, 71, 759763 .

8. Oster, G. K. Acta Phys. Polon. 1964, 26, 435.

9. Carroll, F. A.; Whitten, D. A. J. Phys. Chem. 1976, 80, 2046-2047.

10. Birks, J. B. Photophysics of Aromatic Molecules; WileyIntersceince: New York, 1970; pp 439-441.

11. Dong, D. C.; Winnik, M. A. Can. J. Chem. 1984, 62, 2560-2565.

12. Waris, R.; Rembert, M. A.; Sellers, D. M.; Acree, W. E., Jr.; Street, K. W., Jr.; Fetzer, J. C. Analyst 1989, 114, 195-199.

13. Waris, R.; Street, K. W., Jr.; Acree, W. E., Jr.; Fetzer, J. C. Appl. Spectrosc. 1989, 43, 845-850.

14. Acree, W. E., Jr.; Zvaigzne, A. I.; Fetzer, J. C. AppI. Spectrosc. 1990, 44, 1193-1195.

15. Kalyanasundaram, K.; Thomas, J. K. J. Phys. Chem. 1977, $81,2176-2180$. 
16. Hite, P.; Krasanansky, R.; Thomas, J. K. J. Phys. Chem. $1986,90,5795-5799$.

17. Pankasem, S.; Thomas, J. K. J. Phys. Chem. 1991, 95, $7385-7393$.

18. Dederen, J. C.; Coosemans, L.; De Schryver, F. C.; Van Dormael, A. Photochem. Photobiol. 1979, 30, 443-447.

19. Mazumdar, S.; Manoharan, R.; Dogra, S. J. Photochem. Photobiol. 1989, 46A, 301-314.

20. Hanson, A. W. Acta Crystallogr. 1965, 18, 599-604.

21. Mitchell, R. H. AdV. Theor. Interesting Mol. 1989, 1, 135-199.

22. Ashwood-Smith, M. J.; Mitchel1, R. H.; Kennedy, A. Mutat. Res. 1978, 57, 123-125.

23. Pullman, A.; Pullman, B. Cancèrisation par les Substances Chimiques et Structure Molèculaire; Masson et Cie: Paris; 1955.

24. Pullman, A. In The Theory of Chemical Carcinogenesis and The Problem of Hydrocarbon-Protein Interactions. Biopolymers symp I 1964, 47.

25. Daudel, P.; Daudel, R. Chemical Carcinogenesis and Molecular Biology; Wiley-Interscience: New York; 1966.

26. Lijinsky, W.; Garcia, H; Terracini, B.; Saffiotti, U. J. Nat1. Cancer Inst. 1965, 34, 1-6.

27. Lijinsky, W.; Saffioti, U. Ann. It. Derm. C1in. sper. $1965,19,34-44$.

28. Scott, L. T.; Hashemi, M. M.; Bratcher, M. S. J.Am. Chem. Soc. 1992, 114, 1920-1921.

29. Huiszoon, C.; Van de Waal, B. W.; Van Egmond, A. B.; Harkema, s. Acta Crystallogr. 1972, 28B, 3415-3419.

30. Huiszoon, C.; Van Hummel, G. J.; Van den Ham, D. M. W. Acta Crystallogr. 1977, 33B, 1867-1870.

31. Andreetti, G. D. ; Herbich, J.; Lipkowski, J. Cryst. struct. Commun. 1981, 10, 1233-1238.

32. Cundall, R. B.; Grant, D. J. W. ; Shulman, N. H. J. Chem. Soc., Faraday Trans. 2 1982, 78, 737-750. 
33. Bortolus, P.; Galiazzo, G.; Gennari, G. Anal Chim. Acta 1990, 234, 353-358.

34. Markgraf, J. H.; Katt, R. J. J. Org. Chem. 1972, 37, $717-718$.

35. Pachtner, I. J.; Kloetzel, M. C. J. Amer. Chem. Soc. $1951,73,4958-4960$.

36. Rowe, I.; Post, B. Acta Crystallogr. 1958, 372-374.

37. Lynton, H.; Cox, E. G. J. Chem. Soc. 1956, $4887-4895$.

38. Hosoya, s. Acta Crystallogr. 1963, 310-312.

39. Lumbroso, H.; Montaudo, G. Bull. Chem. Soc. Fr. 1964, 2119-2124.

40. Aroney, R. J.; Lefevre, J. W.; Saxby, J. D. J. Chem. SOC. 1965, 571-575.

41. Distefano, G.; Galasso, V.; Irgolic, K. J.; Pappalardo, G. C. J. Chem. SoC., Perkin Trans. 1983, 2, 1109-1112.

42. Rodin, O. G.; Traven, V. F.; Redchenko, V. V.; Eismont, M. Y.; Stepanov, B. I. Zh. Obshch. Khim. 1983, 53, 2537-2543.

43. Nakasuji, K.; Kubota, H.; Kotoni, T.; Murata, I.; Saito, G.; Enoki, T.; Imaeda, K.; Inokuchi, H.; Honda, M.; Kataymama, C.; Tanka, J. J. Am. Chem. Soc. 1986, $108,3460-3466$.

44. Acree, W. E., Jr.; Tucker, S. A.; Fetzer, J. C. Polycyclic Aromat. Compds. 1991, 2, 75-105.

45. Zander, M.; Kirsch, G. Z. Naturforsch. 1989, 44A, 205209 .

46. Zander, M. Z. Naturforsch. 1989, 44A, 1116-1118.

47. Buisson, J.-P.; Demerseman, P. J. Heter. Chem. 1990, $27,2213-2214$.

48. Schneider, F.; Lippert, E. Ber. Bunsen-Ges. Phys. Chem. $1968,72,1155$.

49. Visser, R. J.; Weisenborn, P. C. M.; van Kan, P. J. M.; Huizer, B. H.; Varma, C. A. G. O.; Warman, J. M.; DeHaas, M. P. J. Chem. Soc., Faraday Trans. 1985, 81, 689-704. 
50. Lueck, H.; windsor, M. W.; Rettig, W. J. Phys. Chem. $1990,94,4550-4559$.

51. Jarzeba, W.; Kahlow, M; Barbara, P. F. J. Imaging Sci. $1989,33,53-57$.

52. Kabouchi, B.; Benali, B.; Fadouach, M.; Kadiri, A.; -Cazeau-Dubroca, C.; Nouchi, G. Spectrochim. Acta 1992, $48 A, 1015-1020$.

53. Bystryak, S. M.; Liktenshten, G. I.; Kotel'niko, A. I. Khim. Fiz. 1992, 11, 374 .

54. Wortmann, R.; Lebus, S.; Elich, K.; Assar, S.; Detzer, N.; Liptay, W. Chem. Phys. Lett. 1992, 198, 220-228.

55. Grabowski, Z. R.; Rotiewicz, R.; Siemiarczuk, A.; Cowley, D. J.; Baumann, W. Nouv. J. Chim. 1979, 3, 443.

56. Rettig, W.; Zander, M., Ber. Bunsen-Ges. Phys. Chem. $1883,87,1143$.

57. Castellan, A.; Kessab, L.; Grelier, S.; Nourmamode, A.; Cotrait, M.; Marsau, P. J. Chem. Soc. Perkin Trans. 2 1993, 953-962. 
CHAPTER IV

CORRELATIONS CONCERNING SOLVENT POLARITY PROBE CHARACTER

Examination of Molecular structure Versus Probe Character Correlations

It is important to try to understand the phenomena of selective enhancement of the vibronic bands in the fluorescence emission spectra of polycyclic aromatic compounds (PACs) at the molecular level utilizing the large spectral database. Not all PAC solutes exhibit similar behavior, and only 42 of the more than 200 compounds are classified as solvent polarity probes. Published papers (15) attribute solvent-dependent intensity ratios to reduction of molecular symetry of the polycyclic aromatic hydrocarbons (PAHs) caused by complexation with surrounding polar solvent molecules. The different molecular elements examined include point groups, number of $\pi$-electrons, molecular families, planarity, and Kekulé structure counts (SC) .

Examination of Tables XLII-LI reveals that the overall molecular symmetry, while important, is not the sole criterion for determining whether a given PAC exhibits probe behavior. There is no apparent correlation between point groups (or symmetry elements) and probe character. For example, coronene $\left(D_{6 h}\right.$ point group) has a center of 


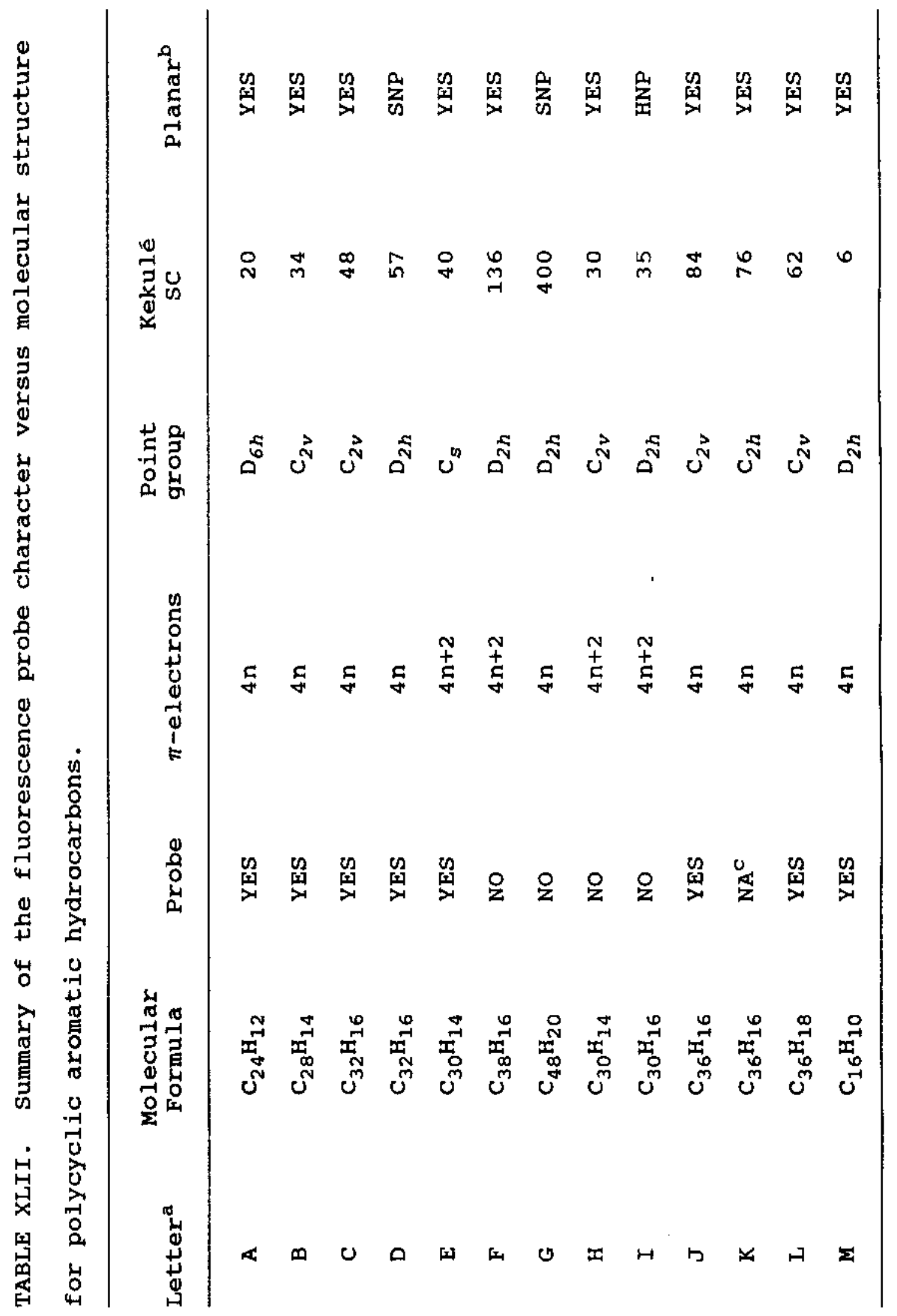




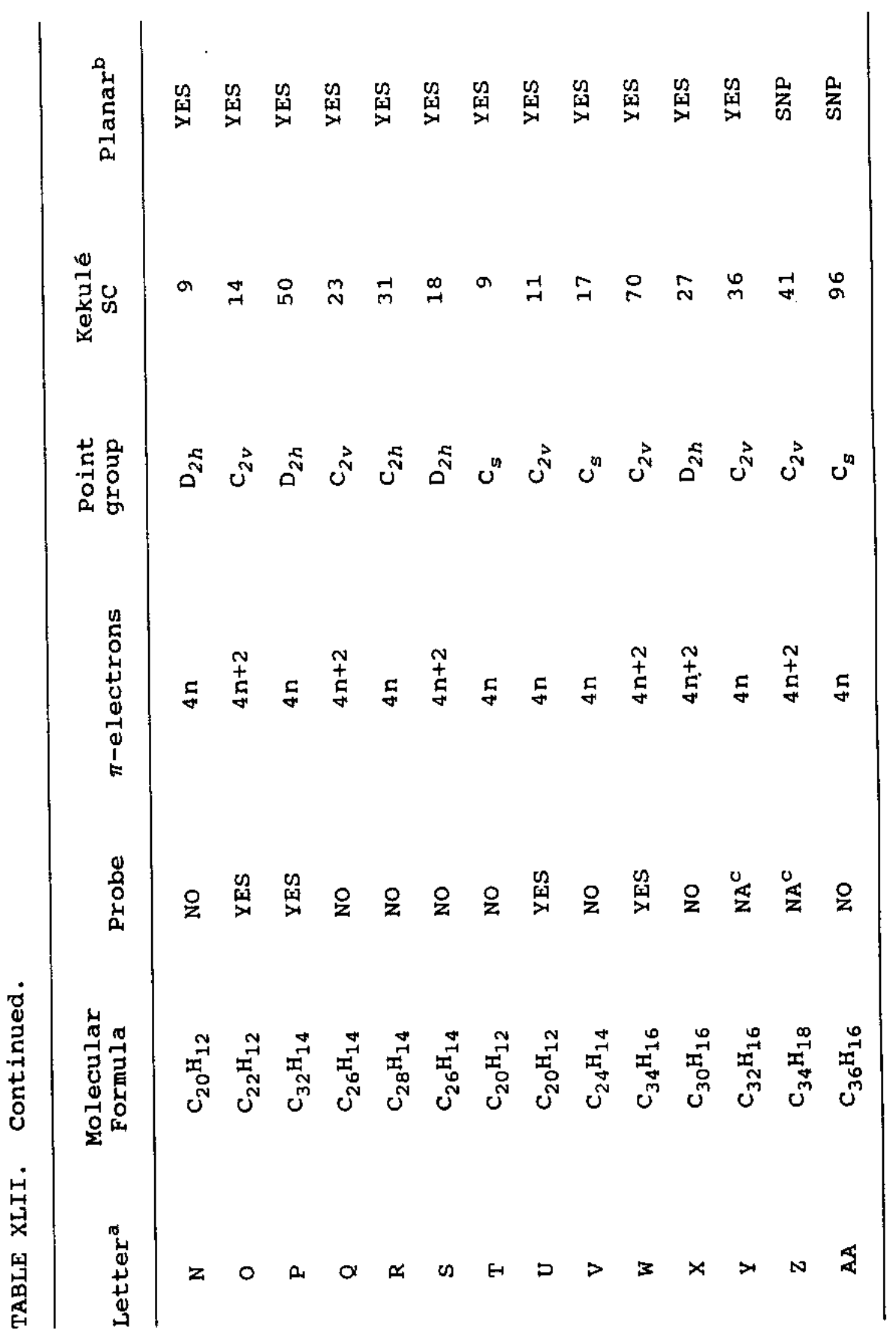




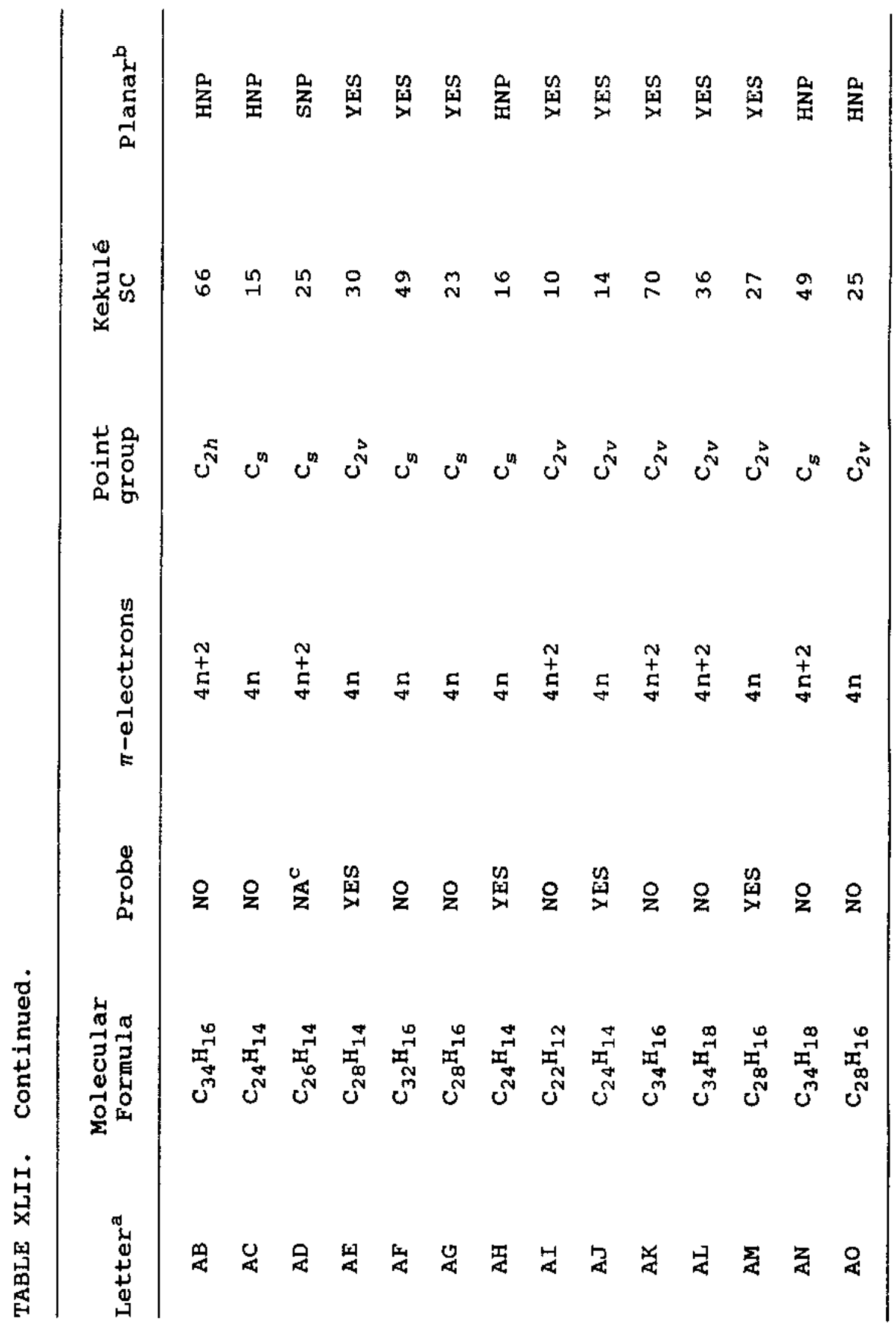




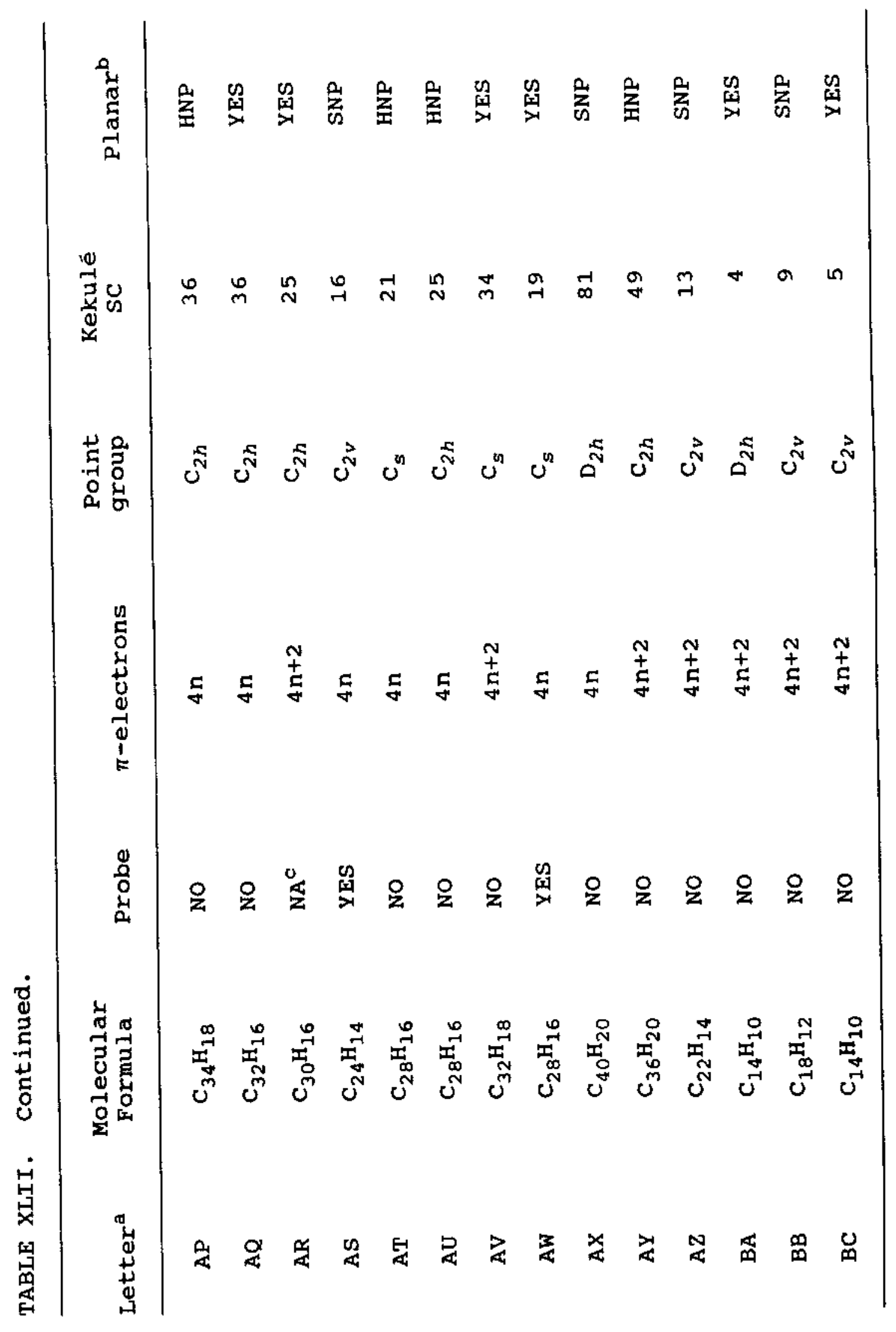




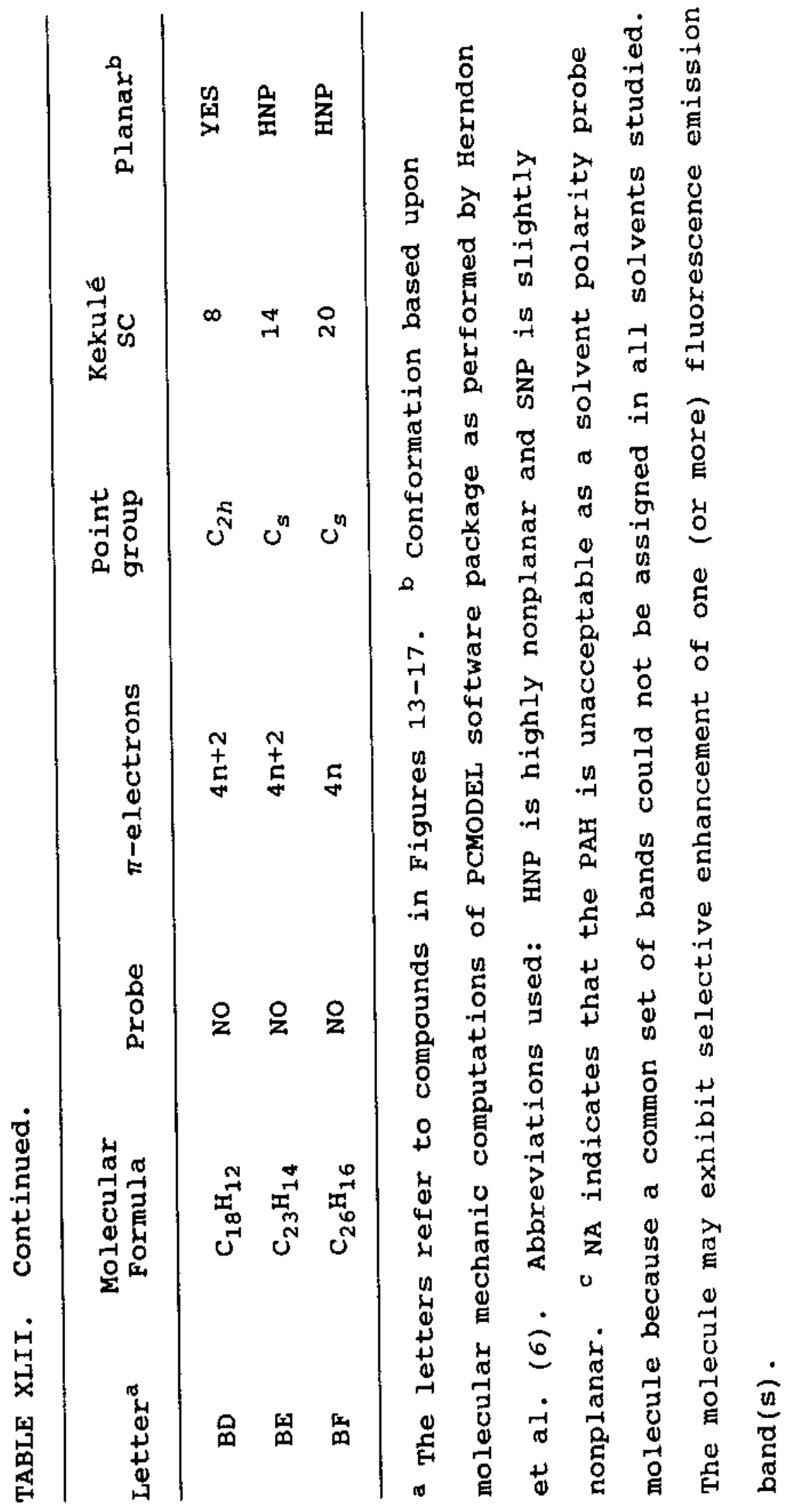




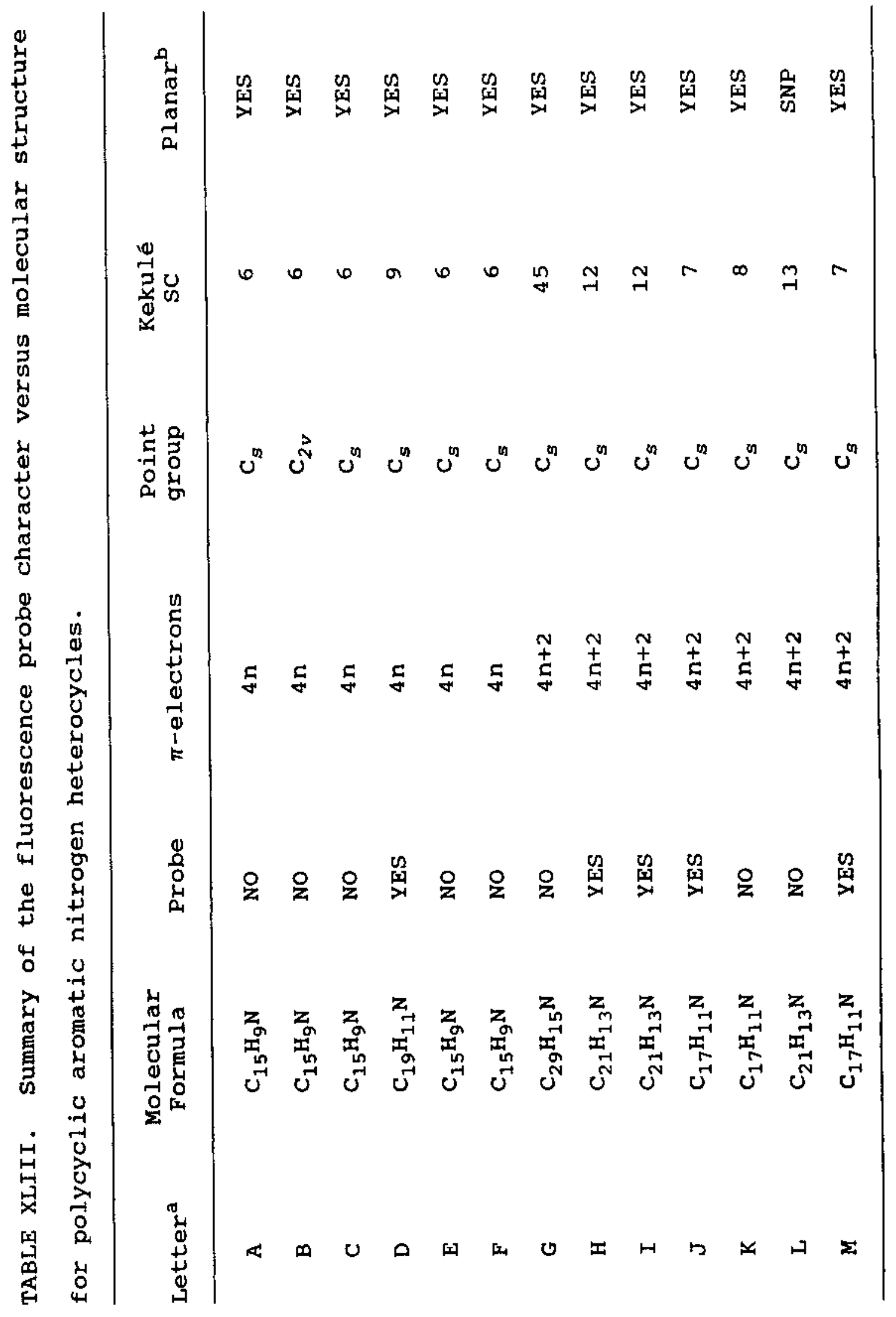




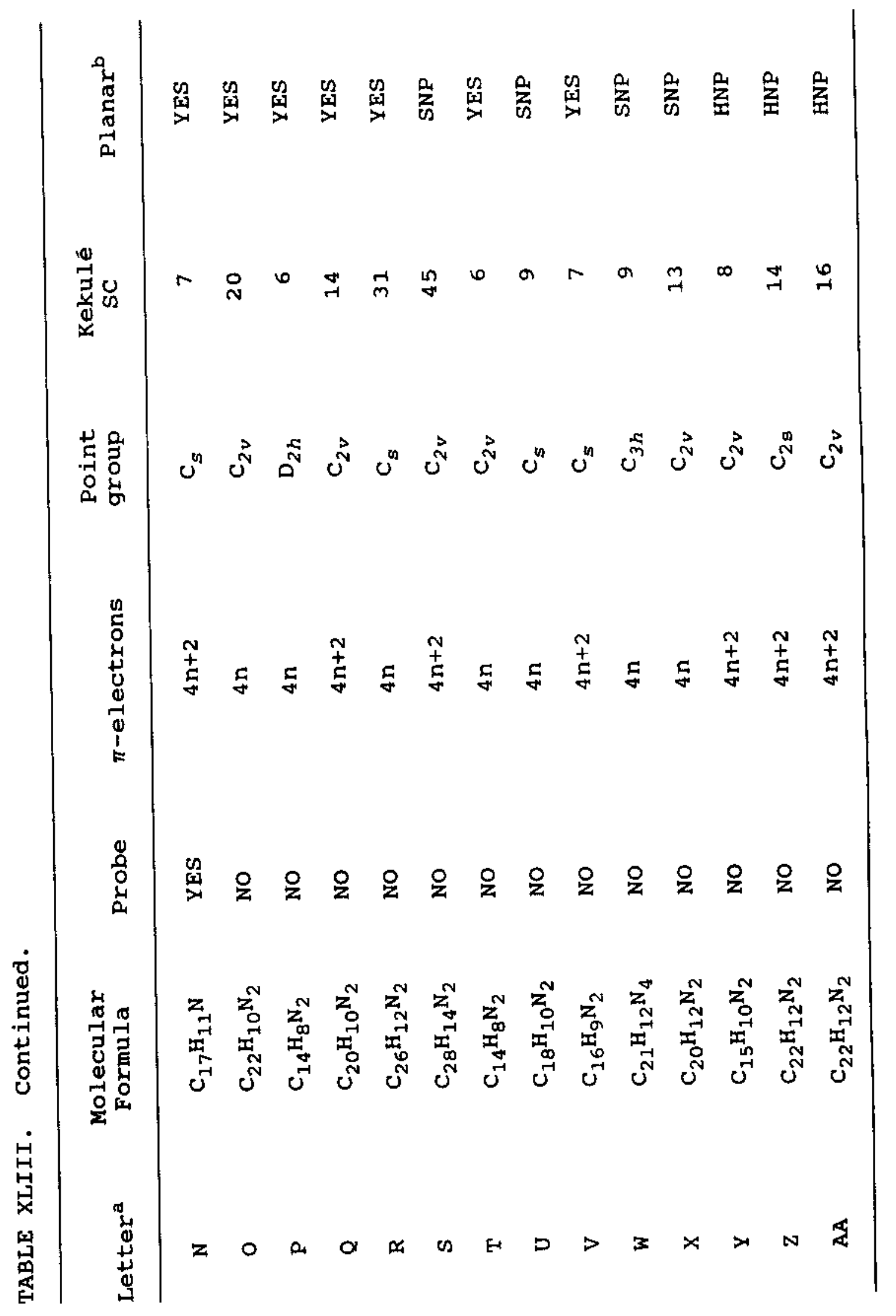




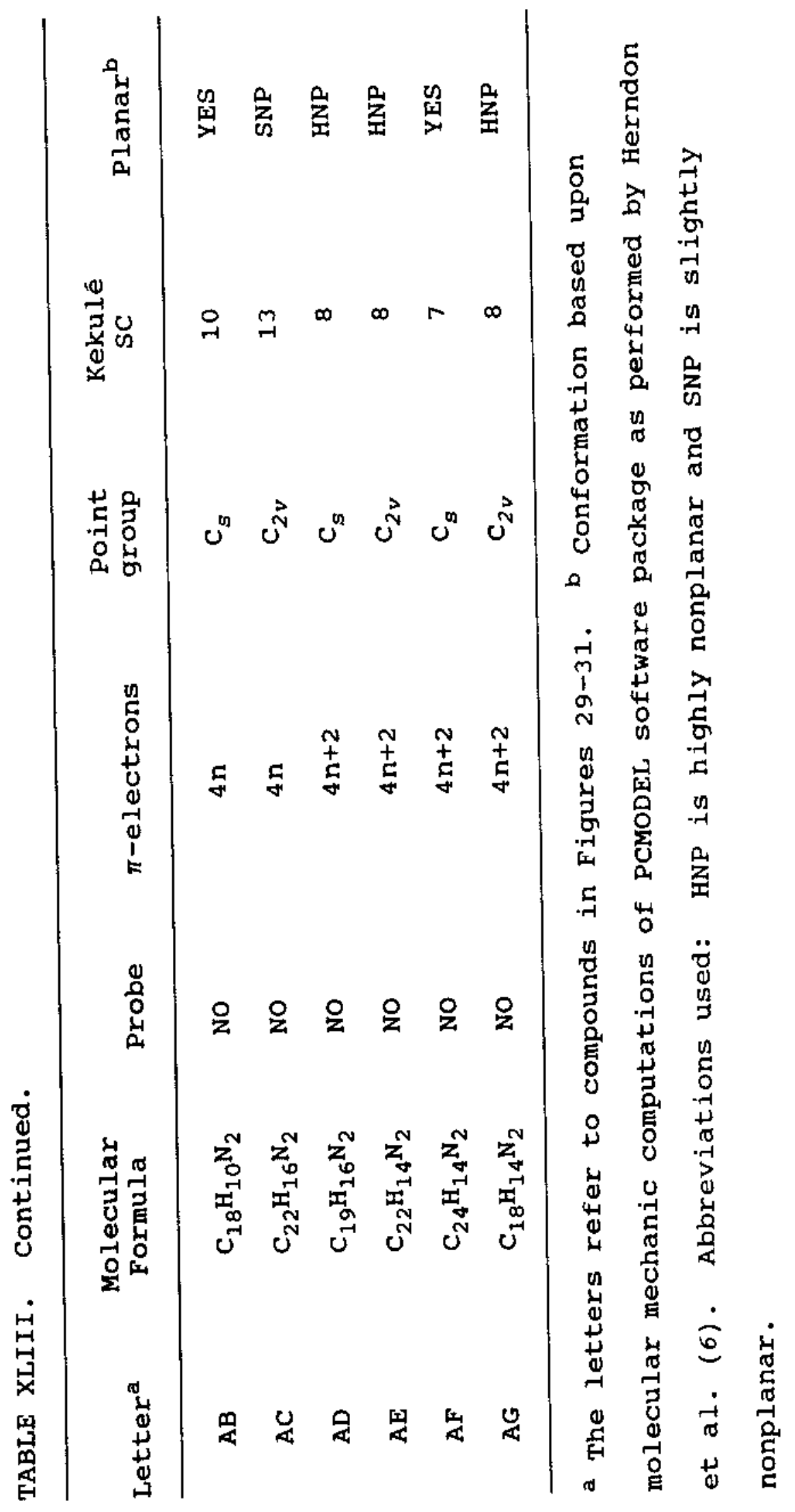




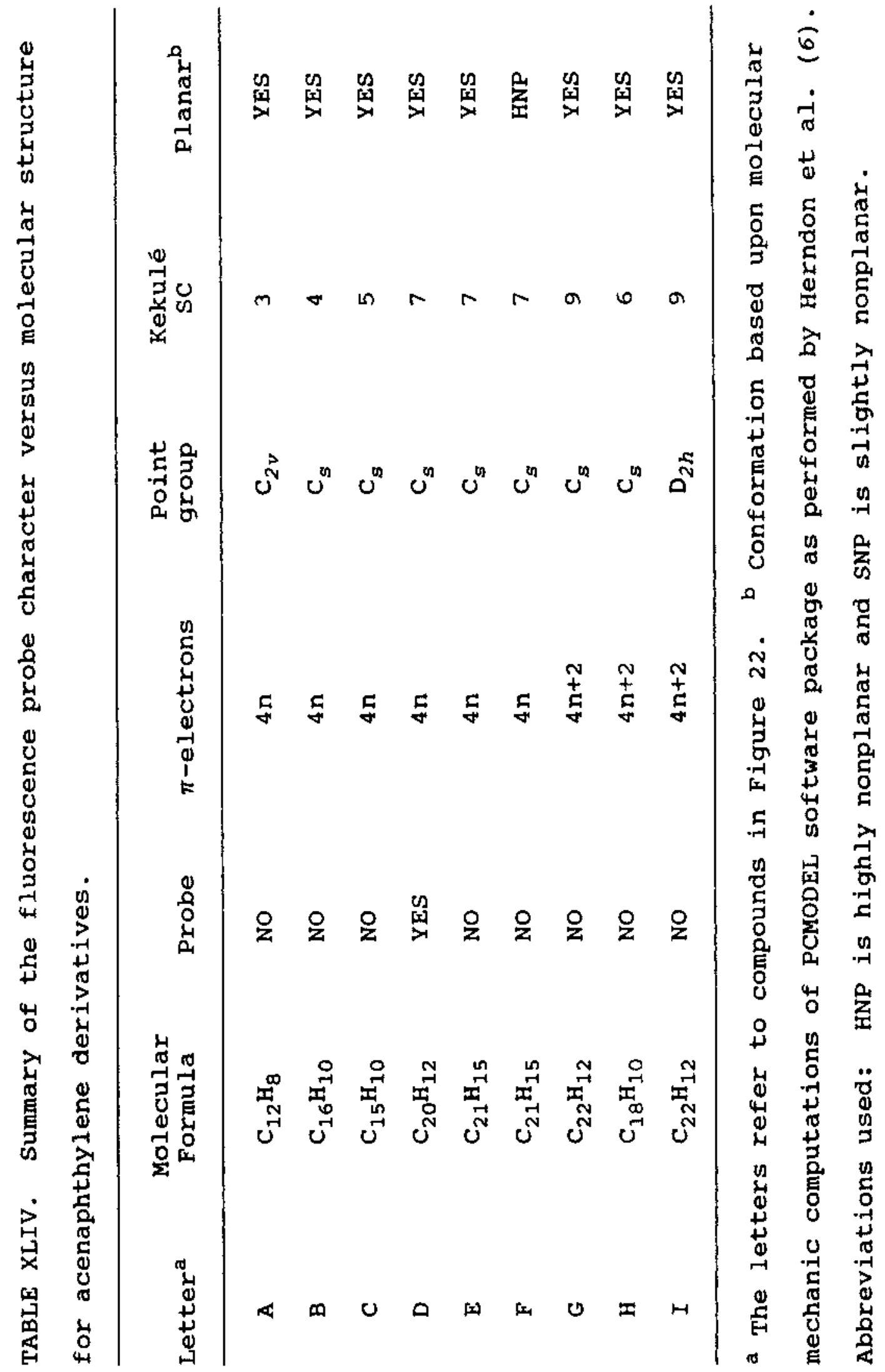




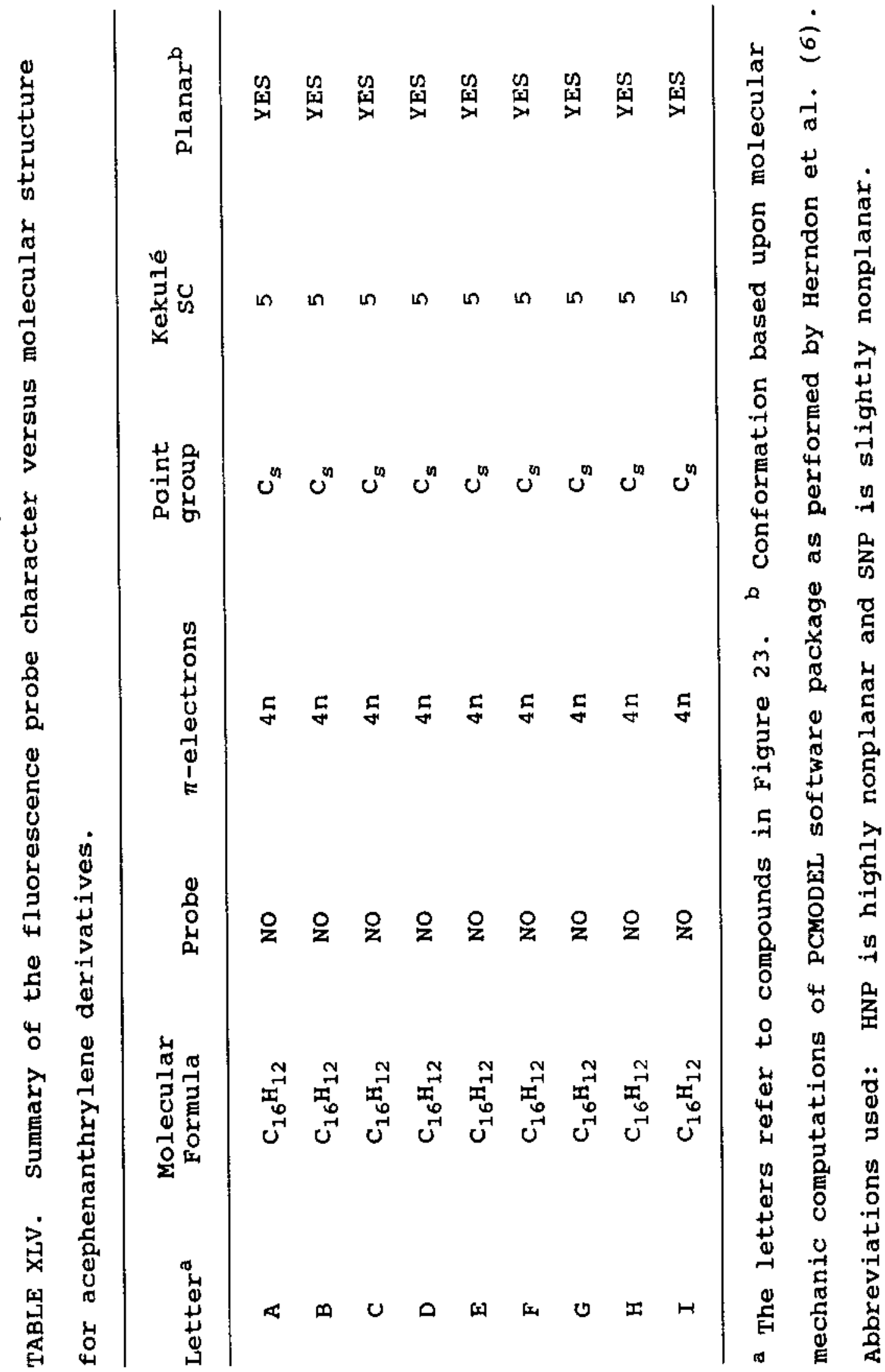




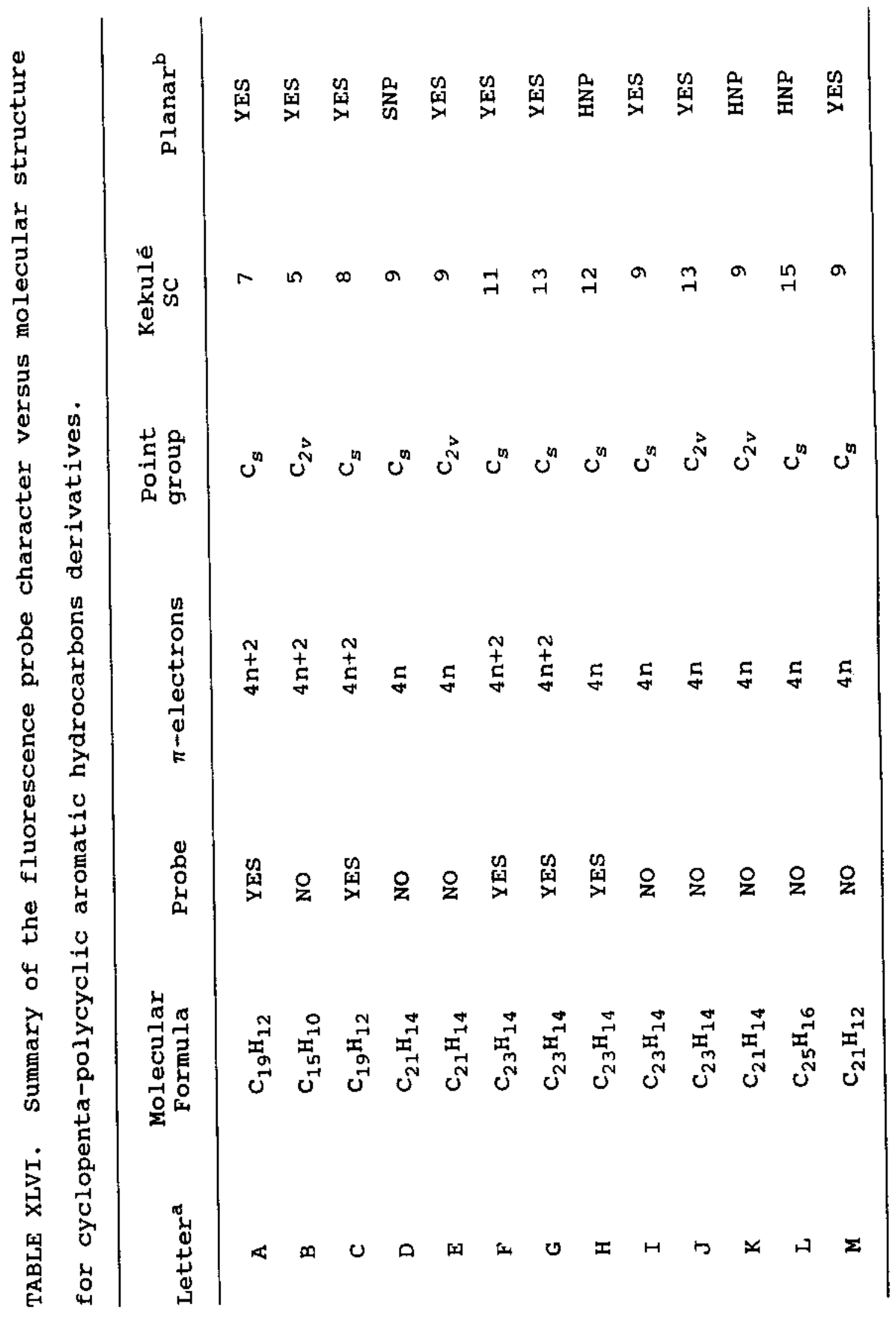




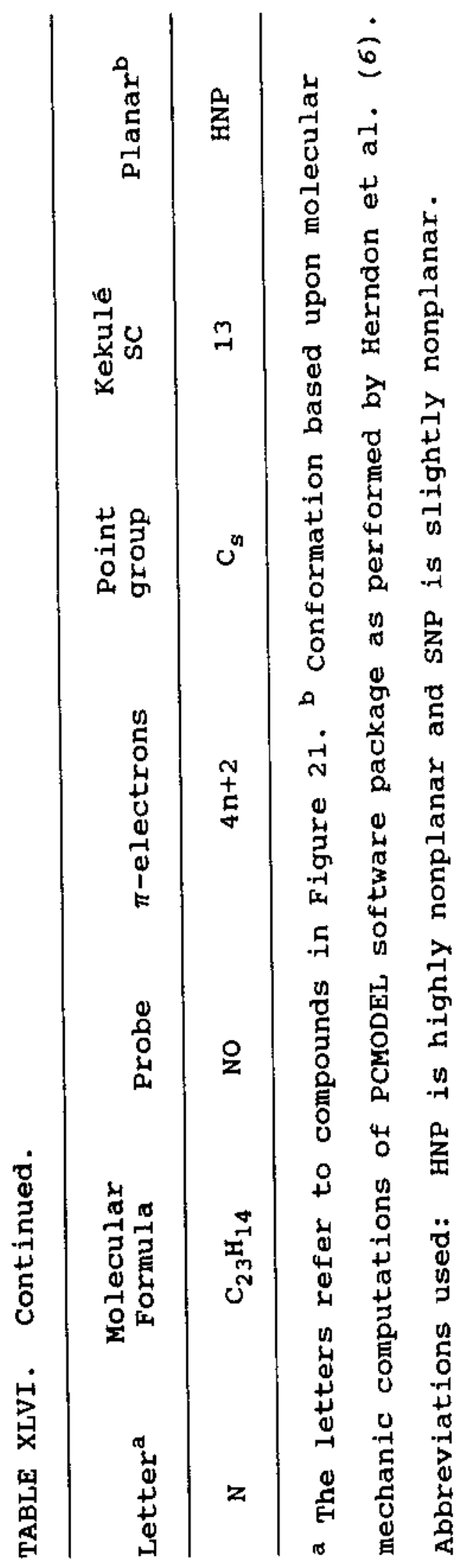




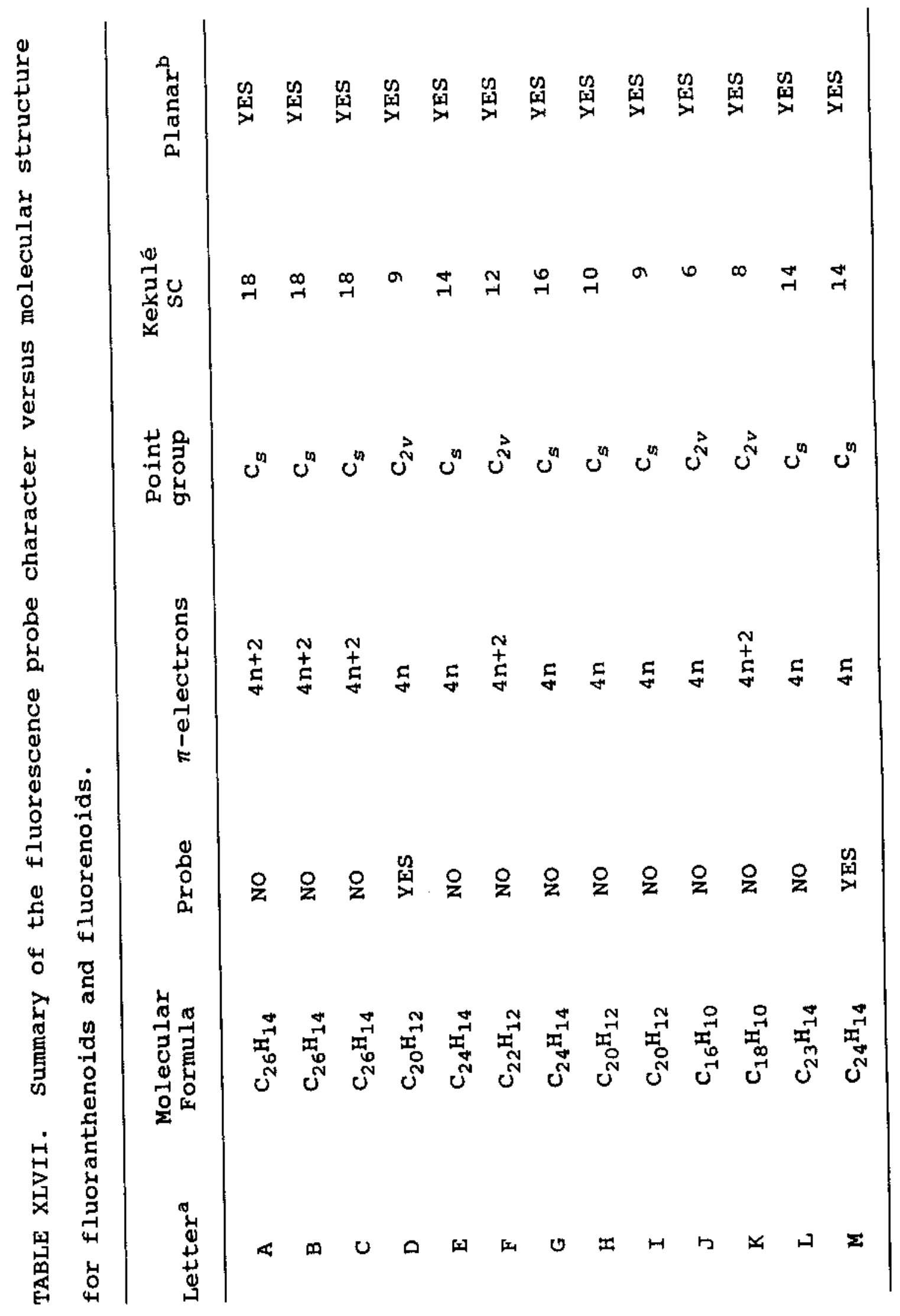




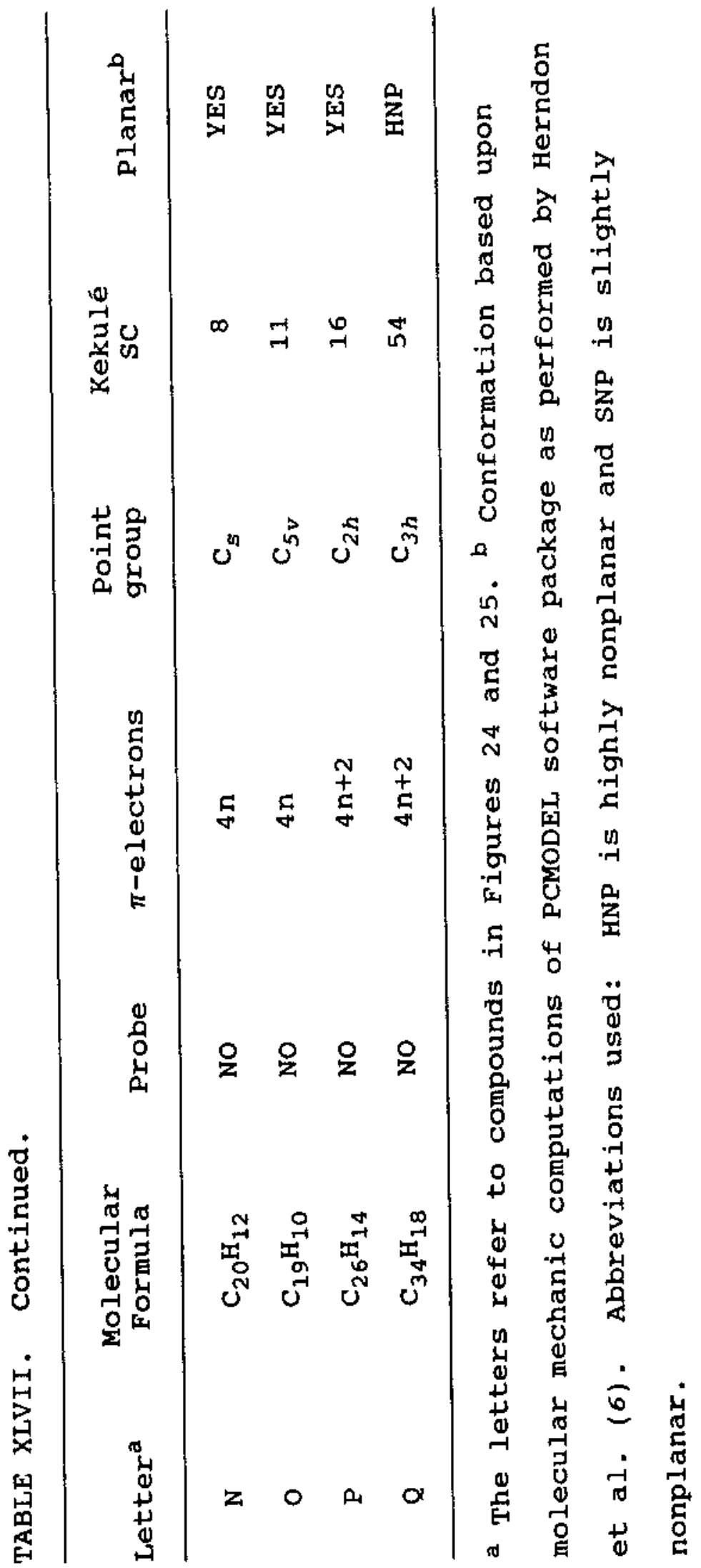




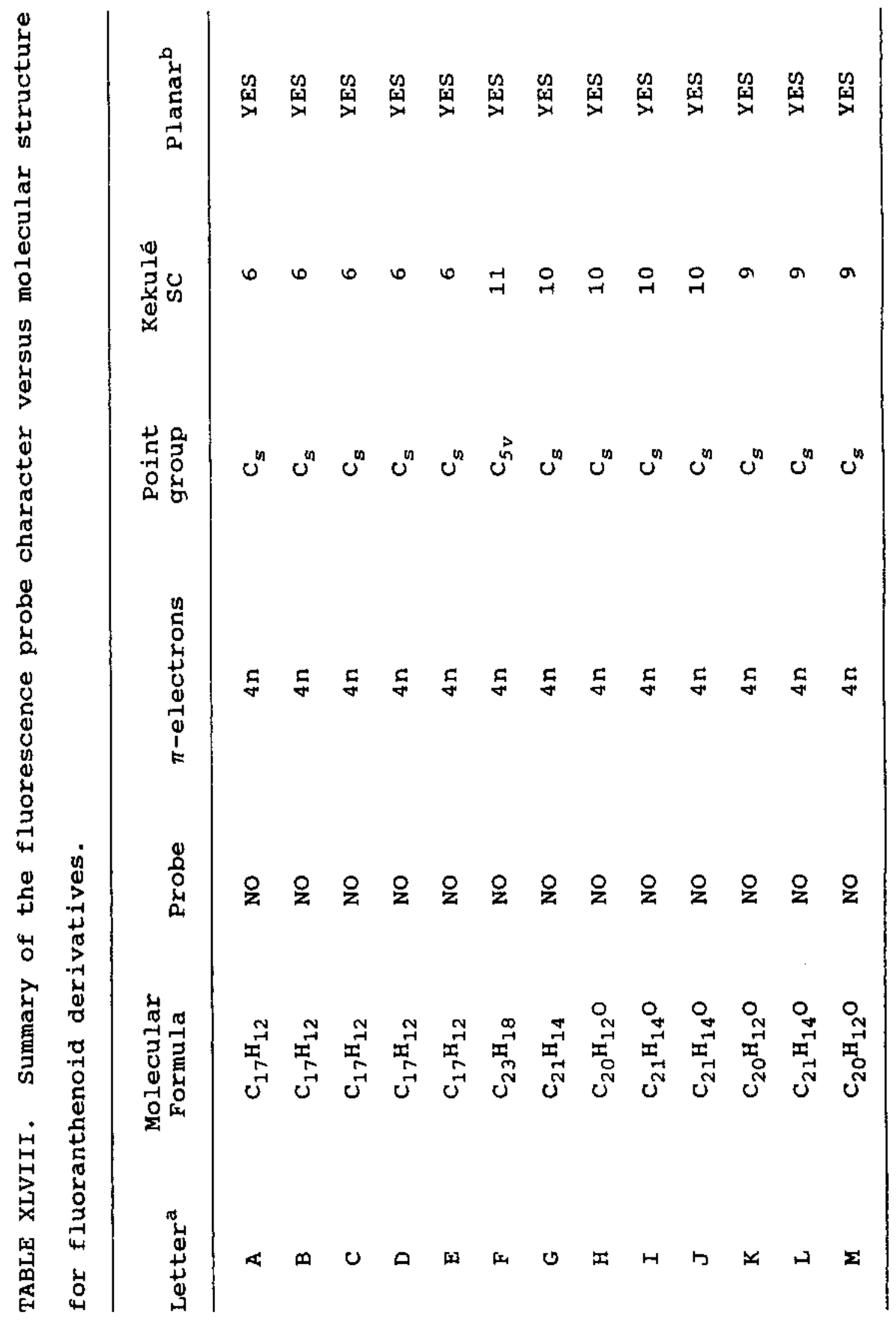




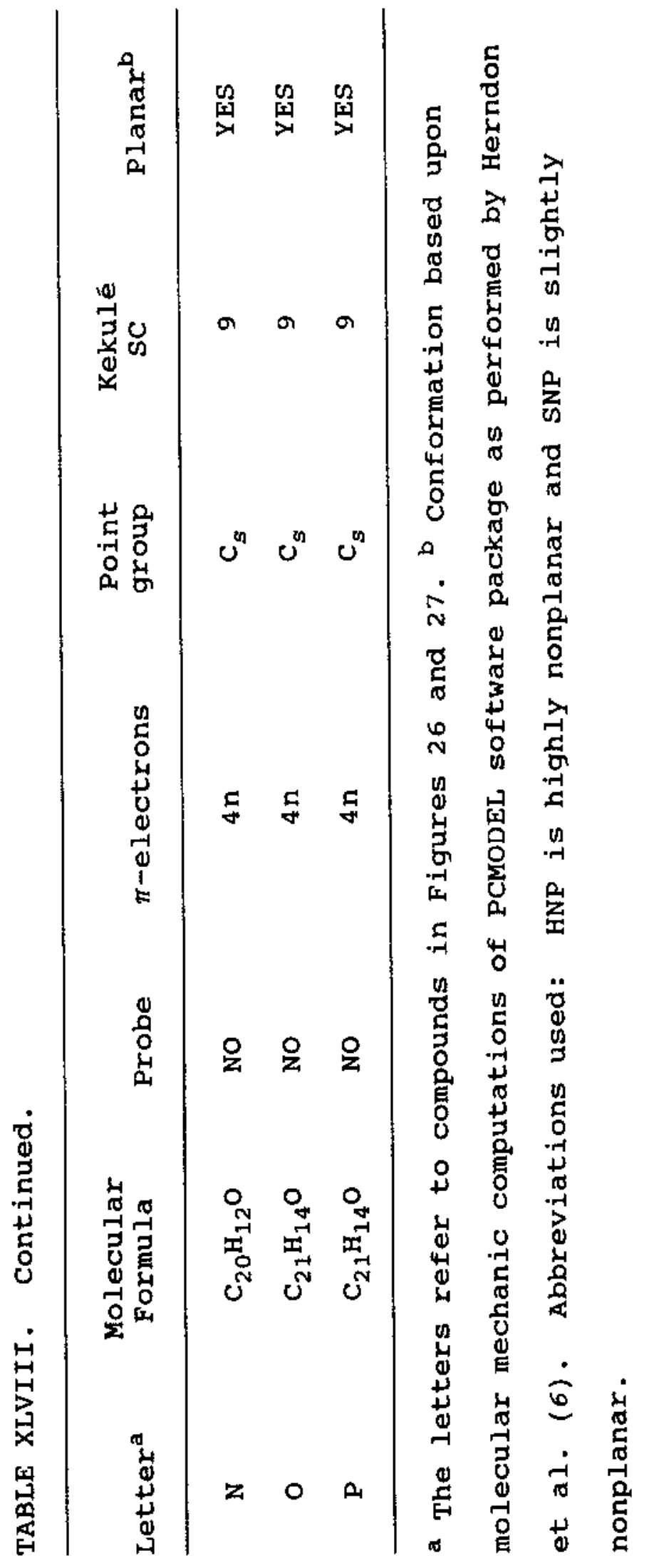




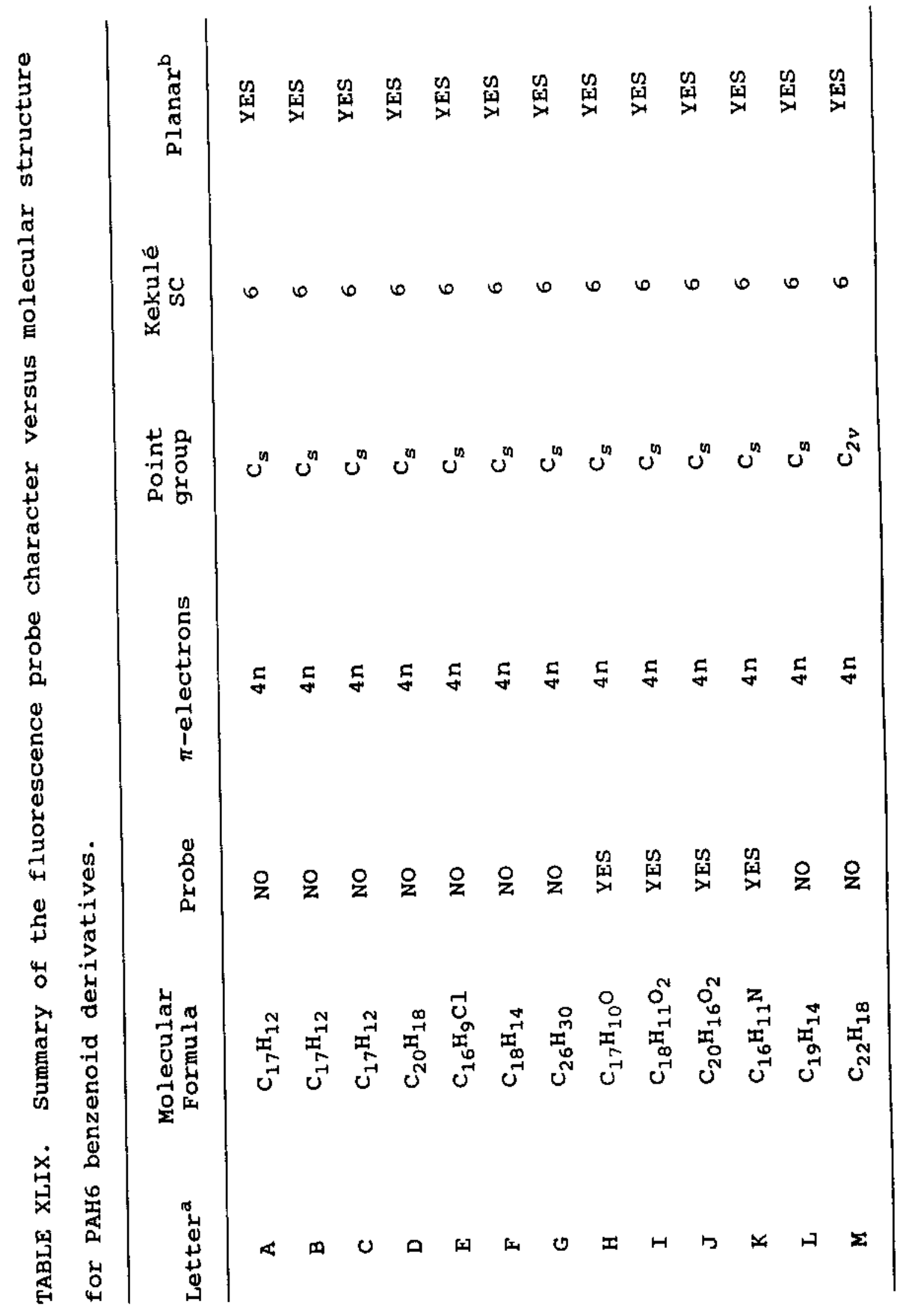




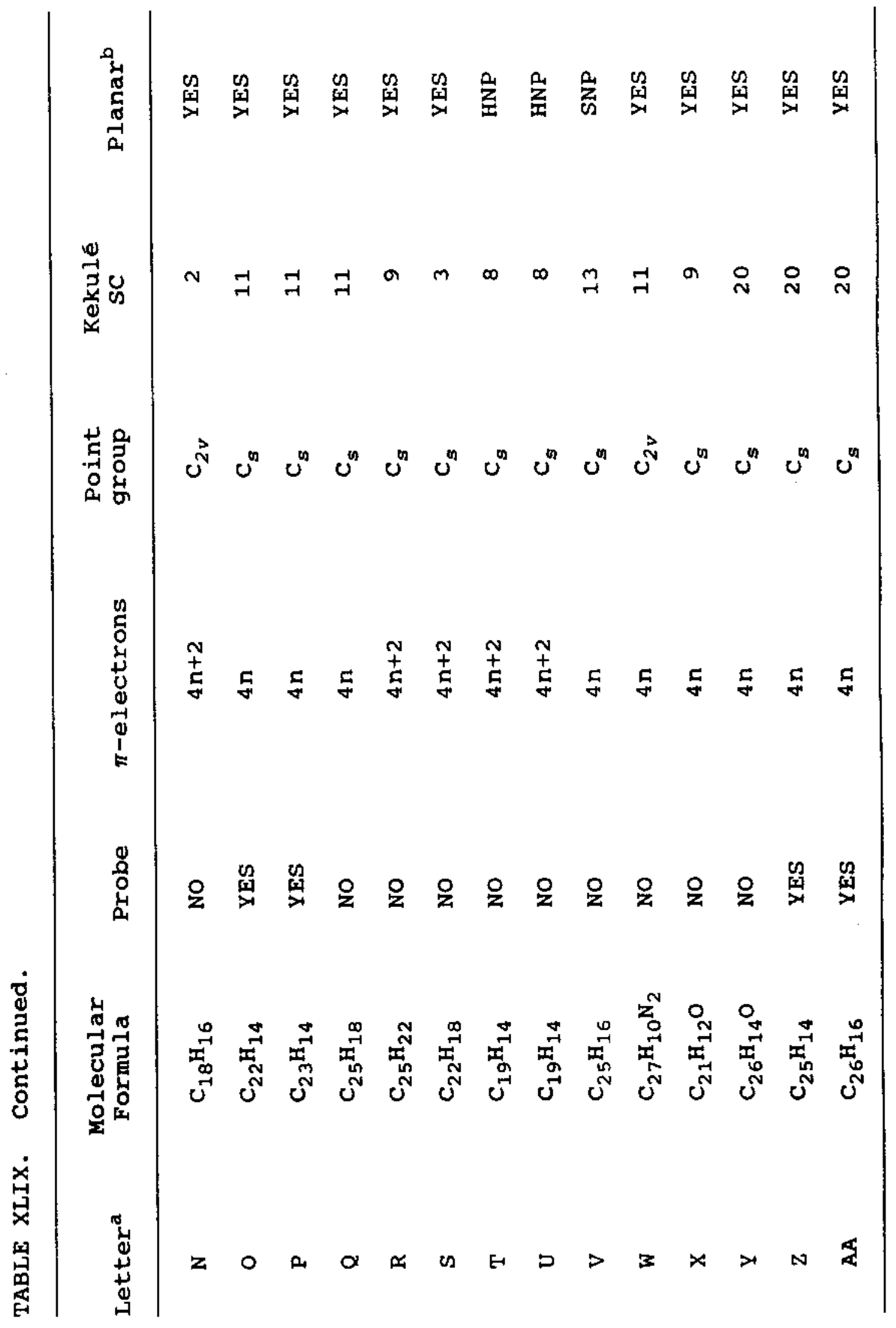




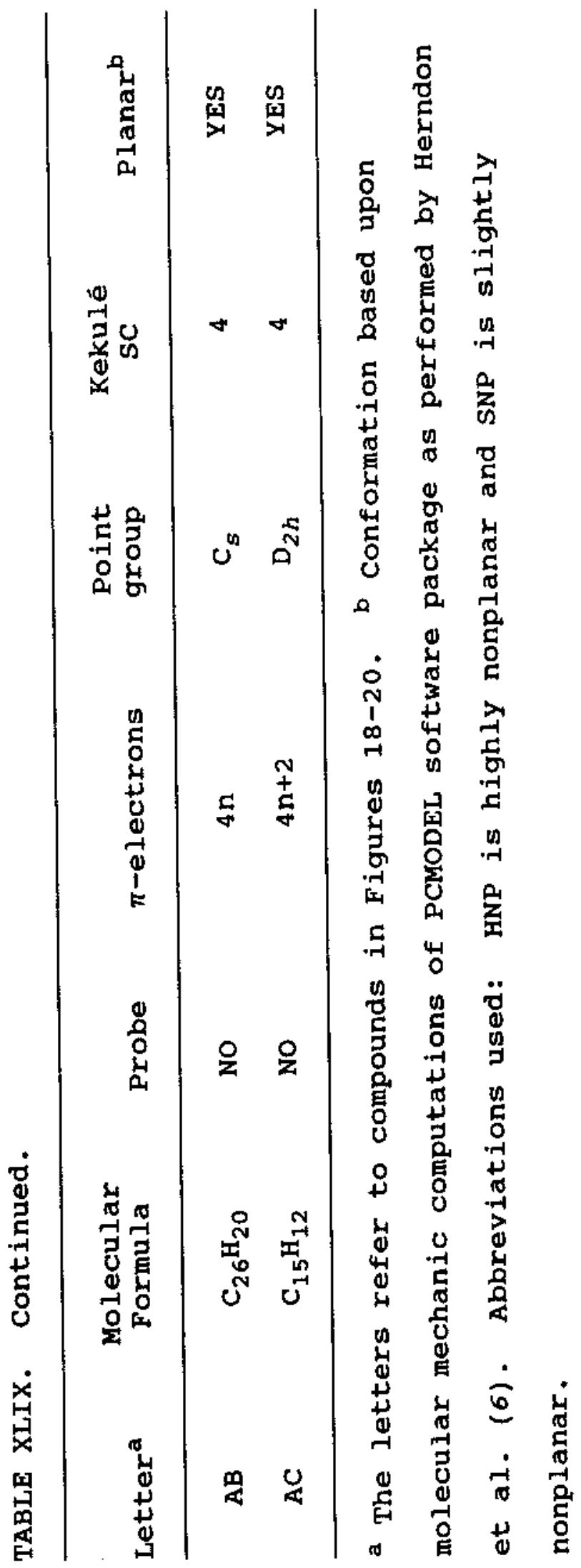




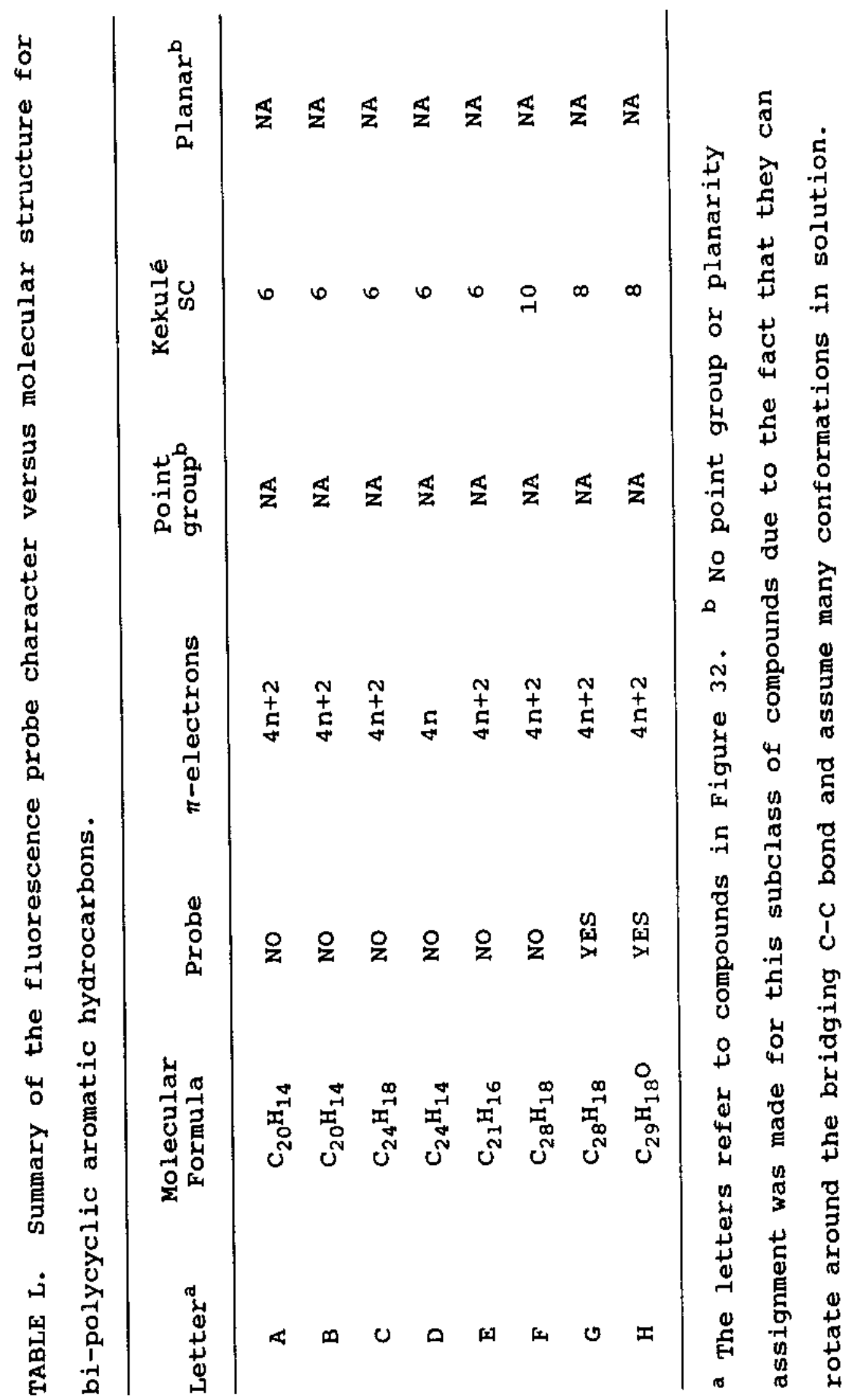




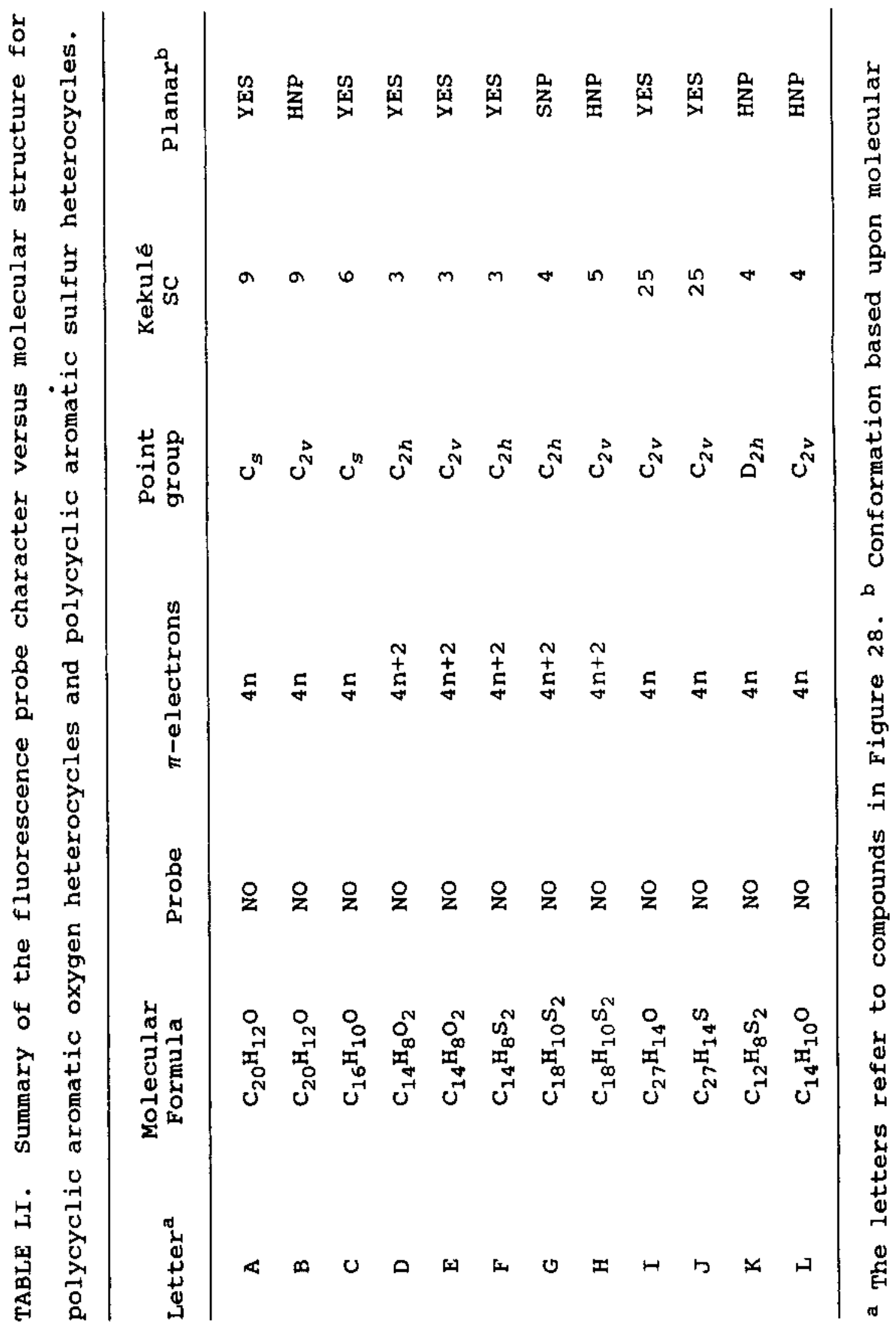


TABLE LI. Continued.

mechanic computations of PCMODEL software package as performed by Herndon et al. (6). Abbreviations used: HNP is highly nonplanar and SNP is slightly nonplanar.

symmetry, a six-fold rotation axis, a horizontal plane, and several vertical mirror planes. In comparison, benzo[ghi]perylene is a six-ring $\mathrm{PAH}$ whose symmetry elements $\left(\mathrm{C}_{2 \mathrm{v}}\right.$ point group) included only a single 2-fold rotation axis and the mirror plane that is common to all planar PAH molecules. The fluorescence emission intensity of the I/III band ratio of both molecules does vary with solvent polarity.

Furthermore, coronene, benzo[a]coronene, naphtho[2,3a]coronene, and dibenzo[a,j]coronene show probe character, irrespective of the position of the added naphtho- and benzo-aromatic rings (refer back to Figures 13-17 for molecular structures). This contrasts with the fluorescence behavior of perylene, which has a 2 -fold major rotation axis and belongs to the $D_{2 h}$ point group. Like coronene and dibenzo[a,j]coronene, perylene also has a center of symmetry. The fluorescence spectra of perylene does not show the selective enhancement of the $I$ band emission intensity in polar solvents.

In light of Tables XLII-LI, similar symmetry arguments can be made in the case of the other PAC subclasses. Point 
group assignments assume a planar molecular structure, which seems to be reasonable in the absence of direct structural information. Published $x$-ray crystallographic data on benzo[c]phenanthrene (7-9), dibenzo[c,g]phenanthrene $(10)$, phenanthro $[4,3 c]$ phenanthrene (11), dibenzo $[9, p]$ chrysene (12), naphtho[1,2,34rst $]$ pentaphene (13), hexahelicene, and higher helicenes $(14,15)$, indicate that not all PACs are planar. Intermolecular solute/solute crystal packing forces and/or the presence of sterically hindered bay-region fragments ( $S_{4 \text { (or higher) }}, T, 3 T$; see Figure 72 for definition of terms) in the molecule can lead to a nonplanar geometry. Both effects are important in the case of solid samples. Molecular structure/geometries based upon $\mathrm{x}$-ray crystallographic data may differ from those in fluid solution where the dissolved PAC is completely surrounded by solvent molecules.

Recent molecular mechanic computations by Herndon et al. (6) on the smaller $\mathrm{PAH}$ molecules containing seven phenyl-rings or less, allow one to examine the possible probe character versus planarity correlations in greater detail without imposing crystal packing constraints. Geometries of all PACs contained in Tables XLII-LI were predicted with PCMODEL software. Compounds having dihedral angles of $0-2^{\circ}$ are designated planar, $2-10^{\circ}$ are designated slightly nonplanar, and $>10^{\circ}$ are designated highly nonplanar (16). For example, dibenzo[a,j]coronene should be slightly 

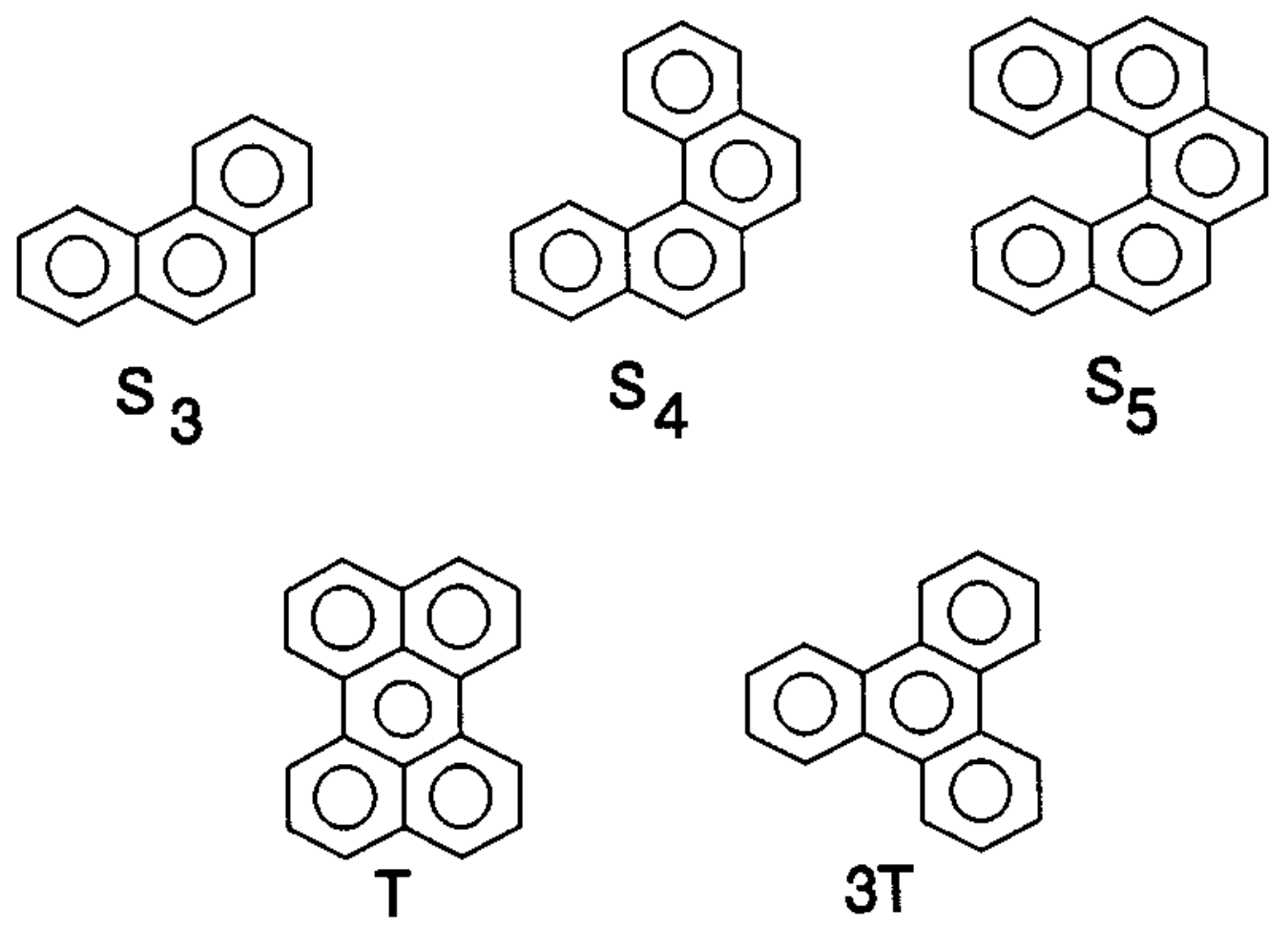

FIGURE 72: Sterically hindered bay-region moieties contained in nomplanar polycyclic aromatic hydrocarbons. The $T$ parameter is characterized by condensation of phenanthroid $s_{3}$ fragments sharing a common central ring that locks the $s_{3}$ bay-region hydrogen atoms in a restricted molecular environment. one $s_{3} s_{3}$ pair (perylene) is denoted as $T$, whereas three $S_{3} S_{3}$ pairs (triphenylene) equal $3 T$. 
nonplanar (SNP) as it has $T$ bay regions (at least one $S_{3} S_{3}$ pair) created at the point of the added benzo-substituent rings. An SNP molecular geometry is consistent with that found in the corresponding pyrene derivative, dibenzo[e, I]pyrene (formally named dibenzo[de, $q r]$ naphthacene).

Inspection of Tables XLII-LI reveals that there are several planar (i.e., pyrene and coronene), sightly nonplanar (i.e., dibenzo[de, $q r]$ naphthacene) and highly nonplanar (i.e., dibenzo[def,p]chrysene) molecules in each subclasses that are probes/nonprobes. To date there appears to be no correlation between solvent polarity probe behavior and planarity or point group (molecular symetry). Further inspection of Tables XIII-LI also reveals that there is no correlation between solvent polarity probes and $\pi$-electron numbers, which is based upon the so-called "aromatic" $(4 n+2)$ and "anti-aromatic" (4n) classification schemes used to divide the PAHs according to chemical reactivity.

Limited correlation can be discovered if attention is restricted to subset families, rather than all 205 PACs. within the coronene family, it is interesting to note that only those derivatives having four to six benzene rings in the center chain exhibited probe behavior. Furthermore, the length of the center chain always exceeded the remaining two chain lengths (see Figure 73). Dinaphtho[8,1,2abc;$\left.2^{\prime}, 1^{\prime}, 8^{\prime} j k l\right]$ coronene possessed these two structural features as well, but unfortunately the appearance of an intense new 

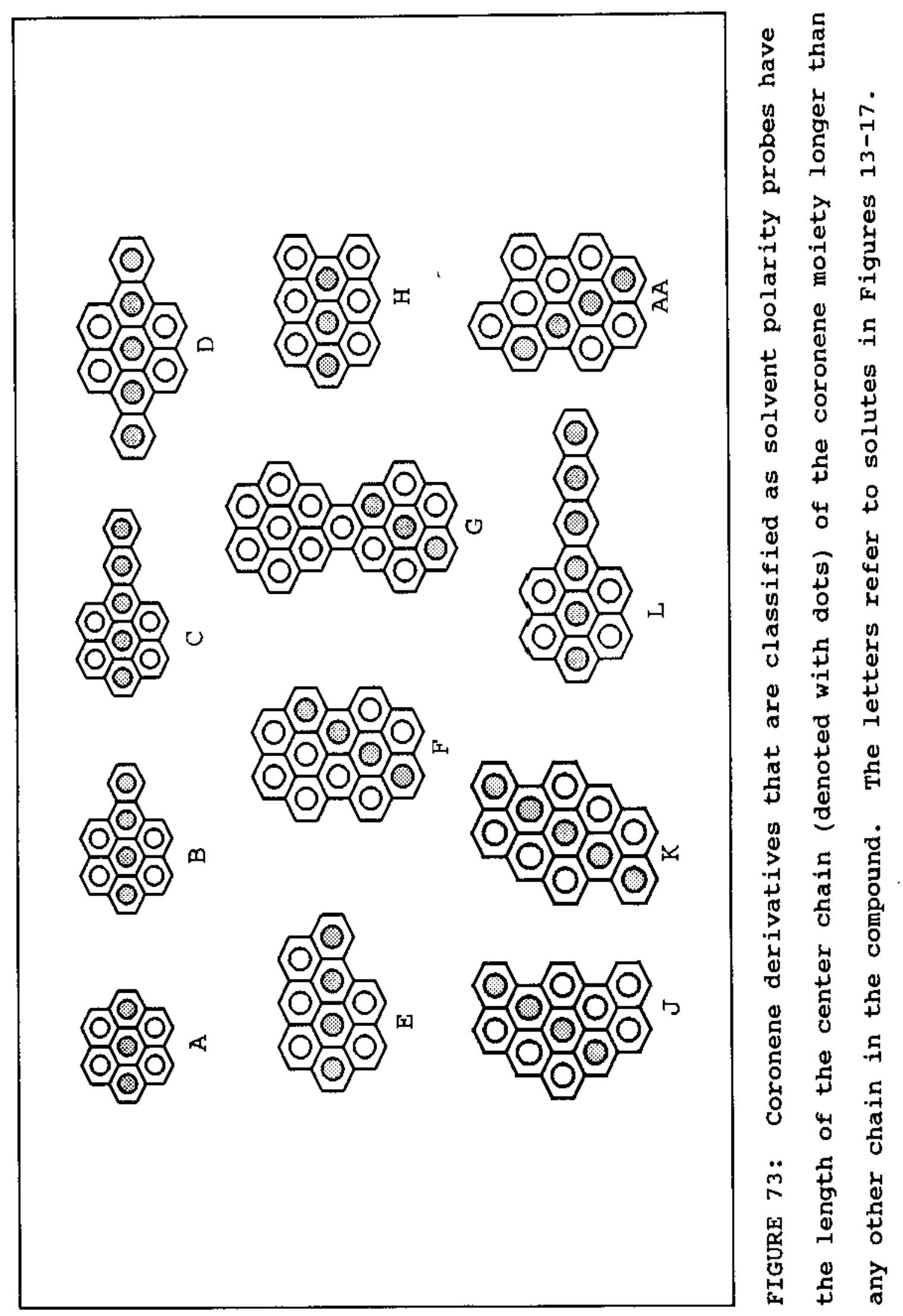
spectral band in several solvents keeps it from being listed as a fluorescent probe.

Two nine-ring PAH6 benzenoids, dibenzo[a,j]coronene and dibenzo[bc, ef]coronene, behave quite differently, depending upon where the substituent aromatic rings are placed. In dibenzo[a,j]coronene the two added benzo-groups extended the center row to five benzene rings; whereas, in dibenzo[bc, ef]coronene a benzo-group was located on both the top and bottom row. Only dibenzo[a,j]coronene showed selective enhancement of the $I$ band emission intensity in polar solvents. Both of the naphthocoronene isomers studied show probe behavior, and by careful examination of their molecular structures (see Figure 13 or 73 ) one notes that either all (naphtho[2,3a]coronene) or part (naphtho[8,1,2$a b c]$ coronene) of the added naphtho-group is used to extend the center row. The molecule's length-to-width ratio does provide a clue to fluorescence behavior in the coronene family only. Unfortunately, the length-to-width ratio method does not extended to other families, such as the pyrene and perylene families.

The underlying concept of arranging PAHs according to molecular formula initially showed promise. Considering the three $\mathrm{C}_{20} \mathrm{H}_{12}$ isomers, one notes that only benzo[e]pyrene exhibited probe character. Interesting, benzo[e]pyrene (SC= 11) has two more Kekulé resonance structures than either perylene or benzo[a]pyrene, which each have Kekulé structure 
counts of nine (SC $=9$ ) (17). The higher number of Kekule structure counts for probe molecules also is true for the $\mathrm{C}_{30} \mathrm{H}_{14}$ and $\mathrm{C}_{22} \mathrm{H}_{12}$ molecular family.

Unfortunately, as the data base became larger and larger the number of exceptions also grew. The molecular families that do not obey this rule are numerous, like $\mathrm{C}_{28} \mathrm{H}_{16}, \mathrm{C}_{32} \mathrm{H}_{16}, \mathrm{C}_{36} \mathrm{H}_{16}, \mathrm{C}_{24} \mathrm{H}_{14}, \mathrm{C}_{34} \mathrm{H}_{16}$, and $\mathrm{C}_{28} \mathrm{H}_{16}$. With the exclusion of the $\mathrm{C}_{28} \mathrm{H}_{16}$ and $\mathrm{C}_{36} \mathrm{H}_{16}$, the number of structure counts between probes and nonprobes among the exceptions are very close in number. Normally, they only differ by one resonance structure. For example, in the molecular formula family $\mathrm{C}_{24} \mathrm{H}_{14}$, dibenzo[def,p]chrysene is a probe with 16 resonance structures, and dibenzo[a,e]pyrene is not a probe with 17 structure counts. It is the large number of compounds in the $\mathrm{C}_{28} \mathrm{H}_{16}$ molecular family that put this rule to rest. This particular molecular family consists of six members - two of which are probes and four of which are nonprobes. The two probes, dibenzo[h,rst]pentaphene and benzo[VwX]hexaphene, have $\mathrm{SCs}=27$ and 19 , respectively. The four nonprobes benzo[a] naphtho[ $8,1,2$ cde] naphthacene, dibenzo[fg,ij]pentaphene, dibenzo[de, st]pentacene, and dibenzo[fg, $q r]$ pentacene have $\operatorname{SCs}=23,25,21$, and 25, respectively.

After several years of continued studies, I am still unable to predict from structural considerations which PACs will exhibit solvent polarity probe character. Preliminary 
observations concerning what structural features and spectral features are common in known PAC solute probes can only suggest molecules for future investigations. At the present time, the only definitive method for determining whether a given PAC behaves as a solvent polarity probe is to actually measure its fluorescence properties in various organic solvents.

Mathematical Functions Versus Probe Character Correlations

From a more quantitative standpoint, solvatochromic wavelength shifts of PACs, like 9,9'-bianthracene, 9,9'bianthracene-10-carboxaldehyde, 1-aminopyrene, 1-pyrenecarboxaldehyde, and 1,3-pyrenedicarboxaldehyde can be explained in terms of solvational differences between the excited and ground state fluorophore. These solvational energies are governed to a large extent by general solvent effects resulting form dipole-dipole, dipole-induced dipole, and induced dipole-induced dipole interactions, and by the more specific solvent effects such as hydrogen-bonding and/or charge transfer type interactions, which lead to "molecular complexes" having fixed stoichiometry and geometry. Theoretical interpretation of solute-solvent interactions at the molecular level is very difficult, often subject to interpretation, and as one may surmise there have been numerous competing mathematical expressions published 
in the spectroscopic literature to explain observed wavelength shifts associated with known solute probes.

To a first approximation specific effects are ignored. The energy difference (in $\mathrm{cm}^{-1}$ ) between the solvated ground and excited state fluorophore can be described by the Lippert equation $(18-21)$ :

$$
\begin{gathered}
\left(1 / \lambda_{\mathrm{ex}}\right)-\left(1 / \lambda_{\mathrm{em}}\right) \approx(2 / \mathrm{hc})[(\epsilon-1) /(2 \epsilon+1)- \\
\left.\left(\mathrm{n}^{2}-1\right) /\left(2 \mathrm{n}^{2}+1\right)\right]\left[\left(\mu^{*}-\mu\right)^{2} / \mathrm{a}^{3}\right]+\text { constant } \\
\left(1 / \lambda_{\mathrm{ex}}\right)-\left(1 / \lambda_{\mathrm{em}}\right) \approx \mathrm{k}[(\epsilon-1) /(2 \epsilon+1)- \\
\left.\left(\mathrm{n}^{2}-1\right) /\left(2 \mathrm{n}^{2}+1\right)\right]+ \text { constant }
\end{gathered}
$$

where $\epsilon$ and $\mathrm{n}$ refer to the dielectric constant and refractive index of the solvent, respectively, $c$ is the speed of light, $h$ denoted Plank's constant, and $a$ is the radius of the solvent cavity wherein the fluorophore resides. The latter three quantities, along with the dipole moments of the fluorophore's excited $\left(\mu^{*}\right)$ and ground state $(\mu)$, are assumed solvent independent (i.e., no major change in electronic transition and no major geometrical reorganization within the excited state) and are thus combined into the proportionality constant, $k$. The first bracketed quantity in equation 4.2 , the $[(\epsilon-1) /(2 \epsilon+1)]$ term, accounts for the Stoke's spectral shift caused by both reorientation of solvent dipoles and redistribution of electrons in the solvent molecules, which is describes separately by the $\left[\left(n^{2}-1\right) /\left(2 n^{2}+1\right)\right]$ ratio. According to the Lippert equation, solvent dipole reorganization is 
primarily responsible to observed stoke's spectral shifts. Electron redistribution occurs almost instanteously, with both ground and excited states being equally stabilized by the process.

Modified forms of the Lippert equation $(1,22,23)$,

$$
\begin{gathered}
\left(1 / \lambda_{e x}\right)-\left(1 / \lambda_{e m}\right) \approx k[(\epsilon-1) /(2 \epsilon+1)- \\
\left.B\left(n^{2}-1\right) /\left(2 n^{2}+1\right)\right]+ \text { constant }
\end{gathered}
$$

as well as other semitheoretical and/or strictly empirical functions of $\epsilon$ and $\mathrm{n}$ such as (24-32),

$$
\begin{gathered}
\left(1 / \lambda_{\mathrm{ex}}\right)-\left(1 / \lambda_{\mathrm{em}}\right) \approx \mathrm{k}\left[(\epsilon-1) /(2 \epsilon+1)-\left(\mathrm{n}^{2}-1\right)\right. \\
\left./\left(2 \mathrm{n}^{2}+2\right)\right] /\left\{\left[1-\beta\left(\mathrm{n}^{2}-1\right) /\left(2 \mathrm{n}^{2}+2\right)\right]^{2}\right. \\
[1-\beta(\epsilon-1) /(2 \epsilon+2)]\}+ \text { constant } \\
1 / \lambda_{\mathrm{abs}} \approx \mathrm{k}[(\epsilon-1) / \epsilon]\left[\left(\mathrm{n}^{2}-1\right) / \mathrm{n}^{2}\right]+\text { constant } \\
1 / \lambda_{\mathrm{abs}} \approx \mathrm{k}\left[\left(\mathrm{n}^{2}-1\right) /\left(\mathrm{n}^{2}+2\right)\right]+\text { constant }
\end{gathered}
$$

have been used successfully to describe spectral properties of various aromatic molecules. For example, Kalyanasundaram and Thomas (33) observed a direct linear relationship between $\lambda_{\text {em }}$ and solvent dielectric constant for 1 -pyrenecarboxaldehyde dissolved in several neat alcohols and in binary aqueous-methanol, aqueous-ethanol, and aqueousdioxane solvent mixtures. Mazumdar et al. (24) reported that if hydroxylic solvents and chloroform were excluded from the correlations, then Bilot-Kawski Equation (equation 4.4 - with $B$ equal to unity) was superior to the Lippert equation for mathematically describing the stoke's shifts of 
o-phenylenediamine, $m$-phenylenediamine, $p$-phenylenediamine, 2,3-diaminonaphthalene, and 2,7-diaminofluorene.

To provide greater insight into the spectrofluorometric properties of 9,9'-bianthracene, 9,9'-bianthracene-10carboxaldehyde, 1-aminopyrene, 1-pyrenecarboxaldehyde, and 1,3-pyrenedicarboxaldehyde possible correlations between experimental Stoke's shifts and the various combined $\epsilon-n$ functions discussed above were checked. Representative Figures 74-77 graphically depict stoke's shifts (in $\mathrm{cm}^{-1}$ ) versus Lippert $\Delta f$ function (equation 4.2 ) and Stoke's shifts versus Bilot-Kawski $F(\epsilon, n)$ (equation 4.4 ) for 9,9bianthracene and 9,9-bianthracene-10-carboxaldehyde, respectively (see Tables XLI for experimental excitation and emission wavelengths). Forty-two of the forty-five solvents studied are included in the plots and subsequent statistical analysis. Methyl tert-butyl ether, dimethyl adipate, and dibutyl oxalate had to be excluded from Table LII because refractive indices and static dielectric constants for these three solvents could not be found in the chemical literature.

In the case of aminopyrene (APy), 1-pyrenecarboxaldehyde (PYCA), and 1,3-pyrenecarboxaldehyde (PYDCA) (graphs not shown) linear least squares regressional analysis of stoke's shifts (in $\mathrm{cm}^{-1}$ ) versus Lippert $\Delta f$ function (equation 4.2) and stoke's shift versus BilotKawski $F(\epsilon, n)$ function (equation 4.4 ) showed that the 


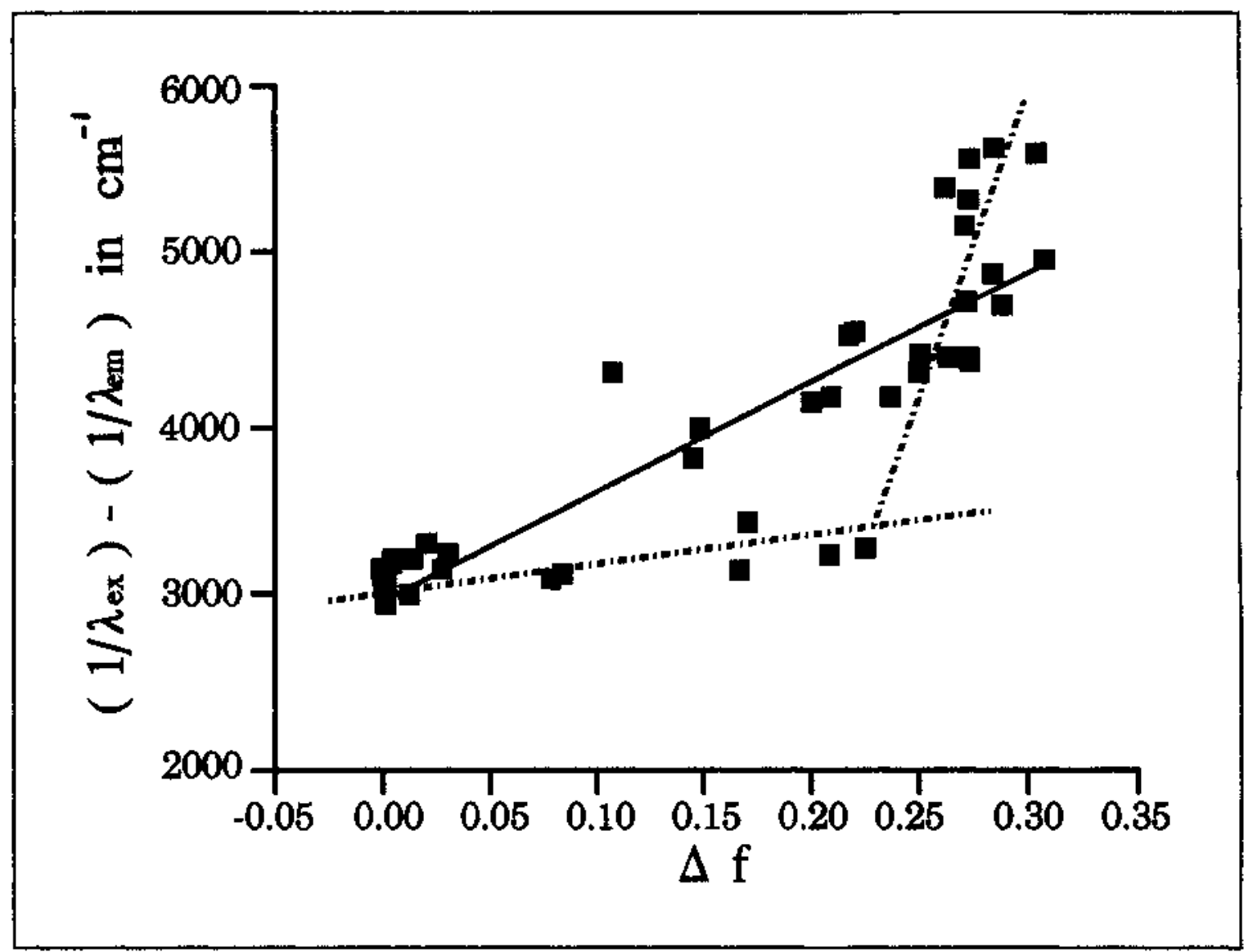

FIGURE 74: Graphical plot of stoke's shift versus Lippert $\Delta$ function for 9,9'-bianthracene dissolved in 42 nonelectrolyte solvents listed in Table LII. The Lippert function is mathematically defined as $\Delta f=(\epsilon-1) /(2 \epsilon+1)$ $-\left(n^{2}-1\right) /\left(2 n^{2}+1\right)$. The solid straight line was determined via linear least squares analysis using all 42 data points for which $\mathrm{n}$ and $\epsilon$ values exist. Dashed lines represent a plausible separation of solvents according to fluorophore excited state, either the anthracene-like LE (nearly horizontal) or the TICT excited state (nearly vertical). 


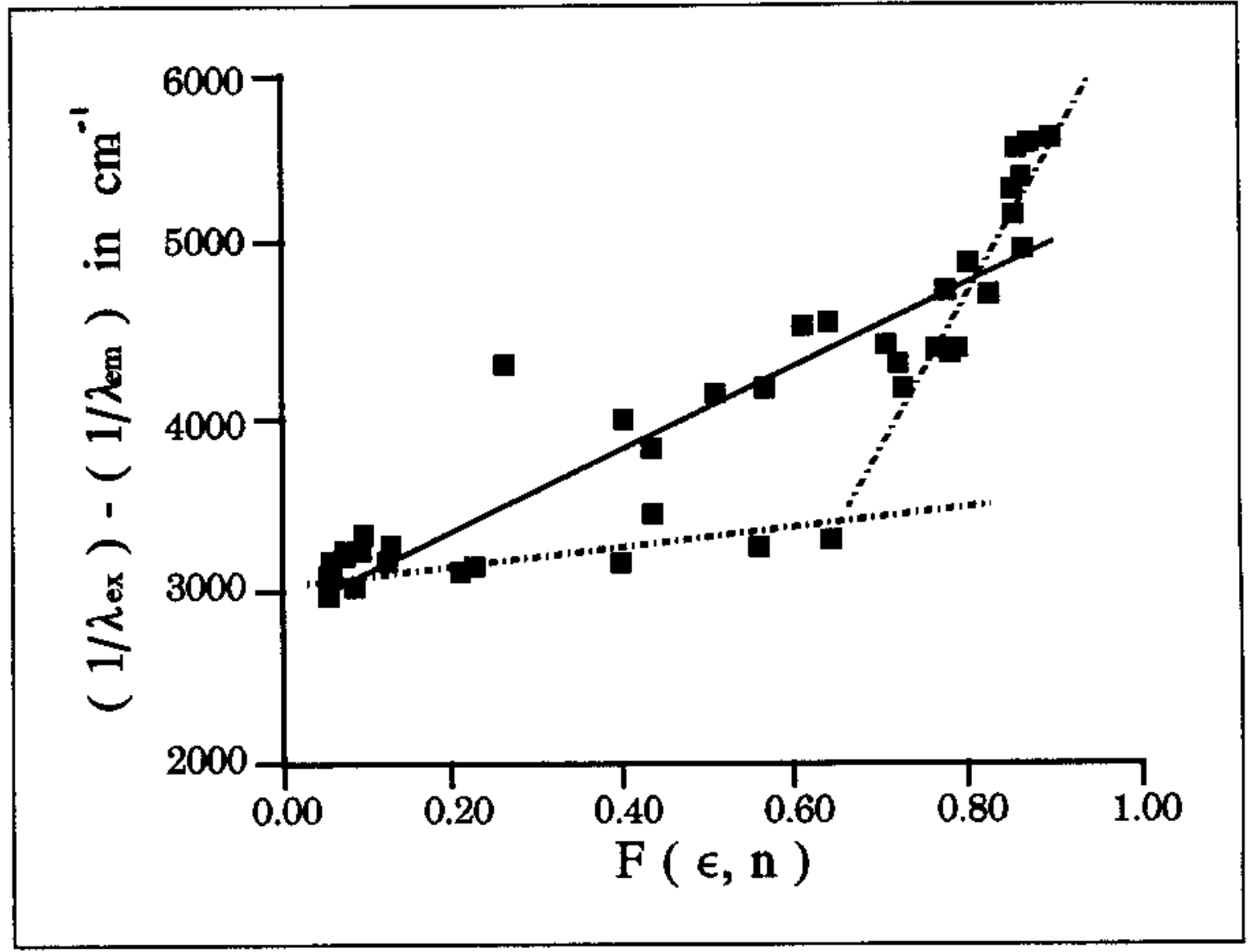

FIGURE 75: Graphical plot of stoke's shift versus BiotKolwski $F(\epsilon, n)$ parameter for 9,9'-bianthracene dissolved in 42 nonelectrolyte solvents listed in Table LII. The BiotKolwski parameter is mathematically defined as $F(\epsilon, n)=[(\epsilon$ $\left.-1) /(2 \epsilon+1)-\left(n^{2}-1\right) /\left(2 n^{2}+2\right)\right] /\left[\left[1-\beta\left(n^{2}-1\right) /\left(2 n^{2}+\right.\right.\right.$ 2) $\left.]^{2}[1-\beta(\epsilon-1) /(2 \epsilon+2)]\right\}$ with $B=1$. The solid straight line was determined via linear least squares analysis using all 42 data points for which $\mathrm{n}$ and $\epsilon$ values exist. Dashed lines represent a plausible separation of solvents according to fluorophore excited state, either the anthracene-like LE (nearly horizontal) or the TICT excited state (nearly vertical). 


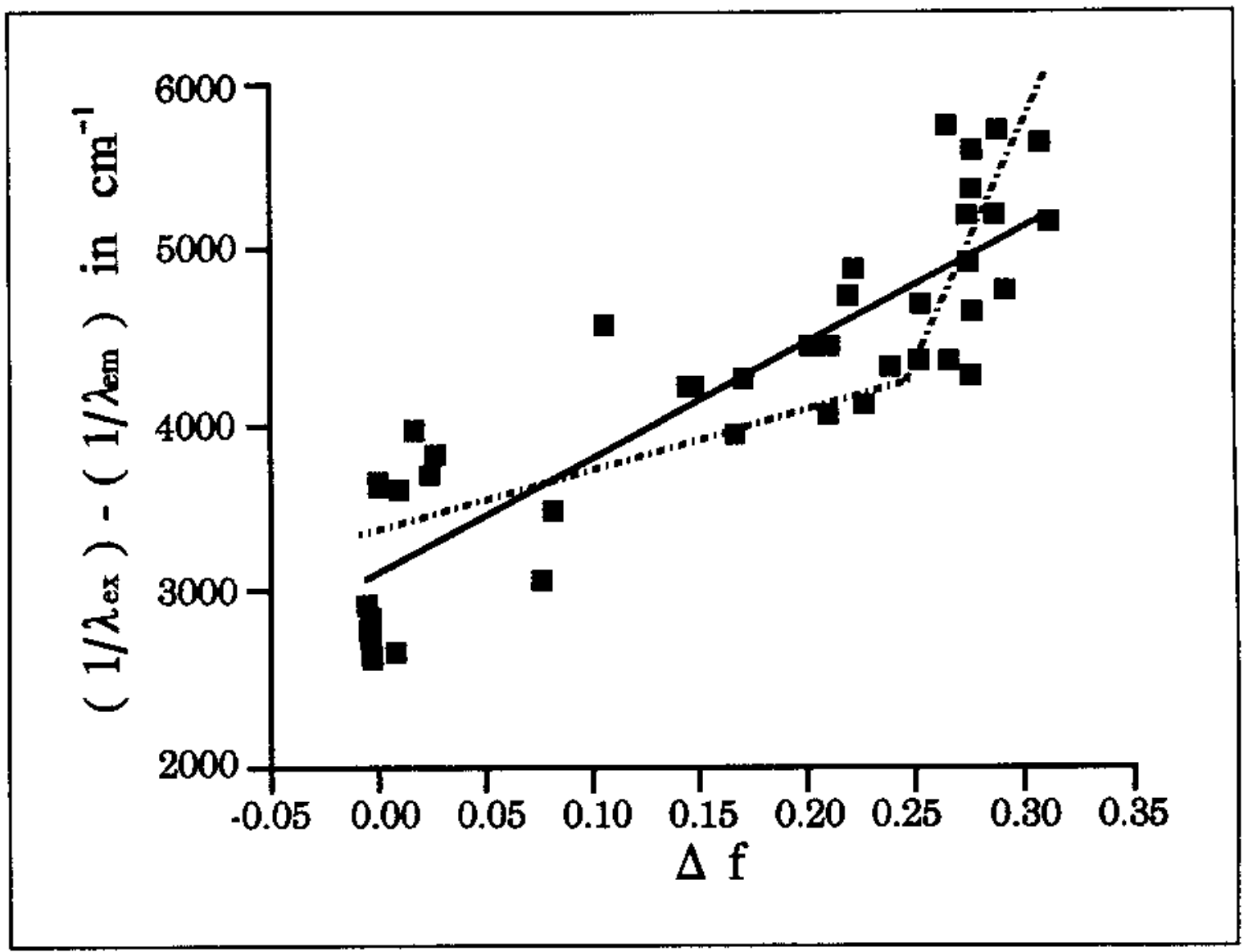

FIGURE 76: Graphical plot of Stoke's shift versus Lippert $\Delta$ function for 9,9'-bianthracene-10-carboxaldehyde dissolved in 42 nonelectrolyte solvents listed in Table LII. The Lippert function is mathematically defined as $\Delta f=(\epsilon-$ 1) $/(2 \epsilon+1)-\left(n^{2}-1\right) /\left(2 n^{2}+1\right)$. The solid straight line was determined via linear least squares analysis using all 42 data points for which $\mathrm{n}$ and $\epsilon$ values exist. Dashed lines represent a plausible separation of solvents according to fluorophore excited state, either the anthracene-like LE (nearly horizontal) or the TICT excited state (nearly vertical). 


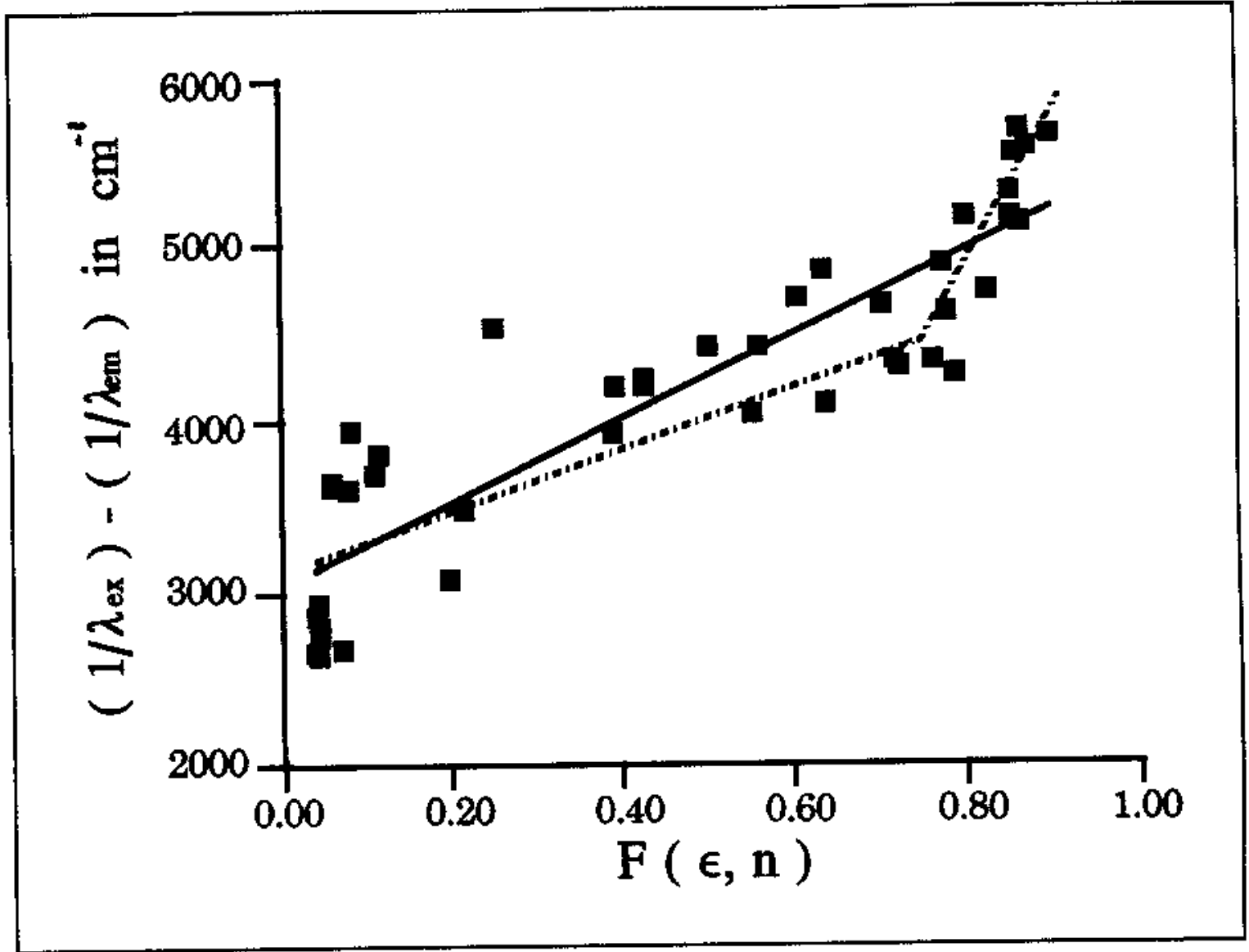

FIGURE 77: Graphical plot of Stoke's shift versus BiotKolwski $F(\epsilon, n)$ parameter for 9,9'-bianthracene-10carboxaldehyde dissolved in 42 nonelectrolyte solvents listed in Table LII. The Biot-Kolwski parameter is mathematically defined as $F(\epsilon, n)=\left[(\epsilon-1) /(2 \epsilon+1)-\left(n^{2}-\right.\right.$ 1) $\left./\left(2 n^{2}+2\right)\right] /\left\{\left[1-B\left(n^{2}-1\right) /\left(2 n^{2}+2\right)\right]^{2}[1-B(\epsilon-1) /(2 \epsilon\right.$ +2 ) ] with $B=1$. The solid straight line was determined via linear least squares analysis using all 42 data points for which $\mathrm{n}$ and $\epsilon$ values exist. Dashed lines represent a plausible separation of solvents according to fluorophore excited state, either the anthracene-like LE (nearly horizontal) or the TICT excited state (nearly vertical). 
TABLE LII. Refractive indices and dielectric constants for various organic solvents.

\begin{tabular}{|c|c|c|}
\hline organic solvent & $n^{a}$ & $\epsilon^{\mathrm{b}}$ \\
\hline$n$-Hexane & 1.3749 & 1.890 \\
\hline$n$-Heptane & 1.3876 & 1.92 \\
\hline Cyclohexane & 1.4262 & 2.023 \\
\hline Methylcyclohexane & 1.4231 & 2.02 \\
\hline $2,2,4$-Trimethylpentane & 1.3915 & 1.94 \\
\hline$n$-Hexadecane & 1.4345 & 2.04 \\
\hline Dibutyl Amine & 1.4177 & 2.978 \\
\hline Carbon Tetrachloride & 1.4602 & 2.238 \\
\hline Dibutyl Ether & 1.3992 & 2.8 \\
\hline 1-octanol & 1.4295 & 10.34 \\
\hline$p$-Xylene & 1.4958 & 2.270 \\
\hline o-xylene & 1.5055 & 2.568 \\
\hline$m$-Xylene & 1.4972 & 2.374 \\
\hline 1-Pentanol & 1.4100 & 14.27 \\
\hline Ethyl Ether & 1.3524 & 4.335 \\
\hline Toluene & 1.4968 & 2.568 \\
\hline Benzene & 1.5011 & 2.284 \\
\hline 1-Butanol & 1.3993 & 17.8 \\
\hline 1-Chlorobutane & 1.4021 & 7.39 \\
\hline Chlorobenzene & 1.5241 & 5.708 \\
\hline 1-Propanol & 1.3856 & 20.33 \\
\hline
\end{tabular}


TABLE LII. Continued.

\begin{tabular}{|c|c|c|}
\hline Organic solvent & $\mathrm{n}^{\mathrm{a}}$ & $\epsilon^{\mathrm{b}}$ \\
\hline Cyclohexanol & 1.4641 & 15.77 \\
\hline 2-Propanol & 1.3772 & 18.97 \\
\hline Ethanol & 1.3614 & 25.41 \\
\hline Chloroform & 1.4459 & 4.806 \\
\hline Butyl Acetate & 1.3942 & 5.01 \\
\hline Methanol & 1.3284 & 33.62 \\
\hline Tetrahydrofuran & 1.4071 & 7.61 \\
\hline Dichloromethane & 1.4242 & 9.08 \\
\hline Ethyl Acetate & 1.3723 & 6.095 \\
\hline 1,2-Dichloroethane & 1.4448 & 10.4 \\
\hline Dimethyl Carbonate & 1.3682 & 3.084 \\
\hline 4-Methyl-2-pentanone & 1.3957 & 13.11 \\
\hline 1,4-Dioxane & 1.4224 & 2.2 \\
\hline 2-Butanone & 1.3788 & 18.51 \\
\hline Ethylene Glycol & 1.4318 & 38.66 \\
\hline Acetone & 1.3587 & 21.19 \\
\hline Acetonitrile & 1.3441 & 37.5 \\
\hline$N, N$-Dimethylacetamide & 1.4380 & 37.8 \\
\hline$N, N$-Dimethylformamide & 1.4305 & 36.7 \\
\hline Propylene Carbonate & 1.4210 & 64.80 \\
\hline Dimethyl sulfoxide & 1.4783 & 46.7 \\
\hline
\end{tabular}

a Experimental values taken from the literature at $20^{\circ} \mathrm{C}(34-$ 
TABLE LII. Continued.

37). b Experimental values taken from the literature at $20^{\circ} \mathrm{C}(36-53)$.

observed values

APY

$$
\begin{array}{ll}
\left(1 / \lambda_{\text {ex }}\right)-\left(1 / \lambda_{\text {em }}\right)=\Delta v=3040.5+4295.5 \Delta f & \left(r^{2}=0.54\right) \\
\left(1 / \lambda_{\text {ex }}\right)-\left(1 / \lambda_{\text {em }}\right)=\Delta v=2995.6+1552.4 F(\epsilon, n) & \left(r^{2}=0.55\right)
\end{array}
$$

PyCA

$$
\begin{array}{ll}
\left(1 / \lambda_{\text {ex }}\right)-\left(1 / \lambda_{\text {em }}\right)=\Delta v=2700.1+6186.7 \Delta f & \left(r^{2}=0.48\right) \\
\left(1 / \lambda_{\text {ex }}\right)-\left(1 / \lambda_{\text {em }}\right)=\Delta v=2628.5+2216.1 \mathrm{~F}(\epsilon, n) & \left(\mathrm{r}^{2}=0.47\right)
\end{array}
$$

PYDCA

$$
\begin{array}{ll}
\left(1 / \lambda_{\text {ex }}\right)-\left(1 / \lambda_{\text {em }}\right)=\Delta v=1386.8+2803.9 \Delta f & \left(x^{2}=0.44\right) \\
\left(1 / \lambda_{\text {ex }}\right)-\left(1 / \lambda_{\text {em }}\right)=\Delta v=1341.6+1029.3 \mathrm{~F}(\epsilon, \mathrm{n}) & \left(r^{2}=0.45\right)
\end{array}
$$

are not correlated. There is considerable scatter in the data points about each of the so-called "best straight lines", which were determined via least squares analysis. This result is not surprising given the fact that it is well documented that both APY and PYCA and presumably PyDCA have two chromophores of similar energy resulting from the excited $\pi \pi^{*}$ (in polar solvents) and $n \pi^{*}$ (in nonpolar solvents) states (54). Such nonlinear behavior is typical of fluorophores having two (or more) excited states in that each state would have a different dipole moment. The multiplicative $\left[\left(\mu^{*}-\mu\right)^{2} / \mathrm{a}^{3}\right]$ factor in equation 4.1 would 
be constant for only a single excited state, not for both the excited states.

In the case of 9,9-bianthracene (BA) and 9,9-bianthracene-10-carboxaldehyde (BACA), linear least squares regressional analysis showed that the observed values are BA

$$
\begin{array}{lll}
\left(1 / \lambda_{\mathrm{ex}}\right)-\left(1 / \lambda_{\mathrm{em}}\right)=\Delta v=2993.4+6322.7 \Delta \mathrm{f} & \left(\mathrm{r}^{2}=0.73\right) \\
\left(1 / \lambda_{\mathrm{ex}}\right)-\left(1 / \lambda_{\mathrm{em}}\right)=\Delta v=2873.6+2381.2 \mathrm{~F}(\epsilon, \mathrm{n}) & \left(\mathrm{r}^{2}=0.79\right)
\end{array}
$$

BACA

$$
\begin{array}{ll}
\left(1 / \lambda_{\text {ex }}\right)-\left(1 / \lambda_{\text {em }}\right)=\Delta v=3116.1+6791.1 \Delta f & \left(r^{2}=0.77\right) \\
\left(1 / \lambda_{\text {ex }}\right)-\left(1 / \lambda_{\text {em }}\right)=\Delta v=3017.8+2493.4 F(\epsilon, n) & \left(r^{2}=0.80\right)
\end{array}
$$

only marginally correlated at best. There is considerable scatter in the data points about each of the so-called "best straight lines", which were determined via least squares analysis. One can argue that such nonlinear behavior is typical of fluorophores having two (or more) excited states (ie., an anthracene-like locally excited, LE, state and a twisted intramolecular charge transfer, TICT, excited state) in that each state would have a different dipole moment. The multiplicative $\left[\left(\mu^{*}-\mu\right) / a^{3}\right]^{2}$ factor in equation 4.1 would be constant for only a single excited state, not for both the LE and TICT excited states of 9,9'-bianthracene parent.

It should be theoretically possible though, to separate into two groups based upon whether the LE of TICT excited state is observed. Examining the 9,9'-bianthracene data, 
the dashed lines in Figures 74 and 75 show one plausible separation scheme. Least squares analysis gives for each of the separate lines the following mathematical equations

$$
\begin{aligned}
& \left(1 / \lambda_{\text {ex }}\right)-\left(1 / \lambda_{\text {em }}\right)=\Delta v=3136.5+722.5 \Delta \mathrm{f} \\
& \left(1 / \lambda_{\text {ex }}\right)-\left(1 / \lambda_{\text {em }}\right)=\Delta v=-857.0+2077.1 \Delta \mathrm{f}
\end{aligned}
$$

for regressional analysis according to the Lippert Equation. Data points for 1,2-dichloroethane, dichloromethane, chlorobenzene, chloroform, cyclohexanol, tetrahydrofuran, dimethyl carbonate, and ethyl acetate do not visually appear to belong to either dashed line, and these eight solvents were excluded from the statistical treatment. Kabouchi et. al. (22) presented a similar two-line graph; however, the authors only studied five solvents.

It should be noted that separation of solvents by fluorophore excited state is very difficult, and in many instances subject to interpretation. The experimental maximum intensity emission wavelength, $\lambda_{\text {em, }}$ does not always correspond to $\lambda_{\mathrm{em}}^{\mathrm{LE}}$ or $\lambda_{\mathrm{em}}^{\mathrm{TICT}}$. This may be the case for several of the solvents in the present fluorescence study. Alternately, 9,9'-bianthracene may have several excited state dipole moments resulting for different partial charges and/or rotational geometries necessitated by solvent molecular shape or packing considerations, or there even may be chemical artifacts present in several of the solvents which serve to distort the emission spectrum. Visser and coworkers (55) proposed eight years ago that the short- 
wavelength fluorescence observed in 9,9'-bianthracene's emission spectrum for apolar solvents is not caused by photon emission from a state with excitation localized on one of the two anthracene rings, but rather results from a polar charge transfer state. The authors further postulated that fluorescence in alkane solvent arises from two different excited states in which the molecular conformation of 9,9'-bianthracene is not the same; the ratio of interconversion between conformations is faster than $10^{10}$ $\mathrm{sec}^{-1}$. The long-wavelength fluorescence observed in solutions of polar and select apolar solvents was rationalized in terms of 9,9'-bianthracene-solvent exciplexes, which can then dissociate into ions if the dielectric constant is large enough. The nonlinearity noted in Figures 74 and 75 is also consistent with ideas put worth by Visser and coworkers. Unfortunately, these steady-state measurements are unable to answer the question concerning why 9,9'-bianthracene compounds exhibit dual fluorescence. Interestingly, half of the eight solvents that were excluded from the dashed line least squares treatment contained at least one chlorine atom. As mentioned previously, abnormal fluorescence behavior of PAH and PANH, polycyclic aromatic nitrogen heterocycles, solute probes in select chloroalkane solvents are rationalized in terms of photochemical reactions, and carbon tetrachloride, and to a much lesser extent chloroform and dichloromethane, are known 
to undergo irreversible photochemical reactions with several PAH solutes. To determine if an undesired photochemical reaction was responsible for several chlorinated solvents being off the dashed lines, solutions of 9,9'-bianthracene dissolved in carbon tetrachloride and chloroform were irradiated at 300 and $360 \mathrm{~nm}$ ( $\lambda_{\text {ex }}$ for the bi-PAH solute) for a prolonged period of time. Fluorescence enission spectra were periodically recorded and any differences from the original spectrum (at time zero) were noted. As shown in Figure 78 , there is a rather dramatic reduction in the measured emission signal for 9,9'-bianthracene dissolved in carbon tetrachloride with increasing irradiation time. After sixty-five minutes of irradiation at $360 \mathrm{~nm}$ (and 300 nm, not shown) the emission signal was approximately onesixth of its original value, and the maximum intensity emission wavelength had redshifted from $418 \mathrm{~nm}$ to $435 \mathrm{~nm}$, with a fairly broad shoulder beginning to appear at $454 \mathrm{~nm}$. Fifty-five minutes of additional irradiation decreased the $418 \mathrm{~nm}$ emission intensity by another 50\%, while the $454 \mathrm{~nm}$ peak grew slightly. The emission spectrum remained constant for the third hour of irradiation. In the case of the chloroform solvent, there were no visual changes noted in 9,9'-bianthracene's emission spectrum, even after two hours continued irradiation at both 300 and $360 \mathrm{~nm}$. Based upon the fact that it took about five minutes (or less) to record the emission spectrum, it is concluded that while 


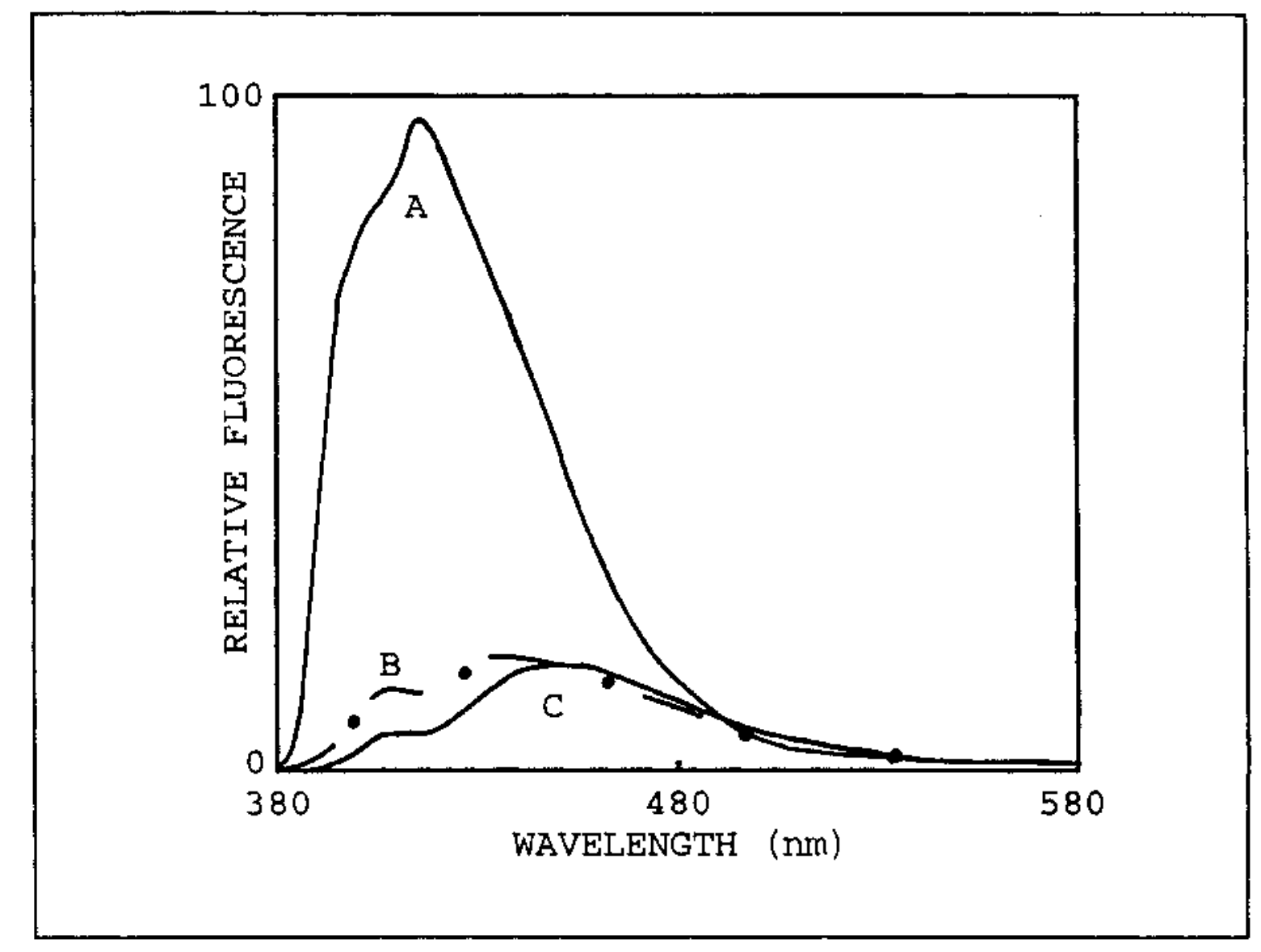

FIGURE 78: Fluorescence emission spectra for 9,9'-bianthracene dissolved in carbon tetrachloride as a function of irradiation time. Curves $A, B$, and $C$ correspond to spectra recorded at 0,65 , and 120 minutes of irradiation time, respectively. Results shown were for irradiation at $360 \mathrm{~nm}$. Similar spectra were obtained 9,9'-bianthracene-carbon tetrachloride solutions irradiated at $300 \mathrm{~nm}$. 
photochemical reactions may occur, they are much too slow to be responsible for the chlorinated solvents deviating from the Lippert and Bilot-Kawski relationship.

Correlations between calculated intensity ratios of select emission bands and combined $\epsilon-n$ functions have been tried. Chen and Frank (56) employed spectrofluorometric probe techniques to study the effects of incorporating "guest molecules" into organized organic self-assembled monolayers formed by spontaneous adsorption from solution. As part of the experimental methodology, the authors first documented that the observed $I_{1} / I_{3}$ intensity ratio for bands $I$ and III in the fluorescence emission spectrum of pyrene and 1-pyrenebutanoic acid dissolved in $n$-hexane, diethyl ether, toluene, ethanol, chloroform, methylene chloride, ethylene glycol, acetone, and water varied systematically with solvent polarity. Measured $I_{1} / I_{3}$ values were found to correlate well linearly with the Lippert function,

$$
\begin{gathered}
I_{1} / I_{3}=(\epsilon-1) /(2 \epsilon+1)-\left(n^{2}-1\right) /\left(2 n^{2}+1\right) \\
+ \text { constant }
\end{gathered}
$$

such intensity correlations, however lack theoretical merit and therefore were not pursued in this work. Moreover, a more extensive set of intensity measurements (57) involving 79 nonelectrolyte solvents for pyrene (not shown) and forty solvents for 1-pyrenebutanoic acid has been completed (see Table XXXII), and the experimental results show only a marginal correlation (at best), with squared correlation 
coefficients of $r^{2}=0.5729$ (pyrene) and $r^{2}=0.6919(1-$ pyrenebutanoic acid), contrary to earlier contention made by Chen and Frank.

Recommendations Concerning Solvent Polarity Probes

Examination of the entire spectral data file including 205 PACs reveals that about 40 are solvent polarity probes. Six solvent polarity probes are recommended for general use in determining solvent polarity - pyrene (Py), benzo[e]pyrene (BPy), benzo[ghi]perylene (BPe), 3,4-dihydrobenzo[ghi]perylene (DHBPe), coronene (Co), and ovalene (OV). All six are from the PAH subclass because they do not suffer from as many artifacts as those probes found in other subclasses. For example, the PANHs have abnormally low values in alcoholic solvents due to protonation effects. Almost all of the probes in the other subclasses have much smaller dynamic ranges than those recommended. While 1methylcoronene and 1,2-dimethylcoronene are excellent solvent polarity probes, the lack of commercial availability prohibits them from being recommended for as general use solvent polarity probes.

Examination of Tables XXV and XXVI and Figures 79 and 80 reveals that for the most part the rank ordering of solvents by polarity is identical for the six PAH probes. Linear least squares analysis of the intensity ratio data 


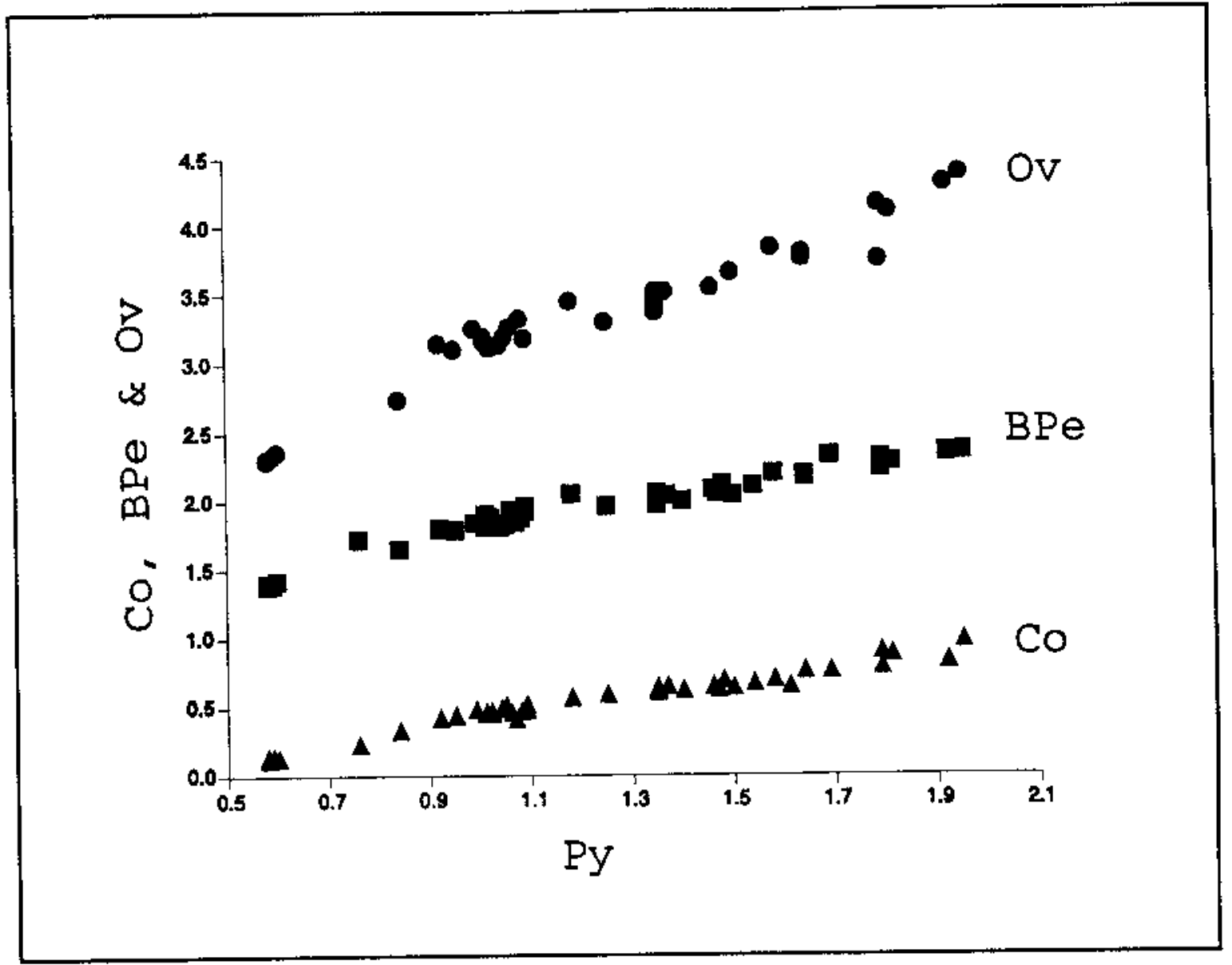

FIGURE 79: Correlations between different PAH solvent polarity probes and pyrene (Py). For graphical purposes, coronene (Co), benzo[ghi]perylene (BPe), and ovalene (Ov) were offset by 0,1 , and 2 units, respectively. 


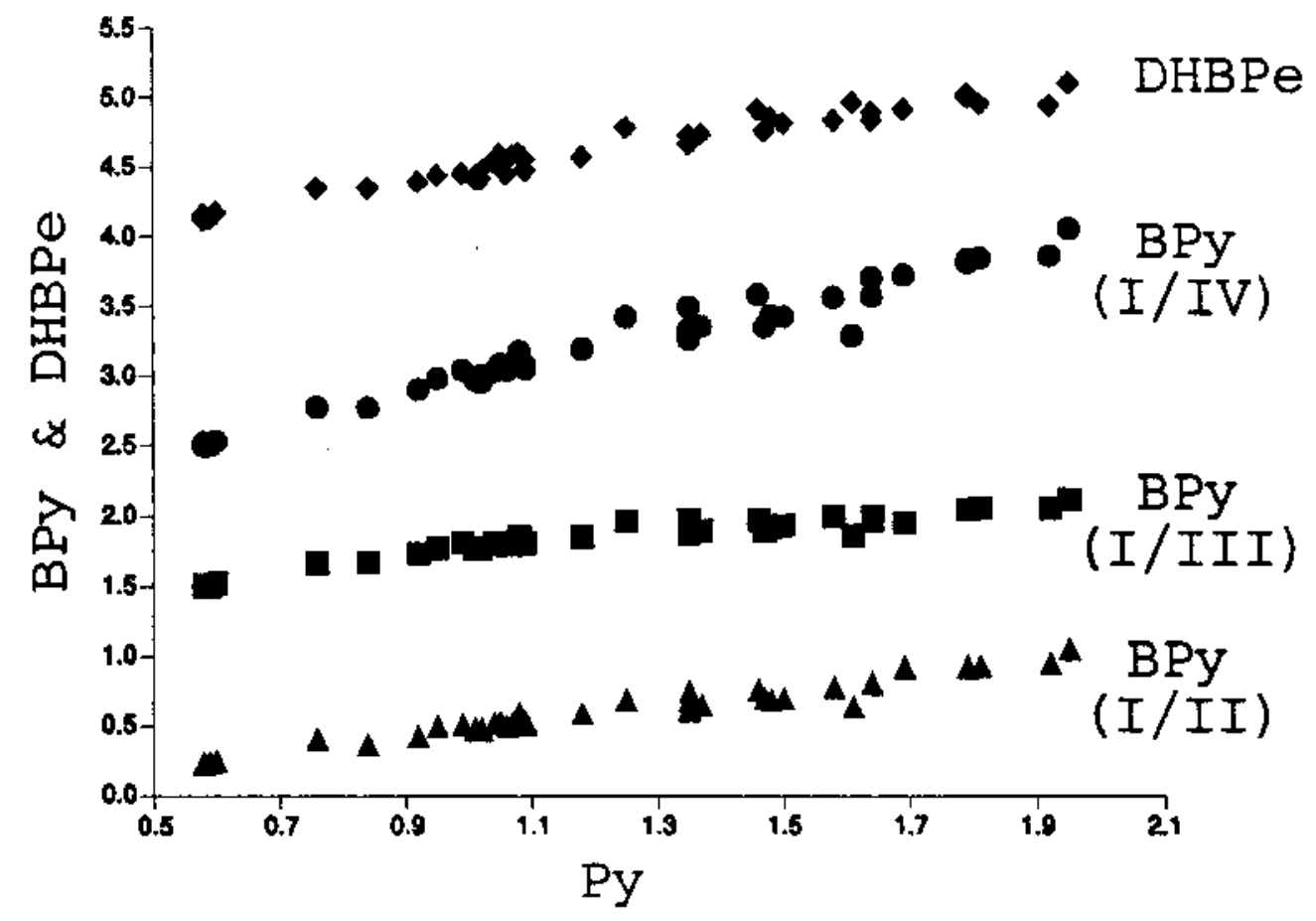

FIGURE 80: Correlations between different PAH solvent polarity probes and pyrene (Py). For graphical purposes, benzo[e]pyrene (BPy) (I/II), BPY (I/III), BPY (I/IV), and 3,4-dihydrobenzo[ghi]perylene (DHBPe) were offset by 0,1 , 2 , and 3 units, respectively. The benzo[e]pyrene bands are I at 375-378 nm, II at 385-388 nm, III at 394-398 nm and IV at 406-410 $\mathrm{nm}$. 
shows that the polarity scales are highly correlated and gives the following mathematical expressions (and squared correlation coefficients)

$$
\begin{array}{ll}
\text { BPy }(I / I I)=0.538 \text { Py }-0.059 & \left(r^{2}=0.959\right) \\
\text { BPy }(I / I I I)=0.399 \text { Py }+0.344 & \left(r^{2}=0.908\right) \\
\text { BPY (I/IV }=1.034 \text { Py }-0.058 & \left(r^{2}=0.963\right) \\
\text { DHBPe }=0.687 \text { PY }+0.773 & \left(r^{2}=0.956\right) \\
\text { BPe }=0.674 \text { PY }+0.109 & \left(r^{2}=0.932\right) \\
\text { CO }=0.550 \text { PY }-0.136 & \left(r^{2}=0.956\right) \\
\text { OV }=1.356 \text { PY }-0.331 & \left(r^{2}=0.945\right)
\end{array}
$$

for converting between scales.

six solvent polarity probes are recommended as one or more may be better suited for a particular application, perhaps because of the spectral characteristics of the solvent (e.g., to avoid inner-filtering artifacts) or solubility and/or molecular size/shape constraints imposed by the system being studied. For example, pyrene is the only PAH without the limitation of water solubility problems. The polarity of new stationary phases in high performance liquid chromatography (HPLC) with binary mobile phases which typically include water can be examined using pyrene.

The six PAH probes have varying fluorescence excitation and emission peaks that may be utilized. They also differ in solubility and molecular size and shape (see Figure 81). on the basis of the experimental data, the best method for 


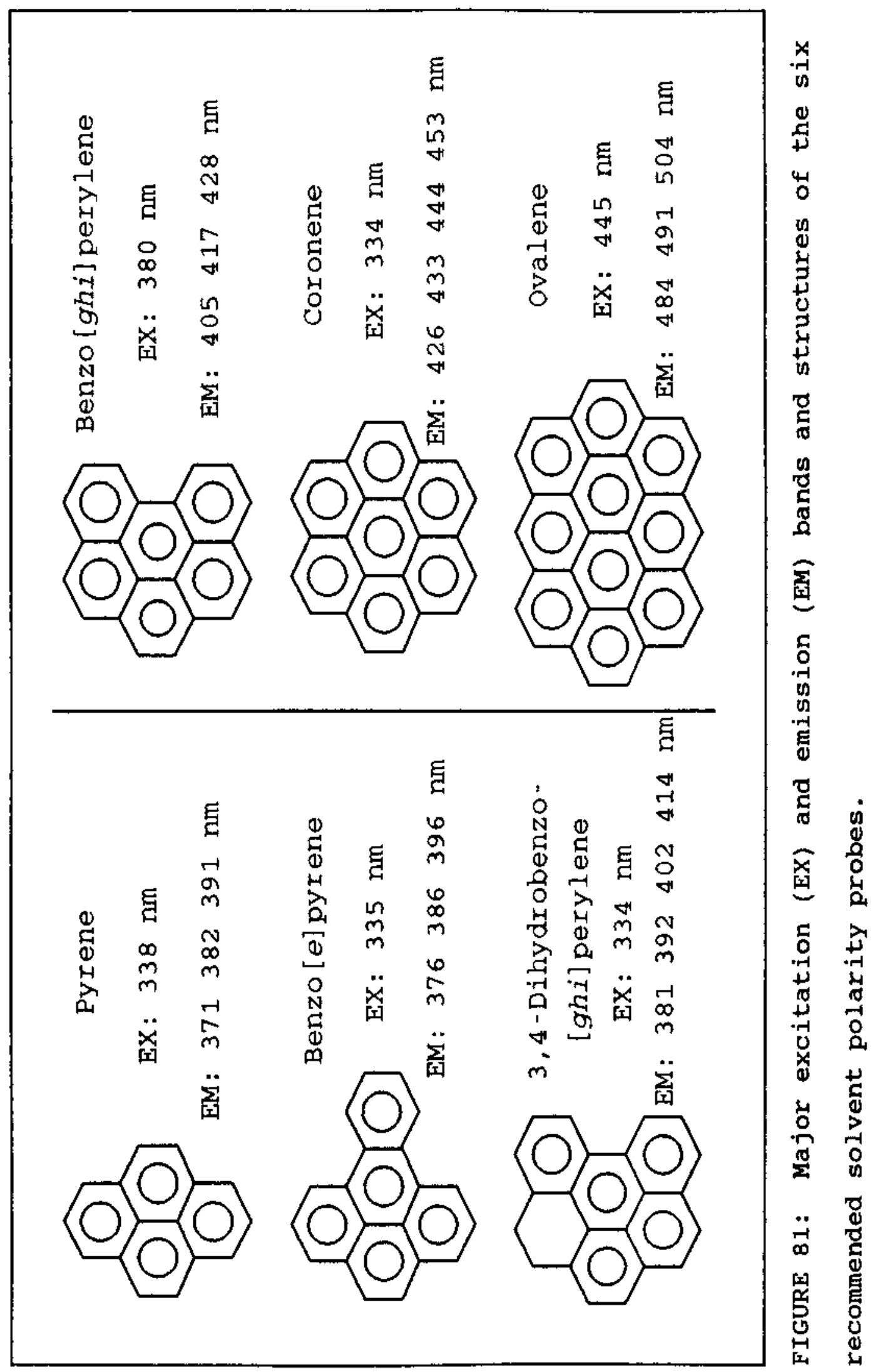


determining solvent polarity and identifying and eliminating the aforementioned undesired artifacts is to utilize several PAH probes. Fluorescence behavior of these six PAHs is well characterized and high quantum efficiencies facilitate fluorescence measurements at low solute concentrations.

Also note that Richard and woo and Mersch-Sundermann et al. reported pyrene $(58,59)$, coronene $(58,59)$, and ovalene (58) to be inactive and noncarcinogenic. 3,4-Dihydrobenzo[ghi]perylene is also reported to posses noncarcinogenic activity $(58,60,61)$. Benzo[e]pyrene and benzo[ghi]perylene, on the other hand, have conflicting reports regarding carcinogenicity. Benzo[ghi]perylene has been reported to be anywhere from inactive (60-62) to active (58, 59) as well as being a known or suspected carcinogen of low moderate potency (58). Benzo[e]pyrene has been reported to be an active (58) to weakly active (59) as well as being a known or suspected carcinogen of low moderate potency. When dealing with PACs, as with any chemical substance, appropriate safety precautions should be taken and human exposure should be minimized as carcinogenic data will always be interpreted differently and lead to conflicting conclusions about health concerns associated with long-term exposure to these compounds.

The two probes showing some carcinogenic activity, benzo[e]pyrene and benzo[ghi]perylene are not abandoned because they both have important properties that no other 
noncarcinogenic recommended PAH probe posses. Benzo[e]pyrene has the advantage of being the only probe with three solvent polarity ratios that can be utilized. Benzo[ghi]perylene has the advantage of being commercially available as well as having excitation and emission bands farther out in the visible, well past UV cut-offs of some solvents, like nitromethane. 
CHAPTER REFERENCES

1. Kalyanasundaram, K.; Thomas, J. K. J. Am. Chem. Soc. 1977, 99, 2039-2044.

2. Lianos, P.; Georghiu, S. Photochem. Photobiol. 1979, $29,843-846$.

3. Lianos, P.; Georghiu, S. Photochem. Photobiol. 1979, $30,355-362$.

4. Lianos, P.; Mukhopadhyay, A. K.; Georghiu, S. Photochem. Photobiol. 1980, 32, 415-419.

5. Langkilde, F. W.; Thulstrup, E. W.; Michl, J. J. Chem. Phys. 1983, 78, 3372-3381.

6. Herndon, W. C.; Nowak, P. C.; Conner, D. A.; Lin, P. J. Am. Chem. Soc. 1992, 114, 41-47.

7. Herbstein, F. H. ; Schmidt, G. M. J. J. Chem. Soc. 1954, 3302-3313.

8. Hirshfeld, F. L.; Sandler, S.; Schmidt, G. M. J. J. Chem. Soc. 1963, 2108-2125.

9. Hirshfeld, F. L. J. Chem. Soc. 1963, 2126-2135.

10. McIntosh, A. O.; Robertson, J. M.; Vand, V. J. Chem. SOC. 1954, 1661-1668.

11. De Rango, C.; Tsourcaris, G.; Declerq, J. P.; Germain, G.; Petzeys, J. P. Cryst. Struct. Comm. 1973, 2, 189192 .

12. Herbstein, F. H. Acta Crystallogr. 1979, 35B, 16611670 .

13. Roberts, P. J.; Ferguson, G. Acta Crystallogr. 1977, $33 B, 1244-1247$.

14. Laarhoven, W. H.; Prinsen, W. J. C. Top. Curr. Chem. $1984,125,63-130$ and references therein.

15. Meurer, K. P.; Vogtle, F. Top. Curr. Chem. 1985, 127, 1-76 and references therein.

16. Herndon, W. C. private communication. 
17. Dias, J. R. Handbook of Polycyclic Aromatic Hydorcarbons; Elsevier: Amsterdam, 1987; Part A.

18. Lakowicz, J. R. Principles of Fluorescence Spectroscopy; Plenum: New York, 1983.

19. Von Lippert, E. Z. Electrochem. 1957, 61, 962 .

20. Kawski, A. Acta Phys. Pol, 1966, 29, 507.

21. Mataga, N; Kaifu, Y.; Koizumi, M Bull. Chem. Soc. Jpn. $1956,29,465$.

22. Kabouchi, B.; Benali, B.; Fadouach, M.; Kadiri, A.; Cazeau-Dubroca, C.; Nouchi, G. Spectrochim. Acta 1992, $48 \mathrm{~A}, 1015-1020$.

23. Nakajima, A. Bull, Chem. Soc. Jpn. 1971, 44, 3272-3277.

24. Mazumdar, S.; Manoharan, R.; Dogra, S. J. Photochem. Photobiol. 1989, 46A, 301-314.

25. Renge, I. Chem. Phys. 1992, 167, 173-184.

26. Suppan, P. J. Chem. Soc., Part A 1968, 3125-3133.

27. Suppan, P. J. Photochem. Photobiol., Part A 1990, 50, 293-330.

28. Bekarek, V.; Bekarek, S.; Pavlet, F. Chem. Pap. 1988, $42,197-203$.

29. Brady, J. E.; Carr, P. W. J. Phys. Chem. 1985, 89, 1813-1822.

30. Kavanagh, R. J.; Iu, K.-K.; Thomas, J. K. Langmuir $1992,8,3008-3013$.

31. Bayliss, N. S. J. Chem. Phys. 1950, 18, 292.

32. Bekarek, V.; Bekarek, S.; Pavlat, F. Z. Phys. Chem., Leipzig 1988, 269, 1147.

33. Kalyanasundaram, K.; Thomas, J. K. J. Phys. Chem. 1977, $81,2176-2180$.

34. TRC Thermodynamic Tables: Hydrocarbons; Marsh, K. N., Ed.; Texas A \& M University: College Station.

35. TRC Thermodynamic Tables: Non-Hydrocarbons; Marsh, K. N., Ed.; Texas A\& M University: College Station. 
36. Linde, D. R. Handbook of Chemistry and Physics, 71st ed.; CRC Press: Boca Raton, 1990.

37. Dean, J. A. Lange's Handbook of Chemistry, 13th ed.; McGraw-Hill: New York, 1985.

38. Papanastasiou, G. E.; Ziogas, I. I. J. Chem. Eng. Data $1991,36,46-51$.

39. Janne11i, L.; Pansini, M. Can. J. Chem. 1988, 66, 662665 .

40. Johnson, J. F.; Cole, R. H. J. Am. Chem. SoC. 1951, 73, $4536-4540$.

41. Musset, E.; Nikuradse, A.; Ulbrich, R. Z. Angew. Phys. $1956,8,8$.

42. Leader, G. R. J. Am. Chem. Soc. 1951, 73, 856-857.

43. Marcheselli, L.; Pistoni, G.; Tagliazucchi, M.; Tassi, L.; Tosi, G. J. Chem. Eng. Data 1993, 38, 204.

44. Ritzoulis, G. Can. J. Chem. 1989, 67, 1105-1108.

45. Lin, R.-Y.; Dannhauser, W. J. Phys. Chem. 1963, 67, $1805-1810$.

46. Vaughn, W. E.; Roeder, S. B.; Provder, T. J. Chem. Phys. 1963, 39, 701.

47. Cole, R. H. J. Chem. Phys. 1941, 9, 251.

48. Davidson, D. W. Can. J. Chem. 1961, 39, 2139.

49. Grove, E. L.; Walden, G. E. J. Chem. Eng. Data 1965, 10,98 .

50. Schmid, R. J. Solution Chem. 1983, 12, 135-152,

51. D'Aprano, A.; Donato, D. I.; Agrigento, V. J. Solution Chem. 1981, 10, 673-680.

52. Thiebaut, J.-M.; Rivail, J.-I.; Greffe, J.-L. J. Chem. Soc., Faraday Trans. II 1976, 72, 2024-2034.

53. Riddick, J. A.; Bunger, W. B. Organic Solvents: Physical Properties and Methods of Purification; 3rd ed.; John Wiley \& Sons: New York, 1970. 
54. Hite, P.; Krasanansky, R.; Thomas,J. K.; J. Phys. Chem. $1986,90,5795-5799$.

55. Visser, R. J.; Weisenborn, P. C. M.; van Kan, P. J. M.; Huizer, B. H.; Varma, C. A. G. O.; Warman, J. M. ; DeHaas, M. P. J. Chem. Soc., Faraday Trans. 1985, 81, 689-704.

56. Chen, S. H.; Frank, C. W. Langmuir 1991, 7, 1719-1726.

57. Tucker, S. A., unpublished results.

58. Richard, A. M.; Woo, Y.-L. Mutat. Res. 1990, 242, 285303 .

59. Mersch-Sundermann, V.; Mochayedi, S.; Kevekordes, s. Mutat. Res. 1992, 278, 1-9.

60. Lijinsky, w.; Saffioti, U. Ann. It. Derm. Clin. Sper. $1965,19,34-44$.

61. Lijinsky, W.; Garcia, H.; Saffiotti, U. J. NatI. Cancer Inst. 1970, 44, 641-649.

62. Andrews, A. W.; Thibault, L. H.; Lijinsky, W. Mutat. Res., 1978, 51, 311-318. 


\section{CHAPTER V}

\section{RESULTS AND DISCUSSION OF SELECTIVE QUENCHING PHENOMENA}

\section{Alternant Polycyclic Aromatic Hydrocarbons}

Nitromethane can be used to simplify observed spectra of complex mixtures by effectively quenching the fluorescence emission of alternant polycyclic aromatic compounds (PACs) as opposed to nonalternant PACs. The alternant polycyclic aromatic hydrocarbons (PAHs) include the PAH6 benzenoids, PAH6 benzenoid derivatives, bi-PAHs, and cyclopenta-PAH derivatives. Although the cyclopentaPAHs with their five-membered ring initially appear to be nonalternants, one must remember that the starring and unstarring takes place only in the aromatic portion of the compound. Closer examination of the molecular structures in Figure 21 reveal that the bridge heads of cyclopenta-PAH's five-membered rings have two hydrogens bonded to the carbon atom and do not contain a double bond at this particular carbon atom; therefore, they are not included in the aromatic portion of the compound.

Figure 82 depicts the fluorescence emission spectra of $4 H$-benzo[b]cyclopenta $[j k l]$ triphenylene (BCYTP) and 13Hdibenz $[b c, k]$ aceanthrylene (DBAA) dissolved in acetonitrile as a function of nitromethane concentration. For BCyTP, 


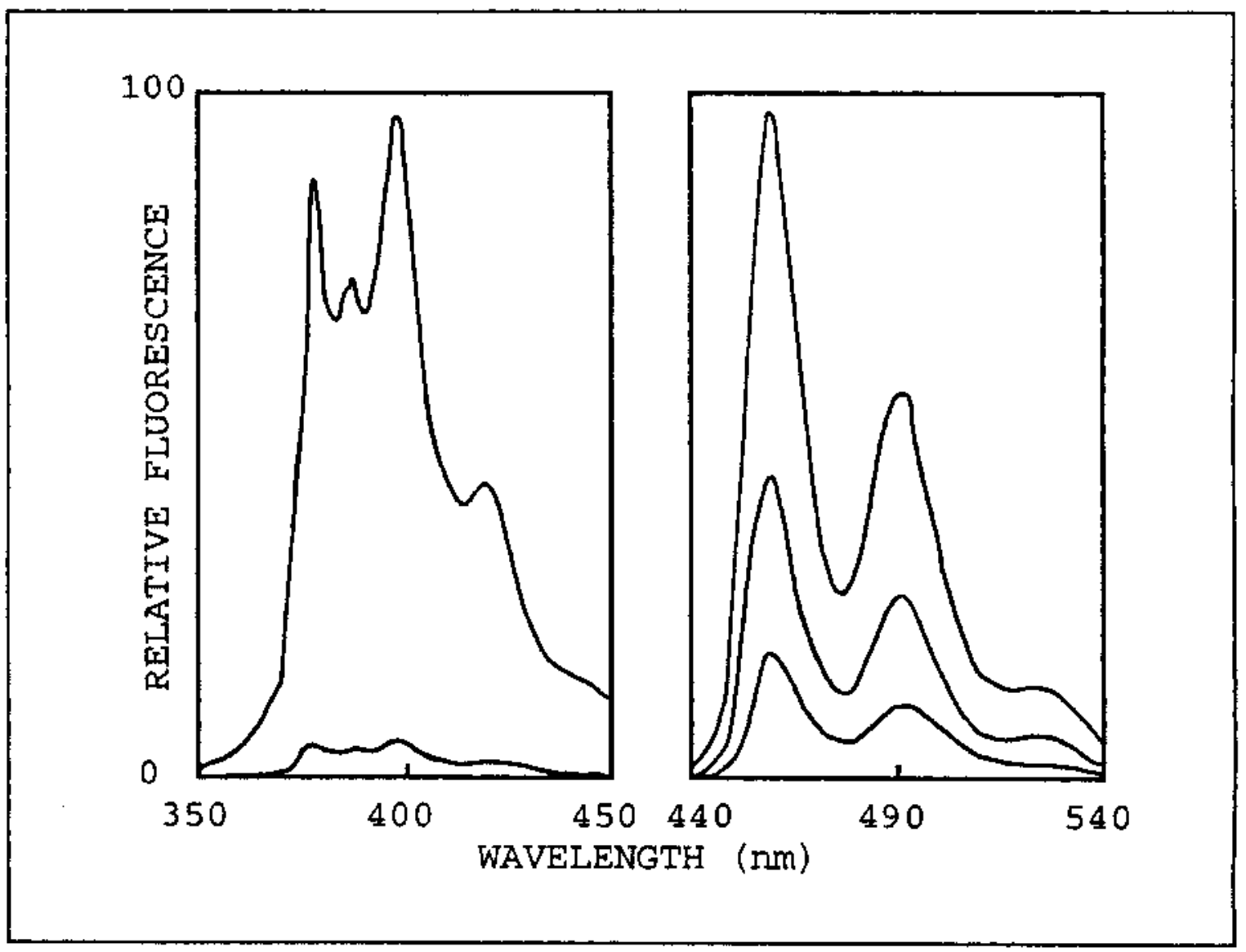

FIGURE 82: Fluorescence emission spectra of $4 \mathrm{H}$-benzo[b]cyclopenta[jkI]triphenylene (right-hand side) and 13Hdibenzo[bc,k]aceanthrylene (left-hand side) dissolved in acetonitrile at various nitromethane concentrations. From top to bottom, the curves correspond to 0 and 1 drop of nitromethane for BCYTP, and 0,3 , and 10 drops of nitromethane for DBAA. 
part of the observed emission intensity reduction results from primary inner-filtering as nitromethane absorbs an appreciable amount of the $300 \mathrm{~nm}$ excitation radiation. Initially, BCYTP has an absorbance of $A=0.000$ and an fluorescence intensity of $F^{\text {obs }}=566.0$. After one pasteur pipet drop of nitromethane the $A=0.986$ and the $F^{\text {obs }}=$ 28.9. Multiplication of the observed signal by $f_{\text {prim }}$ (see Table XXIV) increases the value to $\mathrm{F}^{\text {corr }} \approx 89.6$; however, this is still significantly below the initial emission intensity of $\mathrm{F}^{\mathrm{corr}} \approx 566.0$. Primary inner-filtering corrections are not necessary in the case of DBAA since the excitation wavelength $\left(\lambda_{\mathrm{ex}}=400 \mathrm{~nm}\right)$ now occurs in the spectral region where nitromethane is optically dilute. Secondary inner-filtering corrections for these solutes and all other solutes are not necessary since nitromethane is "optically transparent" in most PACs emission ranges (approximately $>360 \mathrm{~nm}$ ).

In some cases inner-filtering corrections are not even necessary because the solution absorbance are relatively small, and observed emission intensities decrease sharply upon the addition of nitromethane. For example, most of the alkyl-pyrene derivatives have no observable emission spectra after the addition of only 1 drop of nitromethane. 1Methylpyrene and 1,5-dimethylpyrene have initial solution absorbance and emission intensities of $\mathrm{A}=0.003$ and $\mathrm{A}=$ 0.017 and $F^{\text {obs }}=553.7$ and $F^{\text {obs }}=934.6$, respectively. After 
the addition of drop of nitromethane neither solute had any observable fluorescence emission signal and solutions absorbance with their corresponding corrections factors were only $\mathrm{A}=0.169$ and $\mathrm{f}_{\text {prim }}=1.215$ for 1 -methylpyrene and $\mathrm{A}=$ 0.438 and $f_{\text {prim }}=1.655$ for 1,5 -dimethylpyrene. Even if the emission signal had been on the order of 10 or 20 the $F^{\text {corr }}$ still would have indicated quenching by nitromethane.

Table LIII shows the effect of nitromethane on alI available pACs in various solvent systems. Careful examination of Table LIII reveals that nitromethane does quench the fluorescence emission of all PAHs, PAH6 benzenoid derivatives, bi-PAHs, and cyclopenta-PAH derivatives investigated in neat acetonitrile or a binary aqueousacetonitrile mixture (20:80 percent by volume) suggested by Blümer and Zander (1) (other solvents contained in Table LIII will be addressed later). Initial studies utilized the aqueous-acetonitrile solvent mixture recommended, later it was discovered that with the exception of the coronene derivatives the nitromethane selective quenching rule is obeyed in neat acetonitrile as well. The neat acetonitrile is a better solvent for the larger PACs that have very limited water solubility.

of the alternant PAHs in Table LIII, only the fluorescence emission of dibenzo[hi,wX]heptacene and dibenzo[a,rst] naphtho $[8,1,2 c d e]$ pentaphene (at $483 \mathrm{~nm}$ ) were not significantly diminished. The latter compound exhibited 
TABLE LIII. Summary of emission wavelength and fluorescence quenching (by nitromethane) data for alternant and nonalternant PAHs.

\begin{tabular}{|c|c|c|c|c|}
\hline LETTER $^{\mathbf{a}}$ & $\begin{array}{l}\lambda_{\mathrm{em}}^{\mathrm{b}} \\
\mathrm{nm}\end{array}$ & $\begin{array}{c}\text { QUENCHED } \\
\text { ACN }\end{array}$ & $\begin{array}{l}\text { QUENCHED } \\
\text { ACN-TOLN }\end{array}$ & $\begin{array}{l}\text { QUENCHED } \\
\text { ACN-EA }\end{array}$ \\
\hline \multicolumn{5}{|c|}{ Alternant PAHs } \\
\hline A & 425 & YES $^{\mathrm{e}}$ & No & No \\
\hline B & 429 & $\mathrm{YES}^{\mathrm{e}}$ & NO & No \\
\hline$c$ & 442 & $\mathrm{YES}^{\mathrm{e}}$ & No & No \\
\hline $\mathrm{D}$ & 431 & $\mathrm{YES}^{\mathrm{e}}$ & No & No \\
\hline $\mathbf{E}$ & 458 & $\mathrm{YES}^{\mathrm{e}}$ & No & No \\
\hline I & 418 & YES & YES & YES \\
\hline $\mathrm{K}$ & 481 & YES & NO & No \\
\hline $\mathrm{L}$ & 488 & $\mathrm{YES}^{\mathrm{e}}$ & No & NO \\
\hline M & 371 & YES & YES & YES \\
\hline $\mathbf{N}$ & 437 & YES & YES & YES \\
\hline 0 & 405 & YES & YES & YES \\
\hline$Q$ & 416 & YES & YES & YES \\
\hline $\mathbf{R}$ & 418 & $\mathrm{YES}^{\mathrm{e}}$ & YES & YES \\
\hline $\mathrm{T}$ & 402 & YES & YES & YES \\
\hline $\mathrm{U}$ & 386 & YES & YES & YES \\
\hline $\mathrm{V}$ & 394 & YES & YES & YES \\
\hline $\mathrm{AC}$ & 437 & $\mathrm{YES}^{\mathrm{e}}$ & YES & YES \\
\hline $\mathrm{AF}$ & 451 & $\mathrm{YES}^{\mathrm{e}}$ & YES & YES \\
\hline$A G$ & 446 & $\mathrm{YES}^{\mathrm{e}}$ & YES & YES \\
\hline
\end{tabular}


TABLE LIII. Continued

\begin{tabular}{|c|c|c|c|c|}
\hline LETTER ${ }^{a}$ & $\lambda_{\mathrm{em}}^{\mathrm{b}}$ & $\begin{array}{c}\text { QUENCHED } \\
\text { ACN }\end{array}$ & $\begin{array}{l}\text { QUENCHED } \\
\text { ACN-TOLN }\end{array}$ & $\begin{array}{l}\text { QUENCHED } \\
\text { ACN-EA }^{d}\end{array}$ \\
\hline $\mathrm{AH}$ & 419 & YES & YES & YES \\
\hline$A I$ & 430 & YES & YES & YES \\
\hline $\mathrm{AJ}$ & 432 & YES & YES & YES \\
\hline AK & 438 & YES & NO & No \\
\hline$A M$ & 416 & $\mathrm{YES}^{\mathrm{e}}$ & YES & YES \\
\hline AN & 413 & YES & YES & YES \\
\hline$A Q$ & 484 & YES $^{\mathrm{e}}$ & YES & No \\
\hline AR & 467 & YES & YES & YES \\
\hline $\mathrm{AT}$ & 470 & YES & YES & YES \\
\hline $\mathrm{AU}$ & 435 & YES & YES & YES \\
\hline $\mathrm{AV}$ & 446 & YES & YES & YES \\
\hline AW & 477 & YES & YES & YES \\
\hline$A X$ & 437 & YES & YES & YES \\
\hline $\mathrm{AY}$ & 485 & No & No & No \\
\hline $\mathrm{AZ}$ & 375 & YES & YES & YES \\
\hline $\mathrm{BA}$ & 377 & YES & YES & YES \\
\hline $\mathrm{BB}$ & 354 & YES & YES & YES \\
\hline $\mathrm{BC}$ & 363 & YES & YES & YES \\
\hline $\mathrm{BD}$ & 380 & YES & YES & YES \\
\hline $\mathrm{BE}$ & 392 & YES & YES & YES \\
\hline$B F$ & 424 & YES & YES & YES \\
\hline
\end{tabular}


TABLE LIII. Continued

\begin{tabular}{|c|c|c|c|c|}
\hline LETTER $^{\mathrm{a}}$ & $\begin{array}{l}\lambda_{\mathrm{em}}^{\mathrm{b}} \\
\mathrm{nm}\end{array}$ & $\begin{array}{c}\text { QUENCHED } \\
\text { ACN }\end{array}$ & $\begin{array}{l}\text { QUENCHED } \\
\text { ACN-TOLN }\end{array}$ & $\begin{array}{l}\text { QUENCHED } \\
A C N \sim E A^{d}\end{array}$ \\
\hline
\end{tabular}

Alternant Cyclopenta-PAF Derivatives

$\begin{array}{lllll}\text { A } & 389 & \text { YES } & \text { YES } & \text { YES } \\ \text { B } & 362 & \text { YES } & \text { YES } & \text { YES } \\ \text { C } & 360 & \text { YES } & \text { YES } & \text { YES } \\ \text { D } & 381 & \text { YES } & \text { YES } & \text { YES } \\ \text { E } & 384 & \text { YES } & \text { YES } & \text { YES } \\ \text { F } & 398 & \text { YES } & \text { YES } & \text { YES } \\ \text { G } & 395 & \text { YES } & \text { YES } & \text { YES } \\ \text { H } & 398 & \text { YES } & \text { YES } & \text { YES } \\ \text { I } & \text { YES } & \text { YES } & \text { YES } \\ \mathrm{J} & 460 & \text { YES } & \text { YES } & \text { YES } \\ \mathrm{K} & 397 & \text { YES } & \text { YES } & \text { YES } \\ \mathrm{L} & 386 & \text { YES } & \text { YES } & \text { YES } \\ \mathrm{M} & 394 & \text { YES } & \text { YES } & \text { YES } \\ \mathrm{N} & 400 & \text { YES } & \text { YES } & \text { YES }\end{array}$

Alternant Bi-PAHs

\begin{tabular}{lllll} 
A & $363-365$ & YES & YES & YES \\
B & 354 & YES & YES & YES \\
C & 378 & YES & YES & YES \\
E & 334 & YES & YES & YES \\
F & 370 & YES & YES & YES \\
G & $451-461$ & YES & YES & YES \\
\hline
\end{tabular}


TABLE LIII. Continued

\begin{tabular}{|c|c|c|c|c|}
\hline LETTER ${ }^{a}$ & $\begin{array}{l}\lambda_{\mathrm{em}}^{\mathrm{b}} \\
\mathrm{nm}\end{array}$ & $\begin{array}{l}\text { QUENCHED } \\
\text { ACN }\end{array}$ & $\begin{array}{l}\text { QUENCHED } \\
\text { ACN-TOLN }^{\mathrm{C}}\end{array}$ & $\begin{array}{l}\text { QUENCHED } \\
\text { ACN-EA }\end{array}$ \\
\hline
\end{tabular}

\section{Nonalternant Acenaphthylene Derivatives}

$\begin{array}{lllll}\text { A } & 336 & \text { YES } & \text { YES } & \text { YES } \\ \text { B } & 427 & \text { YES } & \text { YES } & \text { YES } \\ \text { C } & 355 & \text { YES } & \text { YES } & \text { YES } \\ \text { D } & 402 & \text { YES } & \text { YES } & \text { YES } \\ \text { E } & 397 & \text { Y.ES } & \text { YES } & \text { YES } \\ \text { F } & 426 & \text { YES } & \text { YES } & \text { YES } \\ \text { G } & 410 & \text { YES } & \text { YES } & \text { YES } \\ \text { H } & 377 & \text { YES } & \text { YES } & \text { YES } \\ \text { I } & 435 & \text { YES } & \text { YES } & \text { YES }\end{array}$

Nonalternant Acephenanthrylene Derivatives

$\begin{array}{lllll}\text { A } & 360 & \text { YES } & \text { YES } & \text { YES } \\ \text { B } & 353 & \text { YES } & \text { YES } & \text { YES } \\ \text { C } & 372 & \text { YES } & \text { YES } & \text { YES } \\ \text { D } & 367 & \text { YES } & \text { YES } & \text { YES } \\ \text { E } & 360 & \text { YES } & \text { YES } & \text { YES } \\ \text { F } & 358 & \text { YES } & \text { YES } & \text { YES } \\ \text { G } & 356 & \text { YES } & \text { YES } & \text { YES } \\ \text { H } & 378 & \text { YES } & \text { YES } & \text { YES } \\ \text { I } & 359 & \text { YES } & \text { YES } & \text { YES }\end{array}$


TABLE LIII. Continued

\begin{tabular}{|c|c|c|c|c|}
\hline LETTER $^{a}$ & $\lambda_{\mathrm{nm}}^{\lambda^{\mathrm{b}}}$ & $\begin{array}{c}\text { QUENCHED } \\
\text { ACN }\end{array}$ & $\begin{array}{l}\text { QUENCHED } \\
\text { ACN-TOLN }\end{array}$ & $\begin{array}{l}\text { QUENCHED } \\
\text { ACN-EA }^{\mathrm{d}}\end{array}$ \\
\hline & Nonalterna & Fluoranthenoids & and Fluor & \\
\hline A & 474 & $\mathrm{NO}^{\mathrm{e}}$ & No & No \\
\hline B & 478 & $\mathrm{NO}^{\mathrm{e}}$ & No & No \\
\hline C & 490 & No & No & No \\
\hline $\mathrm{D}$ & 408 & $Y E S^{e}$ & YES & No \\
\hline$E$ & 482 & $\mathrm{NO}^{\mathrm{e}}$ & No & No \\
\hline $\mathbf{F}$ & 474 & $\mathrm{NO}^{\mathrm{e}}$ & NO & No \\
\hline G & 438 & No ${ }^{e}$ & No & No \\
\hline $\mathrm{H}$ & 446 & $\mathrm{NO}^{\mathrm{e}}$ & No & No \\
\hline I & 508 & $\mathrm{NO}^{\mathrm{e}}$ & No & No \\
\hline $\mathrm{J}$ & 461 & $\mathrm{NO}^{\mathrm{e}}$ & No & No \\
\hline $\mathrm{K}$ & 447 & NO & No & No \\
\hline L & 466 & No ${ }^{e}$ & No & No \\
\hline $\mathbf{M}$ & 422 & $\mathrm{YES}^{\mathrm{e}}$ & NO & No \\
\hline $\mathrm{N}$ & 488 & No & No & No \\
\hline 0 & 433 & No & No & $\mathbf{R R}$ \\
\hline \multirow[t]{2}{*}{$\mathbf{P}$} & 542 & No & No & No \\
\hline & 402 & YES & YES & YES \\
\hline$Q$ & 502 & No & No & NO \\
\hline
\end{tabular}

a Letters refer to molecular structures in Figures 13-32. b Emission wavelength (or wavelength range for those solutes whose emission wavelengths respond to solvent polarity by 
TABLE LIII. Continued

redshifting) used in the nitromethane quenching studies. c Quenching studies performed in a binary acetonitrile-toluene solvent mixture (60:40 percent by volume). ${ }^{d}$ Quenching studies performed in a binary acetonitrile-ethyl acetate solvent mixture (60:40 percent by volume). 'Quenching studies performed in a binary aqueous-acetonitrile solvent mixture (20:80 percent by volume).

abnormal behavior in that nitromethane quenched fluorescence emission signals at 413 and $437 \mathrm{~nm}$, and only at $483 \mathrm{~nm}$ did one observe a nearly constant emission intensity. Nitromethane may undergo a chemical reaction with dibenzo[a,rst $]$ naphtho $[8,1,2 c d e]$ pentaphene, or perhaps may have changed the molecular environment around the dissolved PAH solute in such a fashion that it was responding to solvent polarity changes. Dibenzo[a,rst]naphtho $[8,1,2 c d e]-$ pentaphene was originally classified as a nonprobe molecule, not because its emission intensity ratios remained constant, but rather because the observed changes did not vary systematically with solvent polarity. In Table LIII the emission band at which the quenching is observed is listed. Dibenzo[a,rst $]$ naphtho $[8,1,2 c d e]$ pentaphene is listed as quenched when examined at the $413 \mathrm{~nm}$ emission band. In most cases any emission band can be chosen as they all respond in 
the same manner to the addition of nitromethane. All of the PAHs listed in Table LIII, but dibenzo[hi,wX]heptacene are quenched by nitromethane in neat acetonitrile or the aqueous-acetonitrile mixture at the emission bands listed which is in complete agreement with what would be expected based upon the fact that all solutes are "alternant" polycyclic aromatic hydrocarbons.

Nonalternant Polycyclic Aromatic Hydrocarbons

Nitromethane should not quench the fluorescence emission of nonalternant PACs. The nonalternant PAHs include the fluoranthenoids and fluorenoids, acenaphthylene derivatives, and acephenanthrylene derivatives. The fivemembered ring in the acenaphthylene derivatives, unlike the cyclopenta-PAH derivatives, do contain a double bond; therefore, they are classified as nonalternant compounds. Examination of Table LIII reveals that nitromethane does not, in general, quench the fluorescence emission of nonalternant PAHs. For the fluoranthenoid and fluorenoid solutes studied, the corrected fluorescence emission intensity remained essentially constant, at least to within $\pm 10 \%$ Benzo[k]fluoranthene and naphtho[ $2,3 b]$ fluoranthene are the only major exceptions to the selective quenching rule. Naphtho[ $1,2 b]$ fluoranthene might be considered by some researchers as a "borderline case," since its fluorescence 
intensity did drop by more than $20 \%$, even after primary inner-filtering corrections were made. Once again secondary inner-filtering corrections were not necessary. It is interesting to note that the two fluoranthenoid exceptions to the nitromethane selective quenching rule are the only two solutes in this particular subclasses that are classified as solvent polarity probes.

Figure 83 depicts the fluorescence emission spectra of benzo[b]fluoranthene as a function of nitromethane concentration and demonstrates the importance of innerfiltering corrections. Since nonalternant PAHs should not be quenched by nitromethane according to the selective quenching rule it is imperative to be aware of innerfiltering artifacts.

Neglect of primary inner-filtering leads to erroneous conclusions about the selectivity of nitromethane concerning many fluoranthenoids and fluorenoids. Blumer and Zander (1) and Dreeskamp et al. (2) previously listed benzo[b]fluoranthene as an exception to the nitromethane selective quenching rule. The authors' experimental procedure was only briefly described, and I fail to see how primary innerfiltering was avoided (as claimed) if the sample was irradiated at the optimum excitation wavelength of $346 \mathrm{~nm}$. Initially, the uncorrected spectra in Figure 83 appear to be to support their claim, but upon correction for primary 


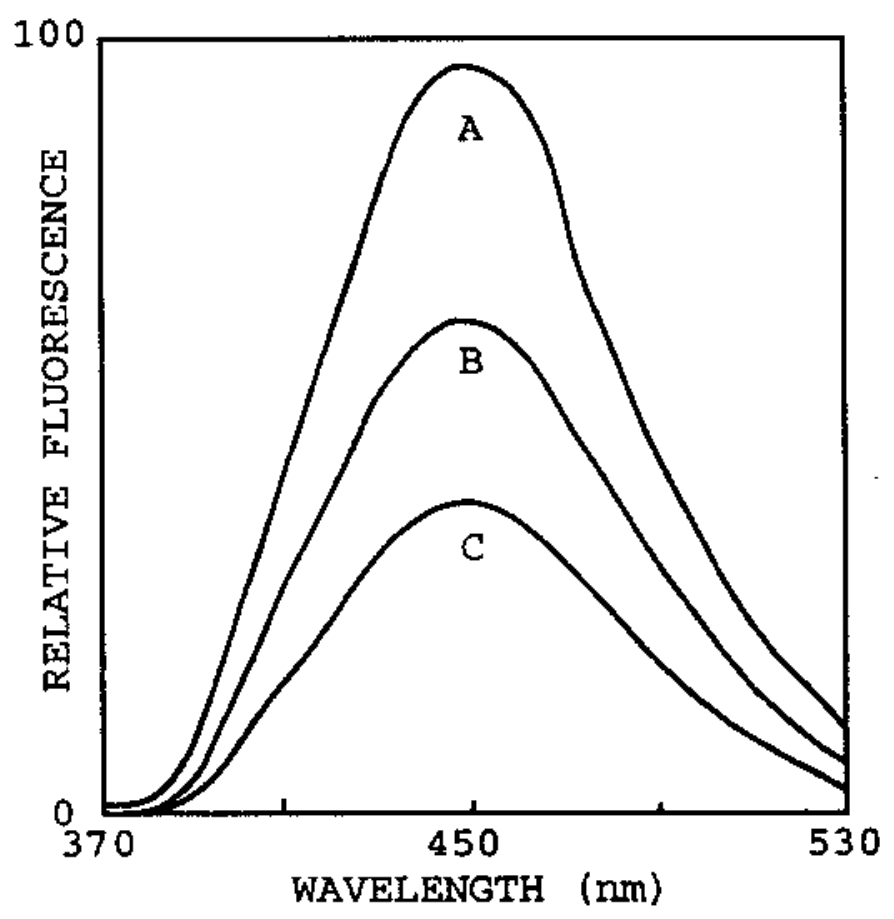

FIGURE 83: Effect of nitromethane on the fluorescence emission behavior of benzo[b]fluoranthene dissolved in a binary aqueous-acetonitrile (20:80 by volume) solvent mixture. Curves $A, B$, and $C$ correspond to 0,2 , and 5 pasteur pipet arops of nitromethane, respectively. 
inner-filtering, the emission intensity is essentially constant.

Examination of Table LIV of select fluoranthenoid and fluorenoids clearly shows the relevance of the innerfiltering corrections. The $F^{\text {obs }}$ of nearly all solutes decreases significantly with addition of nitromethane, but the observed absorbance increases significantly. The $F^{\text {corr }}$ values reveal that in general, this subclass of compounds are not quenched by nitromethane. The initial increase in the $F^{\text {corr }}$ upon the addition of nitromethane may be due to $f_{\text {prim }}$ being calculated on the basis of an incorrect assumed sample cell configuration or, more likely, because nitromethane helped redissolve the small amount of PAH which was still absorbed onto the test tube or cuvette walls.

One of the unexpected "selective quenching" rule exceptions listed in Table LIII is rubicene. Examination of Figure 84 reveals that the quenching of rubicene is very different. Rubicene is a nonalternant fluoranthenoid; therefore, it should not be quenched by nitromethane. The first five emission peaks $(403,416,426,441$, and $453 \mathrm{~nm}$ ) that are narrow and well-defined are quenched, while the two broad peaks $(482$ and $541 \mathrm{~nm})$ remain unquenched. The slight increase in intensity of $541 \mathrm{~nm}$ emission peak via addition of nitromethane may be attributed to enhanced solubility, viscosity or solvent polarity. As mentioned earlier in Chapter 3 , the number of peaks and the surprising amount of 


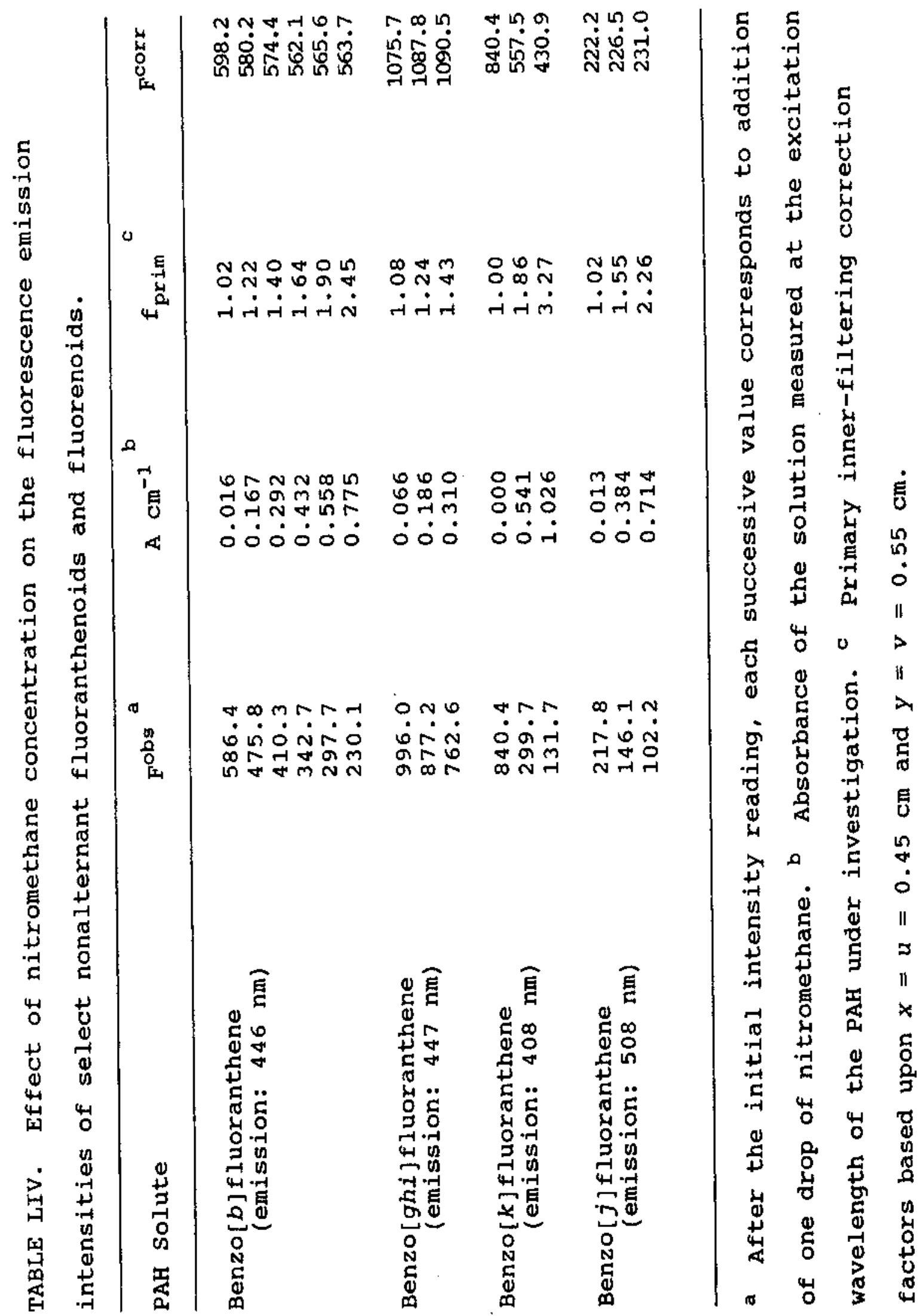




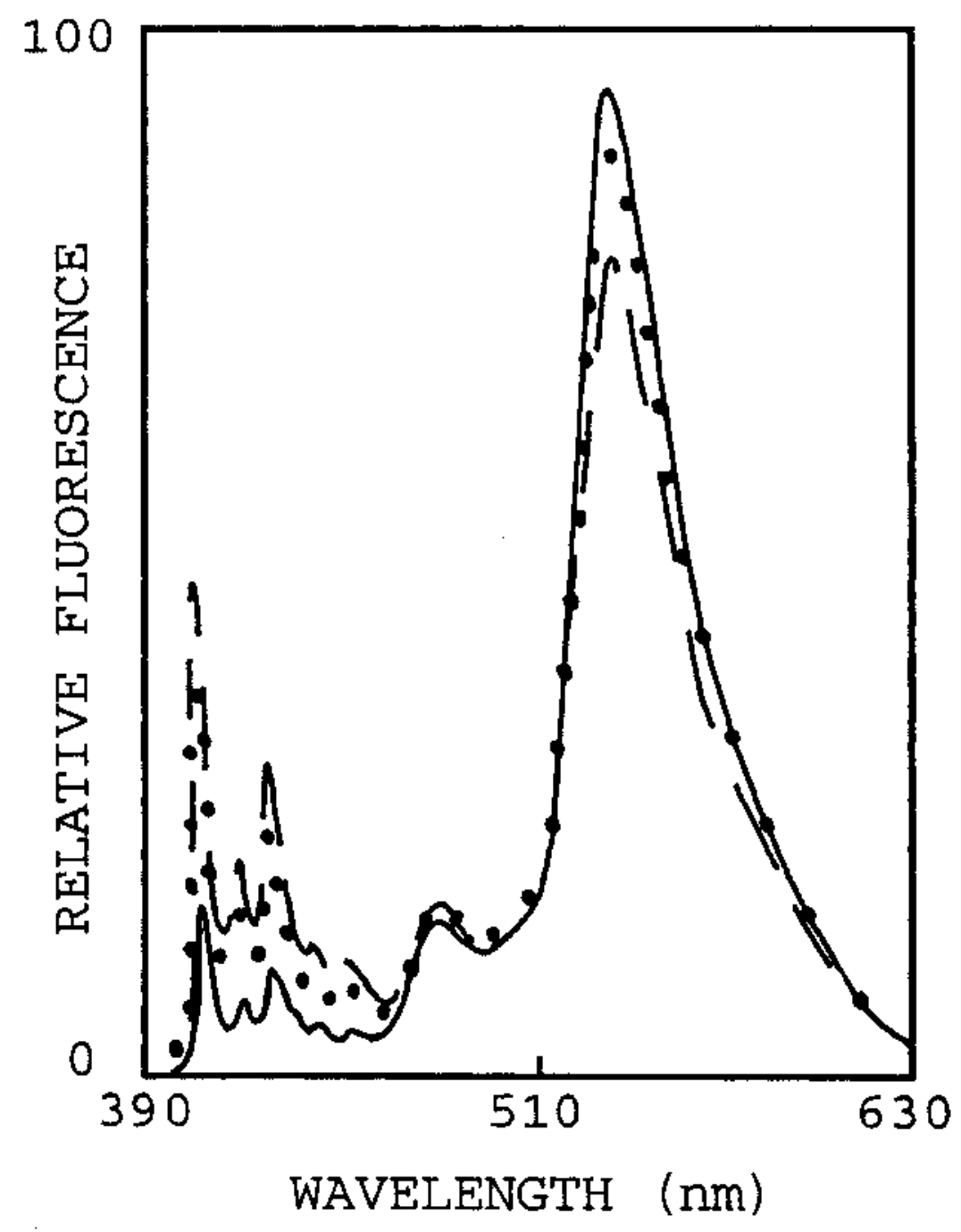

FIGURE 84: Corrected fluorescence emission spectra of rubicene dissolved in acetonitrile at various nitromethane concentrations. From top to bottom in the $390-460 \mathrm{~nm}$ spectral region, the curves correspond to 0,1 , and 5 pasteur pipet drops of nitromethane. 
detailed fine structure of the first five peaks is not indicative of this subclass. The fluorescence emission spectra and the nitromethane quenching behavior suggest that rubicene acts as if it had two electronic centers, the first corresponding to an alternant moiety that would be quenched and the second corresponding to an unquenched nonalternant moiety. The molecular structure would also suggest this possibility since rubicene has an alternant anthracene moiety through the center of the compound as well as two nonalternant fluoranthene moieties on each end. The alternant moiety would explain the fine-structure of the first five bands in the spectra, and the nonalternant moiety could explain the last two broad, unstructured bands. Another plausible explanation of the broad redshifted fluorescence emission peak at $541 \mathrm{~nm}$ in the rubicene spectra is excimer formation. To eliminate this possibility, a concentration study was undertaken. It was found that even when the solute's concentration was so low that it gave only very weak fluorescence emission intensities, all seven or eight bands still remained, including the band at $541 \mathrm{~nm}$. The change in the excitation spectra via changing emission bands was also examined. In differing solvents, the excitation spectra contained the same wavelengths at the various emission peak settings, but the ratios of the bands were significantly different. Also, the excitation spectra with the emission collected at $541 \mathrm{~nm}$ was completely 
different than with the emission setting at $403 \mathrm{~nm}$. In dimethyl sulfoxide and $n$-hexadecane, the excitation spectra did not change when the emission setting was at any of five well-defined peaks (DMSO: 403, 416, 426, 441, and $453 \mathrm{~nm}$ ), but the excitation spectra was not the same as the one obtained at $541 \mathrm{~nm}$. The small broad peak at $482 \mathrm{~nm}$ gave an excitation spectra that appeared to be the addition of the two spectra obtained for the broad band at $541 \mathrm{~nm}$ and welldefined bands at $403,416,426,441$, or $453 \mathrm{~nm}$ as it was not identical to either one, but contained peaks belonging to both. Experimental data from the concentration and excitation studies, combined with the nitromethane quenching phenomena, and the molecular structure arguments support the idea that rubicene acts like two electronic centers. A technique such ultraviolet or fluorescence spectroscopy with polarized light in organized solvent media could perhaps determine if this is true (3).

Figure 85 shows the effect on nitromethane on aceanthrylene dissolved in acetonitrile. All the acenaphthylene and acephenanthrylene derivative solutes examined were quenched by nitromethane which was completely unexpected. Their behavior is indicative of alternant polycyclic aromatic hydrocarbons rather than the nonalternant PAHs they are. These are the first subclasses of PAHs in which all solutes examined behave as exceptions to the nitromethane selective quenching rule. 


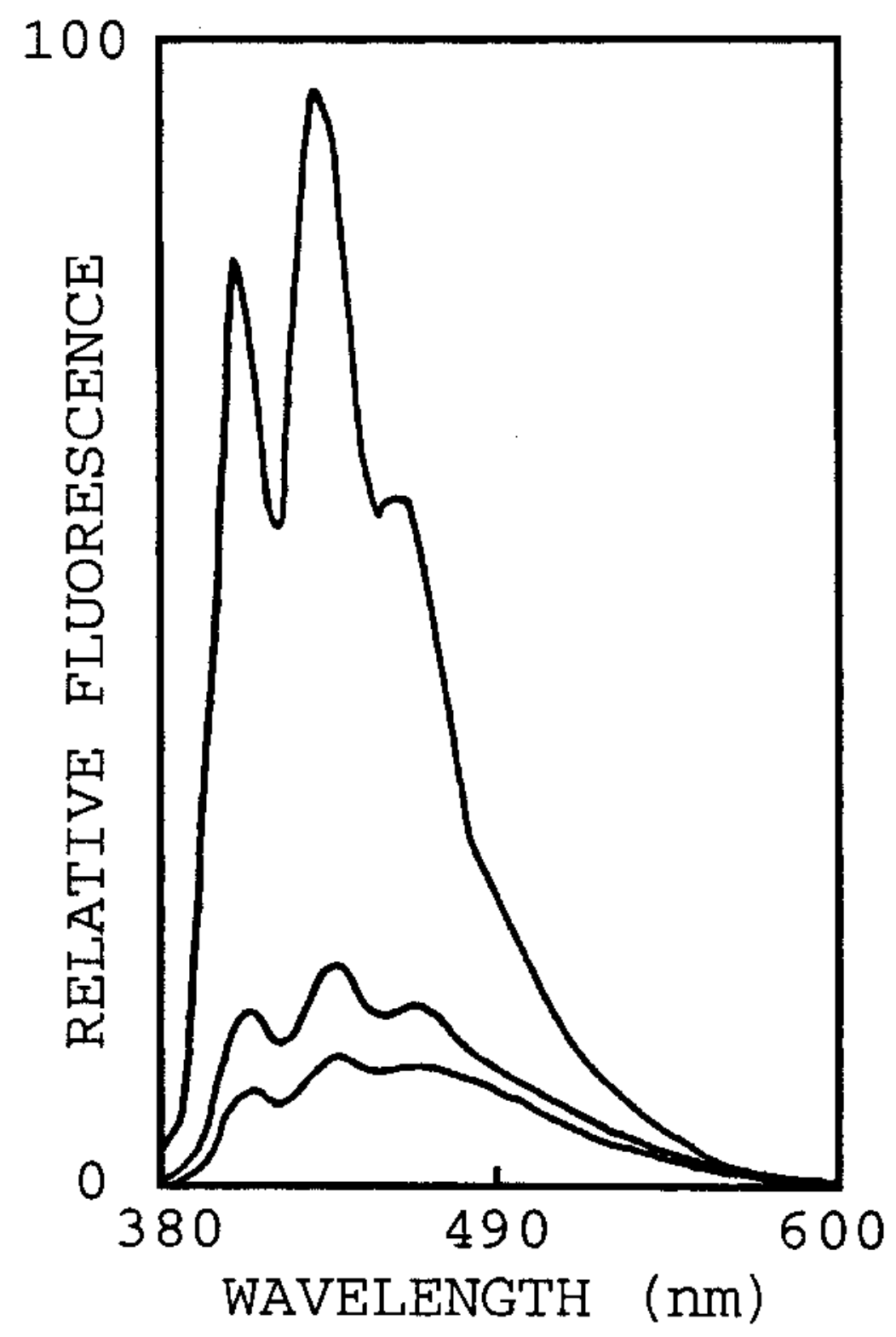

FIGURE 85: Uncorrected fluorescence emission spectra of aceanthrylene dissolved in acetonitrile at various nitromethane concentrations. From top to bottom, the curves correspond to 0,1 , and 2 pasteur pipet drops of nitromethane. 
The peculiar behavior of the acenaphthylene and acephenanthrylene derivatives might be attributed to the fixed double bond in the cyclopenta ring, which is unlike previously examined nonalternant PAH subclasses, e.g. fluorenoids and fluoranthenoids. The fixed double bond will significantly decrease the number of possible resonance structures. Available NMR (nuclear magnetic resonance) data supports the conclusions regarding the fixed double bond in the five-membered ring of the various acenaphthylene derivatives. Figure 86 indicates the coupling constants between adjacent protons in aceanthrylene, acephenanthrylene, and cyclopenta[cd]pyrene. The coupling constants of the protons at the five-membered ring are significantly smaller than the other coupling constants, and their values (5.1-5.3 Hertz, Hz) are outside the region in which vicinal coupling constants of aromatic protons are found (6.6-9.0 $\mathrm{Hz}$ ) A coupling constant of circa $5 \mathrm{~Hz}$ is normal for protons at the double bond of a cyclopentene derivative, and Figure 86 illustrates the olefinic rather than aromatic character of the double bond in the five-membered ring of these acenaphthylene-type PAHs (4).

Also note, the excimer peak in fluorescence emission spectrum of 4-methylacephenanthrylene, 5-methylacephenanthrylene, and 9-methylacephenanthrylene was not quenched by nitromethane. It could be effectively argued that excimer formation could prevent fluorescence quenching of 

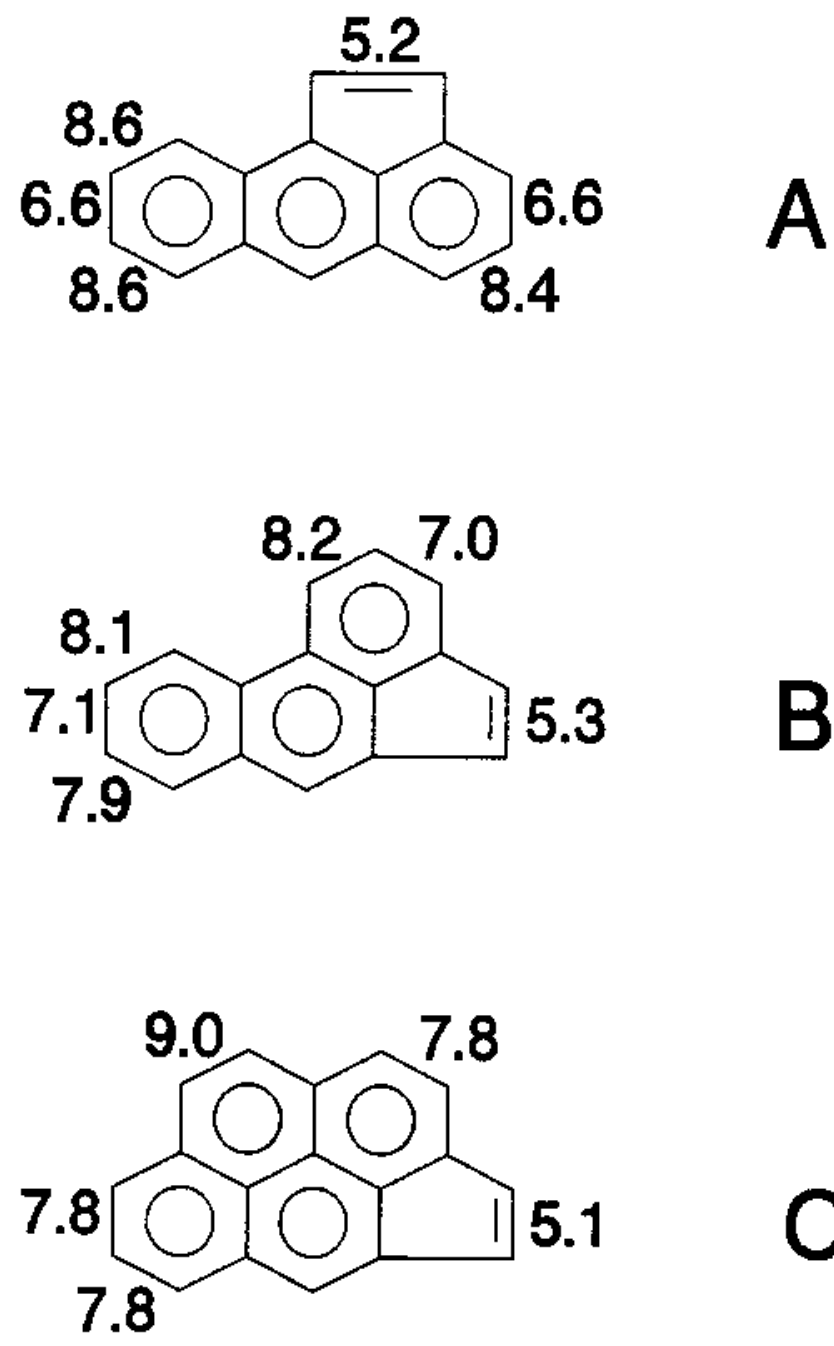

D

FIGURE 86: ${ }^{1}{ }_{\mathrm{H}-{ }^{1} \mathrm{H}}$ NMR coupling constants for (A) aceanthrylene: $J_{1,2}=5.2 \mathrm{~Hz}, J_{3,4}=6.6 \mathrm{~Hz}, J_{4,5}=8.4 \mathrm{~Hz}, J_{7,8}=8.6$ $\mathrm{Hz}, J_{8,9}=6.6 \mathrm{~Hz}$, and $J_{9,10}=8.6 ;$ (B) acephenanthrylene: $\mathrm{J}_{1,2}=8.2 \mathrm{~Hz}, \mathrm{~J}_{2,3}=7.0 \mathrm{~Hz}, \mathrm{~J}_{4,5}=5.3 \mathrm{~Hz}, \mathrm{~J}_{7,8}=7.9 \mathrm{~Hz}$, $J_{8,9}=7.1 \mathrm{~Hz}$, and $J_{9,10}=8.1 \mathrm{~Hz}$; and (C) cyclopenta[cd]pyrene: $J_{1,2}=7.8 \mathrm{~Hz}, J_{3,4}=5.1 \mathrm{~Hz}, J_{6,7}=7.8 \mathrm{~Hz}, J_{7,8}=$ $7.8 \mathrm{~Hz}$, and $\mathrm{J}_{9,10}=9.0 \mathrm{~Hz}(5-7)$. 
the excimer by nitromethane either by a shift of the molecular orbitals so as to result in a more positive reduction potential and/or an increased electron density between the two eclipsed aromatic ring systems and thus making less electron density available to nitromethane for electron transfer.

Two plausible mechanistic schemes for the fluorescence quenching of the excimer by nitromethane are as follows:

$$
\begin{aligned}
& \text { Scheme } 1 \\
& \mathrm{PAH}+\mathrm{h} \mathbf{v}_{0} \rightarrow \mathrm{PAH}^{*} \\
& \mathrm{PAH}^{\star}+\text { Quencher }+\mathrm{PAH}+\text { Quencher } \\
& \mathrm{PAH}^{*}+\mathrm{PAH}+(\mathrm{PAH}){ }_{2}^{*} \\
& \mathrm{PAH}^{*} \rightarrow \mathrm{PAH}+\mathrm{h} v_{1} \\
& (\mathrm{PAH})_{2}^{*} \rightarrow 2 \mathrm{PAH}+\mathrm{h} \mathrm{v}_{2} \\
& \text { Scheme } 2 \\
& \mathrm{PAH}+h v_{0}+\mathrm{PAH}^{\star} \\
& \mathrm{PAH}^{*}+\text { Quencher }-\mathrm{PAH}+\text { Quencher } \\
& 2 \mathrm{PAH}-(\mathrm{PAH})_{2} \\
& (\mathrm{PAH})_{2}+\mathrm{h} v_{1} \rightarrow(\mathrm{PAH})_{2}^{*} \\
& \mathrm{PAH}^{*} \rightarrow \mathrm{PAH}+\mathbf{h} \mathbf{v}_{3} \\
& (\mathrm{PAH})_{2}^{*} \rightarrow 2 \mathrm{PAH}+\mathrm{h} \mathrm{v}_{4}
\end{aligned}
$$

Scheme 1, which is invoked by most researchers, has the formation of the excimer between a ground state and a excited state monomer. If the excited monomer is quenched by nitromethane, as in this case, then the concentration of this particular type excimer should also be reduced. The 
data does not support Scheme 1. Rather, the excimer peaks of 4-methylacephenanthrylene, 5-methylacephenanthrylene, and 9-methylacephenanthrylene were not quenched by nitromethane suggesting scheme 2. In scheme 2, the formation of the excimer occurs between two ground state monomers and should be unaffected by the relative concentration of the excited state monomer. The data concerning excimer formation in 4methylacephenanthrylene, 5-methylacephenanthrylene, and 9methylacephenanthrylene is consistent with Scheme 2 . Fluorescence studies on additional PAHs and bi-PAH cyclophanes would shed more light on this hypothesis. BiPAH cyclophanes are molecules having two aromatic ring systems fixed in space with respect to one another (usually eclipsed or partially eclipsed) via two-alkyl chains.

\section{Bi-Polycyclic Aromatic Hydrocarbons}

One or two pasteur pipette drops of nitromethane (per 3 mLs of solution) was found to effectively quench the fluorescence emission of 1,1'-binaphthalene, 2,2'-binaphthalene, 9,9'-biphenanthrene, 1,1'-methylenebisnaphthalene, and $1,1^{\prime},-2,2^{\prime}$-tetrahydro-5,5'-biacenaphthylene dissolved in neat acetonitrile. After correction for primary innerfiltering, the calculated emission intensities was reduced to roughly 10-25\% of its original value. Emission quenching was less pronounced in the case of 9,9'-bianthracene and 
9,9'-bianthracene-10-carboxaldehyde as five pasteur drops of nitromethane only halved the original emission signal, again after correcting for primary inner-filtering effects. Electron transfer from 9,9'-bianthracene to nitromethane is obviously less favorable, which would be expected based upon the fact that the transferred electron must come from either the twisted intramolecular charge transfer excited state having full electronic charge separation or from the solutesolvent exciplex if one accepts the explanation of visser et al. (8). Structural reorganization would be required in order to accommodate the lost electronic charge. Even so quenching did occur, but to a much lesser degree and at higher nitromethane concentrations. Nitromethane did quench the fluorescence emission of all seven alterant bi-PAH solutes studies, and these results are in complete agreement with the so-called "nitromethane selective quenching rule."

Alternant and Nonalternant Polycyclic Aromatic Heterocycles

There are both alternant and nonalternant, monoaza- and diaza-polycyclic aromatic nitrogen heterocycles (PANHs). For the purposes of this dissertation aromatic carbon atoms and perimeter $-\mathrm{N}=$ atoms are treated identically. Fluorescence emission spectra of dibenzo[c, $f][2,7]$ naphthyridine in Figure 87 depicts the effect of nitromethane on an alternant unprotonated PANH. Examination of Table LV reveals that the 


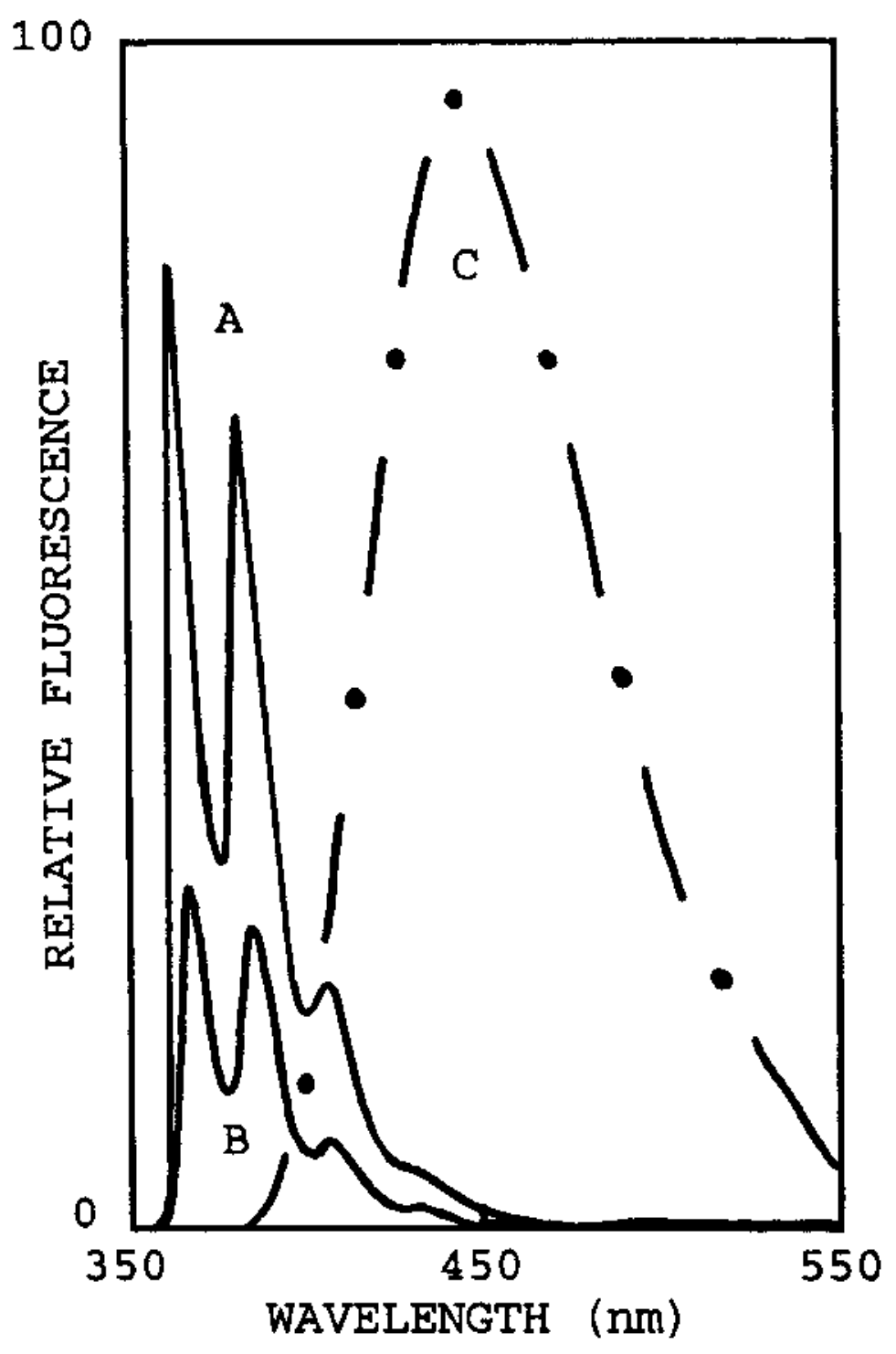

FIGURE 87: Fluorescence emission spectra of dibenzo[c, $f]$ [2,7]naphthyridine dissolved in [A (-)] acetonitxile, [B (-) ] acetonitrile with one pasteur pipet drop of nitromethane, and [C (- -)] acetonitrile-perchlorlc acid protonating solvent. In acetonitrile emission bands occur at about $366,384,405$, and $430 \mathrm{~nm}$. 
TABLE IV. Summary of emission wavelength and fluorescence quenching data for alternant and nonalternant, unprotonated and protonated PANHs.

\begin{tabular}{|c|c|c|c|c|}
\hline Letter ${ }^{a}$ & $\begin{array}{c}\text { PANH }^{b} \\
\text { NM }\end{array}$ & $\begin{array}{c}\text { PANH } \\
\text { TMB }\end{array}$ & $\underset{\mathrm{NM}}{\mathrm{PANH}-\mathrm{H}^{+}}$ & $\underset{\text { TMB }}{\text { PANH }-\mathrm{H}^{+}}$ \\
\hline
\end{tabular}

Alternant Monoaza-PANHs

\begin{tabular}{|c|c|c|c|}
\hline$A^{f}$ & YES & & No \\
\hline$B^{f}$ & YES & & No \\
\hline$c^{f}$ & YES & & NO \\
\hline$D^{f}$ & YES & YES & No \\
\hline$G$ & NO & & No \\
\hline $\mathrm{H}$ & No & & No \\
\hline$I^{f}$ & YES & & No \\
\hline $\mathrm{J}^{f}$ & YES & & No \\
\hline $\mathrm{K}$ & YES & & No \\
\hline $\mathrm{L}$ & YES & & No \\
\hline $\mathbf{M}$ & YES & & No \\
\hline$N$ & NO & & No \\
\hline
\end{tabular}

Nonalternant Monoaza-PANHs

$\begin{array}{lllll}\text { E } & \text { NO } & & \text { NAg } \\ \text { F } & \text { NO } & \text { YES } & \text { NO } & \text { YES }\end{array}$

Alternant Diaza-PANHs

O NO . NO

P YES NO

Q No No No NES 
TABLE LV, Continued.

\begin{tabular}{|c|c|c|c|c|}
\hline Letter ${ }^{a}$ & $\begin{array}{l}\text { PANH }^{b} \\
\text { NM }\end{array}$ & $\begin{array}{c}\text { PANH }^{\mathrm{C}} \\
\text { TMB }\end{array}$ & $\begin{array}{c}\text { PANH }-\mathrm{H}^{+} \\
\text {NM }\end{array}$ & $\begin{array}{c}\text { PANH }-\mathrm{H}^{+} \\
\text {TMB }\end{array}$ \\
\hline $\mathrm{R}$ & No & YES & No & YES \\
\hline $\mathrm{s}$ & YES & No & No & YES \\
\hline $\mathrm{U}$ & No & YES & No & YES \\
\hline $\mathrm{V}$ & NO & YES & No & YES \\
\hline$x$ & No & No & No ${ }^{h}$ & YES $^{h}$ \\
\hline $\mathrm{Y}$ & YES & . & No & \\
\hline$A C$ & No & YES & NAg & \\
\hline$A D$ & No & & No & \\
\hline$A \mathbf{E}$ & YES & & No & \\
\hline $\mathrm{AF}$ & No & YES & No & YES \\
\hline$A G$ & YES & & NO & \\
\hline \multicolumn{5}{|c|}{ Nonalternant Diaza-PANHs } \\
\hline $\mathrm{T}$ & No & YES & No & YES \\
\hline Z & NO & YES & $\mathrm{NA}^{\mathrm{g}}$ & \\
\hline AA & No & & No & \\
\hline $\mathrm{AB}$ & No & YES & No & YES \\
\hline \multicolumn{5}{|c|}{ Alternant Tetraaza-PANH } \\
\hline$W$ & No & NO & No & YES \\
\hline
\end{tabular}

a Letters refer to the molecular structures in Figures 2931. b Quenching data for the unprotonated PANH by nitromethane. c Quenching data for the unprotonated PANH by $1,2,4$-trimethoxybenzene. d Quenching data for the 
TABLE LV. Continued.

protonated PANH by nitromethane in a protonating solvent (1 drop $\mathrm{HClO}_{4}$ in $100 \mathrm{mLs}$ of acetonitrile). e Quenching data for the protonated PANH by 1,2,4-trimethoxybenzene in a protonating solvent ( 1 drop $\mathrm{HClO}_{4}$ in $100 \mathrm{mLs}$ of acetonitrile). f Solvent used was aqueous acetonitrile (20:80 by volume). All other solvents were neat acetonitrile unless noted. $g$ No spectra was observed. $h$ Protonated PANH quenching data was taken in methanol as no spectra was observed in the protonating solvent.

effect of nitromethane on unprotonated PANHs is very different than its effect on the alternant and nonalternant PAH derivatives. Fourteen of the 26 alternant unprotonated PANHs are not quenched by nitromethane which is contrary to the selective quenching rule. There are mono- and diazaPANHs among the exceptions. Interestingly, all five of the nonalternant unprotonated PANHs are not quenched by nitromethane as expected. There are mono- and diaza-PANHs in the nonalternant category as well. The small number of nonalternant PANHs may account for the fact that there are currently no exceptions in this particular subclass.

As argued by Breymann et al. (9) nitromethane quenches the fluorescence emission of alternant PAH6 benzenoids via an electron transfer process. The rate of electron transfer 
depends upon the change in the free energy, with the more favorable electron transfer process proceeding at a much faster reaction rate. The more positive reduction potentials of nonalternant PAHs result in less favorable electron transfer conditions and much smaller quenching rate constants. Emission intensities of nonalternant PAHs are for the most part unaffected by nitromethane, though a few exceptions are known. While electrochemical information on PANH versus PAH reduction potentials in common solvents is relatively scarce in the chemical literature, Fox (10) noted that the reduction of acridine of $E=-1.24 \mathrm{eV}$ (electron volts) in acetonitrile was significantly more positive than the corresponding value of $E=-1.92 \mathrm{eV}$ for the anthracene counterpart. We would expect other PANHs to behave in similar fashion. With approximately $50 \%$ of the alternant PANHs being exceptions to the nitromethane selective quenching rule, it must be concluded that the reduction potential range is fairly broad amongst the PANH solutes. Depending upon the PANH under examination, the PANH Lowest Unoccupied Molecular Orbital (LUMO) falls slightly above (or slightly below) the electron acceptor's LUMO, thereby encouraging (or discouraging) electron transfer.

Knowing that nitromethane reportedly functions as an electron acceptor, investigation into whether nitromethane quenched the fluorescence emission of protonated PANHs seem logical. From simple columbic considerations, it should be 
considerably more difficult to remove an electron from the cationic PANH $-\mathrm{H}^{+}$ion than from the neutral PANH molecule. As expected, the fluorescence emission intensities of the 30 protonated PANHs were unaffected by nitromethane after the observed values were corrected for primary inner-filtering (the other three PANHs contained in Table LV had no observable spectrum upon protonation). The coulombic argument is placed on a more theoretical basis by noting that protonation does cause a decrease in the Highest Occupied-Lowest Unoccupied Molecular Orbital (HOMO-LUMO) energy separation as evidenced by a sizeable redshift in the fluorescence emission wavelength. of the five possible ways to decrease the HOMO-LUMO energy gap, nitromethane quenching data on the protonated PANHs suggests that the LUMO must be moved to give a more positive reduction potential (see Figure 88 for a plausible molecular orbital diagram). This seems reasonable in light of recently published electrochemical data for various quaternized aza-arene compounds. Reduction potential of 4,4'-bipyridine shifted to a more positive value with addition of $\mathrm{CH}_{3}{ }^{+}$group to the nitrogen heteroatom, from $-2.394 \mathrm{eV}$ for $4,4^{\prime}$-bipyridine to $-1.248 \mathrm{eV}$ for the N-methyl-4,4'-bipyridinium ${ }^{+}$cation. A much larger shift was observed for $\mathrm{N}, \mathrm{N}$-dimethyl-4,4'-bipyridinium ${ }^{+2}$ cation $(-0.580 \mathrm{ev})$; however, the authors had to change solvents as the perchlorate salt was insoluble in dimethylformamide (11). 

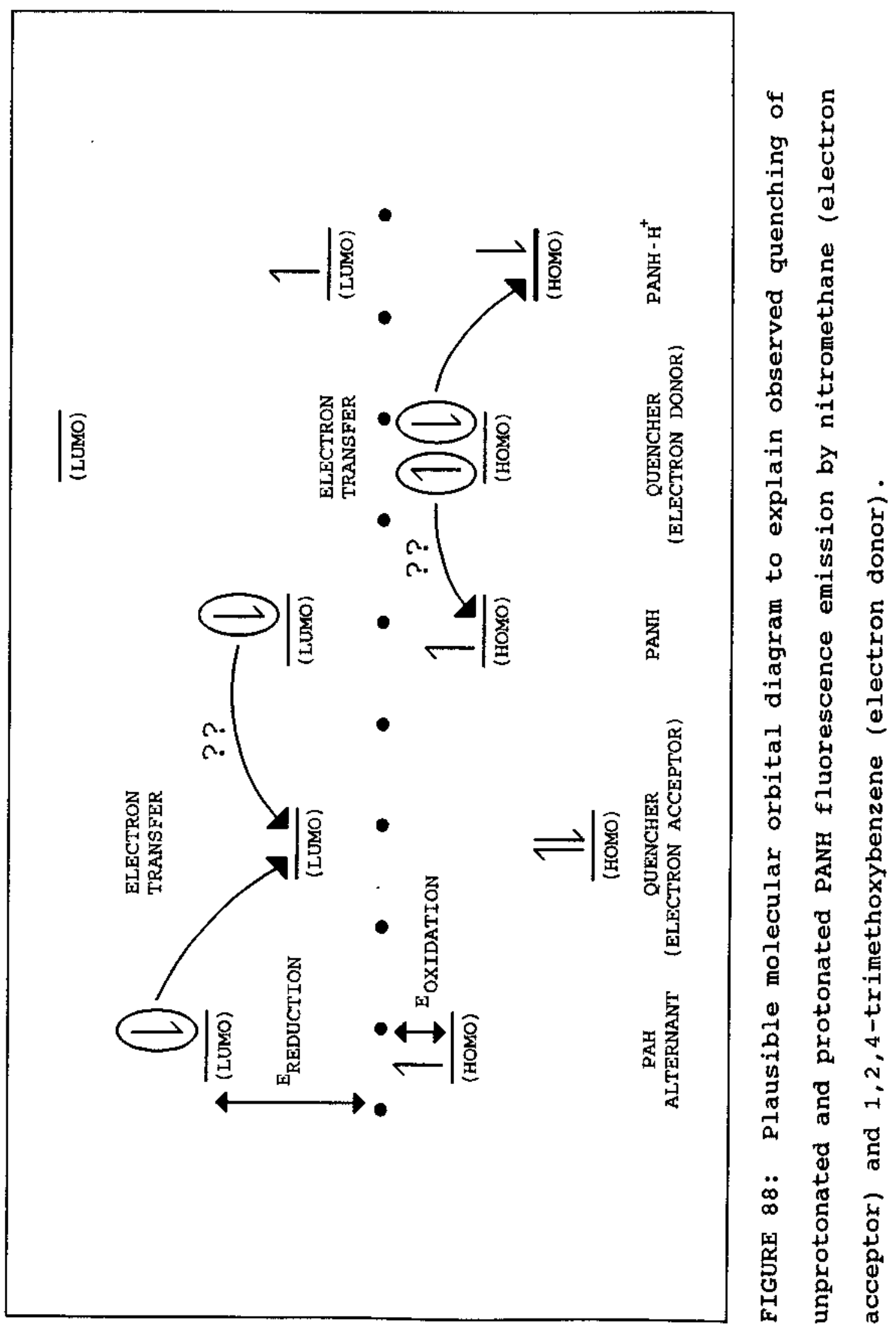
The relative location of the unexcited PANH's and PANH$\mathrm{H}^{+}$'s highest occupied molecular orbital can be ascertained by studying the solute's fluorescence behavior in the presence of an electron donor quenching agent, such as 1,2,4-trimethoxybenzene. Examination of Table LV reveals that trimethoxybenzene quenches the fluorescence emission of the protonated forms of all 12 protonated PANH solutes. Only eight of the 12 were significantly quenched as neutral PANH molecules. Strong absorbance by trimethoxybenzene in the 300-350 $\mathrm{nm}$ spectral region gave primary inner-filtering corrections of $f_{\text {prim }} \geq 3.0$ under the experimental conditions used. To minimize primary inner-filtering corrections studies were restricted to select solutes having their major excitation wavelength greater than $350 \mathrm{~nm}$. The 1,2,4trimethoxybenzene quenching data suggests that upon protonation the PANH's HOMO must move to give a larger oxidation potential. This experimental evidence supports the proposed molecular orbital-quenching mechanism in Figure 88 .

The introduction of a sulfur or an oxygen atom in place of a carbon atom in the perimeter of the PAHs alters the bond character of that position. It is not possible to place a double bond on the oxygen or sulfur thus reducing the number of resonance structures. For that reason, the labeling of the polycyclic aromatic sulfur and oxygen heterocycles (PASHs and PAOHs) as alternant and nonalternant 
is not relevant to the discussion of the selectivity of nitromethane. The effect of nitromethane and trimethoxybenzene on PASHs and PAOHs will not be discussed because it lends no information to the validity of the selective quenching rule. The effect of trimethoxybenzene on the fluorescence emission spectra of PAHs and the many PAH derivatives was not examined as the vast majority have excitation wavelengths in the $300-350 \mathrm{~nm}$ region were absorbances are prohibitive and the $f_{\text {prim }}>3$ where the corrections factors are valid.

\section{Examination of Select High Performance Iiquid Chromatography solvents}

The aforementioned studies involving nitromethane as a selective quenching agent for discriminating between alternant versus nonalternant PAHs utilized either neat acetonitrile or a binary aqueous-acetonitrile mixture (20:80 percent by volume). Such solvents work well as mobile phases in high performance liquid chromatographic (HPLC) separations for those PAHs that contain six rings or less, but different solvent strengths must be employed for larger PAHs having 6-10 rings. Acetonitrile-toluene or acetonitrile-ethyl acetate mixtures have served well in the past as HPLC mobile phase solvents for the separation of the larger PAHs $(12,13)$. Table LIII compares the experimental 
results regarding the ability of nitromethane to act as a selective quenching agent for alternant versus nonalternant PAHs in the aqueous-acetonitrile mixture utilized earlier, a acetonitrile-toluene and acetonitrile-ethyl acetate mixture (60:40 percent by volume). While a published paper (14) has alluded to the fact that solvent polarity affects nitromethane's ability to act as a selective quenching agent, there was no in-depth study ever reported. Examination of Table LIII reveals that the acetonitriletoluene mixture (Py $=1.49$ ) and acetonitrile-ethyl acetate (Py $=1.68)$, although less polar than the aqueousacetonitrile mixture (observed $\mathrm{Py}=1.60$ (15); $\mathrm{Py}=1.80$ after correction for slit width effects) (16) or pure acetonitrile $(P y=1.79)$ (17), can effectively be used as an HPLC solvent when employing nitromethane to selective quench the fluorescence emission signals of alternant versus nonalternant PAHs. Of the solutes examined, only nine behave differently in the acetonitrile-toluene solvent mixture and 11 in the acetonitrile-ethyl acetate solvent mixture as compared to the aqueous-acetonitrile solvent mixture or neat acetonitrile. Interestingly, seven of the nine exceptions in both solvent mixtures are coronene derivatives. Earlier studies utilized the aqueousacetonitrile mixtures, but it was found that for all of the previously mentioned subclasses, except for the 
aforementioned coronene derivatives, the selective quenching rule is obeyed in neat acetonitrile as well.

Two nonalternant compounds, naphtho $[2,3 b]$ fluoranthene and benzo[k]fluoranthene, that were exceptions to the nitromethane selective quenching rule in both aqueousacetonitrile and toluene-acetonitrile mixtures are no longer exceptions when dissolved in the acetonitrile-ethyl acetate solvent. In both acetonitrile-toluene and acetonitrileethyl acetate solvents, benzo[rst]dinaphtho $[2,1,8,7$ defg;$\left.2^{\prime}, 1 ', 81,71 i j k 1\right]$ pentaphene (BNP) was not quenched by nitromethane. BNP should have been quenched based upon the fact that it is an alternant compound. Other than the coronene derivatives, BNP and dinaphtho[2,1,8,7defg;$2^{\prime}, 1^{\prime}, 8^{\prime}, 7 '$ opgr]pentacene remain the only other major exceptions in the acetonitrile-ethyl acetate binary solvent mixture when compared to the more polar aqueous-acetonitrile system. Results of the present investigation clearly indicate that a solute's quenching behavior can be directly affected by the polarity of the surrounding solvent.

Effect of Substituents on Nitromethane's Ability to Selectively Quench Alterant versus Nonalternant Polycyclic Aromatic Hydrocarbons

The electron transfer mechanism postulated in Chapter 1 requires favorable reaction kinetics and thermodynamic 
conditions. From a strictly thermodynamic point-of-view, it is conceivable that the extent of quenching can be altered simply by adding functional groups to the molecule in order to either stabilize or destabilize the charge (or partial charge) that is temporarily formed on the polycyclic aromatic hydrocarbon. Electron donating groups (such as $\left.\mathrm{CH}_{3},-\mathrm{OCH}_{3},-\mathrm{OH}\right)$ should stabilize a positive charge on the PAH ring system, whereas electron withdrawing substituents (such as $-\mathrm{CN},-\mathrm{CHO},-\mathrm{NO}_{2}$ ) would tend to stabilize a negative charge by removing the "extra" electron density from the aromatic $\pi$-cloud system. To date it has not been determined how the various ring-substituents affect nitromethane's selectivity for discriminating between alternant versus nonalternant PAHs.

Electron withdrawing substituents should hinder the electron transfer process from occurring, and electron donating substituent should expedite the electron transfer in alternant PAHs. Inspection of Table LVI reveals that, for the most part strongly deactivating, electron withdrawing groups do effectively hinder the electron transfer process. The electron donating substituents expedite the electron transfer process, but this is no surprise since the electron transfer does take place in all the alternant parent compounds studied. The chloro- group on 1-chloropyrene, being the only weakly deactivating substituent, cannot sufficiently hinder the electron 


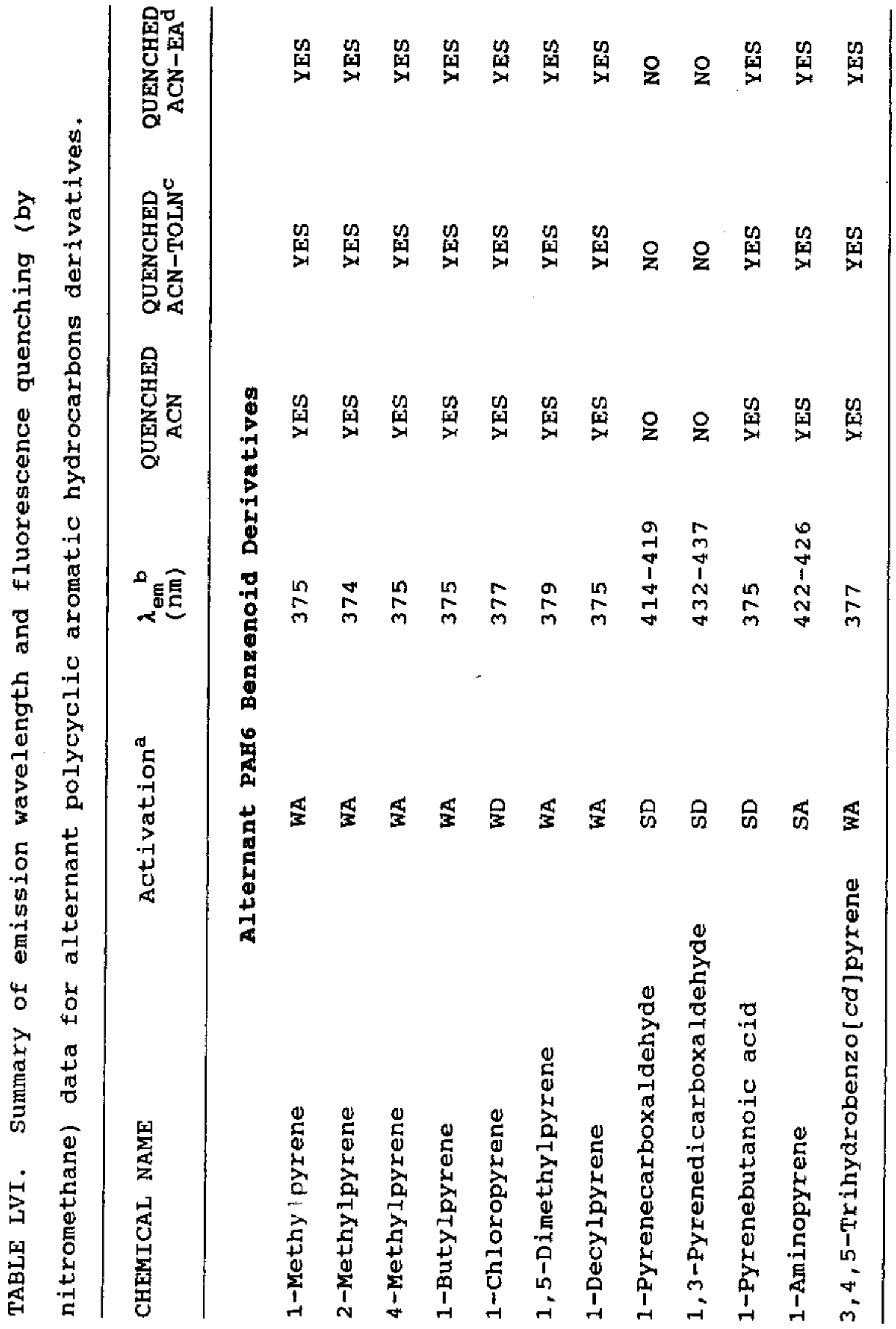




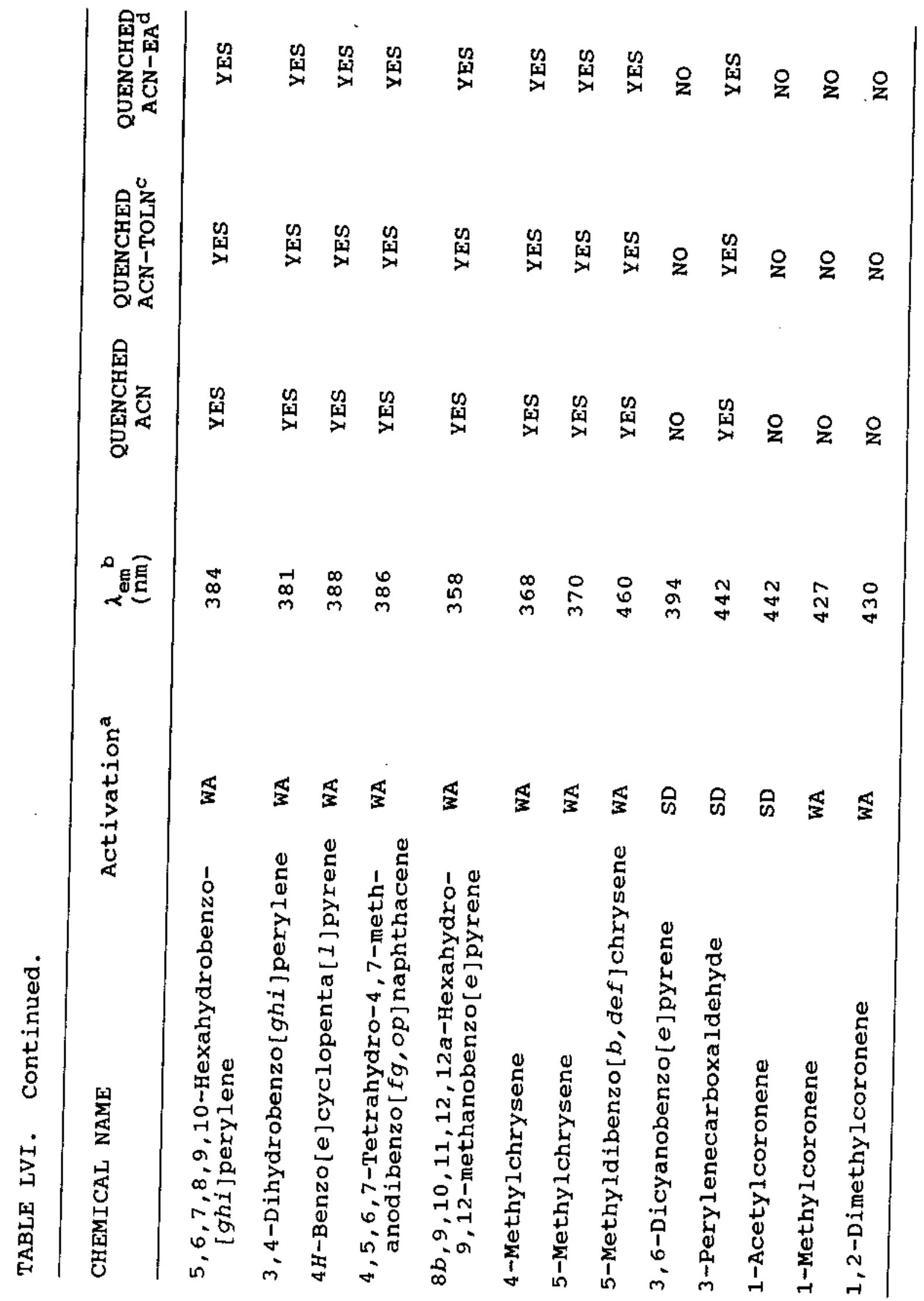




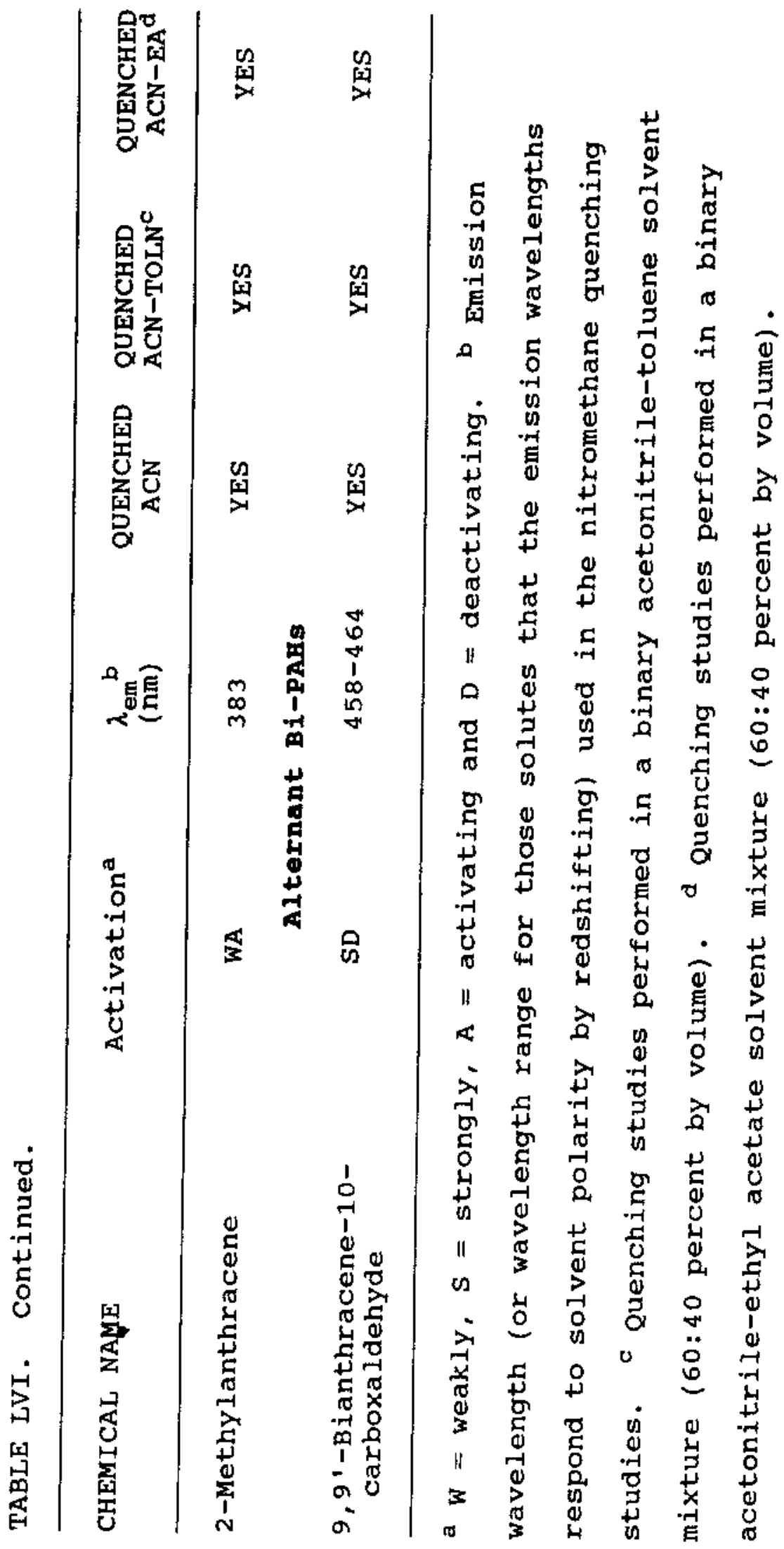


transfer process. It would have been nice to have studied more weakly deactivating compounds, but most halogenated PAHs available (e.g. bromopyrenes) will suffer from internal heavy atom quenching effects.

Three solutes containing strongly deactivating, electron withdrawing substituent are exceptions - 1-pyrenebutanoic acid, 9,9'-bianthracene-10-carboxaldehyde, and 3perylenecarboxaldehyde. In the case of the pyrenebutanoic acid it is conceivable that the electron withdrawing substituent $(-\mathrm{COOH})$ is too far removed (three carbon atoms away) from the PAH to effectively hinder the electron transfer. Also, in the case of 9,9'-bianthracene-10carboxaldehyde the electron withdrawing ability of the aldehyde substituent may be hindered by the fact that this molecule exhibits dual fluorescence resulting from "twisted intramolecular charge transfer" (TICT) excited states, and strong spectral overlap of the TICT band and the anthracenelike "locally excited" (LE) fluorescence band (see chapter 3 for a full discussion). Therefore, the only true exception among the strongly deactivating, electron withdrawing substituents is 3-perylenecarboxaldehyde.

To determine if the electron transfer process can be encouraged through additional electron density on nonalternant PAHs that are not quenched by nitromethane, several fluoranthenoid derivatives were examined. Inspection of Table LVII reveals that, for the most part, 


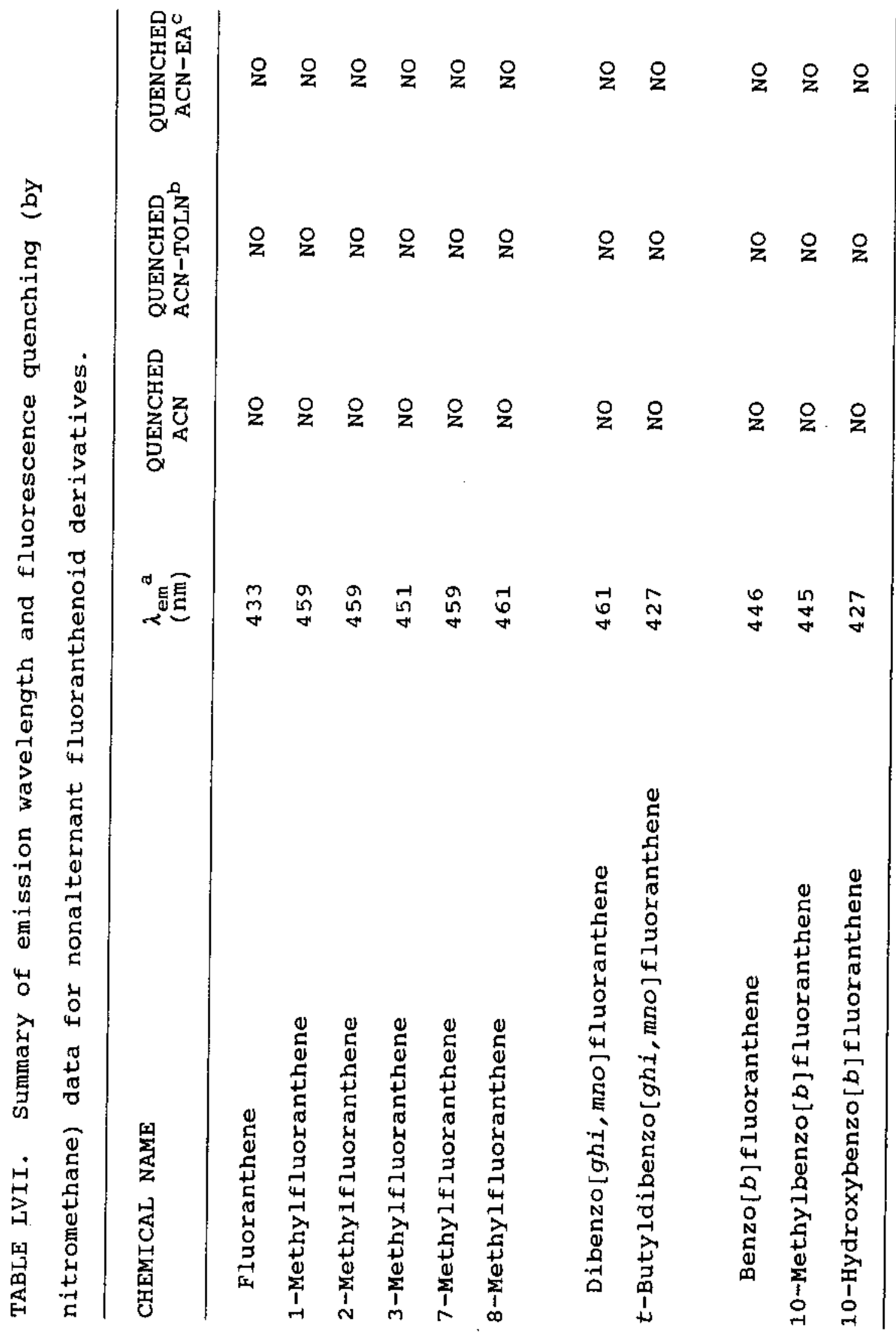




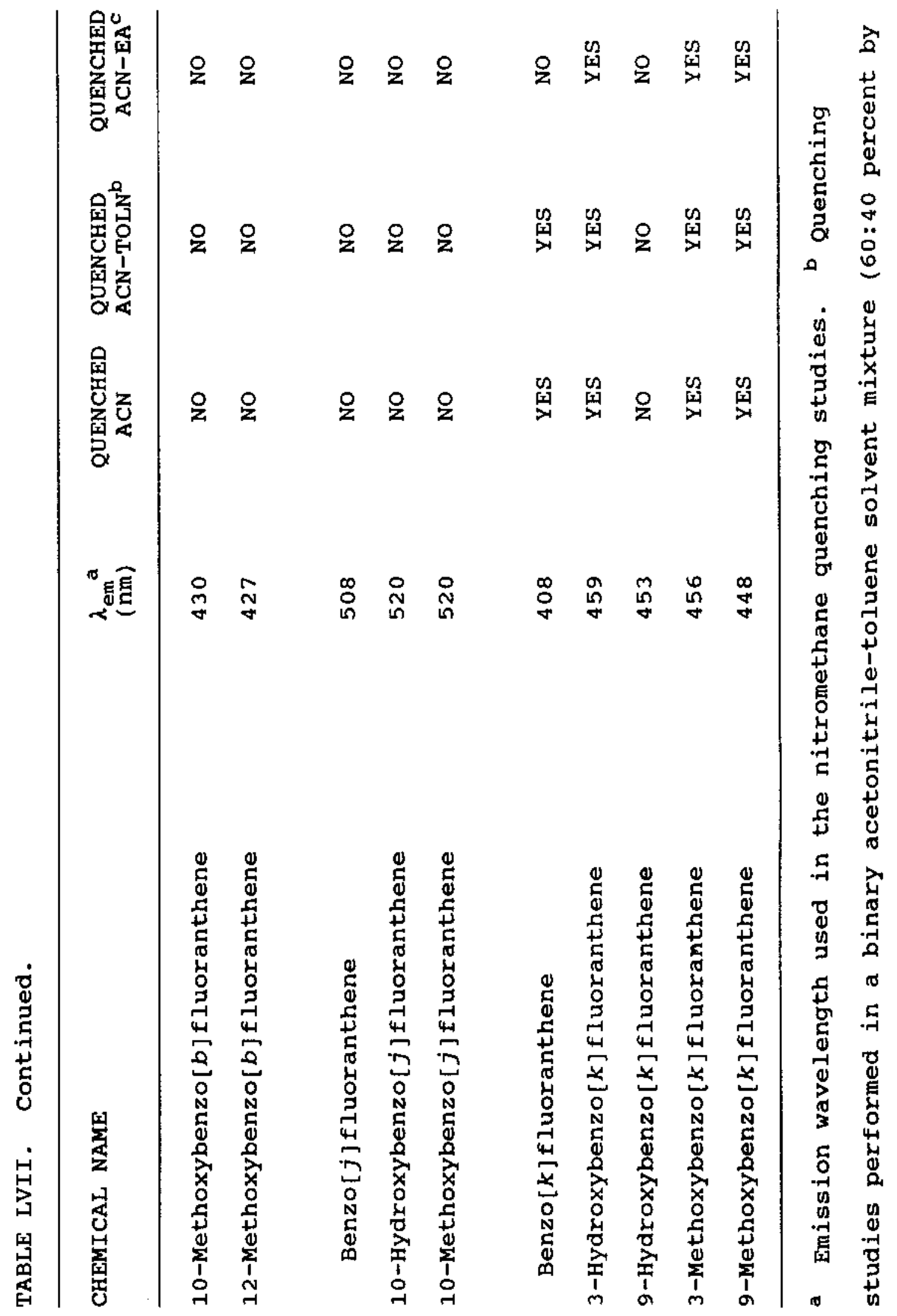


TABLE LVII. Continued.

volume). c Quenching studies performed in a binary acetonitrile-ethyl acetate solvent mixture (60:40 percent by volume) .

the quenching behavior of the derivatized fluoranthenoids is identical to that of the parent compound. With the exception of the benzo[k]fluoranthene subset, none of the nonalternant parent compounds or derivatives are quenched by nitromethane which is what one would expect based upon the selective quenching rule. Thus, neither the additional electron density nor the placement of the LuMo of nonalternant PAH with respect to the nitromethane's LUMO was sufficient to enable electron transfer. These results should not be too surprising in that electron transfer does not normally occur between nitromethane and the parent nonalternant PAHs. It is conceivable that the electron donating substituent does not add electron density to the entire aromatic ring system through delocalization, but instead remains localized on the benzene ring to which the functional group is actually attached. Also, the energy difference between the fluoranthenoid's LUMo and nitromethane's LUMO is not known. It is possible to alter that energy difference, but the alteration may or may not result in conditions favorable for electron transfer during 
the lifetime of the fluorophore's excited state. Electron transfer must occur before the fluorescent photon is emitted if the emission signal is to be quenched.

Nitromethane quenching results for benzo[k]fluoranthene and its derivatives is very different than that of the other fluoranthenoid solutes listed in Table LVII. Benzo[k]fluoranthene's fluorescence emission is quenched in neat acetonitrile and the acetonitrile-toluene $60: 40$ percent by volume) mixture, but not in the acetonitrile-ethyl acetate (60:40 percent by volume) solvent system. The 3-hydroxy-, 3-methoxy-, and 9-methoxybenzo[k]fluoranthenes' fluorescence emission are quenched in all three solvent systems. Only the 9-hydroxybenzo[k]fluoranthene remains unquenched in all three solvent mixtures. In light of the previous nitromethane quenching studies, benzo[k]fluoranthene's quenching behavior is not surprising. Those solutes that show solvent probe behavior tend to be the exceptions to the nitromethane selective quenching rule. For example, of the thirty-six alternant PAH6 benzenoids approximately nine exceptions are seen in each of three solvent systems. Eight of the nine exceptions were solutes that exhibit solvent polarity probe character, and seven of those exceptions are coronene derivatives. In fact, there were only seven coronene derivatives in the entire quenching data set, and all showed solvatochromic behavior. This is also the case among the seventeen nonalternant fluoranthenoid and 
fluorenoid parent compounds. The two exceptions to the nitromethane selective quenching rule, namely those nonalternant solutes that are quenched by nitromethane, are naphtho[ $2,3 b]$ fluoranthene and benzo[k]fluoranthene, both of which are classified as solvent polarity probes.

The Excitation Method

Utilization of the excitation spectra for the examination of selective quenching by nitromethane gives results consistent with the previously discussed "emission method". Representative fluorescence excitation spectra of benzo[ $I m n][3,8]$ phenanthroline, benzo[a]fluoranthene, and naphtho $[2,3 b]$ fluoranthene dissolved in acetonitrile are shown in Figures 89-91. Examination of the spectral data reveals that most PACs have more than one excitation band. For example, benzo[ $1 m n][3,8]$ phenanthroline has a major excitation peak at $326 \mathrm{~nm}$ plus two minor excitation peaks at 360 and $378 \mathrm{~nm}$. Normally one might choose the excitation band with the highest intensity, $\lambda=326 \mathrm{~nm}$, to examine the emission spectra, but one must also take solvent effects into account. Solvents like benzene give rise to an appreciable background when excited near $300 \mathrm{~nm}$. A better choice for the benzo[ $1 \mathrm{mn}][3,8]$ phenanthroline might be the peak at $378 \mathrm{~nm}$ because it is a fairly intense excitation peak that does not excite most solvents. 


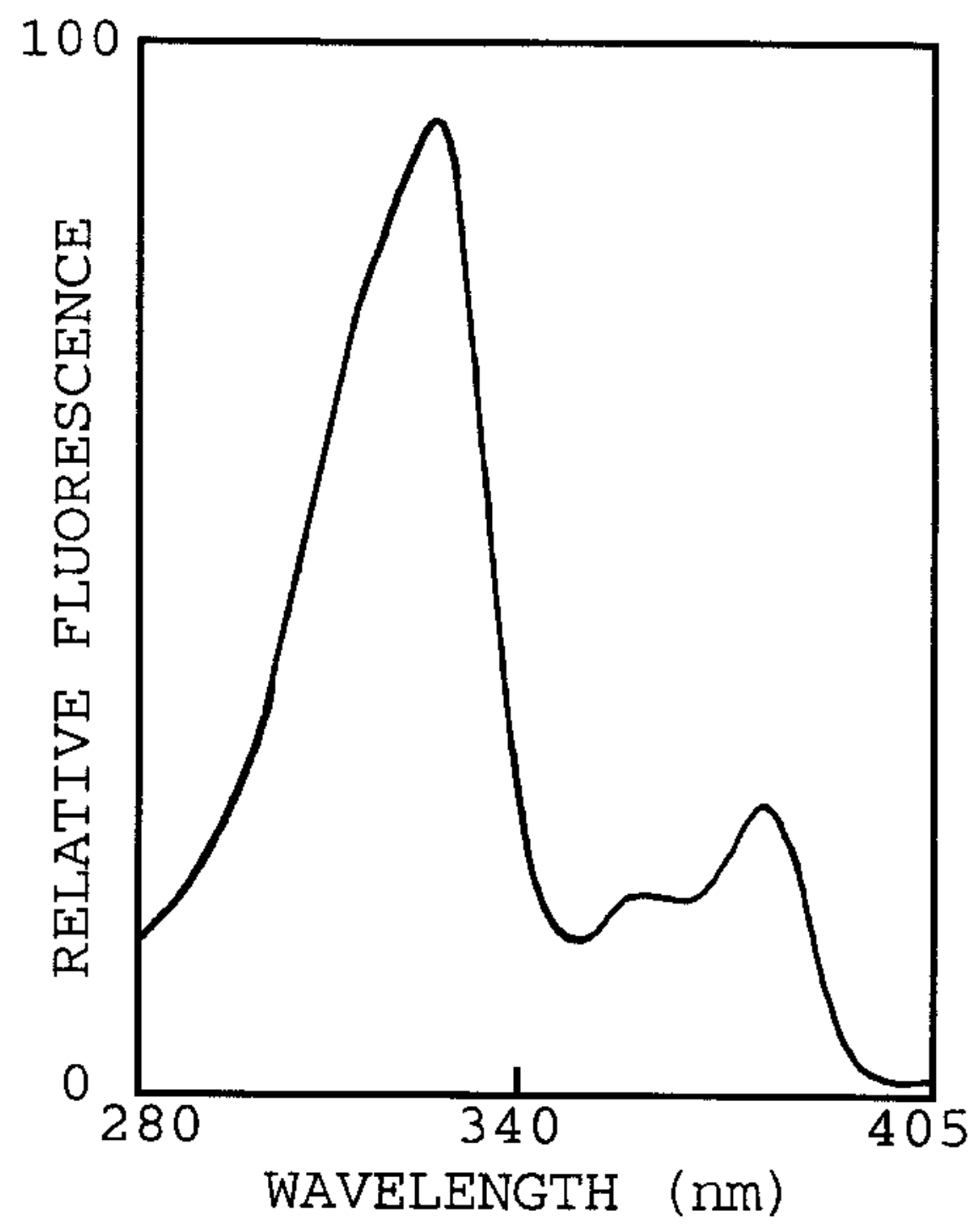

FIGURE 89: Fluorescence excitation spectra of benzo[1mn]$[3,8]$ phenanthroline dissolved in acetonitrile. Major excitation bands occur at 326,360 , and $378 \mathrm{~nm}$. 


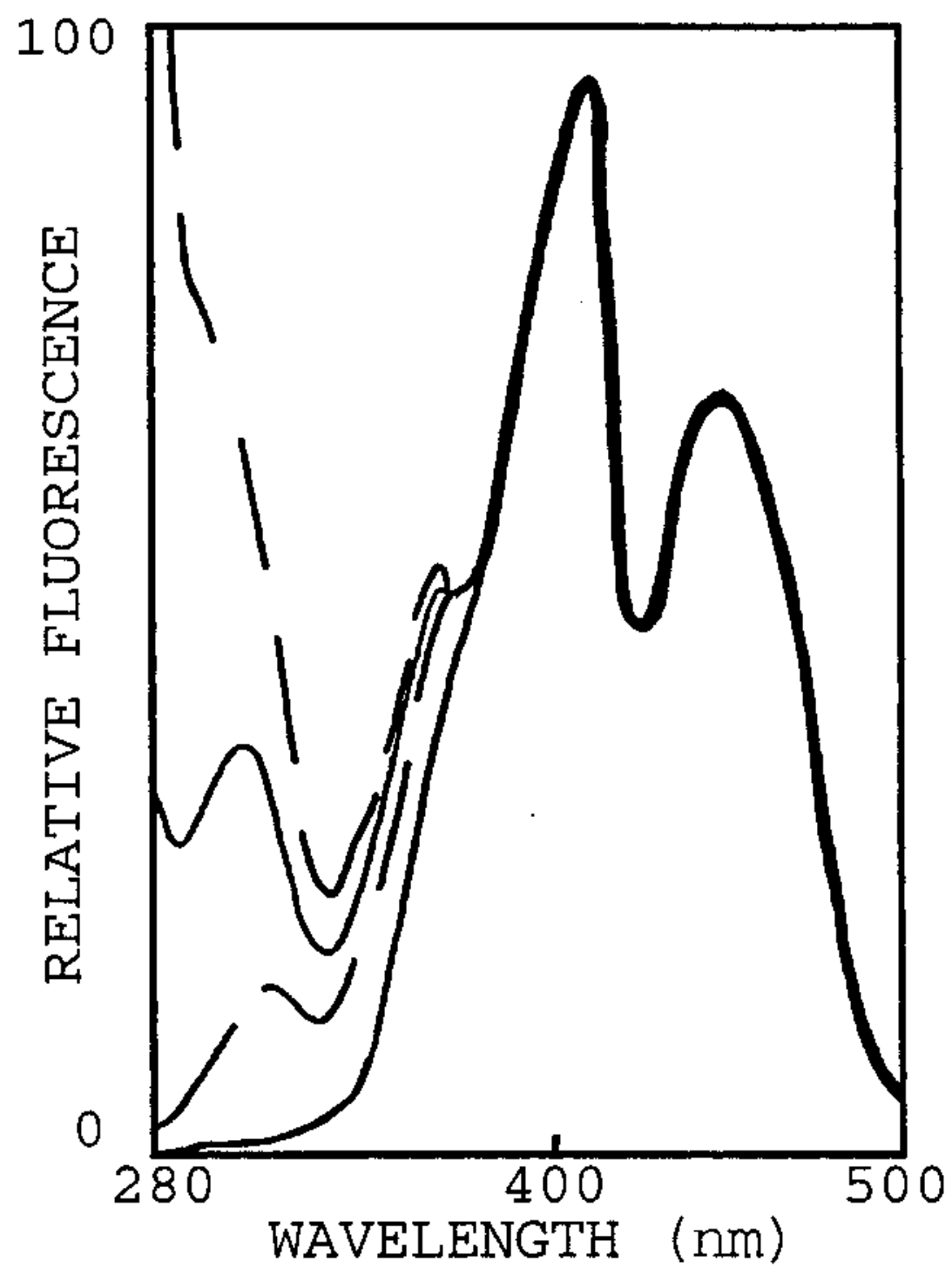

FIGURE 90: Fluorescence excitation spectra of benzo[a]fluoranthene dissolved in acetonitrile at various nitromethane concentrations. From top to bottom, the curves correspond to $0,1,3$, and 10 pasteur pipet drops of nitromethane. 


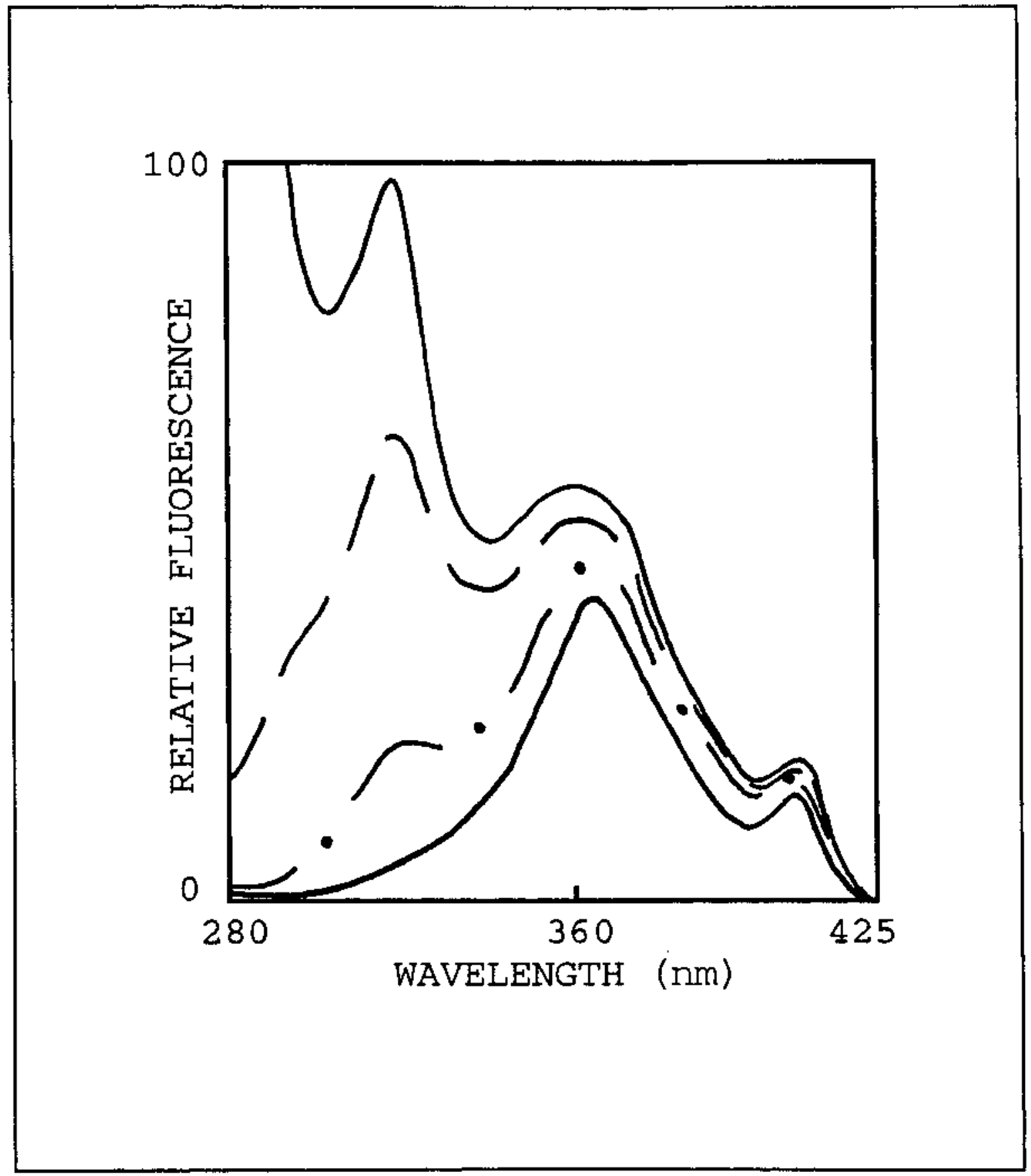

FIGURE 91: Fluorescence excitation spectra of naphtho$[2,3 b]$ fluoranthene dissolved in acetonitrile at various nitromethane concentrations. From top to bottom, the curves correspond to $0,1,3$, and 10 pasteur pipet drops of nitromethane. 
Selection of an appropriate emission peak must also take solvent and quencher spectral characteristics into account. Since this particular study examined the quenching of the excitation by nitromethane and 1,2,4-trimethoxybenzene, one must look at where these molecules absorb radiation in order to minimize primary and/or secondary inner-filtering artifacts. Figure 92 indicates that nitromethane and 1,2,4-trimethoxybenzene both have significant absorbance from $300 \mathrm{~nm}$ to approximately $365 \mathrm{~nm}$. Any excitation or emission peaks in this region will demonstrate inner-filtering artifacts. For example, the first peak in the benzo[k]fluoranthene excitation spectra (Compound I in Figure 92) is heavily inner-filtered by both selective quenching agents. The second and third excitation peaks at 378 and $397 \mathrm{~nm}$ should show minimal (if any) innerfiltering; any decrease in observed fluorescence intensity must be a result of quenching. Examination of Table LVIII shows that this is indeed the case. When one drop of nitromethane is added to a solution of benzo[k]fluoranthene dissolved in acetonitrile, the emission intensity corresponding to the $301 \mathrm{~nm}$ excitation wavelength is reduced by $40 \%$, while intensities of the 378 and 397 nm peaks are only reduced by $4 \%$ each. After ten drops of nitromethane, the first peak is completely removed from the excitation spectra, and the second and third peaks are reduced by approximately $30 \%$. Clearly, nitromethane does marginally 


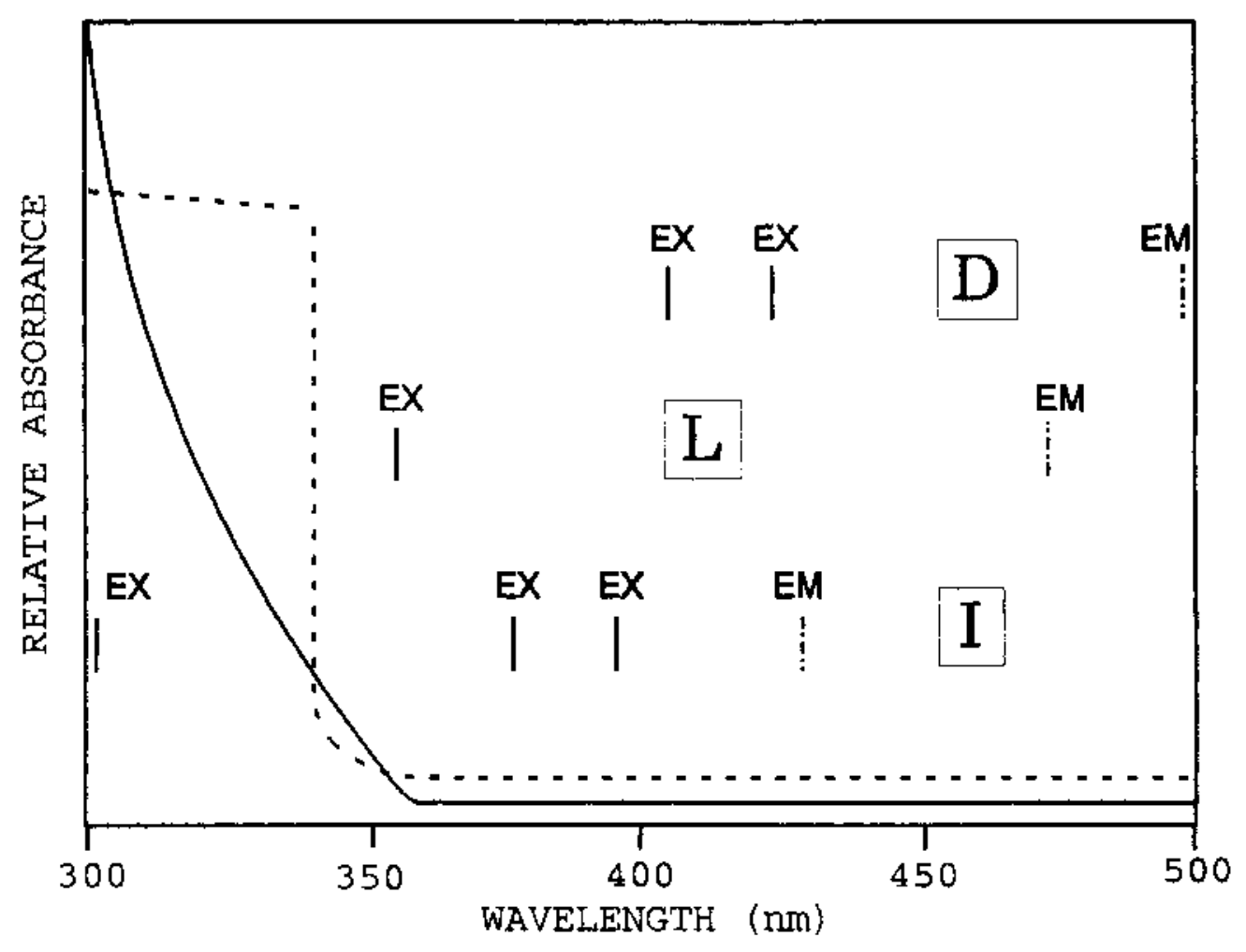

FIGURE 92: Absorption spectra of nitromethane (solid line) and 1,2,4-trimethoxybenzene (broken line). Superimposed on the absorption spectra are the excitation (EX) and emission (EM) wavelengths of benzo[k]fluoranthene (Compound I), indeno $[1,2,3 i j]$ isoquinoline (Compound $L$ ), and perylene (Compound D). To minimize primary and secondary innerfiltering artifacts, the fluorophore's excitation and emission wavelengths must occur in the spectral region in which the quenching agent is "optically transparent". 


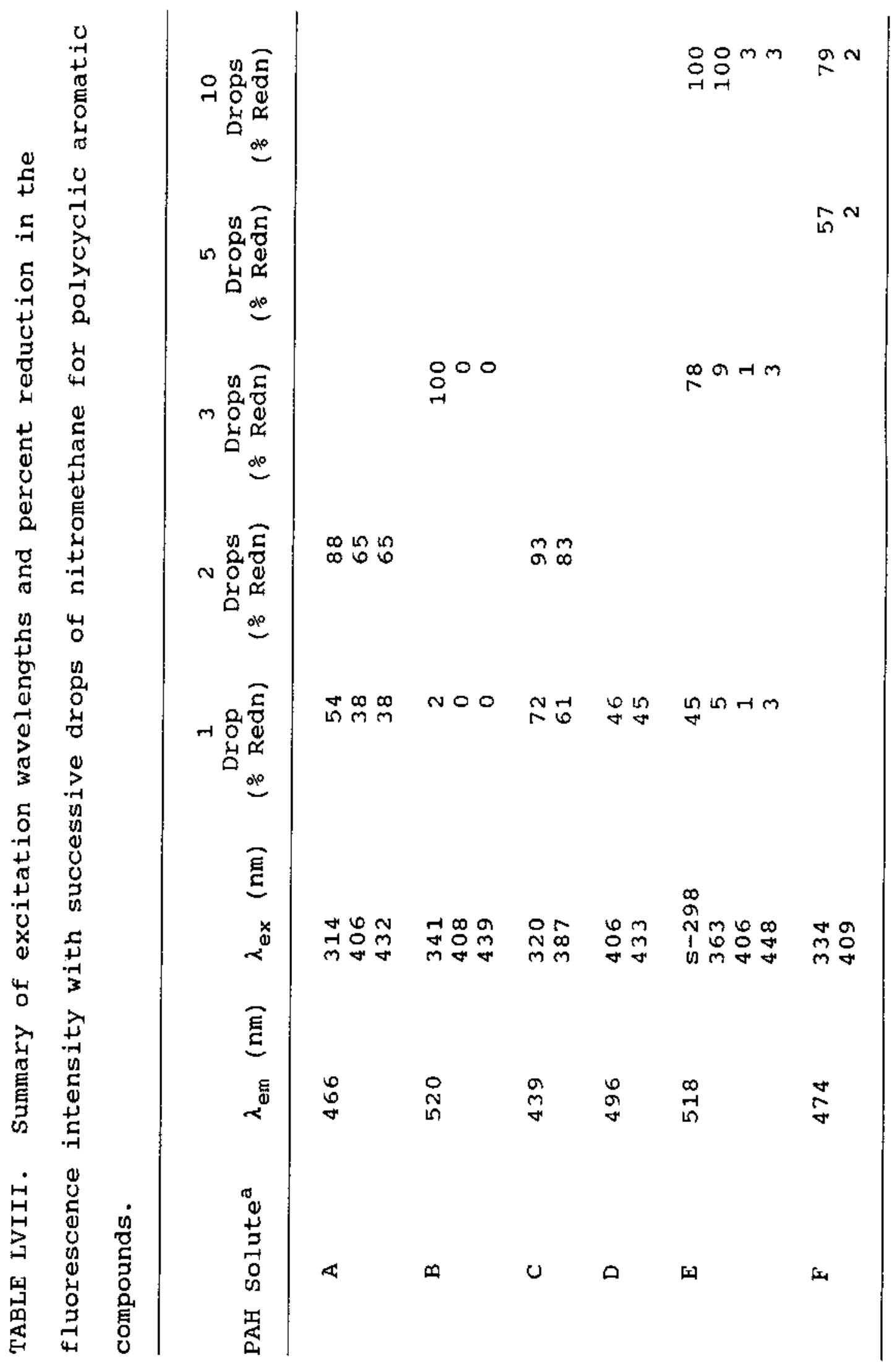




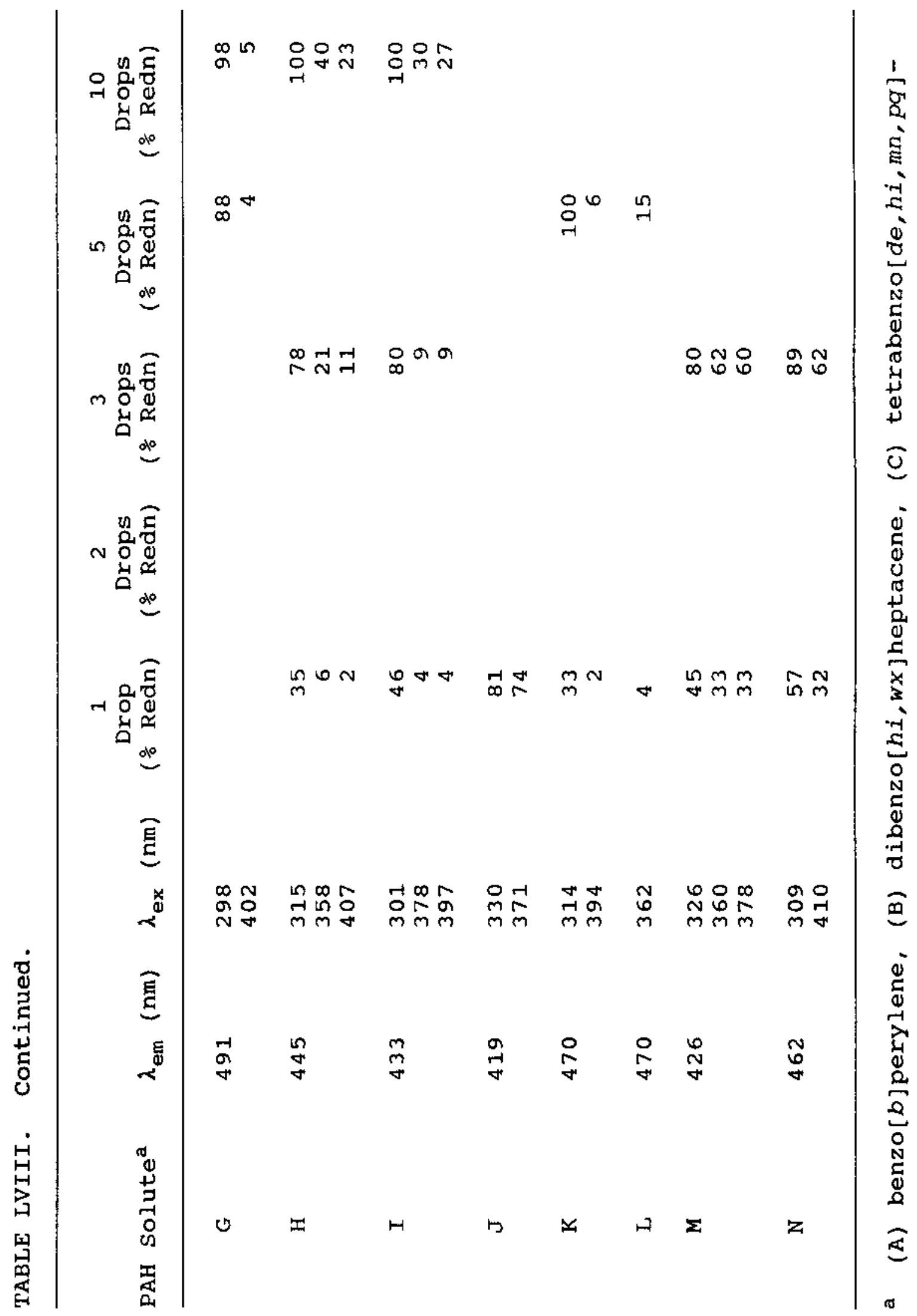


TABLE LVIII. Continued.

naphthacene, (D) perylene, (E) benzo[a]fluoranthene, (F) benzo[def] indeno $[1,2,3 h i]$ chrysene, (G) naphtho[2,1a]fluoranthene, (H) naphtho[2,3b]fluoranthene, (I) benzo[k]fluoranthene, (J) 2-azapyrene, (K) naphtho[8,1,2hij]pyreno$[9,10,1 d e f]$ phthalazine, (L) indeno $[1,2,3 i j]$ isoquinoline, benzo[ $1 m n][3,8]$ phenanthroline, and (N) 5-methyldibenzo$[b$, def $]$ chrysene. Solvent was acetonitrile in all quenching studies.

quench benzo[k]fluoranthene's fluorescence emission, making this PAH solute an exception to the nitromethane selective quenching rule.

Quenching is much more pronounced in the more polar aqueous/acetonitrile solvent mixture (20:80 by volume), which is the recommended solvent for the alternant vs. nonalternant PAH selective quenching studies. As mentioned previously, acetonitrile is substituted for the mixed solvent in order to solubilize the larger PACs. With very few exceptions, identical nitromethane quenching results have been obtained irrespective of whether neat acetonitrile or an aqueous/acetonitrile mixed was used as the solvent. For comparison, Figure 90 depicts the effect of nitromethane on the excitation peaks of an unquenched nonalternant PAF. The first peak $298 \mathrm{~nm}$ in the benzo[a]fluoranthene excitation 
spectra is so heavily inner-filtered that it ceases to exist after ten drops of nitromethane have been added. Peak intensities for excitation at 363,406 , and $446 \mathrm{~nm}$, where nitromethane does not absorb, remain constant.

The "excitation method" for examining quenching phenomena requires that emission peaks must be greater than approximately $400 \mathrm{~nm}$ so that at least one (preferably two) of the excitation peaks 280-380 $\mathrm{nm}$ excitation range is not heavily inner-filtered. Indeno[1,2,3ij]isoquinoline (Compound $\mathrm{L}$ in Figure 92) does have a useable emission peak above $400 \mathrm{~nm}$; however, it would not be a good candidate for this method since its single excitation is at $362 \mathrm{~nm}$. It is questionable whether inner-filtering or quenching artifacts predominate at this particular wavelength. of the 14 PACs listed in Table LVIII, benzo[b]perylene, dibenzo[hi,wx]heptacene, perylene, benzo[a]fluoranthene, benzo[def]indeno$[1,2,3 h i] c h r y s e n e$, naphtho $[2,1 a]$ fluoranthene, naphtho$[2,3 b]$ fluoranthene, benzo $[k]$ fluoranthene, naphtho $[8,1,2 h i j]-$ pyreno $[9,10,1$ def $]$ phthalazine, and 5-methyldibenzo[b, def $]$ chrysene are probably the better suited fluorophores for this type of study as the emission peaks and at least one of the excitation peaks are well out of the inner-filtering region. A decreased fluorescence signal at the higher excitation wavelength will clearly demonstrate only quenching effects. 
The results obtained by the "excitation method" are consistent with past studies on the emission spectra of PACs, but it is not without limitations. Compounds that were quenched by nitromethane previously are still quenched by nitromethane and vice versa. Examination of the excitation spectra is less tedious and time-consuming than the emission spectra examination; however, several PACs cannot be examined this way because the emission peaks are not far enough removed from the inner-filtering region. For example, pyrene, with emission peaks at 371,382 , and 391 $\mathrm{nm}$, has its optimum excitation wavelength(s) in the 280-370 nm spectral region. Strong quencher absorbance would result in appreciable inner-filtering artifacts at all excitation wavelengths considered. For compounds having suitable emission peaks, the "excitation method" offers a very convenient, fast and reliable method to study fluorescence quenching phenomena. The technique does require a prior knowledge of both the excitation and emission spectra.

It should be noted that the "excitation method" can lead to an erroneous conclusions regarding the ability of 1,2,4-trimethoxybenzene to selectively quench the fluorescence emission of nonalternant PACs if one is not careful. Trimethoxybenzene has a fluorescence excitation and emission spectra at several wavelengths employed in Table LVIII. When one or more Pasteur pipet drops of 1,2,4trimethoxybenzene are added to the dissolved PAC solution, 
new peaks may appear in the observed fluorescence spectra. Making exact solutions of 1,2,4-trimethoxybenzene for the blank and solute + blank for back substraction is tiresome and more time-consuming than gathering absorbance data and making the inner-filtering correction on the emission spectra. Nitromethane did not pose this problem, as it does not fluorescence at the excitation and emission wavelengths utilized. The "excitation method" is recommended over the "emission method", when applicable, as it is easier and less time-consuming. 


\section{CHAPTER REFERENCES}

1. Blümer, G.-P.; Zander, M. Fresenius Z. Anal. Chem. $1979,296,1433$.

2. Dresskamp, H.; Koch, E.; Zander, M. Z. Naturforsch. 1975, 30A, 1311-1314.

3. Michl, J.; Thulstrup, E. W. Spectroscopy with Polarized Light; VCH: New York, 1986; Chapter 8.

4. Sangaiah, R.; Gold, A.; Toney, G. E. J. Org. Chem. $1983,48,1632-1638$.

5. Mulder, P. P. J.; Boere, B. B.; Baart, A.; Cornelisse, J.; Lugtenburg, J. Recl. Trav. Chim. Pays-Bas 1993, $112,22-32$.

6. Mulder, P. P. J.; Olde Boerrigter, J.; Boere, B. B.; Zuilhof, H.; Erkelens, C. ; Cornelisse, J.; Lugtenburg, J. Recl. Trav. Chim. Pays-Bas 1993, 112, 287-302.

7. Jans, A. W. H.; Tintel, C.; Cornelisse, J.; Lugtenburg, J. Magn. Reson. Chem. 1986, 24, 101 .

8. Visser, R. J.; Weisenborn, P. C. M.; van Kan, P. J. M.; Huizer, B. H.; Varma, C. A. G. O.; Warman, J. M.; DeHaas, M. P. J. Chem. Soc., Faraday Trans. 1985, 81, 689-704.

9. Breymann, U.; Dreeskamp, H.; Koch, E.; Zander, M. Chem. Phys. Lett. 1978, 59, 68-70.

10. Fox, M. A. In Topics in Organic Electrochemistry; Fry, A. J.; Britton, W. E., Eds.; Plenum Press: New York, 1986 ; Chapter 4 .

11. Braterman, P. S.; Song, J.-I. J. Org. Chem. 1991, 56, 4678-4682.

12. Fetzer, J. C.; Biggs, W. R. Chromatographia 1989, 27, 118-122.

13. Peaden, P. A.; Lee, M. L.; Hirata, Y.; Novotny, M. V. Anal. Chem. 1980, 52, 2268-2271.

14. Blumer, G.-P.; Zander, M. Fresenius Z. Anal. Chem. $1979,296,409-410$. 
15. Street, K. W., Jr.; Acree, W. E. Jr.; J. Liq. Chromatogr. 1986, 9, 2799-2808.

16. Street, K. W. Jr.; Acree, W. E. Jr.; Analyst 1986, I11, $1197-1201$.

17. Dong, D. C.; Winnik, M. A. Can. J. Chem. 1984, 62, 2560-2565. 


\section{BIBLIOGRAPHY}

Acree, W. E., Jr. Environ. Sci. Technol. 1993, 27, 757-758.

Acree, W. E., Jr.; Tucker, S. A.; Cretella, L.; Zvaigzne, A. I.; Street, K. W., Jr.; Fetzer, J. C.; Nakasuji, K.; Murata, I. Appl. Spectrosc. 1990, 44, 951-957.

Acree, W. E., Jr.; Tucker, S. A.; Fetzer, J. C. Polycyclic Aromat. Compds. 1991, 2, 75-105.

Acree, W. E., Ir.; Tucker, S. A.; Zvaigzne, A. I.; Street, K. W., Jr.; Fetzer, J. C.; Grützmacher, H.-F. Appl . Spectrosc. 1990, 44, 477-482.

Acree, W. E., Jr.; Zvaigzne, A. I.; Fetzer, J. C. Appl . Spectrosc. 1990, 44, 1193-1195.

Albrecht, K.; Reiser, 0.; Weber, M.; de Meijere, A. Synlett $1992,521-523$.

Amin, S.; Huia, K.; Hussain, N.; Balanikas, G.; Carmella, S. G.; Hecht, S. S. J. Org. Chem. 1986, 51, 1206-1211.

Ananthapadmanabhan, K. P. ; Goddard, E. D. ; Turro, N. J. ; Kuo, P.-L. Langmuir 1985, 1, 352-355.

Ananthapadmanabhan, K. P.; Leung, P.S.; Goddard, E. D. Colloid surf. 1985, 13, 63-72.

Andreetti, G. D.; Herbich, J.; Lipkowski, J. Cryst. Struct. Commun. 1981, 10, 1233-1238.

Andrews, A. W.; Thibault, L. H.; Linjinsky, W. Mutat. Res. $1978,51,311-318$.

Apfel, M. A.; Finkelmann, H.; Janini, G. M.; Laub, R. J.; Luhmann, B.-H.; Price, A.; Roberts, W. L.; Shaw, T. J.; Smith, C. A. Anal. Chem. 1985, 57, 651-658.

Aroney, R. J.; LeFevre, J. W.; Saxby, J. D. J. Chem. Soc. $1965,571-575$.

Ashwood-Smith, M. J.; Mitchell, R. H.; Kennedy, A. Mutat. Res. 1978, 57, 123-125.

Bachmann, W. E.; Edgerton, R. O. J. Am. Chem. Soc. 1940, 62, $2550-2553$. 
Bachmann, W. E.; Struve, w. S. J. Org. Chem. 1939, 4, 456463.

Bayliss, N. S. J. Chem. Phys. 1950, 18, 292.

Bekarek, V.; Bekarek, S.; Pavlet, F. Chem. Pap. 1988, 42, 197-203.

Bekarek, V.; Bekarek, S.; Pavlat, F. Z. Phys. Chem., Leipzig $1988,269,1147$.

Bertsch, w. In Applications of Glass Capillary Gas Chromatography; Jennings, W. G., Ed.; Marcel Dekker: New York, 1981; p 71.

Birks, J. B. Photophysics of Aromatic Molecules; WileyIntersceince: New York, 1970; pp 439-441.

Bloomfield, D. G.; Upton, C.; Vipond, H. J. J. Chem. Soc., Perkin Trans. I 1986, 857-860.

Blümer, G.-P.; Zander, M. Fresenuis Z. Anal. Chem. 1979, $296,409-410$.

Blümer, G.-P.; Zander, M. Fresenius $Z$. Anal. Chem. 1979, $296,1433$.

Bortolus, P.; Galiazzo, G.; Gennari, G. Anal. Chim. Acta $1990,234,353-358$.

Bowden, B. F.; Picker, K.; Ritchie, E.; Taylor, W. E. Aust. J. Chem. 1975, 28, 2681-2701.

Bowen, E. J.; Brocklehurst, B. J. Chem. Soc. 1954, 38753878 .

Bowen, E. J.; Brocklehurst, B. J. Chem. Soc. 1955, 43204331 .

Bowen, E. J.; Rohatgi, K. K. Disc. Faraday Soc. 1953, 14, 146-150.

Brady, J. E.; Carr, P. W. J. Phys. Chem. 1985, 89, 18131822 .

Braterman, P. S.; Song, J.-I. J. Org. Chem. 1991, 56, 46784682 .

Breymann, U.; Dreeskamp, H.; Koch, E.; Zander, M. Chem. Phys. Lett. 1978, 59, 68-70. 
Brown, S. D. Anal. Chim Acta 1986, 181, 1-29.

Bruggeman, W. A.; van der steen, J.; Hutzinger, O. J. Chromatogr. 1982, 238, 335-346.

Buisson, J.-P.; Demerseman, P. J. Heter. Chem. 1990, 27, 2213-2214.

Buisson, J.-P.; Kotzyba, J.; Lievremont, P.-P.; Demerseman, P.; Platzer, N.; Bideau, J.-P.; Cotrait, M. J. Heter. Chem. 1993, 30,739-747.

Burrell, G. J.; Hurtubise, R. J. Anal . Chem. 1987, 59, 965970 .

Burrell, G. J.; Hurtubise, R. J. Anal. Chem. 1988, 60, 564568 .

Burrell, G. J.; Hurtubise, R. J. Anal. Chem. 1988, 60, 2178-2182.

Butler, H. T.; Coddens, M. E.; Khatib, S.; Poole, C. F. J. Chromatogr. Sci. 1985, 23, 200-207.

Bystryak, S. M.; Liktenshten, G. I.; Kotel'niko, A. I. Khim. Fiz, 1992, 11, 374 .

Carr, J. W. ; Harris, J. M. Anal. Chem. 1986, 58, 626-631.

Carr, J. W.; Harris, J. M. Anal. Chem. 1987, 59, 2546-2550.

Carroll, F. A.; Whitten, D. A. J. Phys. Chem. 1976, 80, 2046-2047.

Castellan, A.; Kessab, L.; Grelier, S. ; Nourmamode, A.; Cotrait, M.; Marsau, P. J. Chem. Soc. Perkin Trans. 2 1993, 953-962.

Cecil, T. L. Ph.D. Dissertation, Virginia Commonwealth University, 1990, p 12-15.

Cecil, T. L.; Rutan, S. C. Anal. Chem. 1990, 62, 1998-2004.

Chen, S. H.; Frank, C. W. Langmuir 1991, 7, 1719-1726.

Cheng, P.-C. M.S. Thesis, University of Nevada, Reno, 1992.

Chin, Y.-P.; Gschwend, P. M. Environ. Sci. Technol. 1993, $27,759-760$.

Cho, B. P.; Harvey, R. G. J. Org. Chem. 1987, 52, 5668-5678. 
Clar, E. Ber. Dtsch. Chem. Ges. 1932, 65, 846.

Clar, E.; Fell, G. S.; Ironside, C. T.; Balsillie, A. Tetrahedron, $1960,10,26-36$.

Clar, E.; Ironside, C. T.; Zander, M. Tetrahedron 1966, 22, $3527-3533$.

Clar, E.; Kelly, W.; Laird, R. M. Mh. Chem. 1956, 80, 391.

Clar, E.; McAndrew, B. A.; Zander M., Tetrahedron 1967, 23, 985-993.

Clar, E.; Zander, M. J. Chem Soc. 1958, 1861-1865.

Clar, E.; Zander, M. Tetrahedron 1963, 19, 521-526.

Cole, R. H. J. Chem. Phys. 1941, 9, 251.

Colmsjo, A. L. Anal. Chim. Acta 1987, 197, 65-70.

Colmsjo, A. L. Anal. Chim. Acta 1987, 197, 71-75.

Colmsjo, A. L.; Ostman, C. E. Anal. Chim. Acta 1988, 208, 183-193.

Colmsjo, A. L.; Stenberg, U. Anal. Chem. 1979, 51, 145-150.

Colmsjo, A. L.; Stenberg, U. Chem. Scr. 1970, 11, 220.

Colmsjo, A. L.; Wise, S. A. Anal. Chim. Acta 1986, 187, 129137 .

Cundall, R. B.; Grant, D. J. W. ; Shulman, N. H. J. Chem. Soc., Faraday Trans. 2 1982, 78, 737-750.

D'Aprano, A.; Donato, D. I.; Agrigento, V. J. Solution Chem. $1981,10,673-680$.

Daudel, P.; Daudel, R. Chemical Carcinogenesis and Molecular Biology; Wiley-Interscience: New York; 1966.

Davidson, D. W. Can. J. Chem. 1961, 39, 2139.

Dean, J. A. Lange's Handbook of Chemistry, 13th ed.; McGrawHill: New York, 1985.

Dederen, J. C.; Coosemans, L.; De Schryver, F. C. ; Van Dormael, A. Photochem. Photobiol. 1979, 30, 443-447. 
De Rango, C.; Tsourcaris, G.; Declerq, J. P.; Germain, G.; Petzeys, J. P. Cryst. Struct. Comm. 1973, 2, 189-192.

Dias, J. R. Handbook of Polycyclic Aromatic Hydorcarbons; Elsevier: Amsterdam, 1987; Part A.

Dias, J. R. J. Chem. Inf. Comput. Sci. 1992, 32, 2-11.

Di Raddo, P.; Hahn, J.-T.; Harvey, R. G. Polycyclic Aromat. Cmpds. 1991, 2, 1-11.

Distefano, G.; Galasso, V.; Irgolic, K. J.; Pappalardo, G. C. J. Chem. Soc., Perkin Trans. 1983, 2, 1109-1112.

Dong, D. C.; Winnik, M. A. Can. J. Chem. 1984, 62, 25602565 .

Dresskamp, H.; Koch, E.; Zander, M. Z. Naturforsch. 1975, $30 A, 1311-1314$.

D'Silva, A. P.; Fassel, V. A. Anal. Chem. 1984, 56, 985A$985 \mathrm{~A}$ and references therein.

Dyker, G. Tetrahedron Lett. 1991, 32 7241-7242.

Dziewoński, K.; Leyko, Z. Ber. Dtsch. Chem. Ges. 1914, 47, 1679-1690.

Eckstein, O. Ber. Dtsch. Chem. Ges. 1905, 38, 3660-3663.

Encinas, M. V.; Rubio, M. A.; Lissi, E. A. J. Photochem. $1982,18,137-150$.

Encinas, M. V.; Rubio, M. A.; Lissi, E. A. Photochem. Photobiol. 1983, 37, 125-130.

Fetzer, J. C. In Chemical Analysis of Polycyclic Aromatic Compounds; Vo-Dinh, T., Ed.; John Wiley and Sons: New York, 1989 ; Chapter 3.

Fetzer, J. C.; Biggs, W. R. Chromatographia 1989, 27, 118122 .

Fetzer, J. C.; Biggs, W. R. J. Chromatogr. 1984, 295, 161169.

Fetzer, J. C.; Biggs, W. R. J. Chromatogr. 1985, 322, 275286.

Fetzer, J. C.; Biggs, W. R. J. Chromatogr. 1987, 386, 87101 . 
Fetzer, J. C.; Biggs, W. R. J. Chromatogr. 1987, 392, 75-82.

Fetzer, J. C.; Zander, M. Z. Naturforsch. 1990, 45A, 727729.

Fetzer, J. C.; Zander, M. Z. Naturforsch. 1990, 45A, 814816.

Fowlie, P. J. A.; Bulman, T. L. Anal. Chem. 1986, 58, 721723 .

Fox, M. A. In Topics in Organic Electrochemistry; Fry, A. J.; Britton, W. E., Eds.; Plenum Press: New York, 1986; Chapter 4 .

Gampp, H.; Maeder, M. ; Meyer, C. J.; Zuberbuhler, A. D. Talanta 1985, 32, 1133-1139.

Garrigues, P. ; Ewald, M. Int. J. Environ. Anal. Chem. 1985, $21,185-197$.

Goltz, M.; Murata, I. Bul1. Chem. Soc. Jpn. 1988, 61, 37673769 .

Gore, P. H. J. Org. Chem. 1957, 22, 135-138.

Grabowski, Z. R.; Dobkowski, J. Pure Appl . Chem. 1983, 55, 245-252.

Grabowski, Z. R. ; Rotiewicz, R.; Siemiarczuk, A.; Cowley, D. J.; Baumann, W. Nouv. J. Chim. 1979, 3, 443.

Grimmer, G.; Jacobs, J.; Naujack, K.-W. Fresenius $Z$. Anal. Chem. 1981, 306, 347-355.

Grove, E. L.; Walden, G. E. J. Chem. Eng. Data 1965, 10, 98 .

Grützmacher; H.-F.; Husemann, w. Tetrahedron Lett. 1985, 26, 2431-2434.

Haky, J. E.; Muschik, G. M. J. Chromatogr. 1981, 214, 161170 .

Hall, D. M.; Ladbury, J. E.; Lesslie, M. S.; Turner, E. E. J. Chem. Soc. 1956, 3475-3482.

Hanson, A. W. Acta Crystallogr. 1965, 18, 599-604.

Hartner, K. C.; Carr, J. W.; Harris, J. M. Appl . Spectrosc. $1989,43,81-87$. 
Harvey, R. G.; Pataki, J.; Cortez, C.; Di Raddo, P.; Yang, C. J. Org. Chem. 1991, 56, 1210-1217.

Harvey, R.G.; Yang, C.; Lee, H. manuscript in preparation.

Hawthorne, S. B.; Langenfeld, J. J.; Miller, D. J.; Burford, M. D. Anal. Chem. 1992, 64, 1614-1622.

Hawthorne, S. B.; Miller, D. J. J. Chromatogr. 1987, 403, $63-76$.

Herbstein, F. H. Acta Crystallogr. 1979, 35B, 1661-1670.

Herbstein, F. H.; Schmidt, G. M. J. J. Chem. Soc. 1954, 3302-3313.

Herndon, w. C. private communication.

Herndon, W. C.; Nowak, P. C.; Conner, D. A.; Lin, P. J. Am. Chem. Soc. 1992, 114, 41-47.

Hirshfeld, F. L. J. Chem. Soc. 1963, 2126-2135.

Hirshfeld, F. L.; Sandler, S.; Schmidt, G. M. J. J. Chem. Soc. 1963, 2108-2125.

Hite, P.; Thomas, J. K. J. Phys. Chem. 1986, 90, 5795-5799.

Ho, S. S. J.; Butler, H. T.; Poole, C. F. J. Chromatogr. $1983,281,330-339$.

Holland, J. F.; Teets, R. E.; Kelly, P. M.; Timnick, A. Anal. Chem. 1977, 49, 706-710.

Hosoya, S. Acta Crystallogr. 1963, 310-312.

Huiszoon, C.; Van de Waal, B. W.; Van Egmond, A. B.;

Harkema, S. Acta Crystallogr. 1972, 28B, 3415-3419.

Huiszoon, C.; Van Hummel, G. J.; Van den Ham, D. M. W. Acta Crystallogr. 1977, 33B, 1867-1870.

Hünig, S.; Gross, J.; Lier, E. F.; Quast, H. Liebigs Ann. Chem. 1973, 339-358.

Hurtubise, R. J.; Phillip, J. D.; Skar, G. T. Anal Chim. Acta 1978, 101, 333-338.

Ivanoff, M. I. Bull. Soc. Chim. Belg. 1962, 71, 759-763. 
Jaeda, M. I.; Parfitt, R. T.; Upton, C. Chung-Hua Yao Hseuh Tsa Chih, 1990, 42, 403-410.

Janini, G. M.; Johnston, K.; Zielinski, W. L., Jr. Anal. Chem. 1975, 47, 670-674.

Janini, G. M.; Muschik, G. M.; Zielinski, W. L., Jr. Anal. chem. 1976, 48, 809-813.

Jannelli, L.; Pansini, M. Can. J. Chem. 1988, 66, 662-665.

Jans, A. W. H.; Tintel, C.; Cornelisse, J.; Lugtenburg, J. Magn. Reson. Chem. 1986, 24, 101.

Jarzeba, W.; Kahlow, M; Barbara, P. F. J. Imaging Sci. 1989, $33,53-57$.

Johnson, J. F.; Cole, R. H. J. Am. Chem. Soc. 1951, 73, $4536-4540$.

Junk, G. A. ; Richard, J. J. Anal Chem. 1986, 58, 962-965.

Kabouchi, B.; Benali, B.; Fadouach, M.; Kadiri, A.; CazeauDubroca, C.; Nouchi, G. Spectrochim. Acta 1992, 48A, 1015-1020.

Kalman, R. E. J. Basic Eng. 1964, 82, 34 .

Kalyanasundaram, K.; Thomas, J. K. J.Am. Chem. SoC. 1977, $99,2039-2044$.

Kalyanasundaram, K.; Thomas, J. K. J. Phys. Chem. 1977, 81, 2176-2180.

Katz, M.; Chau, C. Environ. Sci. Technol. 1980, 14, 838-843.

Kavanagh, R. J.; Iu, K.-K.; Thomas, J. K. Langmuir 1992, 8, 3008-3013.

Kawski, A. Acta Phys. Pol. 1966, 29, 507.

Kehrmann, F.; Mermond, C. Helv. Chim. Acta 1927, 10, 62-66.

Khundkar, L. R. ; Zewail, A. H. J. Chem. Phys. 1986, 84, $1302-1311$.

Klopman, G.; Frierson, M. R.; Rosenkranz, H. S. Mutat. Res. $1990,228,1-50$.

Kong, R. C.; Lee, M. L.; Tominaga, Y.; Pratrap, R.; Iwao, M.; Castle, R. N. Anal. Chem. 1982, 54, 1802-1806. 
Konieczny, M. ; Harvey, R. G. J. Org. Chem. 1979, 44, 21582160 .

Kraft, J.; Hartung, A.; Lies, K.-H.; Schulze, S. J. J. High Resolut. Chromatogr. Chromatogr. Commun. 1982, 5, 489494 .

Kruber, O. Chem. Ber. 1949, 82, 199-201.

Kusumoto, Y. Chem. Phys. Lett. 1987, 136, 535-538.

Laarhoven, W. H.; Prinsen, W. J. C. Top. Curr. Chem. 1984, $125,63-130$ and references therein.

Laitinen H. A.; Harris, W. E. Chemical Analysis; McGrawHill: New York, 1960; pp 84-88.

Lakowicz, J. R. Principles of Fluorescence Spectroscopy; Plenum: New York, 1983.

Lang, K. F.; Zander, M. Chem. Ber. 1964, 97, 218.

Langkilde, F. W.; Thulstrup, E. W.; Michl, J. J. Chem. Phys. $1983,78,3372-3381$.

Leader, G. R. J. Am. Chem. Soc. 1951, 73, 856-857.

Lee, H.; Harvey, R. G. J. Org. Chem. 1990, 55, 3787-3791.

Lee, M. L.; Novotny, M. V.; Bartle, K. D. Analytical Chemistry of Polycyclic Aromatic Compounds; Academic: New York, 1981.

Lee, M. L.; Yang, F. J.; Bartle, K. D. Open Tubular Column Gas Chromatography: Theory and Practice; John wiley and Sons: New York, 1984; pp 249, 312.

Levitz, P.; van Damme, H.; Keravis, D. J. Phys. Chem. 1984, $88,2228-2235$.

Lianos, P.; Georghiu, S. Photochem. Photobiol. 1979, 29, 843-846.

Lianos, P.; Georghiu, S. Photochem. Photobiol. 1979, 30, $355-362$.

Lianos, P.; Mukhopadhyay, A. K.; Georghiu, S. Photochem. Photobiol. 1980, 32, 415-419.

Lijinsky, W.; Garcia, H.; Saffiotti, U. J. Nat. Cancer Inst. $1970,44,641-649$. 
Lijinsky, W.; Garcia, H.; Terracini, B.; Saffiotti, U. J. Nat. Cancer Inst. 1965, 34, 1-6.

Lijinsky, w.; Saffiotti, U. Ann. It. Derm. Clin. Sper. 1965, $19,34-44$.

Lin, R.-Y.; Dannhauser, W. J. Phys. Chem. 1963, 67, 18051810 .

Linde, D. R. Handbook of Chemistry and Physics, 71st ed.; CRC Press: Boca Raton, 1990.

Lohberger, c. Thesis, Technical University of Clausthal, 1983 .

Longhi, P.; Mussini T.; Rondinini, S. Anal. Chem. 1986, 58, 2290-2292.

Lueck, H.; Windsor, M. W.; Rettig, W. J. Phys. Chem. 1990, $94,4550-4559$.

Lumbroso, H. ; Montaudo, G. BuIl. Chem. Soc. Fr. 1964, 21192124 .

Lynton, H.; Cox, E. G. J. Chem. Soc. 1956, 4886-4895.

McIntosh, A. O.; Robertson, J. M.; Vand, V. J. Chem. Soc. 1954, 1661-1668.

Maeder, M. Anal. Chem. 1987, 59, 527-530.

Mallaris, A. Int. Reviews in Phys. Chem. 1988, 7, 95-121.

March, J. Advanced Organic Chemistry: Reactions, Mechanisms and Structure; McGraw-Hill: New York, 1968; pp 46-48.

Marcheselli, L.; Pistoni, G.; Tagliazucchi, M.; Tassi, L.; Tosi, G. J. Chem. Eng. Data 1993, 38, 204.

Markgraf, J. H.; Katt, R. J. J. Org. Chem. 1972, 37, 717718 .

Markides, K. E.; Nishioka, M.; Tarbet, B. J.; Bradshaw, J. S.; Lee, M. L. Anal. Chem. 1985, 57, 1296-1299.

Mason, G; Gustafsson, J.-A. ; Westerholm, R. N.; Li, H. Environ. Sci, Technol. 1992, 26, 1635-1638.

Mataga, N; Kaifu, Y.; Koizumi, M Bull. Chem. Soc. Jpn. $1956,29,465$. 
Matsui, K.; Nakazawa, T. Bull. Chem. Soc. Jpn. 1990, 63, 1116.

Matsui, K.; Usuki, N. Bull. Chem. Soc. Jpn. 1990, 63, 35163520 .

Matsushita, H.; Esumi, Y.; Suzuki, A.; Handa, T. Bunseki Kagaku 1972, 21, 1471-1478.

Mazundar, S.; Manoharan, R.; Dogra, S. J. Photochem. Photobiol. 1989, 46A, 301-314.

Merch-Sundermann, V.; Mochayedi, S.; Kevekordes, S. Mutat. Res. 1992, 278, 1-9.

Meurer, K. P.; Vogtle, F. Top. Curr. Chem. 1985, 127, 1-76 and references therein.

Michl, J.; Thulstrup, E. W. Spectroscopy with Polarized Light; VCH: New York, 1986; Chapter 8.

Migue1, A. H. Int. J. Environ. Chem. 1982, 12, 17-25.

Millican, D. W.; McGown, L. B. Anal. Chem. 1989, 61, 580583 .

Millican, D. W.; McGown, L. B. Anal Chem. 1990, 62, 22422247 .

Millican, D. W.; McGown, L. B. Appl. Spectrosc. 1992, 46, 28-33.

Mitchell, R. H. Adv. Theor. Interesting Mol. 1989, 1, 135199 .

Mitchell, R. H.; Boekelheide, V. J. Am. Chem. Soc. 1974, 96, 1547-1557.

Mitchell, R. H. ; Carruthers, R. J.; Mazuch, L.; Dingle, T. W. J. Am. Chem. SOC. 1982, 104, 2544-2551.

Mitchell, R. H.; Chaudhary, M.; Williams, R. V.; Fyles, R.; Gibson, J; Ashwood-Smith, M. J.; Fry, A. J. Can. J. Chem. 1992, 70, 1015-1022.

Mitchell, R. H. ; Zhou, P. Tetrahedron Lett. 1990, 31, 52775280 .

Mulder, P. P. J.; Boere, B. B.; Baart, A.; Cornelisse, J.; Lugtenburg, J. Recl. Trav. Chim. Pays-Bas 1993, 112, 22-32. 
Mulder, P. P. J.; Olde Boerrigter, J.; Boere, B. B.; Zuilhof, H.; Erkelens, C.; Cornelisse, J.; Lugtenburg, J. Rec1. Trav. Chim. Pays-Bas 1993, 112, 287-302.

Musset, E.; Nikuradse, A.; Ulbrich, R. Z. Angew. Phys. $1956,8,8$.

Nagoa, M.; Sugimura, T.; Yang, S. K.; Gelboin, H. V. Mutat. Res. 1978, 58, 361-365.

Nakajima, A. Bu11. Chem. Soc. Jpn. 1971, 44, 3272-3277.

Nakashima, K.; Tanaka, I. Langmuir 1993, 9, 90-95.

Nakasuji, K.; Kubota, H.; Kotani, T.; Murata, I.; Saito, G.; Enoki, T.; Imaeda, K.; Inokuchi, H.; Honda, M.;

Katayama, C.; Tanaka, J. J. Amer. Chem. Soc. 1986, 108, 3460-3466.

Nakasuji, K.; Oda, A.; Murata, I.; Imaeda, K.; Inokuchi, H.; J. Chem. Soc., Chem. Commun. 1989, 1553-1554.

Naumann, C.; Langhals, H. Chem. Ber. 1990, 123, 1881-1884.

Nelson, G.; Patonay, G.; Warner, I. M. Anal. Chem. 1988, 60, 274-279.

Nelson, G.; Patonay, G.; Warner, I. M. J. Incl. Phenom. $1988,6,277-289$.

Nelson, G.; Warner, I. M. J. Phys. Chem. 1990, 94, 576-581.

Nobel, D. Anal. Chem., 1993, 65, 353A-355A.

Oberkobusch, R. Chem. Ber. 1953, 86, 975-979.

Oster, G. K. Acta Phys. Polon. 1964, 26, 435.

Pachtner, I. J.; Kloetzel, M. C. J. Amer. Chem. Soc. 1951, $73,4958-4960$.

Pankasem, S.; Thomas, J. K. J. Phys. Chem. 1991, 95, 73857393 .

Papanastasiou, G. E.; Ziogas, I. I. J. Chem. Eng. Data $1991,36,46-51$.

Parker, C. A. Photoluminscence of Solutions: With Application to Photocemistry and Analytical Chemistry; Elesevier: Amsterdam, 1968; Chapter 4 . 
Parker, C. A.; Barnes, W. J. Analyst 1957, 82, 606-618.

Partridge, M. W.; Slorach, S. A.; Vipond, H. J. J. Chem. SoC. 1964, 3670-3673.

Partridge, M. W.; Vipond, H. J. J. Chem. Soc. 1962, 632-635.

Peaden, P. A.; Lee, M. L.; Hirata, Y.; Novotny, M. V. Anal. Chem. 1980, 52, 2268-2271.

Peck, D. W. U.S. Patent 3095426, 1963; Chem. Abstr. 1963, $59,12761 f$.

Phillips, R. E.; Drub, G. H.; Hunt, J. A. J. Org. Chem. $1972,37,2030-2033$.

Poole, C. F.; Butler, H. T.; Coddens, M. E.; Khatib, S.; Vandervennet, R. J. Chromatogr. 1984, 302, 149-158.

Poole, S. K.; Dean, T. A.; Poole, C. F. J. Chromatogr. 1987, $400,323-341$.

Pullman, A. In The Theory of Chemical Carcinogenesis and the Problem of Hydrocarbon-Protein Interactions. Biopolymers Symp $11964,47$.

Pullman, A.; Pullman, B. Cancèrisation par les Substances Chimiques et Structure Molèculaire; Masson et Cie: Paris; 1955.

Pummerer, R.; Prell, E.; Rieche, A. Ber. Dtsch. Chem. Ges. 1926, 59, 2159-2161.

Ramis Ramos, G.; Garcia Alvarez-Coque, M. C.; Bertod, A.; Winefordner, J. D. Anal. Chim. Acta 1988, 208, 1-19.

Rein, J.; Cork, C. M.; Furton, K. G. J. Chromatogr. 1991, $545,149-160$.

Renge, I. Chem. Phys. 1992, 167, 173-184.

Rettig, w. Agnew. Chem., Int. Ed. Engl. 1986, 25, 971-988 and references therein.

Rettig, w. In Modern Models of Bonding and Delocalization; Liebman, J.; Greenberg, A., Eds.; VCH: New York, 1988; Chapter 5 .

Rettig, W.; Zander, M. Ber. Bunsen-Ges. Phys. Chem. 1983, 87, 1143-1149. 
Rice, J. E.; Shih, H. C.; Hussain, N.; La Voie, E. J. J. org. Chem. 1987, 52, 849-855.

Richard, A. M.; Woo, Y. Mutat. Res. 1990, 242, 285-303.

Riddick, J. A.; Bunger, W. B. Organic Solvents: Physical Properties and Methods of Purification; 3rd ed.; John Wiley \& Sons: New York, 1970.

Ritzoulis, G. Can. J. Chem. 1989, 67, 1105-1108.

Roberts, P. J.; Ferguson, G. Acta Crystallogr. 1977, 33B, 1244-1247.

Rodin, O. G.; Traven, V. F.; Redchenko, V. V.; Eismont, M. Y.; Stepanov, B. I. Zh. Obshch. Khim. 1983, 53, 25372543 .

Rowe, I.; Post, B. Acta Crystallogr. 1958, 372-374.

Rutan, S. C. J. Chemom. 1987, 1, 7-18.

Rutan, S. C.; Gerow, D. D.; Hartmann, G. Intell. Lab. Sys. $1988,3,61$.

Sander, L. C.; Parris, R. M.; Wise, S. A.; Garrigues, P. Anal. Chem. 1991, 63, 2589-2597.

Sander, L. C.; Wise, S. A. Anal. Chem. 1987, 59, 2309-2313.

Sander, L. C.; Wise, S. A. Anal. Chem. 1989, 61, 1749-1754.

Sander L. C.; Wise, S. A. J. High Resolut. Chromatogr. Chromatogr. Commun. 1988, 11, 383-387.

Sangaiah, R.; Gold, A.; Toney, G. E. J. Org. Chem. 1983, 48, 1632-1638.

Schmid, R. J. Solution Chem. 1983, 12, 135-152.

Schneider, F.; Lippert, E. Ber. Bunsen-Ges. Phys. Chem. $1968,72,1155$.

Schönberg, A.; Brosowski, K.-H. Chem. Ber. 1960, 93, 21492151 .

Schönberg, A.; Brosowski, K.-H.; Singer, E. Chem. Ber. 1962, 95, 1910-1916.

Scott, L. T.; Hashemi, M. M. ; Bratcher, M. S. J. Am. Chem. Soc. 1992, 114, 1920-1921. 
Scott, L. T.; Hasehemi, M. M. ; Meyer, D. T.; Warren, H. B. J. Am. Chem. Soc. 1991, 113, 7082-7084.

Seifert, B. J. Chromatogr. 1977, 131, 417-421.

Selvarajan, N.; Panicker, M. M.; Vaidyanathan, S.; Ramakrishnan, V. Indian J. Chem. 1979, 18A, 23-26.

Shpol'skii, E. V. Soviet Phys. Usp. 1959, 2, 378 .

Shpol'skii, E. V.; Il'ina, A.A.; Klimova, L.A. DokI. Akad. Nauk SSSR 1952, 87, 935-938.

Shpol'skii, E. V.; Klimova, L.A. Bull. Acad. Sci. SSSR Ser. Fiz. 1954, 18, 357 .

Shukla, D.; Wan, P. J. Am. Chem. Soc. 1993, 115, 2990-2991.

Skoog, D. A; West, D. M. Principles of Instrumental Analysis; Saunders College: Philadelphia, 1980; second ed., Chapters 6 and 9 .

Skoog, D. A; West, D. M. Principles of Instrumental Analysis; Saunders College: Philadelphia, 1980; sixth ed. $;$ pp $430-432$.

Stahlberg, J.; Almergen, M. Anal. Chem. 1985, 57, 817-821.

Street, K. W., Jr. J. Liq. Chromatogr. 1987, 10, 655-662.

Street, K. W., Jr.; Acree, W. E., Jr. Analyst 1986, 111 , 1197-1201.

Street, K. W., Jr.; Acree, W. E., Jr. Appl. Spectrosc. 1988, $42,1313-1318$.

Street, K. W., Jr.; Acree, W. E., Jr. J. Liq. Chromatogr. $1986,9,2799-2808$.

Street, K. W., Jr.; Acree, W. E., Jr.; Fetzer, J. C.; Shetty, P. H.; Poole, C. F. Appl. Spectrosc. 1989, 43, 1149-1153.

Street, K. W., Jr.; Acree, W. E., Jr.; Poole, C. F.; Shetty, P. H. Analyst 1988, 113, 1869-1871.

Sullivan, R. F.; Boduszynski, M. M. ; Fetzer, J. C. Energy \& Fuels 1989, 3, 603-612.

Suppan, P. J. Chem. SoC., Part A 1968, 3125-3133. 
Suppan, P. J. Photochem. Photobiol., Part A 1990, 50, 293330 .

Tanga, M. J.; Almquist, R. G.; Smith, T. H.; Wu, H. Y.; Reist, E. J. J. Heterocyclic Chem. 1985, 22, 1597-1598.

Tanga, M. J.; Bupp, J. E. J. Org. Chem. 1993, 58, 4173-4174.

Tanga, M. J.; Davis, R. F.; Reist, E. J. J. Heterocyclic Chem. 1987, 24, 39-41.

Tanga, M. J.; Reist, E. J. J. Heterocyclic Chem. 1986, 23, $747-752$.

Tanga, M. J.; Reist, E. J. J. Heterocyclic Chem. 1991, 28, 29-32.

Tanga, M. J.; Reist, E. J. J. Org. Chem. 1982, 47, 13651366 .

Thiebaut, J.-M.; Rivail, J.-L.; Greffe, J.-L. J. Chem. Soc., Faraday Trans. II 1976, 72, 2024-2034.

Thompson, R. B.; Shaw McBee, S. E. Langmuir 1988, 4, 106110 .

Tokita, S.; Hiruta, K.; Ishikawa, S.; Kitahara, K.; Nishi, H. Synthesis 1982, 854-855.

Tokita, S.; Hiruta, K.; Kitahara, K.; Nishi, H. BulI. Chem. Soc. Jpn. 1982, 3933-3934.

Tokita, S.; Hiruta, K.; Kitahara, K.; Nishi, H. Synthesis 1982, 299-231.

Tokita, S.; Hiruta, K.; Yaginuma, Y.; Ishikawa, S.; Nishi, H. Synthesis 1983, 270-271.

Tong, T. H.; Wong, H. N. C. Synth. Commun. 1992, 22, 17731782 .

TRC Thermodynamic Tables: Hydrocarbons; Marsh, K. N., Ed.; Texas A\& M University: College station.

TRC Thermodynamic Tables: Non-Hydrocarbons; Marsh, K. N., Ed.; Texas A \& M University: College station.

Tucker, S. A., unplublished results.

Tucker, S. A.; Acree, W. E., Jr. J. Chem. Educ. in press. 
Tucker, S. A.; Acree, W. E., Jr.; Street, K. W., Jr.; Fetzer, J. C. Appl. Spectrosc. 1989, 43, 162-164.

Tucker, S. A.; Amszi, V. L.; Acree, W. E., Jr. J. Chem. Educ. 1992, 69, A8, A11, and A12.

Tucker, S. A.; Cretella, L. E.; Waris, R.; street, K. W., Jr.; Acree, W. E., Jr.; Fetzer, J. C. Appl. Spectrosc. $1990,44,269-273$.

Turro, N. J.; Baretz, B. H.; Kuo.; P.-L. Macromolecules $1984,17,1321-1324$.

Turro, N. J.; Kuo, P.-L. J. Phys. Chem. 1986, 90, 837-841.

Turro, N. J.; Okubo, T. J. Am. Chem. SoC. 1981, 103, 72247228 .

Turro, N. J.; Okubo, T. J. Phys. Chem. 1982, 86, 159-161.

Turro, N. J.; Pierola, I. F. Macromolecules 1983, 16, 906910 .

Upton, C. J. Chem. Soc., Perkin Trans. I 1986, 1225-1229.

van Haeringen, C. J. Ph.D. Thesis, Leiden University, 1993.

van Haeringen, C. J.; Aten, N. F.; Cornelisse, J.; Lugtenburg, J. Recl. Trav. Chim. Pays-Bas 1992, 111, 335 .

van Haeringen, C. J.; Cornelisse, J.; Lugtenburg, J. unpublished results.

Vaughn, w. E.; Roeder, S. B.; Provder, T. J. Chem. Phys. $1963,39,701$.

Visser, R. J.; Weisenborn, P. C. M.; van Kan, P. J. M.; Huizer, B. H.; Varma, C. A. G. O.; Warman, J. M.; DeHaas, M. P. J. Chem. Soc., Faraday Trans. 1985, 81, $689-704$.

Vo-Dinh, T. In Modern Fluorescence Spectroscopy; Wehry, E. L., Ed.; Plenum: New York, 1981; Vol. 4, pp 167-192.

Vo-Dinh, T.; Gammage, R. B.; Hawthrone, A. R.; Thronegate, J. H. Environ. Sci. Technol. 1978, 12, 1297-1302.

Vo-Dinh, T.; Martinex, P. R. Anal . Chim. Acta 1981, 125, 1319 . 
Von Lippert, E. Z. Electrochem. 1957, 61, 962.

Waris, R.; Acree, W. E., Jr.; Street, K. W., Jr. Analyst $1988,113,1465-1467$.

Waris, R.; Rembert, M. A.; Sellers, D. M. ; Acree, W. E., Jr.; Street, K. W., Jr.; Fetzer, J. C. Analyst 1989, $114,195-199$.

Waris, R.; Rembert, M. A.; Sellers, D. M.; Acree, W. E., Jr.; Street, K. W., Jr.; Poole, C. F.; Shetty, P. H.; Fetzer, J. C. Appl. Spectrosc. 1988, 42, 1525-1531.

Waris, R.; Street, K. W., Jr.; Acree, W. E., Jr.; Fetzer, J. C. Appl. Spectrosc. 1989, 43, 845-850.

West, W. Chemical Application of Spectroscopy; Interscience: New York, 1968; Chapter 4.

Westcott, c. C. $p H$ Measurements; Academic Press: New York, $1978 ;$ pp 112-123.

Weyand, E. H.; Geddie, N.; Rice, J. E.; Czech, A.; Amin, S.; La Voie, E. J. Carcinogenesis 1988, 9, 1277.

Wiczk, W. M.; Latowski, T. Z. Naturforsch. 1987, 42A, 12901295.

Wiczk, W. M.; Latowski, T. Polish J. Chem. 1990, 64, 373.

Willard, H. H.; Merritt, L. L., Jr.; Dean, J. A.; Settle, F. A., Jr. Instrumental Methods of Analysis, 7th ed.; Wadsworth: Belmont, 1988; Chapter 8 .

Wise, S. A.; Sander, L. C.; Chang, H. K.; Markides, K. E.; Lee, M. L. Chromatographia 1988, 25, 473-479.

Wise, S. A.; Sander, L. C.; Lapouyande, R.; Garrigues, P. J. Chromatogr. 1990, 514, 111-122.

Woodberry, R.; Ransom, S.; Chen, F.-M. Anal. Chem. 1988, 60, $2621-2625$.

Wortmann, R.; Lebus, S.; Elich, K.; Assar, S.; Detzer, N.; Liptay, W. Chem. Phys, Lett. 1992, 198, 220-228.

Wright, B. W.; Frye, S. R.; McMinn, D. G.; Smith, R. D. Anal. Chem. 1987, 59, 640-644.

Wright, B. W.; Wright, C. W.; Gale, R. W. ; Smith, R. D. Anal. Chem. 1987, 59, 38-44. 
Yamasaki, K.; Arita, K.; Kajimoto, o.; Hara, K. Chem. Phys. Lett. 1986, 123, 277-281.

Yang, C.; Yang, D. T. C.; Harvey, R. G. Synlett 1992, 799800 .

Yappert, M. C.; Ingle, J. D. ; Appl. SpectrosC. 1989, 43, $759-767$.

Zana, R.; Lang, J. Colliod Surf. 1990, 48, 153-171.

Zana, R.; Yiv, S.; Strazielle, C.; Lianos, P. J. Colloid Interface Sci. 1981, 80, 208-223.

Zander, M. Chemikerztg. 1988, 112, 142-142.

Zander, M. Z, Naturforsch, 1989, 44A, 1116-1118.

Zander, M.; Breymann, U.; Dreeskamp, H.; Koch, E. Z. Naturforsch. 1977, 32A, 1561-1563.

Zander, M. ; Franke, W. Chem. Ber. 1961, 94, 446-450.

Zander, M.; Franke, w. Chem. Ber. 1973, 106, 2752-2754.

Zander, M.; Kirsch, G. Z. Naturforsch. 1989, 44A, 205-209.

Zielinski, W. L., Jr., Scanlan, R. A.; Miller, M. M. J. Chromatogr. 1981, 209, 87-90.

Zimmerman, H. E. Quantum Mechanics for Organic Chemists; Academic: New York, 1975; pp 145-146. 